\title{
ASSESSING NEAR-FIELD BLACK CARBON VARIABILITY DUE TO WOOD BURNING AND EVALUTING REGRESSION MODELS AND ISC DISPERSION MODELING
}

\author{
A Thesis \\ presented to \\ the Faculty of California Polytechnic State University, \\ San Luis Obispo
}

\author{
In Partial Fulfillment \\ of the Requirements for the Degree \\ Master of Science in Civil and Environmental Engineering
}

by

Stella Hing Tan

August 2011 
(C) 2011

Stella Hing Tan

ALL RIGHTS RESERVED 


\section{COMMITTEE MEMBERSHIP}

TITLE: $\quad$ ASSESSING BLACK CARBON VARIABILITY DUE TO NEARFIELD WOOD BURNING AND EVALUATING REGRESSION MODELS AND ISC DISPERSION MODELING
AUTHOR:
Stella Hing Tan
DATE SUBMITTED:
September 2011
COMMITTEE CHAIR: $\quad$ Tracy Thatcher, Ph.D.
COMMITTEE MEMBER: Beth Chance, Ph.D.
COMMITTEE MEMBER: Yarrow Nelson, Ph.D. 


\begin{abstract}
Assessing black carbon variability due to near-field wood burning and evaluating regression models and ISC dispersion modeling
\end{abstract}

\title{
Stella Hing Tan
}

$\mathrm{PM}_{2.5}$ variability within the neighborhood scale has not been thoroughly studied for wood burning communities. High variability in near-field $\mathrm{PM}_{2.5}$ concentration may lead to harmful public exposure since monitoring does not occur on that scale. This study measures near-field $\mathrm{PM}_{2.5}$ variability by measuring black carbon (BC), a component of $\mathrm{PM}_{2.5}$, in a $1 \mathrm{~km}^{2}$ area located in Cambria, California. BC and meteorological data (when meteorological instruments were available) were measured over thirteen 12-hour intensive operation periods (IOPs) occurring over the winters of 2009 and 2010. Nearfield BC variability was measured to understand the type of exposures found in communities where many homes are burning wood simultaneously within a small area. In addition, relationships between meteorological, geographical, and burning source characteristics and $\mathrm{BC}$ were observed as tools for understanding $\mathrm{BC}$ concentration. The computer air dispersion modeling programs, ISC-PRIME and ISCST3, were also evaluated for applicability to the near field.

$\mathrm{BC}$ concentrations were measured using 1- to 2-minute resolution aethalometers and 12 hour resolution Personal Environmental Monitors (PEMs). On average, over all IOPs and sites, aethalometer and PEM BC averages were very similar, ranging between 200 and $250 \mathrm{ng} / \mathrm{m}^{3}$, or 4 and $5 \mu \mathrm{g} / \mathrm{m}^{3}$ for $\mathrm{PM}_{2.5}$, and standard deviations were often high. Averaging all BC measurements, aethalometer BC standard deviation values were 360 
percent of the average BC concentration and PEM BC standard deviations were 120 percent the average $\mathrm{BC}$ concentration. The average standard deviation detected during each IOP was 190 percent of the average BC concentration for aethalometers and 79 percent of the average $\mathrm{BC}$ concentration for PEMs. The average standard deviation detected at each site was 220 percent of the average $\mathrm{BC}$ concentration for aethalometers and 76 percent of the average $\mathrm{BC}$ concentration for PEMs. The larger standard deviations measured by higher resolution aethalometers demonstrated that low resolution instruments, such as PEMs, are unable to detect high concentrations that may occur.

In addition to examining $\mathrm{BC}$ variability, multiple linear regression analyses were conducted to determine the impact of meteorological variables and geographic and burning source characteristics on $\mathrm{BC}$ concentration and a weighted $\mathrm{BC}$ deviation function (BC standard deviation divided by average BC concentration). Time impacts, humidity, and wind speed, accounted for about 50 percent of variability in aethalometer average BC and BC deviation. However, because all model assumptions were not satisfied, improvements are needed. Regression models based on PEM BC found wind speed and direction to account for about 80 percent of average PEM BC variability and number of burning sources to account for about 30 percent of PEM BC deviation. Although PEM $\mathrm{BC}$ models accounted for a high percentage of $\mathrm{BC}$ variability, few data points were available for the PEM analyses and more IOPs are needed to determine their accuracy.

When evaluating correlations between geographic and burning source characteristics and PEM BC concentrations, specific IOP and PEM sampling location explained almost 70 
percent of variability in $\mathrm{BC}$ concentration, though model residuals suggested model bias. IOP likely explained variation in burning patterns and meteorology over each night while sampling location was likely a proxy for housing density, tree coverage, and/or elevation. Because all regression model assumptions could not be satisfied, the predictors were also observed graphically. Plotting BC concentration versus the number of burning sources suggested that number of burning sources may affect BC concentration in areas of low tree coverage and high housing density and in the case that the level of surrounding vegetation and structures are minimal. More data points will be needed to determine whether or not these relationships are significant.

ISC-PRIME and ISCST3 modeling overall tended to under predict BC concentrations with average modeled-to-measured ratios averaging 0.25 and 0.15 , for ISC-PRIME and ISCST3, respectively. Correction factors of 9.75 and 18.2 for ISC-PRIME and ISCST3, respectively, were determined to bring modeled $\mathrm{BC}$ concentrations closer to unity, but the range of ratios was still high. Both programs were unable to consistently capture BC variability in the area and more investigation will be needed to improve models.

The results of the study indicate high BC variability exists on the near-field scale, but that the variability is not clearly explained by existing regression and air dispersion models. To prevent public exposure to harmful concentrations, more investigation will be needed to determine factors that largely influence pollutant variability on the neighborhood scale.

Keywords: black carbon, $\mathrm{PM}_{2.5}$, near-field, multiple regression, ISCST3, ISC-PRIME 


\section{ACKNOWLEDGMENTS}

I would like to thank Dr. Tracy Thatcher for her encouragement, patience, and enthusiasm. Her knowledge, advice, and feedback were always motivating and immensely helpful for guiding me in the right direction. I feel incredibly grateful to have had her as my thesis advisor. Tracy's wonderful teaching and passion for her work have also been an inspiration for me.

I would also like to thank my committee members, Dr. Yarrow Nelson and Dr. Beth Chance. Yarrow has been a wonderful mentor for me during the course of my studies at Cal Poly and his enthusiasm for learning is infectious to all his students. His thorough and thoughtful input have been invaluable. The statistical analyses conducted in this thesis would not have been possible without Beth. I want to thank her for her patience in responding to all of my e-mails and guidance through all the intricacies that are statistics.

I must also thank Carrie Esaki and Aristotle Ou for their enthusiastic assistance and support through all of the Cambria field work. I do not know what I would have done without your optimistic and hard working attitudes. I have thoroughly enjoyed all the late nights and early mornings in Cambria with you both.

I also want to thank Dr. Hal Cota for his help with this project's dispersion modeling and Courtney Ward and Chris Malejan for their hard work in developing this project. Dr. Cota's encouragement and outreach are always inspiring. Courtney and Chris' work made the field work run many times more smoothly than it would have. I would also like to thank them both for taking the time to answer all of my questions and taking the time to familiarize me with the project.

The Cambria residents were also essential to this project's success. I would like to thank Eugene and Margarita, Jackie, Gary, and the fire fighters of the CDF Fire Station for their friendliness, hospitality, and enthusiasm for the project. I very much enjoyed and looked forward to speaking with them all.

Finally, I want to thank my family and friends for their support. I feel very fortunate to have all of their encouragement, and I want to thank them all for believing the best of me. 


\section{SUPPORT}

This work was supported by Contract 07-308 Assessing Near-Field Exposures from Distributed Residential Wood Smoke Combustion Sources By California Polytechnic University San Luis Obispo and Lawrence Berkeley National Laboratory and under the sponsorship of the California Air Resources Board.

The statements and conclusions in this Report are those of the contractor and not necessarily those of the California Air Resources Board. The mention of commercial products, their source, or their use in connection with material reported herein is not to be construed as actual or implied endorsement of such products. 


\section{TABLE OF CONTENTS}

LIST OF TABLES ...................................................................................................

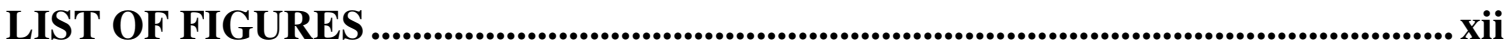

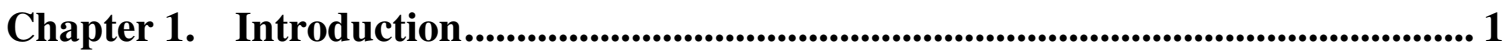

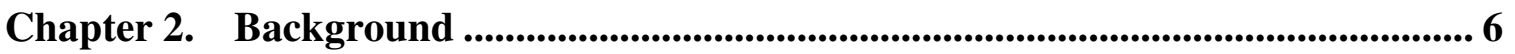

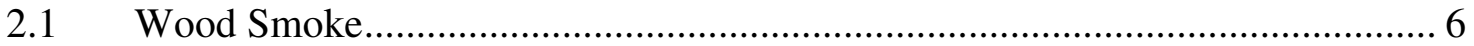

2.1.1 Wood Smoke and $\mathrm{PM}_{2.5}$ Impacts on Health ........................................... 6

2.1.2 Wood Smoke $\mathrm{PM}_{2.5}$ Characterization ..................................................... 9

2.1.3 Wood Smoke Identification ......................................................................... 12

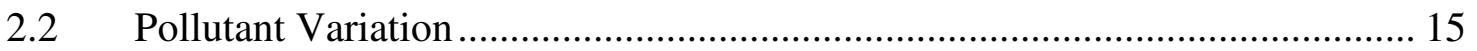

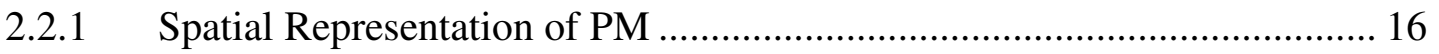

2.2.2 Estimation of Pollutant Variability and Concentrations ............................ 21

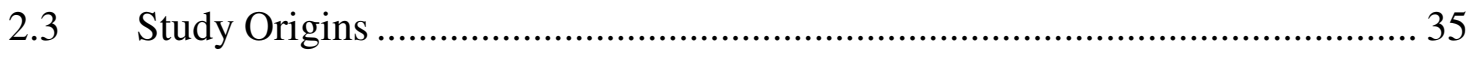

2.3.1 Conclusions from Ward (2009): Spatial Variation and Correlation

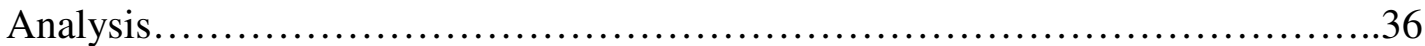

2.3.2 Conclusions from Malejan (2009): Indoor/Outdoor Infiltration and Spectral Dependence of Organic Carbon ............................................................ 38

Chapter 3. Materials and Methods................................................................................ 41

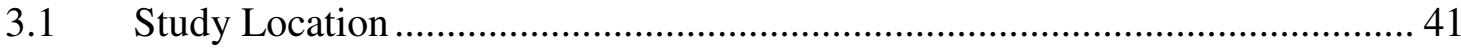

3.1.1 Existing Controls and Regulations..................................................... 46

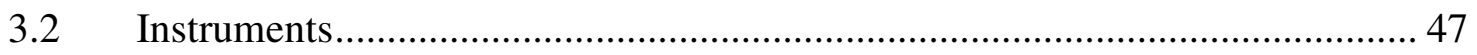

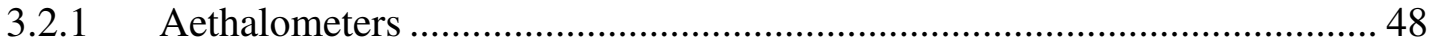

3.2.2 Personal Environmental Monitors ........................................................ 52

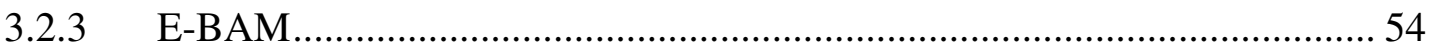

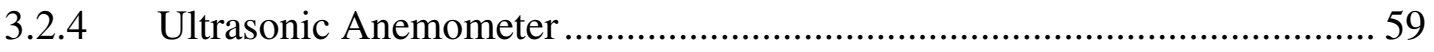

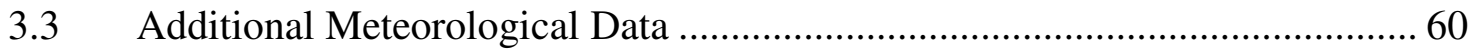

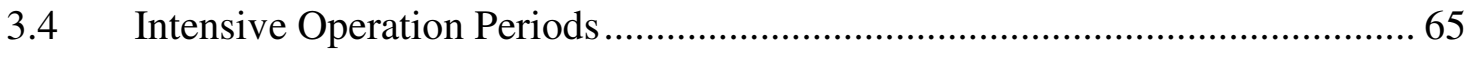

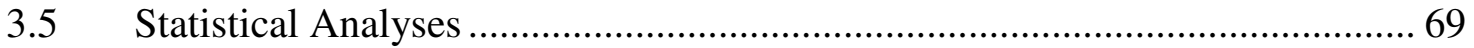

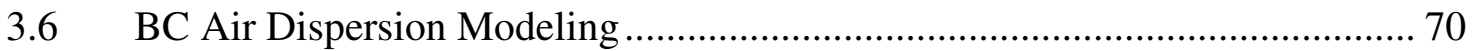

Chapter 4. Results and Discussion............................................................................................ 72 


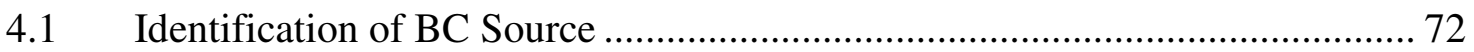

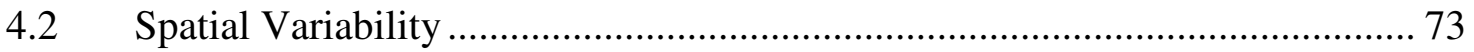

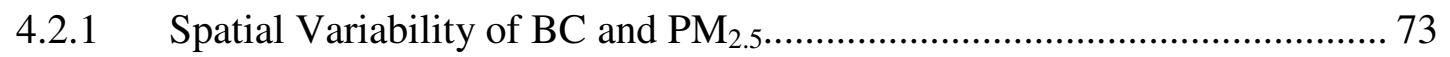

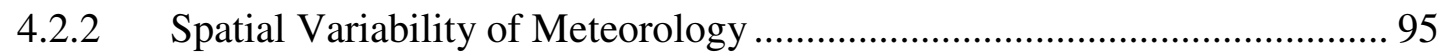

4.3 Statistical Relationship of Meteorology and Source Properties with BC ....... 100

4.3.1 Aethalometer BC and Meteorological Data............................................. 104

4.3.2 PEM BC Relationship to Meteorology and Burning Sources .................. 118

4.3.3 Summary of Aethalometer and PEM BC Regression Results .................. 134

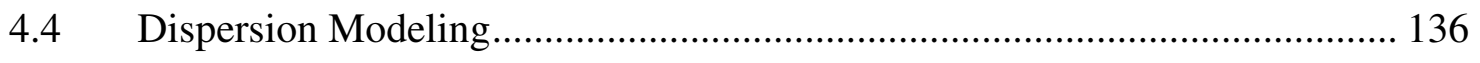

4.4.1 Comparison of ISC-PRIME and ISCST3 Modeled and Measured PEM

BC Concentrations ................................................................................. 141

4.4.2 Comparison of ISC-PRIME and ISCST3 Modeled BC Contours to

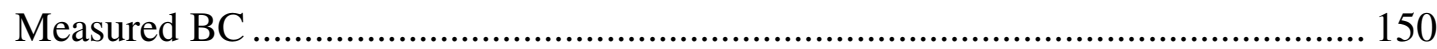

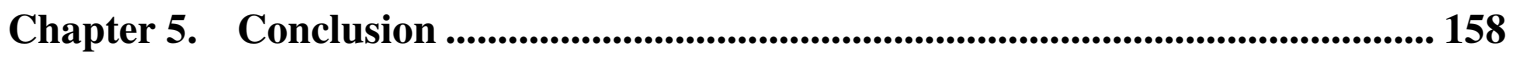

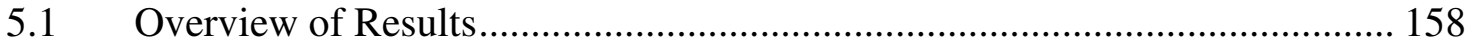

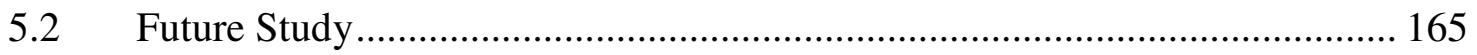

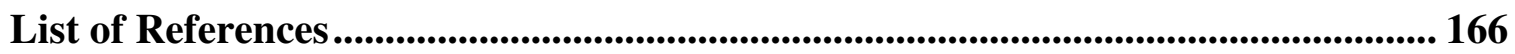

Appendix A. Aethalometer Calibration.............................................................. 184

Appendix B. Adsorption Ångström exponent (AAE) for all PEMs ........................ 197

Appendix C. Aethalometer BC Measurements over Time ................................... 198

Appendix D. Spatial Variability .................................................................................... 205

Appendix E. Summary of IOP Wind Speeds and Direction................................. 210

Appendix F. Graphic Summary for all IOPs.................................................. 265

Appendix G. Aethalometer BC Regression Models ..................................................... 280

Appendix H. PEM BC Linear Regression Models................................................. 297

Appendix I. ISC-PRIME and ISCST3 Dispersion Modeling ................................ 313 


\section{LIST OF TABLES}

Table 1. Required Meteorological Inputs for ISCST3 and AERMOD.......................... 31

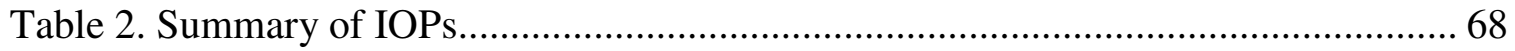

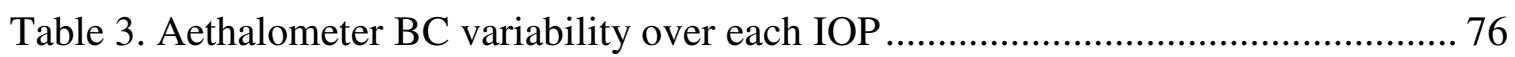

Table 4. Aethalometer BC variability over sites based on 30 minute averaged BC........ 80

Table 5. PEM variability over each IOP ................................................................. 84

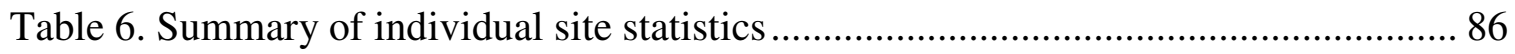

Table 7. Average and median BC concentration and standard deviation for PEMs in

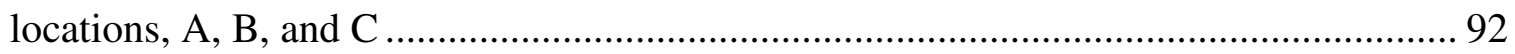

Table 8. 12 hour averaged PEM and aethalometer (aeth) BC comparison...................... 94

Table 9. Height of meteorological instrument above ground ................................... 95

Table 10. Meteorological variability over stations (IOPs 8b through $10 \mathrm{~b}$ only) .............. 97

Table 11. Meteorological data available at each station .......................................... 102

Table 12. Multiple regression models for average aethalometer BC concentrations ..... 110

Table 13. Multiple regression model for aethalometer BC deviation.......................... 115

Table 14. Linear regression models for average PEM BC concentration...................... 120

Table 15. Linear regression models for PEM BC deviation ...................................... 124

Table 16. Directional MLR model for PEM BC concentration.................................... 129 


\section{LIST OF FIGURES}

Figure 1. Approximate determination of tree coverage in study area............................... 42

Figure 2. Map of Cambria with Study Area Selected ..................................................... 45

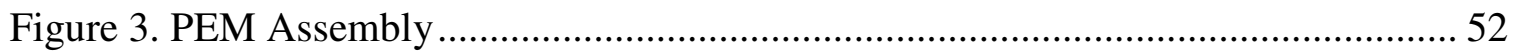

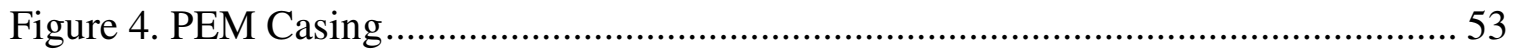

Figure 5. Aerial (left) and ground (right) view of EBAM meteorological site located in

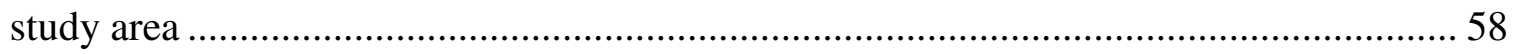

Figure 6. Ultrasonic anemometer set-up within study area ........................................... 60

Figure 7. Map of meteorological stations relative to study area..................................... 62

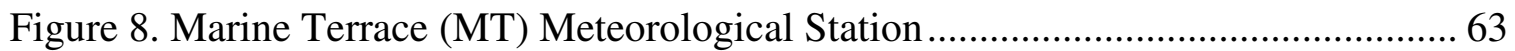

Figure 9. Community Services District Fire Department (CSDF) meteorological

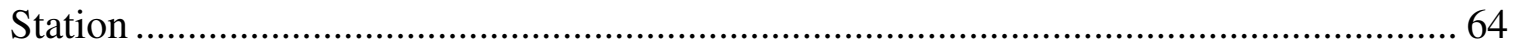

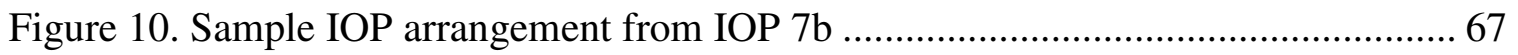

Figure 11. Sample Infrared Camera Image of a Chimney …………………………....... 69

Figure 12. Box plot of all adsorption Ångström exponent (AAE) values ......................... 73

Figure 13. Aethalometer BC variability over each IOP based on 30 minute averaged

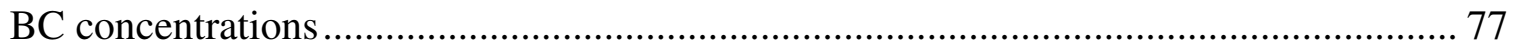

Figure 14. IOP $7 \mathrm{~b}$ aethalometer $\mathrm{BC}$ concentration versus time based on 30 minute

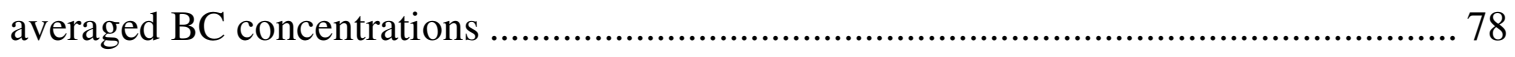

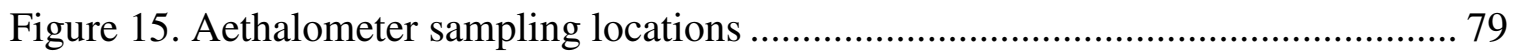

Figure 16. Aethalometer $\mathrm{BC}$ variability over sites based on 30 minute averaged $\mathrm{BC}$..... 81

Figure 17. Box plots comparing aethalometer BC variability between sites..................... 82

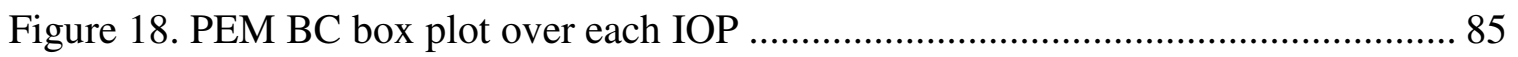


Figure 19. Average PEM BC concentration at each site 87

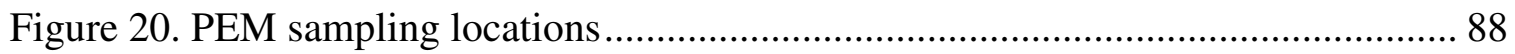

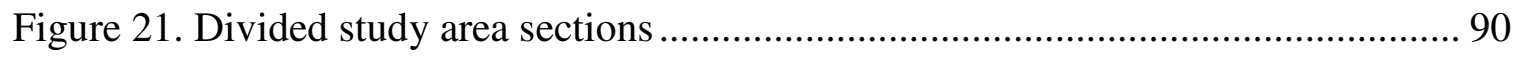

Figure 22. Average PEM BC concentration based on location .................................... 92

Figure 23. Distance between meteorological stations............................................ 96

Figure 24. Meteorological variables compared between stations ................................ 99

Figure 25. ACF Analysis Example for CSDF Meteorological Station.......................... 106

Figure 26. Comparison between residual charts for normalized (right) and un-normalized (left) average aethalometer BC concentration .................................. 109

Figure 27. EBAM variable plots for aethalometer average BC concentration ............... 112

Figure 28. EBAM-LH CSDF combination variable plots for aethalometer average

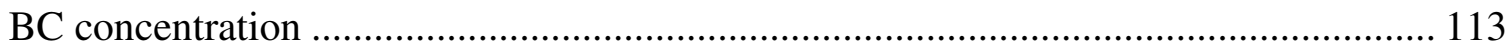

Figure 29. CSDF variable plots for aethalometer BC deviation ................................ 117

Figure 30. Marine Terrace variable plots for wind speed and wind direction versus

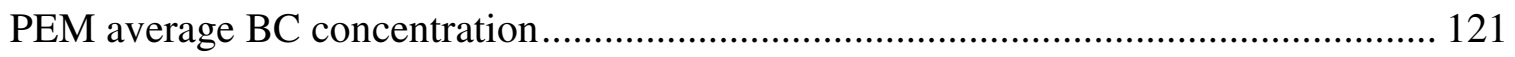

Figure 31. CSDF variable plots for PEM average BC concentration .......................... 122

Figure 32. Marine Terrace and CSDF combination variable plots for PEM average

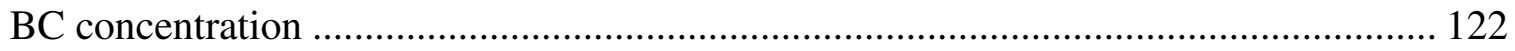

Figure 33. $\ln \left(\mathrm{BC}_{\mathrm{dev}}\right)$ versus number of wood burning sources ................................. 124

Figure 34. Directional MLR residuals versus fits plot............................................. 130

Figure 35. PEM BC versus number of burning sources in location sorted by location and level of vegetation and structures surrounding PEM for IOPs $2 \mathrm{~b}$ through $10 \mathrm{~b} \ldots \ldots . . .131$ 
Figure 36. PEM BC versus number of upwind burning sources in location sorted by location and level of vegetation and structures surrounding PEM for IOPs 4b through $10 \mathrm{~b}$

Figure 37. PEM BC Box Plot for Location and Degree of PEM Obstruction for IOPs 2b through $10 b$ 133

Figure 38. Homes within study area as represented in BEEST 138

Figure 39. Box plots of modeled-to-measured BC 142

Figure 40. ISC-PRIME modeled versus measured PEM BC concentration 144

Figure 41. ISCST3 modeled versus measured PEM BC concentration 144

Figure 42. Box plots of modeled-to-measured $\mathrm{BC}$ ratios after $\mathrm{BC}$ concentration correction factors 148

Figure 43. Corrected modeled BC versus measured BC concentration.

Figure 44. IOP $6 \mathrm{~b}$ modeled contours demonstrating similarity between ISC-PRIME and ISCST3

Figure 45. IOP 4b modeled contours demonstrating differences between

ISC-PRIME and ISCST3

Figure 46. ISC-PRIME contours demonstrating distributions similar to measured distributions.

Figure 47.ISCST3 and ISC-PRIME contours demonstrating distributions differing from measured distributions 154

Figure 48. IOP 5B sources and receptor BC concentrations 157 


\section{Chapter 1. Introduction}

Wood burning fireplaces and stoves, while less common throughout the United States than in previous decades, remain common in many communities. The use of wood for heating and as a fuel has been widely popular for reasons ranging from wood being an inexpensive fuel in highly forested areas to wood burning providing desired aesthetic qualities. Despite these benefits, in areas where meteorology and terrain encourage trapping of atmospheric pollutants, the prevalence of wood burning may lead to high pollutant exposures for surrounding populations inside and outside of homes (Branis et al., 2000; Turpin et al., 2007).

Incomplete wood combustion has been found to include a variety of harmful byproducts including particulate matter less than $2.5 \mu \mathrm{m}\left(\mathrm{PM}_{2.5}\right)$, carbon monoxide, polycyclic aromatic hydrocarbons (PAHs), and nitrogen oxides $\left(\mathrm{NO}_{\mathrm{X}}\right)$ (which are a precursor to secondary $\mathrm{PM}_{2.5}$ ). This study focuses on black carbon (BC) emissions as an extension of $\mathrm{PM}_{2.5}$, which has been a major constituent of health concerns. Its incredibly small size makes $\mathrm{PM}_{2.5}$ capable of evading the lungs' defense mechanisms and penetrating deep into the lungs. Several studies have linked $\mathrm{PM}_{2.5}$ to respiratory and pulmonary symptoms and illnesses, and in some cases even death. In children, $\mathrm{PM}_{2.5}$ had been shown to hinder respiratory growth in addition to causing wheezing, coughing, and asthma (Gauderman et

al., 2004; Honicky et al., 1985). In adults, inflammation of the respiratory and pulmonary tract has caused tissue damage and dramatically increased the chance of death in those with pre-existing illnesses (Barregard et al., 2006; Barregard et al., 2007; Pope et al., 2002). 
To protect the public from high concentrations of detrimental wood smoke byproducts such as $\mathrm{PM}_{2.5}$, it is important to understand, monitor and limit public exposure.

Currently, air monitoring occurs on the regional scale and is intended to capture overall exposure concentrations. However, regional monitoring often fails to provide the high spatial resolution needed to understand local exposures on the neighborhood or near-field scale. Existing studies that attempt to bridge the gap between regional and near-field scales have been limited to observing near-field $\mathrm{PM}_{2.5}$ variability in areas where traffic emissions are abundant, and exposure in wood burning communities remain largely misunderstood. Studies observing wood smoke contributions to ambient pollutants focus mainly on source apportionment instead of spatial variability (Branis et al., 2000).

Ward (2009) and Malejan's (2009) parallel studies taking place in a $1 \mathrm{~km}^{2}$ area in Cambria, California appear to be the only study that has evaluated near-field spatial variability for areas containing many wood burning sources. Notable conclusions from their study include the demonstration of high spatial variability and large similarity in indoor to outdoor exposures. Ward (2009) found average evening $\mathrm{PM}_{2.5}$ concentrations in the area to vary widely with the largest difference in $\mathrm{PM}_{2.5}$ concentrations over an IOP estimated between 54.5 and $81.8 \mu \mathrm{g} / \mathrm{m}^{3}$. Malejan (2009) found indoor to outdoor ratios to average about 75 percent. Additionally, available studies observing small scale variability in traffic sources indicate high variability in PM concentrations over distances less than 10 meters, where some concentrations are higher than those measured by a central monitoring station (Gulliver and Briggs, 2004; Nerriere et al., 2005). These 
results suggest that locally measured $\mathrm{PM}_{2.5}$ concentrations resulting from high density wood burning have potential to be similarly higher than concentrations represented regionally.

Because local monitoring may not be economical, other means of determining pollutant concentrations have been used. Tools designed to represent pollutant exposures include meteorology based statistical regression models and air dispersion models. By using weather characteristics including wind speed, temperature, humidity, and precipitation, correlation-based multiple linear regression analyses have been found to explain between about 20 to 80 percent of the variability in $\mathrm{PM}_{2.5}$ concentrations in locations throughout the world (detailed in Section 2.2.2.1.1). Air dispersion modeling programs such as Industrial Source Complex (ISC) and American Meteorological Society/U.S. Environmental Protection Agency Regulatory Modeling System (AERMOD) have also been commonly used in regulatory applications due to their ability to determine the highest ground level pollutant concentrations. This is accomplished often by simulating site conditions based on inputs of meteorology and source characteristics. When used in optimum scenarios, both ISC and AERMOD programs can predict concentrations within a factor of 2 of the measured values. Statistical regression and air dispersion models are both relatively economical analytical methods that have high potential to help improve understanding of pollutant concentrations and variability within the neighborhood scale provided the availability of detailed inputs. 
This study was conducted as a segment of a larger study whose goal was to contribute to the understanding of near-source variability for primary air pollutants generated within a residential area. The larger study focused on wood smoke particulate matter generated within a residential area in a region where residential wood burning is the primary source of particles. The research addressed four primary questions:

- What is the concentration variability associated with wood smoke burning within a residential neighborhood?

- Can near source contributions be properly estimated based on information on burning patterns, meteorology, and regional monitoring site data?

- Does indoor exposure to outdoor wood smoke sources correlate with expected values based on simple indoor-outdoor models?

- How does the near source contribution affect intake fraction calculations for wood smoke exposures?

The results of the larger study were intended to improve our understanding of the contribution of wood smoke variability to overall $\mathrm{PM}_{2.5}$ exposure, and can be used to aid in the development of $\mathrm{PM}_{2.5}$ source reduction prioritization, increase our understanding of the importance of variability of acute exposures, and improve the effectiveness of source control programs for reducing $\mathrm{PM}_{2.5}$ exposures. To the extent that wood smoke behavior can be generalized to other locally distributed emission sources (such as distributed power generation) this work will aid in decision making regarding siting of sources, 
improve impact calculations, and be a valuable resource for determining the potential for environmental justice concerns.

This study directly addresses the first two questions of the larger study by continuing Ward (2009) and Malejan's (2009) field study in Cambria, California and by evaluating the performance of existing regression and air dispersion modeling tools. By addressing the first two questions, the exposure and intake fractions may also be addressed, as is of interest in question four. By conducting the same air sampling procedures used in Ward (2009) and Malejan's (2009) parallel studies, the increased sample size could be used to conduct regression and modeling analyses. Goals for this study specifically include (1) further measuring pollutant variability within the near-field study area, (2) evaluating the relationship between pollutant variability and meteorology, geography, and burning sources, and (3) identifying the performance of existing dispersion models in application to the near-field based on available input variables. 


\section{Chapter 2. Background}

\subsection{Wood Smoke}

Although the products of ideal and complete biomass combustion are carbon dioxide, steam, and heat, the composition of wood and actual combustion conditions result in products very different than the ideal. By-products including particulate matter less than $2.5 \mu \mathrm{m}\left(\mathrm{PM}_{2.5}\right)$, carbon monoxide, volatile organic compounds (sometimes including carcinogens like polycyclic aromatic hydrocarbons), and nitrogen oxides $\left(\mathrm{NO}_{\mathrm{X}}\right)$ are also typically generated in the form of wood smoke due to complex organic molecules found in wood and non-ideal burning conditions such as low combustion temperatures. $\mathrm{PM}_{2.5}$ may also form as secondary emissions in the atmosphere, such as when $\mathrm{NO}_{\mathrm{X}}$ interacts with atmospheric constituents like ammonia. Many of the by-products can have negative short and/or long term effects on human health (Morandi et al, 2010).

\subsubsection{Wood Smoke and $\mathbf{P M}_{2.5}$ Impacts on Health}

The health impacts of wood smoke have been demonstrated through observation of wood smoke composition and wood smoke's immediate effect on sensitive and healthy populations. Davy et al. (2007) found aerodynamic particle diameters in wood smoke from the combustion of pine and blue gum to aggregate into clumps ranging from 0.010 to $0.30 \mathrm{~nm}$, much smaller than the particle diameter of $2.5 \mu \mathrm{m}$. $\mathrm{PM}_{2.5}$ is capable of evading our lung's natural defense mechanisms and penetrating deep into the lungs. Harmful organic compounds are often attached to carbon soot in wood smoke and have been shown to induce pulmonary reactions. Kocbach et al. (2008) found 20 times more 
carcinogenic polycyclic aromatic hydrocarbons (PAHs) in wood smoke PM than in diesel PM. Kobach et al. also found the presence of organic compounds in wood smoke PM to encourage the release of inflammatory cytokines, a type of signaling protein, which may encourage tissue damage due to prolonged inflammation. Because $\mathrm{PM}_{2.5}$ has demonstrated negative pulmonary impacts, the US Environmental Protection Agency (EPA) established annual and 24-hour National Ambient Air Quality standards (NAAQS) for $\mathrm{PM}_{2.5}$ in 1997. EPA limits for $\mathrm{PM}_{2.5}$ are currently $15.0 \mu \mathrm{g} / \mathrm{m}^{3}$ averaged over 1-year and $35 \mu \mathrm{g} / \mathrm{m}^{3}$ for a 24 hour average (US EPA, 2010). EPA standards are meant to protect public health, including sensitive populations such as children, asthmatics, and the elderly.

Children have been an emphasis of many exposure health studies, especially due to respiratory growth concerns. In a study measuring pulmonary development of children from the age of 10 to 18 years of age in 12 communities, Gauderman et al. (2004) compared the percentage of children with low forced expiratory volume at one second $\left(\mathrm{FEV}_{1}\right)$, a measure of pulmonary function, in a community with a high level of exposure to $\mathrm{PM}_{2.5}$ to the percentage of children with low $\mathrm{FEV}_{1}$ in the community with a low $\mathrm{PM}_{2.5}$ exposure. The percentage of children with low $\mathrm{FEV}_{1}$ in the community exposed to the highest $\mathrm{PM}_{2.5}$ levels was 4.9 times (7.9 percent) the percentage of children exposed to the lowest $\mathrm{PM}_{2.5}$ levels (1.6 percent). Koenig et al. (1993) also found a $34 \mathrm{~mL}$ decline in $\mathrm{FEV}_{1}$ for asthmatic children with each $20 \mu \mathrm{g} / \mathrm{m}^{3}$ increase in $\mathrm{PM}_{2.5}$ concentration and concluded that acute respiratory irritation in asthmatic elementary school children is correlated with wood burning. The use of wood burning as a home heating source in a 
study amongst 62 children between the ages of 1 and 7 years in Michigan indicated a significantly higher prevalence of symptoms of acute respiratory illness (Honicky et al. 1985). Honicky et al.'s study found 77 percent of children were reported to show symptoms of wheezing with cold or having at least one attack of wheezing in the wood burning community during the winter compared to 29 percent in the non-wood-burning community. Although the study found no significant difference in the occurrence of asthma, allergies in the wood burning community occurred in 19.4 percent of the children as opposed to 3.2 percent of the children in the non-wood burning community. One study conducted on asthmatic children in Seattle, Washington found a 14 percent increase in the odds of experiencing asthma symptoms with increases in $\mathrm{PM}_{1.0}$ (Yu et al., 2000).

Studies among the general population have also found associations between $\mathrm{PM}_{2.5}$ and negative health impacts. Pope et al.'s (2002) study on individuals in United States metropolitan areas associated a $10 \mu \mathrm{g} / \mathrm{m}^{3}$ increase in long-term $\mathrm{PM}_{2.5}$ concentrations with about 4, 6, and 8 percent increased risk of death from all-cause, cardiopulmonary, and lung cancer, respectively. In a study with healthy human subjects, Barregard et al. (2006, 2007) exposed 13 people to 240 through 280 micrograms per meter cubed of fine wood smoke particles, the concentration typical in a smoky indoor environment, and found wood smoke to cause inflammation and oxidative stress in the respiratory tract.

Many negative health impacts have been associated with wood smoke $\mathrm{PM}_{2.5}$. For this reason, it is important to understand wood smoke variability within residential 
neighborhoods where frequent exposure may occur in the winter time. This is especially true under meteorological conditions that may elevate exposure levels.

\subsubsection{Wood Smoke PM$_{2.5}$ Characterization}

This study focuses on wood smoke $\mathrm{PM}_{2.5}$ because of the major health concerns associated with the fine particles. The contribution of $\mathrm{PM}_{2.5}$ by wood burning has often been estimated through modeling methods that separate $\mathrm{PM}_{2.5}$ mass produced from gasoline, diesel, and other contributors from wood smoke $\mathrm{PM}_{2.5}$. These studies have found wood smoke to contribute $\mathrm{PM}_{2.5}$ mass ranging from 20 to 60 percent of total fine particulate mass during the wintertime and 10 to 30 percent of total annual $\mathrm{PM}_{2.5}($ Schauer \& Cass, 2000; Favez et al., 2009; Wu et al., 2007; Aarhus University NERI, 2010; Zhang et al. 2010; Bari et al., 2010).

$\mathrm{PM}_{2.5}$ has been found to be composed of 63 through 96 percent elemental carbon (EC) and organic carbon (OC), with OC often being the more dominant component in conventional wood burning (Watson et al., 2001). Although the concentrations of EC and OC in a specific sample can differ by factors of 2 and 10, respectively, based only on measurement methods (Watson et al., 2005), generally accepted definitions exist. OC is commonly defined as compounds oxidized below a temperature threshold (usually ranges between 350 and $550^{\circ} \mathrm{C}$ ) in the absence of an oxidizing agent (Watson et al., 2005), and encompasses thousands of organic compounds. EC is usually defined as compounds oxidized above a higher temperature threshold than OC (usually ranges between 650 and $1100{ }^{\circ} \mathrm{C}$ ) in the presence of an oxidizing agent, often oxygen (Watson et al., 2005), and is 
characterized by having "near-elemental soot-carbon-like composition" (Andreae et al., 2006). Black carbon (BC) is often used interchangeably with $\mathrm{EC}$, but differs because BC concentrations are determined optically, or based on particles' ability to absorb light (Andreae et al., 2006). Both the "BC" and "EC" terms include similar carbon fractions, but differ slightly due to the methods used to determine their presence.

The emission of particulate matter in wood smoke can vary significantly depending on the wood type and burning conditions. Fine et al. (2001) found 6 types of hard and soft woods burned in a conventional masonry fireplace to have average fine particle emission rates ranging between 2.7 to 11.4 grams per kilogram of wood burned. Particulate matter composition can vary significantly based on burning temperature and oxygen content. Bølling et al. (2009) evaluated several studies to determine the impact of combustion temperature and oxygen content. Low temperature combustion was found to typically yield high OC to total carbon (EC plus OC) ratios, with OC comprising 89 to 99 percent of the total wood smoke carbon content. Alternately, high temperature wood combustion with limited oxygen content yields more EC than OC, with EC comprising between 50 to 75 percent of the total carbon content. In the presence of high temperature and an adequate supply of oxygen, less harmful inorganic particles are more dominantly emitted instead. These inorganic particles are primarily composed of alkali salts, sometimes with trace elements such as zinc and magnesium (Johansson et al., 2003). They can be less harmful due to their higher tendency to grow in size in the presence of high humidity (Löndahl et al, 2007). In addition, their solubility makes them less difficult for the lungs to remove than insoluble particles (Bølling et al., 2009). 
Bølling et al. also found that open fireplaces, conventional wood stoves, and masonry heaters, as are more likely found in this study, tend to emit organic or elemental carbondominant particulate matter. Open fireplaces reviewed by Bølling et al. yielded EC/TC ratios of 0.04 through 0.46 with higher fractions of OC. Conventional wood stoves and conventional masonry heaters, however, were less consistently OC- or EC- dominant with different studies finding some emissions more OC-dominant and some emission more EC-dominant. Modern wood stoves and masonry heaters alternatively have been found to emit more inorganic ash with less than 10 percent of emissions being comprised of OC and EC (Jokiniemi et al., 2008).

Ponderosa and Slash Pines are both found in California and are both of the same genus as the Monterey Pines found in the chosen study area. When combusted in a conventional masonry fireplace, Ponderosa and Slash pines emit higher OC than EC content by mass; Fine et al. (2002) found on average 90.1 percent $\mathrm{OC}$ and 7.3 percent EC by mass in Ponderosa Pine wood smoke and 100.6 percent OC and 14.7 percent EC by mass in Slash Pine (Fine et al., 2002; Fine et al., 2004). Percentages greater than 100 were explained by organic gases adsorbing to the quartz fiber. These gases were considered insignificant because backup filters indicated adsorption was less than 20 percent of the measured mass. Residents within the study area also mentioned the use of oak wood in their fireplace (Leimert resident, personal communication, February 13, 2010). Fine et al. (2002) reported Midwestern White and Black oak wood smoke to contain 76.5 percent OC and 1.1 percent EC and 76.0 percent $\mathrm{OC}$ and 2.3 percent EC, respectively. Wood 
smoke particulate matter generated during the study should demonstrate similar OC and EC fractions. Malejan (2009) and Ward's (2009) parallel studies, which this study follows upon, found EC to comprise between 4 and 8 percent in wood smoke samples.

\subsubsection{Wood Smoke Identification}

To attribute collected particles to wood combustion, several techniques are typically used. These methods utilize the fact that wood combustion emits a higher organic carbon fraction than most other common particle emission processes such as gasoline or diesel vehicle fuel combustion, where EC comprises closer to 50 percent of total carbon (Watson et al., 2001). The compounds present in organic fractions of wood smoke and the optical nature of organic carbon have been valuable tools for wood smoke identification.

\subsubsection{Light Attenuation Approach}

The abundance of organic compounds in wood smoke allows for its identification through light attenuation. Particle light attenuation in the UV and visible spectrum can be better understood by using Beers Law or the power law relationship of $\sigma=\mathrm{K} \lambda^{-\alpha}$, where $\sigma$ is the spectrally dependent mass adsorption efficiency proportional to light

attenuation, $\mathrm{K}$ is a constant, $\lambda$ is the wavelength in $\mathrm{nm}$, and $\alpha$ is the adsorption Ångström exponent (AAE). Based on the power law relationship, the adsorption Ångström exponent can indicate the degree of spectral dependence on light attenuation.

Kirchstetter et al. (2004) evaluated the impact of traffic and biomass combustion particles on light absorption throughout the wavelength spectrum. They found that organic 
compounds typically present in biomass combustion display high spectral dependence, with high light attenuation at wavelengths less than $600 \mathrm{~nm}$. Aerosols generated by motor vehicle exhaust displayed an average spectral dependence of $\lambda^{-1}$, and often range from $\lambda^{-0.9}$ to $\lambda^{-1.1}$, while aerosols contributed by biomass smoke typically had a spectral dependence of $\lambda^{-2}$. By evaluating the spectral dependence of light attenuation, there is potential to infer the contribution of $\mathrm{BC}$ from biomass combustion.

\subsubsection{Detection of Organic Compounds}

Because some particulate organic compounds are specific to biomass burning, they have been used to apportion atmospheric particles contributed by wood combustion. When burned, cellulose and lignins, both essential components of vegetation, are converted into cellulose-specific monosaccarides (simple sugars) such as levoglucosan and ligninspecific phenolics (derivative of an aromatic hydrocarbon with an -OH group) such as methoxyphenols. Methoxyphenols, while emitted abundantly during lignin combustion, may be a less effective tracer due to their moderately reactive characteristics (Simpson et al., 2005). Levoglucosan alternatively has been observed to be produced abundantly during wood combustion and to be stable in the atmosphere, making it suitable as a tracer for wood smoke (Locker, 1988; Fraser \& Lakshmanan, 2000; Jordan et al., 2006).

Studies have found traces of levoglucosan in all wood burning samples (Simoneit et al., 1999) and low concentrations in the non-winter ambient environment, due to high temperatures of about $300^{\circ} \mathrm{C}$ required to generate it. Despite its abundance and stability, levoglucosan concentrations can still be difficult to use quantitatively due to wide 
variations in emissions depending on combustion conditions (Hedberg et al., 2006). A recent study conducted by Hoffmann et al. (2010) has even found that levoglucosan is not as stable as previously considered. Hoffmann et al. noted that while previous studies have found no significant levoglucosan degradation for up to 8 hours under ambient conditions (Locker, 1988) and no significant hydrolysis for 10 days under acidic conditions (Fraser \& Lakshmanan, 2000), degradation by free radicals from deliquescent particles was not considered. Deliquescent particles can form hydroxide $(\mathrm{OH})$ radicals when dissolved into atmospheric vapors. Evaluation of levoglucosan degradation by $\mathrm{OH}$ radicals indicated higher winter time degradation fluxes than previously expected (around $4.7 \mathrm{ng} \mathrm{m}^{-3} \mathrm{~h}^{-1}$ ). Still, levoglucosan remains the best known organic tracer for wood smoke because it is highly specific to biomass burning and is typically emitted in abundance.

\subsubsection{Thermal Optical Approach}

Thermal optical methods are primarily used to determine EC and OC fractions, and thus can be used to trace the presence of wood smoke; higher OC content would suggest particles originating from biomass combustion. EC and OC content are determined by collecting samples on quartz fiber filters and heating the samples in a ramp of temperatures specific to each method. The Interagency Monitoring of Protected Visual Environments (IMPROVE), the National Institute of Occupational Safety and Health (NIOSH) 5040, and the Lawrence Berkeley National Laboratory Effluent Gas Analysis (LBNL EGA) methods utilize the thermal optical approach to determine EC and OC fractions (Chow et al., 2001; Ellis \& Novakov, 1982). 
IMPROVE and NIOSH methods both heat filter compounds in a step wise manner (different temperature steps are used for each method) under a oxygen-free environment for $\mathrm{OC}$ and a 2 percent oxygen environment for EC as evolved carbon compounds are measured at each temperature. The temperatures used for IMPROVE and NIOSH methods range from 120 to $850^{\circ} \mathrm{C}$ and 250 to $850^{\circ} \mathrm{C}$, respectively. LBNL EGA instead uses a constant temperature increase rate of $20^{\circ} \mathrm{C}$ each minute from 50 to $600^{\circ} \mathrm{C}$ in an oxygen environment. For each method, carbon content is measured by catalyzing evolved carbon compounds into carbon dioxide on a magnesium dioxide probe. Carbon dioxide is then converted to methane and measured with a flame ionization detector (FID). FID results are correlated with light penetration to understand peaks in methane measurements and total carbon is determined through integration of peaks.

\subsection{Pollutant Variation}

In addition to developing NAAQS, the EPA also requires states to establish a network of air pollution monitoring. These networks are meant to: (1) include sites that represent the highest concentrations in the area, (2) measure concentrations in areas of high population density, (3) identify the impact of a significant source or sources, (4) represent background concentrations, (5) characterize regional pollutant transport, and (6) evaluate air pollution impacts on visibility (US EPA, 2008b). These network characteristics demonstrate compliance to NAAQS and help to protect public health. Although these state-wide monitoring networks have been successful in characterizing pollutant emissions in most communities, no studies have focused on near-field pollutant 
variability in areas where wood burning is common in the winter time. According to the US Census estimates, approximately 2.1 million households in the United States used wood as a heating source between 2005 and 2009 (US Census Bureau, 2009). Because the impacts of wood smoke exposure are understood to have detrimental respiratory impacts, it is important to understand neighborhood scale pollutant variability with in areas where wood burning is prevalent.

\subsubsection{Spatial Representation of PM}

While some studies have evaluated PM variability for urban environments primarily originating from diesel or gasoline exhaust and/or industrial sources, no studies have evaluated the impact of wood burning sources on near-field PM variability. Studies demonstrating small scale variability tend to involve mobile sampling methods and tend not to use simultaneous sampling strategies. For example, Robinson et al.'s study (2007) found large differences in $\mathrm{PM}_{2.5}$ concentration averages over only a 41 meter distance (35 $\mu \mathrm{g} / \mathrm{m}^{3}$ and $90 \mu \mathrm{g} / \mathrm{m}^{3}$ ) when sampling with a mobile nephelometer, a device measuring light scattering by particles which can then be related to PM concentrations, in Armidale, New South Wales Australia. Higher concentrations were found in areas where wood burning was prevalent. Although mobile sampling can well demonstrate differences in concentrations over short distances, changes to concentrations over time remains unaccounted for.

Studies evaluating wood smoke contributions to PM concentrations in small areas have only measured pollutant variability at one location and have had to differentiate between 
PM contributors. One study evaluated both coal and wood burning contributions to winter $\mathrm{PM}_{10}$ and black smoke (similar in definition to $\mathrm{BC}$ ) in the Czech Republic within a small rural village, Žloukovice, of 300 inhabitants (Branis et al., 2000). Resident surveys for fuel type used was conducted in conjunction with air sampling and indicated that 19.2 percent of households were found to use mainly coal and 14.5 percent of households were found to use mainly wood. Winter 1997/1998 $\mathrm{PM}_{10}$ concentrations were found to widely range between 9.71 and $106.4 \mu \mathrm{g} / \mathrm{m}^{3}$ and averaged $33.6 \mu \mathrm{g} / \mathrm{m}^{3}$. Similarly, winter 1998/1999 $\mathrm{PM}_{10}$ concentrations widely ranged between 8.48 and $118.7 \mu \mathrm{g} / \mathrm{m}^{3}$ and averaged $41.2 \mu \mathrm{g} / \mathrm{m}^{3}$. A strong correlation between black smoke and $\mathrm{PM}_{10}$ concentrations indicated that the main source of $\mathrm{PM}_{10}$ was likely from household combustion processes.

In contrast to the scarcity of near-field PM variability studies, there are a larger number of reports investigating spatial variability in PM from vehicular or unspecified sources. Many of these studies evaluated PM variability within urban scales, defined as 4 through $100 \mathrm{~km}$ in diameter, length, or width, and neighborhood scales, defined as $500 \mathrm{~m}$ to $4 \mathrm{~km}$ in diameter, length, or width (40 CFR part 58). Although some researchers have found low variability within urban scales (Gorin et al., 2006; Goswami et al., 2002), other studies have found substantial variability on the smaller neighborhood scale.

PM concentrations have been found to vary considerably over short distances, especially in the presence of abundant pollutant emissions. Gulliver and Briggs (2004) found large standard deviations when measuring $\mathrm{PM}_{10}, \mathrm{PM}_{2.5}$, and $\mathrm{PM}_{1}$ concentrations at 1 second 
resolution over two routes comprising high traffic and low traffic areas while walking and driving. Walking through each route required 15 to 20 minutes and resulted in $\mathrm{PM}_{10}$, $\mathrm{PM}_{2.5}$, and $\mathrm{PM}_{1}$ concentrations of $38.18 \pm 25.17 \mu \mathrm{g} / \mathrm{m}^{3}, 15.06 \pm 16.15 \mu \mathrm{g} / \mathrm{m}^{3}$, and $7.14 \pm$ $9.62 \mu \mathrm{g} / \mathrm{m}^{3}$, respectively. Concentrations for $\mathrm{PM}_{10}, \mathrm{PM}_{2.5}$, and $\mathrm{PM}_{1}$ detected while driving over a 6 minute period were $43.16 \pm 22.71 \mu \mathrm{g} / \mathrm{m}^{3}, 15.54 \pm 15.92 \mu \mathrm{g} / \mathrm{m}^{3}$, and 7.03 $\pm 9.67 \mu \mathrm{g} / \mathrm{m}^{3}$, respectively. Higher variability in PM has been shown to occur in the presence of numerous gasoline and diesel vehicles, while variability declines when only background PM levels are observed (Weijers et al., 2004).

In Gulliver and Briggs' (2004) study discussed above, one of the main objectives was to compare $\mathrm{PM}_{10}$ concentrations measured at a fixed-road monitoring location to concentrations measured using a portable monitoring device, OSIRIS, within an $8 \mathrm{~km}^{2}$ area. $\mathrm{PM}_{10}$ was measured as a subject carried an OSIRIS and walked two different routes and as a vehicle carrying another OSIRIS was driven along the same routes. The routes traveled through both main and minor roads to represent areas both with and without major PM emitting sources. The fixed-road monitor was located about 10 meters from a major road on the first route. The average $\mathrm{PM}_{10}$ concentrations measured during walking and measured during driving were found to be 30 and 67 percent higher than the fixed monitoring site, respectively. Gulliver and Briggs also found a greater difference between $\mathrm{PM}_{10}$ concentrations measured at the fixed site and the second route than with the fixed site and the first route. The second route, located much further away from the monitoring location (about $1 \mathrm{~km}$ away) thus demonstrated a decrease in representation with an increase in distance. 
A study conducted in four French metropolitan areas of contrasting urban settings and air quality also found fixed monitoring locations to under-represent individual exposure to $\mathrm{PM}_{2.5}$ (Nerriere et al., 2005). Three areas within each city were selected to represent locations with high traffic emission exposure, abundant influence by local industry, and urban background levels. Volunteers within each area carried personal monitoring devices, a Harvard ChemPass along with a portable pump, and comparisons were made between the individuals and fixed monitoring stations set up within each area over a period of between 24 and 48 hours. Significant wintertime average differences were found between personal and fixed station $\mathrm{PM}_{2.5}$ concentrations ranging between 1.77 and $26.2 \mu \mathrm{g} / \mathrm{m}^{3}$ for four cities, with all winter average concentrations reading lower at fixed stations. It is important to note that measured $\mathrm{PM}_{2.5}$ may have been contributed by indoor activities, rather than external sources, although volunteers were all non-smokers who had declared no exposure to environmental tobacco smoke. Several other studies have also attributed the difficulty of representing personal pollutant exposure with fixedmonitoring stations to the abundance of indoor pollutant sources (Wilson et al., 2000; Zeger et al., 2000; Turpin et al., 2007).

While large differences in personal and ambient concentrations have been attributed to indoor pollutant sources, it is also important to understand outdoor pollutant variability and its contribution to indoor environments. The infiltration of ambient pollutants into homes and microenvironments (such as cars and buses) has been well demonstrated and can have significant implications for public health given the large percentage of time 
spent indoors. A survey funded by the California Air Resources Board from 1987 through 1988 found that on average people spend 87 percent of their time indoors (Jenkins et al., 1992).

A wide range of indoor/outdoor ratios (I/O) have been observed in several studies. Lunden et al. (2008) reported $\mathrm{PM}_{2.5} \mathrm{I} / \mathrm{O}$ ratios for the months of December and January to be $0.39 \pm 0.2$ and $0.38 \pm 0.3$, respectively. Geller et al. (2002) found $\mathrm{PM}_{2.5} \mathrm{I} / \mathrm{O}$ ratios of $1.03 \pm 0.29$ and explained that outdoor concentrations accounted for 37 percent of the variation with the remaining fraction likely associated with indoor sources. Turpin et al. (2007) found outdoor $\mathrm{PM}_{2.5}$ to contribute 25 to 30 percent to personal exposure and 73 percent on average to indoor concentrations within residential areas located near industrial sources in Los Angeles, California, Elizabeth, New Jersey, and Houston, Texas. Allen et al. (2003) alternately found a $79 \pm 17$ percent contribution of outdoor $\mathrm{PM}_{2.5}$ to indoor $\mathrm{PM}_{2.5}$. Several factors can contribute to differing outdoor pollutant contribution to indoor environments including particle properties, housing characteristics, and near-surface air flows and turbulence (Turpin et al., 2007). Infiltration of outdoor PM into indoor environments can have significant implications for public exposure, especially in locations where indoor PM concentrations are high and PM emissions are elevated due to wood burning. Because areas tend be represented by a single monitoring station, often located relatively far from residential activity, this study was designed to contribute information on $\mathrm{PM}_{2.5}$ spatial variability within a small area containing multiple wood burning sources. 


\subsubsection{Estimation of Pollutant Variability and Concentrations}

Because the number of monitoring stations used in communities may be limited by available funds, modeling methods have been developed to facilitate the prediction of pollutant concentrations in locations where monitoring does not take place. The most commonly used methods for predicting pollutant concentrations either focus on the relationship between ambient sampling data and external factors or predict pollutant concentrations based on mechanical and physical behavior (Georgopoulos et al., 2009). Both categories have their advantages and disadvantages which must be considered based on the intended use of model results. Ambient-data-based models often offer simplicity and require less detailed descriptions of environmental conditions to produce valuable information. Mechanical models, provided adequate model inputs, can offer high accuracy and high resolution. Alternatively, ambient-data-based methods sometimes fail to consider spatial and temporal variation while mechanical methods can misrepresent real conditions depending on the accuracy of assumed conditions. These two model categories are explored briefly below.

\subsubsection{Ambient-Data-Based Models}

Models based on ambient sampling data typically utilize pollutant concentrations at measured sites to interpolate concentrations at locations where sampling did not occur. The simplest of these models are mathematically linear, but more complex, non-linear models have emerged to improve predictions where linear relationships are inappropriate. An evaluation of $\mathrm{PM}_{10}$ prediction methods in the large city of Greece, Thessaloniki, inhabited by 16,000 people tested the statistical performance of four methods: Multiple 
Linear Regression (MLR), Classification and Regression Trees (CART), Principle Component Regression (PCR), and the Multi-Layer Perceptron type of neural network (NN) (Slini et al., 2006). Amongst these methods, MLR and PCR are linear, while NN and CART are non-linear.

MLR is a parametric method (assumes a certain probability distribution for the data) that aims to predict a desired variable $\mathrm{Y}$ using other predictor variables such as wind speed and temperature. In Slini et al.'s (2006) study, the $\mathrm{PM}_{10}$ concentration was the desired variable, Y. Each predictor variable is selected and weighted based on their ability to reduce the difference between the measured Y-value and the predicted Y-value.

The CART method is nonparametric and allows for the consideration of a pool of predictor variables that may or may not be used. The selection of predictor variables occurs through binary recursive partitioning (a continuous splitting of data into two groups) that is based on the satisfaction of conditions previously set. The conditions are modified based on what yields the most accurate predictions and based on previous knowledge about the variables.

PCR is a combination of Principle Component Analysis (PCA) and a linear regression. PCA evaluates the relationship between variables using a covariance matrix, selecting variables that are most independent (have little or no relationship to one another) and account for the most variance. Selected variables are then used in a linear regression analysis. 
NN methods typically employ weights and functions in series that yield a predicted value. The major advantage to NN methods is that information about errors in predictions is used to improve the applied weights and functions through the use of algorithms.

In application of the four forecasting methods to Thessaloniki, a densely populated city with many traffic and industrial sources and complex topography, $\mathrm{PM}_{10}$ and meteorological data from 1994 through 2000 was used. Slini et al.'s evaluation found $\mathrm{NN}$ to have the most reliable predictions containing the smallest errors in modeling performance and variables, yet $\mathrm{NN}$ was unable to predict peak $\mathrm{PM}_{10}$ values and may sometimes underestimate concentrations. PCR and MLR, alternatively, were able to correctly predict actual episode days and did so with a low percentage of false positives. However, MLRs had the highest significant differences between predicted and observed values. The statistical evaluation of modeling methods evaluated by Slini et al. demonstrated advantages and disadvantages to each of the methods. Perez et al. (2000) also demonstrated more accurate predictions for $\mathrm{PM}_{2.5}$ concentrations using $\mathrm{NN}$ in comparison to MLR, although prediction errors were more similar for both methods than in Slini et al.'s research. Still, while NN offered the most accurate predictions, its inability to identify peak concentrations can make it less suitable for some practical purposes. Method selection should be based on the intended use of predicted values.

Although MLR may not offer the highest level of accuracy in pollutant predictions, this study will explore the method, because it is a simple linear model that is readily interpretable and operated (Pires et al., 2008), while also being relatively robust or flexible to deviations from model assumptions (Demuzere \& van Lipzig, 2010). These 
characteristics make MLR readily applicable and understood, in contrast to black box models such as NN that often require complex algorithms. In addition, the limited data inputs available for this study may be restrictive for more complex models. Evaluating the MLR method also expands upon Ward's (2009) research by increasing the sample size that may be used to develop a MLR model.

\subsection{Multiple Regression Models for PM Variability}

Many studies have tested the MLR's ability to explain pollutant variability, though often on a larger-than-neighborhood scale and not always with an emphasis on $\mathrm{PM}_{2.5}\left(\mathrm{PM}_{10}\right.$ has instead been the focus of some MLR studies). These studies reveal that MLR models are site specific, though similarities between developed models offer information generally applicable to other locations. Tai et al. (2010) used the MLR to model daily $\mathrm{PM}_{2.5}$ concentration throughout the United States using air quality and meteorological data collected from 1998 to 2008. Tai et al. found meteorological variables including surface air temperature, relative humidity, precipitation, cloud cover, geopotential height at 850 $\mathrm{hPa}$, local rate of change of sea-level pressure, surface wind speed, a east-west wind direction indicator (cosine of wind direction), and a north-south wind direction indicator (sine of wind direction) to be significant predictors. These variables accounted for up to 50 percent of variability in $\mathrm{PM}_{2.5}$ concentration. Vukovich \& Sherwell (2002) also used MLR to evaluate $\mathrm{PM}_{2.5}$ variability from 1991 through 1997 data, but on a small scale at two specific sites located in Washington, DC and Shenandoah National Park, Virginia. At the Washington, DC site, dew point accounted for the most $\mathrm{PM}_{2.5}$ variability, 53 percent, and the addition of sky cover and $\mathrm{SO}_{2}$ emissions increased explained variation to 
77 percent. At the Shenandoah site, temperature accounted for the most variability in $\mathrm{PM}_{2.5}, 59$ percent, and the addition of a solar radiation variable resulted in a model that accounted for 82 percent of $\mathrm{PM}_{2.5}$ variability. A principle component analysis also demonstrated wind speed and temperature to explain similar percentages of variation in $\mathrm{PM}_{2.5}$ concentrations at both sites.

Studies relating meteorology to $\mathrm{PM}_{10}$ have found similar meteorological variables to explain variation in $\mathrm{PM}_{10}$ concentrations. Variability in daily winter $\mathrm{PM}_{10}$ concentrations have been correlated to meteorological conditions using MLRs for sites within the Netherlands (Demuzere et al., 2009) and within parts of the western Alpe-Adria region in central Europe (Stadlober et al., 2008). Meteorology accounted for 23 percent of $\mathrm{PM}_{10}$ variability in areas within the Netherlands based on data collected between 2001 and 2006 and between 55 and 70 percent of variability within cities of the Alpe-Adria region based on data collected during different time intervals within 2001 and 2007. Wind speed and direction, maximum temperature, shortwave downward radiation, lagged wind speed and direction, lagged cloud cover, precipitation, relative humidity, and lagged precipitation were all significant predictors for Netherland sites, while Alpe-Adria region MLR models indicated lagged $\mathrm{PM}_{10}$ concentration, inversion, day of week and month, winds speed, and precipitation to be significant predictors.

Site specific predictors inevitably result due to differences in the location's terrain and the behavior of the location's population. Despite differences in the percentage of variability accounted for in different MLR models, relative humidity, temperature, 
precipitation, and wind speed all tended to be significant predictors in many of the studies. Developing a MLR model for this study allows for the determination of variables that relate to local $\mathrm{PM}_{2.5}$ variability. In addition to understanding the relationship between meteorological and source impacts and $\mathrm{PM}_{2.5}$ variation at the study site, an evaluation of the MLR method on a neighborhood scale will offer information about its performance for future $\mathrm{PM}_{2.5}$ applications. The MLR method will be discussed in greater detail in Section 4.3. In addition to the MLR method, non-linear relationships will be briefly explored as necessary to improve the developed models.

\subsubsection{Mechanical/Physical Models}

Mechanical and physical modeling methods, of which chemistry-transport models (CTM) and chemical mass balances (CMB) are common, are an alternative to ambient-databased models. These models may rely on thorough chemical analysis of air samples, the understanding of specific source contributions, air mass behavior, and/or thermal impacts on particle chemistry (Chow \& Watson, 2002; Sportisse, 2007). Although mechanical methods such as CTM and CMB can provide pollutant variability in high resolutions within small areas, they tend to require highly detailed and accurate mechanistic information that may be economically impractical (Valari \& Menut, 2010).

Dispersion models belong to a category of mechanical models which does not require as much sampling and chemical analysis as CTM and CMB methods and rely instead on information more readily available in the United States, such as meteorological (i.e. wind speed and direction) and geographical (i.e. elevation) data. Dispersion models are 
mathematical simulations of pollutant travel as it leaves the original source location. The use of dispersion models may allow for the determination of pollutant concentrations over differing spatial and time characteristics. Through the use of measured terrain, weather, and land use information, pollutant concentrations can be predicted at specific locations where air monitoring does not take place.

Two dispersion modeling programs were considered for use in this study, Industrial Source Complex Short Term 3 (ISCST3) and American Meteorological Society/U.S. Environmental Protection Agency Regulatory Modeling System (AERMOD). Both have been developed under the EPA's request due to regulatory need and the importance of protecting public health, and both have been thoroughly evaluated. They are discussed below.

\subsection{AERMOD and ISCST3/ISC-PRIME}

American Meteorological Society/U.S. Environmental Protection Agency Regulatory Modeling System (AERMOD) is the current EPA-preferred dispersion modeling program for near-field regulatory application (US EPA, 2008a), officially replacing its predecessor, Industrial Source Complex Short Term 3 (ISCST3), on December $9^{\text {th }}, 2006$. Under the EPA's Guideline on Air Quality Models, the EPA designated system is the basis of many regulatory programs and its use is required in conjunction with State Implementation Plan (SIP) development and certain instances of federal source permitting, risk assessments, and exposure analyses. Both AERMOD and ISCST3 predict the highest concentrations over a selected averaging period and specified days and 
months based on source, terrain, and meteorological data input into the programs. To select the highest pollutant concentration, the two programs model all concentrations that result as the emission source constantly releases pollutants over the different meteorological conditions and as the plume interacts with the terrain.

ISCST3 was developed in 2002 by the US EPA as the third revision to the first ISCST program. Like ISCST and ISCST2, ISCST3 can evaluate plume rise and downwash for Good Engineering Practice stack heights and for shorter stacks, but it has also been refined to improve algorithms that calculate ground level pollutant concentrations at a one hour time-step or higher for area sources and for instances of dry and wet deposition. The program assumes steady-state Gaussian plume dispersion from stationary point, line, area, or volume sources and is capable of considering conditions such as complex terrain, stack tip downwash, buoyancy induced dispersion, and final plume rise. Equation 1 below shows the steady-state Gaussian dispersion model in its basic form for point sources as presented by Cooper and Alley (2002).

$$
C=\frac{Q}{2 \pi \mathrm{u} \sigma_{\mathrm{y}} \sigma_{\mathrm{z}}}\left[\exp \left(-\frac{1}{2} \frac{(z-H)^{2}}{\sigma_{\mathrm{z}}^{2}}\right)+\exp \left(-\frac{1}{2} \frac{(z+H)^{2}}{\sigma_{\mathrm{z}}^{2}}\right)\right]
$$

where $\quad \mathrm{C}=$ steady-state concentration at a point $(\mathrm{x}, \mathrm{y}, \mathrm{z}), \mu \mathrm{g} / \mathrm{m}^{3}$

$\mathrm{Q}=$ emissions rate, $\mu \mathrm{g} / \mathrm{s}$

$\sigma_{\mathrm{y}}, \sigma_{\mathrm{z}}=$ horizontal and vertical spread variables, $\mathrm{m}$ (as functions of distance, $\mathrm{x}$, and atmospheric stability) $\mathrm{u}=$ average wind speed at stack height, $\mathrm{m} / \mathrm{s}$ 


$$
\begin{aligned}
& \mathrm{z}=\text { vertical distance from ground level, } \mathrm{m} \\
& \mathrm{H}=\text { effective stack heat }(\mathrm{H}+\Delta \mathrm{h}, \text { where } \mathrm{h}=\text { physical stack height and } \Delta h= \\
& \quad \text { plume rise, } \mathrm{m})
\end{aligned}
$$

To calculate ground level concentrations, ISCST3 considers meteorology, including wind profiles, vertical temperature gradients, and high or calm winds (US EPA, 1995a; US EPA, 1995b).

With the help of Electric Power Research Institute (EPRI), the US EPA also developed ISC-Plume Rise Model Enhancement (ISC-PRIME), to improve predictions of building downwash where shorter stacks may cause plumes to become entrained around nearby structures. ISC-PRIME uses the same algorithms as ISCST3 with the exception of the building profile input program (BPIP), which has succeeded in improving representation of the building downwash effect (EPRI, 1997). These improvements are largely a result of considering properties and interactions such as stack location relative to the building, streamline deflection over the building, speed deficits or shear impacts on a plume in a wake, and connections between two downwash algorithms (US EPA, 2003).

While a number of options are available on ISCST3 and ISC-PRIME (ISCST3 combined with BPIP), the program has several shortcomings often resulting in an overestimation of pollutant concentrations in unstable conditions (US EPA, 2003; Trinity Consultants, 2001). The American Meteorological Society/Environmental Protection Agency Regulatory Model Improvement Committee (AERMIC) was formed in 1991 to produce a 
program with more representative modeling capabilities than the US EPA's ISC programs. The result of AERMIC's work was AERMOD, designed to overcome ISCST3's weaknesses while also being suitable for complex terrain and application over distances less than $50 \mathrm{~km}$ (40 CFR Part 51). Although AERMOD also uses a steady-state Gaussian dispersion model, it was developed to manage deviations from Gaussian distributions, largely by considering variations in the planetary boundary layer (PBL) and through more realistic representation of meteorology and plume rise. For example, to account for vertical variations, a bi-Gaussian distribution is used in the vertical component of the convective PBL, where turbulence results due to considered characteristics such as friction and convective velocity, mechanical and convective mixing height, and sensible heat flux. In comparison to ISCST3, which considers only wind profiling, AERMOD additionally considers temperature and turbulence profiles. Changes in plume rise and dispersion are also better represented in AERMOD, because plume variables are considered in at least two locations for the plume rise and throughout the plume depth for plume dispersion. ISCST3 considers only emission variables at one point, the stack top, for plume rise and plume dispersion.

While AERMOD's modeling capabilities are often superior to ISCST3, the model type used in this study depended on the availability of meteorological data. Table 1 below summarizes the meteorological inputs required for both models. 


\section{Table 1. Required Meteorological Inputs for ISCST3 and AERMOD}

\begin{tabular}{|l|l|}
\hline \multicolumn{1}{|c|}{ ISCST3 } & \multicolumn{1}{c|}{ AERMOD } \\
\hline Hourly Flow Vector & Hourly Flow Vector (deg.) \\
Hourly Wind Speed & Hourly Wind Speed (m/s) \\
Hourly Ambient Temperature & Hourly Ambient Temperature (K) \\
Hourly Stability Class & Hourly Solar Radiation \\
Hourly Rural Mixing Height & Hourly Cloud Cover \\
Hourly Urban Mixing Height & Surface characteristics \\
& Twice daily upper air soundings \\
\hline
\end{tabular}

\subsection{Review of ISCST3/ISC-PRIME and AERMOD Performance}

A quantitative evaluation between AERMOD and ISCST3 performance and differences was conducted to understand typical modeling results. Because AERMOD is the current EPA preferred model, this study included a review of AERMOD to ISCST3 ratios and AERMOD and ISCST3 performance in scenarios similar to those found in this study's evaluated site. The characteristics of focus include rural locations, complex terrain, short stacks, and cases were building downwash are significant.

Several studies and field experiments have been conducted, compiled, and reviewed to compare predictions and performance of AERMOD and ISCST3. The US EPA has been a driving force in these studies to develop a regulatory procedure intended to protect public health. Using AERMOD (version 02222), ISCST3, and ISC-PRIME, the US EPA (2003) tested a variety of scenarios in Pittsburg, PA and Oklahoma City, OK observing the impacts of several site and source characteristics: simple and complex terrain, building downwash, rural and urban areas, and source type (point, line, area, and volume). Among the EPA evaluations, 28 cases were for complex terrain and 60 cases focused on building downwash. 
The US EPA study noted ISC programs to perform less accurately for locations with complex terrain than for locations with simple and flat terrain (ISCST3) and locations where building downwash played a significant role (ISC-PRIME). When terrain was complex, ISCST3 has been seen to largely over predict concentrations and to predict concentrations much higher than AERMOD. The average AERMOD to ISCST3 ratio for short stacks over 1 hour and 3 hour averaging times was 0.26 with ratios ranging from 0.1 to 0.51 . The large difference in concentrations estimated by AERMOD and ISCST3 are attributed to the COMPLEXI modeling component found in ISCST3 that was originally designed to be a conservative screening program for complex terrain (US EPA, 2003). Over all cases of complex terrain, differences between AERMOD and ISC-PRIME concentrations were also found to increase with increasing concentration averaging time.

The building downwash component of the US EPA analyses compared AERMOD (with BPIP) and ISC-PRIME results and found that AERMOD to ISC-PRIME ratios were on average close to 1 , ranging from 0.87 to 1.05 , including all averaging times ( 1 hour, 3 hour, 24 hour, annual) when building downwash was known to be significant. When including both significant and insignificant building downwash sources, the average AERMOD to ISC-PRIME ratio for all cases and all averaging times was around 1.12, though ratios ranged widely between 0.28 and 3.46. Similarities in ISC-PRIME and AERMOD with BPIP were expected to result when downwash was significant due to similarities in the PRIME algorithms used. Thus, ratios close to 1 often resulted from running both ISC-PRIME and AERMOD when building downwash was significant. 
The performance of AERMOD and ISC programs was also evaluated based on 17 field studies by Perry et al. (2005) over flat and complex terrain, urban and rural conditions, different stack heights, and with and without wake effects.

Field studies evaluated by Perry et al. for complex terrain all taking place in rural locations often found AERMOD to perform more accurately than ISCST3. For example, an elevated weakly buoyant release of $\mathrm{SF}_{6}$ in mountainous terrain in Tracy, Illinois found a predicted-to-measured concentration ratio of 1.07 for 1 hour AERMOD concentrations, while ISCST3's predicted-to-measured concentration ratio was on average about 2.81 . AERMOD predicted-to-measured ratios were close to unity for 3-hour highly buoyant releases of $\mathrm{SO}_{2}$ where building downwash was not expected, but ISCST3 predicted-tomeasured ratios varied widely at 0.98 for Clifty Creek, Indiana, 8.20 for Lovett, New York, and 11 for Westvaco, Maryland. Two studies for Duane Arnold Energy Center (DAEC) and Experimental Organic Cooled Reactor (EOCR) in Idaho Falls, Idaho conducted in locations containing terrain variations, short stacks, and non-buoyant releases of $\mathrm{SF}_{6}$, however, resulted in greater similarity between ISC-PRIME and AERMOD. In those cases, building downwash was significant and ISC-PRIME predicted-to-measured ratios were on average 0.38 and 1.69, for DAEC and EOCR, respectively, fairly similar to AERMOD predicted-to-measured ratios of 0.51 and 1.72 , respectively. When ISC-PRIME was run instead of ISCST3, the degree of over prediction of concentrations was reduced, and under prediction instead occurred more often. 
In comparing the accuracy of AERMOD and ISCST3 predicted ground level concentration, Perry et al., similar to the US EPA (2003) found that overall, ISCST3 tends to over predict concentrations and predicted concentrations tend to be higher than AERMOD estimates. Additionally, Perry et al. found that AERMOD and ISCST3 are capable of simulating near-field dispersion for near surface releases, but are less accurate in convective and stable conditions. Paine et al. (1998), in a study prior to Perry et al. and using one of the same field studies, also mentioned that concentrations occurring within a kilometer of the stack were under predicted, but noted predictions over longer distances to be fairly accurate. Paine et al. also recognized that under predicted near-field concentrations were small and thus modeled-to-observed concentration ratios were insignificant. Still, although AERMOD's near-field performance could use some improvement, its use of a bi-Gaussian distribution in convective conditions makes it better able to predict concentrations than ISCST3.

Venkatram et al. (2004) conducted a much smaller study to evaluate the performance of ISC-PRIME and AERMOD with and without PRIME in predicting concentrations under downwash conditions. Within a $900 \mathrm{~m}^{2}$ flat parking lot area surrounded by buildings, a small line source on top of a building was simulated using a trailer and a series of tubes. $\mathrm{SF}_{6}$ tracer was emitted and measured at 24 receptors at 2 through 5 meters and at 10 and 20 meters away from the trailer. Meteorology was also measured at one location. Although the study intended to simulate a small line source as may be found in urban 
rather than rural environments, the study evaluated dispersion modeling with ISC and AERMOD over a small area similar in size to the Cambria study site.

Results from Venkatram et al.'s study indicated that both ISCST3 and AERMOD, when used in conjunction with PRIME, overestimated high concentrations and underestimated lower concentrations. This similarity between modeled ISCST3 and AERMOD concentrations run with PRIME was expected as in US EPA's (2003) study, due to the matching PRIME algorithms used. For higher observed concentrations, ISC-PRIME and AERMOD used with PRIME often predicted concentrations greater than a factor of 2 above the observed concentration. When AERMOD was used without the PRIME algorithm, modeled concentrations were higher than measured values, but typically within a factor of two.

\subsection{Study Origins}

Because studies contributing to the understanding of near-field spatial variability are limited, in the winter months between November 2008 and March 2009, Ward (2009) and Malejan (2009) conducted parallel studies to better understand wood smoke BC exposure within a $1 \mathrm{~km}^{2}$ area in Cambria, California. Their study utilized an array of personal environmental monitors (PEMs) and aethalometers to measure $\mathrm{BC}$ concentrations generated by winter wood burning over several 12 hour time periods. Air samples were collected both indoors and outdoors and wood burning sources within the study area were identified using an infrared camera, which identified high chimney temperatures. Each 12-hour sampling and source identification period was identified as 
an intensive operation period (IOP). Ward's analysis of the data included observation of the spatial variation of $\mathrm{BC}$ concentrations within the study area and determination of correlations between measured black carbon concentrations, meteorological conditions, and burning source data over six IOPs. Malejan's analysis observed the source of BC through the analysis of light attenuation over the spectrum of wavelengths and modeled $\mathrm{BC}$ infiltration based on indoor and outdoor aethalometer measurements during IOPs. This study aimed to expand on Ward and Malejan's study, and thus their results and conclusions are summarized below. Goals for this study include (1) further understanding pollutant variability within a near-field area in an effort to identify potential public exposure (2) further evaluating the relationship between meteorological conditions and burning source information, in addition to geographic details, and (3) identifying the performance of existing dispersion models in application to the near-field based on available input variables. In this study, the same sampling methodology as conducted in Ward and Malejan's study was used, but meteorological data was also collected at two locations where sampling took place. This is explained in detail in Chapter 3.

\subsubsection{Conclusions from Ward (2009): Spatial Variation and Correlation Analysis}

To characterize near-field BC variability, Ward (2009) evaluated statistics of measured BC samples (i.e. averages and standard deviations) and used statistical methods to identify variables that influence $\mathrm{BC}$ variability. Using the repeated measures analysis of variance (ANOVA) statistical test, Ward found statistically significant differences between $\mathrm{BC}$ concentrations at sampling sites within the $1 \mathrm{~km}^{2}$ study area. Over a single 
IOP, aethalometers detected BC concentration differences up to $3.27 \mu \mathrm{g} / \mathrm{m}^{3}$. BC concentrations measured using Personal Environmental Monitors (PEMs) differed at most by $0.76 \mu \mathrm{g} / \mathrm{m}^{3}$ over a single IOP. The average standard deviation and relative standard deviation of PEM BC concentrations was $0.10 \mu \mathrm{g} / \mathrm{m}^{3}$ and 77.18 percent, respectively, over all IOPs. A comparison of 12 hour BC concentrations measured by colocated PEMs and aethalometers found four out of six samples to be identical. The differences between the other two samples were attributed to differences in the optical absorption cross-sectional area used.

To determine the effect of burning sources and available meteorological variables on $\mathrm{BC}$ concentrations, the development of multiple linear regression (MLR) analyses were attempted, but proved unsuccessful. Meteorological data was acquired from an existing Weather Underground station, Marine Terrace, located 1.89 miles south of the study area, and evaluated variables included wind speed, wind speed gust, wind direction, temperature, dew point, barometric pressure, humidity, and surface inversion conditions. The number of homes burning wood and their locations were also used to find correlations with $\mathrm{BC}$ concentrations. Attempts to understand $\mathrm{BC}$ concentrations using MLR include the reduction of $\mathrm{BC}$ concentration averaging time, the addition of the burning source information, and the removal of variables based on variance inflation factors (VIFs). VIFs are a statistical tool to identify correlations between variables. Correlated variables are typically removed to improve an MLR. The MLR's inability to find relationships between $\mathrm{BC}$ concentrations and meteorological variables even where relationships are known to exist was attributed to a shortage of available data and/or 
missing variables that may contribute to $\mathrm{BC}$ concentration variability. In another attempt to understand the effect of meteorological variables on BC concentrations, Ward utilized a weighted function which indicated $\mathrm{BC}$ concentration to be proportionally related to the numbers of homes burning and inversely proportional to distance from burning sources, the sine function of the angle between the source and the wind direction, and wind speed. All tests found no suitable MLR models.

This study expands upon the aethalometer and PEM BC data set available to better identify the magnitudes of near-field BC variability in the Cambria study site. In addition, the larger sample size was used to again evaluate the relationship between meteorological and geographic predictors through statistical means, and different strategies were taken to observe predictor impacts on $\mathrm{BC}$ variability.

\subsubsection{Conclusions from Malejan (2009): Indoor/Outdoor Infiltration and Spectral Dependence of Organic Carbon}

Malejan's (2009) study, unlike Ward's (2009) study, evaluated the relationship of indoor and outdoor BC exposures and observed the spectral dependence of BC light attenuation. Based on indoor and outdoor BC concentrations measured by aethalometers, Malejan found the average indoor to outdoor $(\mathrm{I} / \mathrm{O})$ ratio was $0.75 \pm 0.05$ for 16 burning events. This was comparable to previous studies where observed average EC I/O ratios were 0.85 \pm 0.46 (Geller et al., 2002) and BC I/O ratios were observed at $0.6 \pm 0.2$ (Lunden et al., 2008). In exploring infiltration models, Malejan determined a simplified version of the infiltration equation developed by the Lawrence Berkley National Laboratory (LBNL) 
(Thatcher et al., 2001) to best represent measured indoor and outdoor BC concentrations (Equation 2 below).

$$
\frac{\partial C_{I}}{\partial t}=C_{0} P \lambda_{V}-C_{1}\left(\lambda_{V}-k_{d e p}\right)
$$

Equation 2

$$
\text { where } \begin{array}{ll}
\mathrm{C}_{\mathrm{I}}\left(\mu \mathrm{g} / \mathrm{m}^{3}\right) & =\text { indoor particle concentration at time } t, \\
\mathrm{C}_{0}\left(\mu \mathrm{g} / \mathrm{m}^{3}\right) & =\text { outdoor particle concentration at time } t, \\
\mathrm{P}(\text { dimensionless }) & =\text { penetration factor, } \\
\Lambda_{\mathrm{V}}\left(\mathrm{h}^{-1}\right) & =\text { air exchange rate due to infiltration, } \\
\mathrm{k}_{\mathrm{dep}}\left(\mathrm{h}^{-1}\right) & =\text { deposition loss rate }
\end{array}
$$

Infiltration variables including air exchange rate, deposition rate, and penetration factors were determined using SOLVER, a sum of squared difference minimizing tool, in Microsoft Excel. Under the assumptions of constant deposition rate and penetration factor and a varying infiltration rate, deposition rate was $0.10 \pm 0.02 \mathrm{hr}^{-1}$, penetration factor was $0.94 \pm 0.03$, and air exchange rate was $0.35 \pm 0.07 \mathrm{hr}^{-1}$.

Using Beer's law as discussed in Section 2.1.3.1, Malejan confirmed the assumption that most BC samples collected were contributed by wood smoke. Adsorption Ångström exponents (AAE) observed by Malejan were close to the value of 2 for six out of eight PEM filter samples. The average specific attenuation coefficients at 370 and $450 \mathrm{~nm}$ wavelengths for the six wood smoke samples were $6.1 \pm 1.4 \mathrm{~m}^{2} / \mathrm{g}$ and $2.1 \pm 0.6 \mathrm{~m}^{2} / \mathrm{g}$, respectively. The average spectral dependence across all wavelengths between 350 and 
$880 \mathrm{~nm}$ was $\lambda^{-1.7}$ and at wavelengths less than $600 \mathrm{~nm}$, spectral dependence was $\lambda^{-2.24}$ based on power law regressions.

In addition to observing spectral dependence of light attenuation to confirm that $\mathrm{BC}$ was of wood smoke origin, Malejan's analysis also included a comparison of measured EC and BC concentrations using the NIOSH 5040 and LBNL's thermal optical analysis (TOA) method, respectively, and observation of levoglucosan analysis (see section 2.1.3). Total carbon (TC) collected was also measured using both methods. The average ratio of LBNL TOA/NIOSH 5040 for TC was $1.44 \pm 0.91$ using a 95\% confidence interval. The LBNL TOA EC/NIOSH $5040 \mathrm{BC}$ ratio was $2.11 \pm 0.67$, supporting past research that indicated large variation in EC fractions based on methods used (see section 2.1.2). A comparison of $B C$ measured from $L B N L E G A$, aethalometers, and LBNL light attenuation yielded an average LBNL TOA/aethalometer ratio of $1.21 \pm 0.25$ and an average LBNL TOA/LBNL attenuation ratio of $1.05 \pm 0.30$. Levoglucosan was found to comprise $5.9 \pm 3.7$ percent of total NIOSH OC and $5.4 \pm 3.1$ percent of NIOSH TC.

While this study does not delve into indoor BC exposure, Malejan's results draw several valuable conclusions for this study: the relationship between indoor and outdoor BC is important for understand exposure within the study area, wintertime Cambria BC originates primarily from wood smoke, and analytical methods for measuring BC may differ significantly from one another. This study expanded the sample size of observable AAE values, further demonstrating wood smoke as the principal source of BC. 


\section{Chapter 3. Materials and Methods}

The BC sampling process and periods, instrument and meteorological station details, and analytical methods are summarized in this section.

\subsection{Study Location}

Situated off of Highway 1, a two-lane road running north and south along the California coast, Cambria lies 35 miles north of San Luis Obispo in San Luis Obispo (SLO) County, in between San Francisco and Los Angeles. The town spans about $3 \mathrm{~km}^{2}$, is populated by approximately 6,500 people, and lists tourism, light industry, and agriculture as its main industrial activities (Cambria Chamber of Commerce, 2010). The 2000 US census indicated a median population age of 50.9, and 46 percent of structures (including homes) built before 1980 and 32 percent built between 1980 and 1989 (US Census Bureau, 2000).

Several characteristics combine to make Cambria a suitable location for this study. Cambria contains very little commercial diesel traffic and industrial pollution, and is located in a county noted to have some of the lowest $\mathrm{PM}_{2.5}$ concentrations in California (American Lung Association, 2010). Thus, for the majority of the year, Cambria experiences low $\mathrm{PM}_{2.5}$ concentrations. Still, a significant fraction of homes in Cambria contain chimneys and/or wood burning stoves. US Census surveys conducted between 2005 and 2009 suggest about $3.1 \pm 2.5$ percent of homes in Cambria use wood as a primary heating fuel (US Census Bureau, 2009). The use of wood as a heating source and the abundance of homes with chimneys and wood stoves in Cambria may be a result 
of tree availability in the area in addition to the local demographic. The tree cover in the chosen $1 \mathrm{~km}^{2}$ study area comprises about 60 percent based on a visual estimate from Google Earth satellite imagery. Figure 1 details an approximated estimate of tree coverage. The prevalence of trees in Cambria offers an inexpensive alternative to petroleum heating. This may be especially relevant to a population that is largely retired or approaching retirement age. Cambria's population has a median age of 55.9, and 30.6 percent of its population is above the age of 65 years (US Census Bureau, 2009). Fixed income amongst retired populations may encourage lower cost fuel sources such as wood (Tian et al., 2004).

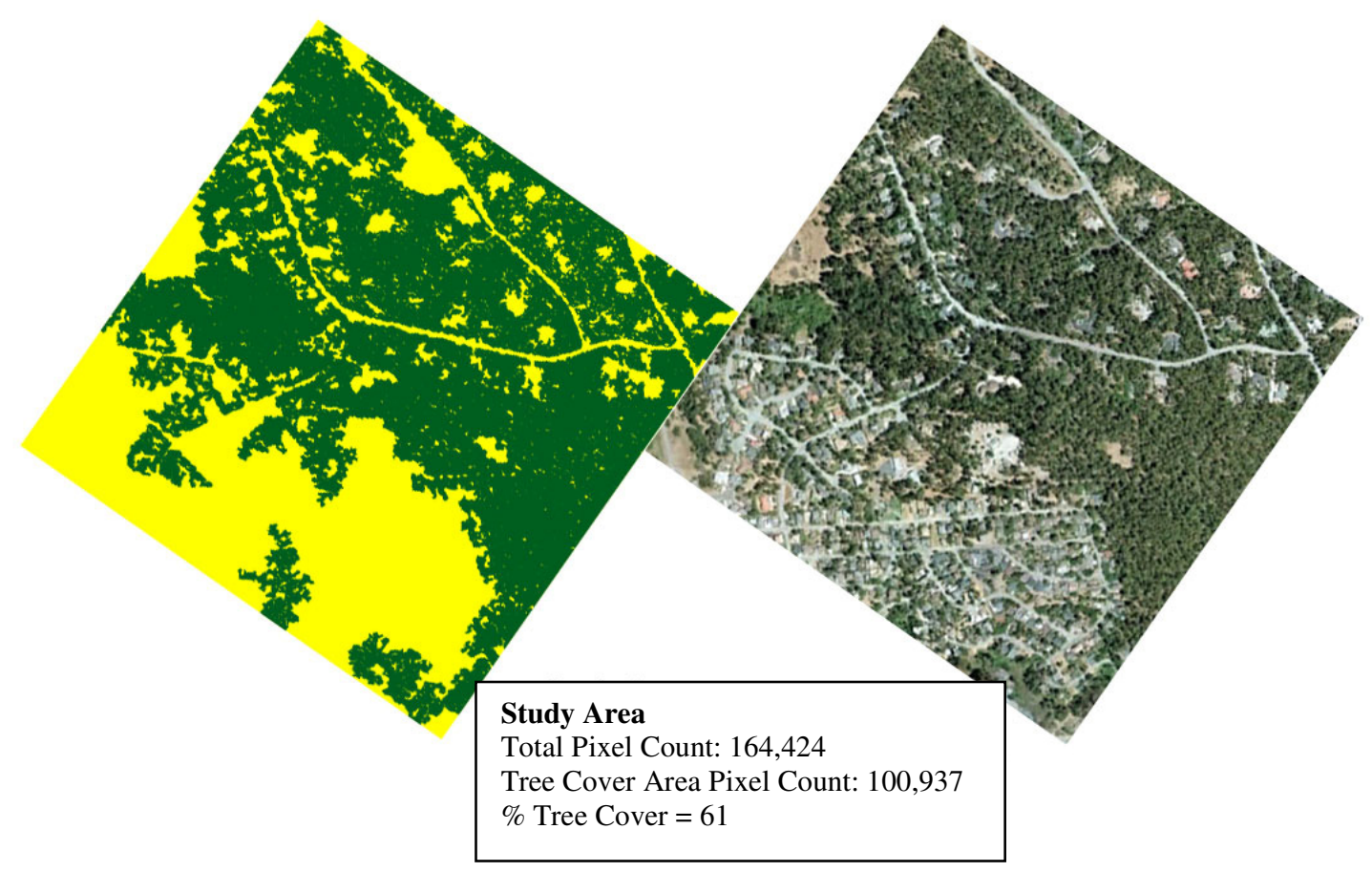

Figure 1. Approximate determination of tree coverage in study area 
While wood burning households make up a small percentage of the population, the prevalence of wood burning concentrated within residential neighborhoods may lead to frequent exposure. In SLO county, residential wood combustion was estimated to constitute 26 percent of the total winter $\mathrm{PM}_{2.5}$ in 2005 (CA ARB, 2006). Wood smoke exposure can also be enhanced by winter time coastal inversion layers in Cambria which occur due to its proximity to the ocean. Coastal inversion layers may result when air above the ocean surface is cooled by ocean upwelling and then transported to land by horizontal movement or advection. Because the transported ocean air is then situated above the land surface and is cooler than the air above it, there is no upward air movement and mixing, trapping pollutants close to human receptors. Wood smoke detected by residents has brought about health concerns, and in the winter of 2008 , the San Luis Obispo Air Pollution Control District (SLO APCD) received several complaints from Cambria residents.

Several factors made Cambria an ideal location for this study. First, Cambria's typically low $\mathrm{PM}_{2.5}$ concentrations and lack of industrial and traffic source impacts allow virtually all of the $\mathrm{BC}$ and $\mathrm{PM}_{2.5}$ in the air during the winter to be attributed to wood burning. In addition, concerned residents also provide a group of participants willing to permit the placement of air sampling equipment at their residences; volunteers from Ward and Malejan's (2009) study were again happy to permit instrument placement on their property. A low average precipitation each month of about 3.3 inches during winter 
months, December through March (The Weather Channel, 2010), also reduces removal of pollutants from the air, allowing for more potential sampling days during the winter.

A $1 \mathrm{~km}^{2}$ area within the Leimert and Happy Hills neighborhoods as shown in Figure 2 was selected for the study. The selected neighborhoods were a suitable site, because they share similar characteristics with other Cambria neighborhoods. The area contains about 400 homes, many with constructed chimneys. The terrain is both flat and hilly at elevations between about 90 and 250 feet and all streets have only single lanes in both directions, often without sidewalks. Most homes contain front yards that separate the homes from the road. In areas of higher housing density (mostly the Happy Hills neighborhood), homes are spaced about 10 feet apart, often with the backs of two houses facing one another, and almost every house contains trees on at least two sides of the residence. In the area of lower housing density (mostly the Leimert neighborhood), homes are often spaced greater than 50 feet apart with trees surrounding the homes on all sides. Cambria's downtown is located just south of the study area comprising mostly one long street running south east from the study site. The downtown area located closest to the study site consists of restaurants, novelty shops, art galleries, hotels, a couple of gas stations, and other local businesses. 


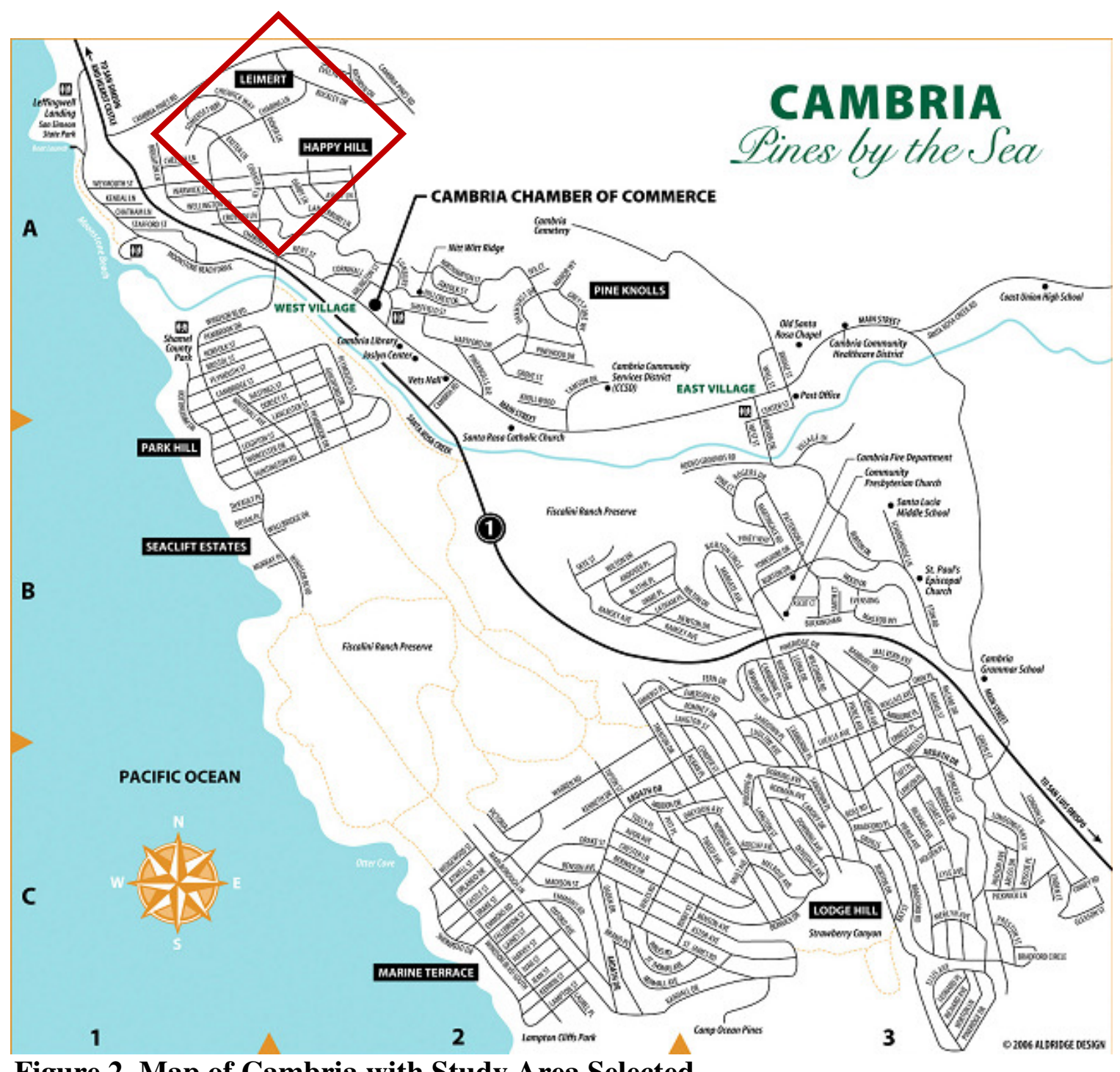

Figure 2. Map of Cambria with Study Area Selected

While Cambria provides a suitable study site for the reasons discussed above, it is important to note that pollutant variability is site specific. There is no single area that will necessarily represent all other locations where heating with wood is common. Cambria shares both geographic differences and similarities with areas where winter wood burning has contributed to high $\mathrm{PM}_{2.5}$ levels. The Central Valley, including San Joaquin Valley, for example, has been the subject of many studies due to its failure to meet $\mathrm{PM}_{2.5}$ National Ambient Air Quality Standards (Park et al., 2006; Chen et al., 2007; 
Cahill, 2010). The Central Valley primarily experiences high pollution exposure due to the prevalence of many $\mathrm{PM}_{2.5}$ emitting sources and surrounding mountains. Temperature inversions that occur within or just above the valley act to trap the particles in the valley. Although Cambria may have significant sources of $\mathrm{PM}_{2.5}$ from wood burning during the winter, its coastal location often allows for better pollutant dispersion. Cambria's neighborhoods also differ from many Central Valley locations in its neighborhood scale topography. Although many Central Valley cities are relatively flat, Cambria neighborhoods have more hills and slopes. Central Valley cities also tend to be surrounded by agriculture fields while areas around Cambria have remained undeveloped, containing expanses of woodlands.

With the exception of topography, many homes in Central Valley cities such as Fresno, Chowchilla, Sacramento, and Clovis contain neighborhood characteristics similar to Cambria. As with the higher density Happy Hills area in the Cambria study site, these Central Valley cities have similar house spacing, homes situated back to back, and trees often line at least two sides of each home. These characteristics may result in similar pollutant transport behavior including particle deposition within trees and the generation of recirculation cavities in between homes.

\subsubsection{Existing Controls and Regulations}

In California, stationary air pollution sources such as wood stoves and chimneys are controlled by air pollution control districts or air quality management districts. Cambria, California falls under the jurisdiction of the San Luis Obispo Air Pollution Control 
District (SLO APCD). SLO APCD Rule 504, Residential Wood Combustion, was adopted October 19, 1993 and became effective February 1, 1994. The rule regulates the purchase and sale of wood burning devices and the sale of wood, while also allowing the APCD to declare and notify residents of voluntary curtailment during poor air quality events. Once effective, only EPA-certified, Oregon-Certified, Pellet-Fueled Wood Heaters, or other district-approved devices could be purchased and sold. In addition, retailers of wood combustion devices were required to provide instructions for proper device installation and operation and inform customers about the health benefits of lowemitting devices. Rule 504 also requires that only wood with 20 percent or less moisture by weight can be advertised as "seasoned" or "dry" wood, and prohibits the burning of materials that produce noxious or toxic compounds including garbage, treated wood, plastic, rubber, petroleum products, paints, and coals in wood burning devices.

\subsection{Instruments}

To achieve a quantitative analysis of wood smoke concentration and the effects of meteorology on that variability, instruments were used for the purpose of measuring particles, including black carbon (BC) and PM and meteorological variables. The measurement of $\mathrm{BC}$ was chosen because it is a significant component of wood smoke and its concentration is determined using optical methods. These methods tend to be more economical than chemical methods that detect emitted organics like levoglucosan. The use of optical methods can also offer high resolution BC measurements while providing information about the combustion sources from which the particles originate.

Aethalometers and Personal Environmental Monitors (PEMs) were the selected sampling 
instruments. Meteorological instruments were selected based on their availability in the educational and local community. A Met One Instruments, Inc. E-BAM and a Young Model 81000 Ultrasonic Anemometer were obtained for this study.

\subsubsection{Aethalometers}

Three to four Magee Scientific Aethalometers were used to measure BC at 1- and 2minute resolution during the study. Aethalometers measure light attenuation (ATN) transmitted through a quartz fiber filter based on Equation 3 below

$$
A T N=100 \times \ln \left(\frac{I_{O}}{I}\right) \quad \text { Equation } 3
$$

where $\mathrm{I}_{\mathrm{O}}$ is the intensity of light transmitted in the absence of filter deposit and $\mathrm{I}$ is the intensity of light transmitted in the presence of filter deposit. BC concentration is then determined by evaluating the change in attenuation over a select period of time $(\triangle \mathrm{ATN})$ and measured based on Equation 4 below

$$
B C=\frac{\Delta A T N A}{V \sigma_{S G}}
$$

\section{Equation 4}

where $\mathrm{A}$ is the area of the filter deposit $\left(\mathrm{cm}^{2}\right), \mathrm{V}$ is the sample volume passed through the filter $\left(\mathrm{m}^{3)}\right.$, and $\sigma_{\mathrm{SG}}$ is the specific attenuation cross-section $\left(\mathrm{m}^{2} / \mathrm{g}\right)$ (typically referred to as just $\sigma$, but the "SG" subscript was added to differentiate it from the spectrally dependent mass adsorption efficiency discussed in Section 2.1.3.1). Equation 4 assumes a constant attenuation cross-section (that particles have been deposited evenly) and a linearly proportional relationship between light attenuation and mass. As discussed by Malejan 
(2009), these assumptions have been found to be false in some studies. Petzold et al. (1997) and Liousse et al. (1993) found $\sigma_{\mathrm{SG}}$ to range between 8 and $19 \mathrm{~m}^{2} / \mathrm{g}$ and 14 and 20 $\mathrm{m}^{2} / \mathrm{g}$, respectively, and filter loading was found to affect light attenuation in Weingartner et al.'s (2003) study. Weingartner et al. observed a decrease in the optical path through the filter as filter loading increased, resulting in lower reporting of concentrations. Due to the observed impact of filter loading on concentration readings, Malejan (2009) ran aethalometers in close proximity simultaneously and compared different attenuations to their respective concentrations. No filter loading impacts were observed. The same check was conducted for this study and similarly no filter loading impacts were observed.

Of the four different Magee Scientific Aethalometers used in this study, one was multiwavelength, measuring light attenuation at $370,470,520,590,660,880$, and $950 \mathrm{~nm}$ and three were dual wavelength, measuring light attenuation at 370 and $880 \mathrm{~nm}$. The wavelength of $370 \mathrm{~nm}$ is well absorbed by EC and OC, while $880 \mathrm{~nm}$ is well absorbed almost exclusively by the $\mathrm{BC}$ component of PM. The multi-wavelength and one of the dual wavelength aethalometers were of the portable variety.

Aethalometers drew air samples at flow rates of about 4 or $7 \mathrm{~L} / \mathrm{min}$, depending on aethalometer, and $\mathrm{BC}$ concentration was determined based on light attenuation measured at $880 \mathrm{~nm}$. Concentrations were then calculated based on the Aethalometer handbook recommendation for $\sigma_{\mathrm{SG}}$ of $16.6 \mathrm{~m}^{2} / \mathrm{g}$ (Hansen, 2005), which also falls well into previously observed ranges for $\sigma_{\mathrm{SG}}$ as discussed above. It is important to note that due to the algorithm used in the aethalometer, extremely low BC concentrations can be read as 
negative values. This has been attributed to desorption of organic vapors from the filter surface as relatively clean air passes through the aethalometer. This desorption results in a decrease of UV absorbing material, which is then interpreted as a negative value in the aethalometer algorithm (Hansen, 2005). To correct for negative BC readings, the lowest detection limit (LDL) was used to replace each negative value. In accordance with the aethalometer manual, the LDL was calculated assuming 1 ng noise per 20 liters air flow (Hansen, 2005).

For this study, the aethalometers were calibrated to more precisely represent one another as done by Malejan (2009) and Ward (2009). Aethalometers were simultaneously run side-by-side over a period of 24 hours once early in the winter sampling period and then a second time after sampling was completed. The multi-wavelength aethalometer was not tested after sampling completion because the aethalometer filter tape was unexpectedly used up and changing the tape would have changed calibrated $\sigma_{\mathrm{SG}}$ values. Determined $\sigma_{S G}$ values for winter 2010 demonstrated a percent difference from between 17 and 31 percent in comparison to values for winter 2009. The difference between 2009 and 2010 $\sigma_{\mathrm{SG}}$ values may be attributed to variation in flow rates and conditions for each aethalometer run.

For each side-by-side aethalometer run, the $\sigma_{S G}$ values for each aethalometer was reduced or increased over one time interval of measured $\mathrm{BC}$ concentrations to achieve the average $\mathrm{BC}$ concentration of all aethalometer $\mathrm{BC}$ readings. The $\sigma_{\mathrm{SG}}$ values were then applied to the second time interval of $\mathrm{BC}$ concentrations and a statistical Analysis of Variance 
(ANOVA) conducted in Minitab on the adjusted readings in the second time interval to determine whether significant differences existed between the different aethalometers. The ANOVA test evaluates whether or not significant differences exist under the assumption that the responses, or measured $\mathrm{BC}$ concentrations in this case, are independent from one another, are normally distributed, and that the samples from each machine have equal standard deviations. Based on probability distributions, the ANOVA test determines whether the mean response for each machine deviates enough from the mean response of all machines to be considered different. If differences were found based on the ANOVA test, $\sigma_{\mathrm{SG}}$ was adjusted until the test indicated no statistical difference between machines. $\sigma_{\mathrm{SG}}$ values were only adjusted using time segments where all machines were similar in concentration-trends. Selecting only segments displaying the same behavior eliminates influences of readings that deviated significantly from the others. $\mathrm{BC}$ readings were averaged over ten minutes to reduce noise in the calibration. Values for $\sigma_{S G}$ determined for the two simultaneous runs were averaged and used for the study readings. Minitab analysis and graphical representation of data before and after calibration are detailed in Appendix A.

Available aethalometers were run over each 12 hour sampling period. All aethalometers were co-located with a PEM and placed outside of the homes of volunteers in locations where they would not be disturbed and where an outdoor power source was available. 


\subsubsection{Personal Environmental Monitors}

BC was also measured using SKC Inc. Model 200 Personal Environmental Monitors (PEMs). The PEMs select for $\mathrm{PM}_{2.5}$ and their main components include an outer-casing with inlet holes, an air outlet, where the pump is connected, an impaction ring, onto which particles larger than $2.5 \mu \mathrm{m}$ land and stick, and a stainless steel screen that supports a quartz fiber filter. An additional mask containing a $2 \mathrm{~cm}$ opening was used to decrease the deposit area and thus increase measurement sensitivity. Before each sampling period, PEMs were cleaned with methanol and allowed to dry completely to ensure all carbon particles were removed. In assembling the PEMs, filters were transferred with sanitized tweezers to minimize contamination, and a mineral oil was applied to the impaction ring to improve the capture of particles larger than $2.5 \mu \mathrm{m}$. A PEM assembly is shown in Figure 3. Once assembled, all PEMs were placed inside of a single plastic bag for transport.

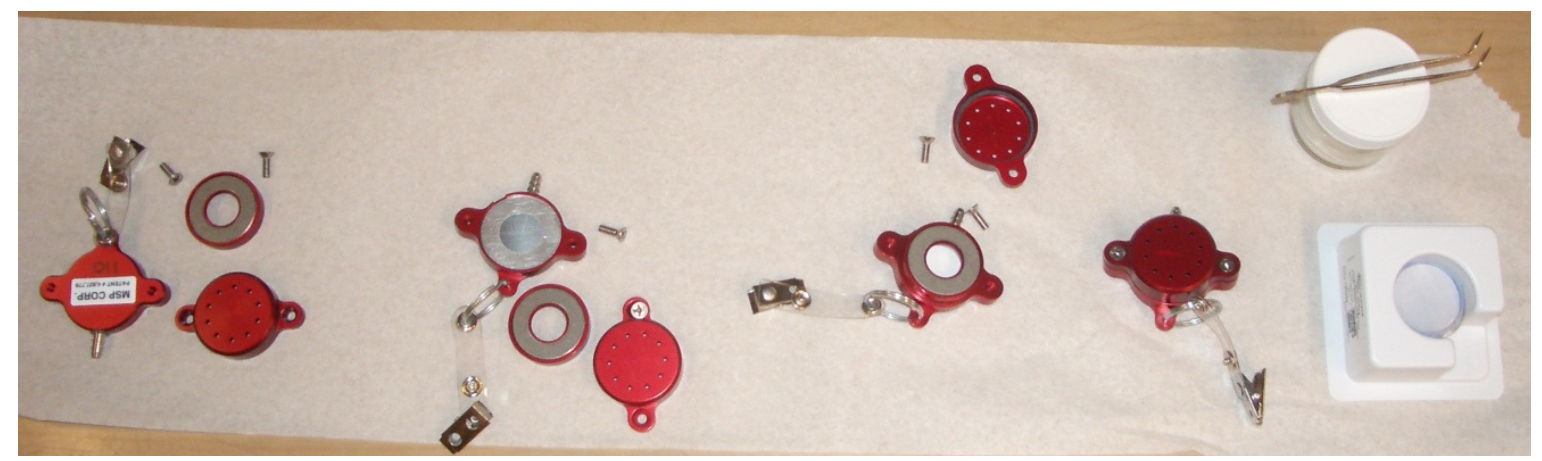

\section{Figure 3. PEM Assembly}

Photograph was taken by Malejan (2009)

The Model $200 \mathrm{PEM}$ is designed to operate at a $10 \mathrm{~L} / \mathrm{min}$ flow rate. Leland Legacy flowcontrolled pumps (SKC Inc.) were used to achieve the desired flow rate. The pumps 
were calibrated using a Model DC-HC-1 Bios DryCal DC-2 calibrator. To protect the pumps and decrease noise, the pumps were placed inside of a plastic container surrounded by pieces of foam during sampling. A tube-sized circular opening was made in the plastic cases to allow its connection to a PEM. Figure 4 shows this configuration.

\section{Figure 4. PEM Casing}

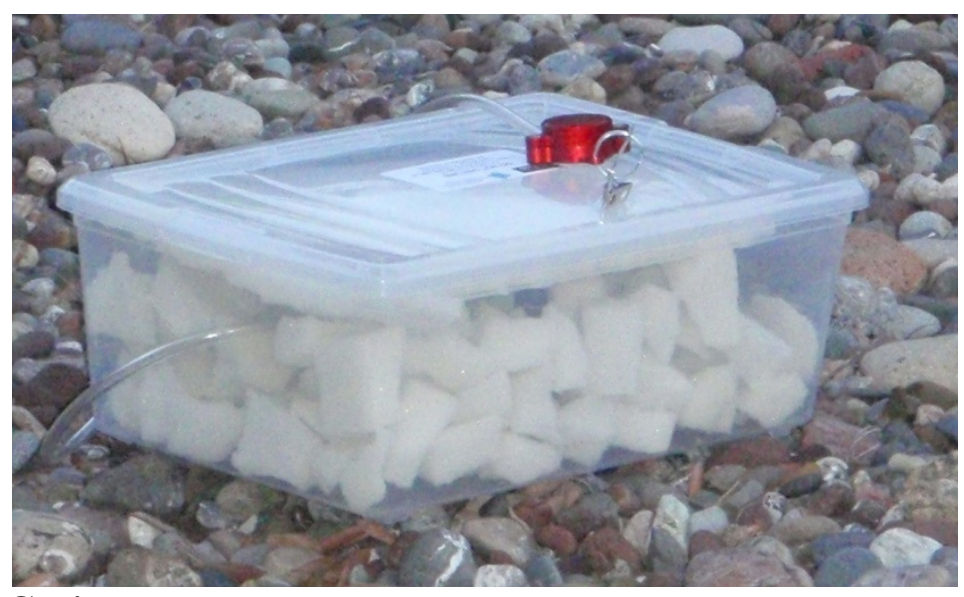

Photograph was taken by Malejan (2009)

In preparation for each IOP, all SKC pumps were programmed to run for 12 hours either between 6 p.m. and 6 a.m. or 5 p.m. and 5 a.m. Each cleaned PEM was connected to an SKC pump at the PEM sampling location and a 12-hr integrated air sample was taken. Each aethalometer used was co-located with a PEM for comparison purposes.

After each IOP, PEM filters were placed in individual petri dishes, each petri dish was sealed with Teflon tape, all petri dishes were bundled together, wrapped in foil, and the samples were sent to Lawrence Berkeley National Laboratory (LBNL) to be analyzed. Light attenuation of filter samples over the near ultraviolet to infrared spectrum, 350 to $1000 \mathrm{~nm}$, respectively, was determined using an LBNL custom built transmissometer 
which uses light emitting diodes (LEDs) to send out light. To prevent high leveraging in analyses by PEM filters containing BC at concentrations lower than the detection limit, $\mathrm{BC}$ readings were assumed to be midway between zero and the lower detection limit (lower detection limit divided by two), $30.2 \mathrm{ng} / \mathrm{m}^{3}$.

\subsubsection{E-BAM}

A Met One Instruments, Inc. E-BAM (Beta Attenuation Monitor) 9800 Rev K borrowed from the San Luis Obispo Air Pollution Control District (SLO APCD) was used to both measure $\mathrm{PM}_{10}$ and meteorological variables. Meteorological variables including wind speed, wind direction, relative humidity, and temperature were measured to better characterize the impacts of local weather patterns on pollutant dispersion. The E-BAM has been found to satisfy EPA Class III criteria for $\mathrm{PM}_{2.5}$ and $\mathrm{PM}_{10}$, potentially making it suitable as a substitute for Federal Reference Methods (MDEQ \& MCCHD, 2010).

The E-BAM measures particles by emitting beta rays onto a glass fiber filter through the decay of ${ }^{14}$ Carbon. ${ }^{14}$ Carbon is a naturally occurring radioactive isotope whose nucleus constantly emits low to medium energy electrons known as beta particles. These beta particles lose their energy when they come into contact with nearby matter and are collectively known as beta rays. As the beta rays are emitted, beta particles are first counted by a Photo Multiplier Tube (PMT) for 4 minutes on a clean glass fiber filter. The PMT then takes a second count of beta particles over another 4 minutes as air is sampled. When particles in the air are deposited on the filter, beta particles easily lose their energy to and sometimes absorb to matter from the incoming air. In the presence of 
particles from the air, a reduction in beta ray intensity occurs, the PMT detects this, and the mass of absorbing matter is determined as a function of the change in beta particles detected. The difference in beta particles is used to determine the PM concentration based on Equation 5 and Equation 7. The mass density of the absorbing material is measured by solving for $\mathrm{x}$ in Equation 5 below:

$$
I^{\prime}=I_{O}{ }^{\prime} e^{-\mu x}
$$

\section{Equation 5}

where I' is the beta ray intensity in counts per unit time after sampling, Io' is the beta ray intensity in counts per unit time before sampling, $\mu$ is the absorption cross-section of the material absorbing the beta rays in $\mathrm{cm}^{2} / \mathrm{g}$, and $\mathrm{x}$ is the mass density of the absorbing material in $\mathrm{g} / \mathrm{cm}^{2}$. The absorption cross-section is determined during calibration, while $\mathrm{x}$ is determined by Equation 6 below. Having solved for $\mathrm{x}$, the collected particle concentration is calculated by multiplying $\mathrm{x}$ by the area and dividing by the flow rate and time as displayed in Equation 7:

$$
\begin{array}{rlr}
x & =\frac{1}{\mu} \ln \left(\frac{I_{O}{ }^{\prime}}{I^{\prime}}\right) & \text { Equation 6 } \\
P M_{i} & =\frac{10^{6} A}{Q \Delta \operatorname{t\mu }} \ln \left(\frac{I_{O}^{\prime}}{I^{\prime}}\right) & \text { Equation 7 }
\end{array}
$$


where $\mathrm{PM}_{\mathrm{i}}$ is the concentration of $\mathrm{PM}$ less than $\mathrm{i} \mu \mathrm{m}$ in $\mu \mathrm{g} / \mathrm{m}^{3}$ (for this study a $\mathrm{PM}_{10}$ Virtual Impactor inlet was used, so $\mathrm{i}$ is equal to 10), $\mathrm{A}$ is the cross sectional area of particle deposition in $\mathrm{cm}^{2}, \mathrm{Q}$ is the air flow rate in $\mathrm{L} / \mathrm{min}$, and $\Delta \mathrm{t}$ is the sampling time in minutes. Although Equation 5 is a simplification of the actual process, Met One Instruments, Inc. (2001) found no substantial error during operation.

Although the E-BAM can be run using a $\mathrm{PM}_{2.5}, \mathrm{PM}_{10}$, or TSP inlet, a $\mathrm{PM}_{10}$ inlet was used because a $\mathrm{PM}_{2.5}$ inlet was unavailable. As discussed in Section 3.1, wood smoke should be the only major contributor to $\mathrm{PM}_{2.5}$ in the study area. Additionally, $\mathrm{PM}_{10}$ is not expected to be present in the study area. In the absence of major traffic, industrial, and agricultural pollution sources, $\mathrm{PM}_{10}$ is typically found only in the form of geologic and mineral dusts. For coastal areas, sea salt can be a major contributor to $\mathrm{PM}_{10}$ during wind speeds higher than 4 meters per second (8.9 miles per hour) (Arasa et al., 2008). However, because sampling periods were selected to have low wind speeds, sea salt should not be a significant contributor to $\mathrm{PM}_{10}$ in this study. Thus, it will be assumed that $\mathrm{PM}_{2.5}$ contributed by wood smoke is the only source of $\mathrm{PM}$, and $\mathrm{PM}_{10}$ readings taken by the E-BAM will be considered to be equivalent to $\mathrm{PM}_{2.5}$ concentrations.

The E-BAM uses several sensors to measure meteorological variables. Wind speed and direction are measured using a wind combination sensor Model 034B mounted on the E$\mathrm{BAM}$; the ambient temperature sensor has a range between -50 and $50^{\circ} \mathrm{C}$ with an accuracy of $0.1^{\circ} \mathrm{C}$; and the Model EX-593 relative humidity sensor has a range between 0 and 100 percent with an accuracy of 3 percent (Met One Instruments, Inc., 2001). 
For this study, the E-BAM recorded meteorological data at the Cambria California Department of Fire (CDF) station at an elevation of 227 feet within an open field approximately 20 feet away from the closest trees and fences. Although the Met One Instruments, Inc. manual (2001) recommends placement at least 20 meters from the drip line of trees, due to particle deposition on tree surfaces, such a large amount of space was unavailable within the study area. Figure 5 shows an aerial and ground view of the EBAM site. Before operation, the E-BAM was flow and leak checked, the temperature was compared to an external thermometer, and the E-BAM was properly aligned. The EBAM was operated using the standard $\mathrm{PM}_{10}$ measurement mode, which uses a flow rate of $16.7 \mathrm{~L} / \mathrm{min}$, and $\mathrm{PM}_{10}$ measurements were taken at 10 minute and 1 hour intervals. Because the E-BAM is capable of withstanding even rainy conditions, the E-BAM was left to run during and in between IOPs, starting with the third successful IOP (IOP 4b). Meteorological data was recorded by the E-BAM in seven out of nine IOPs (4b-10b), but $\mathrm{PM}_{10}$ was only measured during six out of nine IOPs $(4 \mathrm{~b}-8 \mathrm{~b}, 10 \mathrm{~b})$ with IOP $9 \mathrm{~b}$ data unavailable because the filter tape ran out before the IOP began. Figure 7 on page 62 illustrates the E-BAM location within the study area, in addition to its proximity to other meteorological stations used for this study. The E-BAM station was expected to be one of the more representative meteorological stations used in this study due to its location within the study area and its relatively unobstructed placement in the fire station field. 


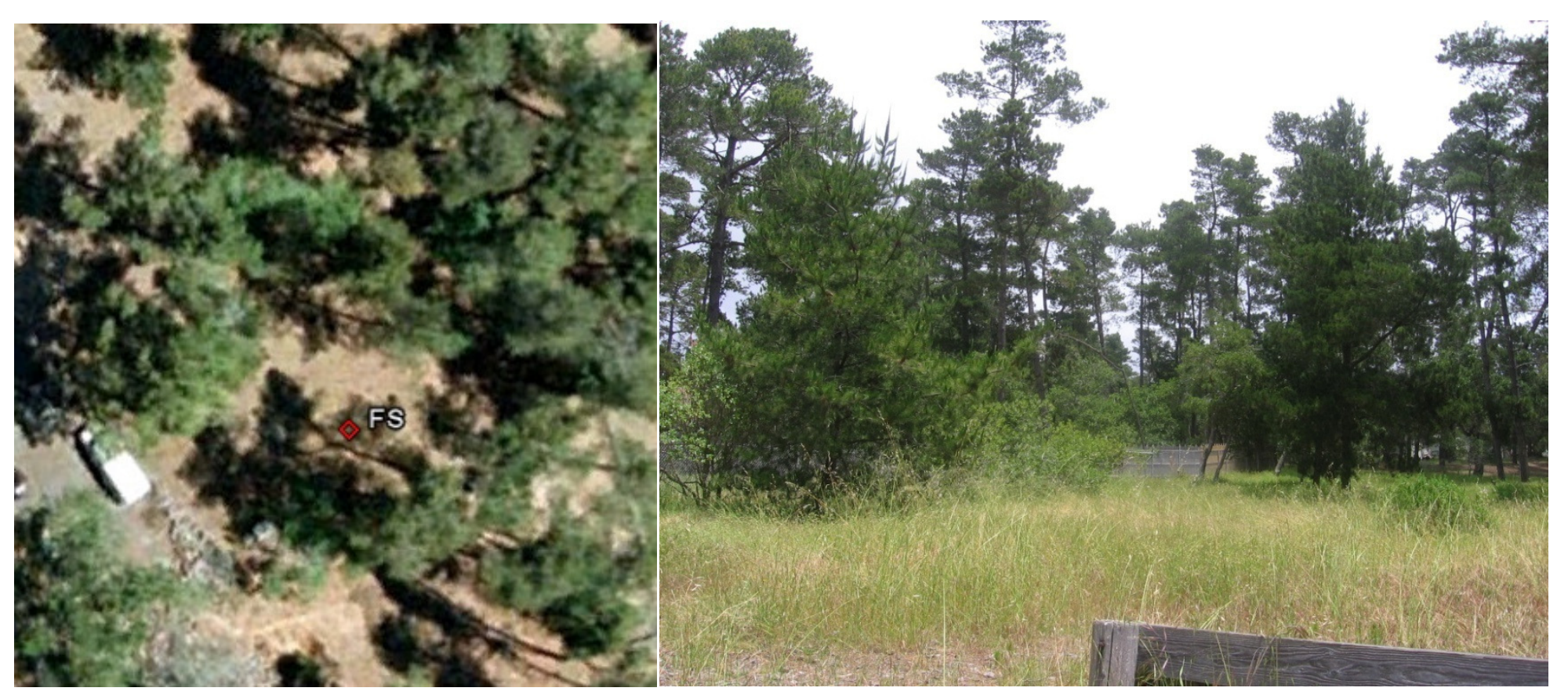

Figure 5. Aerial (left) and ground (right) view of EBAM meteorological site located in study area 


\subsubsection{Ultrasonic Anemometer}

A Young Model 81000 Ultrasonic Anemometer (Sonic) borrowed from the Lawrence Berkeley National Laboratory was also used to measure meteorological data within the study area. To determine 3-dimensional wind speed and direction, the sonic anemometer measures the transit time of ultrasonic acoustic signals between transducers. The capability to read 3-dimensional wind speed allows for observation of turbulence. The ultrasonic anemometer tracks temperature ranges between -50 and $50^{\circ} \mathrm{C}$ to correct for wake effects caused by the anemometer structure and agrees with ambient temperature within $2^{\circ} \mathrm{C}$. For this reason, the anemometer temperature was used as a proxy for ambient temperature. The ultrasonic anemometer is capable of high resolution readings, up to $32 \mathrm{~Hz}$ and its wind speed threshold is $0.2 \mathrm{~m} / \mathrm{s}$, or $0.45 \mathrm{mph}$, (any lower wind speeds are read as $0 \mathrm{~m} / \mathrm{s}$ ). Wind speed accuracy between 0 and $30 \mathrm{~m} / \mathrm{s}$ is $\pm 0.05 \mathrm{~m} / \mathrm{s}$ and wind direction between 1 and $30 \mathrm{~m} / \mathrm{s}$ is $\pm 2^{\circ}$.

In this study, the anemometer was placed in the backyard of a Cambria resident at an elevation of 207 feet approximately 5 feet away from any obstructions as shown in Figure 6. Although fewer obstructions would have been favorable, options were limited based on the configuration of the yard and availability of power. Before operation, the anemometer was aligned with true north and the temperature was compared to a thermometer. The sonic anemometer was run during three of the nine IOPs $(8 b-10 b)$ taking measurements 4 times each second. Readings were recorded directly onto a Dell laptop computer using the program HyperTerminal. The ultrasonic anemometer was colocated with an aethalometer and a PEM. Figure 7 on page 62 illustrates the Ultrasonic 
Anemometer location within the study area in addition to its proximity to other meteorological stations used for this study.

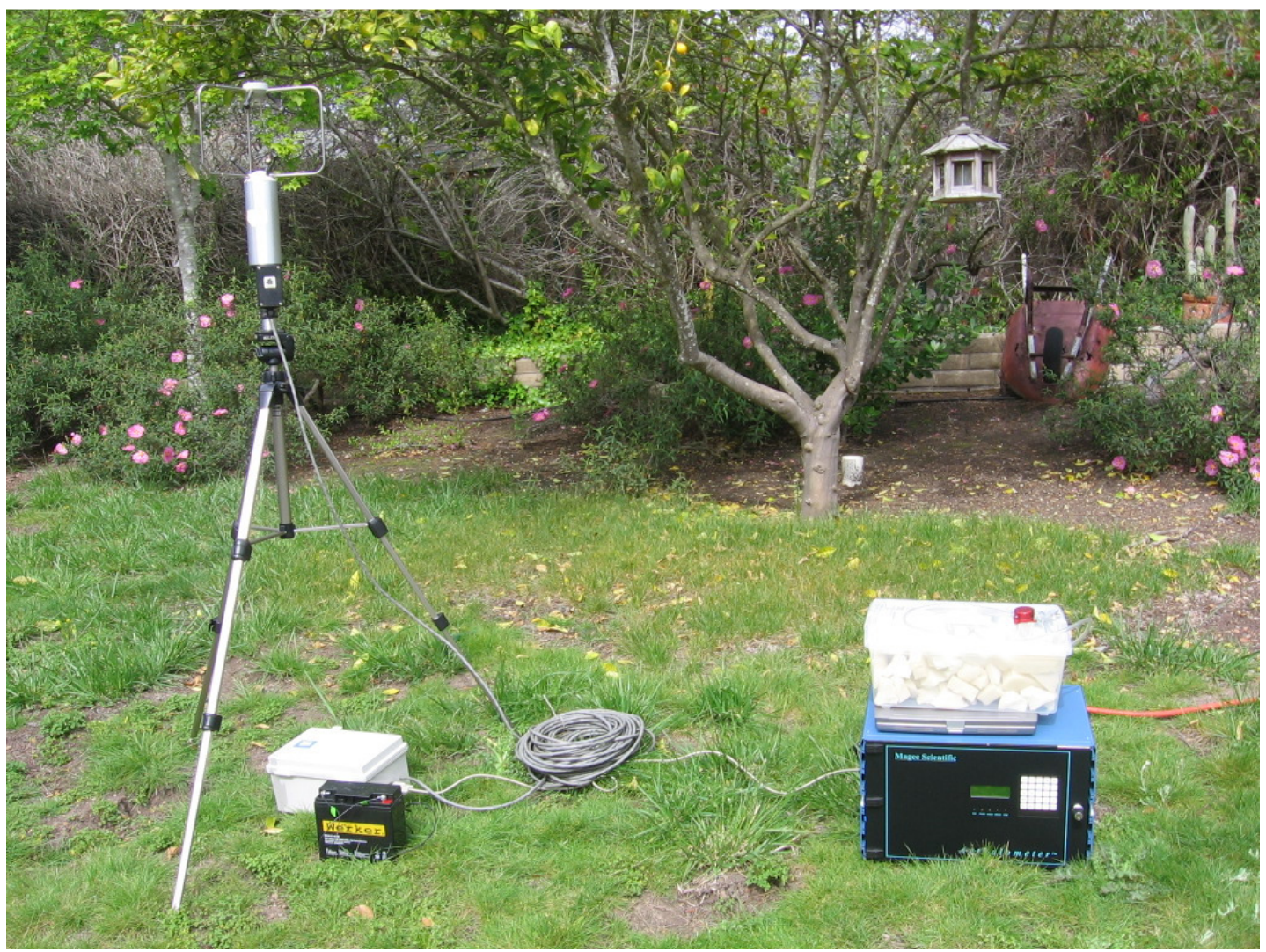

Figure 6. Ultrasonic anemometer set-up within study area

\subsection{Additional Meteorological Data}

Additional meteorological data was required for both statistical and modeling analyses. Cambria weather data was available through the Weather Underground website which provides free historical and real-time weather data in both national and international locations. In addition to providing meteorological data from its own stations, Weather Underground's Personal Weather Station (PWS) project allows any resident, organization, or business to contribute meteorological measurements in their area to the 
Weather Underground website. Although PWSs are typically not certified, Weather Underground provides free meteorological archives in areas within close proximity to the Cambria study area.

Two Weather Underground PWS stations located southeast of the study site, one in the Marine Terrace (MT) neighborhood and one in the Lodge Hill neighborhood, were chosen for statistical analysis. The MT station is located about 2 miles from the study area on the roof of a home located in a residential neighborhood close to the coast. The Lodge Hill station is located at the Cambria Community Services District Fire Department (CSDF) that is 1.8 miles from the study site. The CSDF station contains trees on the west side of the fire station building, but because the meteorological instruments are located on the fire station radio tower, approximately 25 feet above the ground, there are fewer physical obstructions. Meteorological data from an additional meteorological station was available in the Lodge Hill neighborhood at Whispering Pines Bed and Breakfast about 2.8 miles from the study area, but because the station, located on the roof of the bed and breakfast house, is surrounded by woods which would act as obstructions to wind movement, the station was not used for analysis in this study. The elevations for the MT and CSDF stations are 53 and $260 \mathrm{ft}$, respectively, and meteorological readings are recorded every 10 minutes and every 30 minutes for MT and CSDF, respectively. Weather Underground stations are displayed in a Google map image in Figure 7 along with the study area, boxed in red, to demonstrate proximity and geographic characteristics. Figure 8 and Figure 9 below shows the Marine Terrace and CSDF stations, respectively. 


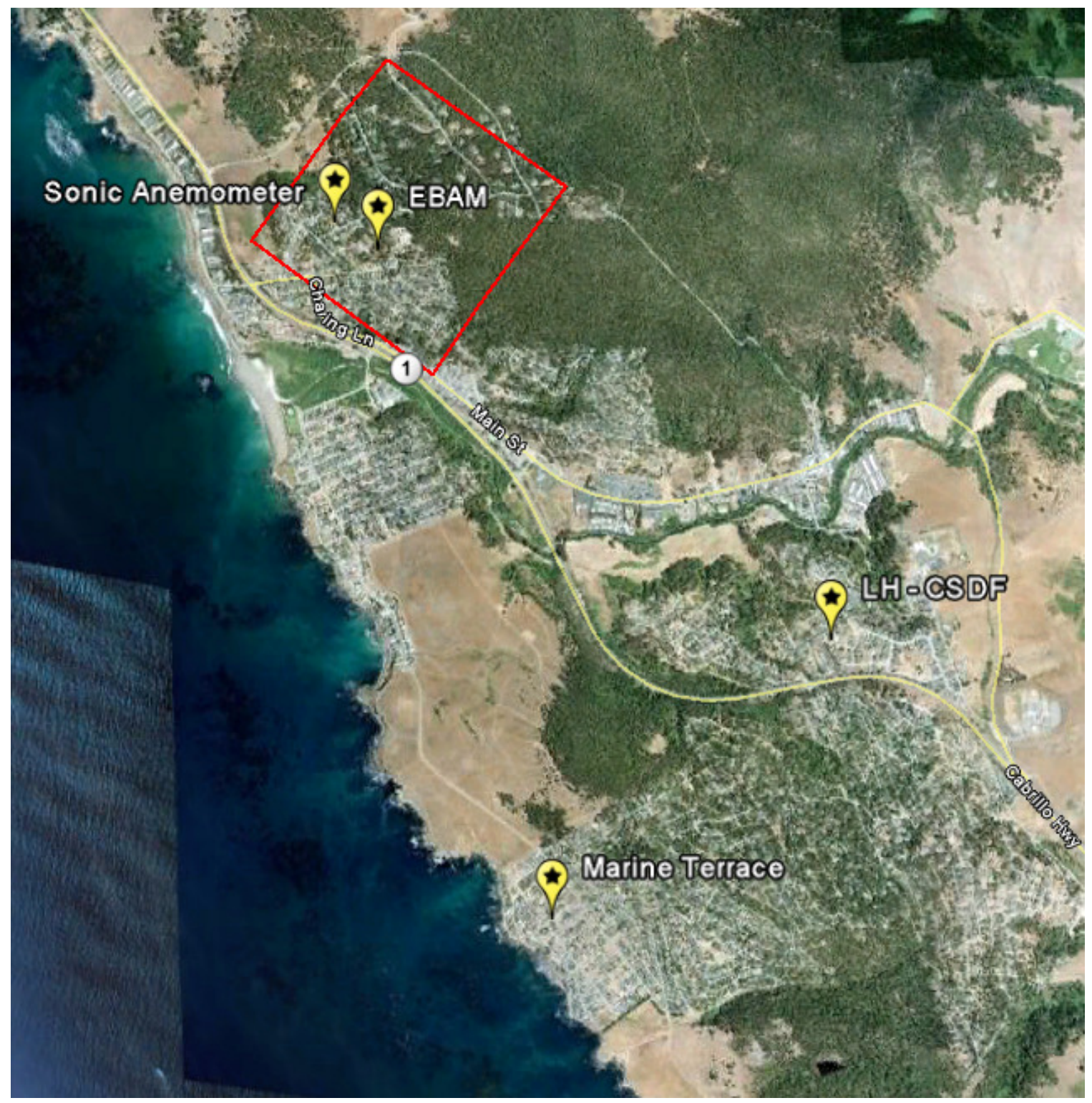

Figure 7. Map of meteorological stations relative to study area

The study area is boxed in red, while yellow-starred locations refer to meteorological stations 


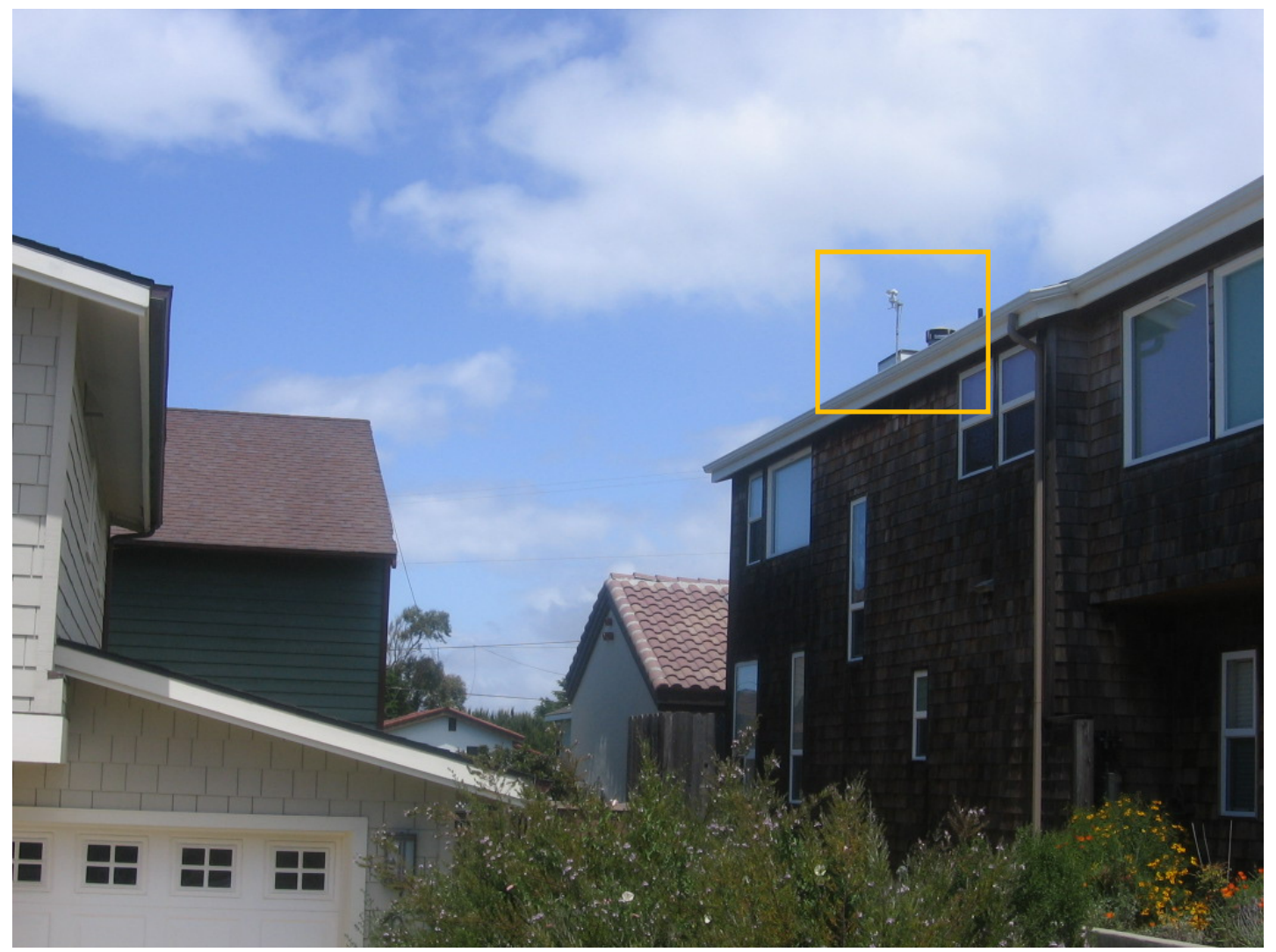

Figure 8. Marine Terrace (MT) Meteorological Station

Meteorological equipment is boxed in yellow. 


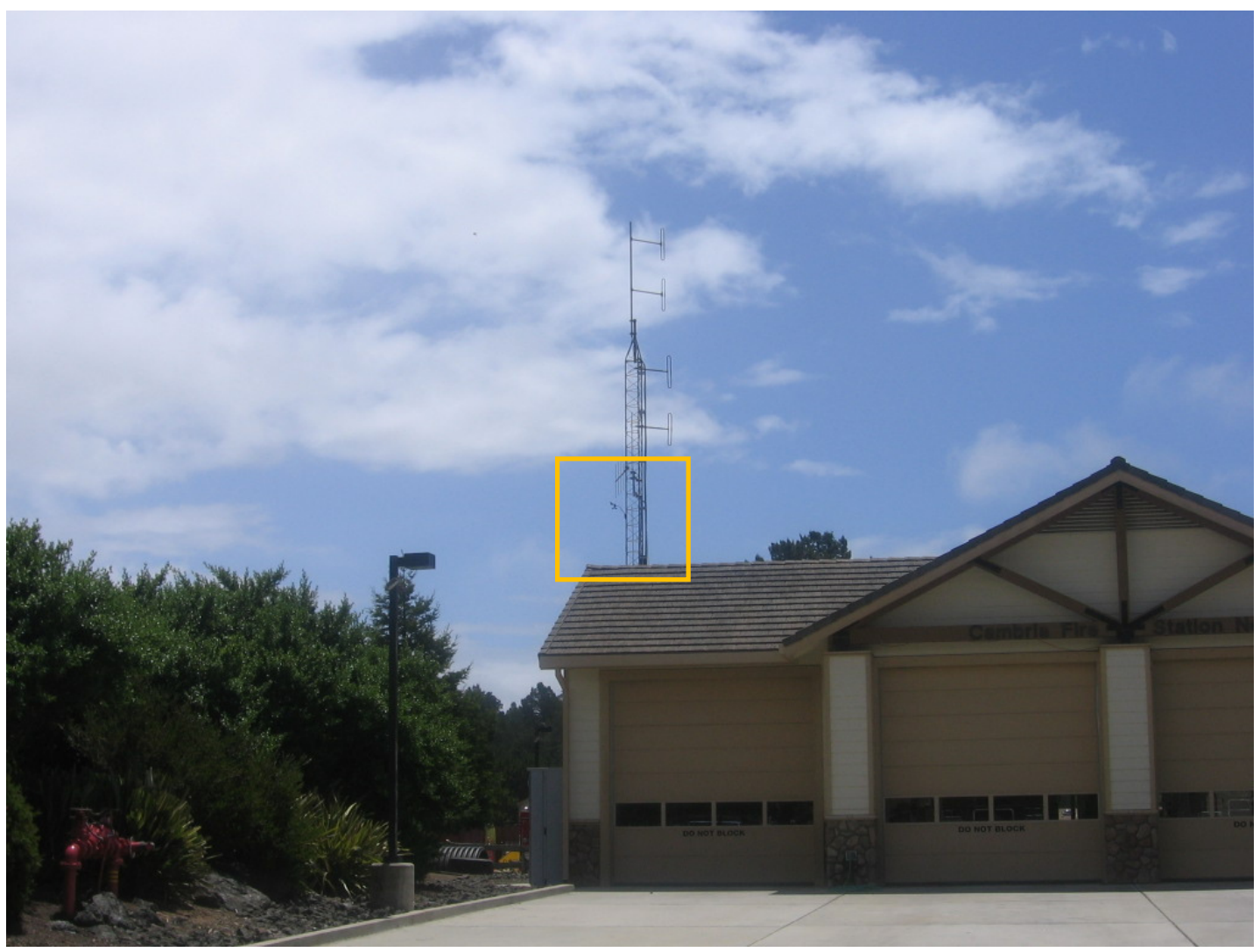

Figure 9. Community Services District Fire Department (CSDF) meteorological Station

Meteorological equipment is boxed in yellow.

While the CSDF station has not undergone certification similar to many other PWS stations, it has been evaluated for quality control through the National Oceanic and Atmospheric Administration (NOAA) Meteorological Assimilation Data Ingest System (MADIS) project, providing information about data accuracy. The results have been posted on the Citizen Weather Observer Program (CWOP) website and have indicated that over the largest available analysis period of the previous 52 weeks (approximately one year), pressure and wind vectors are correctly calibrated, while dew point readings and temperature readings demonstrated large errors (CWOP, 2010). For this reason, 
CSDF site dew point and temperature were not used. The CSDF station was expected to be most representative of the study area among available Weather Underground stations due to its similarity to the study area in topography, terrain, and proximity to the ocean.

Because dispersion modeling requires more detailed meteorological data than available through Weather Underground and on-site measurement instruments, the San Luis Obispo Air Pollution Control District (SLO APCD) was contacted for any available data. The only meteorological data available was for the ISCST3 program for the location of Morro Bay, California, located about 20 miles south from the study site. This meteorological data was provided for the years of 1994 through 1996 by the SLO APCD.

\subsection{Intensive Operation Periods}

An "intensive operation period" (IOP) refers to a 12 hour air sampling period where BC was collected throughout the study area. IOPs were first chosen to occur between 6 p.m. and 6 a.m., as conducted by Ward (2009) and Malejan (2009). However, because peaks in aethalometer BC concentrations appeared to begin earlier in the evening, IOPs $4 b$ through $7 \mathrm{~b}$ were conducted between 5 p.m. and 5 a.m. Once daylight savings time began on March $14^{\text {th }}, 2010$, shifting time 1 hour ahead, IOPs $8 \mathrm{~b}$ through $10 \mathrm{~b}$ were run between 6 p.m. and 6 a.m. During each IOP, available aethalometers were co-located with a PEM as well as a meteorological instrument when available.

Each IOP required instrument and equipment preparation and placement, wood burning source identification, and equipment and instrument pickup. PEMs, aethalometers, and 
meteorological instruments were placed in an array that was distributed as evenly as possible over the whole the study area. Sampling locations were restricted to volunteers' homes and locations where the PEMs would be less likely disturbed. The sample arrangement from IOP $7 \mathrm{~b}$ is displayed in Figure 10. Table 2 below summarizes sampling period date and time, number of PEMs and aethalometers deployed, and meteorological instruments used. IOPs conducted by Ward (2009) and Malejan (2009) are also included because data from IOPs conducted during their research was included in the analysis of IOP data from this study. 


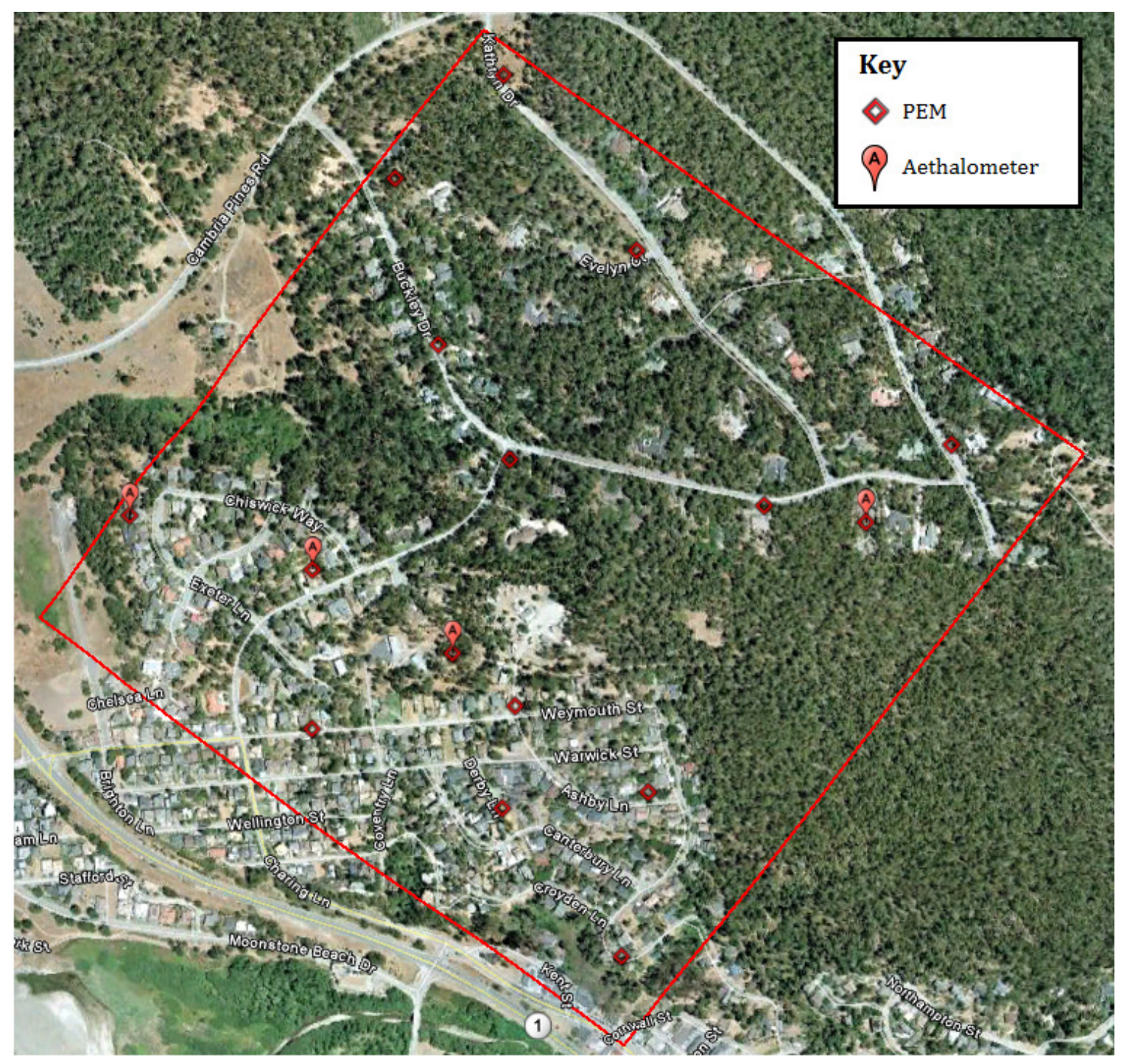

Figure 10. Sample IOP arrangement from IOP 7b 
Table 2. Summary of IOPs

\begin{tabular}{|c|c|c|c|c|c|c|}
\hline \multirow[t]{2}{*}{ IOP } & \multirow[t]{2}{*}{ Date } & \multirow{2}{*}{$\begin{array}{l}\text { Sample } \\
\text { Time }\end{array}$} & \multirow{2}{*}{$\begin{array}{c}\text { Number of } \\
\text { Functioning } \\
\text { PEMs }\end{array}$} & \multirow{2}{*}{$\begin{array}{c}\text { Number of } \\
\text { Aethalometers }\end{array}$} & \multicolumn{2}{|c|}{$\begin{array}{c}\text { Meteorological Data } \\
(\text { Yes }=y, \text { No }=n)\end{array}$} \\
\hline & & & & & EBAM & Ultrasonic \\
\hline \multicolumn{7}{|c|}{ Winter 2009 (Ward 2009 \&Malejan 2009) } \\
\hline $1 \mathrm{a}$ & $\begin{array}{c}\text { Jan } 31- \\
\text { Feb } 1\end{array}$ & $\begin{array}{c}6 \text { PM - } 6 \\
\text { AM }\end{array}$ & 9 & 2 & $\mathrm{n}$ & $\mathrm{n}$ \\
\hline $2 \mathrm{a}$ & $\begin{array}{l}\text { Feb } 18- \\
\text { Feb } 19\end{array}$ & $\begin{array}{c}6 \mathrm{PM}-6 \\
\mathrm{AM}\end{array}$ & 11 & 2 & $\mathrm{n}$ & $\mathrm{n}$ \\
\hline $3 a$ & $\begin{array}{l}\text { Feb } 26- \\
\text { Feb } 27\end{array}$ & $\begin{array}{c}6 \mathrm{PM}-6 \\
\mathrm{AM}\end{array}$ & 8 & 1 & $\mathrm{n}$ & $\mathrm{n}$ \\
\hline $4 \mathrm{a}$ & $\begin{array}{l}\text { Feb } 27- \\
\text { Feb } 28\end{array}$ & $\begin{array}{c}6 \mathrm{PM}-6 \\
\mathrm{AM}\end{array}$ & 11 & 2 & $\mathrm{n}$ & $\mathrm{n}$ \\
\hline $7 a^{*}$ & $\begin{array}{l}\text { Mar } 15- \\
\text { Mar } 16\end{array}$ & $\begin{array}{c}6 \mathrm{PM}-6 \\
\mathrm{AM}\end{array}$ & 12 & 1 & $\mathrm{n}$ & $\mathrm{n}$ \\
\hline $8 a^{*}$ & $\begin{array}{c}\operatorname{Mar} 20- \\
\operatorname{Mar} 21\end{array}$ & $\begin{array}{c}6 \mathrm{PM}-6 \\
\mathrm{AM}\end{array}$ & 12 & 2 & $\mathrm{n}$ & $\mathrm{n}$ \\
\hline \multicolumn{7}{|c|}{ Winter 2010} \\
\hline $2 b$ & $\begin{array}{c}\text { Jan } 23- \\
\text { Jan } 24\end{array}$ & $\begin{array}{c}6 \mathrm{PM}-6 \\
\mathrm{AM}\end{array}$ & 11 & 3 & $\mathrm{n}$ & $\mathrm{n}$ \\
\hline $3 b$ & $\begin{array}{c}\text { Jan } 30- \\
\text { Jan } 31\end{array}$ & $\begin{array}{c}6 \text { PM - } 6 \\
\text { AM }\end{array}$ & 16 & 3 & $\mathrm{n}$ & $\mathrm{n}$ \\
\hline $4 b$ & $\begin{array}{l}\text { Feb } 13- \\
\text { Feb } 14\end{array}$ & $\begin{array}{c}5 \mathrm{PM}-5 \\
\mathrm{AM}\end{array}$ & 15 & 3 & $\mathbf{y}$ & $\mathrm{n}$ \\
\hline $5 b$ & $\begin{array}{l}\text { Feb 28- } \\
\text { Mar } 1\end{array}$ & $\begin{array}{c}5 \mathrm{PM}-5 \\
\mathrm{AM}\end{array}$ & 15 & 4 & $\mathbf{y}$ & $\mathrm{n}$ \\
\hline $6 b$ & $\begin{array}{l}\text { Mar } 4- \\
\text { Mar } 5\end{array}$ & $\begin{array}{c}5 \mathrm{PM}-5 \\
\mathrm{AM}\end{array}$ & 14 & 4 & $\mathbf{y}$ & $\mathrm{n}$ \\
\hline $7 b$ & $\begin{array}{l}\text { Mar } 11- \\
\text { Mar } 12\end{array}$ & $\begin{array}{c}5 \mathrm{PM}-5 \\
\mathrm{AM}\end{array}$ & 15 & 4 & $\mathbf{y}$ & $\mathrm{n}$ \\
\hline $8 \mathrm{~b}$ & $\begin{array}{l}\text { Mar } 15- \\
\text { Mar } 16\end{array}$ & $\begin{array}{c}6 \mathrm{PM}-6 \\
\mathrm{AM}\end{array}$ & 15 & 4 & $\mathbf{y}$ & $\mathbf{y}$ \\
\hline $9 b$ & $\begin{array}{l}\text { Apr } 3 \text { - } \\
\text { Apr } 4\end{array}$ & $\begin{array}{c}6 \mathrm{PM}-6 \\
\mathrm{AM}\end{array}$ & 15 & 4 & $\mathbf{y}$ & $\mathbf{y}$ \\
\hline $10 b$ & $\begin{array}{l}\text { Apr } 23- \\
\text { Apr } 24\end{array}$ & $\begin{array}{c}6 \mathrm{PM}-6 \\
\mathrm{AM}\end{array}$ & 15 & 3 & $\mathbf{y}$ & $\mathbf{y}$ \\
\hline
\end{tabular}

*These IOPs were not included in results and analyses due to a high prevalence of PEM BC concentrations lower than the detection limit.

The identification of wood burning sources was conducted using a Fluke Ti25 Thermal Imager. The Fluke Ti25 can measure the temperature of objects ranging from $-4^{\circ} \mathrm{F}$ to $662^{\circ} \mathrm{F}$ within accuracy of $\pm 2^{\circ} \mathrm{C}$ or $2 \%$ and capture thermal images. To identify wood 
burning sources, the Fluke Ti25 was used to determine chimney temperature. Chimney temperatures detected above $75^{\circ} \mathrm{F}$ were considered to be burning sources and images of burning sources were captured. A sample infrared image from IOP $3 b$ taken on January $30^{\text {th }}$ is displayed in Figure 11 below.

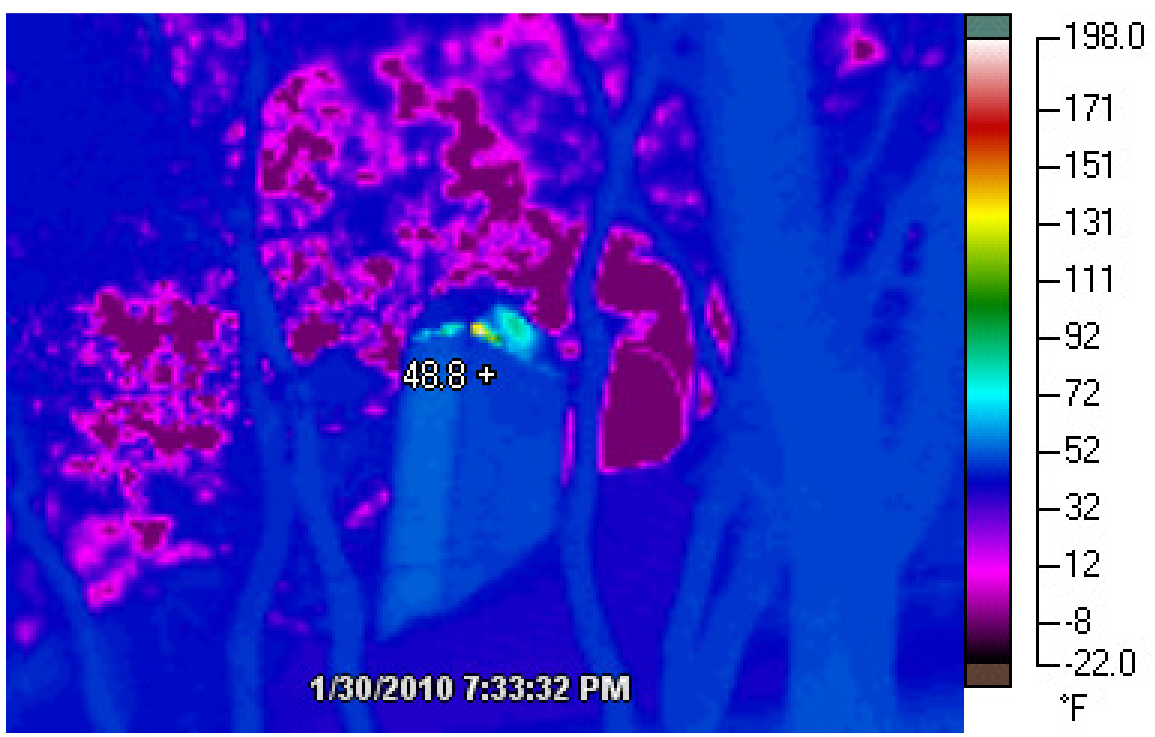

\section{Figure 11. Sample Infrared Camera Image of a Chimney}

At the end of each IOP, the aethalometers, PEMs, and ultrasonic anemometer were collected, while the E-BAM was left at the CDF fire station. PEM filters were then sealed and sent to LBNL for analysis as described in Section 3.2.2.

\subsection{Statistical Analyses}

Several statistical analyses were conducted to understand BC variability and interactions with meteorology and site characteristics. BC variability was observed through averages, medians, and standard deviations at each sampling location, between sampling locations, 
between IOPs, and over all IOPs and locations. Spatial variability between

meteorological data at different sites was observed through averages, medians, and standard deviations for wind speeds, wind directions, temperature, and humidity.

Variability within sampling locations and between IOPs was then evaluated relative to meteorology and site characteristics through the use of multiple regression analyses.

These analyses were used to determine the most representative meteorological stations and whether location relative to wind direction and burning sources was significant in the study area. Regression analyses conducted are described in detail in Section 4.3.

\subsection{BC Air Dispersion Modeling}

As discussed in Section 2.2.2.2, dispersion modeling can be a useful tool for understanding pollutant exposures. To identify the performance of dispersion modeling in the case where detailed data may not always be available, ISC and AERMOD were considered for application in this study. Although both programs assume steady state conditions which may not always be applicable, they have both been US EPA preferred short-distance models and are currently the best option where limited data is available. Although both programs require less data than CTM and CMB models, meteorological data obtained in this study was still insufficient for conducting model dispersion analyses. For this reason, the local air quality regulatory agencies were contacted for available meteorological data that may be applied in either program. ISCST3 was ultimately selected for this study because only data for Morro Bay, CA (located about 20 miles south from the study site) was available from the San Luis Obispo County Air Pollution Control District (SLO APCD). Morro Bay meteorological data was available for the 
years, 1994, 1995, and 1996. Because AERMOD has been found to be an improvement upon ISCST3 and ISC-PRIME, a review of available literature was conducted for comparisons of ISC and AERMOD results and performance. ISC-PRIME and ISCST3 were then used to predict BC concentrations in the study area and predicted concentrations were compared to measured PEM and aethalometer BC concentrations and distributions. Dispersion modeling methods will be discussed in greater depth in Section 4.4. 


\section{Chapter 4. Results and Discussion}

\subsection{Identification of $\mathrm{BC}$ Source}

To confirm that measured $\mathrm{BC}$ concentrations originated from wood burning sources, the adsorption Ångström exponent (AAE) was evaluated over the wave lengths of $350 \mathrm{~nm}$ to $700 \mathrm{~nm}$ as described in Section 2.1.3.1 for all PEM samples. As discussed in Section 2.1.3.1, AAE values around 1 or lower tend to be associated with $\mathrm{BC}$ originating from motor vehicle exhaust, while AAE values larger than 1 are found for wood smoke. For this study, AAE values greater than 1.2 were assumed to originate from wood smoke, while AAE values less than 1.2 were assumed to indicate motor vehicle exhaust. AAE values for PEM samples collected by both Ward (2009) and Malejan (2009) in the winter of 2009 and in this study during the winter of 2010 averaged 1.89 , with a median value of 1.89 , and a range of 0.28 to 3.98 . PEM samples lower than the detection limit were excluded. A box plot detailing the distribution of AAE values is shown in Figure 12, showing the $1^{\text {st }}$ quartile value of 1.6 and the $3^{\text {rd }}$ quartile value of 2.2. Among all samples with $\mathrm{BC}$ concentrations measured above the lower detection limit, 91 percent of $\mathrm{AAE}$ values were associated with wood smoke while 9 percent were associated with AAE values for motor vehicle exhaust. Because Cambria, CA does not contain major sources of vehicle exhaust, it is suspected that PEM samples with AAE values less than 1.2 may have originated from an upwind source. The AAE values for all PEMs are detailed in Appendix B. 


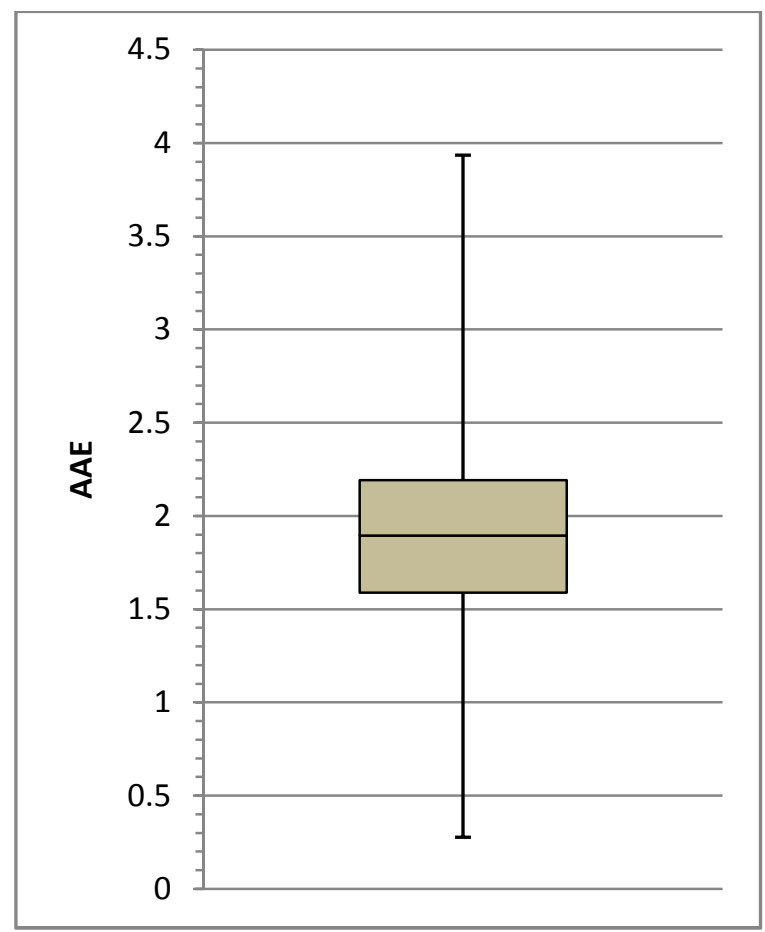

Figure 12. Box plot of all adsorption Ångström exponent (AAE) values

Box plots denote the minimum, maximum, $1^{\text {st }}$ and $3^{\text {rd }}$ quartile, and median value for all concentrations. The whiskers indicate the maximum and minimum values, while the top, middle, and bottom line of the box represent the $3^{\text {rd }}$ quartile, median, and $1^{\text {st }}$ quartile value, respectively.

\subsection{Spatial Variability}

The spatial variability of black carbon (BC) and meteorological data was observed to better understand near-field exposures and regional representation. Averages, medians, and standard deviations were computed for BC and meteorological data and comparisons between sites and instruments were observed.

\subsubsection{Spatial Variability of $\mathrm{BC}$ and $\mathrm{PM}_{2.5}$}

BC concentrations measured throughout the study area by both aethalometers and PEMs were evaluated and are presented in this section along with a comparison between 
concentrations measured by both instruments. $\mathrm{BC}$ concentrations were converted to $\mathrm{PM}_{2.5}$ based on Equation 8 below, as determined by Ward (2009) to be an approximate relationship between $\mathrm{BC}$ and $\mathrm{PM}_{2.5}$ in the study area.

$$
P M_{2.5}=\frac{B C}{0.05}
$$

\section{Equation 8}

\subsubsection{Aethalometer Measured BC}

Based on data collected every minute, BC concentrations over all IOPs ranged between lower than the detection limit and $174,000 \mathrm{ng} / \mathrm{m}^{3}$. The average BC concentration measured over the study area by aethalometers was $386 \mathrm{ng} / \mathrm{m}^{3}$, the median BC concentration was $125 \mathrm{ng} / \mathrm{m}^{3}$, and the standard deviation was large at 2,640 ng/m $\mathrm{m}^{3}$. Because the higher standard deviation in the data is likely the result of noise in the one minute readings, statistics based on 30 minute averages were evaluated. Observing variability based on 30 minute averages reduced noise and resulted in an average BC concentration of $229 \mathrm{ng} / \mathrm{m}^{3}$, a median of $122 \mathrm{ng} / \mathrm{m}^{3}$, and a standard deviation of 827

$\mathrm{ng} / \mathrm{m}^{3}$. The still large (though substantially smaller) standard deviation, is a testament to the large variability in $\mathrm{BC}$ concentrations due to factors including diurnal variations in wood burning and meteorology and differences in meteorology and burning patterns between sampling periods or IOPs.

$\mathrm{BC}$ variability was also observed over each IOP using all 30 minute averaged readings measured by aethalometers in operation during each IOP. Table 3 and 
details the average, median, and standard deviation of 30 minute $\mathrm{BC}$ concentrations over each IOP. The variability within IOPs increases when high BC concentrations are detected and alternately decreases when only background concentrations are being measured. The number of aethalometers in operation may also affect the magnitude of the standard deviation since averaging $\mathrm{BC}$ at more sites increases the spatial variability captured by the statistic. This spatial variability can be seen in aethalometer BC concentrations versus time graphs as show in IOP $7 \mathrm{~b}$ in Figure 14 for the different aethalometer locations. Still, an observation of IOPs $5 \mathrm{~b}$ through $9 \mathrm{~b}$, all containing the same number of aethalometers, in Table 3 and Figure 13, seems to indicate that standard deviation is more highly influenced by sudden peaks in BC concentration than spatial variability alone. Spatial variability likely accounts for a smaller portion of the standard deviation, because typical differences in $\mathrm{BC}$ concentration between locations are much smaller than the difference between the highest BC concentrations and typical background $\mathrm{BC}$ concentrations measured during the study periods. Overall, the average 30 minute-based $\mathrm{BC}$ concentration found during an IOP was $226 \mathrm{ng} / \mathrm{m}^{3}$, or $4.5 \mu \mathrm{g} / \mathrm{m}^{3}$ for $\mathrm{PM}_{2.5}$, the average median value was $132 \mathrm{ng} / \mathrm{m}^{3}$, or $2.6 \mu \mathrm{g} / \mathrm{m}^{3}$ for $\mathrm{PM}_{2.5}$, and the average standard deviation was $438 \mathrm{ng} / \mathrm{m}^{3}$, or $8.8 \mu \mathrm{g} / \mathrm{m}^{3}$ for $\mathrm{PM}_{2.5}$. Detailed BC readings over time during each sampling period and at each site can be found in Appendix C. 
Table 3. Aethalometer BC variability over each IOP

Statistics are based on 30 min averaged BC concentrations measured during each IOP.

\begin{tabular}{|c|c|c|c|c|c|c|}
\hline IOP \# & $\begin{array}{c}\text { Number of } \\
\text { Aethalometers } \\
\text { in Operation }\end{array}$ & $\begin{array}{c}\text { Average } \\
\left(\mathbf{n g} / \mathbf{m}^{\mathbf{3}}\right)\end{array}$ & $\begin{array}{c}\text { Median } \\
\left(\mathbf{n g} / \mathbf{m}^{\mathbf{3}}\right)\end{array}$ & $\begin{array}{c}\mathbf{M a x} \\
\left(\mathbf{n g} / \mathbf{m}^{\mathbf{3}}\right)\end{array}$ & $\begin{array}{c}\mathbf{M i n} \\
\left(\mathbf{n g} / \mathbf{m}^{\mathbf{3}}\right)\end{array}$ & $\begin{array}{c}\text { Standard } \\
\text { Deviation } \\
\left(\mathbf{n g} / \mathbf{m}^{\mathbf{3}}\right)\end{array}$ \\
\hline 1a & 2 & 385 & 359 & 903 & 238 & 132 \\
\hline $2 \mathrm{a}$ & 2 & 110 & 69 & 397 & 32 & 89 \\
\hline $3 \mathrm{a}$ & 1 & 54 & 32 & 139 & 14 & 42 \\
\hline 4a & 2 & 137 & 99 & 883 & 6.9 & 191 \\
\hline $2 \mathrm{~b}$ & 3 & 141 & 125 & 298 & 77 & 47 \\
\hline $3 \mathrm{~b}$ & 3 & 184 & 91 & 1060 & 4.4 & 245 \\
\hline $4 \mathrm{~b}$ & 3 & 626 & 237 & 16700 & 13 & 2000 \\
\hline $5 \mathrm{~b}$ & 4 & 511 & 194 & 18200 & 6.9 & 1800 \\
\hline 6b & 4 & 167 & 78 & 1060 & 6.9 & 227 \\
\hline $7 \mathrm{~b}$ & 4 & 151 & 131 & 896 & 4.4 & 99 \\
\hline $8 \mathrm{~b}$ & 4 & 141 & 82 & 2010 & 6.9 & 252 \\
\hline 9b & 4 & 192 & 100 & 4100 & 3.6 & 462 \\
\hline 10b & 3 & 144 & 123 & 553 & 87 & 62 \\
\hline & Average & 226 & 132 & 3630 & 39 & 438 \\
\hline
\end{tabular}




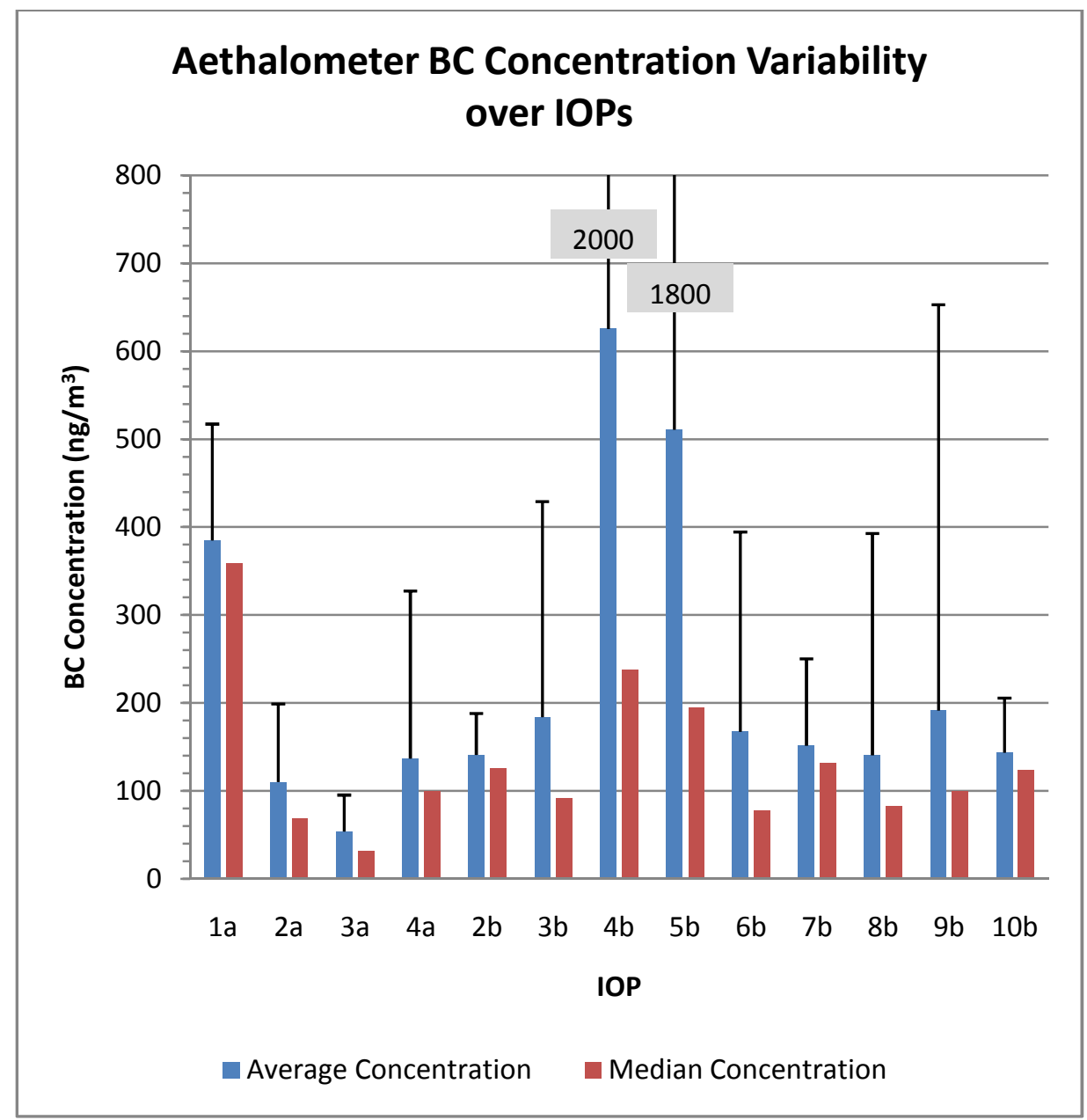

Figure 13. Aethalometer BC variability over each IOP based on 30 minute averaged BC concentrations

Capped lines represent the standard deviation. Numbers in gray boxes give the standard deviation values that lie above the axis value. 


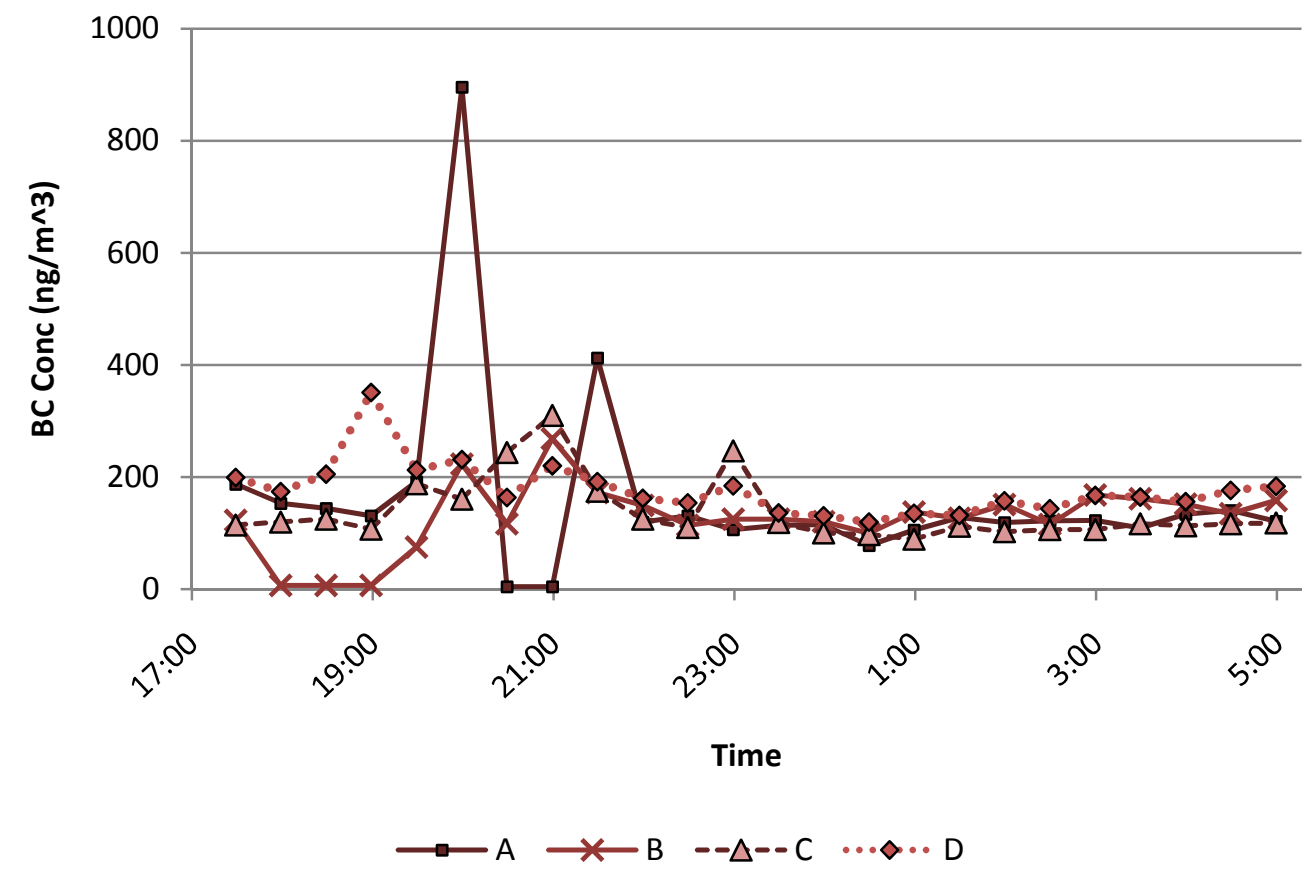

Figure 14. IOP 7b aethalometer $B C$ concentration versus time based on 30 minute averaged $B C$ concentrations

Locations A through D are labeled in Figure 15.

Variability over sampling sites A through $\mathrm{E}$ as labeled in Figure 15 was observed as displayed in Table 4, Figure 16, and Figure 17 based on aethalometer data averaged over 30 minutes. The highest $\mathrm{BC}$ average, median, and standard deviation was observed at site $\mathrm{B}$ where the highest $\mathrm{BC}$ concentrations were often detected. The average and median concentration over all other sites were fairly similar, with the exception of the lower median concentration at site $\mathrm{E}$, which may be a result of there being fewer sampling periods available and/or the site being surrounded by many trees. Standard deviations were also fairly similar with the clear exception of site B and a slightly higher standard deviation for site A. The higher variation at site A may represent the slight shifting of the aethalometer approximately 10 feet from the original location that occurred after IOP $8 \mathrm{~b}$. 
Thus, the increase in standard deviation may represent the change in variability due to a

10 foot location move.

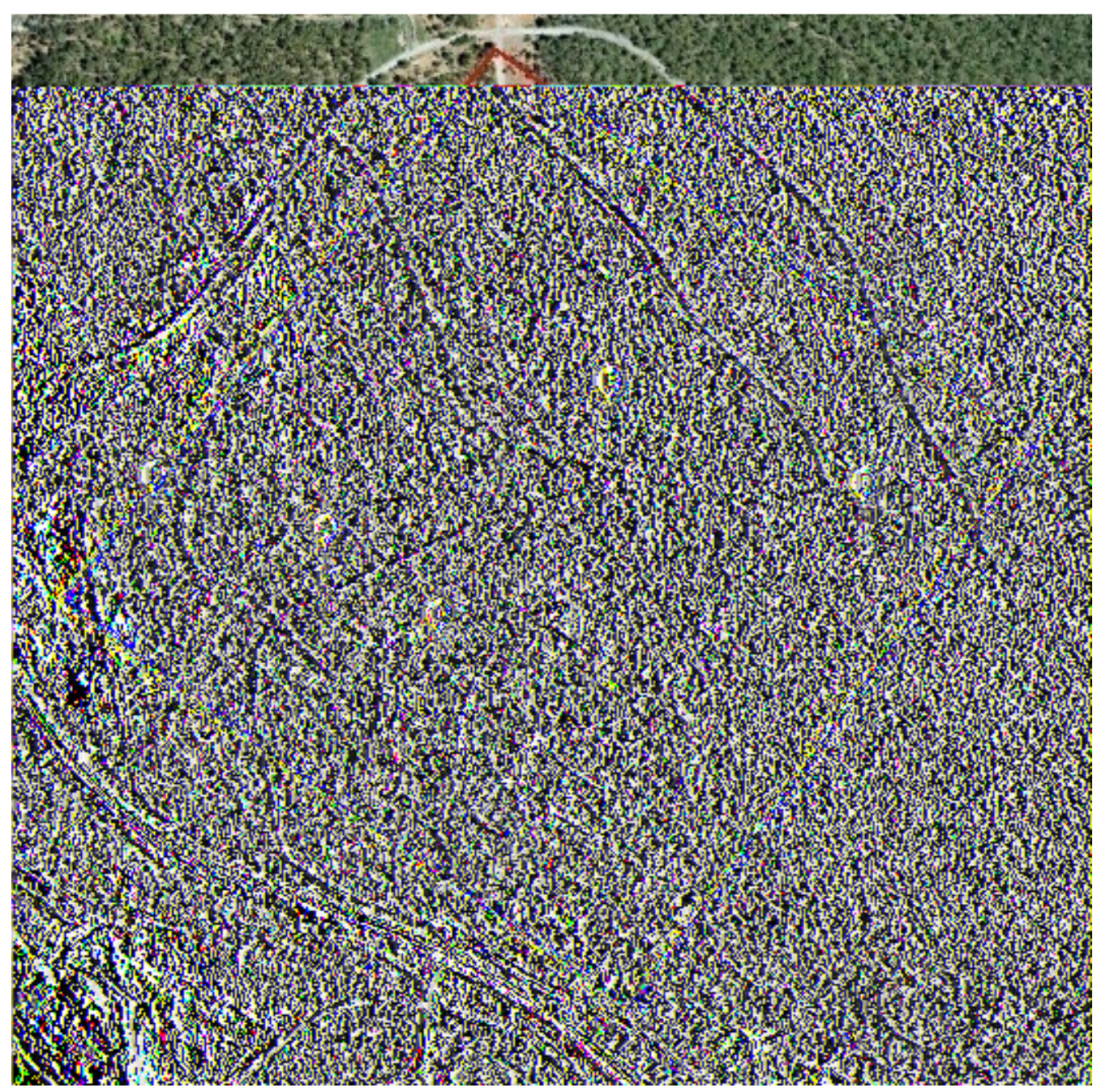

\section{Figure 15. Aethalometer sampling locations}

As summarized in Table 4, the average of BC averages for all sites was $211 \mathrm{ng} / \mathrm{m}^{3}$, or 4.2 $\mu \mathrm{g} / \mathrm{m}^{3}$ for $\mathrm{PM}_{2.5}$, the average median concentration was $111 \mathrm{ng} / \mathrm{m}^{3}$, or $2.2 \mu \mathrm{g} / \mathrm{m}^{3}$ for $\mathrm{PM}_{2.5}$, and the average standard deviation was $470 \mathrm{ng} / \mathrm{m}^{3}$, or $9.4 \mu \mathrm{g} / \mathrm{m}^{3}$ for $\mathrm{PM}_{2.5}$. To observe the impact of site B's higher BC concentrations on the overall statistics, the 
average, median, and standard deviation were observed for all other sites. When

excluding site $\mathrm{B}$, the average $\mathrm{BC}$ concentration between sites was $169 \mathrm{ng} / \mathrm{m}^{3}$, the average median was $108 \mathrm{ng} / \mathrm{m}^{3}$, and the average standard deviation was $179 \mathrm{ng} / \mathrm{m}^{3}$. When site B is not considered, the average concentration is half the magnitude of the average when site $\mathrm{B}$ is included and standard deviation decreases substantial. Thus, BC variability increased spatially when pollutant concentrations increase. The box plots in Figure 17 demonstrate the $\mathrm{BC}$ concentration distribution, also illustrating the large difference between the highest and lowest concentration (greater than $174,000 \mathrm{ng} / \mathrm{m}^{3}$, or 3,480 $\mu \mathrm{g} / \mathrm{m}^{3}$ for $\left.\mathrm{PM}_{2.5}\right)$.

Table 4. Aethalometer BC variability over sites based on 30 minute averaged BC

\begin{tabular}{|c|c|c|c|c|}
\hline Site & Cases & $\begin{array}{c}\text { Average } \\
\left(\mathbf{n g} / \mathbf{m}^{\mathbf{3}}\right)\end{array}$ & $\begin{array}{c}\text { Median } \\
\left(\mathbf{n g} / \mathbf{m}^{\mathbf{3}}\right)\end{array}$ & $\begin{array}{c}\text { Std } \\
\text { Dev }\end{array}$ \\
\hline A & 11 & 214 & 142 & 228 \\
\hline B & 10 & 381 & 125 & 1632 \\
\hline C & 6 & 157 & 112 & 169 \\
\hline D & 9 & 175 & 126 & 168 \\
\hline E & 3 & 128 & 50 & 151 \\
\hline \multicolumn{2}{|c|}{ Average } & 211 & 111 & 470 \\
\hline
\end{tabular}




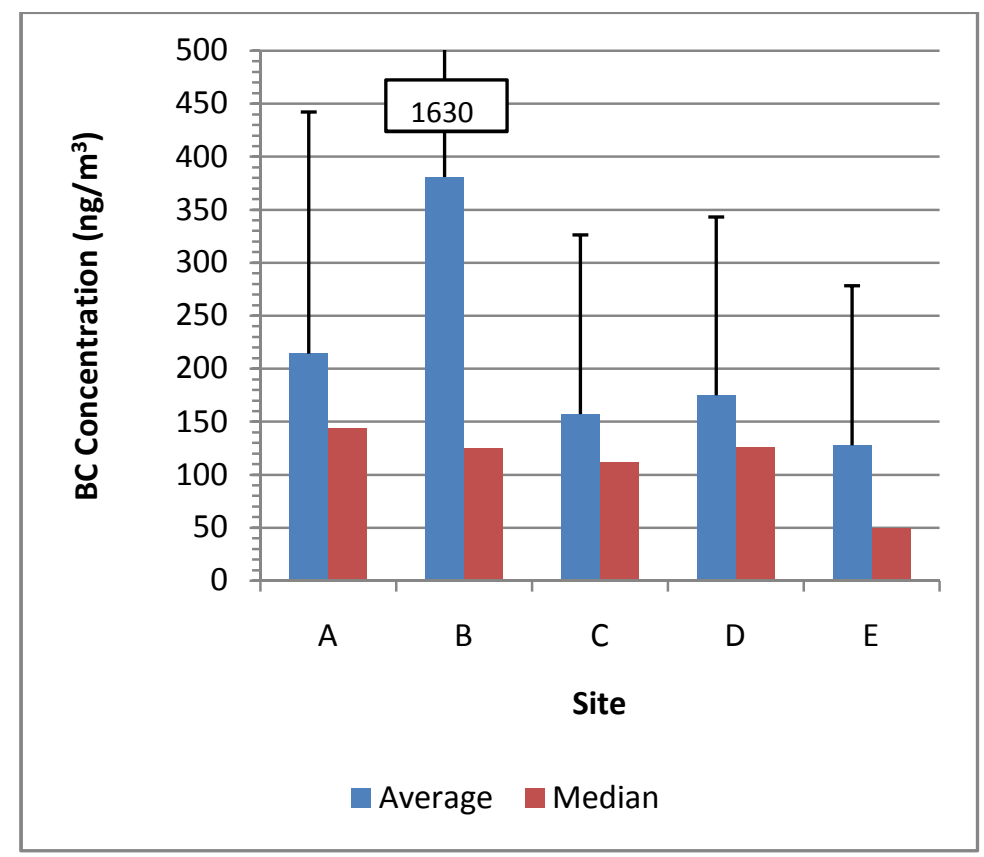

Figure 16. Aethalometer BC variability over sites based on 30 minute averaged BC Capped lines represent the positive standard deviation. Numbers in gray boxes give the standard deviation for values that lie above the axis value. 


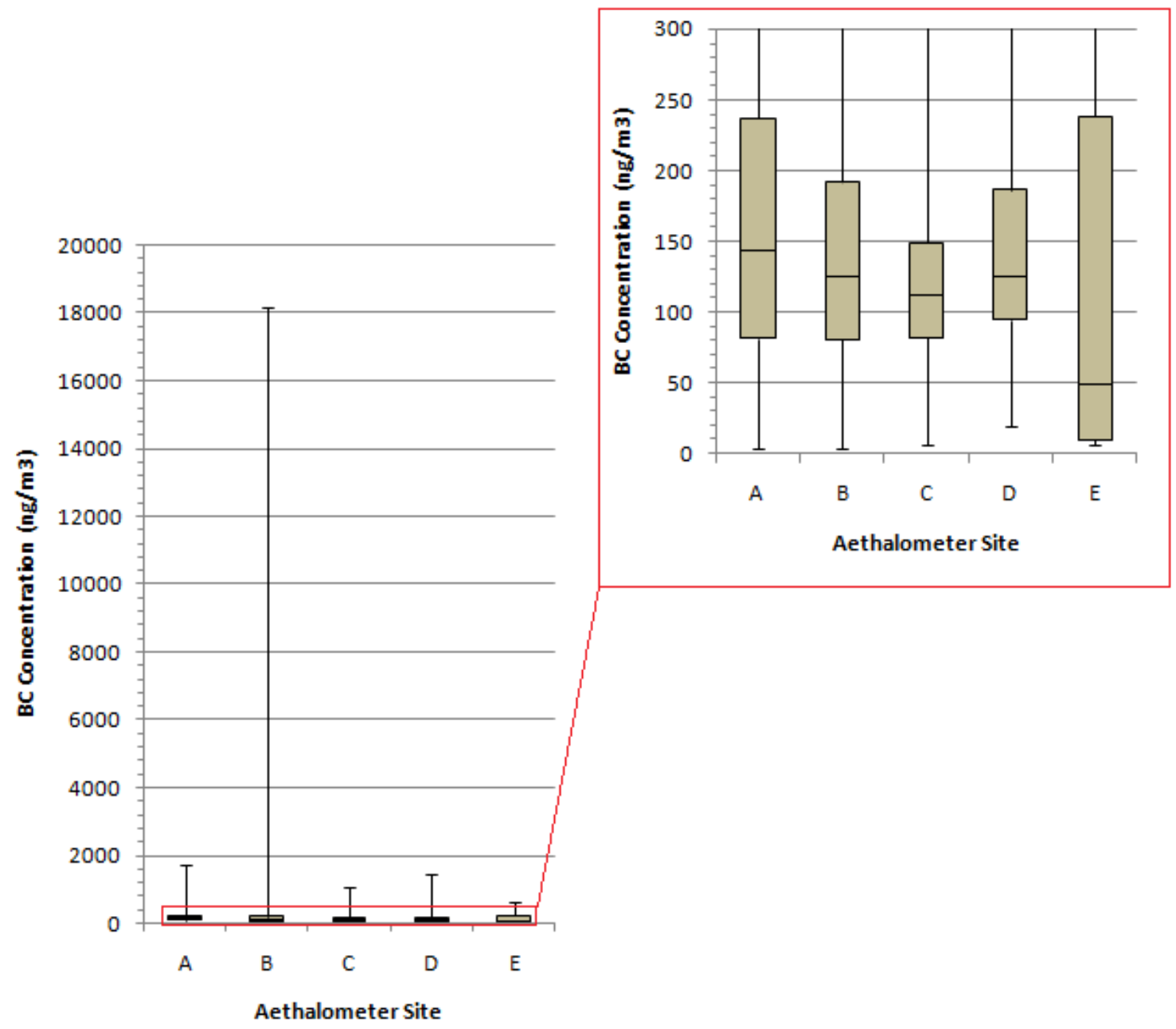

Figure 17. Box plots comparing aethalometer BC variability between sites

Box plots denote the minimum, maximum, $1^{\text {st }}$ and $3^{\text {rd }}$ quartile, and median value for all concentrations. The whiskers indicate the maximum and minimum values, while the top, middle, and bottom line of the box represent the $3^{\text {rd }}$ quartile, median, and $1^{\text {st }}$ quartile value, respectively.

\subsubsection{Personal Environmental Monitor Measured BC}

BC concentrations analyzed from Personal Environmental Monitor (PEM) 12 hour filter sampling periods in the study area ranged from a maximum of $2,790 \mathrm{ng} / \mathrm{m}^{3}$, or 55.8 $\mu \mathrm{g} / \mathrm{m}^{3}$ for $\mathrm{PM}_{2.5}$, to a minimum below the detection limit of $60.52 \mathrm{ng} / \mathrm{m}^{3}$, or $1.2 \mu \mathrm{g} / \mathrm{m}^{3}$ for $\mathrm{PM}_{2.5}$. The average PEM BC concentration over all locations and IOPs was $224 \mathrm{ng} / \mathrm{m}^{3}$, 
or $4.5 \mu \mathrm{g} / \mathrm{m}^{3}$ for $\mathrm{PM}_{2.5}$, with a median concentration of $163 \mathrm{ng} / \mathrm{m}^{3}$, or $3.3 \mu \mathrm{g} / \mathrm{m}^{3}$ for $\mathrm{PM}_{2.5}$, and a standard deviation of $262 \mathrm{ng} / \mathrm{m}^{3}$, or $5.2 \mu \mathrm{g} / \mathrm{m}^{3}$ for $\mathrm{PM}_{2.5}$. Table 5 summarizes PEM BC averages, medians, and standard deviations for each IOP. PEM BC concentrations averaged by IOP ranged between 80.5 and $441 \mathrm{ng} / \mathrm{m}^{3}$, or 1.6 and 8.8 $\mu \mathrm{g} / \mathrm{m}^{3}$ for $\mathrm{PM}_{2.5}, \mathrm{BC}$ medians ranged between the lower detection limit and $343 \mathrm{ng} / \mathrm{m}^{3}$, or $8.8 \mu \mathrm{g} / \mathrm{m}^{3}$ for $\mathrm{PM}_{2.5}$, and standard deviations ranged from 31.7 to $651 \mathrm{ng} / \mathrm{m}^{3}$, or 0.63 and $13.0 \mu \mathrm{g} / \mathrm{m}^{3}$ for $\mathrm{PM}_{2.5}$. Higher average $\mathrm{BC}$ concentrations tended to have larger standard deviations and standard deviation also increased with an increase in the number of samples. Figure 18 shows box plots for each IOP, demonstrating that concentrations tended to vary widely with most concentrations much lower than the maximum concentrations for each IOP. 
Table 5. PEM variability over each IOP

\begin{tabular}{|c|c|c|c|c|c|}
\hline IOP & $\begin{array}{c}\text { Number of PEMs } \\
\text { in Operation }\end{array}$ & $\begin{array}{c}\text { Average } \\
\left(\mathbf{n g} / \mathbf{m}^{\mathbf{3}}\right)\end{array}$ & $\begin{array}{c}\text { Median } \\
\left(\mathbf{n g} / \mathbf{m}^{\mathbf{3}}\right)\end{array}$ & $\begin{array}{c}\text { Standard } \\
\text { Deviation }\end{array}$ & $\begin{array}{c}\text { Number of } \\
\text { Burning Sources }\end{array}$ \\
\hline $1 \mathrm{a}$ & 9 & 371 & 343 & 60 & 5 \\
\hline $2 \mathrm{a}$ & 11 & 165 & 86 & 223 & 7 \\
\hline $3 \mathrm{a}$ & 8 & 82 & 76 & 32 & 5 \\
\hline $4 \mathrm{a}$ & 11 & 308 & 262 & 185 & 9 \\
\hline $7 \mathrm{a}^{*}$ & 16 & 60 & 30 & 36 & 11 \\
\hline $8 \mathrm{a}^{*}$ & 12 & 33 & 30 & 6.7 & 10 \\
\hline $2 \mathrm{~b}$ & 11 & 82 & 30 & 86 & 18 \\
\hline $3 \mathrm{~b}$ & 16 & 203 & 181 & 168 & 15 \\
\hline $4 \mathrm{~b}$ & 15 & 331 & 281 & 214 & 7 \\
\hline $5 \mathrm{~b}$ & 15 & 441 & 273 & 651 & 8 \\
\hline $6 \mathrm{~b}$ & 14 & 220 & 174 & 183 & 10 \\
\hline $7 \mathrm{~b}$ & 15 & 272 & 159 & 251 & 14 \\
\hline $8 \mathrm{~b}$ & 15 & 187 & 154 & 111 & 7 \\
\hline $9 \mathrm{~b}$ & 15 & 136 & 119 & 48 & 5 \\
\hline $10 \mathrm{~b}$ & 15 & 81 & 88 & 47 & 12 \\
\hline & Average & 221 & 171 & 174 & 9.4 \\
\hline
\end{tabular}

"These IOPs were not included in analyses due to a high prevalence of PEM BC concentrations lower than the detection limit. Average concentrations listed in this table assume values lower than the detection limit to be the average of a zero concentration and the detection limit $\left(60.5 \mathrm{ng} / \mathrm{m}^{3}\right)$, or 30.2 $n g / m^{3}$.

${ }^{* *}$ IOP $7 a$ and $8 a$ are excluded. 


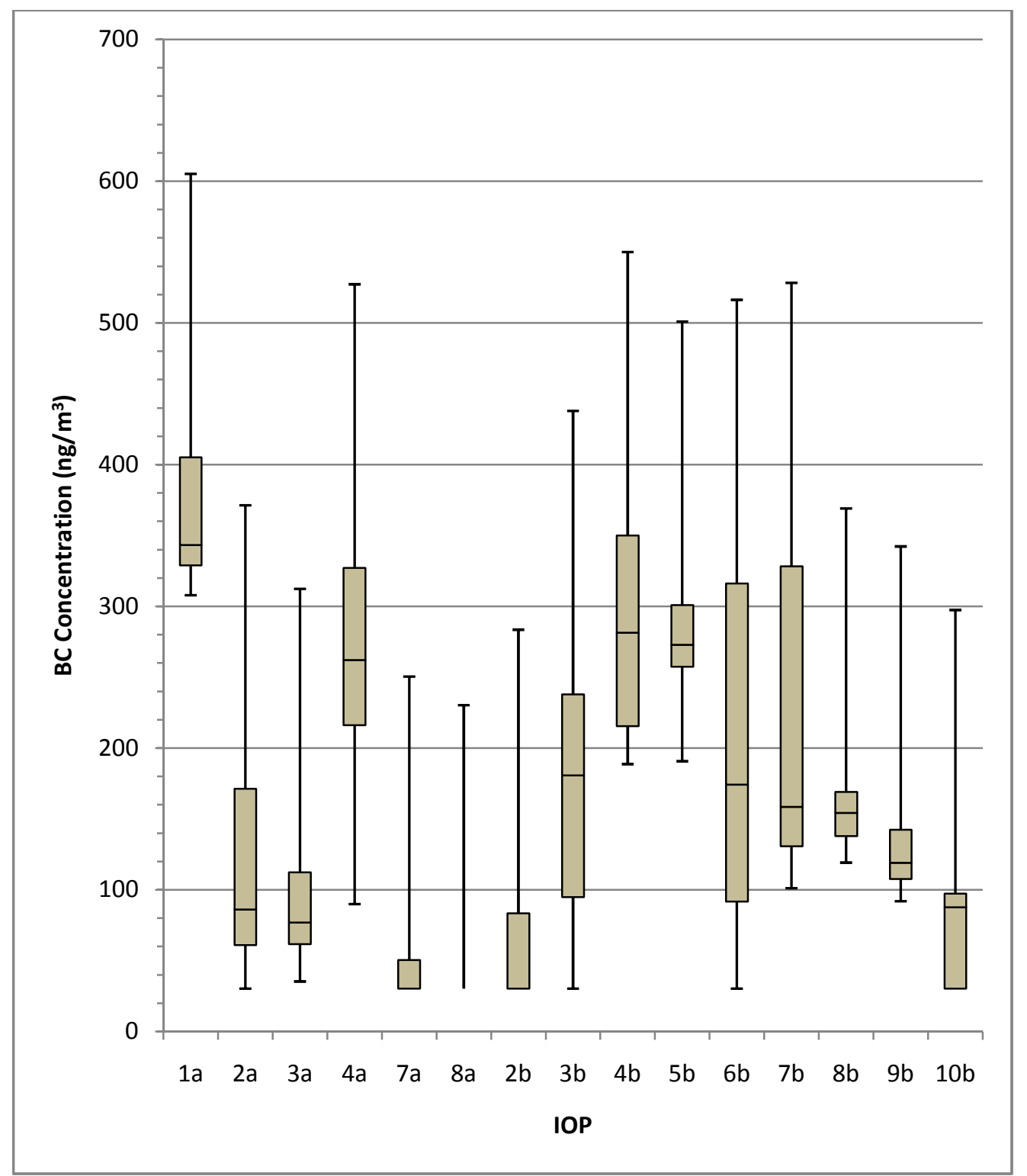

Figure 18. PEM BC box plot over each IOP

Box plots denote the minimum, maximum, $1^{\text {st }}$ and $3^{\text {rd }}$ quartile, and median value for all concentrations. The whiskers indicate the maximum and minimum values, while the top, middle, and bottom line of the box represent the $3^{\text {rd }}$ quartile, median, and $1^{\text {st }}$ quartile value, respectively. IOPs $7 a$ and 8 a differ in appearance because minimum, $1^{\text {st }}$ quartile, and median values are all equal to the lowest concentration $\left(30.2 \mathrm{ng} / \mathrm{m}^{3}\right)$. In addition, the $3^{\text {rd }}$ quartile value is also equal to $30.2 \mathrm{ng} / \mathrm{m}^{3}$ for IOP 8 .

The summary table for site variability was not included in this section due to the large number of sites used for sampling. The summary table is instead included in Appendix D 
under PEM Variability and the results are discussed here. In addition, PEM sites were simplified into three locations and the BC concentration statistics for each location was observed. Table 6 details the range and average of average $\mathrm{BC}$ concentrations, medians, and standard deviations observed at each PEM sampling site, and Figure 19 illustrates the average BC concentration at each site. PEM sites are labeled in Figure 20. In the case of PEM sites, variability did not tend to increase with the number of samples available. In fact, the largest standard deviation had only 3 cases (as opposed to the highest number of samples, 12). As with aethalometers, PEMs demonstrate high spatial variability with the highest and lowest $\mathrm{BC}$ concentrations differing by more than $2,800 \mathrm{ng} / \mathrm{m}^{3}$ over all IOPs and more than $2,500 \mathrm{ng} / \mathrm{m}^{3}$ over a single IOP.

\section{Table 6. Summary of individual site statistics}

The average, median, and standard deviation at each PEM site was determined over all IOPs and the results briefly summarized in this table. The $1^{\text {st }}$ column refers to the statistic observed at each site, the $2^{\text {nd }}$ column refers to the minimum value of the statistic designated in column 1, the $3^{\text {rd }}$ column, refers to the maximum value of the statistic in column 1, and the $4^{\text {th }}$ column refers to the average value the statistic in column 1. For example, for the row titled "Average," the "Minimum" column refers to the smallest average BC concentration observed among all sites averaged over all IOPs.

\begin{tabular}{|c|c|c|c|}
\hline $\begin{array}{c}\text { BC Concentration } \\
\text { for Site }\left(\mathrm{ng} / \mathrm{m}^{3}\right)\end{array}$ & Minimum & Maximum & Average \\
\hline Average & LDL & 520 & 233 \\
\hline Median & LDL & 486 & 206 \\
\hline Standard Deviation & 23.4 & 863 & 165 \\
\hline
\end{tabular}




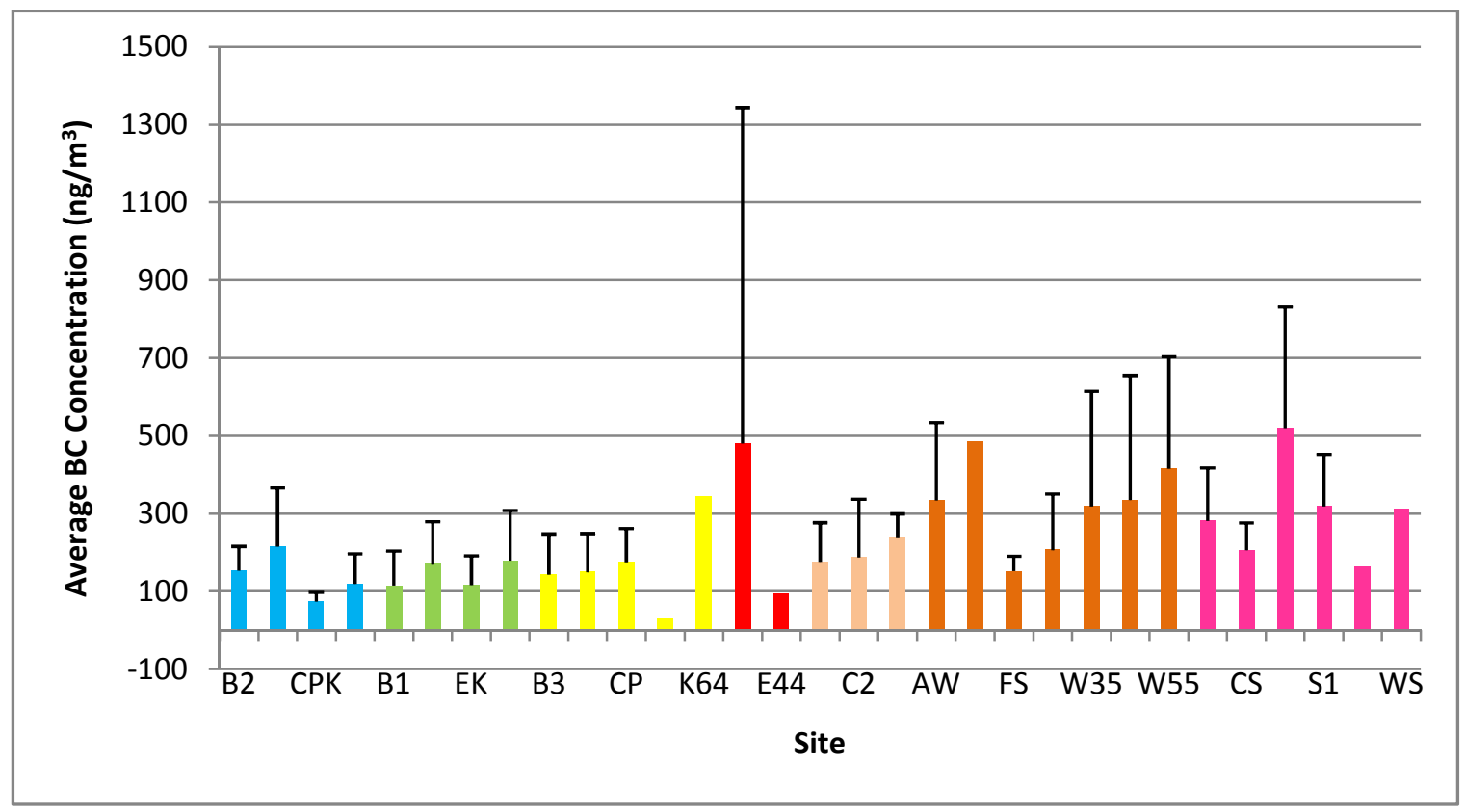

Figure 19. Average PEM BC concentration at each site

PEM sites are color coded based on sampling locations as shown in Figure 20. The bars represent the positive standard deviation. 


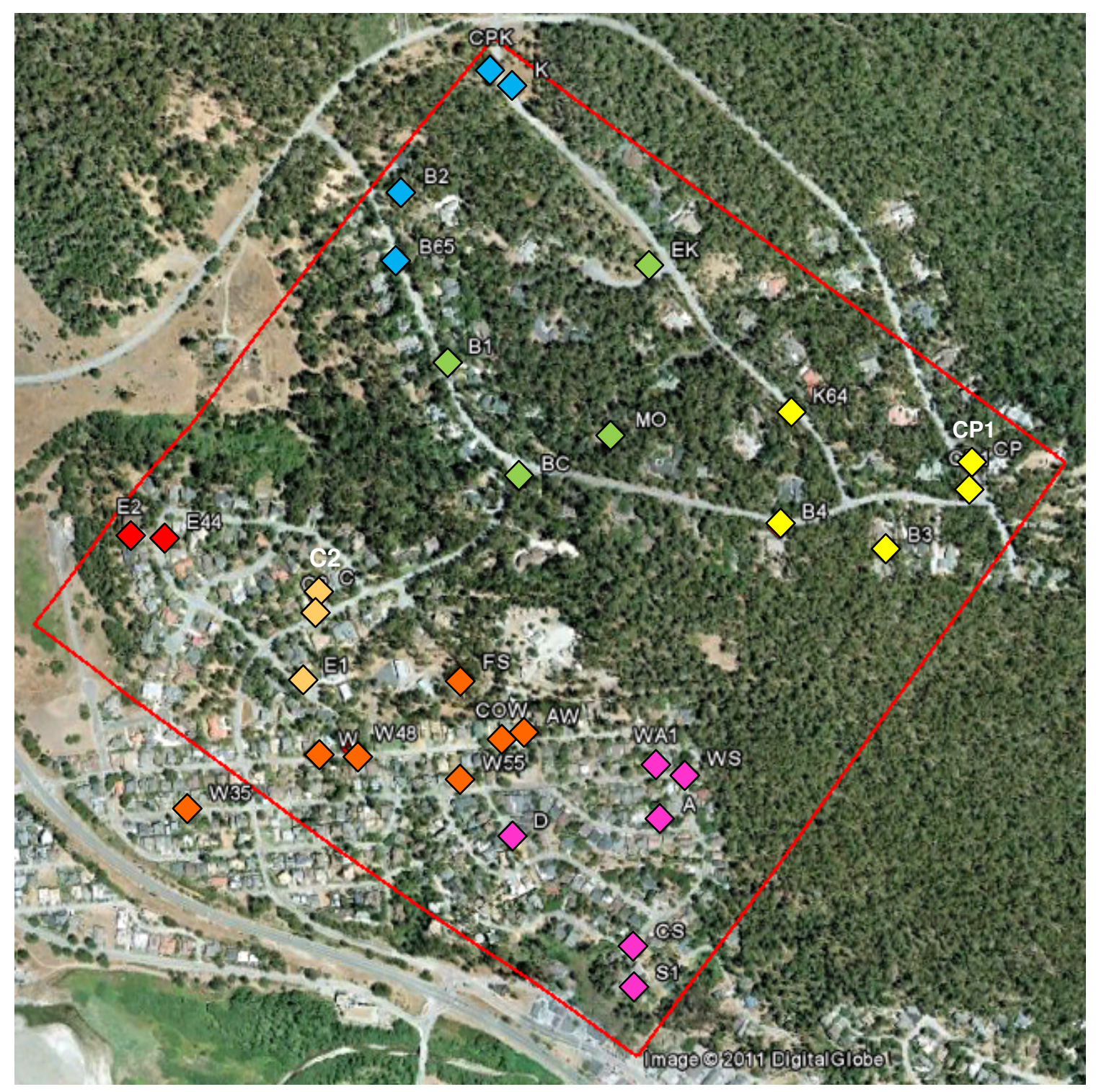

Figure 20. PEM sampling locations

To evaluate spatial variation between PEM locations, the study area was divided into three locations, A, B, and C, as illustrated in Figure 21. The division was made at locations that differ in terrain, housing density, and elevation, with location A on average having the lowest housing density and highest elevations, location $\mathrm{C}$ on average having the highest housing density and lowest elevation, and location B on average containing 
elevations in between location $\mathrm{A}$ and $\mathrm{C}$ and housing density about midway between locations A and C. Location characteristics become more important in Section 4.3.2.2 and are described in greater detail there. Table 7 and Figure 22 detail the PEM BC statistics by IOP in addition to the locations' average, median, and standard deviation over all IOPs. Observing BC concentrations during each IOP in each location, location C's average and median BC concentrations were higher than location A's average and median BC concentration. Location B, however, was less consistently comparable to location A and C. Average BC concentrations occurring in location B were highest during IOP $4 \mathrm{~b}$ and $5 \mathrm{~b}$, but the lowest average PEM BC concentrations also occurred during IOPs 1a, 2a, 6b, and 9b. 


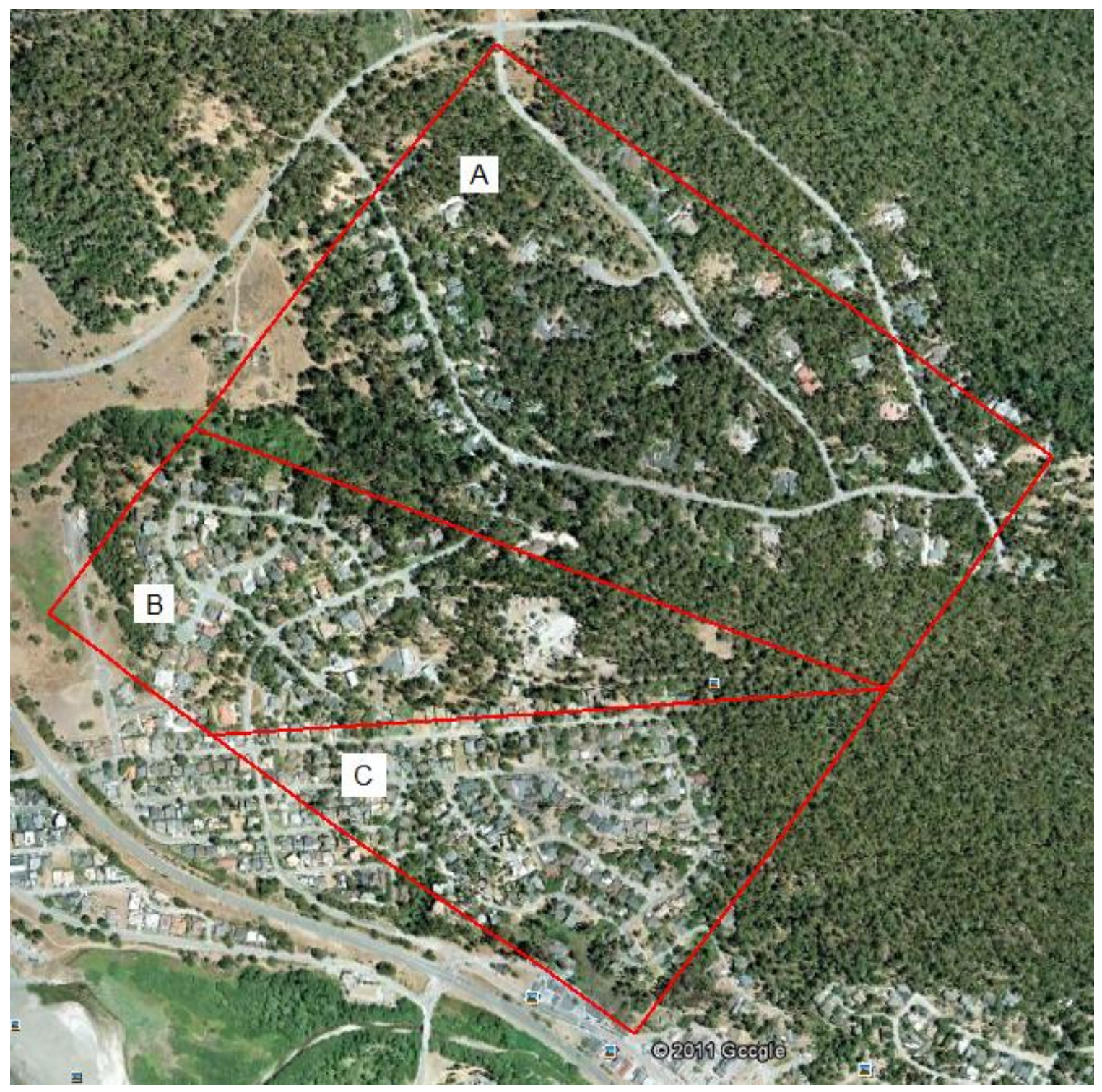

Figure 21. Divided study area sections

In contrast, when averaging PEM BC concentrations over all IOPs for each location, location A had the lowest average BC concentration, followed by location B, and then location $\mathrm{C}$. The difference between $\mathrm{BC}$ averages over each IOP and over all IOPs demonstrates that lower resolution data measured over longer time periods will tend to smooth out peaks in $\mathrm{BC}$ concentration. By observing the standard deviations for concentrations averaged over all IOPs for each location, the high concentrations 
occurring in location B can still be observed. Standard deviation was highest at location $\mathrm{B}$ when compared to location $\mathrm{A}$ and $\mathrm{C}$ due to the detection of the highest concentrations in location B. Standard deviation at location A over all IOPs was the lowest, about twice as low as the standard deviation detected in location $\mathrm{C}$ over all IOPs. This is in turn a demonstration of the higher concentrations found in location $\mathrm{C}$ than in location $\mathrm{A}$. The large difference between the average, median, and standard deviation of $\mathrm{BC}$ concentration between locations $\mathrm{A}$ and $\mathrm{C}$ was likely due to the difference in housing density and tree coverage in the locations. Because there are more homes per square area in location $\mathrm{C}$, wood smoke was more concentrated, resulting in higher BC concentrations. In addition to the having lower housing density with fewer burning sources per square area, the higher number of trees found in location A also may act to slow or prevent dispersion of wood smoke into the area, contributing to lower concentrations detected in areas further away from wood burning. 
Table 7. Average and median BC concentration and standard deviation for PEMs in locations, A, B, and C

\begin{tabular}{|c|c|c|c|c|c|c|c|c|c|}
\hline & \multicolumn{3}{|c|}{ Location A } & \multicolumn{3}{c|}{ Location B } & \multicolumn{3}{c|}{ Location C } \\
\hline & $\begin{array}{c}\text { Ave } \\
\left(\mathbf{n g} / \mathbf{m}^{3}\right)\end{array}$ & $\begin{array}{c}\text { Med } \\
\left(\mathbf{n g} / \mathbf{m}^{3}\right)\end{array}$ & $\begin{array}{c}\text { Std } \\
\text { I.P }\end{array}$ & $\begin{array}{c}\text { Ave } \\
\left(\mathbf{n g} / \mathbf{m}^{3}\right)\end{array}$ & $\begin{array}{c}\text { Med } \\
\left(\mathbf{n g} / \mathbf{m}^{3}\right)\end{array}$ & $\begin{array}{c}\text { Std } \\
\text { Dev }\end{array}$ & $\begin{array}{c}\left(\mathbf{n g} / \mathbf{m}^{3}\right. \\
)\end{array}$ & $\begin{array}{c}\text { Med } \\
\left(\mathbf{n g} / \mathbf{m}^{3}\right)\end{array}$ & $\begin{array}{c}\text { Std } \\
\text { Dev }\end{array}$ \\
\hline $\mathbf{1 a}$ & 355 & 343 & 34 & 308 & 308 & $0^{*}$ & 402 & 405 & 75 \\
\hline $\mathbf{2 a}$ & 101 & 81 & 76 & 69 & 82 & 34 & 301 & 171 & 347 \\
\hline $\mathbf{3 a}$ & 58 & 60 & 20 & 76 & 76 & $0^{*}$ & 116 & 115 & 7 \\
\hline $\mathbf{4 a}$ & 196 & 216 & 74 & 242 & 293 & 99 & 469 & 439 & 215 \\
\hline $\mathbf{2 b}$ & 30 & 30 & $0^{*}$ & 84 & 84 & 2.7 & 183 & 163 & 117 \\
\hline $\mathbf{3 b}$ & 103 & 78 & 86 & 206 & 193 & 27 & 362 & 451 & 202 \\
\hline $\mathbf{4 b}$ & 219 & 210 & 35 & 552 & 326 & 430 & 354 & 374 & 76 \\
\hline $\mathbf{5 b}$ & 246 & 263 & 47 & 1112 & 283 & 1451 & 311 & 294 & 62 \\
\hline $\mathbf{6 b}$ & 143 & 102 & 139 & 107 & 107 & 109 & 334 & 293 & 193 \\
\hline $\mathbf{7 b}$ & 135 & 136 & 29 & 142 & 142 & 23 & 475 & 420 & 306 \\
\hline $\mathbf{8 b}$ & 154 & 138 & 52 & 155 & 155 & 1.4 & 254 & 176 & 174 \\
\hline $\mathbf{9 b}$ & 119 & 115 & 19 & 111 & 111 & 13 & 173 & 169 & 69 \\
\hline $\mathbf{1 0 b}$ & 67 & 82 & 31 & 93 & 93 & 1.3 & 97 & 104 & 72 \\
\hline $\mathbf{A l l}$ & 144 & 125 & 95 & 287 & 156 & 516 & 303 & 269 & 201 \\
\hline
\end{tabular}

* A standard deviation value of " 0 " indicates that only one PEM BC value was available

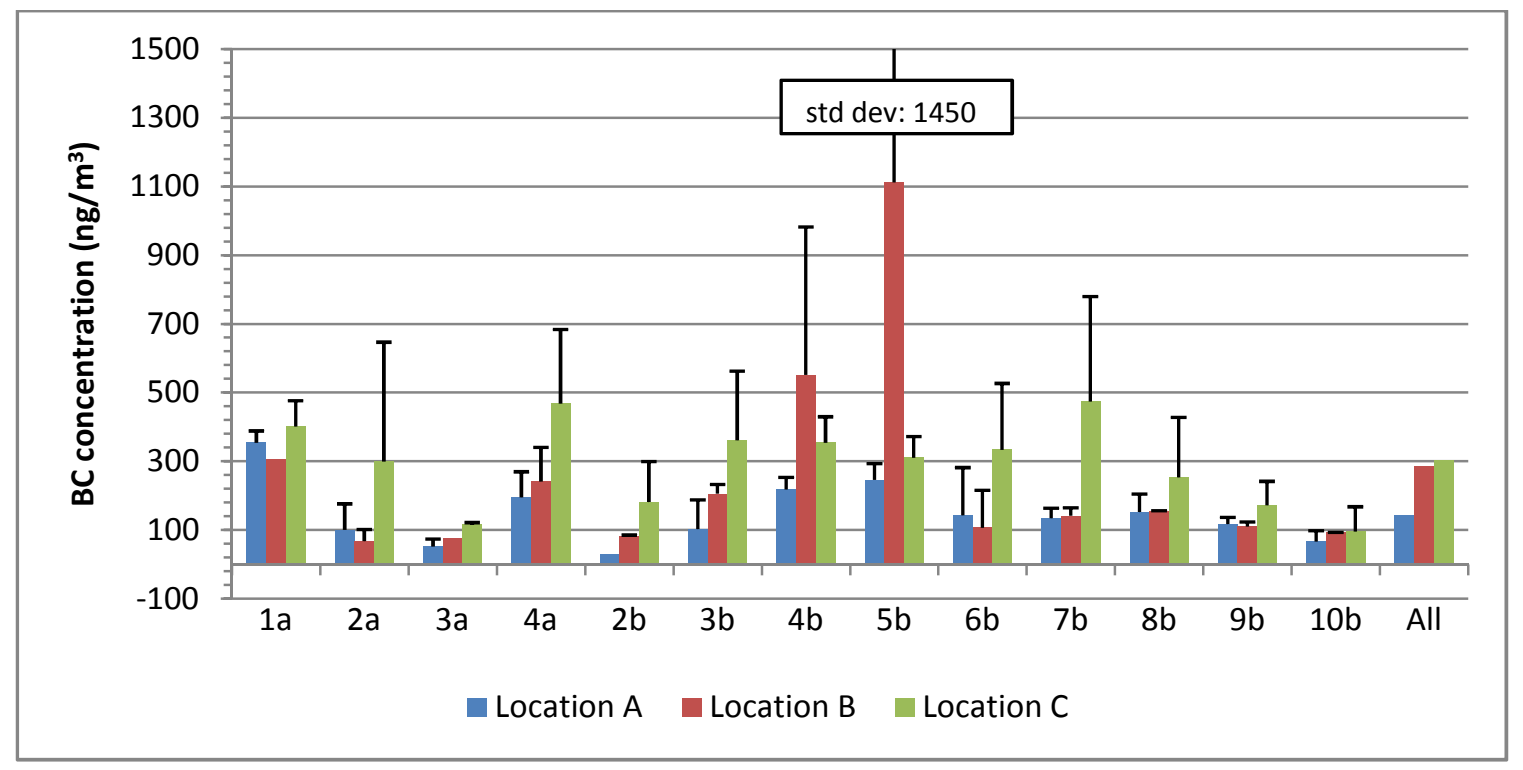

Figure 22. Average PEM BC concentration based on location

Capped lines represent standard deviation as detailed in Table 7. 


\subsubsection{Aethalometer versus PEM BC Variability}

A comparison of PEM and aethalometer 12 hour averaged BC concentrations was made as seen in Table 8 . The average difference between all co-located aethalometer and PEM measured $\mathrm{BC}$ concentrations was 39 percent, with a median of 25 percent, a minimum difference of 1 percent, a maximum difference of 83 percent, and a standard deviation of 25 percent when the unusually large differences for the locations $\mathrm{B}$ and $\mathrm{E}$ was eliminated due to suspected aethalometer measurement issues. Differences between PEM and aethalometer concentrations were largely variable with aethalometer measured BC concentrations often higher than PEM BC. Percent differences between aethalometer and PEM BC concentrations were explored in correlation with wind speed and magnitude of PEM and aethalometer concentration magnitudes were evaluated, but no correlations were found. Large differences in PEM and aethalometer measurements may be attributed to the absorption of organic gases or changes in humidity. Other meteorological factors may have also resulted in differing concentrations over distances less than the distance between aethalometer and inlets. 
Table 8. 12 hour averaged PEM and aethalometer (aeth) BC comparison

Aethalometer locations labeled in this table refer to sites marked in Figure 15. Aethalometer and PEM Concentrations are in $n g / m^{3}$.

\begin{tabular}{|c|c|c|c|c|c|c|c|c|c|c|c|c|c|c|c|}
\hline & \multicolumn{3}{|c|}{ Location A } & \multicolumn{3}{|c|}{ Location B } & \multicolumn{3}{|c|}{ Location C } & \multicolumn{3}{|c|}{ Location D } & \multicolumn{3}{|c|}{ Location E } \\
\hline IOP & $\begin{array}{c}\text { Aeth } \\
\text { BC }\end{array}$ & $\begin{array}{c}\text { PEM } \\
\text { BC }\end{array}$ & $\begin{array}{c}\% \\
\text { diff }\end{array}$ & $\begin{array}{c}\text { Aeth } \\
\text { BC }\end{array}$ & $\begin{array}{c}\text { PEM } \\
\text { BC }\end{array}$ & $\begin{array}{c}\% \\
\text { diff }\end{array}$ & $\begin{array}{c}\text { Aeth } \\
\text { BC }\end{array}$ & $\begin{array}{c}\text { PEM } \\
\text { BC }\end{array}$ & $\begin{array}{c}\% \\
\text { diff }\end{array}$ & $\begin{array}{c}\text { Aeth } \\
\text { BC }\end{array}$ & $\begin{array}{c}\text { PEM } \\
\text { BC }\end{array}$ & $\begin{array}{c}\% \\
\text { diff }\end{array}$ & $\begin{array}{c}\text { Aeth } \\
\text { BC }\end{array}$ & $\begin{array}{c}\text { PEM } \\
\text { BC }\end{array}$ & $\begin{array}{c}\% \\
\text { diff }\end{array}$ \\
\hline $1 \mathrm{a}$ & 413 & 308 & 25 & & & & & & & & 329 & & 357 & 343 & 4 \\
\hline $2 \mathrm{a}$ & 125 & 30 & 76 & & & & & & & & 32 & & 95 & 75 & 21 \\
\hline $3 a$ & & 76 & & 39 & & & & & & & & & & 77 & \\
\hline $4 a$ & & 306 & & 249 & 128 & 49 & & & & & 262 & & -662 & 222 & $134^{*}$ \\
\hline $2 b$ & 121 & 82 & 33 & 89 & 85 & 4 & & & & 32 & 30 & 5 & & & \\
\hline $3 b$ & 198 & 188 & 5 & 225 & 237 & -5 & & & & 107 & 30 & 72 & & & \\
\hline $4 \mathrm{~b}$ & 437 & 326 & 25 & 802 & 1048 & -31 & & & & 237 & 210 & 11 & & & \\
\hline $5 b$ & 377 & 283 & 25 & 1590 & 265 & 83 & 280 & & & 287 & 270 & 6 & & & \\
\hline $6 \mathrm{~b}$ & 186 & 185 & 1 & 122 & 30 & 75 & 160 & 164 & -2 & 174 & 153 & 12 & & & \\
\hline $7 b$ & 157 & 159 & -1 & 115 & 125 & -9 & 139 & 184 & -33 & 177 & 115 & 35 & & & \\
\hline $8 b$ & 125 & 154 & -24 & 234 & 156 & 33 & 96 & 162 & -69 & 88 & 119 & -35 & & & \\
\hline $9 \mathrm{~b}$ & 153 & 102 & 33 & -60 & 120 & $302 *$ & 111 & 105 & 5 & 166 & 137 & 18 & & & \\
\hline $10 \mathrm{~b}$ & 159 & 94 & 41 & & 92 & & 118 & 104 & 12 & 135 & 30 & 78 & & & \\
\hline $\begin{array}{l}\text { Ave } \\
\% \\
\text { diff }\end{array}$ & & 29 & & & 45 & & & 22 & & & 32 & & & 12 & \\
\hline
\end{tabular}

*This aethalometer and PEM percent difference was excluded from averaged due to large fluctuations in aethalometer attenuation 


\subsubsection{Spatial Variability of Meteorology}

Because meteorological stations may often be used to represent areas greater than a few miles in diameter, meteorological variability was evaluated in this study. Figure 23 shows the distance of all meteorological stations from the EBAM station located within the study area, with the largest distance being 1.9 miles and the shortest distance being 0.1 miles. Despite the relatively short distances between stations, significant differences were found between meteorological stations. As shown in Figure 23, Table 10, and Figure 24, the Marine Terrace (MT) station wind speeds on average were almost twice as high as CSDF wind speeds, and more than three times as high as Sonic and EBAM stations. The lower EBAM and Sonic wind speeds are likely due to their placement closer to ground level, where obstructions may readily slow velocities. Table 9 details the elevation and height above ground level for the instruments from each meteorological station. A detailed summary of wind speed over time for all stations and station combinations are available in Appendix E under Wind Speed Comparisons for All

\section{Stations.}

\section{Table 9. Height of meteorological instrument above ground}

Heights with “ ” are approximate estimations of instrument height due to access limitations.

\begin{tabular}{|l|c|c|}
\hline Met Station & Elevation & Instrument Height (feet) \\
\hline EBAM & 227 & 6.9 \\
\hline Ultrasonic Anemometer & 207 & 6.1 \\
\hline CSDF & 260 & $\sim 25$ \\
\hline Marine Terrace & 50 & $\sim 20$ \\
\hline
\end{tabular}




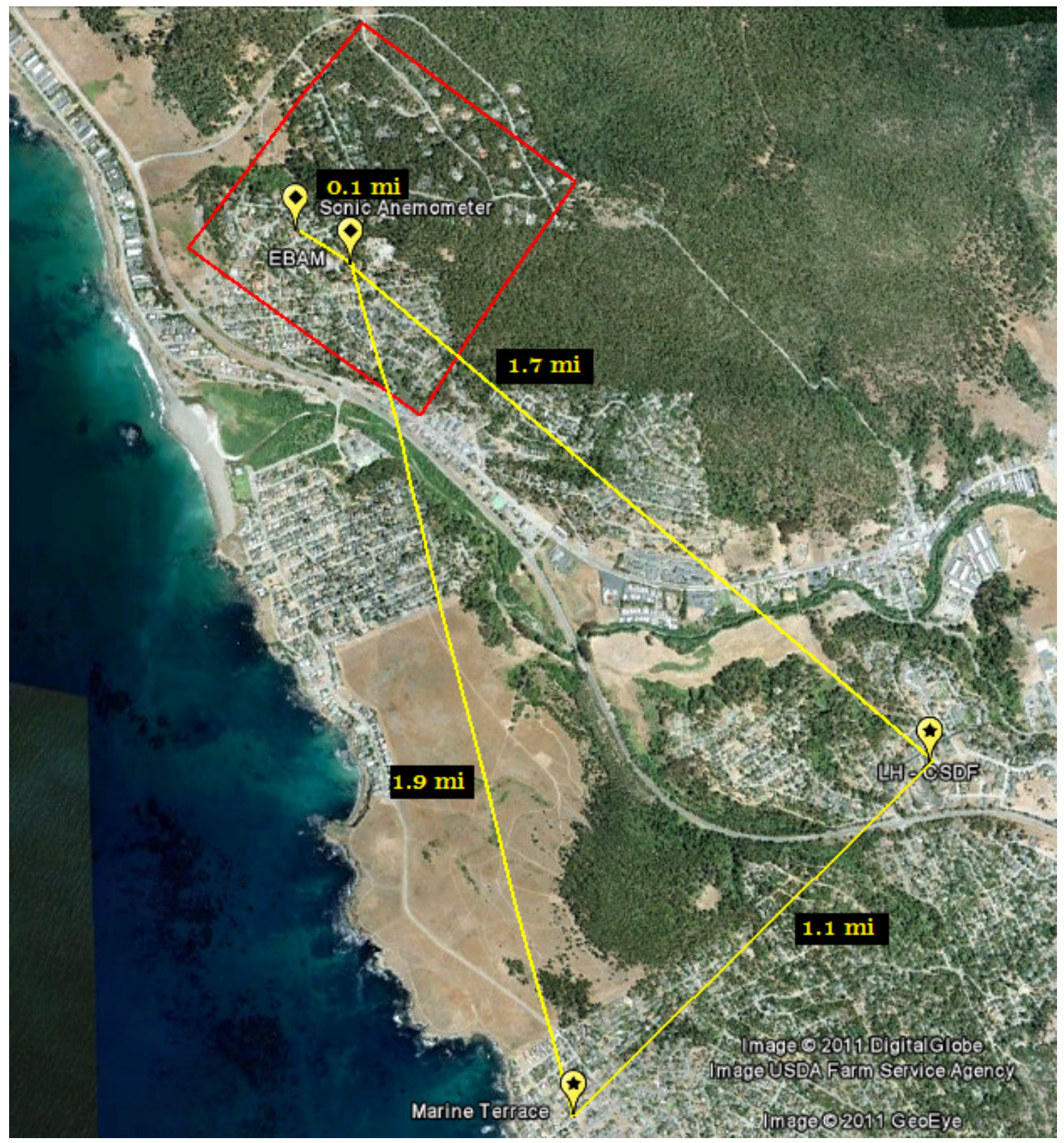

Figure 23. Distance between meteorological stations 
Table 10. Meteorological variability over stations (IOPs 8b through 10b only)

Only IOPs $8 b$ through $10 b$ are detailed to allow for comparison between meteorological stations.

\begin{tabular}{|c|c|c|c|c|c|c|c|c|c|c|c|c|}
\hline \multirow{2}{*}{ Station } & \multirow{2}{*}{$\begin{array}{l}\text { Time } \\
\text { Basis }\end{array}$} & \multicolumn{3}{|c|}{ Wind speed (mph) } & \multicolumn{2}{|c|}{ Wind Direction } & \multicolumn{3}{|c|}{ Temperature (F) } & \multicolumn{3}{|c|}{ Humidity (\%) } \\
\hline & & Average & Median & $\begin{array}{l}\text { Std } \\
\text { Dev }\end{array}$ & Average & $\begin{array}{l}\text { Std } \\
\text { Dev }\end{array}$ & Average & Median & $\begin{array}{l}\text { Std } \\
\text { Dev }\end{array}$ & Average & Median & $\begin{array}{l}\text { Std } \\
\text { Dev }\end{array}$ \\
\hline MT & $10 \min$ & 4.3 & 4.3 & 2.2 & 299 & 47.7 & 49.8 & 50.2 & 5.4 & 61.1 & 71.0 & 18.4 \\
\hline CSDF & $30 \mathrm{~min}$ & 2.4 & 2.0 & 2.4 & 254 & 49.1 & & & & 77.0 & 88.0 & 21.2 \\
\hline EBAM & $10 \mathrm{~min}$ & 1.1 & 0.9 & 0.5 & 277 & 42.4 & 49.7 & 51.1 & 4.9 & 69.0 & 81.0 & 18.7 \\
\hline Sonic & $10 \mathrm{~min}$ & 0.6 & 0.5 & 0.3 & 89.7 & 78.0 & 49.7 & 50.8 & 5.8 & & & \\
\hline
\end{tabular}


On average, wind direction did not vary widely for the MT, CSDF, and EBAM stations, but it did vary widely for the Sonic station; MT, CSDF, and EBAM wind directions varied within a 90 degree range, while Sonic wind directions varied within a 270 degree range. Observing each IOP's dominant wind direction, as shown in Appendix E under Wind Roses for All IOPs, for almost all cases, dominant wind directions for each station were not greater than 90 degrees from the dominant wind directions of other stations. Temperature, with measurements available at all stations except CSDF, was fairly consistent over all locations, varying about $\pm 5^{\circ} \mathrm{F}$ over all IOPs. Relative humidity across all sites was also similar in standard deviation (about 20 percent), but differed on average at the MT, CSDF, and EBAM stations where measurements were available. The highest relative humidity was always found at the CSDF site, which is also most inland of all stations, followed by the EBAM site which is second most inland, and the MT site, which is closest to the coast and lowest in elevation, had the lowest relative humidity. Figure 24 summarizes meteorological variability for all stations over each IOP. Meteorological data is available in greater detail in Appendix D under Meteorological Variability and Appendix E under Wind Roses for All IOPs. 

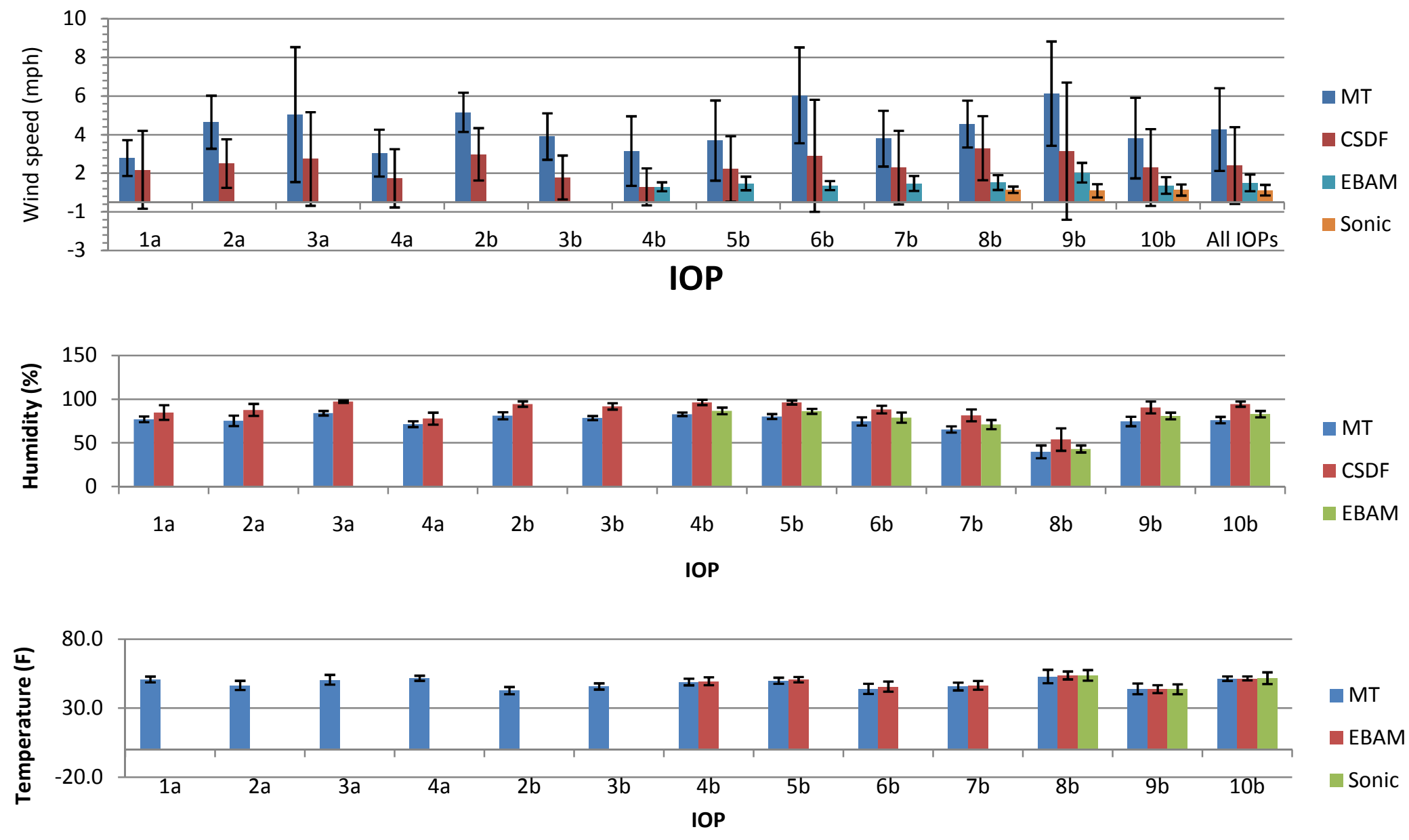

Figure 24. Meteorological variables compared between stations

Capped lines represent standard deviation. 


\subsection{Statistical Relationship of Meteorology and Source Properties with BC}

Multiple linear regression (MLR) and non-linear multiple regression (NMR) analyses were used to evaluate the effect of meteorological and wood burning source variables on BC concentration. The MLR model, a first-order multiple regression model, determines the impact of explanatory, or independent, variables on a response, or dependent, variable as demonstrated in Equation 9 below for $\mathrm{k}$ explanatory predictors.

$$
y=\beta_{0}+\beta_{1} x_{1}+\beta_{2} x_{2}+\beta_{3} x_{3}+\cdots+\beta_{k} x_{k}+\varepsilon \quad \text { Equation } 9
$$

In Equation 9, $y$ is the response variable, $x_{i}$ represents the independent variable $\mathrm{i}, \beta_{\mathrm{i}}$ is a partial slope of variable $i$, and $\varepsilon$ is random error. The partial slopes, $\beta_{\mathrm{i}}$, represent the expected change in the response variable, $y$, with each change in the explanatory variables, $x_{i} \cdot \beta_{0}$, alternatively, is the value of $y$ when all $x_{i}$ 's are equal to zero. The random error, $\varepsilon$, represents variation in $\mathrm{y}$ that cannot be explained by the $\mathrm{x}_{\mathrm{i}}$ 's (explanatory variables). MLR assumes that explanatory variables are linearly related to the response variable and that random error is normally distributed about zero. To minimize the sum of the squared residuals, or difference between the modeled and measured y value, the least-squares method was used. This method first examines the sum of the squared errors that result when different $\widehat{\beta_{1}}$ and $\widehat{\beta 0}$ values are used. The $\widehat{\beta_{1}}$ and $\widehat{\beta 0}$ values that result in the lowest sum of squared error are then selected for the MLR model. The NMR follows the same principles as the MLR except that the explanatory variables, $\mathrm{x}_{\mathrm{i}}$, and $\beta_{\mathrm{i}}$ are included in the model as a more complex, non-linear function such as in $\frac{1}{1+\beta_{i} X}$. 
The 2010 version of the statistical analysis program, Minitab version 16.1.1 (Minitab Inc.) was used to determine the best regression models for this study. BC concentrations and deviations were used as response variables while meteorological data, number of sources, distance from sources, and tree coverage were tested as explanatory variables when suitable. Data from individual meteorological stations and combinations of meteorological stations were used in the analyses, where weather station groups used data that was the average of values available for the stations it combined. Weather stations were grouped based on their geographic proximity and characteristics relative to the study site. Both Weather Underground stations, Marine Terrace and CSDF were combined due to their location within Cambria but outside of the study area. The weather stations thought to be most representative of the study area, CSDF and E-BAM, were grouped. Finally, both stations located within the study area, E-BAM and Sonic, were evaluated as a combination. Meteorological variables evaluated for all sites and combinations are summarized in Table 11. 
Table 11. Meteorological data available at each station

\begin{tabular}{|c|c|c|c|c|c|c|c|c|c|}
\hline $\begin{array}{l}\text { Station/ } \\
\text { Combination }\end{array}$ & $\begin{array}{l}\text { Measurement } \\
\text { intervals }\end{array}$ & $\begin{array}{l}\text { Wind } \\
\text { Speed } \\
(\text { mph })\end{array}$ & $\sin _{(\mathbf{W D}}^{\#)}$ & $\underset{\left(\mathbf{W D}^{\#}\right)}{\cos ^{\prime}}$ & $\begin{array}{c}\text { Humidity } \\
(\%)\end{array}$ & $\begin{array}{l}\text { Temp } \\
\text { (F) }\end{array}$ & $\begin{array}{l}\text { Wind } \\
\text { Gust } \\
\text { Speed } \\
\text { (mph) }\end{array}$ & $\begin{array}{l}\text { Vertical } \\
\text { Wind } \\
\text { Speed } \\
(\text { mph })\end{array}$ & $\begin{array}{c}\text { Average } \\
\text { Difference } \\
\text { between } \\
\text { Wind } \\
\text { Direction } \\
\end{array}$ \\
\hline $\begin{array}{l}\text { Marine } \\
\text { Terrace (MT) }\end{array}$ & $10 \mathrm{~min}$ & $X$ & $X$ & $X$ & $\mathrm{X}$ & $X$ & $X$ & & \\
\hline CSDF & $30 \mathrm{~min}$ & $X$ & $\mathrm{X}$ & $\mathrm{X}$ & $\mathrm{X}$ & & $X$ & & \\
\hline E-BAM & $10 \mathrm{~min}$ & $X$ & $X$ & $X$ & $X$ & $X$ & & & \\
\hline $\begin{array}{l}\text { Ultrasonic } \\
\text { Anemometer } \\
\text { (Sonic) }\end{array}$ & $<1 \min$ & $X$ & $X$ & $\mathrm{X}$ & & $X$ & & $X$ & \\
\hline MT \& CSDF & 30 min* & $\mathrm{X}$ & $X$ & $X$ & $X$ & & $X$ & & $X$ \\
\hline $\begin{array}{l}\text { E-BAM \& } \\
\text { CSDF }\end{array}$ & 30 min* & $X$ & $\mathrm{X}$ & $X$ & $X$ & & & & $X$ \\
\hline $\begin{array}{l}\text { E-BAM \& } \\
\text { Sonic }\end{array}$ & $10 \mathrm{~min} *$ & $X$ & $\mathrm{X}$ & $\mathrm{X}$ & & $X$ & & & $X$ \\
\hline
\end{tabular}

${ }^{\#}$ WD: Wind Direction

*The measurement intervals used for combinations were based on the station with the lowest resolution. For example, for the MT and CSDF combination, meteorological data is available at 10 and 30 minute resolutions, respectively, so 30 minute intervals were used. 
Meteorological data was evaluated in conjunction with both aethalometer and PEM BC, because both offered different characterizations of pollutant concentrations and variability within the study area. Because aethalometers provided BC data at high resolution during each IOP, a larger sample size is available to determine the relationship between $\mathrm{BC}$ concentration and weather characteristics. For this reason, it was possible to use aethalometer data to evaluate all meteorological stations and determine the meteorological station most capable of explaining variability in $\mathrm{BC}$ concentrations. PEM $\mathrm{BC}$ concentrations were also evaluated in a similar manner but for 12 hour averaged $\mathrm{BC}$ concentrations and deviations with 12 hour averaged meteorological data. EBAM and Sonic stations could not be evaluated for PEMs because they only operated during a subset of the IOPs and as a result contain too few data points. The differences and similarities between PEM and aethalometer relationships to weather data from different stations provided information about time averaging's impact on BC concentrations and variability.

While the time resolution of the PEMs (12 hours) was much lower than that of the aethalometers (1 minute), spatial resolution for the PEMs was higher than spatial resolution for aethalometers. Each IOP contained between 8 and 16 outdoor PEMs in different locations, while only between 1 and 4 aethalometers were operated in different outdoor locations during each IOP. PEM BC concentrations were thus used to evaluate the impact of distance from burning sources, number of burning sources, and site characteristics on $\mathrm{BC}$ concentration and variability. 


\subsubsection{Aethalometer BC and Meteorological Data}

Meteorological data collected from Weather Underground and available meteorological instruments, discussed in Chapter 3, were evaluated with aethalometer results to determine the meteorological data which best accounted for variability in BC concentration and $\mathrm{BC}$ deviation. For the analysis of aethalometer data, two response variables were evaluated: $\mathrm{BC}$ concentration averaged over 30 minutes $\left(\mathrm{BC}_{\mathrm{ave}}\right)$ and the weighted deviation function $\left(\mathrm{BC}_{\mathrm{dev}}\right)$ described in Equation 10

$$
B C_{d e v}=\frac{s t d d e v_{t}}{B C_{t, a v e}}
$$

\section{Equation 10}

where $s t d \operatorname{dev}_{\mathrm{t}}$ is equal to the standard deviation of all operating aethalometers at time $\mathrm{t}$ and $\mathrm{BC}_{\mathrm{t}, \mathrm{ave}}$ is the average $\mathrm{BC}$ concentration of $\mathrm{BC}$ measurements from all operating aethalometers at time t. Since concentration variability is most appropriately considered as a percentage of the measurement, $\mathrm{BC}_{\mathrm{dev}}$ allows for comparison of $\mathrm{BC}$ standard deviation over a wide range of concentrations. For instance, a standard deviation of 40 $\mathrm{mg} / \mathrm{m}^{3}$ implies much more variability when the average concentration is $40 \mathrm{ng} / \mathrm{m}^{3}$. The use of the weighted deviation function also reduces the bias toward high concentrations. Both averaged BC concentrations and deviations were additionally transformed as necessary to normalize residuals about zero. Normalization concentrates residuals about zero with a bell shaped frequency distribution, and was conducted by applying functions to the response variables. Because residuals are the difference between the observed response variable (in this study $\mathrm{BC}$ concentration or deviation) and the response variable 
resulting from the regression model, residuals normalized about zero suggests that on average, the error is approximately zero.

To satisfy the independence assumption for MLR models, BC concentrations were averaged over 30 minutes to eliminate major time correlations between $\mathrm{BC}$ readings and a BC concentration delayed (or lagged) by one time point was included as an explanatory variable in the regression models. The presence of time correlations was evaluated using the Autocorrelation Function (ACF) and the Durbin-Watson statistic in Minitab. For the $\mathrm{ACF}$, potential explanatory variables were used to develop a regression model and the residuals were stored. ACF then computes the correlation between residuals by comparing a residual at time $\mathrm{t}$ to the data point at time $\mathrm{t}+\mathrm{k} \Delta$, where $\Delta$ is 30 minutes in this study and $\mathrm{k}$ is any constant. Minitab produces ACF statistics and a graph that indicates whether correlations exist between the response variable at times $\mathrm{t}$ and $\mathrm{t}+\mathrm{k} \Delta$. A comparison of $\mathrm{t}$ and $\mathrm{t}+1 \Delta$ (where $\mathrm{k}$ is equal to 1 ) is referred to as "lag 1 ," and comparing $\mathrm{t}$ to $\mathrm{t}+2 \Delta$ (where $\mathrm{k}$ is a value of 2 ) is called "lag 2 ," and so forth. Figure 25 is a sample graph of residuals resulting from the use of the CSDF meteorological station variables in conjunction with average $\mathrm{BC}$ concentration. The red dotted lines indicate the 95 percent confidence intervals where time correlations are insignificant. An ACF value above the top of or below the bottom of the red dotted line indicates that correlations exist. In the case shown in Figure 25, a lag 1 correlation exists and must be accounted for in the model. Because all IOPs showed correlations with lag 1, BC concentration or deviation lagged by one time point was included as an explanatory variable. To run the ACF analysis, missing meteorological data at single time points were assumed to be the 
average of the previous and following time point value. Because there were very few missing single time points, their effect on the models should be undetectable. When larger intervals of data were missing, the data set had to be excluded from the ACF analyses. Data sets where the ACF could not be conducted were assumed to be correlated at similar lags as data sets where the ACF was used. Large time intervals of missing data were rare, so their impact on all data sets should not be significant.

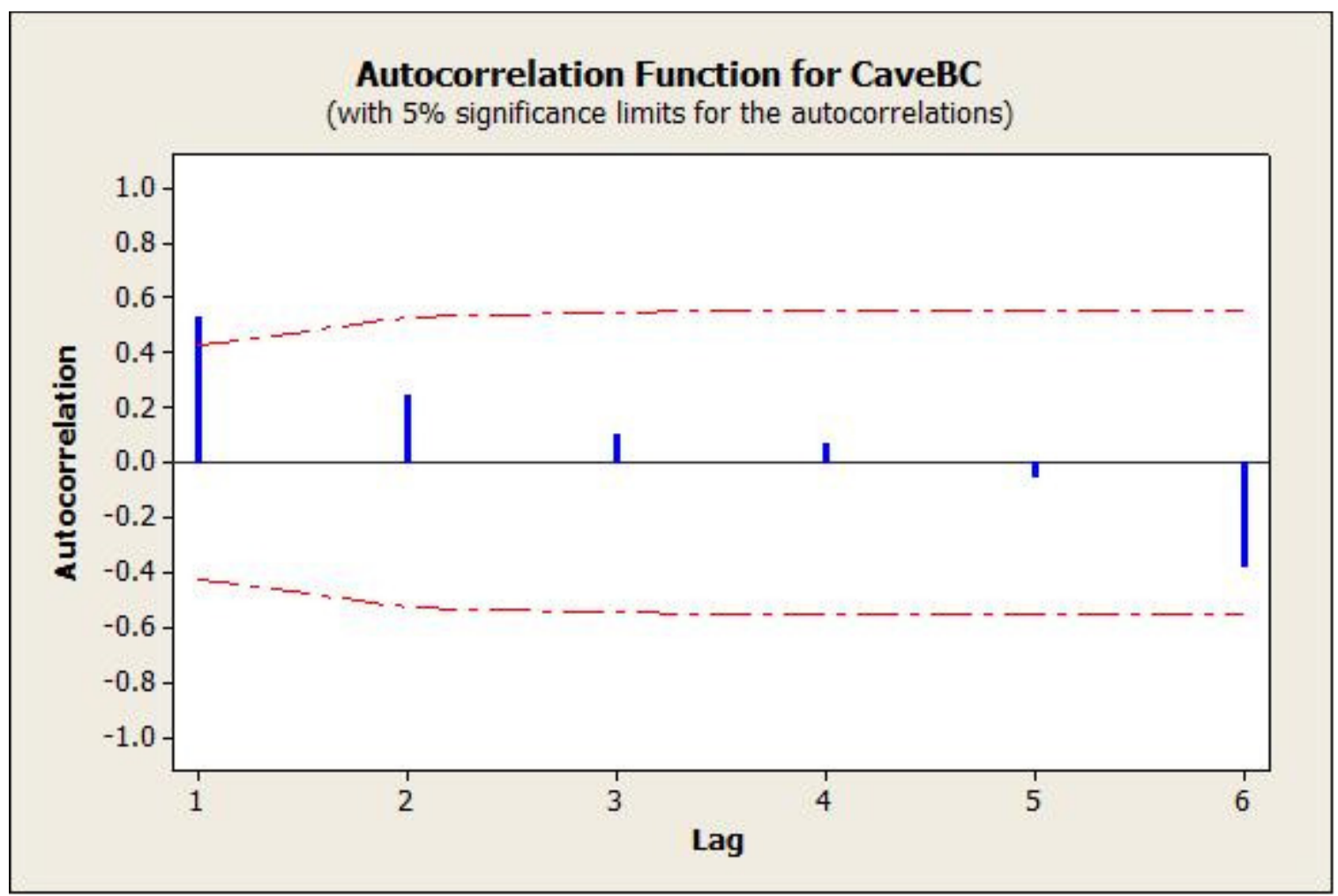

Figure 25. ACF Analysis Example for CSDF Meteorological Station

In developing a suitable regression model, the Durbin-Watson (DW) statistic was also evaluated. Although similar in purpose to the ACF analysis, the DW statistic checks only for correlations in the lag 1 situation and differs mathematically. Critical values developed by Durbin and Watson (1951) indicate when negative and positive correlations 
exist based on the fact that squared differences in sequential residuals will be smaller for positive correlations and larger for negative correlations. A DW statistic of 2 generally indicates no serial correlation while values less than 2 and greater than 2 suggest positive and negative correlations, respectively (Ott \& Longnecker, 2010). Because DW cutoffs for determining correlations are limited to sample sizes of 200 or less (Savin \& White, 1977), specific DW cutoffs could not be used for this study's data sets which in most cases contained sample sizes greater than 300 . For this reason, the DW statistic observed in developed aethalometer models was assumed to have no time correlations if close to the value of 2. This approximate use of the DW statistic offers additional support to the ACF conclusion - that no correlation exists at lag 1 . ACF tests alone are considered to be sufficient on their own due to their ability to evaluate correlations at multiply lags instead of just one as for the Durbin Watson test.

In developing multiple regression models, suitable meteorological predictors for $\mathrm{BC}$ averages or deviations were determined by first including potential predictors (as listed in Table 11) for each meteorological station or combination in the regression models and then eliminating an explanatory variable that both had a p-values greater than 0.05 , the significance level, and a p-value higher than the p-value of all other variables. At a significance level of 0.05 , the confidence of the partial slopes can be determined using one minus the significance level, or 95 percent confidence in this case. Another regression model was then tested using all remaining explanatory variables and a second explanatory variable whose p-value was greater than the significance level and also higher than the p-value of all other independent variables was then eliminated. The same 
process was then repeated until all variables with p-values higher than the significant level were removed. To account for diurnal variations in $\mathrm{BC}$ concentration, a predictor representing the number of time intervals passed during the sampling period was used. For example, when using 30 minute averaged $\mathrm{BC}$ concentrations, the first 30 minutes of the sampling period is time interval 1 , the second 30 minute period is time interval 2 , and so forth. A "lack of fit" test was also used to indicate whether interactions or curvature existed in the models. Minitab's lack of fit test evaluates the applicability of the developed model for future model inputs. If interactions or curvature were found, modifications were made to account for these properties resulting in nonlinearity of the model(s).

\subsubsection{Predictors for Aethalometer Average BC Concentration}

While the relationship between $\mathrm{BC}$ concentrations and meteorological predictors is typically not linear, a MLR model can still be used by normalizing BC concentrations through the use of a transformative function. To normalize average $\mathrm{BC}$ concentrations $\left(\mathrm{BC}_{\mathrm{ave}}\right)$ and residuals, a transformation that best normalize $\mathrm{BC}$ concentrations, raising average $\mathrm{BC}$ concentration to the $-0.7^{\text {th }}$ power, was determined and used for the MLR model. Figure 26 compares the normal probability plots and histograms of average BC concentration (left) to its transformed counterpart (BCave) ${ }^{-0.7}$ (right). A straight sloping line following the blue line is needed for the normal probability plot and a bell shaped pattern is desired for the histogram. However, it is important to note that when the normalizing power was negative as shown in Figure 26, higher concentrations become the lower values of the normalized response variable. 

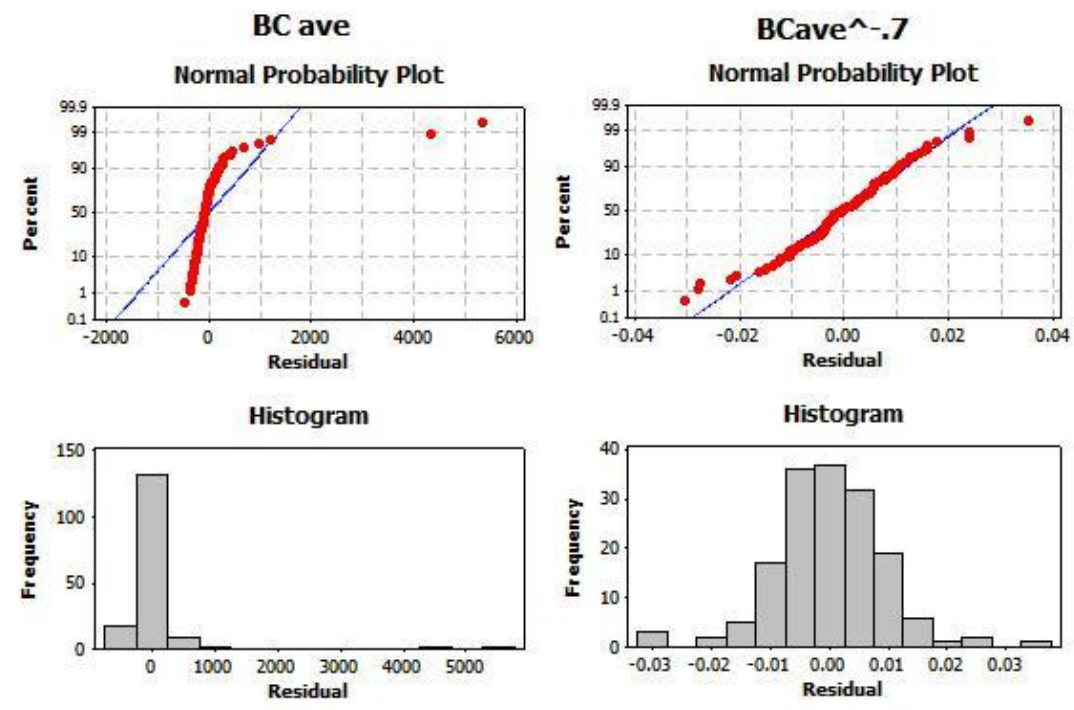

Figure 26. Comparison between residual charts for normalized (right) and unnormalized (left) average aethalometer BC concentration

When using meteorological variables from each station or combination to determine a model for average $\mathrm{BC}$ concentration, it was found that only two meteorological stations or combinations contained variables the explained variability in average $\mathrm{BC}$ concentration: the EBAM station and the EBAM and CSDF station combination. Table 12 summarizes the results of the Minitab analyses including the partial slopes for significant variables and the coefficients of determination $\left(\mathrm{R}^{2}\right)$ for each model. The $\mathrm{R}^{2}$ value indicates the percent of variability in the average $\mathrm{BC}$ concentration accounted for by the explanatory variables included in the model. The $\mathrm{R}^{2}$ value for the average $\mathrm{BC}$ concentration models ranged from 52 to 55 percent, with the EBAM and CSDF combination explaining the most variability for both linear and non-linear models. Among tested explanatory variables, relative humidity and wind speed were the only significant meteorological predictors for aethalometer BC average. Average BC 
concentration lagged by one time interval (Lag 1 (BCave $)^{-0.7}$ ) and time interval, accounting for time of day, also significantly accounted for average BC concentration after transformation.

Table 12. Multiple regression models for average aethalometer $\mathrm{BC}$ concentrations

The table below summarizes explanatory predictors determined to be significantly correlated to aethalometer BC based on Minitab analysis.

\begin{tabular}{|c|c|c|c|c|c|c|}
\hline $\begin{array}{l}\text { Met } \\
\text { Station }\end{array}$ & $\begin{array}{l}\text { Model } \\
\text { Type }\end{array}$ & $\begin{array}{l}\text { Response } \\
\text { Variable }\end{array}$ & $\begin{array}{l}\text { Explanatory } \\
\text { Variable }\end{array}$ & $\begin{array}{l}\text { Coefficient } \\
\left(\widehat{\beta_{1}}\right)\end{array}$ & $\begin{array}{l}\text { Std Error } \\
\text { of } \\
\text { Coefficient }\end{array}$ & $\mathbf{R}^{2}(\%)$ \\
\hline \multirow[t]{9}{*}{ EBAM } & \multirow[t]{4}{*}{ Linear } & \multirow[t]{4}{*}{ (BCave $^{-0.7}$} & Lag 1 (BCave) $)^{-0.7}$ & $5.3 \times 10^{-01}$ & $6.5 \times 10^{-02}$ & \multirow[t]{4}{*}{52} \\
\hline & & & Time Interval & $4.0 \times 10^{-04}$ & $1.2 \times 10^{-04}$ & \\
\hline & & & Humidity (\%) & $-2.1 \times 10^{-04}$ & $5.8 \times 10^{-05}$ & \\
\hline & & & Constant, $\beta$ & $2.5 \times 10^{-02}$ & $5.4 \times 10^{-03}$ & \\
\hline & \multirow{5}{*}{$\begin{array}{l}\text { Non- } \\
\text { linear }\end{array}$} & \multirow[t]{5}{*}{$(\text { BCave })^{-0.7}$} & Lag1 (BCave) $)^{-0.7}$ & $2.5 \times 10^{-01}$ & $1.4 \times 10^{-01}$ & \multirow[t]{5}{*}{54} \\
\hline & & & Time Interval & $-3.8 \times 10^{-04}$ & $3.8 \times 10^{-04}$ & \\
\hline & & & $\begin{array}{l}\text { (Lag } 1 \text { (BCave) } \\
0.7) *(\text { Time } \\
\text { Interval) }\end{array}$ & $2.2 \times 10^{-02}$ & $1.0 \times 10^{-02}$ & \\
\hline & & & $(\text { Humidity } \%)^{2}$ & $-1.4 \times 10^{-06}$ & $4.5 \times 10^{-07}$ & \\
\hline & & & Constant, $\beta$ & $2.8 \times 10^{-02}$ & $5.1 \times 10^{-03}$ & \\
\hline \multirow{11}{*}{$\begin{array}{l}\text { EBAM } \\
\& \mathrm{LH} \\
\mathrm{CSDF}\end{array}$} & \multirow[t]{5}{*}{ Linear } & \multirow[t]{5}{*}{$(\text { BCave })^{-0.7}$} & Lag 1 (BCave) $)^{-0.7}$ & $5.1 \times 10^{-01}$ & $6.5 \times 10^{-02}$ & \multirow[t]{5}{*}{53} \\
\hline & & & Time Interval & $3.8 \times 10^{-04}$ & $1.2 \times 10^{-04}$ & \\
\hline & & & $\begin{array}{l}\text { Wind Speed } \\
(\mathrm{mph})\end{array}$ & $2.3 \times 10^{-03}$ & $9.4 \times 10^{-04}$ & \\
\hline & & & Humidity (\%) & $-1.7 \times 10^{-04}$ & $6.1 \times 10^{-05}$ & \\
\hline & & & Constant, $\beta$ & $2.1 \times 10^{-02}$ & $5.9 \times 10^{-03}$ & \\
\hline & \multirow{6}{*}{$\begin{array}{l}\text { Non- } \\
\text { linear }\end{array}$} & \multirow[t]{6}{*}{$(\text { BCave })^{-0.7}$} & Lag1 (BCave) ${ }^{-0.7}$ & $2.5 \times 10^{-01}$ & $1.3 \times 10^{-01}$ & \multirow[t]{6}{*}{55} \\
\hline & & & Time Interval & $-4.5 \times 10^{-04}$ & $3.7 \times 10^{-04}$ & \\
\hline & & & $\begin{array}{l}\text { (Lag } 1 \text { (BCave) })^{-} \\
0.7) *(\text { Time } \\
\text { Interval) }\end{array}$ & $2.3 \times 10^{-02}$ & $1.0 \times 10^{-02}$ & \\
\hline & & & $\begin{array}{l}\text { Wind Speed } \\
(\mathrm{mph})\end{array}$ & $2.2 \times 10^{-03}$ & $9.3 \times 10^{-04}$ & \\
\hline & & & Log(Humidity \%) & $-8.3 \times 10^{-03}$ & $4.0 \times 10^{-03}$ & \\
\hline & & & Constant, $\widehat{\beta 0}$ & $5.3 \times 10^{-02}$ & $1.8 \times 10^{-02}$ & \\
\hline
\end{tabular}


Although the model $\mathrm{R}^{2}$ values were found to account for more than 50 percent of average $\mathrm{BC}$ variability, lack of fit tests conducted in conjunction with Minitab analyses suggest that the models need improvement. Lack of fit tests provide information about how well a developed model will perform for inputs independent of the variables used to develop the model. All models poorly represent average $\mathrm{BC}$ concentration at higher values of $\mathrm{x}_{\mathrm{i}}$ (i.e. higher wind speeds and humidity). Additionally, all linear models were found to have potential curvature and/or interactions. Figure 27 displays plots of $\left(\mathrm{BC}_{\mathrm{ave}}\right)^{-0.7}$ versus EBAM meteorological variables in addition to applied trend lines. Although the variables are statistically significant, the figure indicates that only a weak relationship exists between $\left(\mathrm{BC}_{\mathrm{ave}}\right)^{-0.7}$ and the variables, time interval and relative humidity. $\mathrm{BC}$ concentration tends to generally decrease as time interval increases with a fairly noisy relationship Relative humidity may potentially follow a parabolic pattern, but data between 50 and 70 percent humidity did not occur during sampling periods. The linear correlation with Lag $1\left(\mathrm{BC}_{\mathrm{ave}}\right)^{-0.7}$ alternately exists because the values directly correspond to $(\mathrm{BCave})^{-0.7}$ values. Figure 28 displays $\left(\mathrm{BC}_{\mathrm{ave}}\right)^{-0.7}$ versus explanatory predictors for the EBAM-CSDF combination in addition to applied trend lines. The response variable, $\left(\mathrm{BC}_{\mathrm{ave}}\right)^{-0.7}$, had similarly ambiguous relationships with relative humidity and time interval, and wind speed also seems to demonstrate a noisy distribution with a mild upward slope. This upward sloping relationship between $\left(\mathrm{BC}_{\mathrm{ave}}\right)^{-0.7}$ and wind speed was expected since higher wind speeds tend to increase mixing and pollutant dispersion, resulting in lower $\mathrm{BC}$ concentrations. 

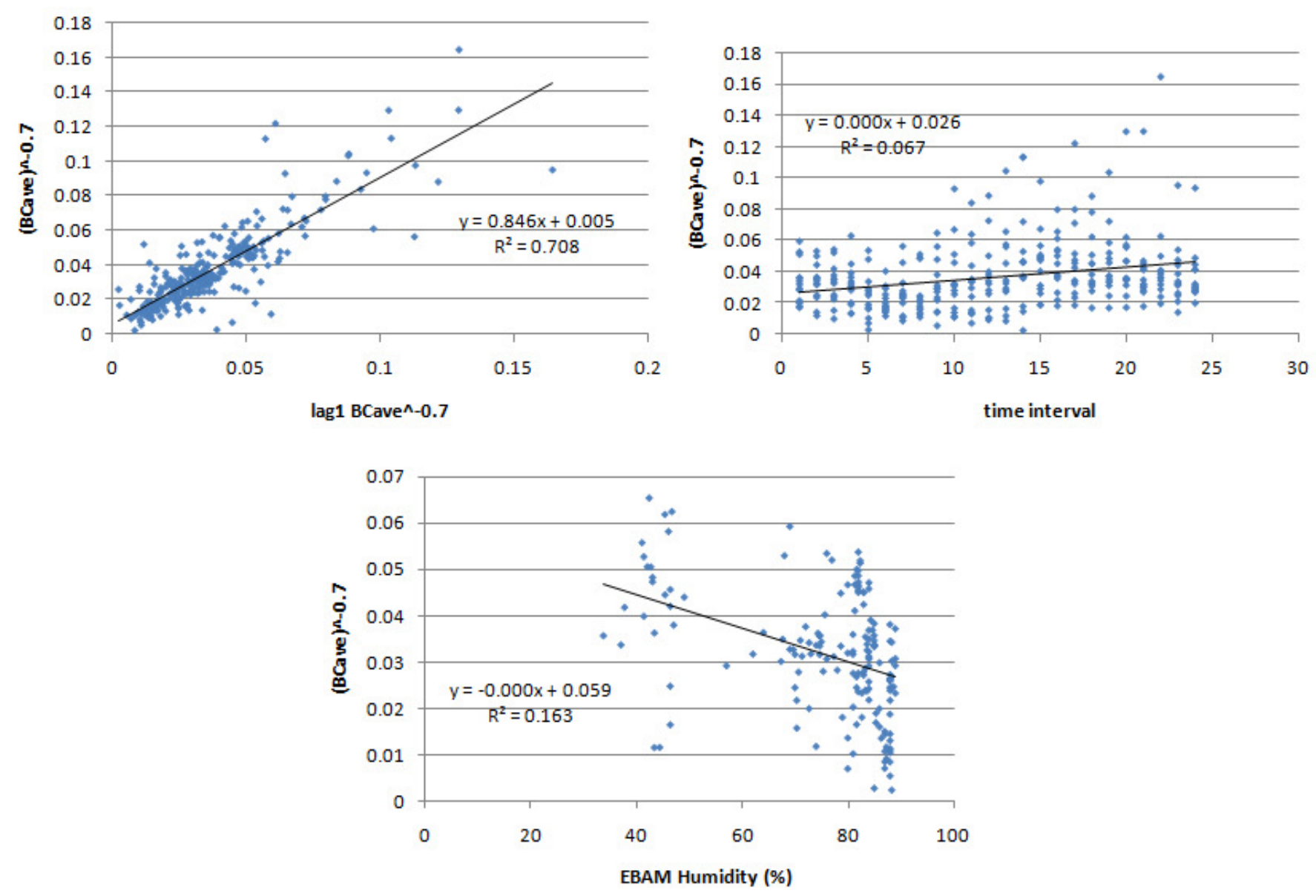

Figure 27. EBAM variable plots for aethalometer average BC concentration

Note that the negative power applied to $B C_{\text {ave }}$ inverses the correlations between average $B C$ and the explanatory variables, time interval and relative humidity. 

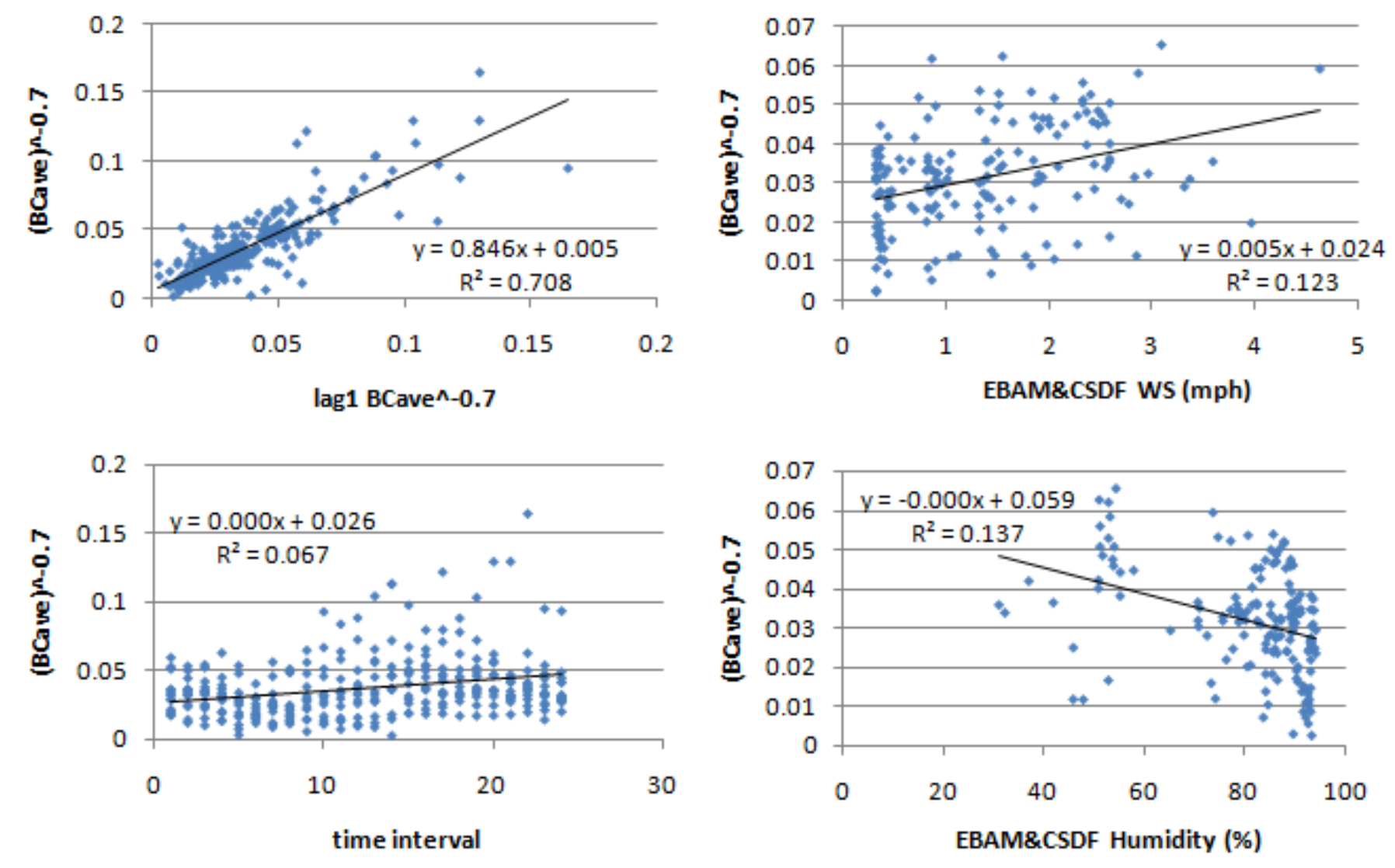

Figure 28. EBAM-LH CSDF combination variable plots for aethalometer average BC concentration

Note that the negative power applied to $B C_{\text {ave }}$ inverses the correlations between average $B C$ and the explanatory variables, wind speed (WS), time interval, and relative humidity. 
The unclear relationship between the meteorological and time variables likely resulted in the detection of possible interactions and curvature by the lack of fit tests. To account for possible interactions and curvature, the models were adjusted to be non-linear. In the cases of the EBAM and EBAM-CSDF combinations, converting the linear model into a non-linear model did not improve the model's $\mathrm{R}^{2}$ value dramatically. Mild improvements to the $\mathrm{R}^{2}$ values may be the result of increasing the number of explanatory variables or only mildly improving the fit. The former is suspected because the standard errors for the linear models tend to be smaller than the standard deviations for the nonlinear models relative to their coefficients. Smaller variations in the linear models suggest higher accuracy than non-linear models. For this reason, the linear models are likely a more suitable model for understanding average aethalometer BC concentration than the non-linear models. In future application of linear models, potential interaction detected in the Lag $1\left(\mathrm{BC}_{\mathrm{ave}}\right)^{-0.7}$ predictor and possible curvature in time interval and relative humidity variables for the EBAM meteorological station should be noted. For the meteorological data combining the EBAM and CSDF stations, potential interactions in the Lag 1 (BCave) $)^{-0.7}$ and wind speed predictors were observed. Minitab outputs for the established models are included in their entirety in Appendix G.

While the results of the models suggest that the EBAM and CSDF combination account for more of the $\mathrm{BC}$ variation than the EBAM station alone, the difference is small. This may indicate that the EBAM station alone would be sufficient to represent average BC concentration when using the above models. The small improvement in $\mathrm{R}^{2}$ values for the EBAM and CSDF combination versus EBAM alone may have resulted from the addition 
of the wind speed predictor in the EBAM and CSDF combination model or because using additional information from the CSDF station helps to account for upper air meteorology (in addition to EBAM's ground level meteorology). Regardless, EBAM meteorological data alone accounted for a similar percentage of average aethalometer $\mathrm{BC}$ concentration.

\subsubsection{Predictors for Aethalometer BC Deviation}

$\mathrm{BC}$ deviation $\left(\mathrm{BC}_{\mathrm{dev}}\right)$ and meteorology were evaluated to determine whether meteorology was correlated with $\mathrm{BC}$ standard deviation, or variance. To normalize $\mathrm{BC}$ deviation $\left(\mathrm{BC}_{\mathrm{dev}}\right)$ as done with average $\mathrm{BC}$, the best transformation was $\mathrm{BC}$ deviation raised to the $0.5^{\text {th }}$ power. Only the CSDF meteorological station's variables were found to be suitable predictors of BC deviation. Table 13 summarizes the results of the Minitab analysis including the partial slopes for significant variables and the coefficient of determination $\left(\mathrm{R}^{2}\right)$ for the resulting linear model. The $\mathrm{R}^{2}$ value for the CSDF model was 47 percent, with wind speed and relative humidity as significant meteorological predictors of variation in $\mathrm{BC}$ deviation and $\mathrm{Lag} 1\left(\mathrm{BC}_{\mathrm{dev}}\right)^{0.5}$ as a variable intended to account for time correlations. Wind speed and lagged BC deviation were positively correlated with BC deviation while relative humidity was negatively correlated.

Table 13. Multiple regression model for aethalometer BC deviation

\begin{tabular}{|c|c|c|c|c|c|c|}
\hline $\begin{array}{l}\text { Met } \\
\text { Station }\end{array}$ & $\begin{array}{l}\text { Model } \\
\text { Type }\end{array}$ & $\begin{array}{l}\text { Response } \\
\text { Variable }\end{array}$ & $\begin{array}{l}\text { Explanatory } \\
\text { Variable }\end{array}$ & $\begin{array}{l}\text { Coefficient } \\
(\widehat{\beta} \mathrm{l})\end{array}$ & $\begin{array}{l}\text { Std Error } \\
\text { of } \\
\text { Coefficient }\end{array}$ & $\mathbf{R}^{2}(\%)$ \\
\hline \multirow[t]{4}{*}{ CSDF } & \multirow[t]{4}{*}{ Linear } & \multirow[t]{4}{*}{$\left(\mathrm{BC}_{\mathrm{dev}}\right)^{0.5}$} & $\begin{array}{l}\text { Lag } 1 \\
(\mathrm{BCdev})^{0.5}\end{array}$ & $6.1 \times 10^{-01}$ & $4.5 \times 10^{-02}$ & \multirow[t]{4}{*}{47} \\
\hline & & & Wind Speed & $-2.8 \times 10^{-02}$ & $8.3 \times 10^{-03}$ & \\
\hline & & & Humidity & $-2.4 \times 10^{-03}$ & $1.1 \times 10^{-03}$ & \\
\hline & & & Constant, $\widehat{\beta 0}$ & $4.7 \times 10^{-01}$ & $1.1 \times 10^{-01}$ & \\
\hline
\end{tabular}


Similar to models for average $\mathrm{BC}$ concentration, the $\mathrm{BC}$ deviation model contained possible curvature and an interaction, but larger predictor variables (i.e. high wind speed and relative humidity) were not recognized by Minitab to fit the model poorly as for $\mathrm{BC}_{\text {ave. }}$ Lag $1\left(\mathrm{BC}_{\mathrm{dev}}\right)^{0.5}$ demonstrated potential curvature and relative humidity showed potential interactions, but attempts to account for the curvature and interaction were unsuccessful. Figure 29 shows plots of $\left(\mathrm{BC}_{\mathrm{dev}}\right)^{0.5}$ versus $\mathrm{CSDF}$ wind speed, relative humidity, and Lag $1\left(\mathrm{BC}_{\mathrm{dev}}\right)^{0.5}$ as well as applied trend lines. Although the Lag 1 $\left(\mathrm{BC}_{\mathrm{dev}}\right)^{0.5}$ predictor demonstrates a general upward sloping trend, the wind speed and relative humidity relationships with $\mathrm{BC}$ deviation are much less apparent which is similar to the trends for the average $\mathrm{BC}$ concentration models. The detection of possible curvature and interactions by the lack of fit tests may have resulted from the unclear relationships between the $\mathrm{BC}$ deviation and the meteorological variables. Minitab outputs for the established models are included in their entirety in Appendix G. 

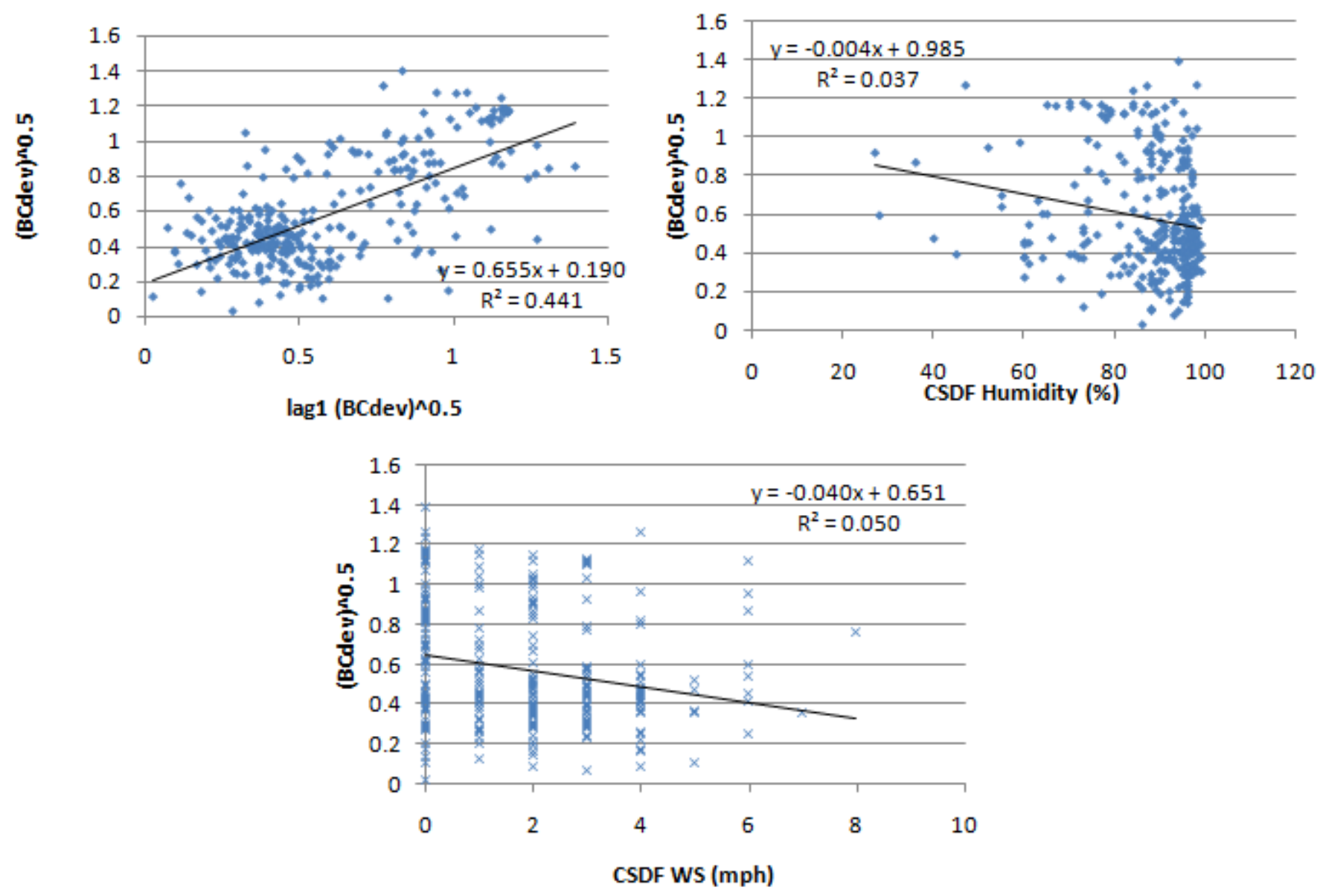

Figure 29. CSDF variable plots for aethalometer BC deviation 


\subsubsection{PEM BC Relationship to Meteorology and Burning Sources}

Two different MLR analyses were conducted for PEM BC data. Similar to the analyses for aethalometer BC data, the first test evaluated which meteorological data best accounted for variability in BC concentration and deviation. Each IOP's average BC concentration was determined by taking the mean of all PEM BC measured during the IOP's sampling period and the IOP's BC deviation was determined by taking the standard deviation of all PEM BC concentrations for that sampling period and then

dividing by the mean $\mathrm{BC}$ concentration (similar to Equation 10, but where $\mathrm{BC}_{\mathrm{t}, \text { ave }}$ is the time integrated concentration for all operating PEMs operating over the same IOP). The second PEM BC analysis investigated the impact of wood burning sources on PEM BC concentration by focusing on geographic proximity to sources and relation to dominant wind direction.

\subsubsection{Meteorological Representation for PEM BC}

To determine which meteorological station contained variables that best explained PEM BC concentrations, a similar statistical approach was taken for PEM BC as was used for aethalometer BC in Minitab. Average PEM BC and BC deviation were used as the response variables, while meteorological characteristics were used as explanatory variables. Because PEM and aethalometer resolution differed, response and explanatory variables were used in a modified manner for the PEM analysis. Instead of using average $\mathrm{BC}$ concentration and weighted deviation for every 30 minutes, the 12 hour integrated BC concentration measured using each PEM was averaged with other PEMs operating during the same IOP to determine $\mathrm{BC}_{\mathrm{ave}}$. The standard deviation of those PEMs was then 
divided by $\mathrm{BC}_{\mathrm{ave}}$ to find the $\mathrm{BC}_{\mathrm{dev}}$ value. Meteorological data (as summarized in Table 11) available at 10 and 30 minute resolution (for Marine Terrace and CSDF, respectively) were also averaged over 12 hours for use as explanatory variables in the regression analyses. Because a substantially smaller sample size was available for PEM analysis than for aethalometer analyses, EBAM and Sonic meteorological stations, which were not available for all IOPs, could not be analyzed. Additional meteorological variables, presence of inversion (yes or no) and difference in wind direction (for combined meteorological stations only), were included in the analysis. The number of wood burning sources detected on each night of sampling was also included as a predictor variable, while lagged $\mathrm{BC}$ concentration and deviation were not needed as in the aethalometer analysis due to the larger time spans between sampling nights. PEM BC concentrations were assumed to be independent from one another and the DW statistical cutoffs determined by Savin and White (1977) were used to confirm independence.

\subsection{PEM Average BC Concentration}

The transformation best able to normalize $\mathrm{BC}$ concentrations was average PEM BC concentration raised to the $0.4^{\text {th }}$ power for the Marine Terrace station and the natural log of BC concentration for the CSDF station and the Marine Terrace and CSDF combination. For average PEM BC concentration, wind speed consistently accounted for variation. The sine of wind direction was found to also be a predictor for the Marine Terrace and the Marine Terrace and CSDF combination and the cosine of the wind direction was an additional predictor for the Marine Terrace station. Table 14 summarizes the results of the Minitab analysis including the partial slopes for significant 
variables and the coefficient of determination $\left(\mathrm{R}^{2}\right)$ for the resulting linear model. The $\mathrm{R}^{2}$ values for the average $\mathrm{BC}$ models ranged from 47 to 81 percent, with the Marine Terrace model explaining the most variability (81 percent) in average PEM BC concentration.

Figure 30, Figure 31, and Figure 32 show scatter plots and an applied trend line for the two stations and their combination and their significant variables. Minitab outputs for the average PEM BC models are included in their entirety in Appendix $\mathrm{H}$.

Table 14. Linear regression models for average PEM BC concentration

\begin{tabular}{|c|c|c|c|c|c|}
\hline $\begin{array}{l}\text { Met } \\
\text { Station }\end{array}$ & $\begin{array}{l}\text { Response } \\
\text { Variable }\end{array}$ & $\begin{array}{l}\text { Explanatory } \\
\text { Variable }\end{array}$ & $\begin{array}{l}\text { Coefficient } \\
\left(\widehat{\beta_{1}}\right)\end{array}$ & $\begin{array}{l}\text { Std Dev of } \\
\text { Coefficient }\end{array}$ & $\mathbf{R}^{2}(\%)$ \\
\hline \multirow{4}{*}{$\begin{array}{l}\text { Marine } \\
\text { Terrace }\end{array}$} & \multirow[t]{4}{*}{$\left(\mathrm{BC}_{\text {ave }}\right)^{0.4}$} & Wind Speed & $-2.3 \times 10^{0}$ & $4.8 \times 10^{-01}$ & \multirow[t]{4}{*}{81} \\
\hline & & $\sin$ (wind direction) & $1.5 \times 10^{01}$ & $5.5 \times 10^{0}$ & \\
\hline & & $\begin{array}{l}\cos (\text { wind } \\
\text { direction })\end{array}$ & $1.3 \times 10^{01}$ & $3.3 \times 10^{0}$ & \\
\hline & & Constant, $\beta$ & $1.8 \times 10^{01}$ & $1.9 \times 10^{0}$ & \\
\hline \multirow[t]{2}{*}{ CSDF } & \multirow[t]{2}{*}{$\ln \left(\mathrm{BC}_{\mathrm{ave}}\right)$} & Wind Speed & $-7.8 \times 10^{-01}$ & $2.5 \times 10^{-01}$ & \multirow[t]{2}{*}{47} \\
\hline & & Constant, $\beta$ & $6.7 \times 10^{0}$ & $5.0 \times 10^{-01}$ & \\
\hline \multirow{3}{*}{$\begin{array}{l}\text { Marine } \\
\text { Terrace } \\
\& \text { CSDF }\end{array}$} & \multirow[t]{3}{*}{$\ln \left(\mathrm{BC}_{\mathrm{ave}}\right)$} & Wind Speed & $-7.3 \times 10^{-01}$ & $1.4 \times 10^{-01}$ & \multirow[t]{3}{*}{74} \\
\hline & & $\sin$ (wind direction) & $2.6 \times 10^{0}$ & $1.0 \times 10^{0}$ & \\
\hline & & Constant, $\widehat{\beta 0}$ & $7.3 \times 10^{0}$ & $4.1 \times 10^{-01}$ & \\
\hline
\end{tabular}

As was expected, PEM BC concentration decreased with increasing wind speed in all models. The physical significance of sine and cosine of wind direction however is more obscure in the PEM models. The Marine Terrace model indicates that average PEM BC decreased as winds originated further northward and westward, while the Marine Terrace and CSDF combination indicated that $\mathrm{BC}$ concentrations decreased as winds originated further southward. The Marine Terrace station suggested wind directions may indicate that the incoming ocean breeze on the north western side of the study area contributes significantly to $\mathrm{BC}$ dispersion which in turn reduces $\mathrm{BC}$ concentration. Alternately, winds originating from the northern direction for the Marine Terrace station were 
consistently higher than winds originating from the southern direction. In that case, the higher magnitude of wind speed may have been the cause of lower BC concentrations. No other wind directions and speeds for Marine Terrace and the Marine Terrace and CSDF combination demonstrated this pattern.
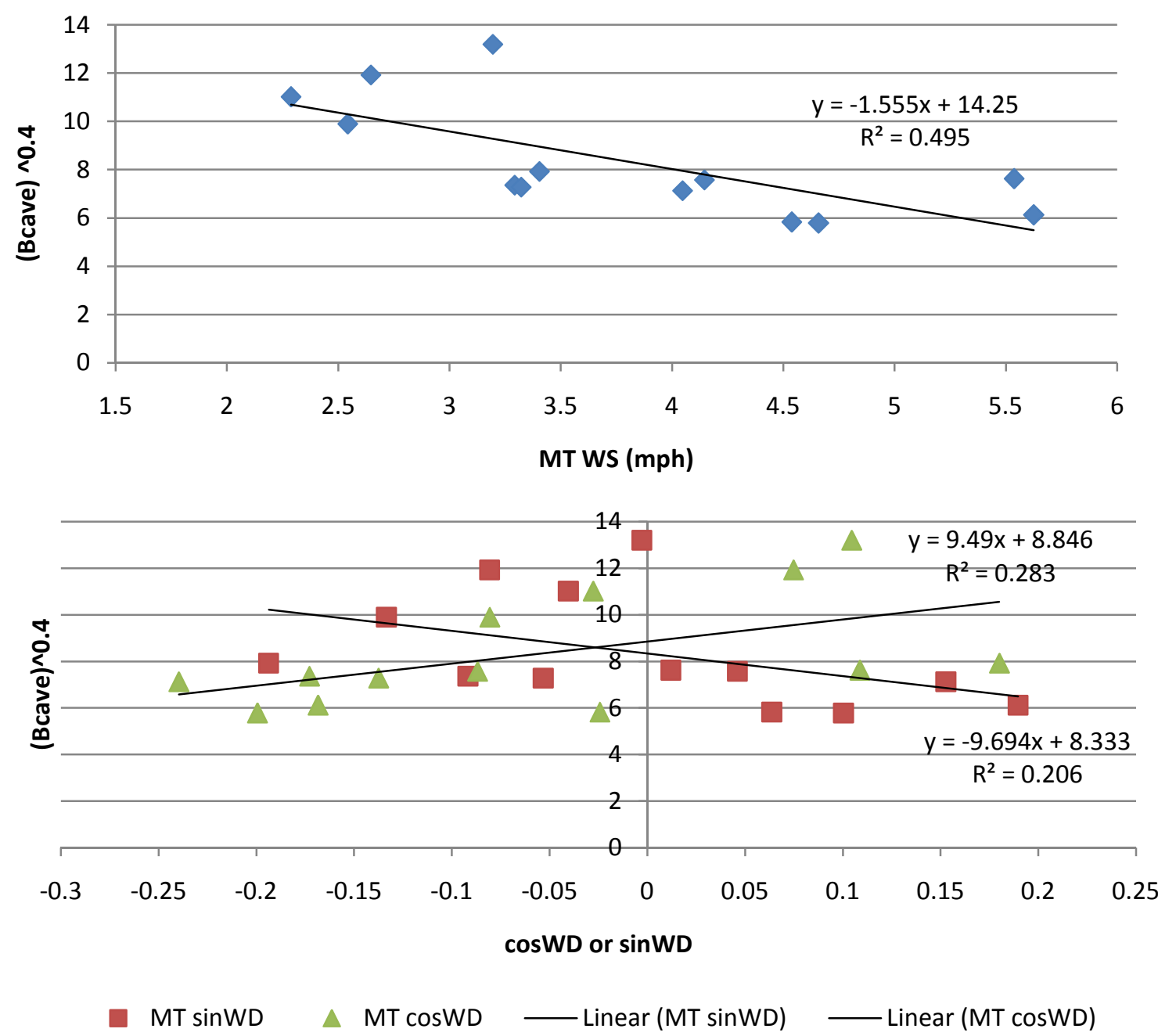

Figure 30. Marine Terrace variable plots for wind speed and wind direction versus $P E M$ average $B C$ concentration 


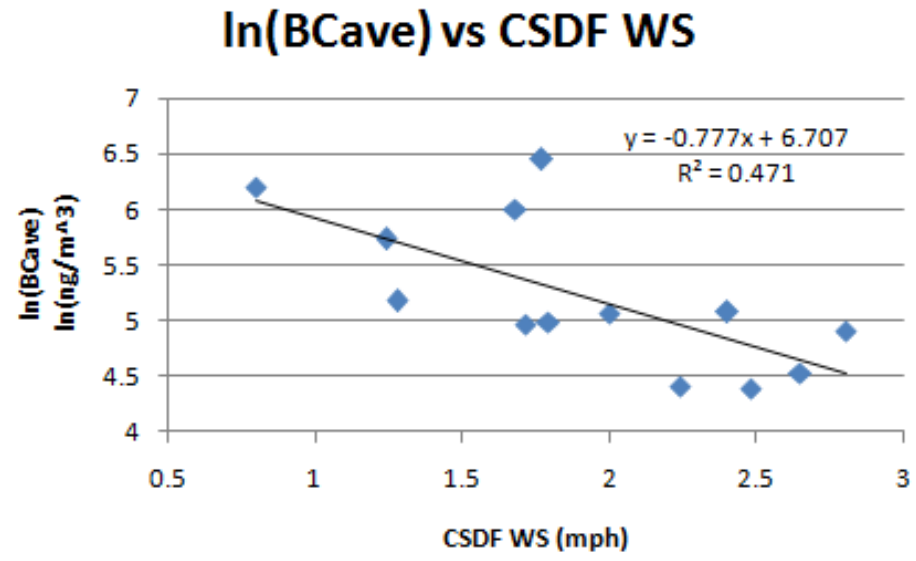

Figure 31. CSDF variable plots for PEM average BC concentration
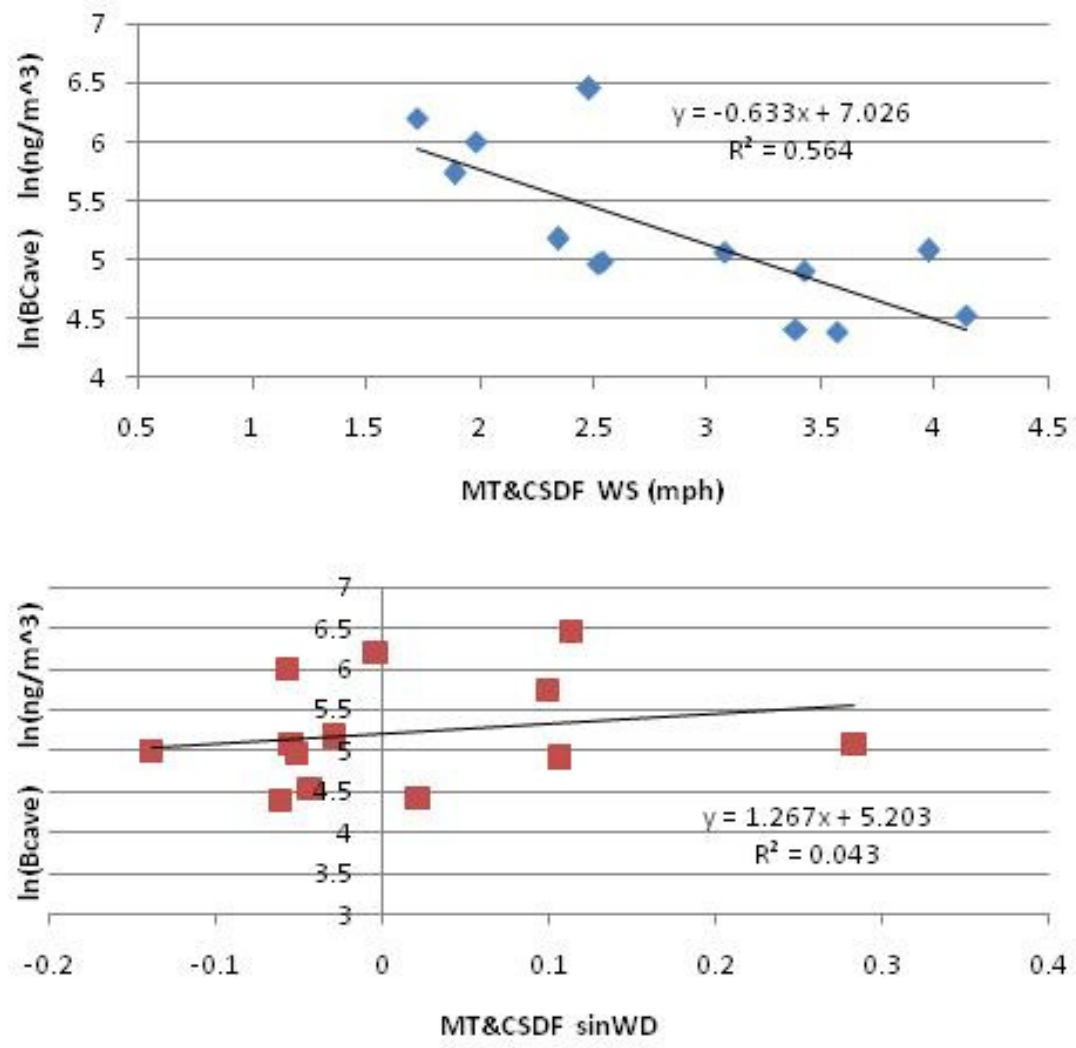

Figure 32. Marine Terrace and CSDF combination variable plots for PEM average BC concentration 
Unlike the aethalometer models, PEM models often lacked repeated values that allow the model to account for variability within predictor variables (i.e. at one wind speed there is only one BC concentration as opposed to many different concentrations). Without repeated values, Minitab could not perform lack of fit tests. Figure 30, Figure 31, and Figure 32 demonstrate the absence of repeated values. Although the $\mathrm{R}^{2}$ values resulting from PEM models are higher than those for aethalometer models, PEM model performance cannot be determined without the addition of repeated predictor variables.

\subsection{PEM BC Deviation}

No meteorological stations contained variables capable of explaining variation in BC deviation. However, the number of wood burning sources was found to be a suitable predictor when a natural log transformation was applied to PEM BC deviation. The number of wood burning sources alone accounted for 30 percent of the variability in PEM BC deviation, though the shortage of repeated values as discussed in Section 4.3.2.1.1 above still apply. As expected, the number of wood burning sources was positively correlated with BC concentration. When the number of burning sources increased, higher concentrations of BC were detected. As discussed in Section 4.2.1, higher detected concentrations tend to result in higher standard deviations. Table 15 and Figure 33 summarize the details of the model and plot the relationship with a trend line, respectively. Minitab outputs for the PEM BC deviation models are included in their entirety in Appendix H. 
Table 15. Linear regression models for PEM BC deviation

\begin{tabular}{|l|l|c|c|c|}
\hline $\begin{array}{l}\text { Response } \\
\text { Variable }\end{array}$ & $\begin{array}{l}\text { Explanatory } \\
\text { Variable }\end{array}$ & Coefficient $\left(\boldsymbol{\beta}_{\mathbf{i}}\right)$ & $\begin{array}{l}\text { Std Dev of } \\
\text { Coefficient }\end{array}$ & \multirow{2}{*}{$\mathbf{R}^{\mathbf{2}(\boldsymbol{\%})}$} \\
\cline { 1 - 4 } $\ln \left(\mathrm{BC}_{\mathrm{dev}}\right)$ & Number of Sources & $7.6 \times 10^{-02}$ & $3.5 \times 10^{-02}$ & \multirow{2}{*}{31} \\
\cline { 2 - 4 } & Constant, $\beta$ & $-1.0 \times 10^{0}$ & $3.5 \times 10^{-01}$ & \\
\hline
\end{tabular}

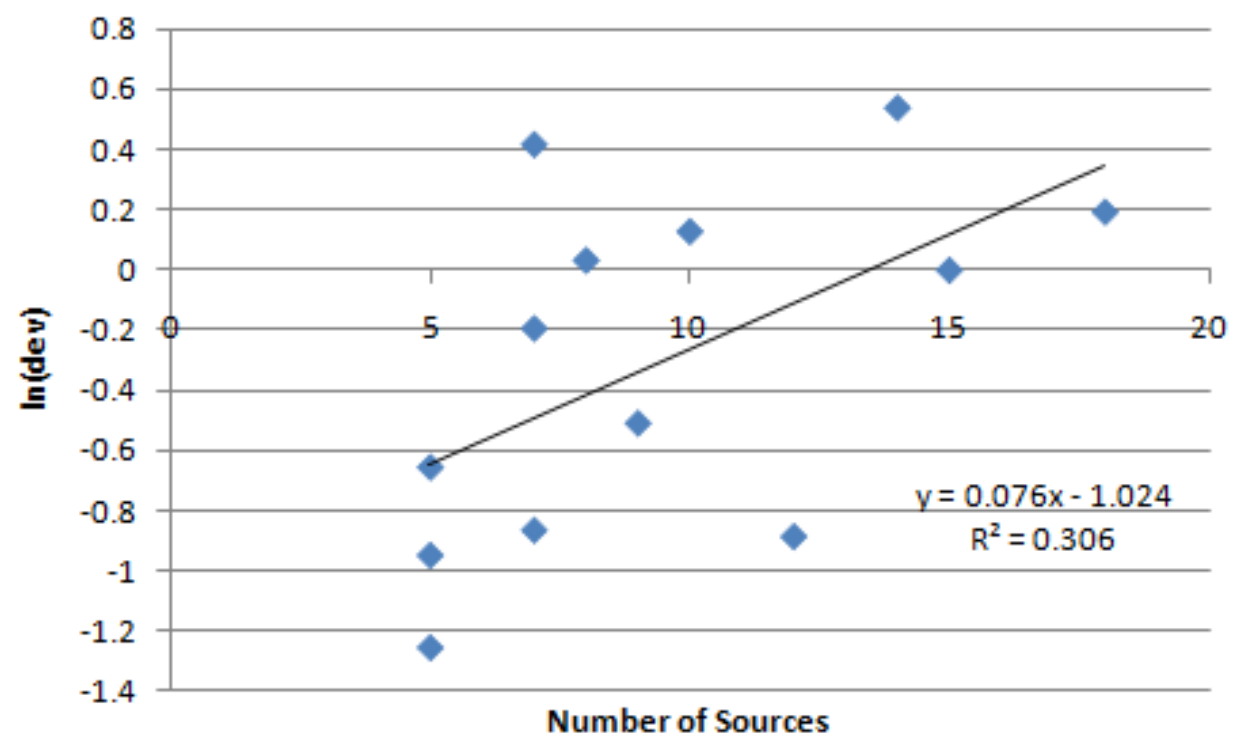

Figure 33. $\ln \left(\mathrm{BC}_{\mathrm{dev}}\right)$ versus number of wood burning sources

\subsubsection{Directional Impacts on PEM BC}

Several factors were considered in the analysis of the relationship between PEM concentrations and directional/geographic impacts. To conduct the MLR analysis and identify characteristics that have the largest effect on PEM BC, the study area was first divided into sections and labeled as shown in Figure 21, included again below for convenience. The study area divisions distinguish between differences in geographic characteristics including tree coverage, elevation, and housing density which may impact pollutant dispersion. Section A typically is higher in elevation, contains more trees surrounding homes, and contains homes that tend to be fairly separated from one another. 
Section C alternately is lower in elevation, contains fewer trees than section A, and has homes in closer proximity to one another. Section B's characteristics tend to be somewhat mid-way between section A and section C in elevation, tree coverage, and housing density, though tree coverage and housing density in section B share more similarities with section C. Figure 21 demonstrates differences and similarities in tree coverage and housing density over each area. The establishment of sections allows for the MLR model to take into account sectional differences in BC dispersion and better account for variability in average $\mathrm{BC}$ concentrations. 


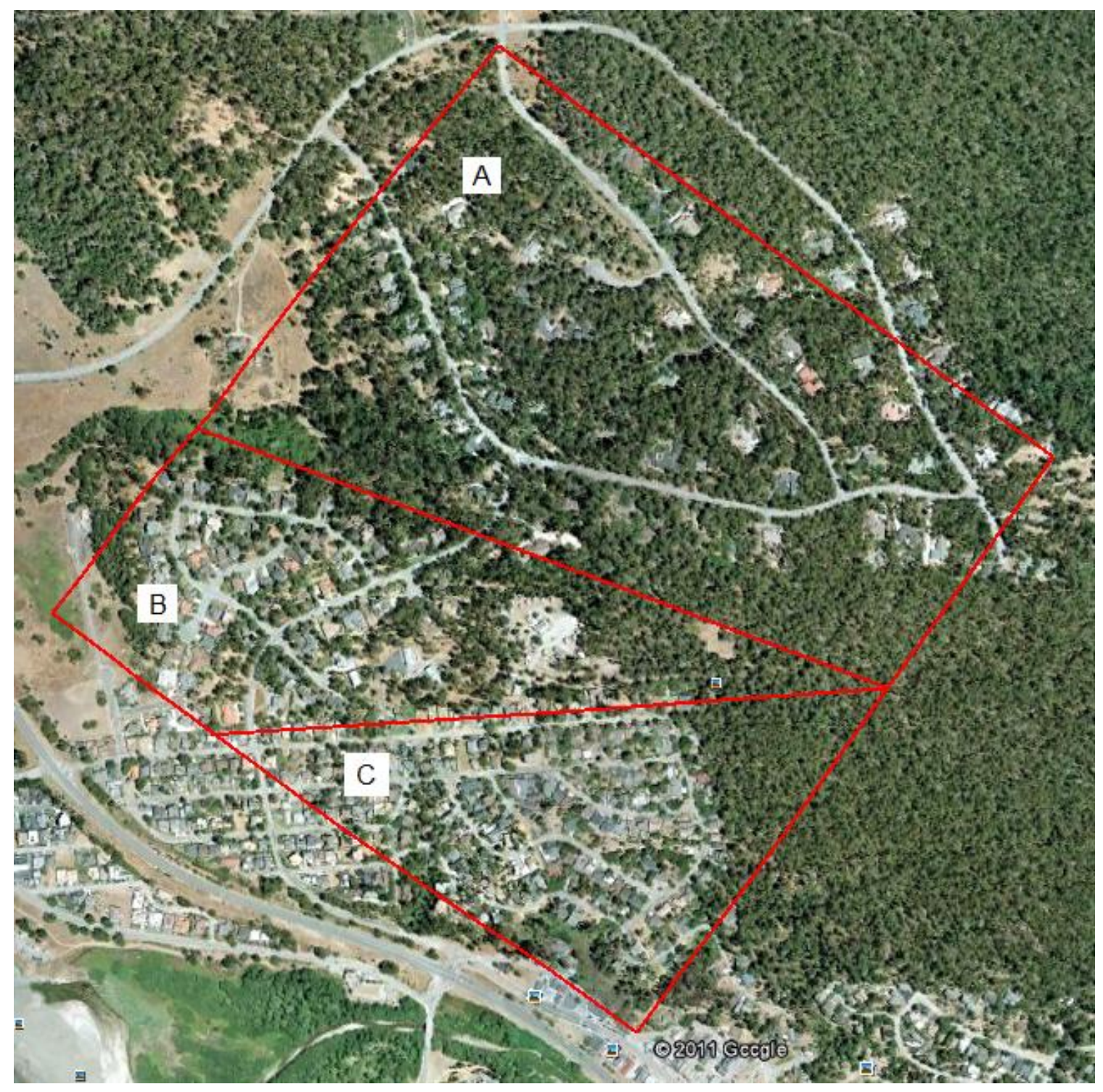

\section{Figure 21. Study area divisions used for directional regression models}

Having divided the study area into sections, the number of burning sources within each location was counted to observe potential relationships with PEM BC concentrations.

The number of upwind burning sources was also considered, along with the closest distance of each sampler to any burning source and to any upwind burning source.

Impacts of wind direction were considered by including the smallest angle between each sampling location and the IOP's dominant wind direction in the model. Impacts due to 
sampler site characteristics were observed through noting the sampling sites' proximity to the roads and the level of vegetation and structures surrounding the sampling instruments during operation. The level of vegetation and surrounding structures, mild, moderate, and heavy, was determined based on sampling location details. A mild level of vegetation and structures was assigned to PEM sites where surrounding vegetation was low and where virtually no structures were in close proximity. A moderate level was assigned where surrounding vegetation or structures taller than the PEM placement height were found on approximately 30 to 60 percent of the area surrounding the PEM. Lastly, a heavy level refers to PEM sites where vegetation taller than the PEM height in addition to rocks, trees, or fences were adjacent to PEM locations. The IOP during which each PEM BC concentration was sampled was also considered in the MLR to account for differences in burning behavior and site meteorology over each night.

Unlike MLRs conducted for relating BC and meteorology, the directional MLR used categorical variables (non-numerical), location (A, B, and C), IOP (4b through 10b), and level of vegetation and structures (i.e. fencing) surrounding PEMs (mild, moderate, and heavy). For categorical variables, the MLR model assumes all options of the categorical variables but one to be a dependent variable, $x_{i}$ (see Equation 9 repeated below), whose value is either 1 (true) or 0 (false). The partial slope, $\beta_{\mathrm{i}}$, of the included variable evaluated then represents the difference between the included option and the excluded option. For example, when considering the categorical variable, 'location,' which has three options, A, B, and C, options A and B are represented by $\mathrm{x}_{\mathrm{i}}$ 's in the MLR model, while option $\mathrm{C}$ is excluded. When a PEM is located in location $\mathrm{A}$, the $\mathrm{x}$ representing 
location $\mathrm{A}$ is equal to 1 and the resulting partial slope is equal to the difference in $\mathrm{BC}$ concentration between location $\mathrm{A}$ and $\mathrm{C}$ (assuming the response variable is equal to $\mathrm{BC}$ concentration). The location $\mathrm{B}$ variable $\mathrm{x}$ would then be equal to 0 and would not be included for consideration in that particular sample. Categorical variables allow the MLR model to quantitatively account for differences between sampling conditions.

$$
y=\beta_{0}+\beta_{1} x_{1}+\beta_{2} x_{2}+\beta_{3} x_{3}+\cdots+\beta_{k} x_{k}+\varepsilon \quad \text { Equation } 9
$$

Considering wind direction played an important role in the variables for the directional analyses, IOPs were only evaluated if the most representative meteorological data was available. For this reason, only IOPs $4 \mathrm{~b}$ through $10 \mathrm{~b}$ were evaluated since aethalometer results described in section 4.3.1.1 indicated EBAM meteorological data (measured only in IOPs $4 \mathrm{~b}$ through $10 \mathrm{~b}$ ) to be most representative for the study area. Due to the lower time resolution of PEM data and the smaller sample size available than for aethalometer analyses, a significance level of 0.1 (90 percent confidence) was used instead of 0.05 (95 percent confidence) for previous analyses.

\subsection{Directional MLR Modeling Results}

The directional MLR was conducted by first normalizing the PEM BC data through the use of the best transformation, taking the natural logarithm of PEM BC concentration. The resulting model explained 69 percent of the variability through the two categorical variables, location and IOP, as detailed in Table 16. The final model indicates that significant differences were found between PEMs in locations A and C and over IOPs 
4b,5b,7b,9b, and 10b. Significant differences between IOPs and locations indicate that 90 percent confidence intervals for BC concentration do not overlap in value for the locations and IOPs. Still, differences in concentration between locations and IOPs may be small, on average around $1 \mathrm{ng} / \mathrm{m}^{3}$ for each variable.

Table 16. Directional MLR model for PEM BC concentration

\begin{tabular}{|c|c|c|c|c|c|}
\hline $\begin{array}{l}\text { Response } \\
\text { Variable }\end{array}$ & $\begin{array}{l}\text { Categorical } \\
\text { Variable }\end{array}$ & Option & $\begin{array}{l}\text { Coefficient } \\
\left(\boldsymbol{\beta}_{\mathrm{i}}\right)\end{array}$ & $\begin{array}{l}\text { Std Dev of } \\
\text { Coefficient }\end{array}$ & $\begin{array}{l}\mathrm{R}^{2} \\
(\%)\end{array}$ \\
\hline \multirow{8}{*}{$\begin{array}{l}\ln (\mathrm{PEM} \\
\mathrm{BC})\end{array}$} & Location & A & $-2.6 \times 10^{-01}$ & $5.7 \times 10^{-02}$ & \multirow[t]{7}{*}{69} \\
\hline & & $\mathrm{C}$ & \multicolumn{2}{|c|}{ excluded } & \\
\hline & IOP & $4 \mathrm{~b}$ & $4.3 \times 10^{-01}$ & $1.1 \times 10^{-01}$ & \\
\hline & & $5 b$ & $4.5 \times 10^{-01}$ & $1.1 \times 10^{-01}$ & \\
\hline & & $7 b$ & $2.8 \times 10^{-11}$ & $1.1 \times 10^{-01}$ & \\
\hline & & $10 \mathrm{~b}$ & $-9.7 \times 10^{-01}$ & $1.1 \times 10^{-01}$ & \\
\hline & & $9 \mathrm{~b}$ & \multicolumn{2}{|c|}{ excluded } & \\
\hline & Constant, $\beta$ & $\mathrm{n} / \mathrm{a}$ & $5.2 \times 10^{0}$ & $5.7 \times 10^{-02}$ & \\
\hline
\end{tabular}

While the model seems to have explained a large percentage of variability, the model's residual versus fitted value plot as shown in Figure 34 does not clearly satisfy the MLR assumption of equal variance in the residuals. Residual values are often smaller at fitted values larger than 4.5 , suggesting a potential non-linear relationship between the independent and dependent variables. Because the data used in the directional MLR did not clearly satisfy all MLR assumptions, even after transformation, the developed model may not be reliable and significant relationships may still exist between PEM BC concentration and other tested explanatory variables. 


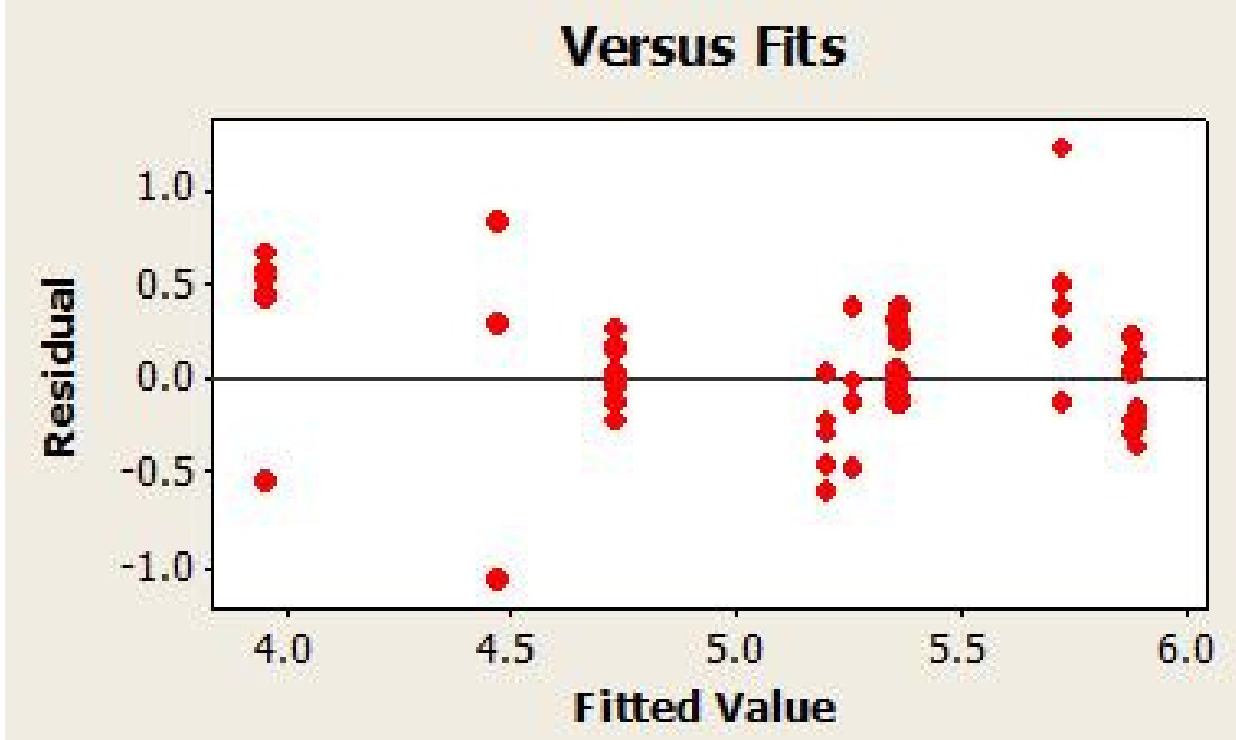

Figure 34. Directional MLR residuals versus fits plot

Observing variables graphically suggests a potential significance of the variables level of vegetation and structures surrounding PEMs, number of burning sources in location, and number of upwind burning sources, after sorting for location and level of vegetation and structures surrounding PEMs. As shown in Figure 35, PEM BC concentration over different locations and under differing level of vegetation and structures surrounding PEMs demonstrate that a notable relationship between PEM BC and number of burning sources in sampling location may be found. PEM BC concentrations measured in location C and at sites where vegetation and structures were minimal ("mild") had a visible tendency to be higher as the number of burning sources in the location increased. A moderate level of vegetation and structures also seemed to have a similar pattern, though less clearly in comparison to the mild level. A similar relationship was seen for the dependent variable, number of upwind burning sources, as shown in Figure 36. Box plots showing differences in PEM BC concentrations are further detailed in Figure 37, 
showing a tendency for $\mathrm{BC}$ concentrations to be higher in location $\mathrm{C}$ and in instances of mild levels of surrounding vegetation and structures.

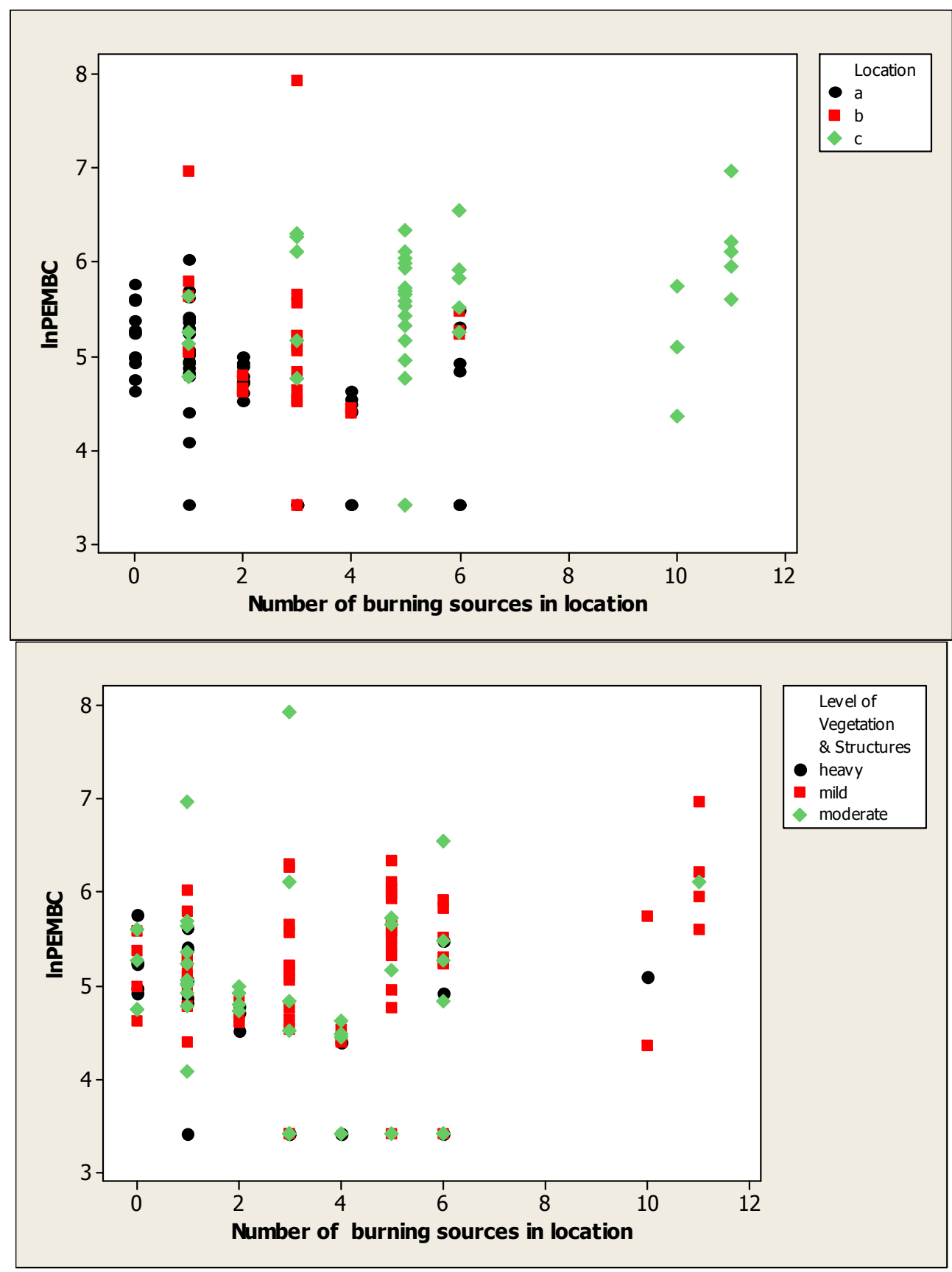

Figure 35. PEM BC versus number of burning sources in location sorted by location and level of vegetation and structures surrounding PEM for IOPs $2 \mathrm{~b}$ through 10b

The InPEMBC transformation (as opposed to untransformed PEM BC concentration), makes the differences between variables more visible. 


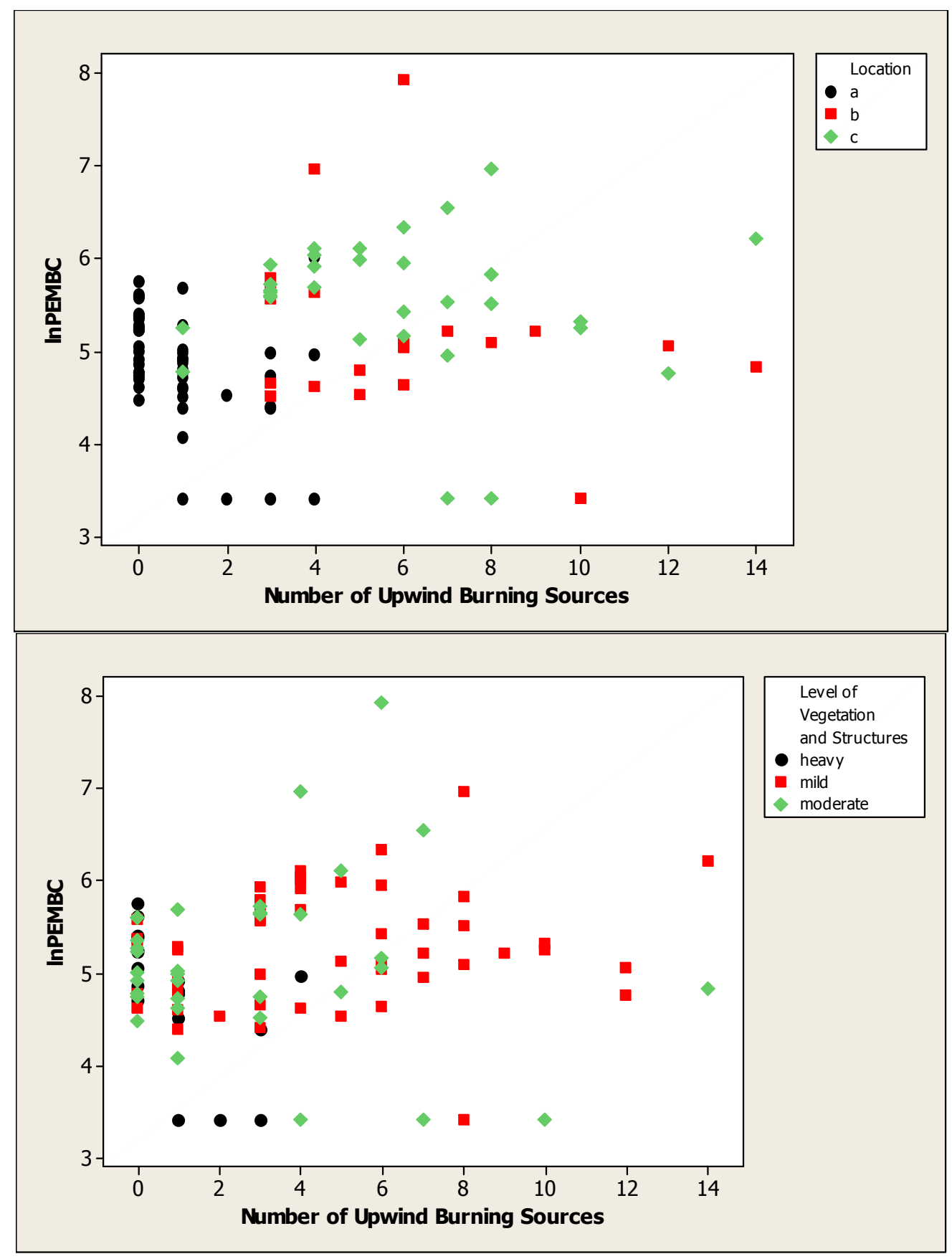

Figure 36. PEM BC versus number of upwind burning sources in location sorted by location and level of vegetation and structures surrounding PEM for IOPs 4b through 10b

The InPEMBC transformation (as opposed to untransformed PEM BC concentration), makes the differences between variables more visible. 


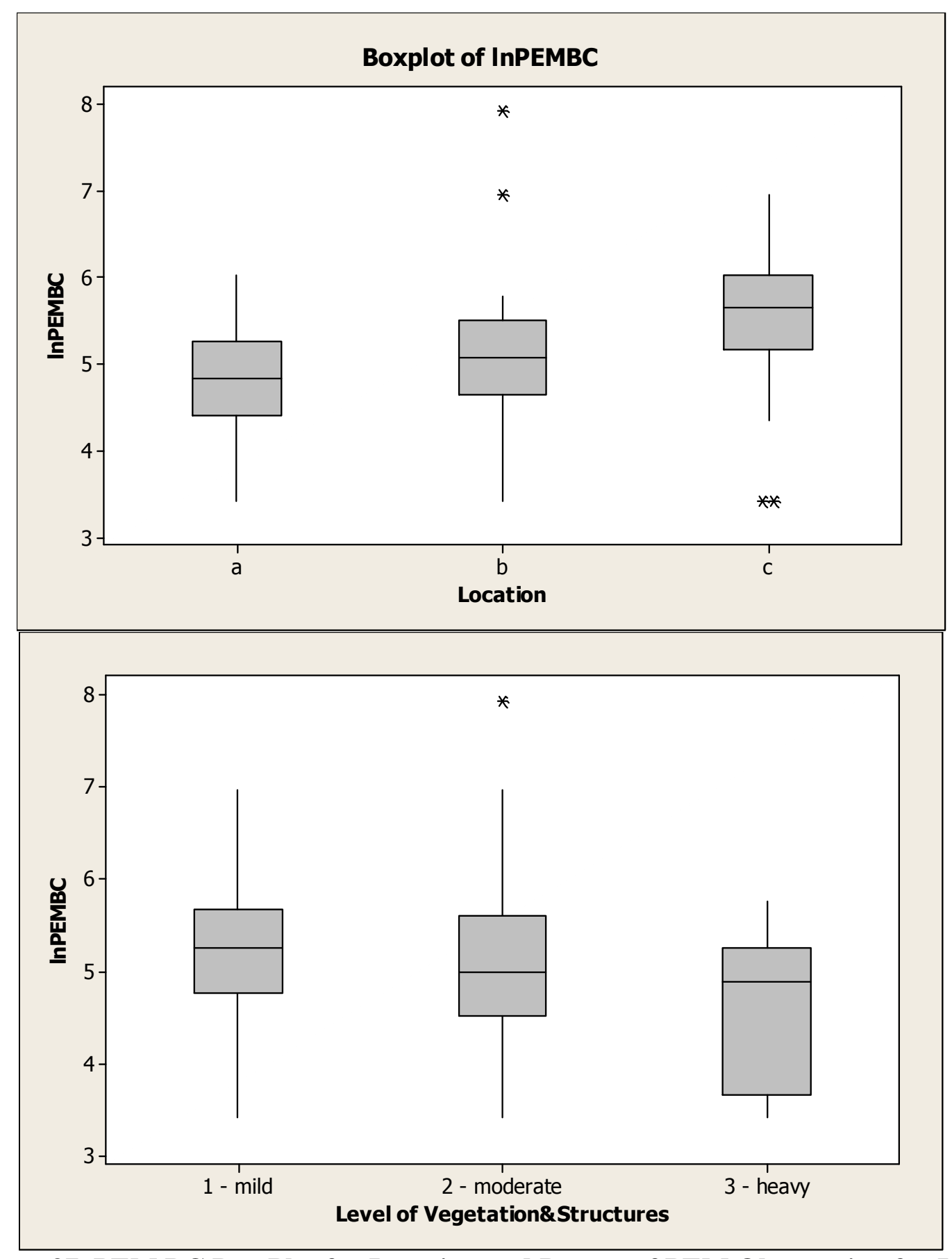

Figure 37. PEM BC Box Plot for Location and Degree of PEM Obstruction for IOPs 2b through 10b

Box plots denote the minimum, maximum, $I^{\text {st }}$ and $3^{\text {rd }}$ quartile, and median value for all concentrations. The whiskers indicate the maximum and minimum values, while the top, middle, and bottom line of the box represent the $3^{\text {rd }}$ quartile, median, and $1^{\text {st }}$ quartile value, respectively. Each star represents an outlier identified by Minitab. The InPEMBC transformation (as opposed to untransformed PEM BC concentration), makes the differences between variables more visible. 


\subsubsection{Summary of Aethalometer and PEM BC Regression Results}

Developed aethalometer and PEM regression models suggest that meteorology, time, and site characteristics can potentially account for between 47 and 81 percent of variability in BC concentration and deviation. Still, these models will need to be improved with more IOPs and samples due to detected curvature and interactions that could not be accounted for in developed models. The correlations between BC concentration and deviation and explanatory variables determined to be significant were often visually unclear with high variability in $\mathrm{BC}$ concentration for the same values of explanatory variables.

Between aethalometer analyses intended to indicate the more representative weather station for the study area, there was little consistency between average BC and BC deviation analyses. Although the EBAM station seemed to explain the most variability in average $\mathrm{BC}$ concentration, the CSDF station was more suitable for detection of BC deviation. For PEM analyses, Marine Terrace accounted for the most variability in average BC concentration (while no stations predicted PEM BC deviation well). The inconsistency in the most representative meteorological station over different analyses suggests a stochastic relationship between meteorology when an intensive grid of weather stations within the area of interest is unavailable. Still, the analyses may indicate that ground level meteorological data measured in the study area is more suitable for the prediction of higher resolution (i.e. 30 minute averaged) BC concentrations, while upper air meteorological data is more suitable for lower (i.e. 12 hour averaged) resolution BC concentrations. 
PEM and aethalometer regression analyses for this study suggest that BC is significantly correlated with sampling days, time of day, BC concentrations occurring 30 minutes apart, location of BC sampling, wind speed, relative humidity, and potentially wind direction and number of burning sources. Correlations were observed as expected for some cases but not in others. The relationship between average BC concentration and sampling day and time of day was expected due to variations in residential wood burning patterns. Residential wood burning often occurs during periods of cold weather and wood burning often begins in the evening when residents return home and when temperatures drop. Likewise, BC concentrations correlated between concentrations occurring 30 minutes apart were expected because low wind speeds in the area means pollutant dispersion occurs over a longer period of time. Other expected associations include the number of wood burning sources and wind direction. The more wood burning sources present during an IOP, the more wood smoke and BC are emitted. This relationship was illustrated in Figure 33, where BC deviation increased with number of wood burning sources. Still, no correlations at 95 percent significance were found between average aethalometer $\mathrm{BC}$ concentration and number of burning sources, though a correlation between PEM BC deviation and number of burning sources was detected. This may indicate that number of burning sources has a larger impact on $\mathrm{BC}$ variability than on average $\mathrm{BC}$ concentration in the near-field. Correlations between average $\mathrm{BC}$ concentration and wind direction were also expected and were demonstrated for PEM BC as shown in Figure 30 and Figure 31 and for aethalometer BC as shown in Figure 27. Average $\mathrm{BC}$ concentrations are expected to decrease with increasing wind speeds because higher wind speeds increase pollutant mixing, dispersion, and dilution as 
observed in studies by Chaloulaku et al. (2003), Clark (2010), Dawson et al. (2007), and Hien et al (2002). Wind directions directing wood smoke toward receptors are expected to result in higher BC concentrations, but this relationship was only detected for PEMs, likely due to the higher fluctuation of high resolution aethalometer data.

While wind speed and direction were expected to correlate with $\mathrm{BC}$ concentration, relative humidity was unexpectedly found to be significantly correlated with aethalometer BC. Relative humidity was found to be positively correlated to aethalometer BC average as shown in Figure 27. Although Dawson et al. (2007) and Sarotis et al. (2008) had previously found positive correlations between humidity and $\mathrm{BC}$ concentration, the pollutants in both studies were from traffic sources and humidity primary played a role in encouraging the production of secondary $\mathrm{PM}_{2.5}$ from ammonium sulfate. Because ammonium sulfate is not typically found in wood smoke and is not expected to be significant in the study area, humidity was not anticipated to be correlated with BC. Thus, it is expected that relative humidity is representative of a meteorological characteristic that was not included in the analysis.

\subsection{Dispersion Modeling}

Dispersion modeling was conducted using ISC-PRIME and ISCST3 through the BEEST version 9.74 (BEE-Line Software) user interface to predict BC concentrations within the

$1 \mathrm{~km}^{2}$ study area. Both ISC programs are capable of computing pollutant concentrations over a specified averaging period based on source and meteorological inputs. The BEEST interface additionally produces contours based on predicted concentrations, 
allowing for a visual interpretation of the results. By using both ISC-PRIME and ISCST3, the impact of building downwash may be observed; ISC-PRIME considers building downwash while ISCST3 does not. Both ISC-PRIME and ISCST3 were used to model 12 hour PEM BC concentrations over each IOP, the modeled concentrations were then compared to measured concentrations, and the results of ISC-PRIME and ISCST3 were compared to one another. The advantages and shortcoming of the two programs are discussed in Section 2.2.2.1, and a review of literature detailing the previously observed performance of ISCST3 and ISC-PRIME and comparisons between ISCST3, ISCPRIME, and AERMOD performance are located in Section 2.2.2.2.2.

To conduct dispersion modeling in ISCST3 and ISC-Prime, several characteristics were categorized and assumptions were made where data was unavailable. The Cambria study area was characterized as a rural location due to the low percentage of residential land use, about 40 percent, highly forested terrain, and the combination of simple and complex terrain. Homes, instead of all being represented individually, were represented as groups when homes were located close to one another. Figure 38 illustrates the representation of homes in the BEEST interface. Structures were represented in groups rather than individually for efficiency and because a high resolution area map is fairly inconsequential without meteorological data specific to the study site. 


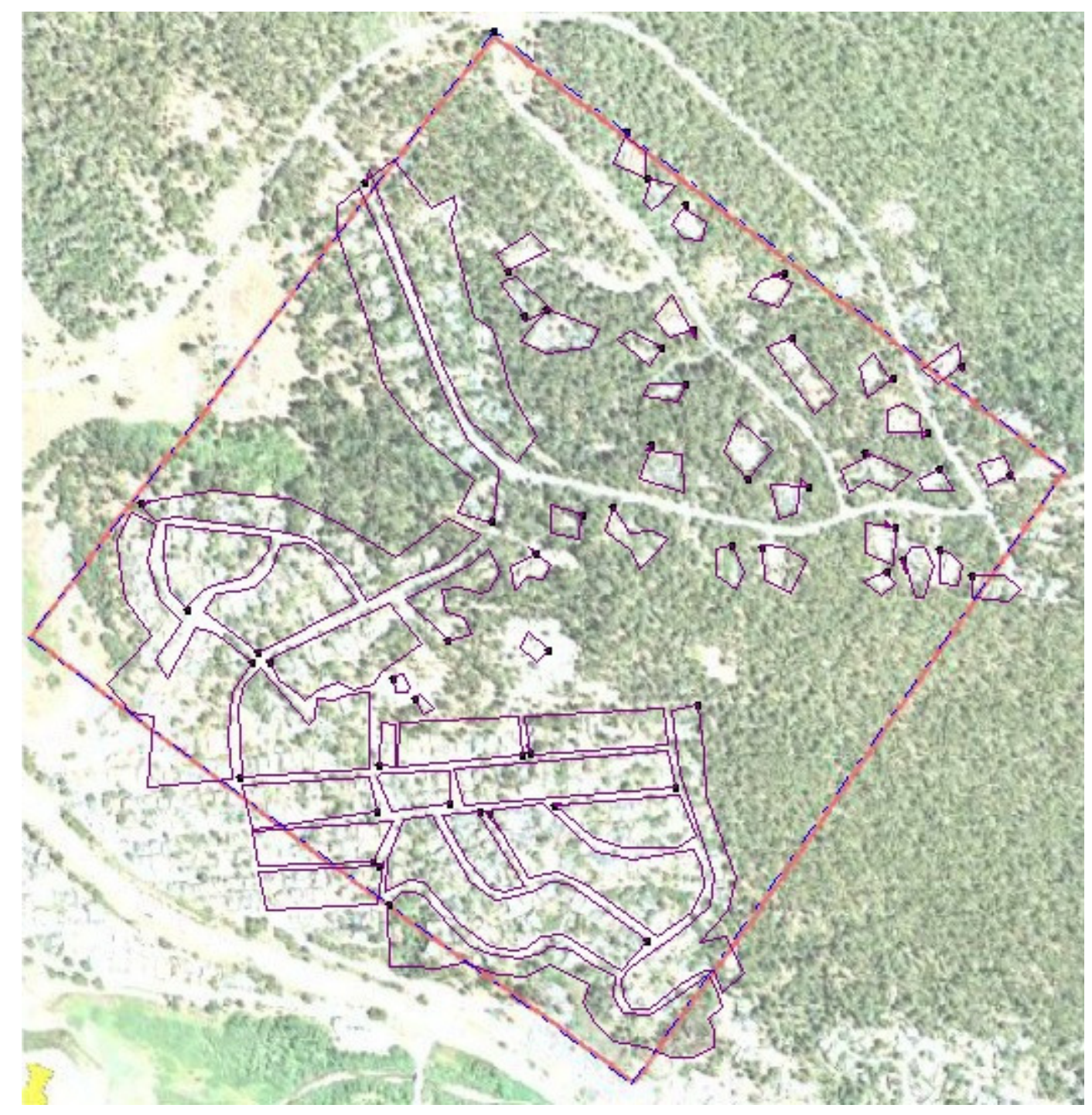

Figure 38. Homes within study area as represented in BEEST

Because $\mathrm{BC}$ fireplace emission data is not readily available, $\mathrm{BC}$ emission rates were determined based on $\mathrm{PM}_{2.5}$ emission data. When Purvis et al. (2000) tested standard and EPA-certified fireplace designs, they found total PM emission rates ranging between 10.3 to 58.4 grams per hr with $\mathrm{PM}_{2.5}$ constituting about 83 percent of total PM. Thus on average, Purvis et al. found $\mathrm{PM}_{2.5}$ emissions of 32.9 grams per hour to result from fireplace wood burning. Gullet et al. (2003) in another study testing EPA-certified 
fireplaces and woodstoves found emission rates to average 23.9 grams per hour, falling into the range observed by Purvis et al. The average of Gullet et al. and Purvis et al.'s observations, a $\mathrm{PM}_{2.5}$ emission rate of 28.4 grams per hour, was assumed in ISC-PRIME modeling. Because $\mathrm{BC}$ emissions were measured, a relationship between $\mathrm{PM}_{2.5}$ and $\mathrm{BC}$ displayed in Equation 8 (modified to determine BC as in Equation 11) as determined by Ward (2009) for this study area was used, yielding a BC emission rate of 1.42 grams per hour per source.

$$
B C=0.05 P M_{2.5}
$$

\section{Equation 11}

Chimneys were assumed to have an exit velocity of 9.3 feet per second, the median velocity determined in Dasch's (1982) study of fireplace emissions. On average, homes were approximately 20 feet in height, chimneys were about 25 feet tall, and chimney exit diameters were estimated to be about 7.5 inches (a compromise between the sizes of wood stoves and fireplaces). A sensitivity analysis indicated small differences in modeled concentrations between exit diameters ranging between 6 and 12 inches for ISCPRIME, but ISCST3 concentrations differed widely. Because the "stacks" in the case of this study are chimneys located slightly above homes at heights much lower than specified for Good Engineering Practice in 40 CFR 51.100, the stacks in the study area were considered short stacks. Burning temperatures were assumed to be the maximum temperature measured by the Fluke Thermal Imager. When Fluke temperatures were unavailable, temperatures were assumed to be the average of all temperatures measured on the specific IOP. 
To model air dispersion, Morro Bay meteorological data was used to represent Cambria, because mixing heights and stability classes required by the ISC programs were unavailable for Cambria. Morro Bay is a coastal community with similar climate to Cambria, located approximately 12 miles to the southeast of Cambria. Twelve hour Morro Bay meteorological data acquired from the SLO APCD was selected for each IOP based on its similarity to each IOP's meteorology. The meteorology for each sampling period as detected by the Marine Terrace station was compared to similar hours of meteorology in Morro Bay, because other Cambria meteorological stations had wind speeds that were too low to compare to Morro Bay. Days between the months of December through April where average and median wind speed and temperature were similar between Morro Bay and the Cambria Marine Terrace location were selected for use.

Once final BC concentrations were modeled by the ISCST3 and ISC-PRIME programs, $\mathrm{BC}$ concentrations had to be adjusted to account for the actual time period of source emissions. ISCST3 and ISC-PRIME assume each emission source to be emitting throughout the 12 hour averaging period, when in fact emissions occur over a much shorter time span. Based on aethalometer data, on average, source emissions occur over about 4.5 hours (37.5 percent) of each IOP, most often in the evening and night time. To approximately adjust BC concentrations modeled by the ISC programs to represent actual emissions, 37.5 percent of the ISC modeled concentrations were taken as the 12 hours 
integrated average $\mathrm{BC}$ concentration in the area. It is important to note that taking a factor of the modeled concentration is an approximate method that does not account for diurnal meteorological variations. Because the ISC programs tend to choose the time interval where meteorological characteristics will yield higher concentrations (i.e. lower wind speeds and mixing height), modeled BC concentrations may be higher than would be expected in actual conditions. Modeled concentrations of zero or lower than the detection limit were assumed to be the average of zero and the lower detection limit, and were therefore set to $30.26 \mathrm{ng} / \mathrm{m}^{3}$ for comparison with measured data.

\subsubsection{Comparison of ISC-PRIME and ISCST3 Modeled and Measured PEM BC Concentrations}

Dispersion modeling using ISC-PRIME and ISCST3 for the Cambria study area resulted in a majority of under predicted concentrations. On average, the modeled-to-measured $\mathrm{BC}$ concentration ratio was 0.25 , ranging from 0 to 3.94 , and 0.15 , ranging from 0 to 1.62, for ISC-PRIME and ISCST3, respectively. Ratios of zero resulted when modeled concentrations were equal to zero. Box plots shown in Figure 39 demonstrate the distribution of ratios and the prevalence of ratios in between the $1^{\text {st }}$ quartile value of 0.03 and the third quartile value of 0.24 for ISC-PRIME and the $1^{\text {st }}$ quartile value of 0.02 and third quartile value of 0.15 for ISCST3. Modeled BC concentrations corresponding to each PEM measurement are included in Appendix I. 


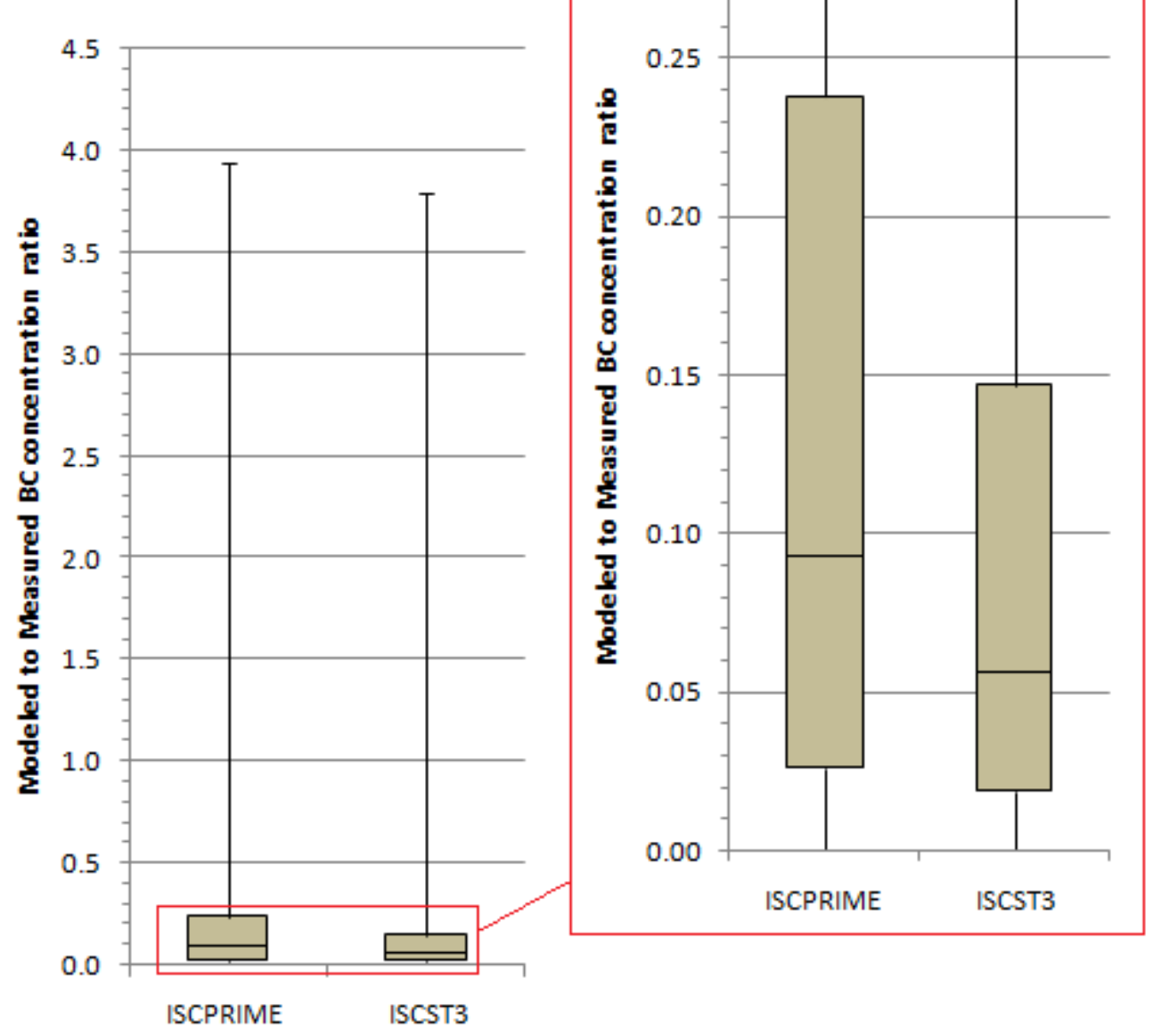

Figure 39. Box plots of modeled-to-measured BC

Box plots denote the minimum, maximum, $I^{\text {st }}$ and $3^{\text {rd }}$ quartile, and median value for all concentrations. The whiskers indicate the maximum and minimum values, while the top, middle, and bottom line of the box represent the $3^{\text {rd }}$ quartile, median, and $1^{\text {st }}$ quartile value, respectively.

While both programs tended to yield $\mathrm{BC}$ concentrations less than the measured $\mathrm{BC}$ concentrations, ISCST3 underestimated concentrations more often than ISC-PRIME. The overall tendency to under predict concentrations was not expected for ISCST3, but has been seen to occur for ISC-PRIME as discussed in Section 2.2.2.2.2. ISCST3 was 
expected to predict higher-than-measured concentrations under complex terrain as discussed by US EPA (2003) and Perry et al. (2005).

Modeled-to-measured $\mathrm{BC}$ ratios for ISC-PRIME were also inconsistent with the findings of Venkatram et al. (2004), who found ISC-PRIME to often over predict concentrations for high-measured concentrations and to under predict concentrations when concentrations were low. When observing the modeled-to-measured $\mathrm{BC}$ ratio relative to the magnitude of measured BC concentrations for ISC-PRIME as shown in Figure 40, $\mathrm{BC}$ concentrations over predicted by ISC-PRIME only resulted when measured concentrations were low. The same tendency was observed for ISCST3 predictions as shown in Figure 41. Figure 40 and Figure 41 also show that the highest measured BC concentrations were always under predicted by both ISC-PRIME and ISCST3. Though this study's results differ than those in Venkatram et al., it is important to point out that Venkatram et al's study took place in a flat area surrounded by buildings. Additionally, high modeled-to-measured $\mathrm{BC}$ ratios are less than 5 percent of all measured ratios and high measured concentrations are less than 5 percent of all measured concentrations. 


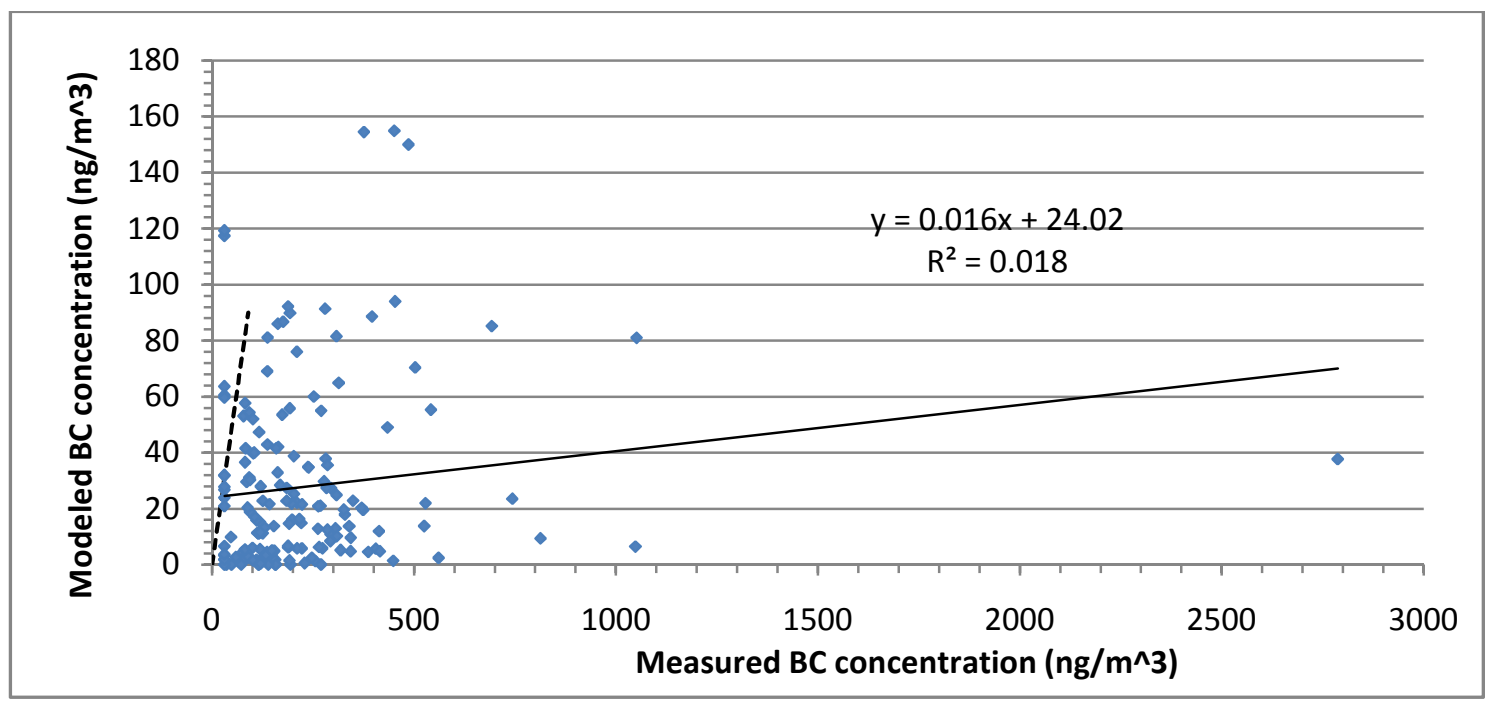

Figure 40. ISC-PRIME modeled versus measured PEM BC concentration

The dashed line represents a one-to-one ratio between measured and modeled $B C$ concentrations. The solid line is an applied trend line with equation and $R^{2}$ value shown. One outlier was removed due to evidence of direct fireplace downwash received at that point.

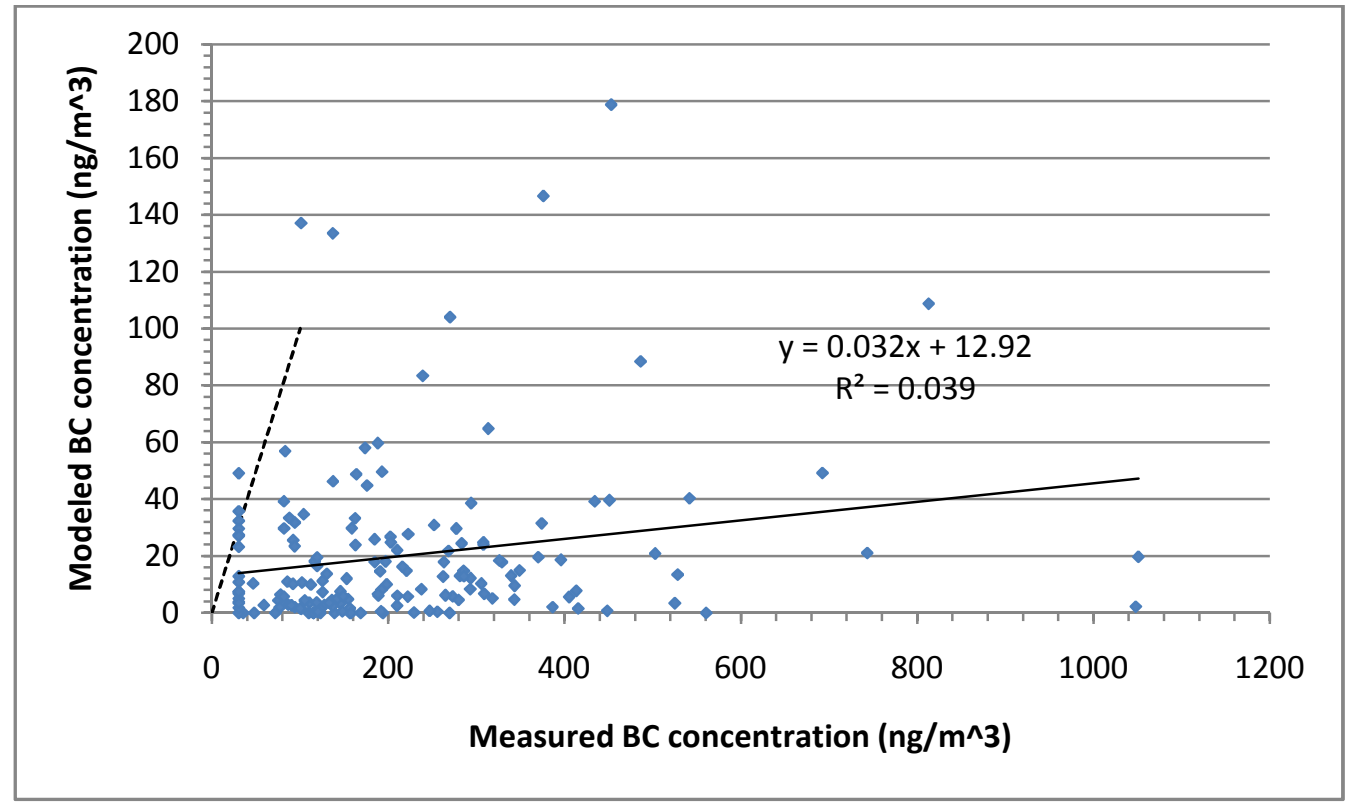

\section{Figure 41. ISCST3 modeled versus measured PEM BC concentration}

The dashed line represents a one-to-one ratio between measured and modeled $B C$ concentrations. The solid line is an applied trend line with equation and $R^{2}$ value shown. One outlier was removed due to evidence of direct fireplace downwash received at that point. 
The disparities between this study's results and the results of the reviewed studies were likely due to the near-field application of the ISC programs and several assumptions made in the modeling process and meteorological data used. Although emission rates and stack characteristics can vary widely, it was only within this study's means to use approximated values. Emission rates are highly variable not only amongst homes, but also amongst fireplaces and even between burning events in the same fireplace, as discussed in Section 2.1.2. In addition, all stacks were approximated to be identical, although it is likely that exit flue heights and diameters differ significantly throughout the area. The approximation of all of these characteristics reduced the accuracy of the modeled results.

In addition to model assumptions, the use of Morro Bay meteorological data likely contributed to lower modeled BC concentrations and higher BC variability in the modeled results. Although the EBAM station explained the most variability in BC concentration (Section 4.3.1.1), the Morro Bay meteorological data was selected based on similarity to the Marine Terrace data because of the availability of more data and similarity between the types of stations. However, the Marine Terrace data had average wind speeds between 3 to 5 times greater than the EBAM station. Higher wind speeds likely increased modeled BC dispersion, resulting in increased mixing and dilution of pollutants which may cause a decrease to BC concentrations determined by ISC-PRIME and ISCST3. Differences in dominant wind direction also would play a role in determining the concentration in each PEM location. 
The difference between patterns observed in previous studies and observations made in this study suggest the need to improve the accuracy of model inputs used for ISCST3 and ISC-PRIME. Representative meteorology and emission and stack characteristics need to be measured within the site to produce reliable predictions of pollutant concentrations. Though dispersion modeling results may have differed significantly from previous studies, study data may still be useful if a correction factor is applied. For this reason, factors were determined for modeled $\mathrm{BC}$ concentrations that would bring median modeled-to-measured $\mathrm{BC}$ concentrations closer to unity. Concentrations that were lower than the detection limit after correction factors were applied were given the value of $30.26 \mathrm{ng} / \mathrm{m}^{3}$ to be consistent with measured PEM concentrations.

To find suitable correction factors, all available IOPs were split into two groups consisting of IOP $1 \mathrm{a}$ through $5 \mathrm{~b}$ for the development group and IOP $6 \mathrm{~b}$ through $10 \mathrm{~b}$ for the test group. The development group was used to determine a correction factor that would bring the median ratio closest to unity, and the factor was then applied to the test group to determine the factor's ability to bring the latter group's median BC concentration ratios close to unity. When the development group medians were adjusted to unity, the resulting factors were 12.5 and 17.1 for ISC-PRIME and ISCST3, respectively. Application to the test group data yielded median values of 1.53 and 0.89 for ISC-PRIME and ISCST3, respectively. Because the test group median ratios were not as close to unity as desired, the correction factors were again adjusted to reduce the difference between the development and test group ratios. The final factor value for ISCPRIME that reduced the difference between the development and test case median was 
9.75 , resulting in a test group ratio differing from 1 by a value of 0.20 instead of 0.53 . To bring the test group median ratio closer to unity, the correction factor for ISCST3 was also adjusted. The final correction factor that reduced the difference between the development and test case median for ISCST3 was 18.2, resulting in a test group ratio differing from 1 by a value of 0.05 instead of 0.11 . When correction factors were adjusted, development group median ratios also differed from unity by the same increment as the test case (about 0.2 for ISC-PRIME and about 0.05 for ISCST3).

When the factors were applied to all IOPs, the resulting median ratios were 0.94 and 1.03 for ISC-PRIME and ISCST3, respectively. The range of modeled-to measured ratios was then 0.054 to 38.4 for ISC-PRIME and 0.011 to 29.5 for ISCST3. Adjusting the modeled concentrations to approach unity allowed for more accurate prediction of a majority of the concentrations, but also dramatically resulted in over prediction of low measured concentrations. The over estimation of extremely low measured concentrations was primarily a problem where PEM concentrations were assumed to be the average of zero and the lower detection limit. These values were not excluded from the analysis, however, because low BC measurements would then be poorly represented. Figure 42 shows box plots to demonstrate the distribution of modeled-to-measured ratios after the correction factor was applied. Figure 43 shows the resulting modeled-to-measured BC concentration graphs. All corrected modeled BC concentrations for each PEM location are included in Appendix I. 


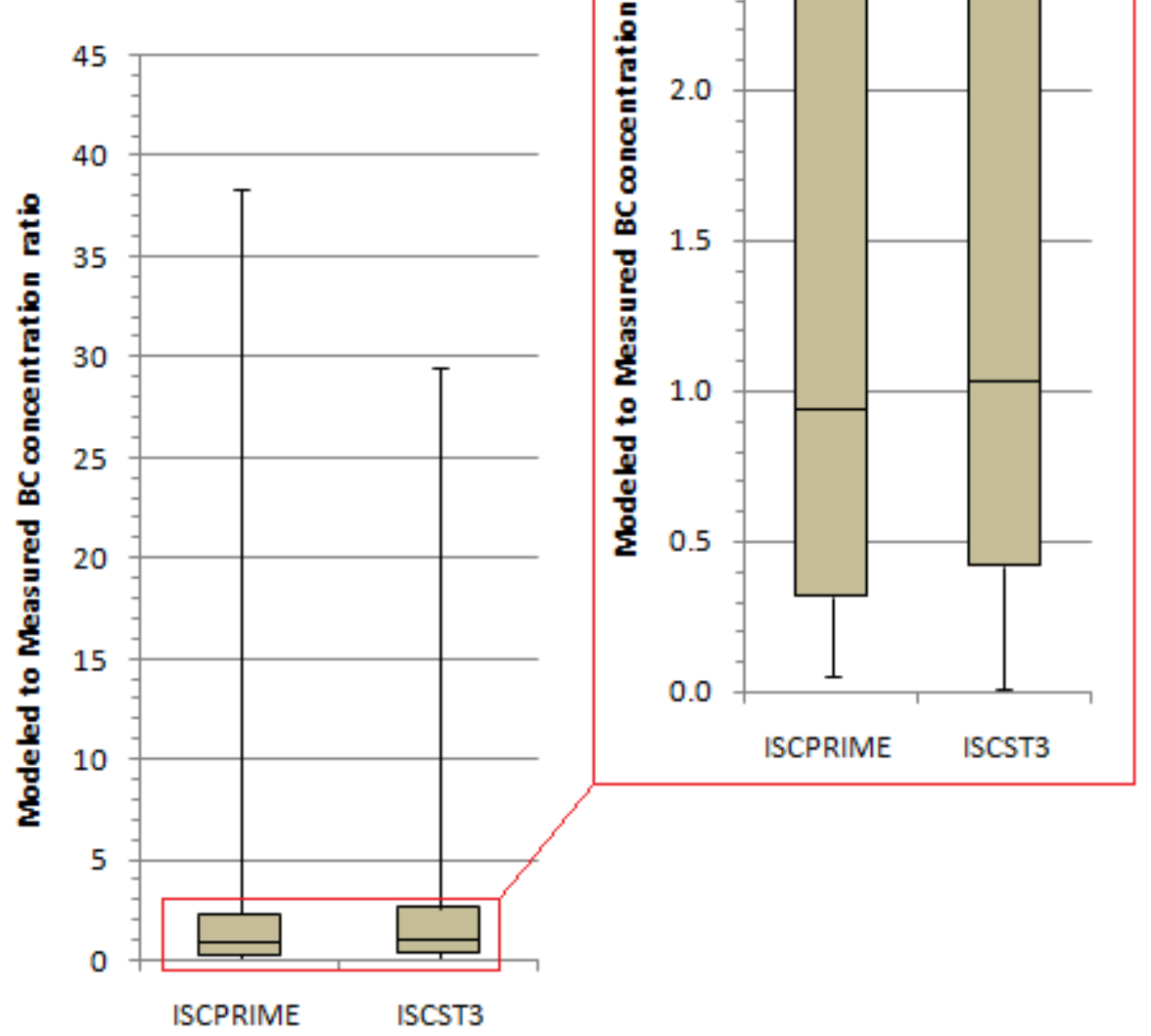

Figure 42. Box plots of modeled-to-measured $\mathrm{BC}$ ratios after $\mathrm{BC}$ concentration correction factors

Box plots denote the minimum, maximum, $1^{\text {st }}$ and $3^{\text {rd }}$ quartile, and median value for all concentrations. The whiskers indicate the maximum and minimum values, while the top, middle, and bottom line of the box represent the $3^{\text {rd }}$ quartile, median, and $1^{\text {st }}$ quartile value, respectively. 


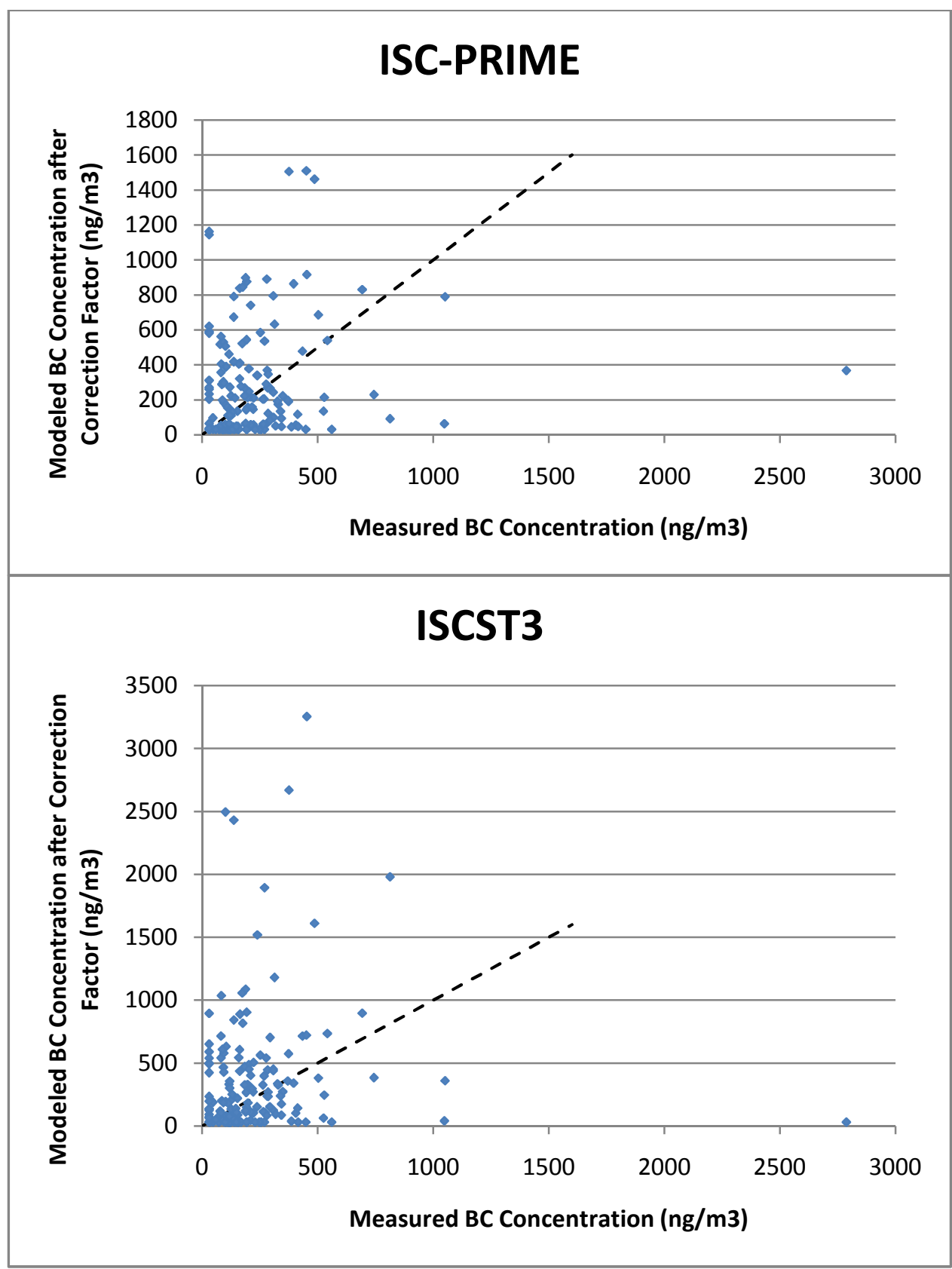

Figure 43. Corrected modeled BC versus measured BC concentration

The dotted line represents a one-to-one ratio between measured and modeled $B C$ concentrations. 


\subsubsection{Comparison of ISC-PRIME and ISCST3 Modeled BC Contours to Measured BC}

Based on the modeled concentrations generated by ISC-PRIME and ISCST3 programs, the BEEST interface generates contours to illustrate the concentration distribution in the modeled area. Contours for all IOPs generated are included in Appendix I, but only a few will be observed in depth in this section. Although ISC-PRIME and ISCST3 modeled $\mathrm{BC}$ concentrations were much lower than measured $\mathrm{BC}$ concentrations, as demonstrated in Section 4.4.1, the contours were evaluated based on their qualitative ability to capture the concentration distribution occurring over different IOPs. To make modeled IOP data comparable to measured IOP data, different factors were applied to the modeled concentrations for each IOP and ISC program such that the average measured $\mathrm{BC}$ concentration was equal to the average modeled $\mathrm{BC}$ concentration. By using this method, it was found that ISC-PRIME and ISCST3 were only occasionally able to generate models with BC distributions similar to measured BC distributions. Figure 44 through Figure 47 illustrate dispersion contours as represented by ISC-PRIME and ISCST3 for selected IOPs. The colors of the contour show the magnitude of the modeled $\mathrm{BC}$ concentration with red representing the highest concentration and white representing the lowest concentration. The superimposed colored circles represent the measured BC concentrations based on the same scale as the modeled concentrations after factors were applied. 

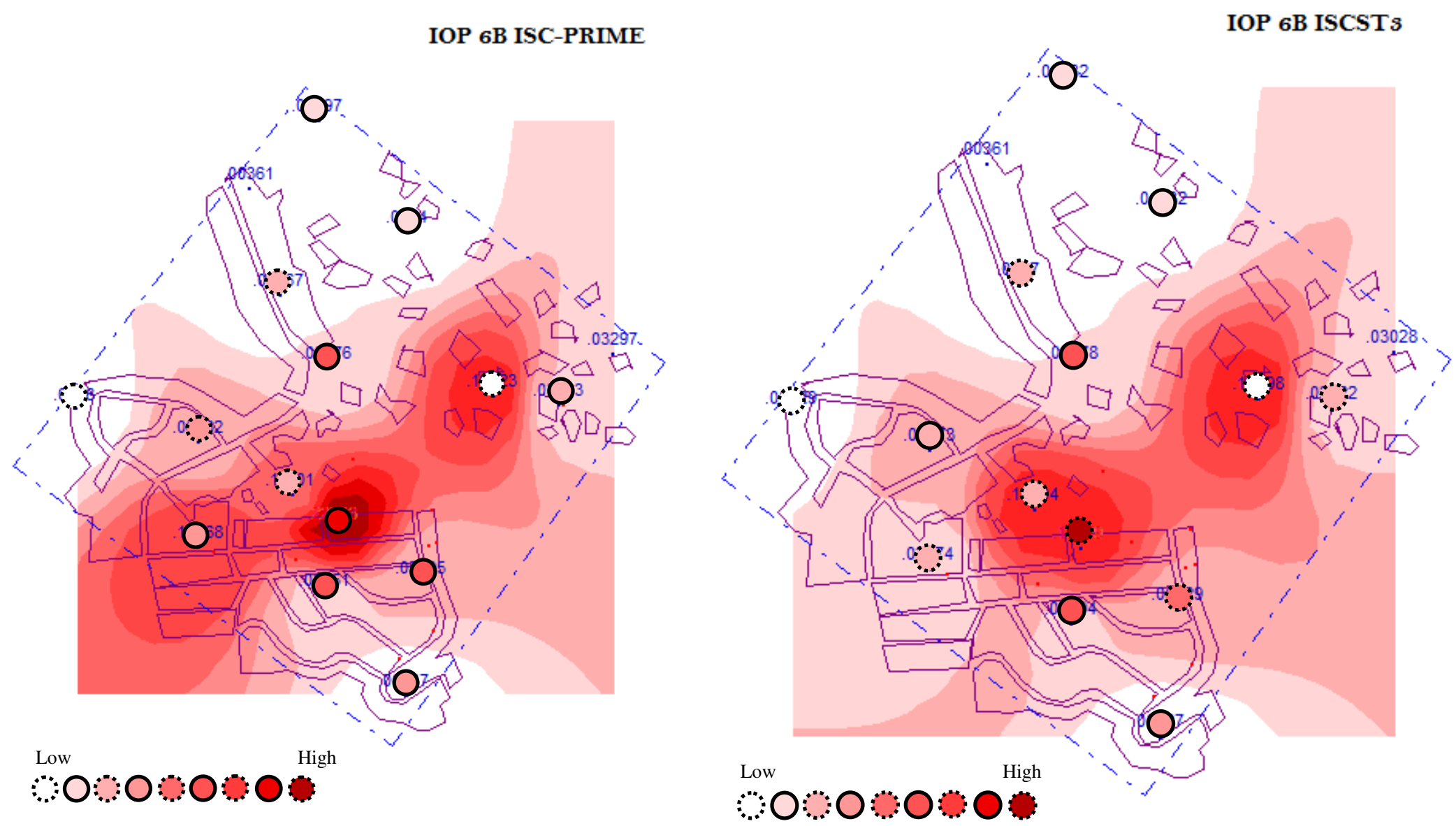

Figure 44. IOP 6b modeled contours demonstrating similarity between ISC-PRIME and ISCST3 

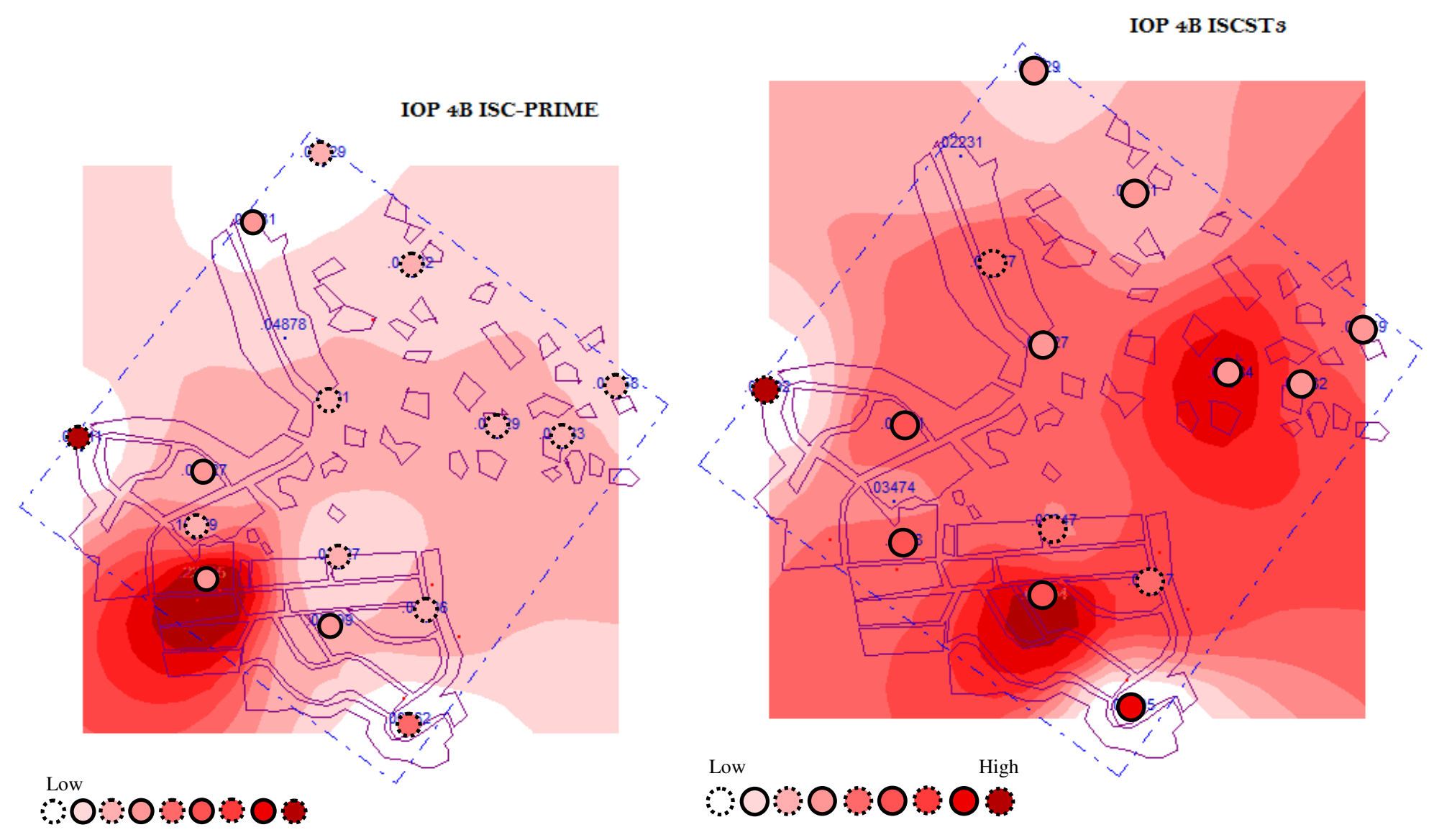

Figure 45. IOP 4b modeled contours demonstrating differences between ISC-PRIME and ISCST3 

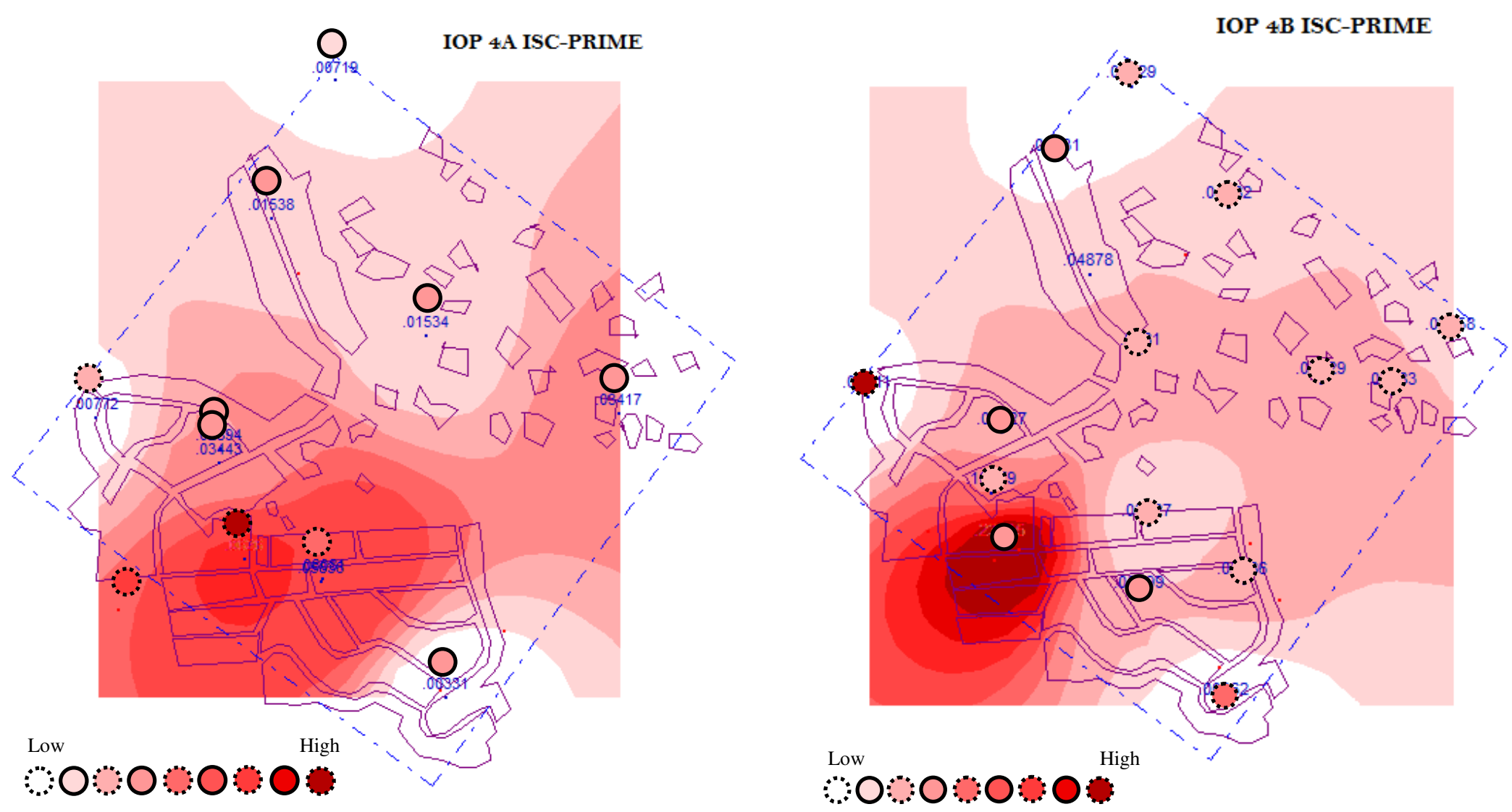

Figure 46. ISC-PRIME contours demonstrating distributions similar to measured distributions 

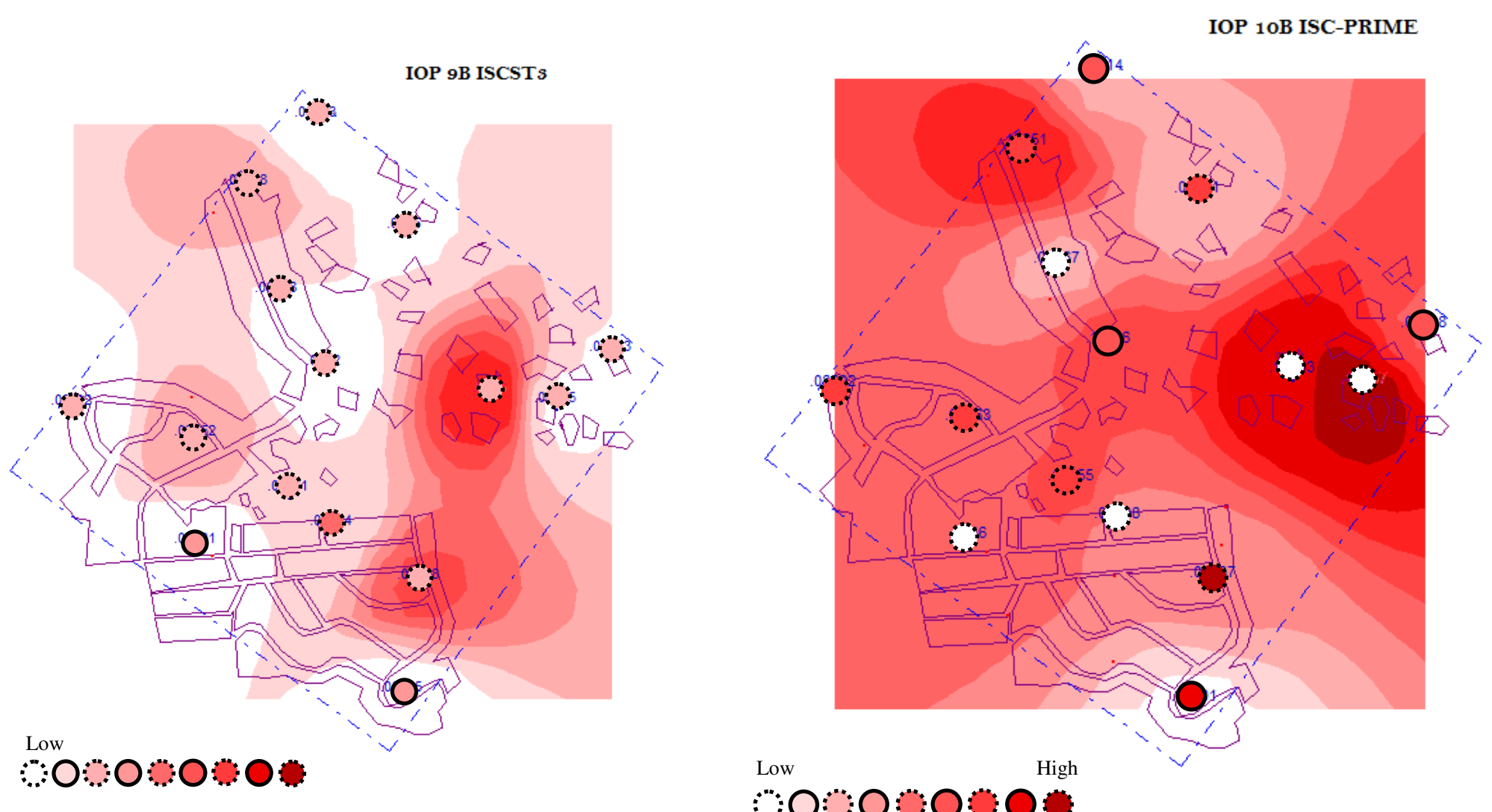

Figure 47.ISCST3 and ISC-PRIME contours demonstrating distributions differing from measured distributions 
For some IOPs, both the ISCST3 and ISC-Prime models yielded contours that were similar in many details. For instance, Figure 44 shows model contours for IOP 6 b, were both models yielded contour maps with similar shapes, although there is still some variability in the details. Each model predicted peak areas in similar locations and low concentrations in the northwestern portion of the study area. The measured concentrations show some features that are similar to the models, however measured concentrations tend to exhibit lower concentrations, indicating more dispersion and dilution. On the other hand, for some IOPs the two models yielded very different contours. Figure 45 shows the modeled contour maps for IOP4b. In this instance, ISCPRIME predicts relatively consistent concentrations over much of the study area with a single peak in the southwest. However, ISCST3 predicts a concentration ridge running along the southeast border of the study area. For this IOP, ISC-PRIME appears to do a better job of representing the measured concentrations, although the model predicts low concentrations where the PEM measurements were highest, in the southwest corners of the study area. Differences between ISC-PRIME and ISCST3 BC dispersion were usually small where average wind speed was less than $3 \mathrm{mph}$ (IOP 4b), but increased when average wind speeds were above $3 \mathrm{mph}$ (IOP 6b). This is an indication that building downwash becomes less significant when wind speeds are low.

In general, both models were able to capture some of the features shown in the PEM measurements during some IOPs, but not during others. For instance, ISC-PRIME captured BC dispersion well for IOP 4A, but not as well for IOP 4B (Figure 46). Still, for IOP 4B, ISC-PRIME identified higher concentrations on the southwest part of the 
study area and then decreasing concentrations in the northeast direction. ISC-PRIME was sometimes able to accurately predict the location where the highest BC concentration occurred, while ISCST3 was less often able to do so. Figure 47 shows two examples (IOPs 9B and 10B) where dispersion was not well modeled by both ISC programs. The locations of the highest measured BC concentrations were not identified by ISC-PRIME and ISCST3 and locations of low concentration were often identified as locations of high concentration.

When observing wind speeds for IOPs 4A, 4B, 9B, and 10B along with other IOP contours and their respective wind speeds, ISC-PRIME and ISCST3 seems to more accurately model pollutant dispersion at average wind speeds less than $3 \mathrm{mph}$. Based on the Marine Terrace meteorological station, IOPs 4A and 4B had wind speeds of 2.5 and $2.6 \mathrm{mph}$, respectively, and IOPs 9B and 10B had wind speeds of 5.6 and $3.3 \mathrm{mph}$, respectively. Unfortunately, neither model consistently provided an accurate picture of variability within the study area.

While there is potentially a relationship between wind speed and model performance, other factors may also have contributed to inaccuracy in modeled dispersion. For example, modeling may have been improved if high tree density surrounding homes could have been considered in area A. Additionally, the inability of the model to properly represent BC concentrations at receptor S1 (see Figure 20 for PEM location map) at the south most corner suggests that sources of BC may have been overlooked. The higher concentration measured at receptor CP (see Figure 20) during IOP 5B, as 
shown in Figure 48, with no surrounding wood burning sources also seems to suggest that burning sources were overlooked. Burning sources may have been missed either because sources were located just outside of the study area or because wood burning began late in the night when the search for wood burning sources had already been completed. Without accurate inputs, the performance of the ISC programs is limited.

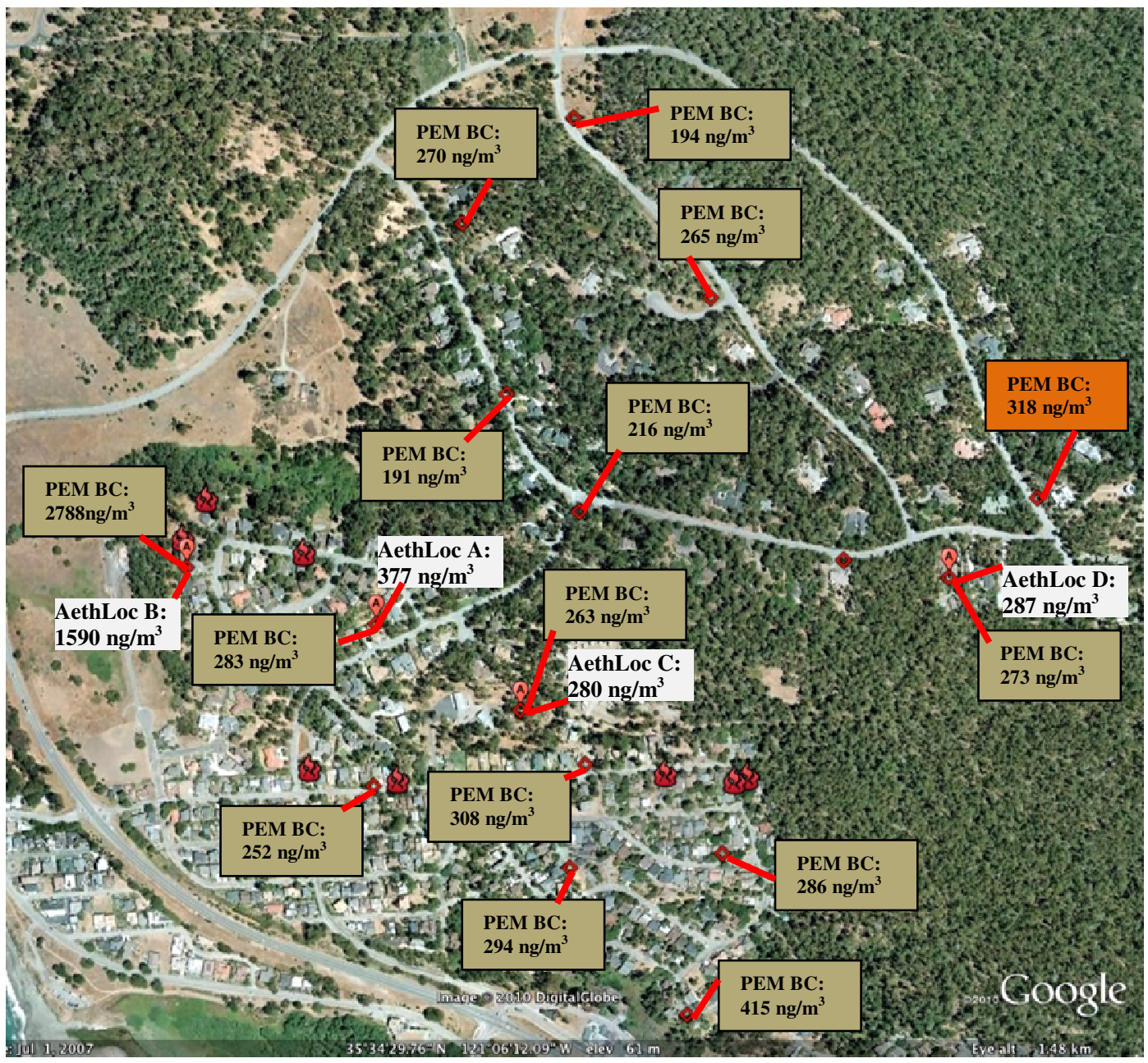

Figure 48. IOP 5B sources and receptor BC concentrations

The magnitude of receptor CP (PEM BC highlighted in orange) potentially suggests that existing burning sources went undetected. 


\section{Chapter 5. Conclusion}

\subsection{Overview of Results}

The objectives of this study included (1) assessing BC spatial variability in the $1 \mathrm{~km}^{2}$ study area containing multiple wood burning sources, (2) determining the impact of meteorology, geography, and burning source characteristics on $\mathrm{BC}$ concentrations and deviation through the use of regression models, and (3) evaluating the performance of ISC-PRIME and ISCST3 in application to near-field analyses where source characteristics are estimated.

The first task indicated high variability near field BC variability, with standard deviations being as high as 3 to 4 times the average $\mathrm{BC}$ concentration detected. Overall BC averages measured by aethalometers and PEMs tended to be fairly similar and low, but standard deviations varied considerably within IOPs, within sites, and over all IOPs and sites. Over all IOPs and sites, BC concentrations measured by aethalometers were on average $229 \mathrm{ng} / \mathrm{m}^{3}$, or $4.6 \mu \mathrm{g} / \mathrm{m}^{3}$ for $\mathrm{PM}_{2.5}$, with a large standard deviation of $827 \mathrm{ng} / \mathrm{m}^{3}$ (360 percent of average), or $16.5 \mu \mathrm{g} / \mathrm{m}^{3}$ for $\mathrm{PM}_{2.5}$. Similarly, between IOPs and sites, aethalometer BC averaged $226 \mathrm{ng} / \mathrm{m}^{3}$, or $4.5 \mu \mathrm{g} / \mathrm{m}^{3}$ for $\mathrm{PM}_{2.5}$, and $211 \mathrm{ng} / \mathrm{m}^{3}$, or 4.2 $\mu \mathrm{g} / \mathrm{m}^{3}$ for $\mathrm{PM}_{2.5}$, respectively, and standard deviations between IOPs and sites were 438

$\mathrm{ng} / \mathrm{m}^{3}$ (190 percent), or $8.8 \mu \mathrm{g} / \mathrm{m}^{3}$ for $\mathrm{PM}_{2.5}$, and $470 \mathrm{ng} / \mathrm{m}^{3}$ (220 percent), or $9.4 \mu \mathrm{g} / \mathrm{m}^{3}$ for $\mathrm{PM}_{2.5}$, respectively. Average aethalometer measured $\mathrm{BC}$ concentrations were fairly low relative to their standard deviation because a majority of the measured $\mathrm{BC}$ during each IOP consisted of background measurements. Burning typically occurred during approximately 38 percent of the IOP, and the high concentrations seen during that time 
are responsible for the large standard deviation of aethalometer BC over all IOPs. Aethalometer averages between IOPs and between sites were similar, but standard deviations between IOPs and between sites were $438 \mathrm{ng} / \mathrm{m}^{3}\left(8.8 \mu \mathrm{g} / \mathrm{m}^{3} \mathrm{PM}_{2.5}\right)$ and 470 $\mathrm{ng} / \mathrm{m}^{3}\left(9.4 \mu \mathrm{g} / \mathrm{m}^{3} \mathrm{PM}_{2.5}\right)$ on average, respectively, almost half the size of standard deviation over all IOPs and sites. Lower variability occurring between IOPs and sites than over all IOPs was expected because BC concentrations are highly specific to the burning patterns and local meteorology affecting the site during each sampling period. For instance, some IOPs experience higher concentrations on average due to the high prevalence of wood burning and lower wind speeds, while some IOPs experience only background concentrations on account of fewer burning sites and higher wind speeds. Still, though the standard deviation is lower over sites and IOPs, a standard deviation of almost $10 \mu \mathrm{g} / \mathrm{m}^{3} \mathrm{PM}_{2.5}$ may be problematic in areas that struggle to meet $\mathrm{PM}_{2.5}$ standards.

Samples obtained from PEM measured BC concentrations averaged $224 \mathrm{ng} / \mathrm{m}^{3}$, or 4.5 $\mu \mathrm{g} / \mathrm{m}^{3}$ for $\mathrm{PM}_{2.5}$, very similar to aethalometers, but had a lower standard deviation of 262 $\mathrm{ng} / \mathrm{m}^{3}$ (120 percent), or $5.2 \mu \mathrm{g} / \mathrm{m}^{3}$ for $\mathrm{PM}_{2.5}$. Similar to aethalometers, average BC concentrations within sites and IOPs were similar to over all IOPs and sites, but standard deviations were lower. Between IOPs and sites, PEM BC averaged 221 ng/m³ , or 4.4 $\mu \mathrm{g} / \mathrm{m}^{3}$ for $\mathrm{PM}_{2.5}$, and $251 \mathrm{ng} / \mathrm{m}^{3}$, or $5.0 \mu \mathrm{g} / \mathrm{m}^{3}$ for $\mathrm{PM}_{2.5}$, respectively, and standard deviations between IOPs and sites were $174 \mathrm{ng} / \mathrm{m}^{3}$ (79 percent), or $3.5 \mu \mathrm{g} / \mathrm{m}^{3}$ for $\mathrm{PM}_{2.5}$, and $190 \mathrm{ng} / \mathrm{m}^{3}$, or $3.8 \mu \mathrm{g} / \mathrm{m}^{3}$ for $\mathrm{PM}_{2.5}$ (76 percent), respectively. The difference between aethalometer and PEM BC standard deviations reflect the higher time resolution of aethalometers, which allow them to capture peak BC concentrations. Differences in PEM 
BC standard deviation between IOPs, between sites, and over all IOPs largely reflect variations in burning patterns on different sampling periods, as was the case for aethalometer BC. A lower standard deviation resulting from PEM samplings than from aethalometer samples indicate that lower resolution samples fail to capture the extent of public exposure.

Regression models in fulfillment of the second objective were conducted with two purposes in mind, determining the relationship between measured $\mathrm{BC}$ and meteorology and the relationship between $\mathrm{BC}$ and geography and burning source characteristics. The analyses indicated that meteorology accounted for only a small fraction of variability in $\mathrm{BC}$ concentration and that geographical and burning source impacts are difficult to identify individually.

Although multiple regression models were conducted for both PEMs and aethalometers, the larger sample size available for aethalometers make aethalometer regression models more reliable than PEM regression models for determining the meteorological station most capable of explaining variability in $\mathrm{BC}$ averages and deviation. Aethalometer regression models all indicated that meteorology and time dependence accounted for about 50 percent of variability in $\mathrm{BC}$ averages and deviation, though time of day and lagged $\mathrm{BC}$ concentration were more significant than meteorological variables. Among aethalometer regression models, meteorological variables significantly accounting for variability in $\mathrm{BC}$ were relative humidity and wind speed, but these correlations were often weak and interactions and curvature were often detected. When non-linear models 
were instead used, no major improvements were seen in the model's ability to account for $\mathrm{BC}$ variability, so linear models were considered preferable. Among all meteorological stations evaluated, variables measured at the EBAM station proved the most suitable for explaining variability in average aethalometer BC concentration. The EBAM station's relative humidity in addition to lagged $\mathrm{BC}$ and time interval sufficiently accounted for variability in average BC. Although relative humidity is not physically expected to affect $\mathrm{BC}$ concentration directly, its significance in the regression model may suggest that changes in relative humidity take place in conjunction with another physically significant variable. Wind speed and relative humidity as measured at the CSDF station in addition to lagged $\mathrm{BC}$ deviation were found to be significant predictors for aethalometer $\mathrm{BC}$ deviation. With the exception of relative humidity, wind speed, time interval, and lagged $\mathrm{BC}$ were all anticipated predictors for average $\mathrm{BC}$ and $\mathrm{BC}$ deviation. $\mathrm{BC}$ concentration has often been observed to decrease with increasing wind speed, wood burning occurs based on a diurnal pattern, and dispersion occurring at one location may change slowly over time. In this study, aethalometer BC was found to be positively correlated with lagged $\mathrm{BC}$ and relative humidity, and negatively correlated with wind speed and time interval.

While less than 15 samples were available for PEM analyses, variables found to significantly explain average PEM BC concentration variability were wind speed, sine of wind direction, and cosine of wind direction. When wind speed increased, average PEM BC consistently decreased for all meteorological stations and combinations. Sine and cosine of wind direction, however, were less consistently correlated with $\mathrm{BC}$ 
concentration. For the Marine Terrace models, winds originating from the north and west directions were correlated with lower PEM BC concentrations, whereas for the Marine Terrace and CSDF combination, BC decreased when winds originated from the south. Higher wind speeds originating from the north for the Marine Terrace station seemed to be the cause of lower BC concentrations, but this pattern was not seen for the winds of western origin or for southern winds from the Marine Terrace and CSDF combination. Number of burning sources was the only predictor to account for variability in PEM BC deviation, and was found to be positively correlated with $\mathrm{BC}$ deviation.

Regression models evaluating the impact of geography and burning source characteristics in connection with meteorology found only IOP and location to significantly account for variability in PEM BC concentration at a 90 percent significance level, but model residuals seemed to indicate that the model was unreliable. Significant differences between locations $\mathrm{A}$ and $\mathrm{C}$ suggest that housing density and tree coverage may play a significant role in exposure, with high housing density and lower tree coverage providing better conditions for higher BC concentrations. Because model residuals were questionable, a graphical approach to observing the impact of number of burning sources within the PEM area and number of upwind burning sources was used and potentially indicated an increase in concentration as number of sources increased. Level of surrounding vegetation and structures may also be significant. By graphically observing changes in PEM BC concentration with an increase in the number of upwind burning sources, it was seen that a positive correlation was potentially present. This trend seemed to be more apparent when the level of surrounding vegetation and structures was mild, 
highlighting the impact of obstructions on $\mathrm{BC}$ concentration; when obstructions are minimal, dispersion patterns are more predictable, but when obstructions are more abundant, $\mathrm{BC}$ concentrations may be more stochastic. More samples, however, will be needed to show more definite correlations.

The third task indicated that ISC dispersion modeling in the near-field case cannot be conducted accurately when approximate inputs are used. Both programs modeled study area BC concentrations much lower than actual concentrations, with ISC-PRIME and ISCST3 modeled-to-measured ratios averaging 0.25 , ranging from 0 to 3.94 , and averaging 0.15 , ranging from 0 to 1.62 , respectively. This under prediction may be associated with application to the near-field in addition to assumptions made about burning sources and meteorological conditions where information was not available. To bring median modeled BC concentrations closer to unity, correction factors of 9.75 and 18.2 were applied to ISC-PRIME and ISCST3 values, respectively. Upon application of correction factors, median ratios were 0.94 and 1.03 for ISC-PRIME and ISCST3, respectively, and ratios ranged from 0.054 to 38.4 for ISC-PRIME and 0.011 to 29.5 for ISCST3.

Modeled PEM BC distributions were further observed through BC concentration contours generated in BEEST. ISC-PRIME typically performed better than ISCST3, especially when modeling the location of the highest measured PEM BC concentration. Still, while ISC-PRIME was sometimes able to model the location of the highest measured PEM BC concentrations and the general direction of decreasing $\mathrm{BC}$ 
concentration, there were also many instances where the highest concentrations were modeled as the lowest concentrations and vice versa. Observed contours indicated that ISC-PRIME and ISCST3 could not consistently model BC variability within the study area.

Additionally, trends typical of other studies using ISC-PRIME and ISCST3 were not seen in this application. For example, both ISC programs typically estimated concentrations conservatively, with ISCST3 tending to be more conservative than ISC-PRIME. In this study application, ISCST3 predicted concentrations lower than ISC-PRIME 75 percent of the time. Additionally, instead of ISC-PRIME over predicting high measured concentrations and under predicting low concentrations as previously observed, this study found the programs to under predict the highest values. The inconsistencies in these results may suggest that ISC programs are not applicable to the near-field case of that more accurate burning source and local meteorological data is needed. When using estimated emission and meteorological data for the site, ISCST3 and ISC-PRIME were unable to reliably model BC concentrations. Because the ISC-PRIME and ISCST3 results were inconsistent with other studies, the developed correction factors are only applicable to this study area. To determine the applicability of ISC-PRIME and ISCST3 to near-field sites, more site specific inputs will have to be evaluated for their ability to improve dispersion modeling methods. 


\subsection{Future Study}

This study found substantial spatial variability within the $1 \mathrm{~km}^{2}$ area over each IOP. Although the average variability may not have large impacts in areas meeting $\mathrm{PM}_{2.5}$ standards, $\mathrm{PM}_{2.5}$ standard deviations of almost $10 \mu \mathrm{g} / \mathrm{m}^{3}$ or about three to four times the measured average concentration may mean harmful exposures in areas like Fresno, CA, where geographic and meteorological characteristics tend to promote high $\mathrm{PM}_{2.5}$ concentrations. This study aimed to better understand variables that affect BC concentrations through the use of regression models and dispersion modeling, but these methods were incapable of pinpointing any single or group of specific meteorological, , or source variables that could easily fulfill the task. The stochastic nature of meteorology and its interaction with terrain are likely the cause of these results. To better relate $\mathrm{BC}$ concentration to meteorology, more investigation will be needed to determine the meteorological, geographical, and source variables that better help explain pollutant variability.

Although this study found ISC air dispersion models to poorly model BC concentrations, several improvements to operating the dispersion modeling programs may still be made. For example, the acquisition of site specific meteorology, geography, and source characteristics would better assess model performance. 


\section{List of References}

Aarhus University National Environmental Research Institute (NERI). (October 20, 2010). Contribution to air pollution from residential wood combustion. Retrieved November 4, 2010, from http://www.dmu.dk/en/news/artikel/contribution_ to_air_pollution_from_residential_wood_combustion/

Allen, R., Larson, T., Sheppard, L., Wallace, L., \& Liu, L. S. (2003). Use of Real-Time Light Scattering Data To Estimate the Contribution of Infiltrated and IndoorGenerated Particles to Indoor Air. Environmental Science \& Technology, 37(16), 3484-3492. doi:10.1021/es021007e

American Lung Association. (2010). State of the Air 2010. Retrieved November 1, 2010, from http://www.stateoftheair.org/2010/assets/SOTA2010.pdf

Andreae, M. O., \& Gelencser, A. (2006). Black carbon or brown carbon? The nature of light absorbing carbonaceous aerosols. Atmospheric Chemistry and Physics, 6(10), 3131-3148.

Arasa, R., Soler, M. R., \& Ortega, S. (2008). The Role of Sea-Salt Emissions in Air Quality Models. In Air Pollution Modeling and Its Application XIX, NATO Science for Peace and Security Series C: Environmental Security (pp. 663-664664). Springer Netherlands. Retrieved from http://dx.doi.org/10.1007/978-1-40208453-9_76

Bari, M. A., Baumbach, G., Kuch, B., \& Scheffknecht, G. (2010). Temporal variation and impact of wood smoke pollution on a residential area in southern Germany. Atmospheric Environment, 44(31), 3823-3832. doi:10.1016/j.atmosenv. 2010.06.031 
Barregard, L., Sällsten, G., Gustafson, P., Andersson, L., Johansson, L., Basu, S., \& Stigendal, L. (2006). Experimental Exposure to Wood-Smoke Particles in Healthy Humans: Effects on Markers of Inflammation, Coagulation, and Lipid Peroxidation. Inhalation Toxicology, 18(11), 845-853. doi:10.1080/ 08958370600685798

Barregard, L., Sällsten, G., Andersson, L., Almstrand, A., Gustafson, P., Andersson, M., \& Olin, A. (2007). Experimental exposure to wood smoke: effects on airway inflammation and oxidative stress. Occupational and Environmental Medicine, 65(5), 319-324. doi:10.1136/oem.2006.032458

Beelen, R., Hoek, G., Pebesma, E., Vienneau, D., de Hoogh, K., \& Briggs, D. J. (2009). Mapping of background air pollution at a fine spatial scale across the European Union. Science of The Total Environment, 407(6), 1852-1867. doi:10.1016/ j.scitotenv.2008.11.048

Bølling, A.K., Pagels, J., Yttri, K. E., Barregard, L., Sallsten, G., Schwarze, P. E., \& Boman, C. (2009). Health effects of residential wood smoke particles: the importance of combustion conditions and physicochemical particle properties. Particle and Fibre Toxicology, 6, 29. doi:10.1186/1743-8977-6-29

Branis, M., Koznarová, J., \& Domasová, M. (2000). Coal and wood burning as main cause of particulate pollution in a rural area: Case study from the Czech Republic. Journal of Aerosol Science, 31(Supplement 1), 889-890. doi:10.1016/S00218502(00)90899-1 
Cahill, T. M. (2010). Size-Resolved Organic Speciation of Wintertime Aerosols in California's Central Valley. Environmental Science \& Technology, 44(7), 23152321. doi:10.1021/es902936v

California Air Resources Board (CA ARB). (2006, September 8). CEPAM: 2009 Almanac - Top 25 Tool. Retrieved January 31, 2010, from http://www. arb.ca.gov/app/emsinv/t25cat/ cat_top25.php

Cambria Chamber of Commerce. (2010). Facts About Cambria. Retrieved November 1, 2010, from http://www.cambriachamber.org/info.php

Cambria Weather. (2008). Retrieved November 2, 2010, from http://countrystudies. us/united-states/weather/California/cambria.htm

Chaloulakou, A., Kassomenos, P., Spyrellis, N., Demokritou, P., \& Koutrakis, P. (2003). Measurements of PM10 and PM2.5 particle concentrations in Athens, Greece. Atmospheric Environment, 37(5), 649-660. doi:10.1016/S1352-2310(02)00898-1

Chen, L. A., Watson, J. G., Chow, J. C., \& Magliano, K. L. (2007). Quantifying PM2.5 Source Contributions for the San Joaquin Valley with Multivariate Receptor Models. Environmental Science \& Technology, 41(8), 2818-2826. doi:10.1021/es0525105

Chow, J. C., Watson, J. G., Crow, D., Lowenthal, D. H., \& Merrifield, T. (2001). Comparison of IMPROVE - and NIOSH - Carbon Measurements. Aerosol Science and Technology, 34(1), 23. doi:10.1080/02786820119073

Chow, J. C., \& Watson, J. G. (2002). Review of PM2.5 and PM10 Apportionment for Fossil Fuel Combustion and Other Sources by the Chemical Mass Balance Receptor Model. Energy \& Fuels, 16(2), 222-260. doi:10.1021/ef0101715 
Christakos, G., \& Serre, M. L. (2000). BME analysis of spatiotemporal particulate matter distributions in North Carolina. Atmospheric Environment, 34(20), 3393-3406. doi:10.1016/S1352-2310(00)00080-7

Cimorelli, A. J., Perry, S. G., Venkatram, A., Weil, J. C., Paine, R., Wilson, R. B., Lee, R. F., et al. (2011). AERMOD: A Dispersion Model for Industrial Source Applications. Part I: General Model Formulation and Boundary Layer Characterization. J. Appl. Meteor., 44(5), 682-693. doi:doi: 10.1175/JAM2227.1

Citizen Weather Observer Program (CWOP). (2010, December 30). CWOP Information for CW8373. Weather Quality Reporter. Retrieved February 2, 2011, from http://weather.gladstonefamily.net/site/C8373

Clark, E. (2010). Impact of Ground-level Aviation Emissions on Air Quality in the Western United States. Boise State University Theses and Dissertations. Retrieved from http://scholarworks.boisestate.edu/td/98

Cooper, C. D., \& Alley, F. C. (2002). Air pollution control: a design approach. Waveland Press.

Dasch, J. M. (1982). Particulate and gaseous emissions from wood-burning fireplaces. Environmental Science \& Technology, 16(10), 639-645. doi:10.1021/ es00104a003

Davy, P. K., Markwitz, A., \& Watherburn, D. C. (2007). Composition, size and Morphological Comparison of Wood Smoke Particles from Source Emissions and in Urban Air by SEMEDS. In International Union of Air Pollution Prevention and Environmental Protection Associations. Presented at the 14th IUAPPA World Congress clean air partnerships, Brisbane. 
Dawson, J. P., Adams, P. J., \& Pandis, S. N. (2007). Sensitivity of PM2.5 to climate in the Eastern US: a modeling case study. Atmos. Chem. Phys., 7(16), 4295-4309.

Demuzere, M., Trigo, R. M., Vila-Guerau de Arellano, J., \& van Lipzig, N. P. M. (2009). The impact of weather and atmospheric circulation on $\mathrm{O}_{3}$ and $\mathrm{PM}_{10}$ levels at a rural mid-latitude site. Atmospheric Chemistry and Physics, 9(8), 2695-2714. doi:10.5194/acp-9-2695-2009

Demuzere, M., \& van Lipzig, N. P. (2010). A new method to estimate air-quality levels using a synoptic-regression approach. Part I: Present-day O3 and PM10 analysis. Atmospheric Environment, 44(10), 1341-1355.

doi:10.1016/j.atmosenv.2009.06.029

Durbin, J., \& Watson, G. S. (1951). Testing for Serial Correlation in Least Squares Regression. II. Biometrika, 38(1-2), 159 -178. doi:10.1093/biomet/38.1-2.159

Electric Power Research Institute (EPRI). (1997, November). Results of the Independent Evaluation of ISCST3 and ISC-PRIME. Retrieved June 2, 2011, from: http://www.epa.gov/scram001/7thconf/iscprime/evalrpt.pdf

Ellis, E. C., \& Novakov, T. (1982). Application of thermal analysis to the characterization of organic aerosol particles. Science of The Total Environment, 23, 227-238. doi:10.1016/0048-9697(82)90139-5

Favez, O., Cachier, H., Sciare, J., Sarda-Estève, R., \& Martinon, L. (2009). Evidence for a significant contribution of wood burning aerosols to PM2.5 during the winter season in Paris, France. Atmospheric Environment, 43(22-23), 3640-3644. doi:10.1016/j.atmosenv.2009.04.035 
Fine, P. M., Cass, G. R., \& Simoneit, B. R. (2001). Chemical characterization of fine particle emissions from fireplace combustion of woods grown in the northeastern United States. Environmental Science \& Technology, 35(13), 2665-2675.

Fine, P. M., Cass, G. R., \& Simoneit, B. R. (2002). Chemical characterization of fine particle emissions from the fireplace combustion of woods grown in the southern united states. Environmental Science \& Technology, 36(7), 1442-1451.

Fine, P. M., Cass, G. R., \& Simoneit, B. R. (2004). Chemical characterization of fine particle emissions from the fireplace combustion of wood types grown in the Midwestern and Western United States. Environmental Engineering Science, 21(3), 387-409.

Fraser, M. P., \& Lakshmanan, K. (2000). Using Levoglucosan as a Molecular Marker for the Long-Range Transport of Biomass Combustion Aerosols. Environmental Science \& Technology, 34(21), 4560-4564. doi:10.1021/es9912291

Gauderman, W. J., Avol, E., Gilliland, F., Vora, H., Thomas, D., Berhane, K., et al. (2004). The Effect of Air Pollution on Lung Development from 10 to 18 Years of Age. The New England Journal of Medicine, 351(11), 1057-1067.

Geller, M. D., Chang, M., Sioutas, C., Ostro, B. D., \& Lipsett, M. J. (2002). Indoor/outdoor relationship and chemical composition of fine and coarse particles in the southern California deserts. Atmospheric Environment, 36(6), 1099-1110.

Georgopoulos, P, Isukapalli, S, Burke, J, Napelenok, S, \& Palma, T. (2009, October). Air quality needs for exposure assessment from the source-to-outcome perspective. , A\&WMA’s Magazine for Environmental Managers, 26-35. 
Gorin, C. A., Collett, J. L., Jr, \& Herckes, P. (2006). Wood smoke contribution to winter aerosol in Fresno, CA. Journal of the Air \& Waste Management Association (1995), 56(11), 1584-1590.

Goswami, E., Larson, T., Lumley, T., \& Liu, L. J. S. (2002). Spatial characteristics of fine particulate matter: identifying representative monitoring locations in Seattle, Washington. Journal of the Air \& Waste Management Association (1995), 52(3), 324-333.

Gullett, B. K., Touati, A., \& Hays, M. D. (2003). PCDD/F, PCB, HxCBz, PAH, and PM Emission Factors for Fireplace and Woodstove Combustion in the San Francisco Bay Region. Environmental Science \& Technology, 37(9), 1758-1765. doi:10.1021/es026373c

Gulliver, J., \& Briggs, D. J. (2004). Personal exposure to particulate air pollution in transport microenvironments. Atmospheric Environment, 38(1), 1-8. doi:10.1016/j.atmosenv.2003.09.036

Hansen, A. D. A. (2005). The Aethalometer, manual. Magee Scientific Company. Berkeley, California, USA.

Hedberg, E., Johansson, C., Johansson, L., Swietlicki, E., \& Brorström-Lundén, E. (2006). Is levoglucosan a suitable quantitative tracer for wood burning? Comparison with receptor modeling on trace elements in Lycksele, Sweden. Journal of the Air \& Waste Management Association (1995), 56(12), 1669-1678.

Hien, P. D., Bac, V. T., Tham, H. C., Nhan, D. D., \& Vinh, L. D. (2002). Influence of meteorological conditions on PM2.5 and PM2.5-10 concentrations during the 
monsoon season in Hanoi, Vietnam. Atmospheric Environment, 36(21), 34733484. doi:10.1016/S1352-2310(02)00295-9

Hoffmann, D., Tilgner, A., Iinuma, Y., \& Herrmann, H. (2010). Atmospheric Stability of Levoglucosan: A Detailed Laboratory and Modeling Study. Environmental Science \& Technology, 44(2), 694-699. doi:10.1021/es902476f

Honicky, R. E., Osborne, J. S., \& Akpom, C. A. (1985). Symptoms of Respiratory Illness in Young Children and the Use of Wood-Burning Stoves for Indoor Heating. Pediatrics, 75(3), 587-593.

Hooyberghs, J., Mensink, C., Dumont, G., Fierens, F., \& Brasseur, O. (2005). A neural network forecast for daily average PM10 concentrations in Belgium. Atmospheric Environment, 39(18), 3279-3289. doi:10.1016/j.atmosenv.2005.01.050

Jenkins, P. L., Phillips, T. J., Mulberg, E. J., \& Hui, S. P. (1992). Activity patterns of Californians. Use of and proximity to indoor pollutant sources. Atmospheric Environment, 26(12), 2141-2148.

Johansson, L. S., Tullin, C., Leckner, B., \& Sjövall, P. (2003). Particle emissions from biomass combustion in small combustors. Biomass and Bioenergy, 25(4), 435446. doi:10.1016/S0961-9534(03)00036-9

Jokiniemi, J, Hytönen, K, Tissari, J, Obernberger, I, \& Brunner, T., et al. (2008). Biomass combustion in residential heating: particulate measurements, sampling, and physicochemcial and toxicological characterization. ISSN 0786-4728. Department of Environmental Science, University of Kupio, Kuopio, Finland. Retrieved from http://www.bios-bioenergy.at/uploads/media/Paper-ObernbergerBiomass-Combustion-in-Residential-Heating-2008-01-01.pdf 
Jordan, T. B., Seen, A. J., \& Jacobsen, G. E. (2006). Levoglucosan as an atmospheric tracer for woodsmoke. Atmospheric Environment, 40(27), 5316-5321. doi:10.1016/j.atmosenv.2006.03.023

Kirchstetter, T. W., Novakov, T., \& Hobbs, P. V. (2004). Evidence that the spectral dependence of light absorption by aerosols is affected by organic carbon. Journal of Geophysical Research-Atmospheres, 109(D21), D21208.

Kocbach, A., Namork, E., \& Schwarze, P. E. (2008). Pro-inflammatory potential of wood smoke and traffic-derived particles in a monocytic cell line. Toxicology, 247(2-3), 123-132. doi:10.1016/j.tox.2008.02.014

Kocbach Bølling, A., Pagels, J., Yttri, K., Barregard, L., Sallsten, G., Schwarze, P. E., \& Boman, C. (2009). Health effects of residential wood smoke particles: the importance of combustion conditions and physicochemical particle properties. Particle and Fibre Toxicology, 6(1), 29. doi:10.1186/1743-8977-6-29

Koenig, J. Q., Larson, T. V., Hanley, Q. S., Rebolledo, V., Dumler, K., Checkoway, H., et al. (1993). Pulmonary function changes in children associated with fine particulate matter. Environmental research, 63(1), 26-38.

Larson, T., Su, J., Baribeau, A., Buzzelli, M., Setton, E., \& Brauer, M. (2007). A Spatial Model of Urban Winter Woodsmoke Concentrations. Environmental Science \& Technology, 41(7), 2429-2436. doi:10.1021/es0614060

Liousse, C., Cachier, H., \& Jennings, S. G. (1993). Optical and thermal measurements of black carbon aerosol content in different environments: Variation of the specific attenuation cross-section, sigma( sigma). Atmospheric Environment; Part A, General Topics, 27(8), 1203-1211. 
Locker, H. B. (1988). The use of levoglucosan to assess the environmental impact of residential wood-burning on air quality. Dartmouth College.

Löndahl, J., Massling, A., Pagels, J., Swietlicki, E., Vaclavik, E., \& Loft, S. (2007). Sizeresolved respiratory-tract deposition of fine and ultrafine hydrophobic and hygroscopic aerosol particles during rest and exercise. Inhalation Toxicology, 19(2), 109-116. doi:10.1080/08958370601051677

Lunden, M. M., Kirchstetter, T. W., Thatcher, T. L., Hering, S. V., \& Brown, N. J. (2008). Factors affecting the indoor concentrations of carbonaceous aerosols of outdoor origin. Atmospheric Environment, 42(22), 5660-5671.

doi:10.1016/j.atmosenv.2008.03.017

MacIntosh, D. L., Stewart, J. H., Myatt, T. A., Sabato, J. E., Flowers, G. C., Brown, K. W., Hlinka, D. J., et al. (2010). Use of CALPUFF for exposure assessment in a near-field, complex terrain setting. Atmospheric Environment, 44(2), 262-270. doi:10.1016/j.atmosenv.2009.09.023

Malejan, C. (2009, June). Assessing Near-Field Outdoor Concentration Variability from Residential Wood Smoke Combustion Sources. California Polytechnic State University San Luis Obispo.

Met One Instruments, Inc. (2001). EBAM Operation Manual. Rowlett, Texas, USA. Montana Department of Environmental Quality \& Missoula City - County Health Department (MDEQ \& MCCHD). (2010, September). Seeley Lake Particulate Matter with an Aerodynamic Diameter of 2.5 Microns or less (PM2.5) Saturation Study. Retrieved from Missoula County Official Website Online: http://www.co. missoula.mt.us/airquality/MissoulasAir/pdfs/SL_PM25_SatStudy2010.doc 
Morandi, M. T., \& Ward, T. J. (2010). Wood smoke risk assessment: Defining the questions. Inhalation Toxicology, 22(2), 94-98. doi:10.3109/08958370903008854

Nerriere, É., Zmirou-Navier, D., Blanchard, O., Momas, I., Ladner, J., Le Moullec, Y., Personnaz, M., et al. (2005). Can we use fixed ambient air monitors to estimate population long-term exposure to air pollutants? The case of spatial variability in the Genotox ER study. Environmental Research, 97(1), 32-42. doi:10.1016/j.envres.2004.07.009

New Jersey Department of Environmental Protection (NJDEP). (2010, February 25). The Existence of Complex Wind Fields in the Region Surrounding the Portland Power Plant Site and the Use of CALPUFF. Retrieved from: http://www.state.nj.us/dep/baqp/petition/Exh\%2011\%20Portland_met_field.pdf

Ott, L., \& Longnecker, M. (2010). An introduction to statistical methods and data analysis. Cengage Learning.

Paine, R. J., Lee, R. F., Brode, R., Wilson, R. B. Cimorelli, A. J., Perry, S. G., Weil, J. C., et al. (1998). Model evaluation results for AERMOD. Retrieved June 8, 2011, from http://www.epa.gov/scram001/

Park, K., Chow, J. C., Watson, J. G., Trimble, D. L., Doraiswamy, P., Park, K., Arnott, W. P., et al. (2006). Comparison of continuous and filter-based carbon measurements at the Fresno supersite. Journal of the Air \& Waste Management Association (1995), 56(4), 474-491.

Pérez, P., Trier, A., \& Reyes, J. (2000). Prediction of PM2.5 concentrations several hours in advance using neural networks in Santiago, Chile. Atmospheric Environment, 34(8), 1189-1196. doi:10.1016/S1352-2310(99)00316-7 
Perry, S. G., Cimorelli, A. J., Paine, R. J., Brode, R. W., Weil, J. C., Venkatram, A., Wilson, R. B., et al. (2005). AERMOD: A Dispersion Model for Industrial Source Applications. Part II: Model Performance against 17 Field Study Databases. $J$. Appl. Meteor., 44(5), 694-708. doi:doi: 10.1175/JAM2228.1

Petzold, A., Kopp, C., \& Niessner, R. (1997). The dependence of the specific attenuation crosssection on black carbon mass fraction and particle size. Atmospheric Environment, 31(5), 661-672.

Pierson, W. E., Koenig, J. Q., \& Bardana, E. J. (1989). Potential adverse health effects of wood smoke. Western Journal of Medicine, 151(3), 339-342.

Pires, J.C.M., Martins, F.G., Sousa, S.I.V., Alvim-Ferraz, M.C.M., Pereira, M.C., (2008). Prediction of the daily mean PM10 concentrations using linear models. American Journal of Environmental Sciences, 4(5), 445-453.

Pope III, C. A., Burnett, R. T., Thun, M. J., Calle, E. E., Krewski, D., Ito, K., et al. (2002). Lung Cancer, Cardiopulmonary Mortality, and Long-term Exposure to Fine Particulate Air Pollution. JAMA, 287(9), 1132-1141. doi:

10.1001/jama.287.9.1132.

Purvis, C. R., McCrillis, R. C., \& Kariher, P. H. (2000). Fine Particulate Matter (PM) and Organic Speciation of Fireplace Emissions. Environmental Science \& Technology, 34(9), 1653-1658. doi:10.1021/es981006f

Radonjic, Z., Chambers, D. B., \& Kirkaldy, J. (2003 October). Modelling Line Sources (Roads) Using CAL3QHCR,ISCST3, AERMOD and CALPUFF. Presented at the A\&WMA Specialty Conference, Guideline on Air Quality Models: The Path Forward, Mystic, CT. 
Robinson, D., Monro, J., \& Campbell, E. (2007). Spatial variability and population exposure to PM2.5 pollution from woodsmoke in a New South Wales country town. Atmospheric Environment, 41(26), 5464-5478. doi:10.1016/ j.atmosenv.2007.01.059

Sarotis, L., Marazioti, C., Marazioti, P., \& Maraziotis. (2008). Statistical analysis of inhalable (PM10) and fine particles (PM2.5) concentrations in urban region of Patras, Greece. GLOBAL NEST JOURNAL, 10(2), 123-131.

Savin, N. E., \& White, K. J. (1977). The Durbin-Watson Test for Serial Correlation with Extreme Sample Sizes or Many Regressors. Econometrica, 45(8), 1989-1996.

Schauer, J. J., \& Cass, G. R. (2000). Source Apportionment of Wintertime Gas-Phase and Particle-Phase Air Pollutants Using Organic Compounds as Tracers. Environmental Science \& Technology, 34(9), 1821-1832. doi:10.1021/es981312t

Simoneit, B. R., Schauer, J. J., Nolte, C. G., Oros, D. R., Elias, V. O., Fraser, M. P., et al. 1999). Levoglucosan, a tracer for cellulose in biomass burning and atmospheric particles. Atmospheric Environment, 33(2), 173-182.

Simpson, C. D., Paulsen, M., Dills, R. L., Liu, L. S., \& Kalman, D. A. (2005). Determination of Methoxyphenols in Ambient Atmospheric Particulate Matter: Tracers for Wood Combustion. Environmental Science \& Technology, 39(2), 631637. doi:10.1021/es0486871

Slini, T., Kaprara, A., Karatzas, K., \& Moussiopoulos, N. (2006). PM10 forecasting for Thessaloniki, Greece. Environmental Modelling \& Software, 21(4), 559-565. doi:10.1016/j.envsoft.2004.06.011 
Sportisse, B. (2007). A review of current issues in air pollution modeling and simulation. Computational Geosciences, 11(2), 159-181.

Stadlober, E., Hörmann, S., \& Pfeiler, B. (2008). Quality and performance of a PM10 daily forecasting model. Atmospheric Environment, 42(6), 1098-1109. doi:10.1016/j.atmosenv.2007.10.073

Tai, A. P., Mickley, L. J., \& Jacob, D. J. (2010). Correlations between fine particulate matter (PM2.5) and meteorological variables in the United States: Implications for the sensitivity of PM2.5 to climate change. Atmospheric Environment, 44(32), 3976-3984. doi:10.1016/j.atmosenv.2010.06.060

Tian, Y. Q., Radke, J. D., Gong, P., \& Yu, Q. (2004). Model development for spatial variation of PM2.5 emissions from residential wood burning. Atmospheric Environment, 38(6), 833-843. doi:10.1016/j.atmosenv.2003.10.040

Thatcher, T. L., McKone, T. E., Fisk, W. J., Sohn, M. D., Delp, W. W., Riley, W. J., et al. (2001). Factors affecting the concentration of outdoor particles indoors (COPI): Identification of data needs and existing data. Retrieved from http://www.osti.gov/energycitations/servlets/purl/820780-8m21pL/native/

Tian, Y. Q., Radke, J. D., Gong, P., \& Yu, Q. (2004). Model development for spatial variation of PM2.5 emissions from residential wood burning. Atmospheric Environment, 38(6), 833-843. doi:10.1016/j.atmosenv.2003.10.040

Trinity Consultants. (2001, December 1). Understanding and Adapting to New Dispersion Models. Retrieved from Trinity Consultants website: http://trinityconsultants.com/WorkArea/DownloadAsset.aspx?id=1251 
Trinity Consultants. (2008, June 24). Performance of AERMOD vs. CALPUFF on fugitive emission sources in the nearfield. Retrieved from Trinity Consultants website: http://trinityconsultants.com/WorkArea/DownloadAsset.aspx ?id=1289

Turpin, B. J., Weisel, C. P., Morandi, M., Colome, S., Stock, T., Eisenreich, S., \& Buckley, B. (2007). Relationships of Indoor, Outdoor, and Personal Air (RIOPA): part II. Analyses of concentrations of particulate matter species. Research Report (Health Effects Institute), (130 Pt 2), 1-77; discussion 79-92.

US Census Bureau (2000). Census Bureau Home Page. Retrieved November 1, 2010, from http://www.census.gov/

US Census Bureau (2009). US Census Bureau American FactFinder Home Page. Retrieved November 1, 2010, from http://factfinder.census.gov/

US Environmental Protection Agency (EPA) (1995a, 27, September). User's guide for the Industrial source complex (ISC3) dispersion models: Volume I - User Instructions. Retrieved May 31, 2011, from http://www.epa.gov/scram001/userg/regmod/isc3v1.pdf

US Environmental Protection Agency (EPA) (1995b, September). User's guide for the Industrial source complex (ISC3) dispersion models: Volume I - Description of Model Algorithms. Retrieved May 31, 2011, from http://www.epa.gov/scram001/userg/regmod/isc3v2.pdf

US EPA (2003, June). Comparison of regulatory design concentrations: AERMOD vs ISCST3, CTDMPLUS, ISC-PRIME. Retrieved May 31, 2011, from http://www.epa.gov/scram001/7thconf/aermod/compar.pdf 
US EPA (2008a, August 13). "Clarification of Regulatory Status of CALPUFF for NearField Applications.” Retrieved January 7, 2011, from http://www.epa.gov/ scram001/clarification $\% 20$ of $\% 20$ regulatory $\% 20$ status $\% 20$ of $\% 20$ calpuff.pdf

US EPA (2008b, December). QA Handbook for Air Pollution Measurement Systems; Volume II; Ambient Air Quality Monitoring Program. Retrieved December 19, 2010, from http://www.epa.gov/ttnamti1/files/ambient/pm25/qa/QA-HandbookVol-II.pdf

US EPA (2010, October 28). PM Standards. Retrieved November 10, 2010, from http://www.epa.gov/air/particlepollution/standards.html

Valari, M., \& Menut, L. (2010). Transferring the heterogeneity of surface emissions to variability in pollutant concentrations over urban areas through a chemistrytransport model. Atmospheric Environment, 44(27), 3229-3238.

doi:10.1016/j.atmosenv.2010.06.001

Venkatram, A., Isakov, V., Yuan, J., \& Pankratz, D. (2004). Modeling dispersion at distances of meters from urban sources. Atmospheric Environment, 38(28), 46334641. doi:16/j.atmosenv.2004.05.018

Vukovich, F. M., \& Sherwell, J. (2002). Comparison of fine particles and the relationship between particle variations and meteorology at an urban site and a remote site in the Eastern United States. Journal of the Air \& Waste Management Association (1995), 52(5), 573-584.

Ward, C. (2009, June). Assessing Near-Field Outdoor Concentration Variability from Residential Wood Smoke Combustion Sources. California Polytechnic State University San Luis Obispo. 
Watson, J. G., Chow, J. C., \& Houck, J. E. (2001). PM2.5 chemical source profiles for vehicle exhaust, vegetative burning, geological material, and coal burning in Northwestern Colorado during 1995. Chemosphere, 43(8), 1141-1151. doi:10.1016/S0045-6535(00)00171-5

Watson, J. G., Chow, J. C., \& Chen, A. (2005). Summary of Organic and Elemental Carbon/Black Carbon Analysis Methods and Intercomparisons. Aerosol and Air Quality Research, 5(1), 65-102.

The Weather Channel. (2010). Retrieved November 2, 2010, from http://www. weather.com/

Weijers, E. P., Khlystov, A. Y., Kos, G. P. A., \& Erisman, J. W. (2004). Variability of particulate matter concentrations along roads and motorways determined by a moving measurement unit. Atmospheric Environment, 38(19), 2993-3002. doi:10.1016/j.atmosenv.2004.02.045

Weingartner, E., Saathoff, H., Schnaiter, M., Streit, N., Bitnar, B., \& Baltensperger, U. (2003). Absorption of light by soot particles: determination of the absorption coefficient by means of aethalometers. Journal of Aerosol Science, 34(10), 14451463.

Wilson, W. E., Mage, D. T., \& Grant, L. D. (2000). Estimating separately personal exposure to ambient and nonambient particulate matter for epidemiology and risk assessment: why and how. Journal of the Air \& Waste Management Association (1995), 50(7), 1167-1183.

Wu, C., Larson, T. V., Wu, S., Williamson, J., Westberg, H. H., \& Liu, L. S. (2007). Source apportionment of $\operatorname{PM}(2.5)$ and selected hazardous air pollutants in Seattle. 
The Science of the Total Environment, 386(1-3), 42-52. doi:10.1016/ j.scitotenv.2007.07.042

Wu, L., Bocquet, M., \& Chevallier, M. (2010). Optimal reduction of the ozone monitoring network over France. Atmospheric Environment, 44(25), 3071-3083. doi:10.1016/j.atmosenv.2010.04.012

Yu, H., Chen, J., Christakos, G., \& Jerrett, M. (2009). BME estimation of residential exposure to ambient PM10 and ozone at multiple time scales. Environmental Health Perspectives, 117(4), 537-544. doi:10.1289/ehp.0800089

Yu, O., Sheppard, L., Lumley, T., Koenig, J. Q., \& Shapiro, G. G. (2000). Effects of ambient air pollution on symptoms of asthma in Seattle-area children enrolled in the CAMP study. Environmental Health Perspectives, 108(12), 1209-1214.

Zeger, S. L., Thomas, D., Dominici, F., Samet, J. M., Schwartz, J., Dockery, D., \& Cohen, A. (2000). Exposure measurement error in time-series studies of air pollution: concepts and consequences. Environmental Health Perspectives, $108(5), 419-426$.

Zhang, X., Hecobian, A., Zheng, M., Frank, N. H., \& Weber, R. J. (2010). Biomass burning impact on $\mathrm{PM}_{2.5}$ over the southeastern US during 2007: integrating chemically speciated FRM filter measurements, MODIS fire counts and PMF analysis. Atmospheric Chemistry and Physics, 10(14), 6839-6853. doi:10.5194/acp-10-6839-2010 


\section{Appendix A. Aethalometer Calibration ANOVA Output for Aethalometer Calibration}

Minitab output for ANOVA of segment 1 of January 19, 2010 readings with adjusted $\sigma_{\mathrm{SG}}$ to achieve a similar aethalometer data average:
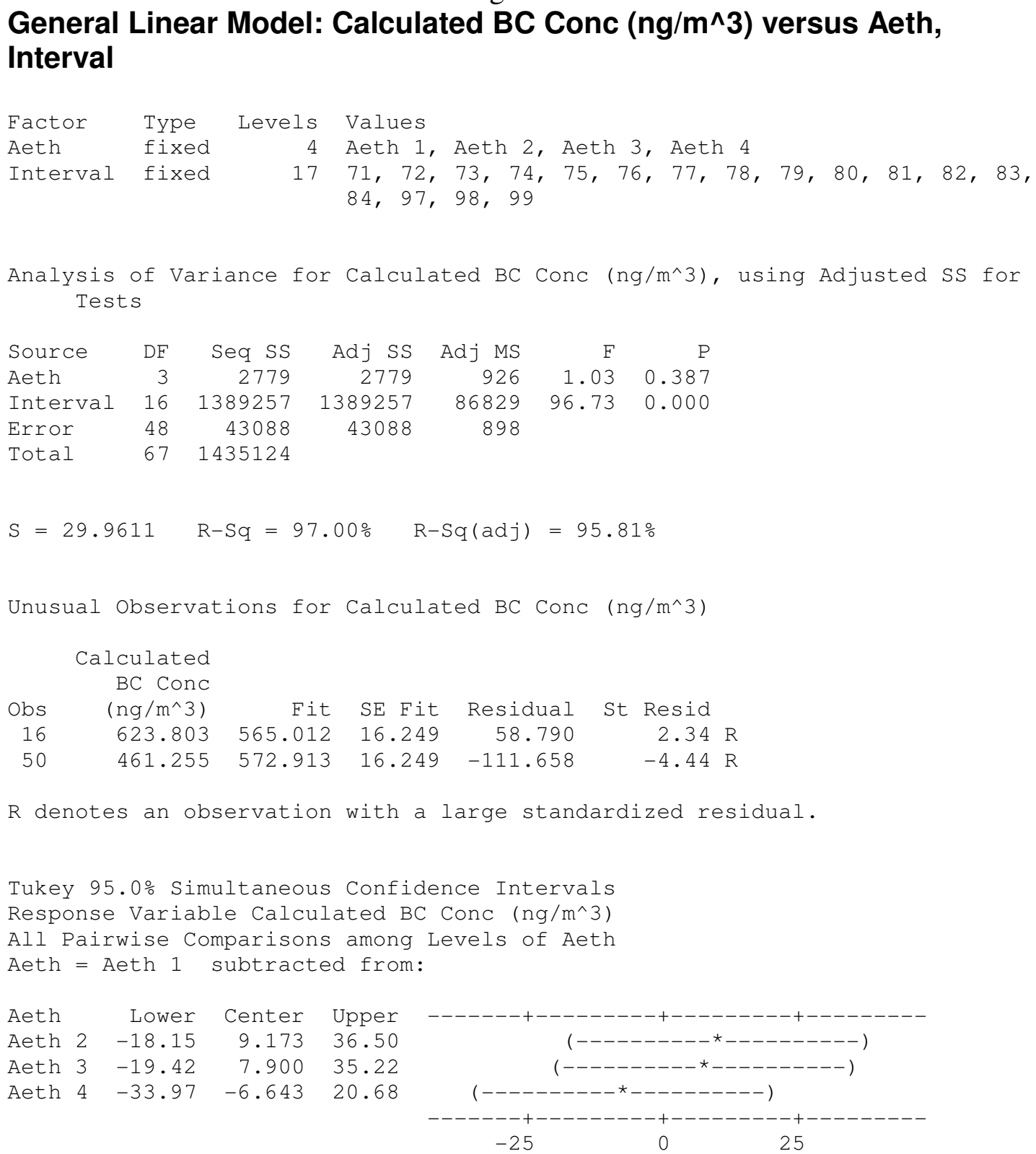

Aeth $=$ Aeth 2 subtracted from:

Aeth Lower Center Upper

$\begin{array}{llll}\text { Aeth } 3 & -28.60 & -1.27 & 26.05\end{array}$

Aeth $4 \quad-43.14 \quad-15.82 \quad 11.51$

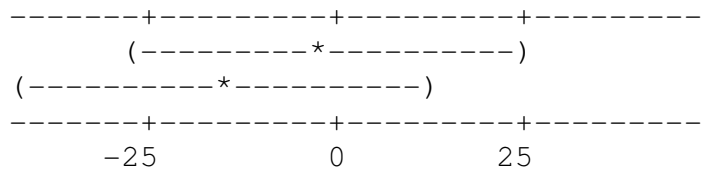

Aeth $=$ Aeth 3 subtracted from: 

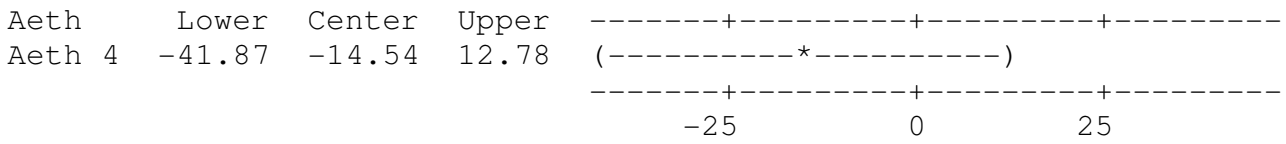

Tukey Simultaneous Tests

Response Variable Calculated BC Conc ( $\mathrm{ng} / \mathrm{m}^{\wedge} 3$ )

All Pairwise Comparisons among Levels of Aeth

Aeth $=$ Aeth 1 subtracted from:

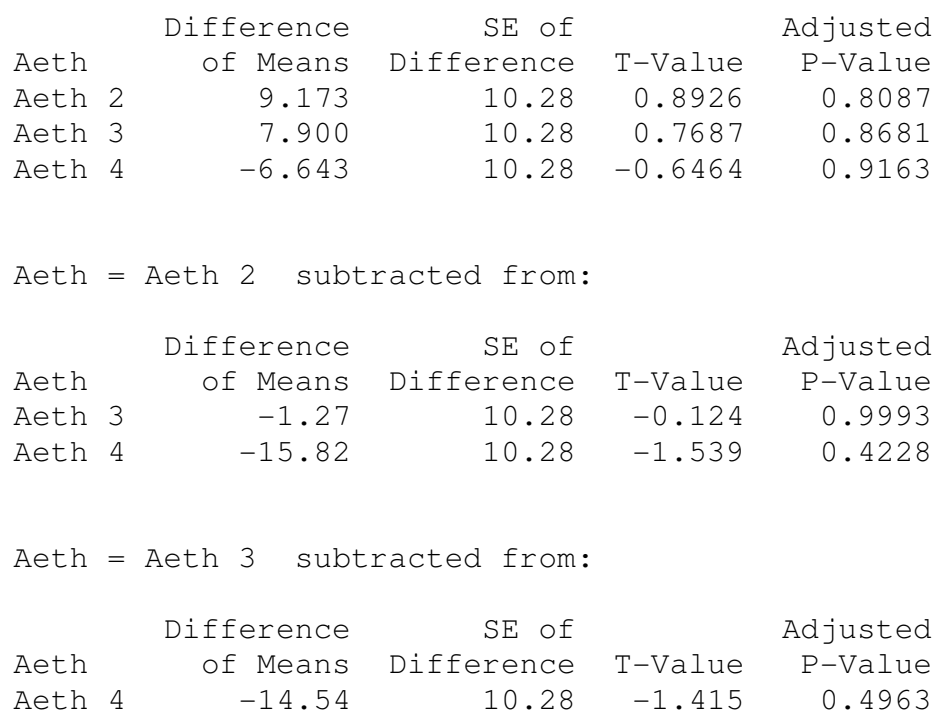

\section{Residual Plots for Calculated BC Conc (ng/m^3)}

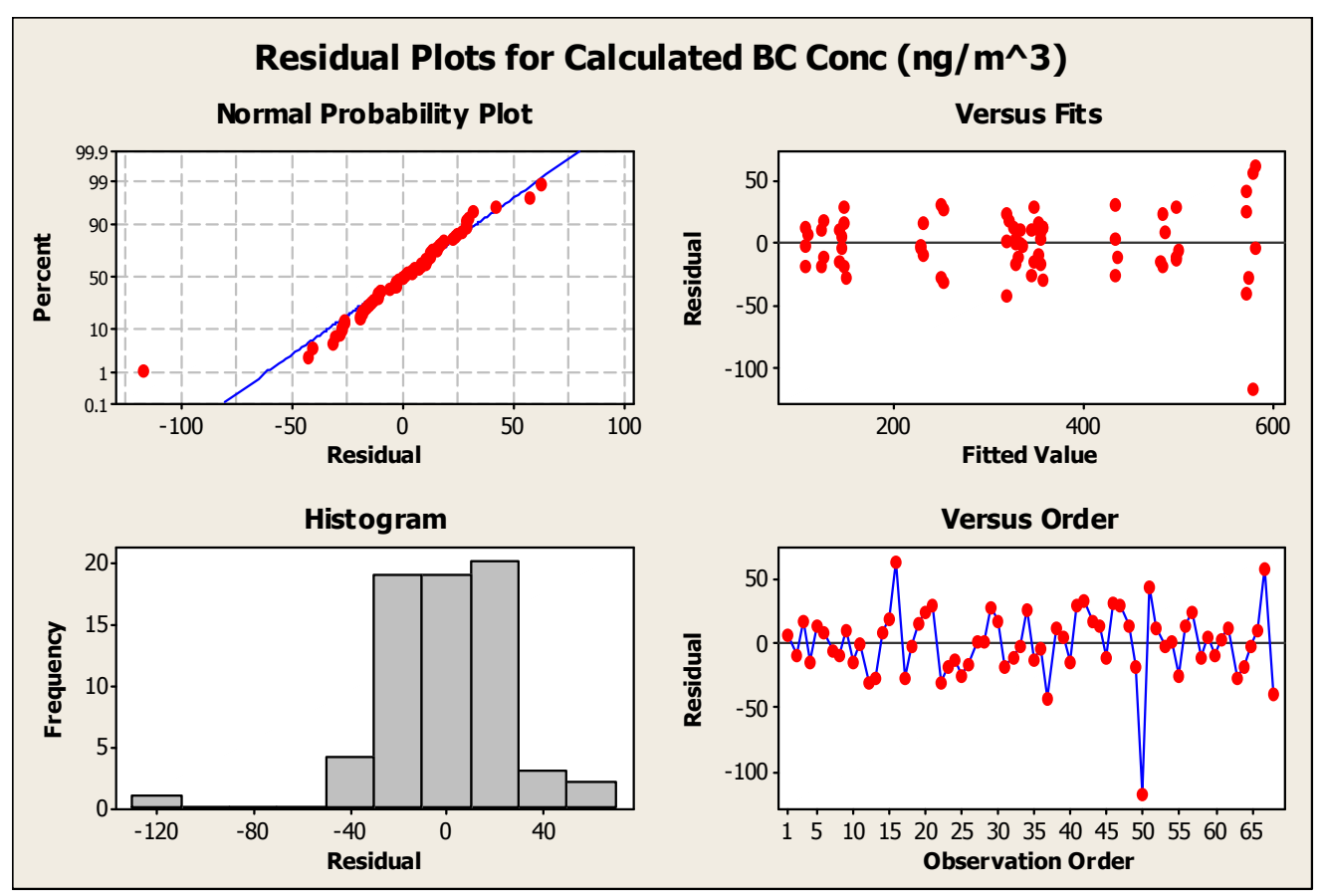


Graph of segment 1 of January 19, 2010 readings before adjusted $\sigma_{\mathrm{SG}}$ to achieve a similar aethalometer data average:

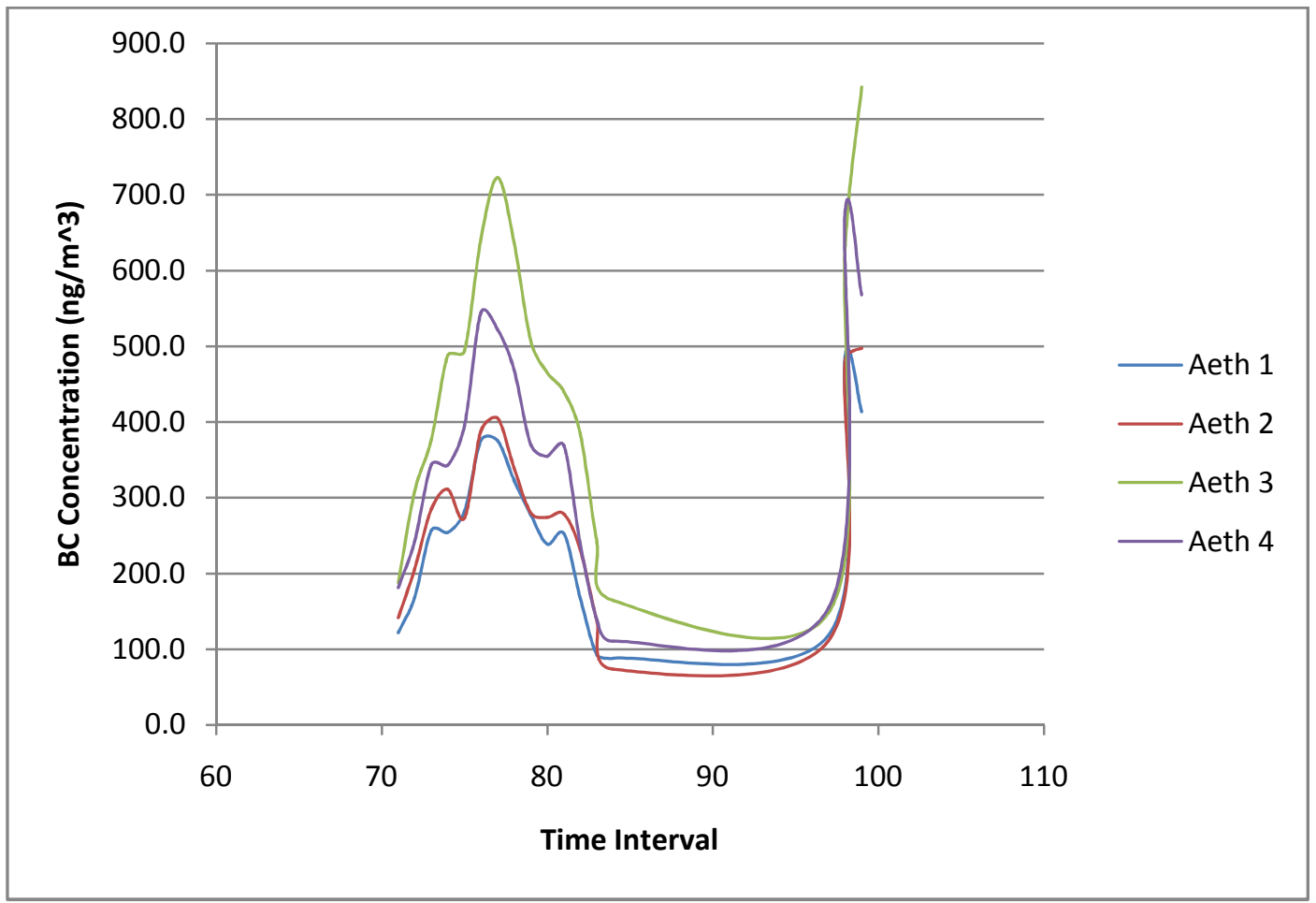

Graph of segment 1 of January 19, 2010 readings with adjusted $\sigma_{\mathrm{SG}}$ to achieve a similar aethalometer data average:

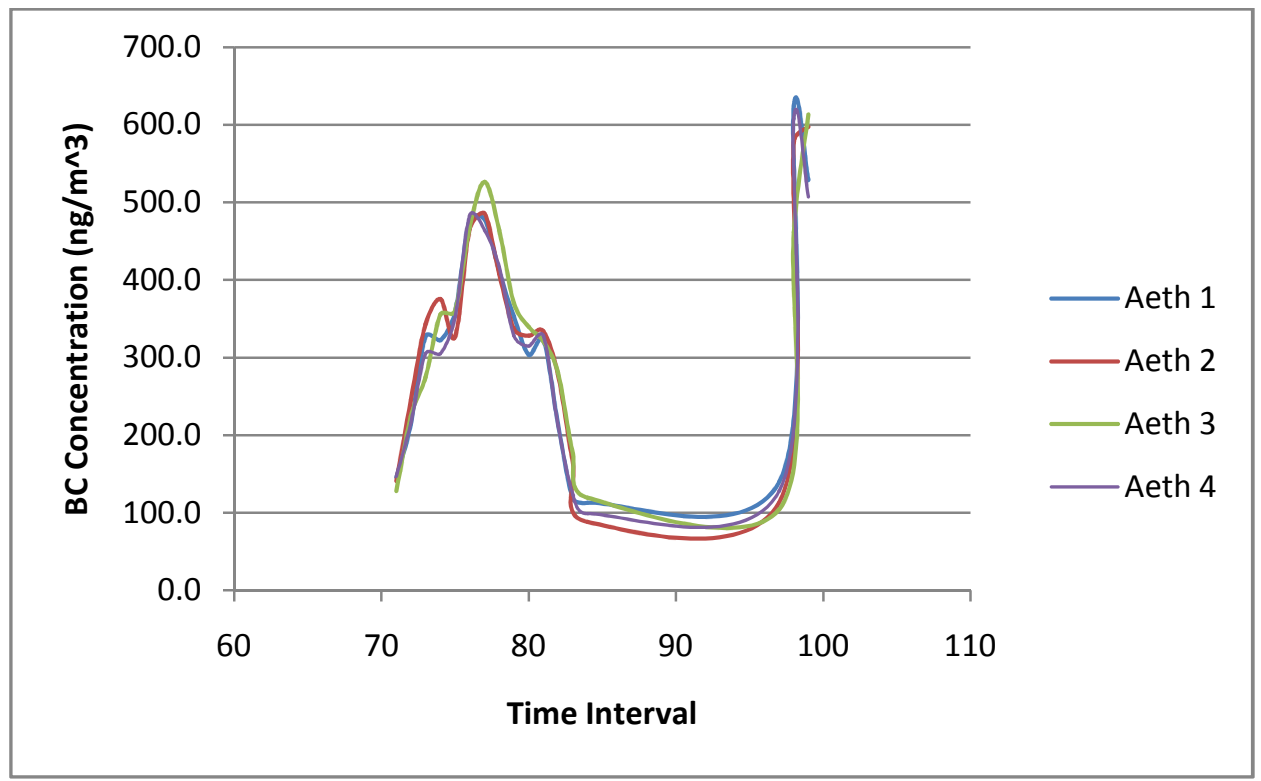


Minitab output for ANOVA of segment 2 of January 19, 2010 readings adjusted to have the same $\sigma_{\mathrm{SG}}$ as segment 1 :

\section{General Linear Model: Calculated BC Conc $\left(\mathrm{ng} / \mathrm{m}^{\wedge} 3\right)$ versus Aeth, Interval}

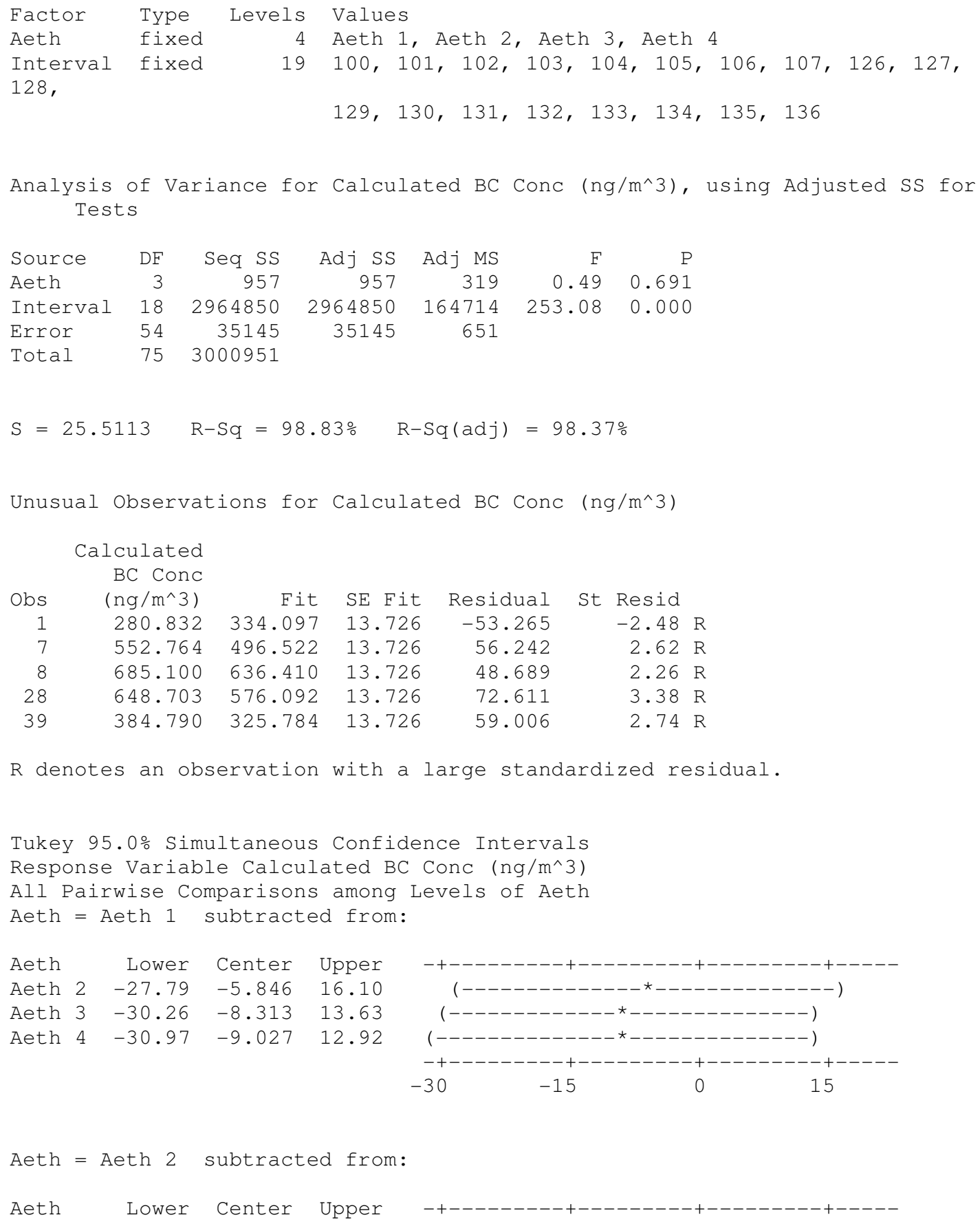

$\mathrm{R}$ denotes an observation with a large standardized residual.

Tukey 95.0\% Simultaneous Confidence Intervals Response Variable Calculated BC Conc ( $\mathrm{ng} / \mathrm{m}^{\wedge} 3$ ) All Pairwise Comparisons among Levels of Aeth Aeth $=$ Aeth 1 subtracted from:

Aeth $=$ Aeth 2 subtracted from:

Aeth Lower Center Upper 


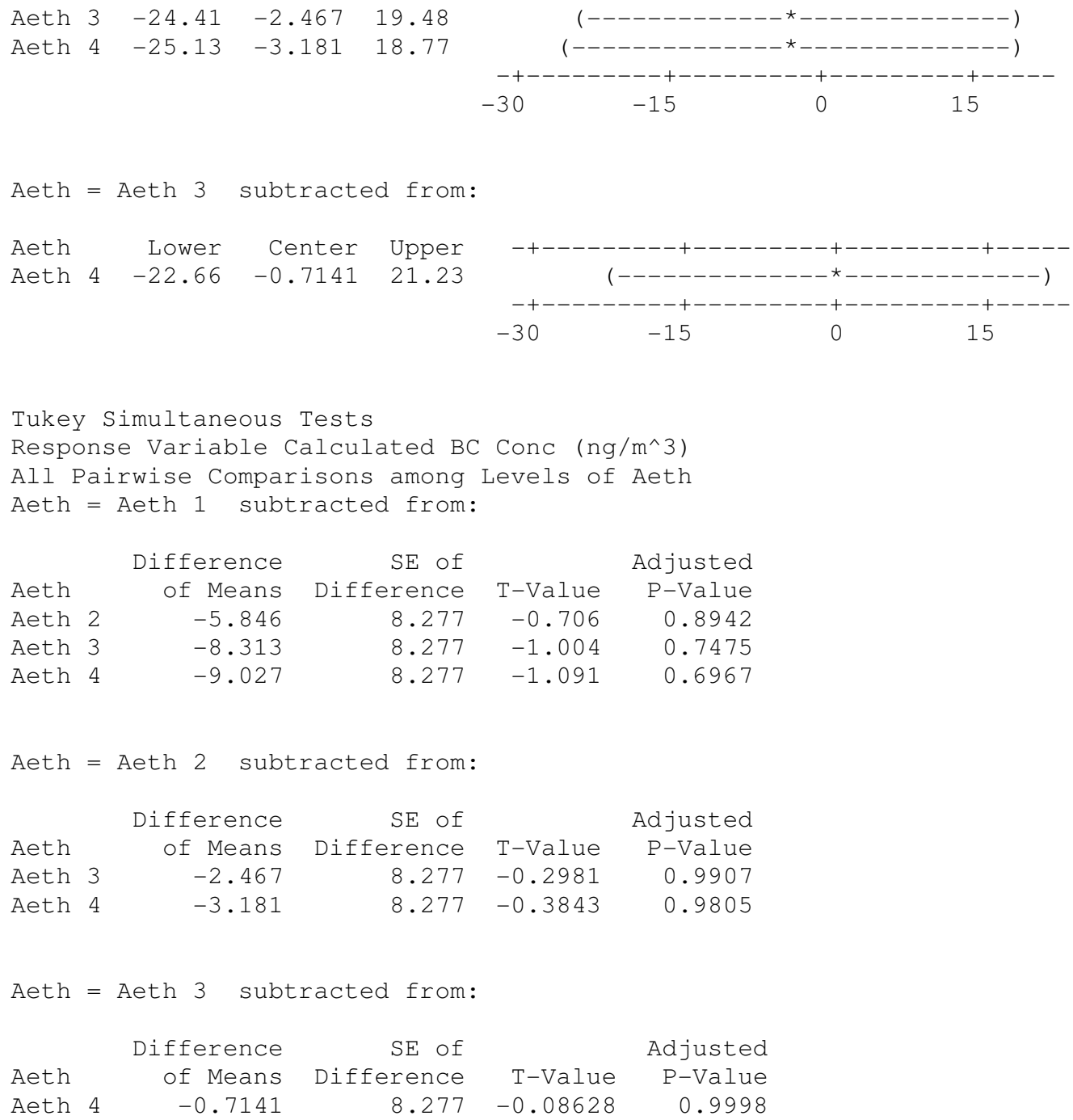

\section{Residual Plots for Calculated BC Conc (ng/m^3)}




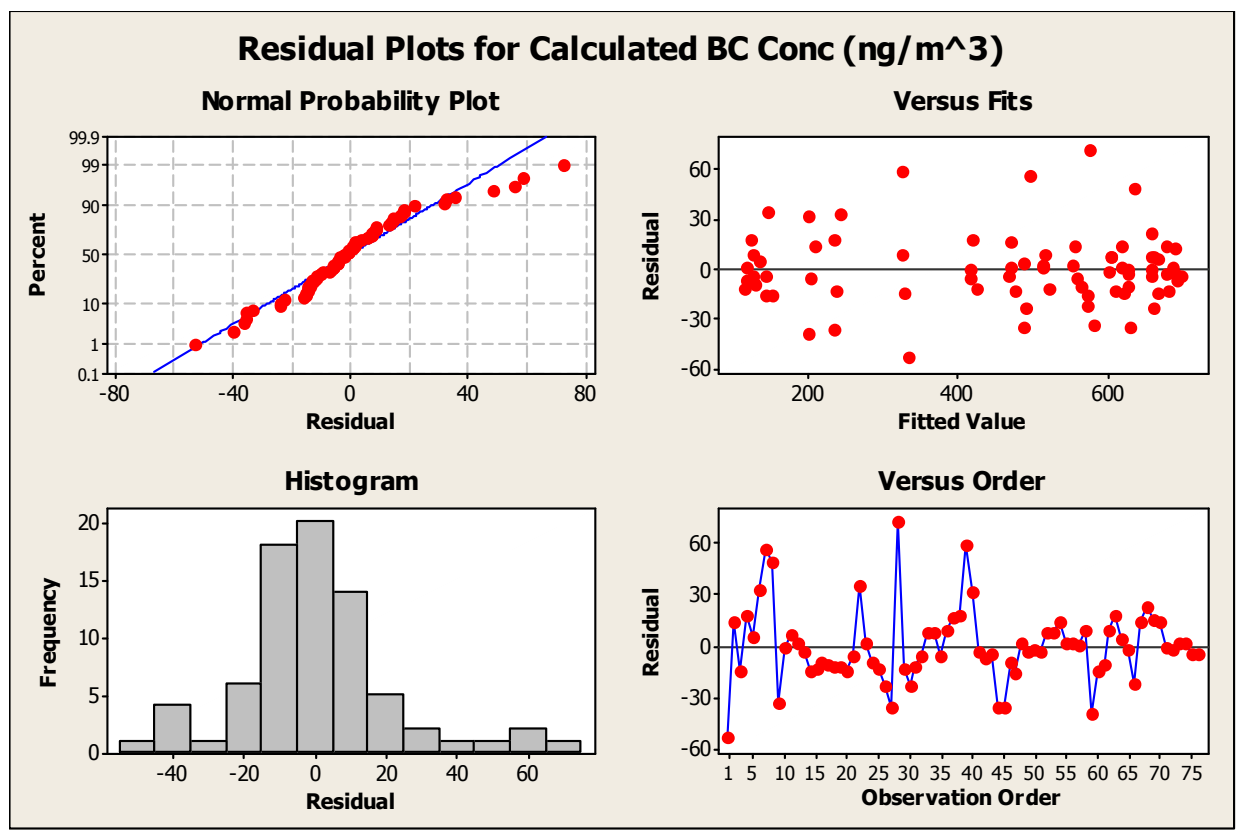

Graph of segment 2 of January 19, 2010 readings before adjusted $\sigma_{S G}$ from segment 1:

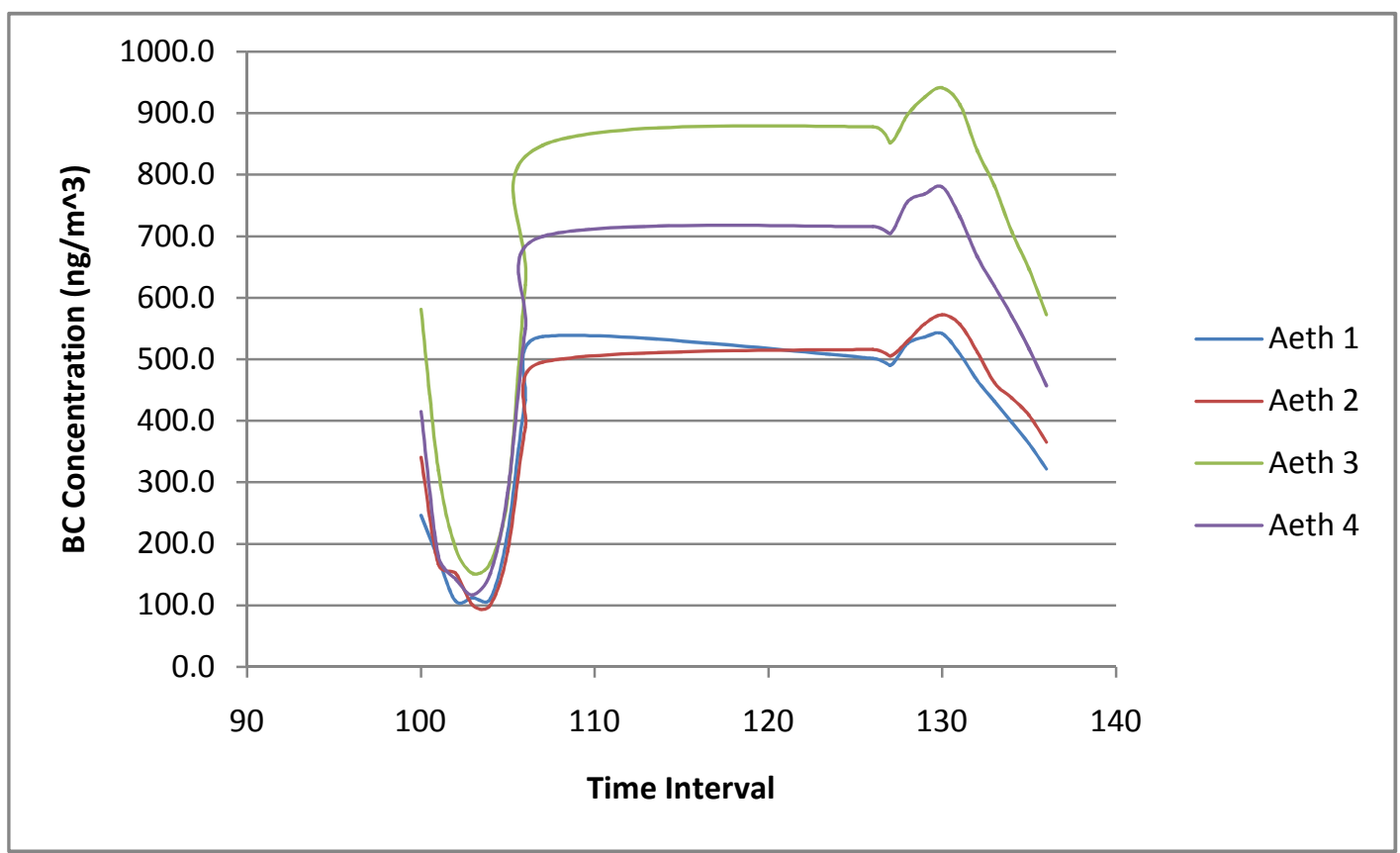

Graph of segment 2 of January 19, 2010 readings with adjusted $\sigma_{\text {SG }}$ from segment 1: 


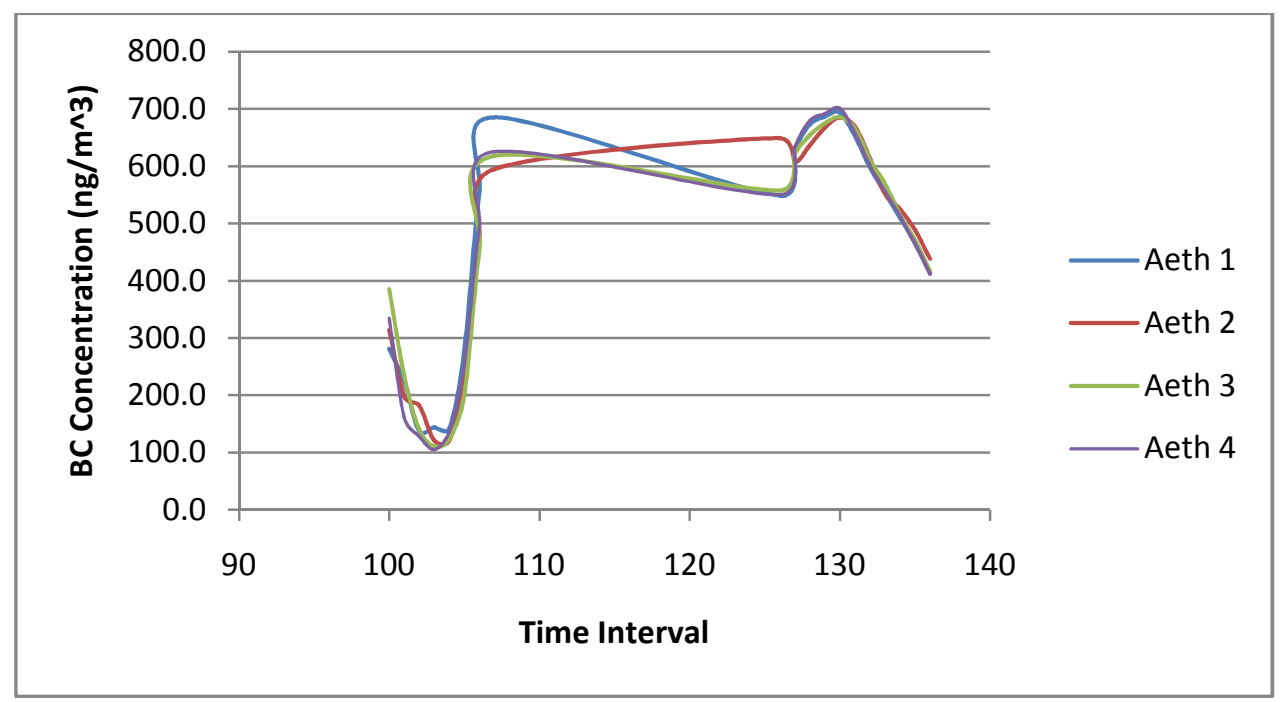

Minitab output for ANOVA of segment 1 of June 22 -23 (2010) readings with adjusted $\sigma_{S G}$ to achieve a similar aethalometer data average:

\section{General Linear Model: P.1Calculated BC versus Aeth, Interval}

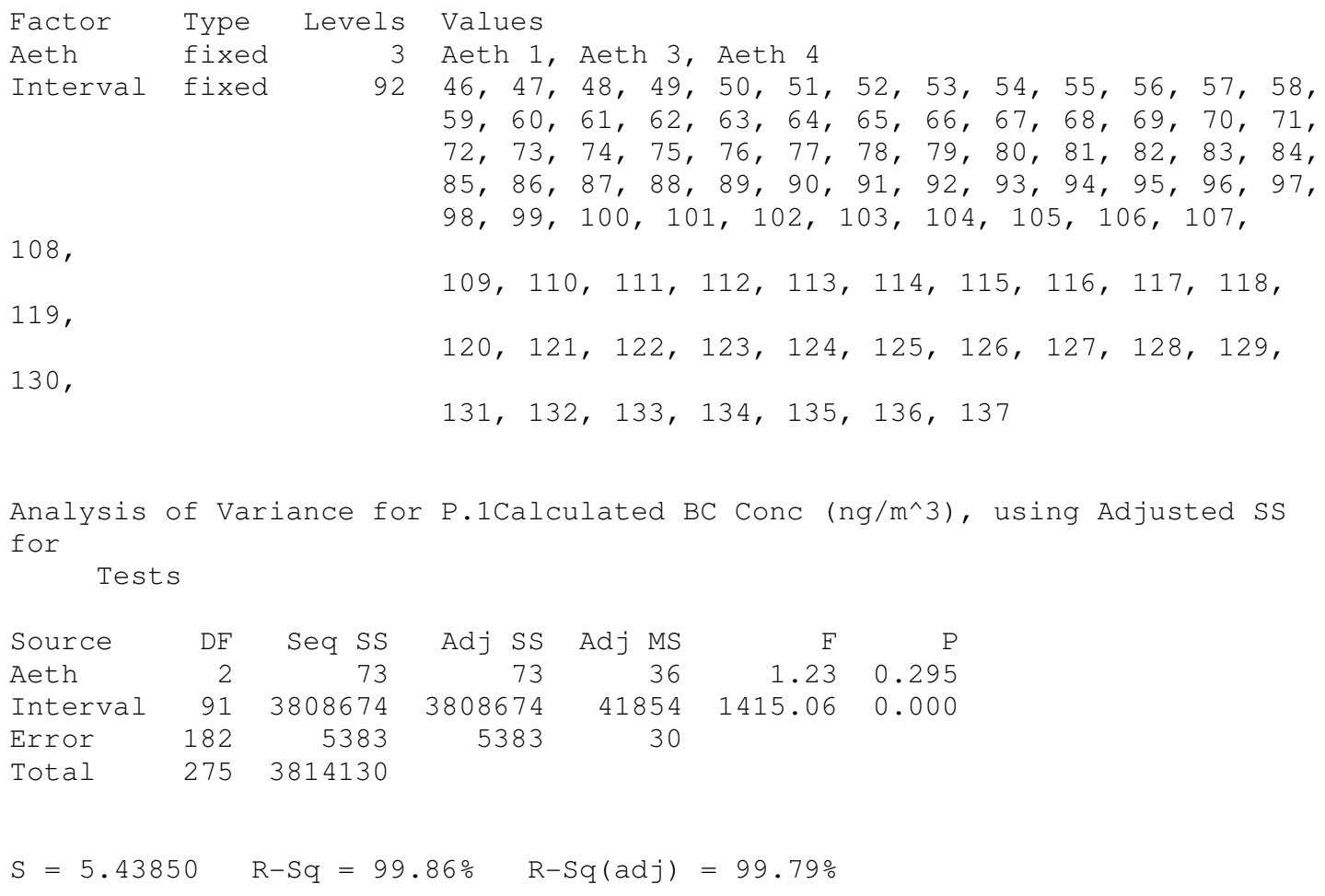




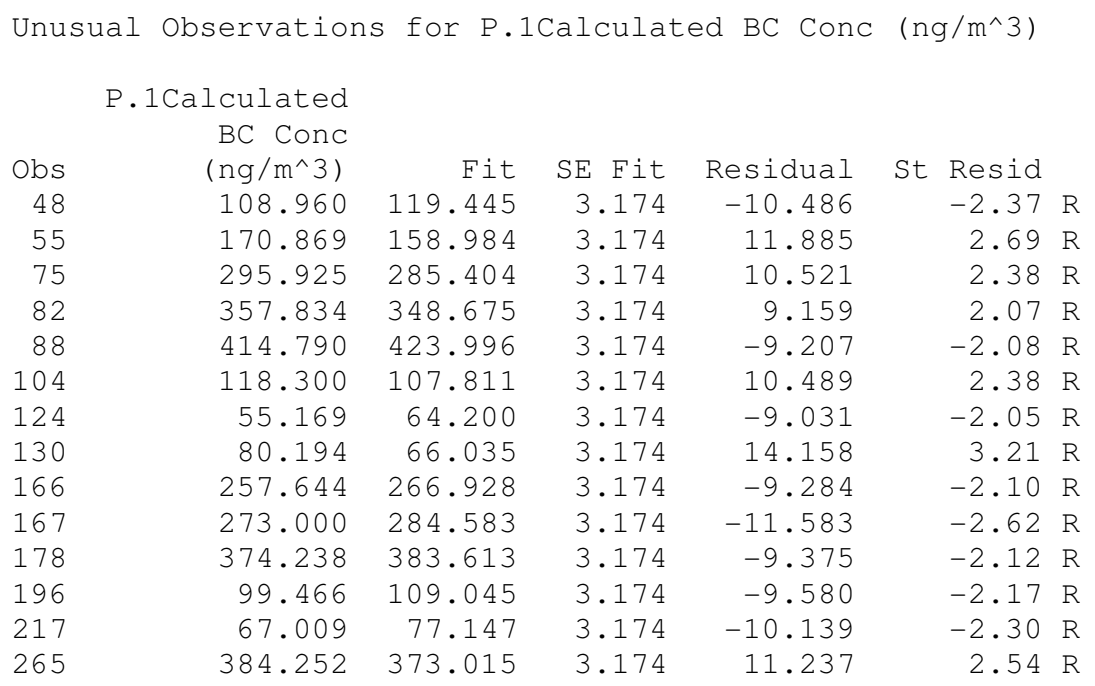

R denotes an observation with a large standardized residual.

Tukey 95.0\% Simultaneous Confidence Intervals Response Variable P.1Calculated BC Conc ( $\mathrm{ng} / \mathrm{m}^{\wedge} 3$ ) All Pairwise Comparisons among Levels of Aeth Aeth $=$ Aeth 1 subtracted from:

$\begin{array}{lrrr}\text { Aeth } & \text { Lower } & \text { Center } & \text { Upper } \\ \text { Aeth } 3 & -2.714 & -0.8207 & 1.073 \\ \text { Aeth 4 } & -1.480 & 0.4141 & 2.308\end{array}$

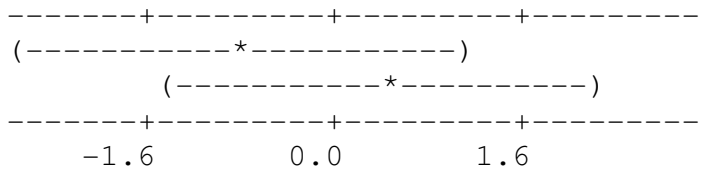

$-1.6$

0.0

1.6

Aeth $=$ Aeth 3 subtracted from:

Aeth Lower Center Upper

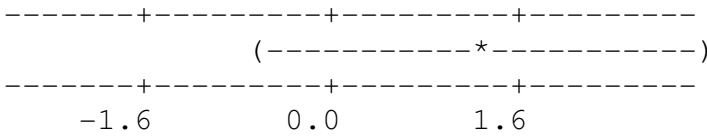

Tukey Simultaneous Tests

Response Variable P.1Calculated BC Conc ( $\mathrm{ng} / \mathrm{m} \wedge 3$ )

All Pairwise Comparisons among Levels of Aeth

Aeth = Aeth 1 subtracted from:

$\begin{array}{lrrrr} & \begin{array}{r}\text { Difference } \\ \text { of Means }\end{array} & \begin{array}{r}\text { SE of } \\ \text { Difference }\end{array} & \text { T-Value } & \text { Adjusted } \\ \text { P-Value } \\ \text { Aeth } 3 & -0.8207 & 0.8019 & -1.023 & 0.5630 \\ \text { Aeth 4 } & 0.4141 & 0.8019 & 0.516 & 0.8634\end{array}$

Aeth $=$ Aeth 3 subtracted from:

$\begin{array}{lrrrr} & \text { Difference } & \text { SE of } & & \text { Adjusted } \\ \text { Aeth } & \text { of Means } & \text { Difference } & \text { T-Value } & \text { P-Value } \\ \text { Aeth 4 } & 1.235 & 0.8019 & 1.540 & 0.2748\end{array}$

\section{Residual Plots for P.1Calculated BC Conc $\left(\mathrm{ng} / \mathrm{m}^{\wedge} 3\right)$}




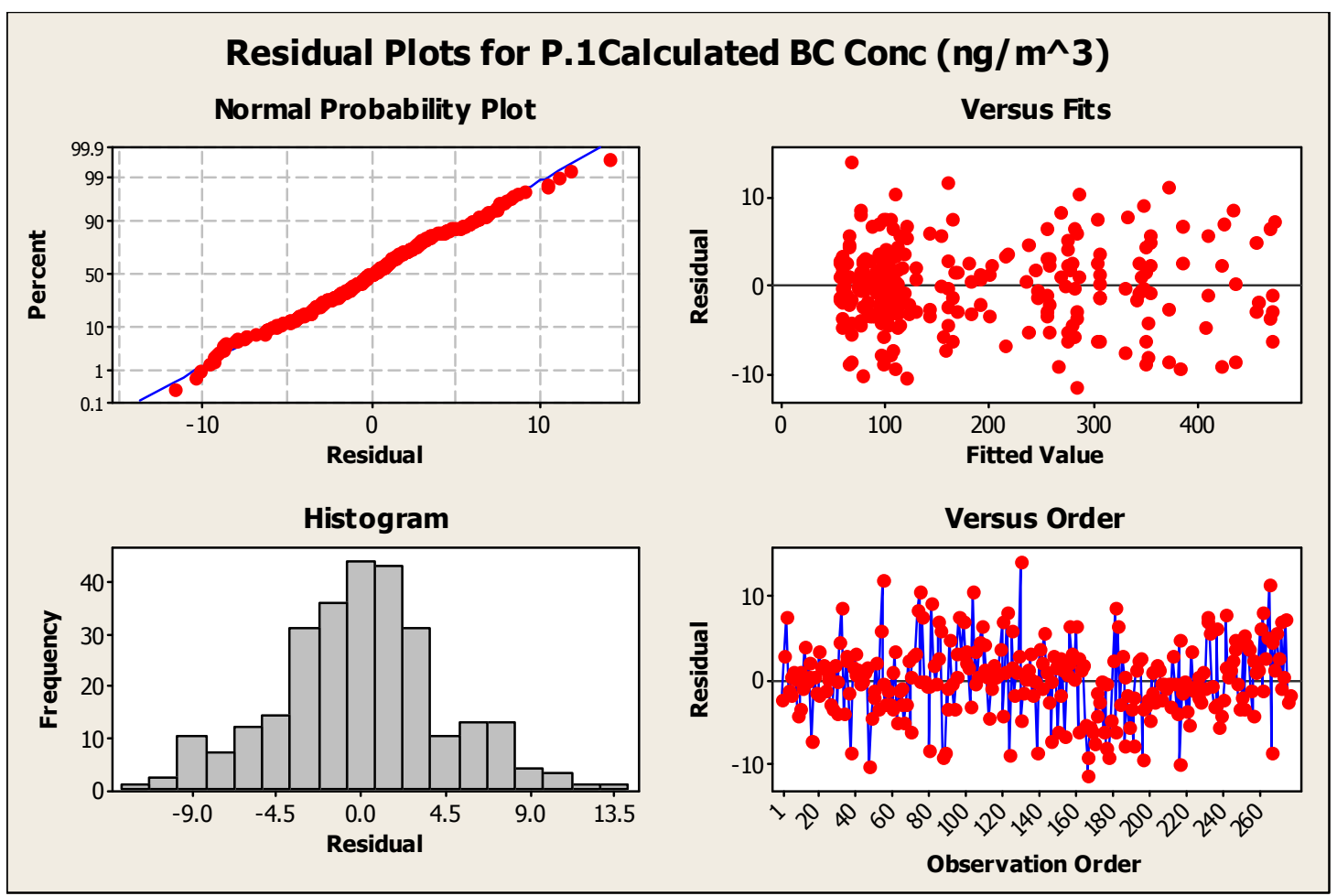

Graph of segment 1 of June 22-23, 2010 readings before adjusted $\sigma_{\mathrm{SG}}$ to achieve a similar aethalometer data average:

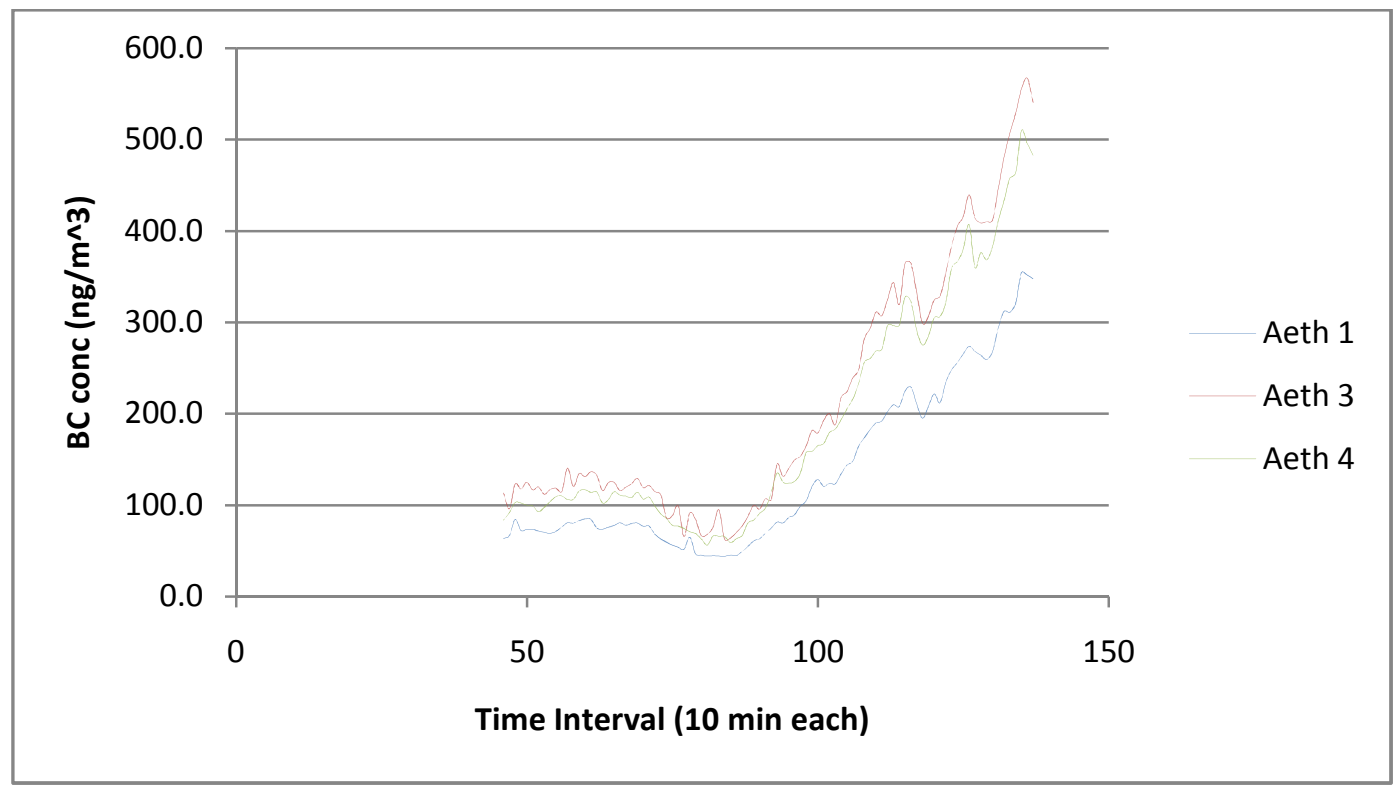


Graph of segment 1 of June 22-23, 2010 readings with adjusted $\sigma_{\mathrm{SG}}$ to achieve a similar aethalometer data average:

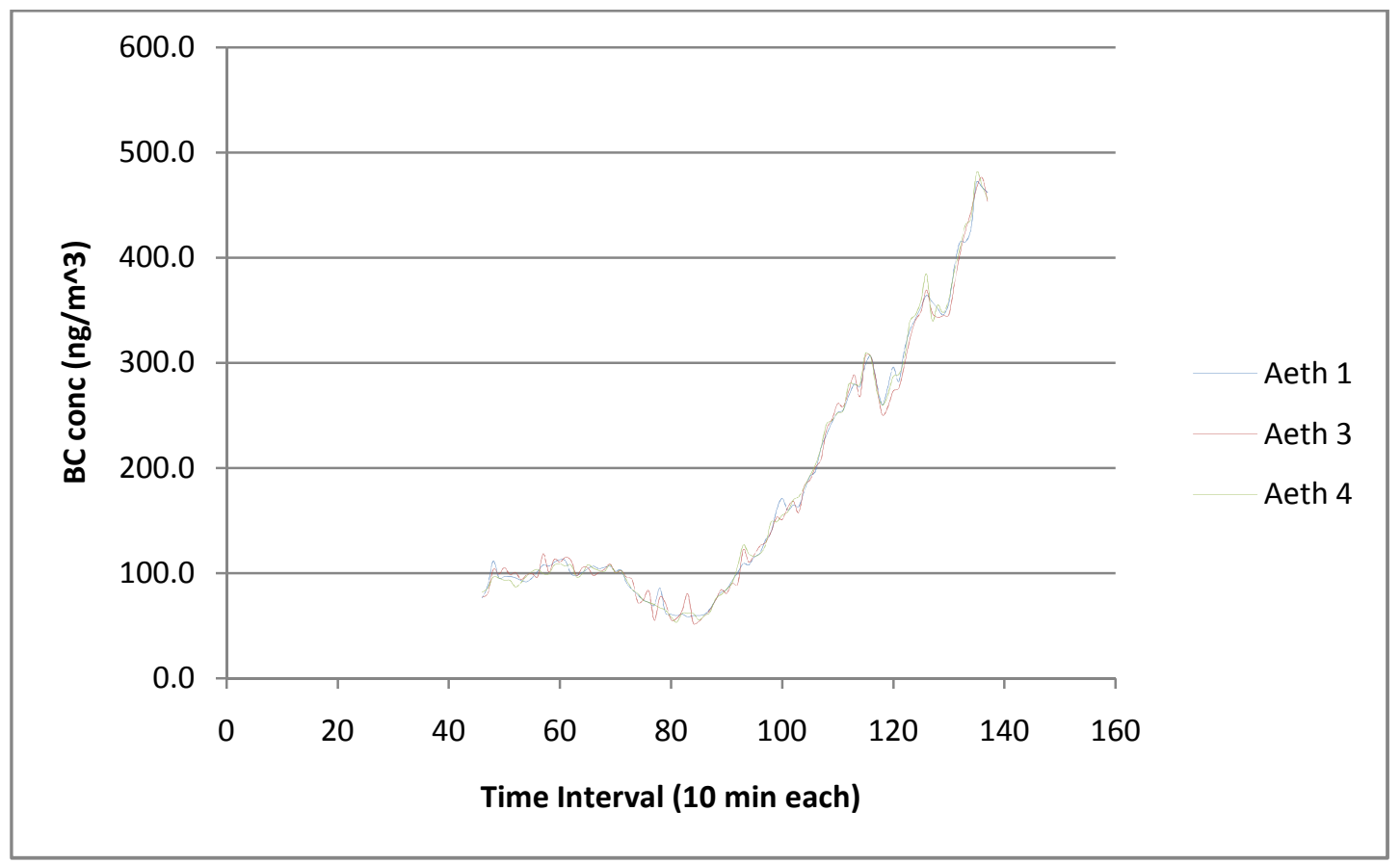

Minitab output for ANOVA of segment 2 of June 22-23, 2010 readings adjusted to have the same $\sigma_{\mathrm{SG}}$ as segment 1 :

\section{General Linear Model: p2-aCalculated B versus Aeth, Interval}

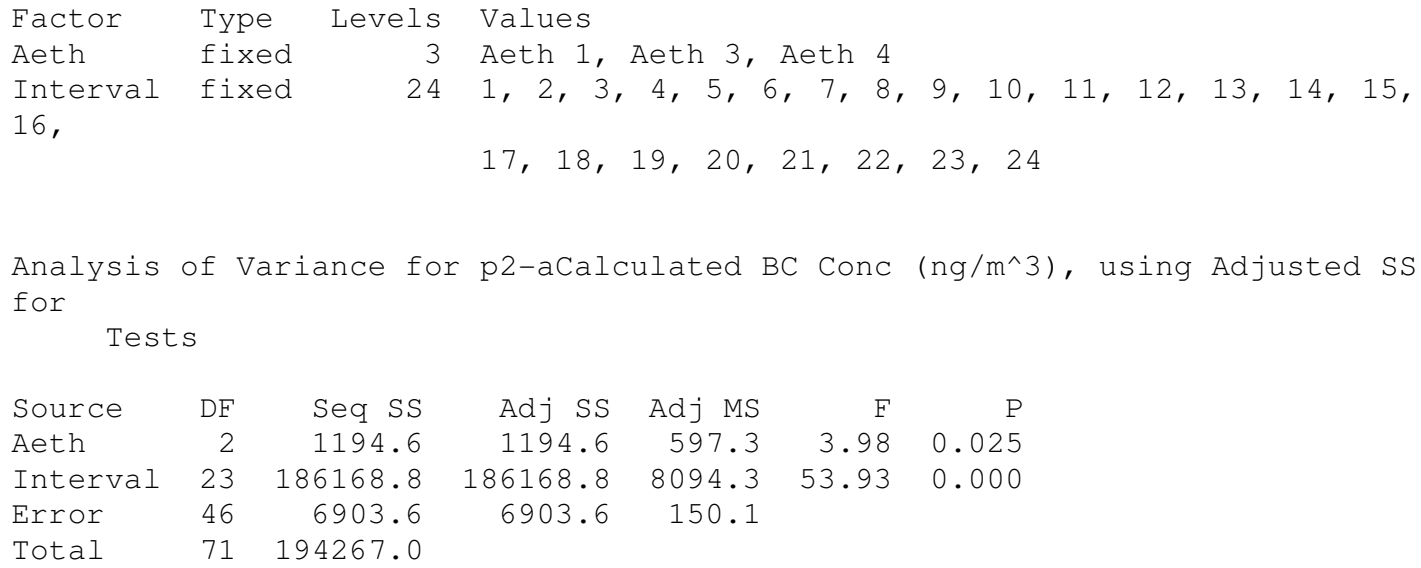

$\begin{array}{rrrr}\text { Adj SS } & \text { AdjMS } & F & P \\ 1194.6 & 597.3 & 3.98 & 0.025 \\ 186168.8 & 8094.3 & 53.93 & 0.000 \\ 6903.6 & 150.1 & & \end{array}$




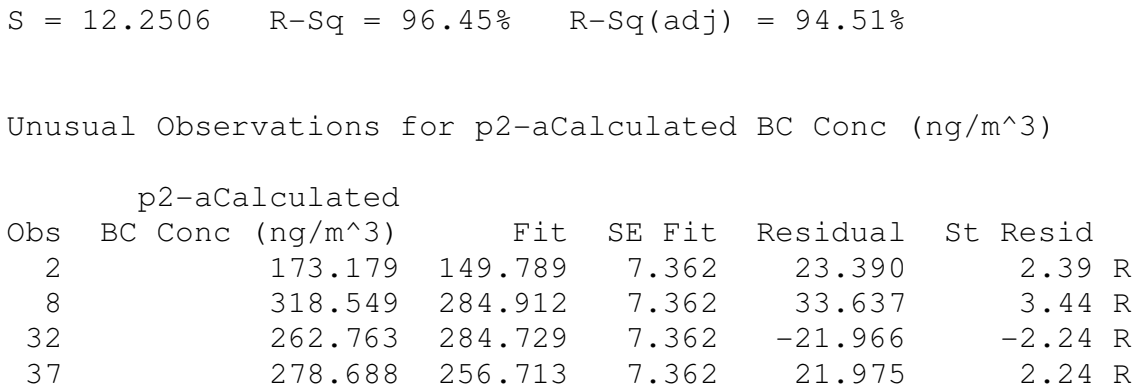

R denotes an observation with a large standardized residual.

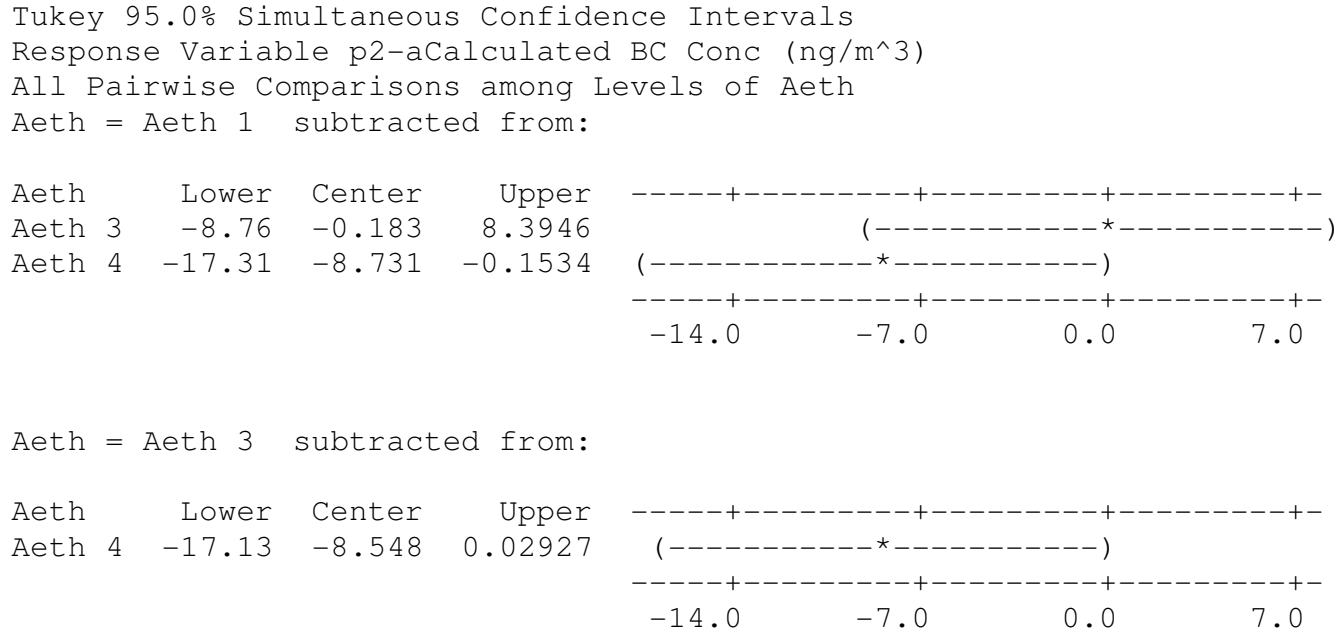

Residual Plots for p2-aCalculated BC Conc $\left(\mathrm{ng} / \mathrm{m}^{\wedge} 3\right)$ 


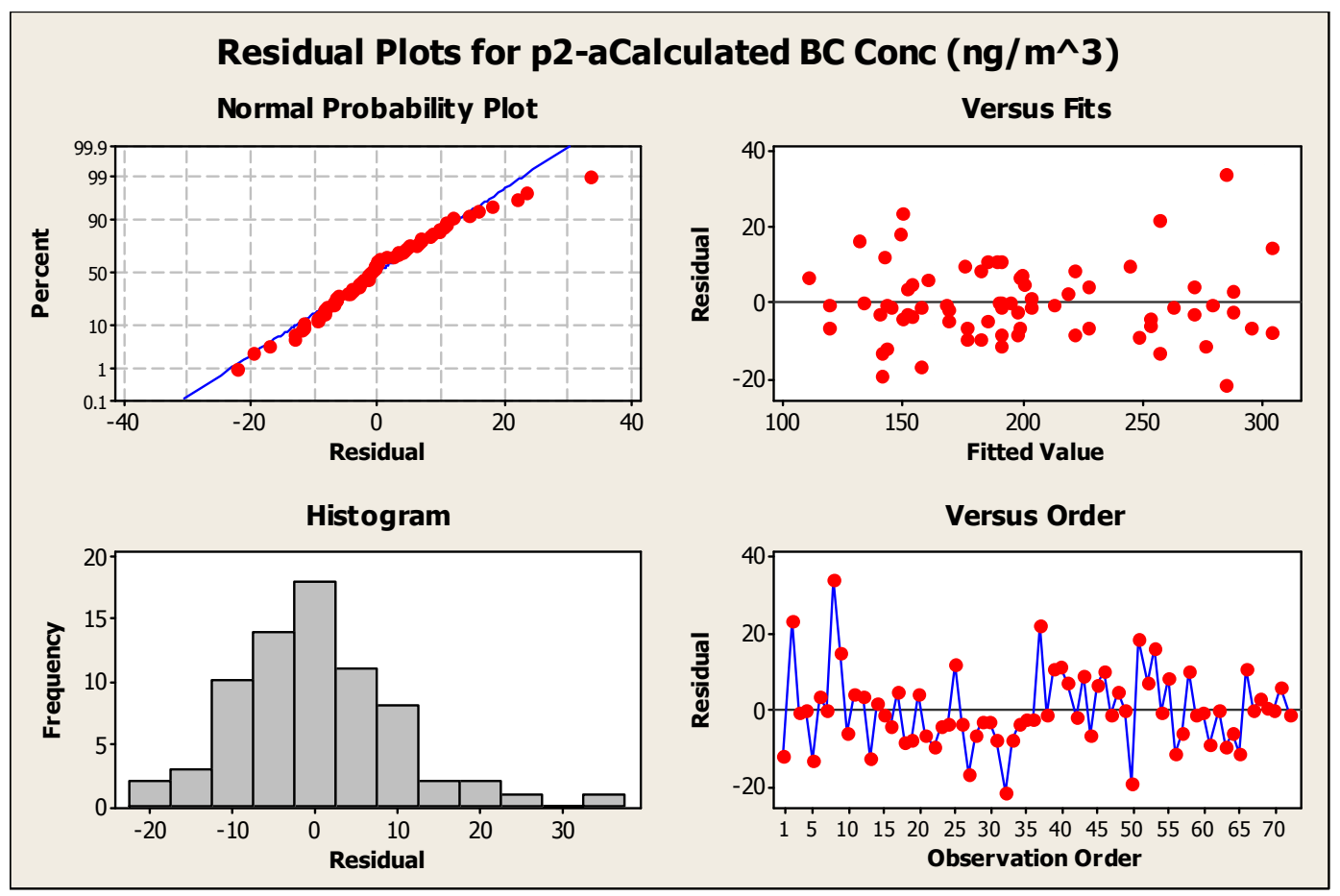

Graph of segment 2 of June 22-23, 2010 readings before adjusted $\sigma_{S G}$ to achieve a similar aethalometer data average:

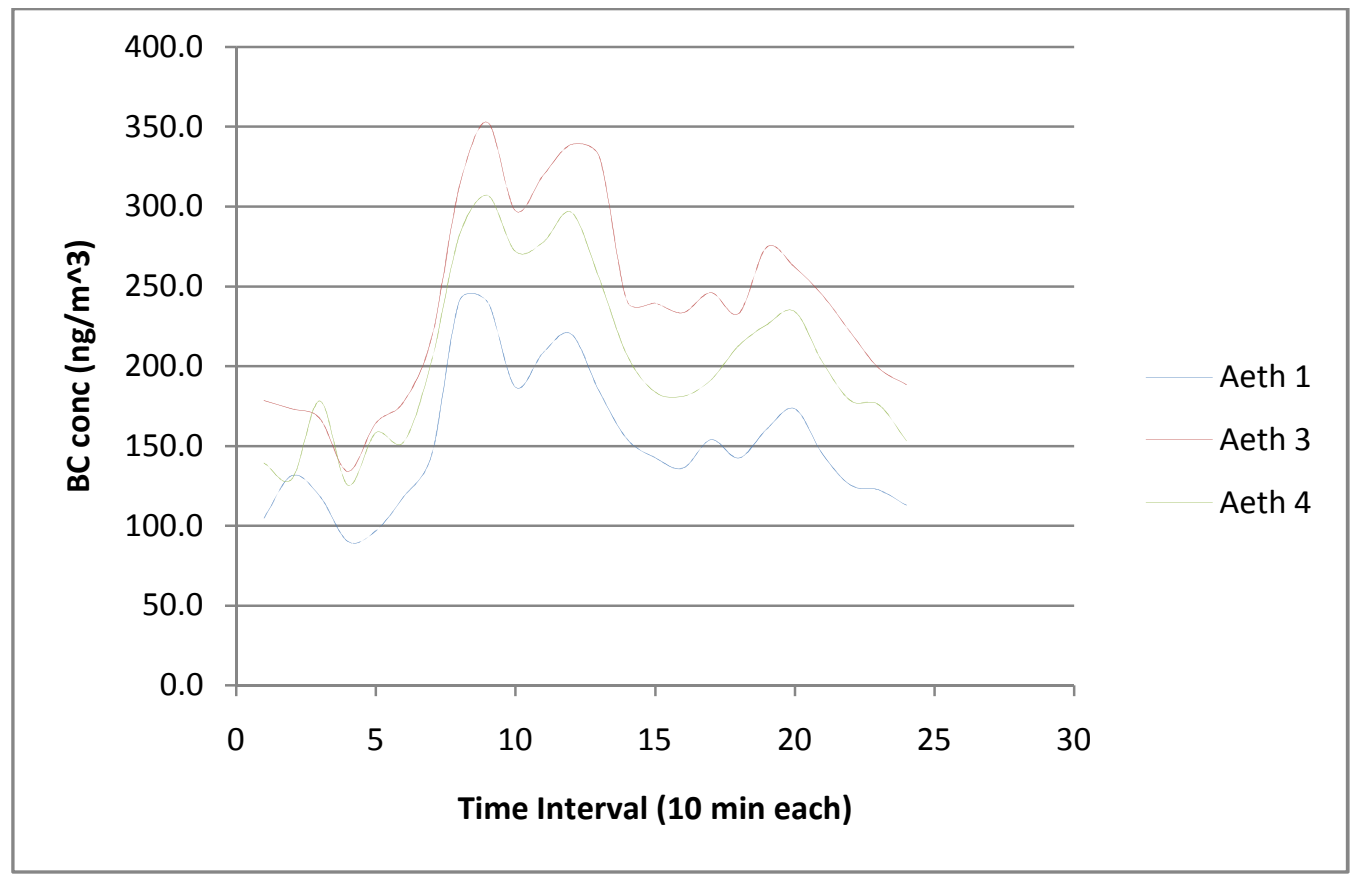


Graph of segment 2 of June 22-23, 2010 readings with adjusted $\sigma_{\mathrm{SG}}$ to achieve a similar aethalometer data average:

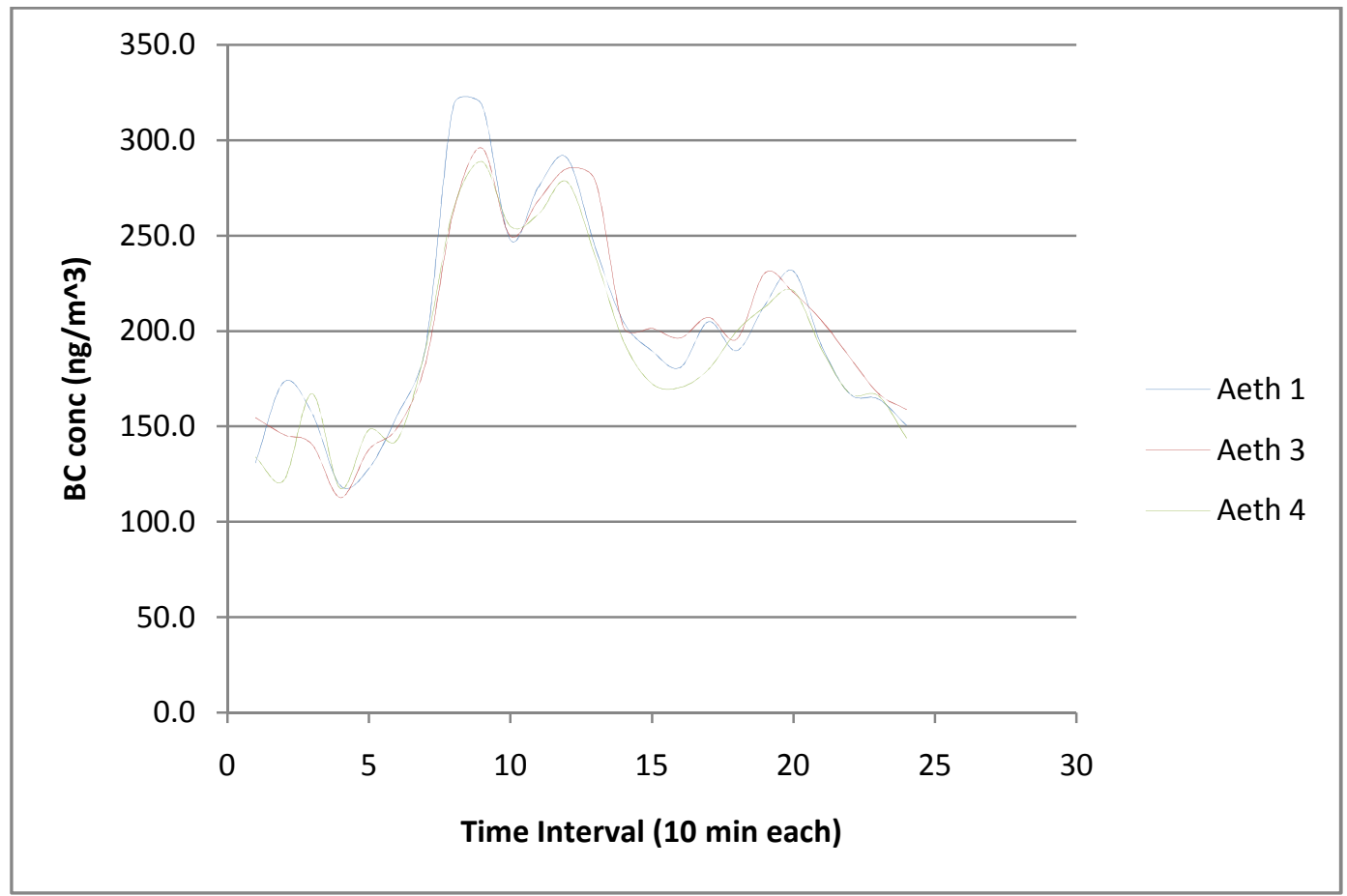

Summary of final $\sigma_{S G}$ values:

\begin{tabular}{|c|c|c|c|}
\hline \multirow{2}{*}{ Aeth } & \multicolumn{3}{|c|}{$\boldsymbol{\sigma}_{\mathbf{S G}}\left(\mathbf{m}^{\mathbf{2}} / \mathbf{g}\right)$} \\
\cline { 2 - 4 } & 19-Jan & 22-Jun & Average \\
\hline 1 & 19 & 18.31 & 18.66 \\
\hline 2 & 16.225 & - & 16.23 \\
\hline 3 & 23.035 & 19.98 & 21.51 \\
\hline 4 & 23 & 21.92 & 22.46 \\
\hline
\end{tabular}


Appendix B. Adsorption Ångström exponent (AAE) for all PEMs

\begin{tabular}{|c|c|c|c|c|c|c|c|c|c|c|c|c|c|}
\hline & \multicolumn{4}{|c|}{ AAE $700-350 \mathrm{~nm}$} & \multicolumn{9}{|c|}{ AAE $700-360 \mathrm{~nm}$} \\
\hline Location & $1 \mathrm{~A}$ & $2 A$ & $3 \mathrm{~A}$ & $4 \mathrm{~A}$ & 2B & 3B & 4B & $5 B$ & $6 B$ & 7B & 8B & $9 \mathrm{~B}$ & $10 \mathrm{~B}$ \\
\hline A & & & & & & 1.70 & 1.94 & 2.48 & 3.28 & 2.57 & 3.37 & 1.94 & 1.55 \\
\hline AW & & & & & & 2.36 & 2.18 & 1.39 & 1.75 & 1.39 & 1.70 & 2.74 & \\
\hline B1 & & & & & & 1.27 & 2.02 & 1.58 & 1.29 & 1.59 & 1.00 & 1.86 & \\
\hline B2 & & & & & & 1.59 & 1.98 & 1.42 & & 1.60 & 0.99 & 1.99 & 2.10 \\
\hline B3 & 1.77 & & 1.95 & 1.71 & & & 2.13 & 1.80 & 2.21 & & 0.95 & 2.19 & \\
\hline B4 & & & & & & 1.61 & 1.97 & & & 1.59 & 1.19 & 2.21 & \\
\hline B65 & 1.90 & 1.55 & & 1.44 & & & & & & & & & \\
\hline$B C$ & & & & & & 1.62 & 2.28 & 1.60 & 1.18 & 1.59 & 0.95 & 2.08 & 2.19 \\
\hline C & 2.13 & & 1.86 & 1.60 & & 2.23 & 2.78 & 1.65 & 1.47 & 1.53 & 0.88 & 1.99 & 2.08 \\
\hline $\mathrm{C} 2$ & & 2.23 & & 1.58 & & & & & & & & & \\
\hline cow & 1.85 & & & & & & & & & & & & \\
\hline $\mathrm{CP}$ & & & & & & & 1.95 & 1.22 & 1.36 & 1.41 & 0.94 & 2.00 & 2.20 \\
\hline CP1 & & & & & & & & & & & & & \\
\hline CPK & & 1.99 & & 1.94 & & & & & & & & & \\
\hline CS & & 2.08 & & 1.58 & & & & & & & & & \\
\hline $\mathrm{D}$ & & & & & & 1.36 & 2.31 & 2.78 & 2.62 & 1.12 & & & \\
\hline E1 & & & & & 2.13 & 1.93 & 2.49 & & & & & & \\
\hline E2 & & & 0.28 & 2.35 & & 2.35 & 1.88 & 1.24 & & 1.79 & 1.04 & 1.89 & 1.99 \\
\hline E44 & & 2.33 & & & & & & & & & & & \\
\hline EK & & & & & & & 2.58 & 1.34 & 1.53 & 1.89 & 0.92 & 1.88 & 2.18 \\
\hline FS & & & & & & & & 1.74 & 2.61 & 1.87 & 2.36 & 2.01 & 1.93 \\
\hline $\mathrm{K}$ & & & & & & & 2.16 & 1.47 & & 1.76 & 0.94 & 1.83 & 2.23 \\
\hline K64 & 1.85 & 1.59 & & & & & & & & & & & \\
\hline MO & 1.77 & 2.18 & 1.76 & 2.09 & & & & & & & & & \\
\hline S1 & & & & & 2.17 & 2.23 & 2.04 & 2.05 & 2.33 & 2.39 & & & \\
\hline W & & & & & & 2.71 & 2.52 & 1.76 & 2.84 & 1.60 & 2.31 & 1.84 & \\
\hline W35 & & 2.75 & & & & & & & & & & & \\
\hline W48 & 1.82 & 3.91 & 1.99 & 2.28 & & & & & & & & & \\
\hline W55 & 1.81 & 2.28 & 2.35 & 1.44 & & & & & & & & & \\
\hline WA1 & & & & & 2.07 & & & & & & & & \\
\hline WS & 1.71 & & & & & & & & & & & & \\
\hline
\end{tabular}




\section{Appendix C. Aethalometer BC Measurements over Time}

The figures below illustrate 30 minute averaged $\mathrm{BC}$ concentration over time during each IOP at each operating aethalometer location. Negative values have been assumed to be half of the lower detection limit (the average of 0 and the lower detection limit), 30.2 $\mathrm{ng} / \mathrm{m}^{3}$.

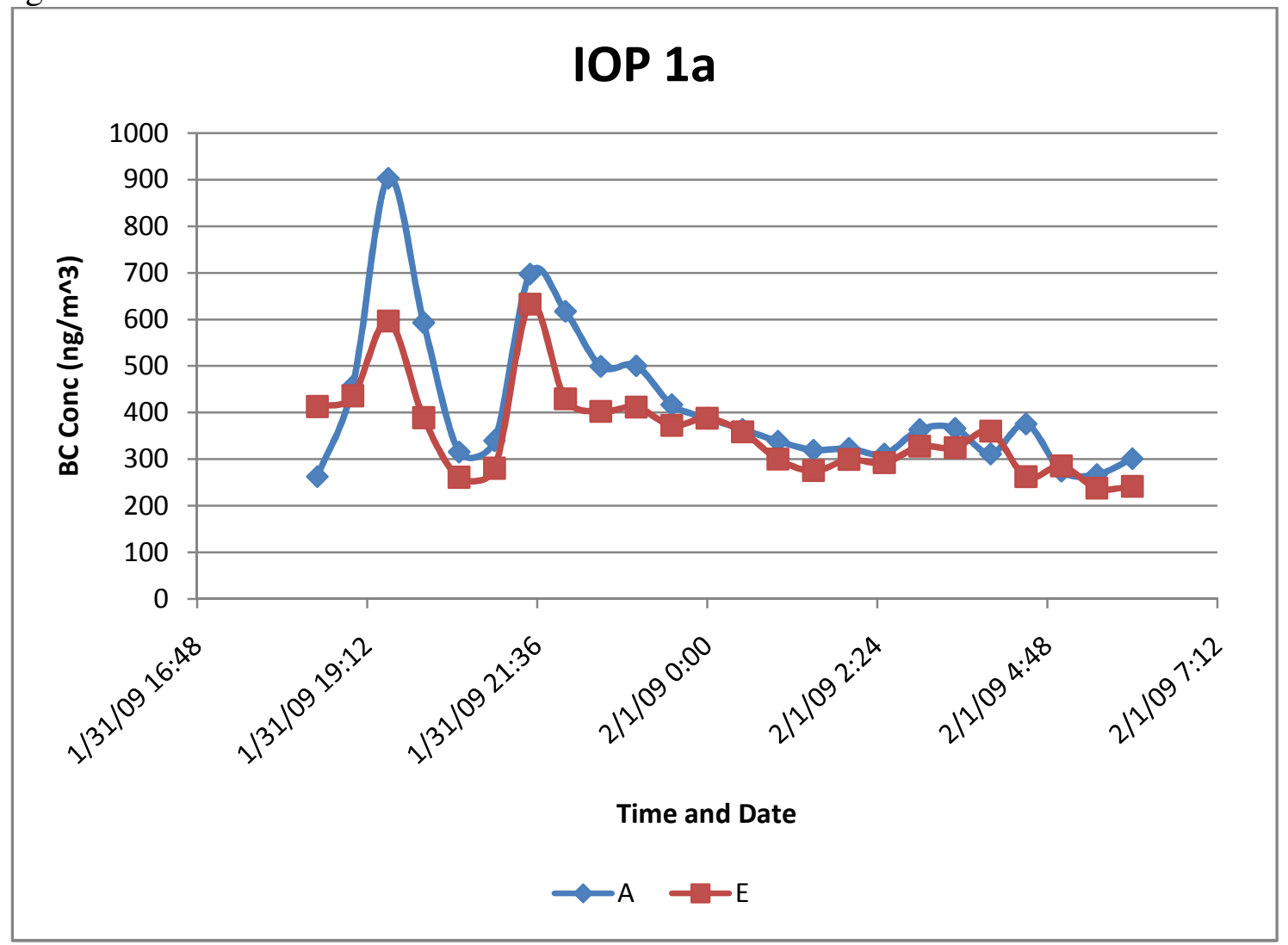




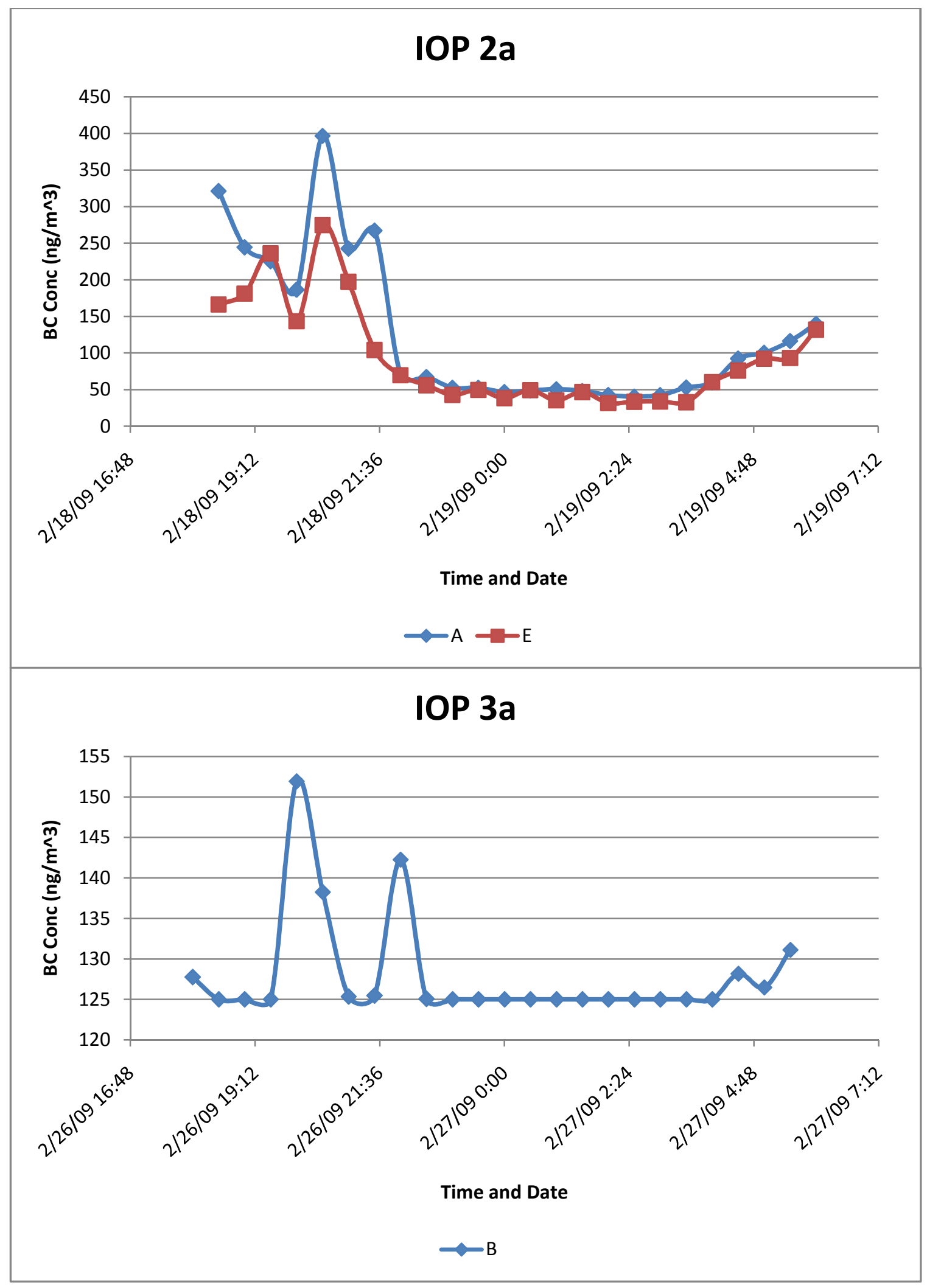




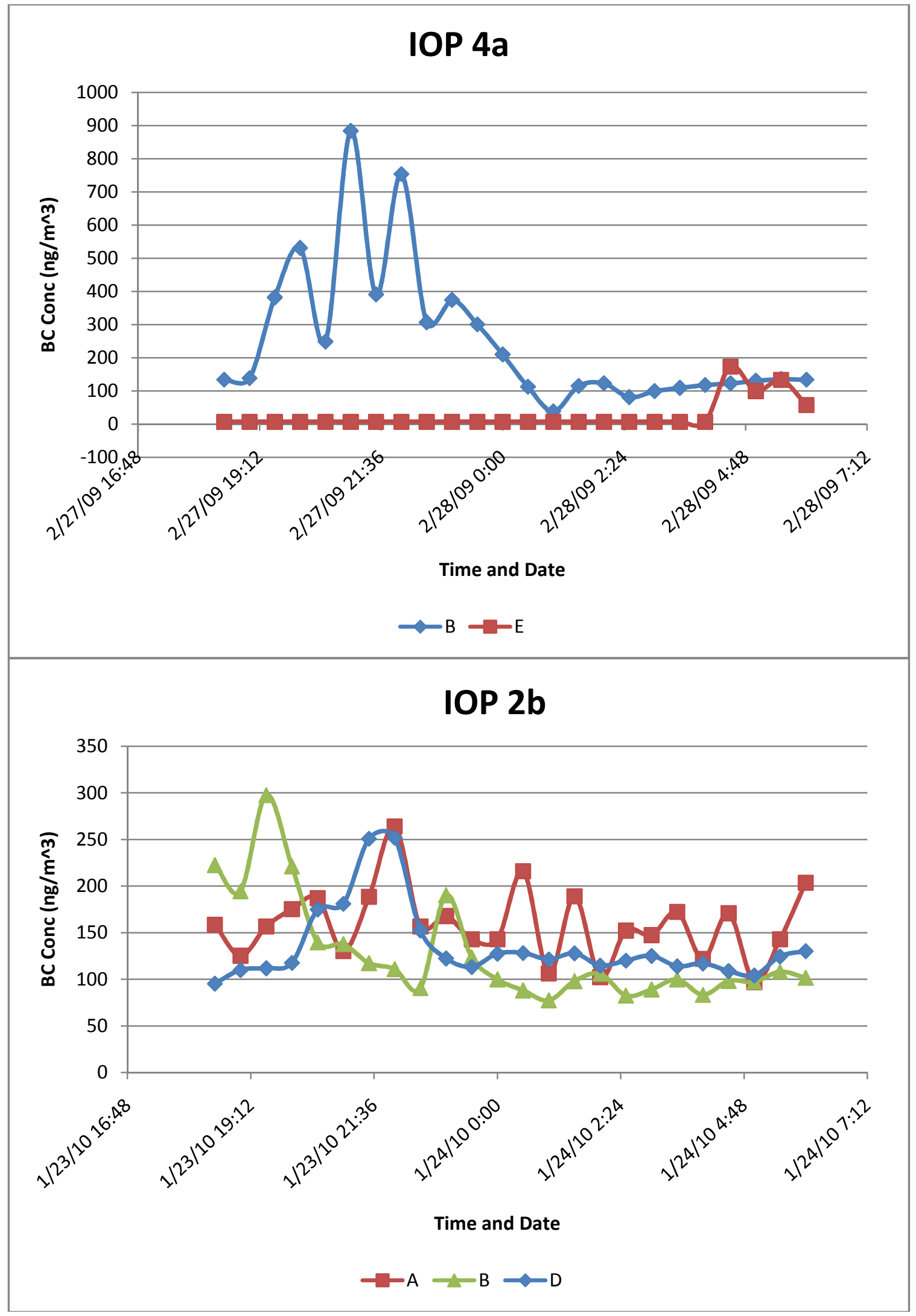




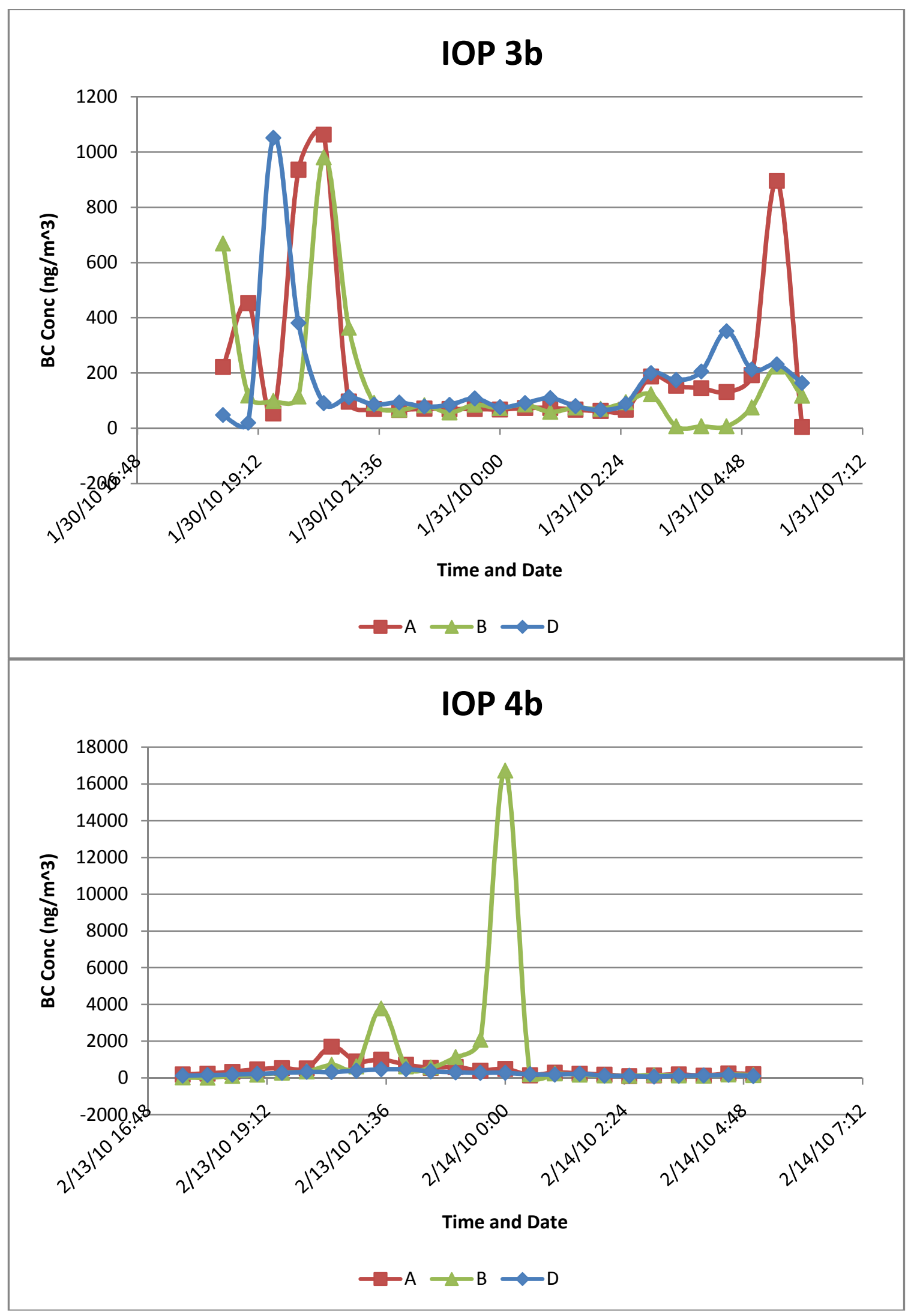




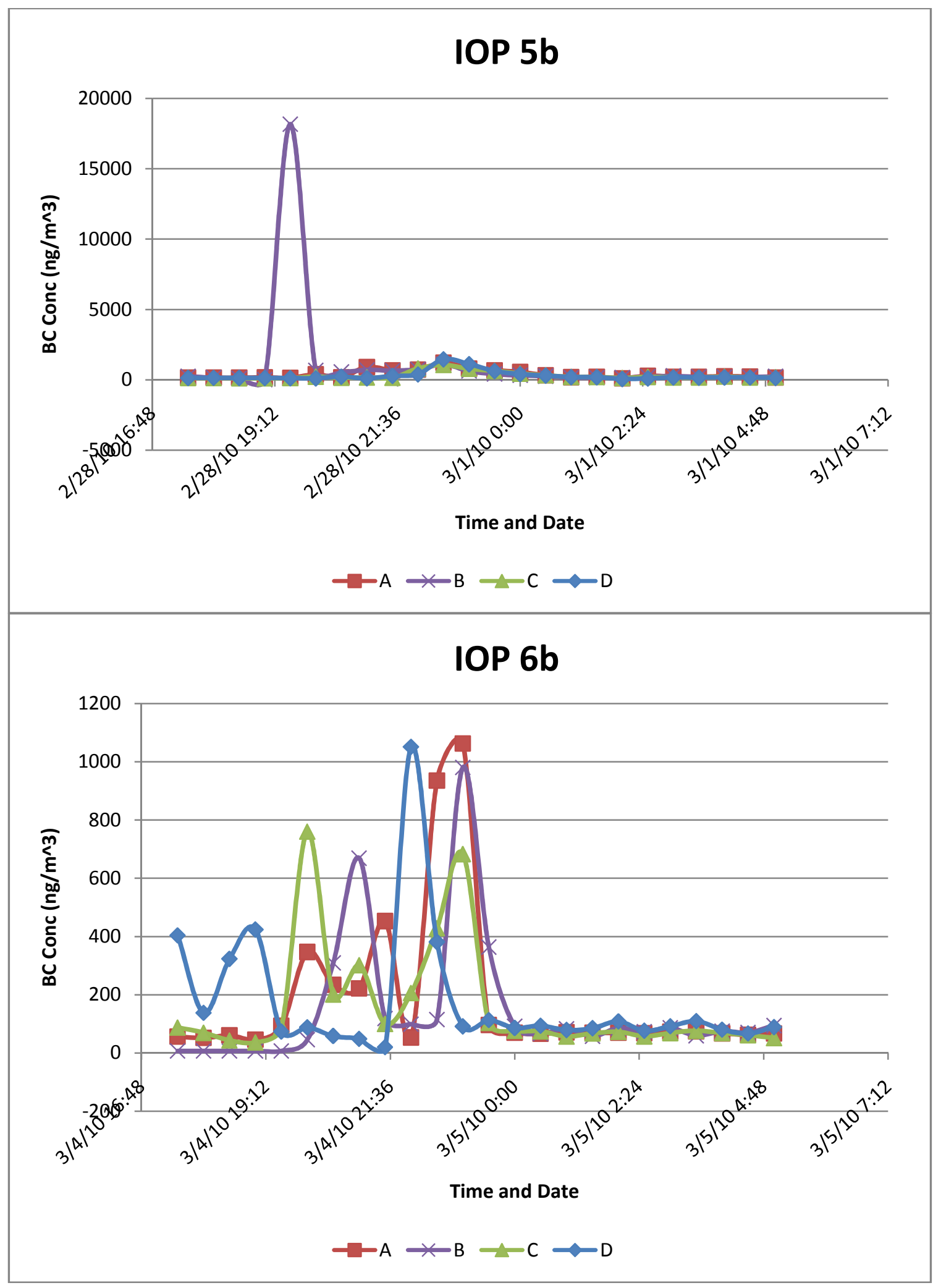




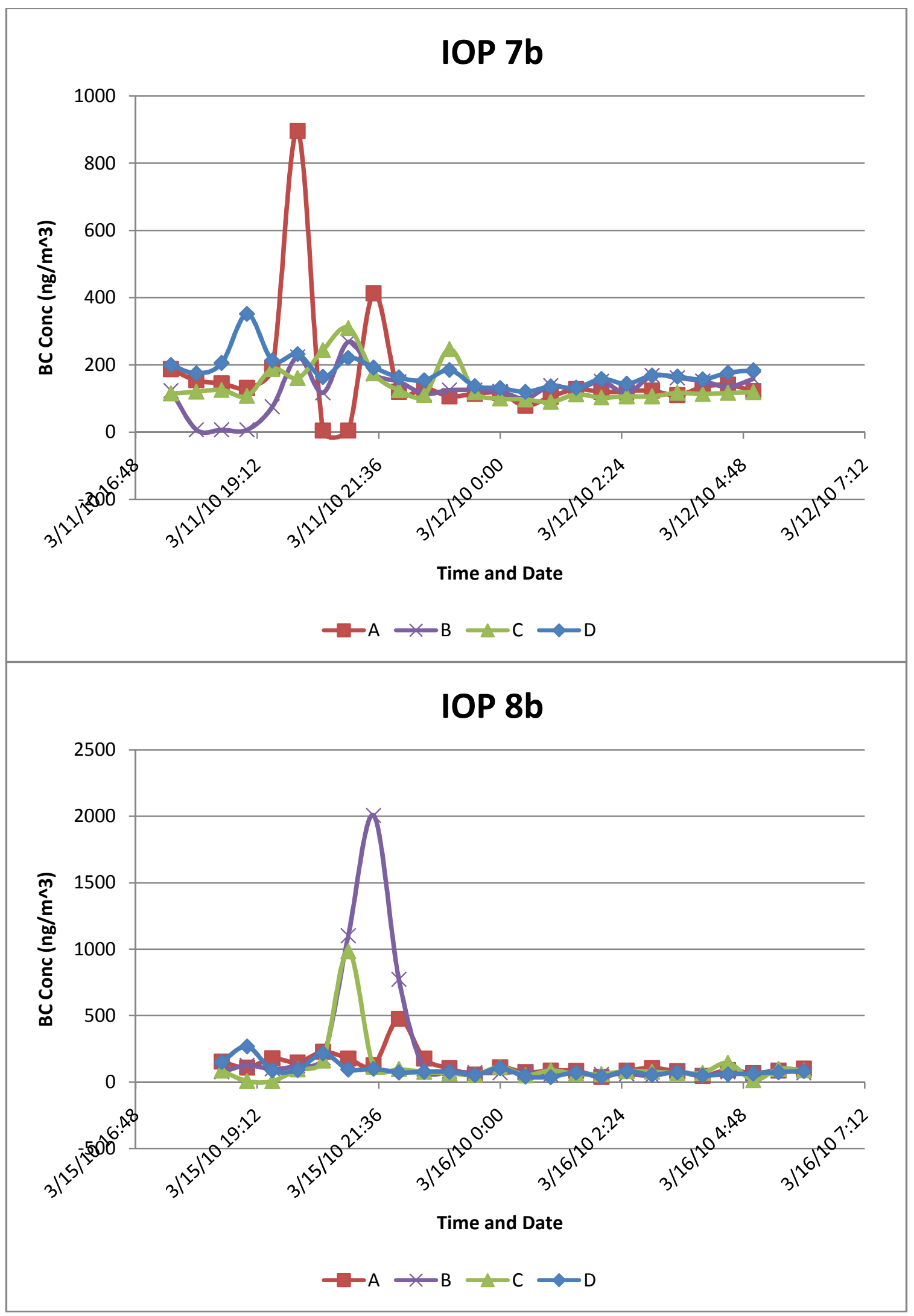




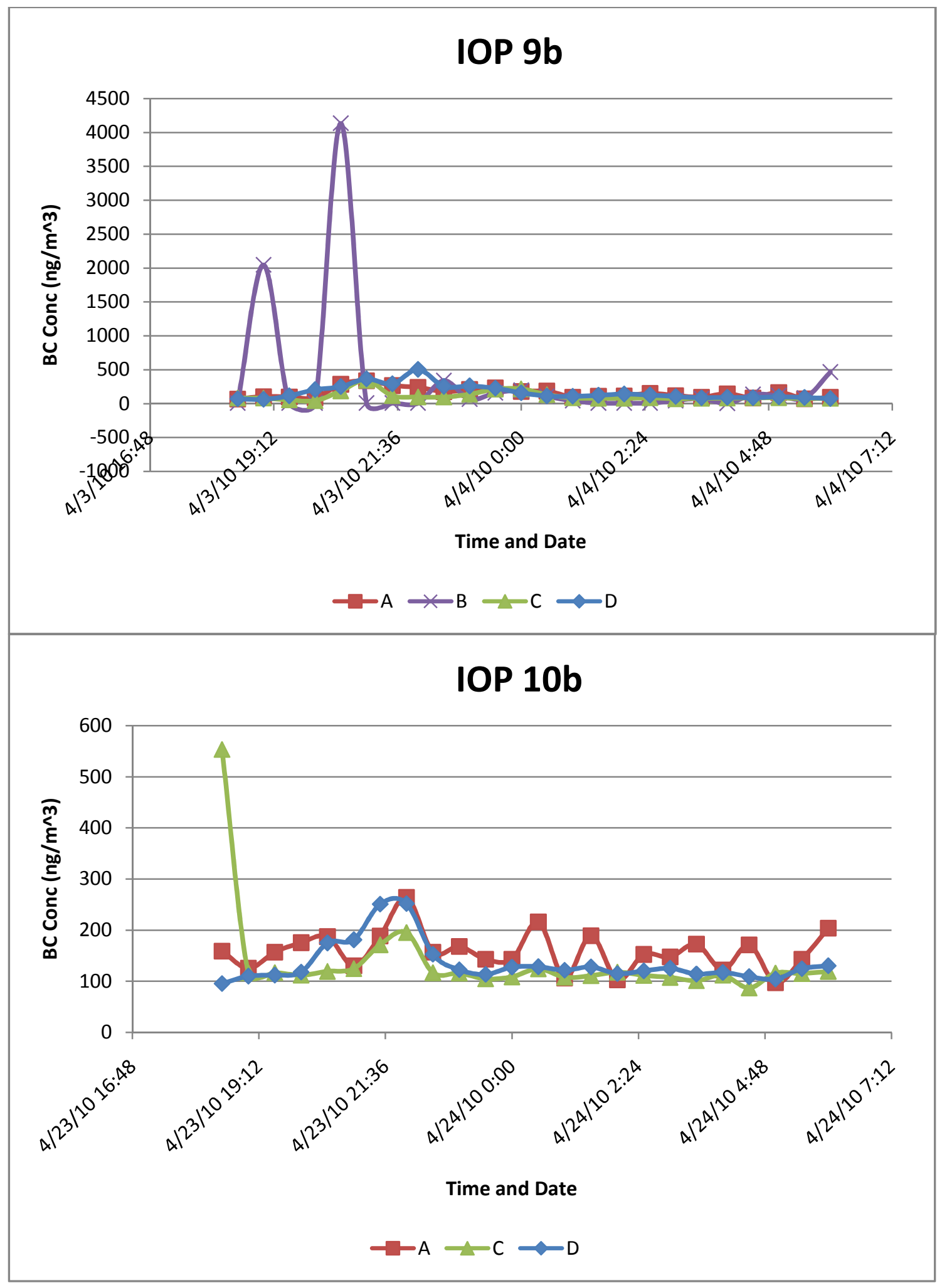




\section{Appendix D. Spatial Variability}

\section{Aethalometer Variability}

The first table below summarizes aethalometer variability for 30 minute averaged $\mathrm{BC}$ concentrations. BC concentrations are in nanograms per meters cubed. The second table details the minimum and maximum concentrations measured during each IOP.

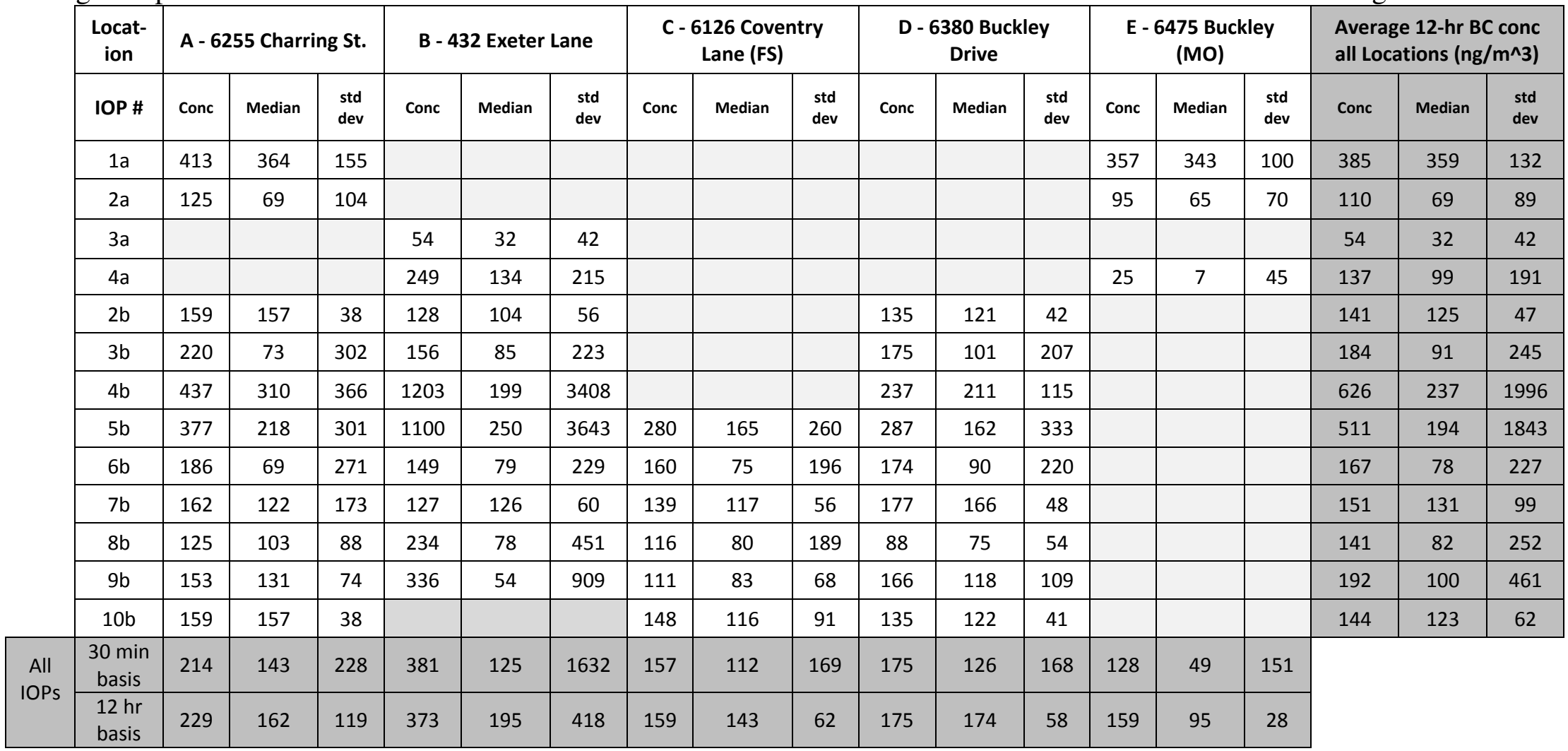

\begin{tabular}{|l|c|c|c|c|c|c|c|c|c|c|c|c|c|c|}
\hline \multicolumn{10}{|c|}{ 1 minute BC Concentration $\left(\boldsymbol{\mu g} / \mathbf{m}^{\mathbf{3}}\right)$} \\
\hline IOP & $1 \mathrm{a}$ & $2 \mathrm{a}$ & $3 \mathrm{a}$ & $4 \mathrm{a}$ & $2 \mathrm{~b}$ & $3 \mathrm{~b}$ & $4 \mathrm{~b}$ & $5 \mathrm{~b}$ & $6 \mathrm{~b}$ & $7 \mathrm{~b}$ & $8 \mathrm{~b}$ & $9 \mathrm{~b}$ & $10 \mathrm{~b}$ & All \\
\hline Max & 2 & 1 & 0.3 & 174 & 0.5 & 3 & 56 & 85 & 15 & 83 & 21 & 70 & 19 & $\mathbf{1 7 4}$ \\
\hline Min & 0.067 & 0.067 & 0.13 & 0.067 & 0.067 & 0.070 & 0.067 & 0.067 & 0.070 & 0.070 & 0.067 & 0.067 & 0.067 & $\mathbf{0 . 0 6 7}$ \\
\hline
\end{tabular}


PEM Variability

The table below summarizes PEM variability for $\mathrm{BC}$ concentrations. $\mathrm{BC}$ concentrations are in nanograms per meters cubed.

\begin{tabular}{|c|c|c|c|c|c|c|c|c|c|c|c|c|c|c|}
\hline \multirow[b]{2}{*}{ Label } & \multirow[b]{2}{*}{ Location } & \multicolumn{13}{|c|}{$12 \mathrm{hr}$ BC Conc (ng/m $\left.\mathrm{m}^{3}\right)$} \\
\hline & & $1 a$ & $2 a$ & $3 a$ & $4 a$ & $2 b$ & $3 \mathbf{b}$ & $4 b$ & $5 b$ & $6 b$ & $7 b$ & $8 b$ & $9 b$ & $10 \mathrm{~b}$ \\
\hline $\mathrm{MO}$ & $\mathrm{A}$ & 343 & 75 & 77 & 222 & & & & & & & & & \\
\hline B65 & $\mathrm{A}$ & 405 & 210 & 35 & 210 & & & & & & & & & \\
\hline W55 & $\mathrm{C}$ & 376 & 813 & 123 & 349 & & & & & & & & & \\
\hline WS & $\mathrm{C}$ & 313 & & & & & & & & & & & & \\
\hline K64 & A & 343 & & & & & & & & & & & & \\
\hline W48 & $\mathrm{C}$ & 434 & 47 & 115 & 744 & & & & & & & & & \\
\hline cow & C & 486 & & & & & & & & & & & & \\
\hline CS & $\mathrm{C}$ & & 156 & & 256 & & & & & & & & & \\
\hline CPK & A & & 86 & 48 & 90 & & & & & & & & & \\
\hline E44 & $\mathrm{B}$ & & 94 & & & & & & & & & & & \\
\hline W35 & $\mathrm{C}$ & & & 110 & 528 & & & & & & & & & \\
\hline $\mathrm{A}$ & $\mathrm{C}$ & & & & & & 174 & 268 & 286 & 370 & 270 & 561 & 119 & 203 \\
\hline AW & $\mathrm{C}$ & & & & & & 451 & 285 & 308 & 693 & 453 & 176 & 280 & 30 \\
\hline $\mathrm{B} 1$ & $\mathrm{~A}$ & & & & & 30 & 239 & & & & & 158 & 119 & 30 \\
\hline B2 & A & & & & & & 125 & 293 & 191 & 123 & 145 & 137 & 112 & 101 \\
\hline B3 & A & 329 & 32 & & 262 & 30 & 30 & 210 & 270 & 153 & 115 & 119 & 137 & 30 \\
\hline B4 & $A$ & & & & & & 137 & 222 & 273 & 30 & 136 & 277 & 92 & 30 \\
\hline$B C$ & $A$ & & & & & 30 & 202 & 197 & 216 & 413 & 146 & 139 & 100 & 83 \\
\hline$C$ & $B$ & 308 & 30 & 76 & 306 & 82 & 188 & 326 & 283 & 185 & 159 & 154 & 102 & 94 \\
\hline $\mathrm{C} 2$ & $B$ & & 82 & & 293 & & & & & & & & & \\
\hline $\mathrm{CP}$ & $A$ & & & & & & & 221 & 318 & & 188 & 130 & 110 & 81 \\
\hline CP1 & $\mathrm{A}$ & & & & & 30 & 30 & & & & & & & \\
\hline $\mathrm{D}$ & $C$ & & & & & & 542 & 374 & 294 & 339 & 1051 & & & \\
\hline E1 & B & & & & & & 193 & 281 & & & & & & \\
\hline E2 & $B$ & & & & 128 & 85 & 237 & 1048 & 2787 & 30 & 125 & 156 & 120 & 92 \\
\hline EK & $A$ & & & & & 30 & 30 & 198 & 265 & 81 & 101 & 120 & 133 & 94 \\
\hline $\mathrm{K}$ & A & & & & & 30 & 30 & 189 & 194 & 59 & 115 & 149 & 148 & 88 \\
\hline S1 & C & & & & & 309 & 525 & 448 & 416 & 247 & 386 & 229 & 192 & 117 \\
\hline W & $C$ & & 187 & & & 78 & 116 & 396 & 252 & 192 & 503 & 142 & 169 & 30 \\
\hline FS & $C$ & & & & & & & & 263 & 164 & 184 & 162 & 105 & 104 \\
\hline WA1 & C & & & & & 163 & & & & & & & & \\
\hline
\end{tabular}




\section{Meteorological Variability}

The tables below summarize meteorological variability for each meteorological station.

\begin{tabular}{|c|c|c|c|c|c|c|c|c|c|c|c|c|c|c|c|c|c|c|c|}
\hline \multirow{3}{*}{$\begin{array}{l}\text { Location } \\
\text { IOP } \\
\end{array}$} & \multicolumn{11}{|c|}{ Marine Terrace ( $10 \mathrm{~min}$ basis) } & \multicolumn{8}{|c|}{ LH CSDF (30 min basis) } \\
\hline & \multicolumn{3}{|c|}{ Wind speed (mph) } & \multicolumn{2}{|c|}{ Wind Direction } & \multicolumn{3}{|c|}{ Temperature (F) } & \multicolumn{3}{|c|}{ Humidity (\%) } & \multicolumn{3}{|c|}{ Wind speed (mph) } & \multicolumn{2}{|c|}{ Wind Direction } & \multicolumn{3}{|c|}{ Humidity (\%) } \\
\hline & Average & Median & Std Dev & Average & Std Dev & Average & Median & Std Dev & Average & Median & Std Dev & Average & Median & Std Dev & Average & Std Dev & Average & Median & Std Dev \\
\hline $1 a$ & 2 & 2 & 1 & 214 & 44 & 51 & 51 & 2 & 77 & 77 & 3 & 2 & 1 & 2 & 250 & 51 & 85 & 86 & 8 \\
\hline $2 a$ & 4 & 5 & 1 & 298 & 45 & 46 & 46 & 3 & 75 & 77 & 6 & 2 & 2 & 1 & 125 & 40 & 88 & 90 & 7 \\
\hline $3 a$ & 5 & 4 & 3 & 339 & 42 & 51 & 52 & 4 & 84 & 85 & 3 & 2 & 2 & 2 & 117 & 47 & 97 & 98 & 1 \\
\hline $4 a$ & 3 & 2 & 1 & 212 & 45 & 52 & 51 & 2 & 71 & 71 & 3 & 1 & 1 & 2 & 347 & 56 & 78 & 78 & 7 \\
\hline $2 b$ & 5 & 5 & 1 & -63 & 42 & 43 & 42 & 3 & 81 & 83 & 4 & 2 & 3 & 1 & 242 & 42 & 95 & 96 & 3 \\
\hline $3 b$ & 3 & 4 & 1 & 137 & 44 & 46 & 45 & 2 & 78 & 79 & 2 & 1 & 1 & 1 & 301 & 46 & 92 & 91 & 4 \\
\hline $4 b$ & 3 & 2 & 2 & 137 & 42 & 49 & 49 & 2 & 83 & 83 & 2 & 1 & 1 & 1 & 288 & 56 & 96 & 97 & 3 \\
\hline $5 b$ & 3 & 3 & 2 & 92 & 42 & 50 & 49 & 2 & 80 & 81 & 3 & 2 & 2 & 2 & 12 & 46 & 96 & 97 & 2 \\
\hline $6 \mathrm{~b}$ & 6 & 5 & 2 & 84 & 46 & 44 & 43 & 4 & 75 & 77 & 5 & 2 & 2 & 3 & 338 & 53 & 88 & 89 & 4 \\
\hline $7 \mathrm{~b}$ & 3 & 3 & 1 & 242 & 45 & 46 & 46 & 3 & 65 & 65 & 3 & 2 & 1 & 2 & 163 & 46 & 82 & 83 & 7 \\
\hline $8 \mathrm{~b}$ & 4 & 4 & 1 & -57 & 47 & 53 & 51 & 5 & 40 & 44 & 7 & 3 & 4 & 2 & 280 & 46 & 54 & 60 & 13 \\
\hline $9 b$ & 6 & 5 & 3 & -42 & 49 & 44 & 45 & 4 & 75 & 74 & 5 & 3 & 2 & 4 & 232 & 53 & 91 & 93 & 7 \\
\hline $10 \mathrm{~b}$ & 3 & 3 & 2 & 249 & 48 & 51 & 51 & 2 & 76 & 77 & 4 & 2 & 1 & 2 & 92 & 49 & 94 & 95 & 3 \\
\hline All IOPs & 4 & 4 & 2 & 264 & 45 & 48 & 48 & 4 & 74 & 77 & 12 & 2 & 2 & 2 & 292 & 47 & 87 & 93 & 14 \\
\hline
\end{tabular}




\begin{tabular}{|c|c|c|c|c|c|c|c|c|c|c|c|c|c|c|c|c|c|c|c|}
\hline \multirow{3}{*}{$\begin{array}{l}\text { Location } \\
\text { IOP } \\
\end{array}$} & \multicolumn{11}{|c|}{ EBAM (10 min basis) } & \multicolumn{8}{|c|}{ Sonic ( $10 \mathrm{~min}$ basis) } \\
\hline & \multicolumn{3}{|c|}{ Wind speed (mph) } & \multicolumn{2}{|c|}{ Wind Direction } & \multicolumn{3}{|c|}{ Temperature (F) } & \multicolumn{3}{|c|}{ Humidity (\%) } & \multicolumn{3}{|c|}{ Wind speed (mph) } & \multicolumn{2}{|c|}{ Wind Direction } & \multicolumn{3}{|c|}{ Temperature (F) } \\
\hline & Average & Median & Std Dev & Average & Std Dev & Average & Median & Std Dev & Average & Median & Std Dev & Average & Median & Std Dev & Average & Std Dev & Average & Median & Std Dev \\
\hline 1a & & & & & & & & & & & & & & & & & & & \\
\hline $2 a$ & & & & & & & & & & & & & & & & & & & \\
\hline 3а & & & & & & & & & & & & & & & & & & & \\
\hline $4 a$ & & & & & & & & & & & & & & & & & & & \\
\hline $2 \mathrm{~b}$ & & & & & & & & & & & & & & & & & & & \\
\hline $3 b$ & & & & & & & & & & & & & & & & & & & \\
\hline $4 \mathrm{~b}$ & 0.8 & 0.7 & 0.2 & 68 & 44 & 49 & 49 & 3 & 87 & 88 & 4 & & & & & & & & \\
\hline $5 b$ & 1.0 & 0.9 & 0.4 & -84 & 44 & 51 & 50 & 2 & 86 & 87 & 3 & & & & & & & & \\
\hline $6 b$ & 0.9 & 0.7 & 0.2 & 158 & 48 & 46 & 44 & 4 & 79 & 82 & 6 & & & & & & & & \\
\hline $7 \mathrm{~b}$ & 1.0 & 0.9 & 0.4 & 110 & 46 & 47 & 46 & 3 & 71 & 71 & 5 & & & & & & & & \\
\hline $8 \mathrm{~b}$ & 1.0 & 0.9 & 0.4 & -66 & 42 & 54 & 53 & 3 & 43 & 44 & 4 & 0.6 & 0.6 & 0.2 & 138 & 43 & 54 & 52 & 4 \\
\hline $9 b$ & 1.5 & 1.6 & 0.5 & 243 & 44 & 44 & 42 & 3 & 81 & 82 & 4 & 0.6 & 0.5 & 0.3 & 89 & 70 & 44 & 42 & 4 \\
\hline $10 \mathrm{~b}$ & 0.9 & 0.7 & 0.4 & -63 & 44 & 52 & 51 & 1 & 83 & 84 & 4 & 0.6 & 0.6 & 0.3 & 90 & 74 & 52 & 51 & 4 \\
\hline All IOPs & 1.0 & 0.9 & 0.4 & 257 & 45 & 49 & 49 & 4 & 76 & 82 & 15 & 0.6 & 0.5 & 0.3 & 90 & 78 & 50 & 51 & 6 \\
\hline
\end{tabular}




\begin{tabular}{|c|c|c|c|c|c|c|c|c|c|c|c|c|c|c|c|c|c|c|c|}
\hline \multirow{3}{*}{$\begin{array}{l}\text { Location } \\
\text { IOP } \\
\end{array}$} & \multicolumn{11}{|c|}{ EBAM (10 min basis) } & \multicolumn{8}{|c|}{ Sonic ( $10 \mathrm{~min}$ basis) } \\
\hline & \multicolumn{3}{|c|}{ Wind speed (mph) } & \multicolumn{2}{|c|}{ Wind Direction } & \multicolumn{3}{|c|}{ Temperature (F) } & \multicolumn{3}{|c|}{ Humidity (\%) } & \multicolumn{3}{|c|}{ Wind speed (mph) } & \multicolumn{2}{|c|}{ Wind Direction } & \multicolumn{3}{|c|}{ Temperature (F) } \\
\hline & Average & Median & Std Dev & Average & Std Dev & Average & Median & Std Dev & Average & Median & Std Dev & Average & Median & Std Dev & Average & Std Dev & Average & Median & Std Dev \\
\hline 1a & & & & & & & & & & & & & & & & & & & \\
\hline $2 a$ & & & & & & & & & & & & & & & & & & & \\
\hline 3а & & & & & & & & & & & & & & & & & & & \\
\hline $4 a$ & & & & & & & & & & & & & & & & & & & \\
\hline $2 \mathrm{~b}$ & & & & & & & & & & & & & & & & & & & \\
\hline $3 b$ & & & & & & & & & & & & & & & & & & & \\
\hline $4 \mathrm{~b}$ & 0.8 & 0.7 & 0.2 & 68 & 44 & 49 & 49 & 3 & 87 & 88 & 4 & & & & & & & & \\
\hline $5 b$ & 1.0 & 0.9 & 0.4 & -84 & 44 & 51 & 50 & 2 & 86 & 87 & 3 & & & & & & & & \\
\hline $6 b$ & 0.9 & 0.7 & 0.2 & 158 & 48 & 46 & 44 & 4 & 79 & 82 & 6 & & & & & & & & \\
\hline $7 \mathrm{~b}$ & 1.0 & 0.9 & 0.4 & 110 & 46 & 47 & 46 & 3 & 71 & 71 & 5 & & & & & & & & \\
\hline $8 \mathrm{~b}$ & 1.0 & 0.9 & 0.4 & -66 & 42 & 54 & 53 & 3 & 43 & 44 & 4 & 0.6 & 0.6 & 0.2 & 138 & 43 & 54 & 52 & 4 \\
\hline $9 b$ & 1.5 & 1.6 & 0.5 & 243 & 44 & 44 & 42 & 3 & 81 & 82 & 4 & 0.6 & 0.5 & 0.3 & 89 & 70 & 44 & 42 & 4 \\
\hline $10 \mathrm{~b}$ & 0.9 & 0.7 & 0.4 & -63 & 44 & 52 & 51 & 1 & 83 & 84 & 4 & 0.6 & 0.6 & 0.3 & 90 & 74 & 52 & 51 & 4 \\
\hline All IOPs & 1.0 & 0.9 & 0.4 & 257 & 45 & 49 & 49 & 4 & 76 & 82 & 15 & 0.6 & 0.5 & 0.3 & 90 & 78 & 50 & 51 & 6 \\
\hline
\end{tabular}




\section{Appendix E. Summary of IOP Wind Speeds and Direction}

Wind Speed Comparisons for All Stations

The graphs below detail wind speed over time during each sampling period at each meteorological station and combination used for analysis. The wind speeds are presented here (as opposed to another meteorological variable) to offer a comparison of a meteorological characteristic that often has a large impact on pollutant dispersion and that can vary largely in small areas.

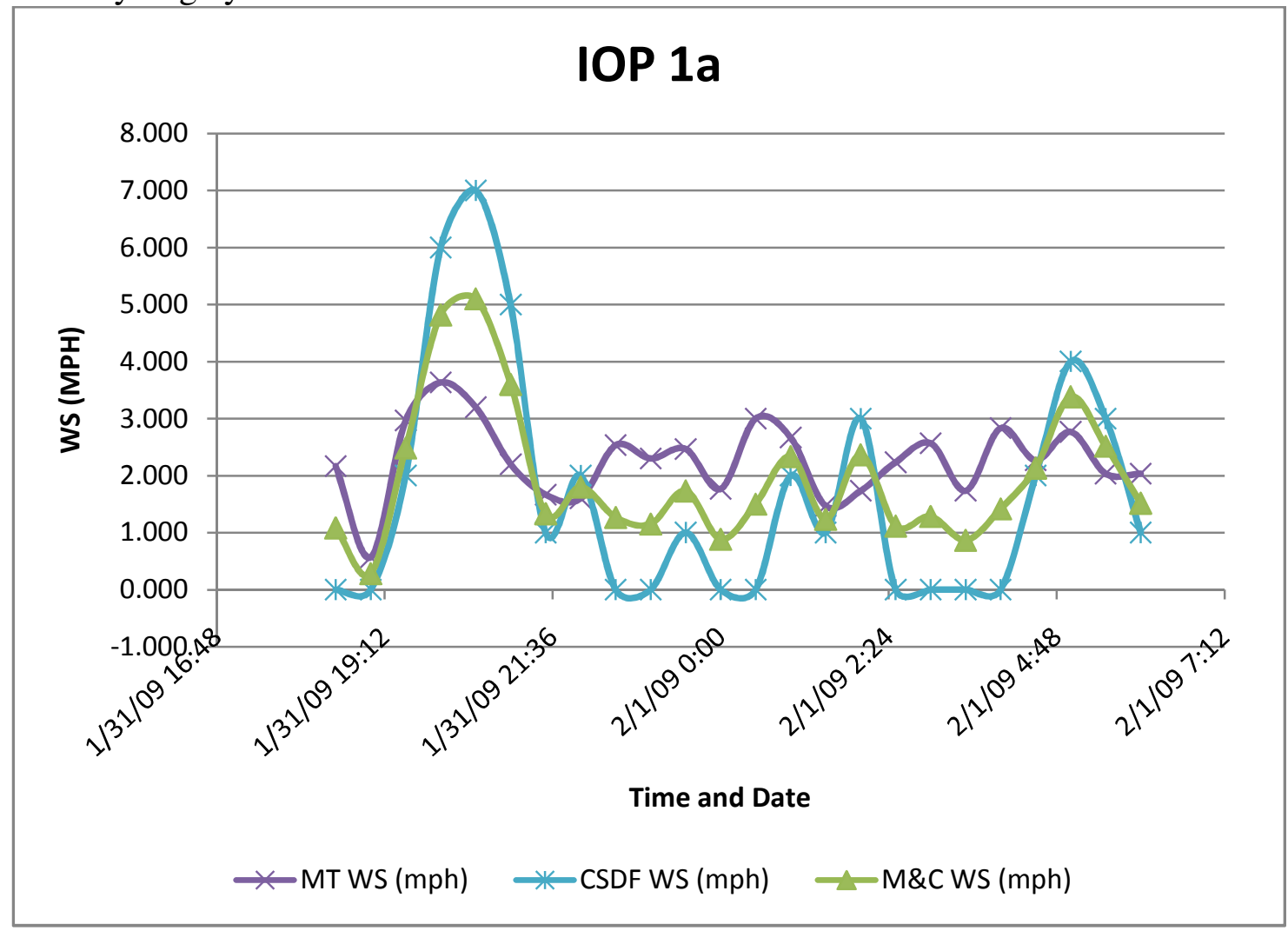




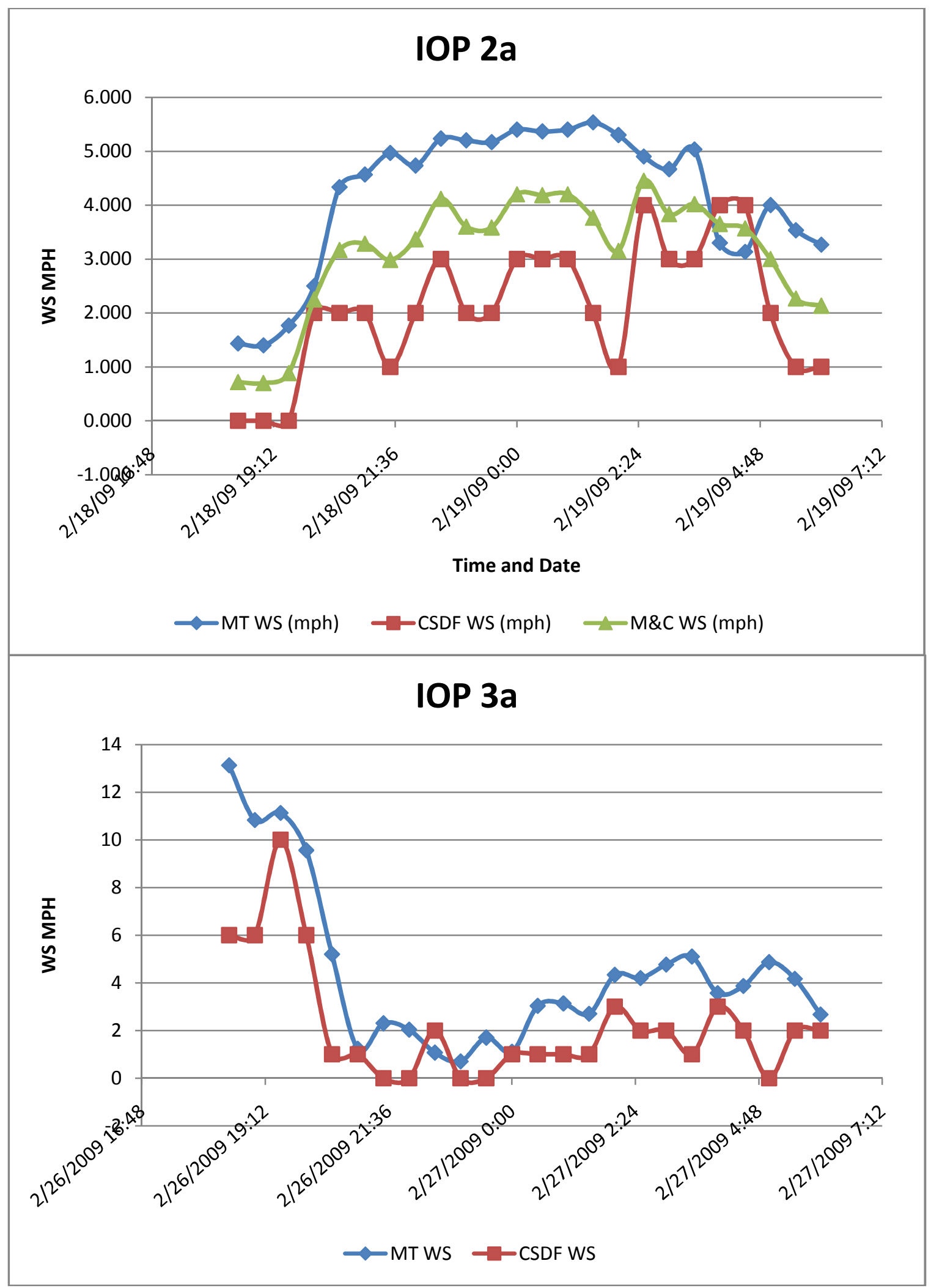




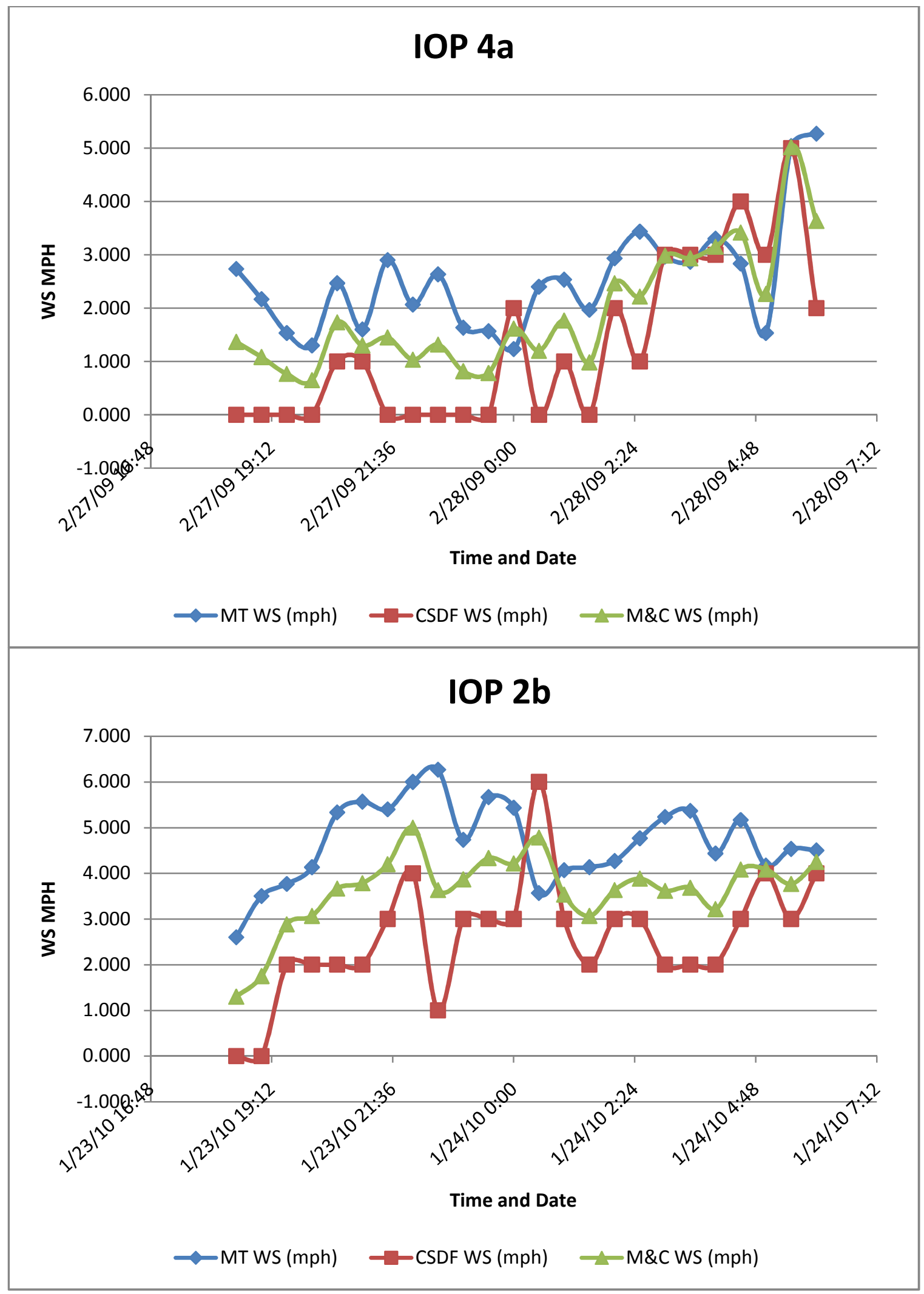




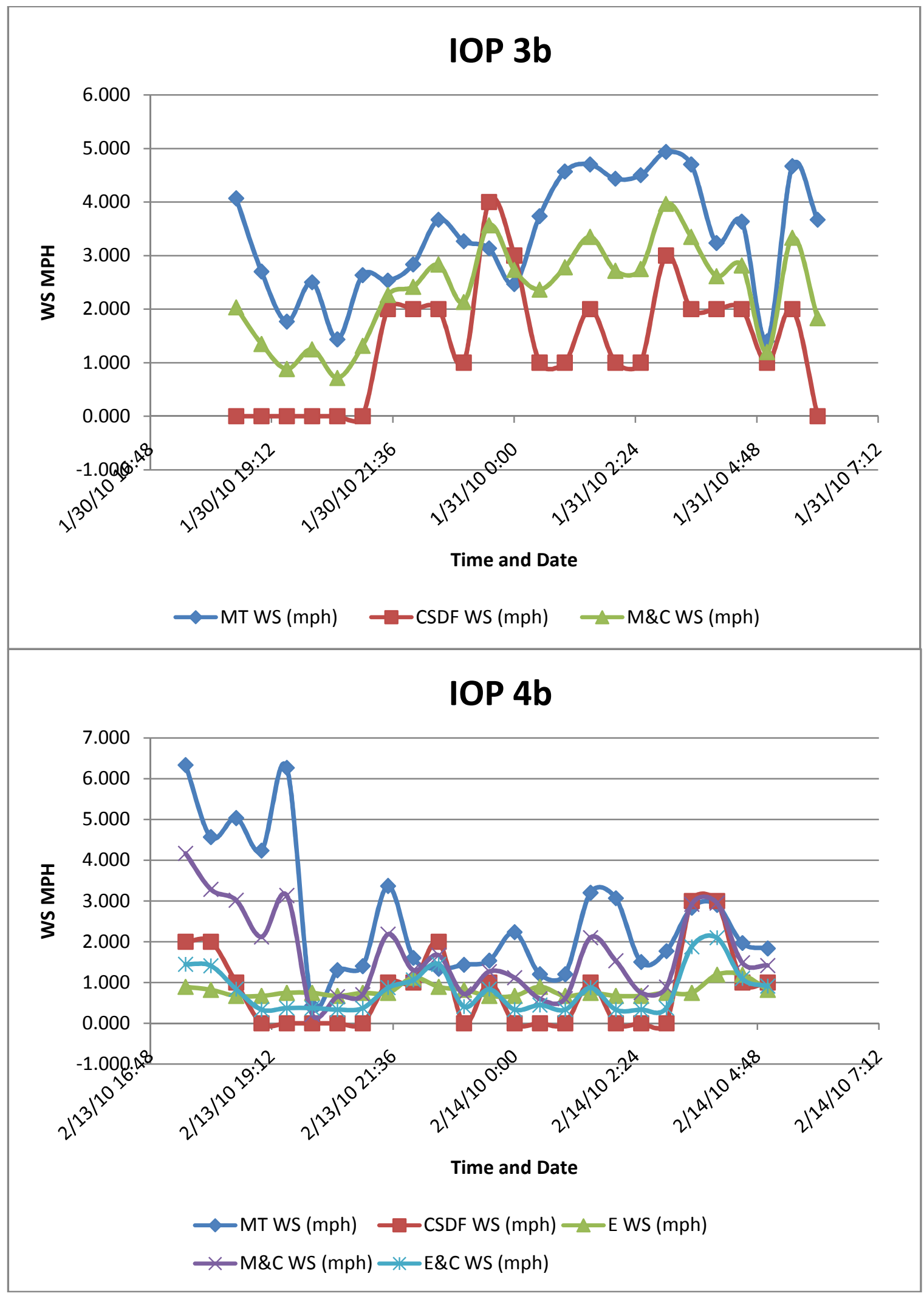




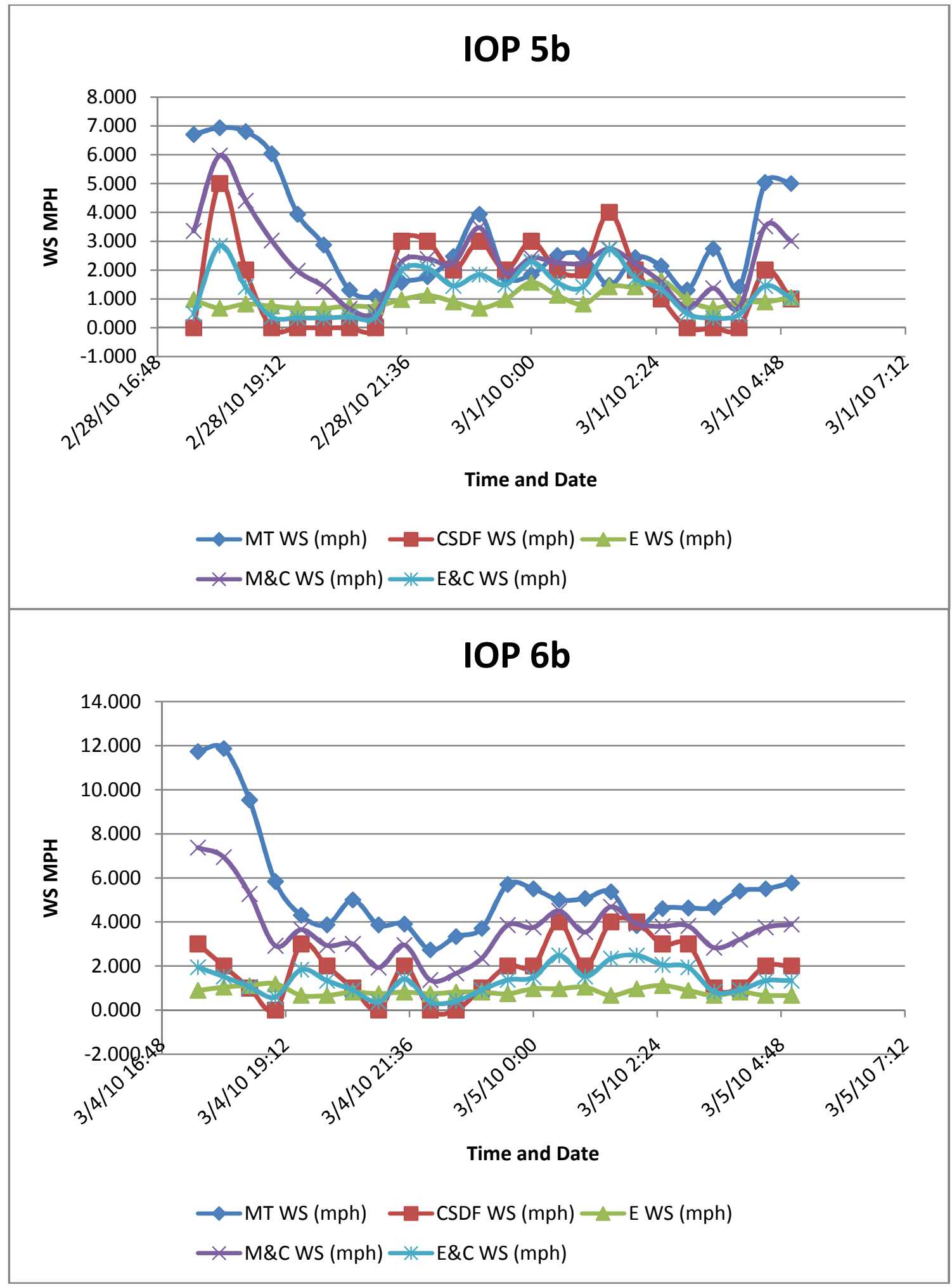




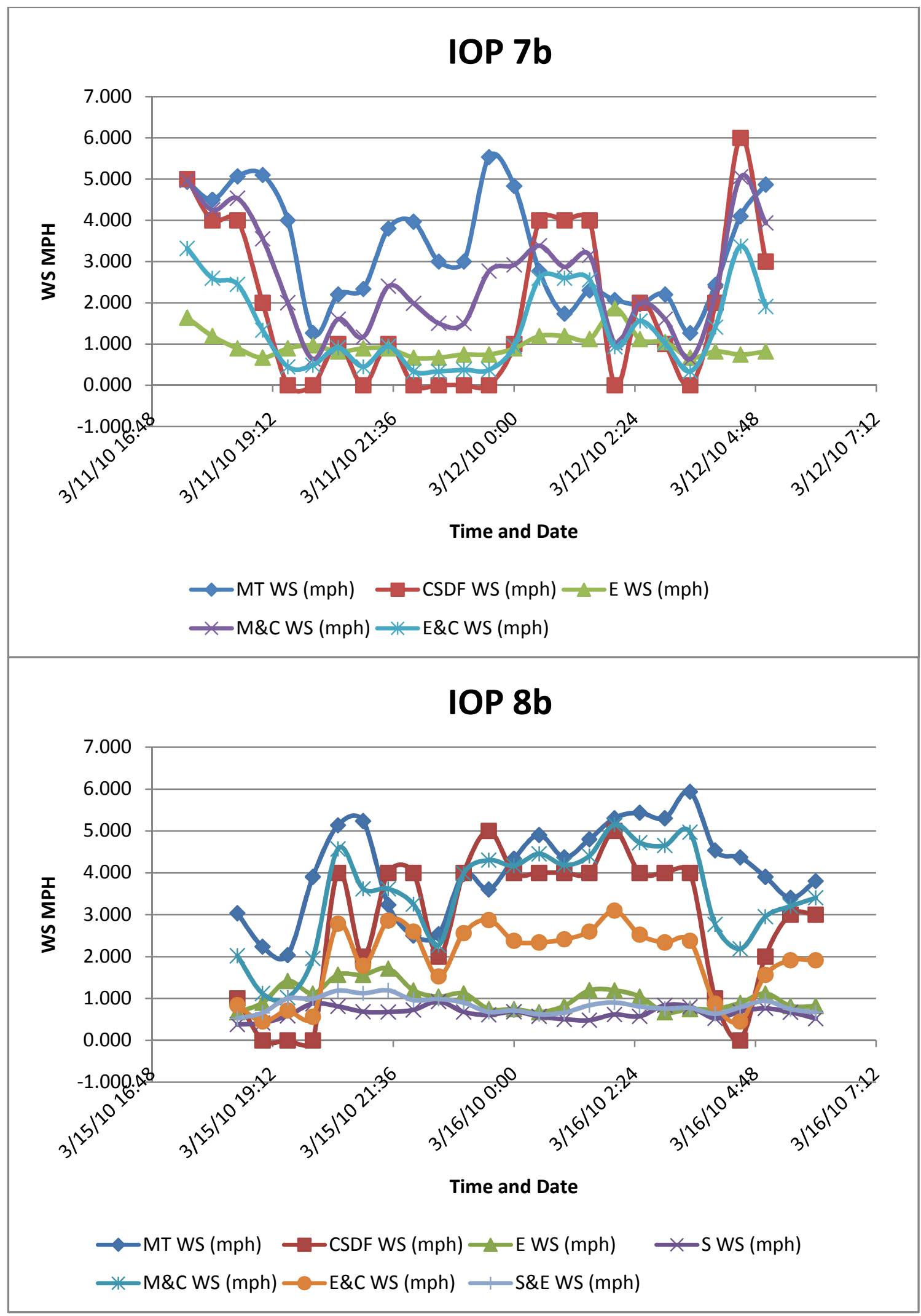




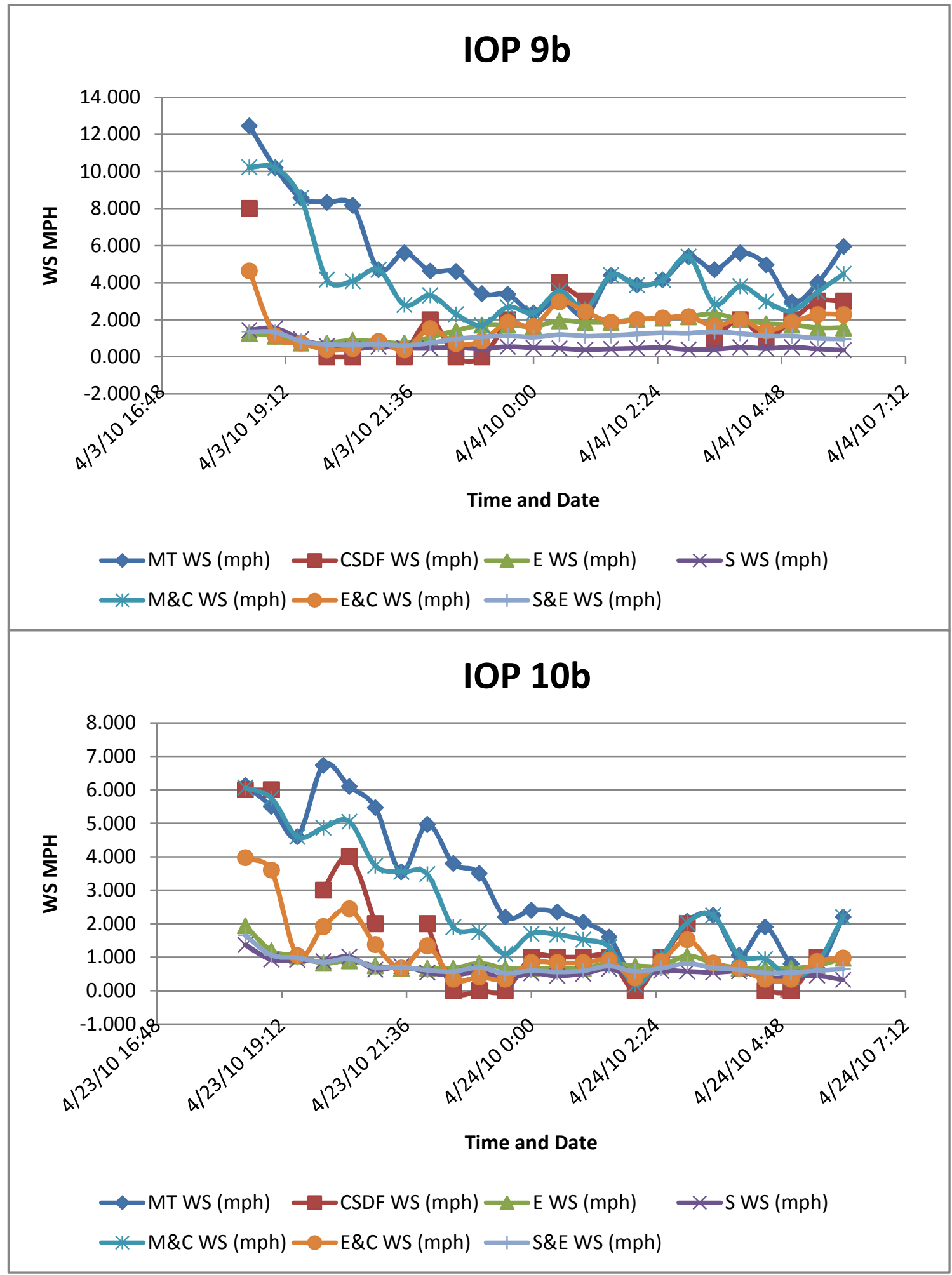




\section{Wind Roses for all IOPs}

\section{IOP 1a: January 31, 2009}

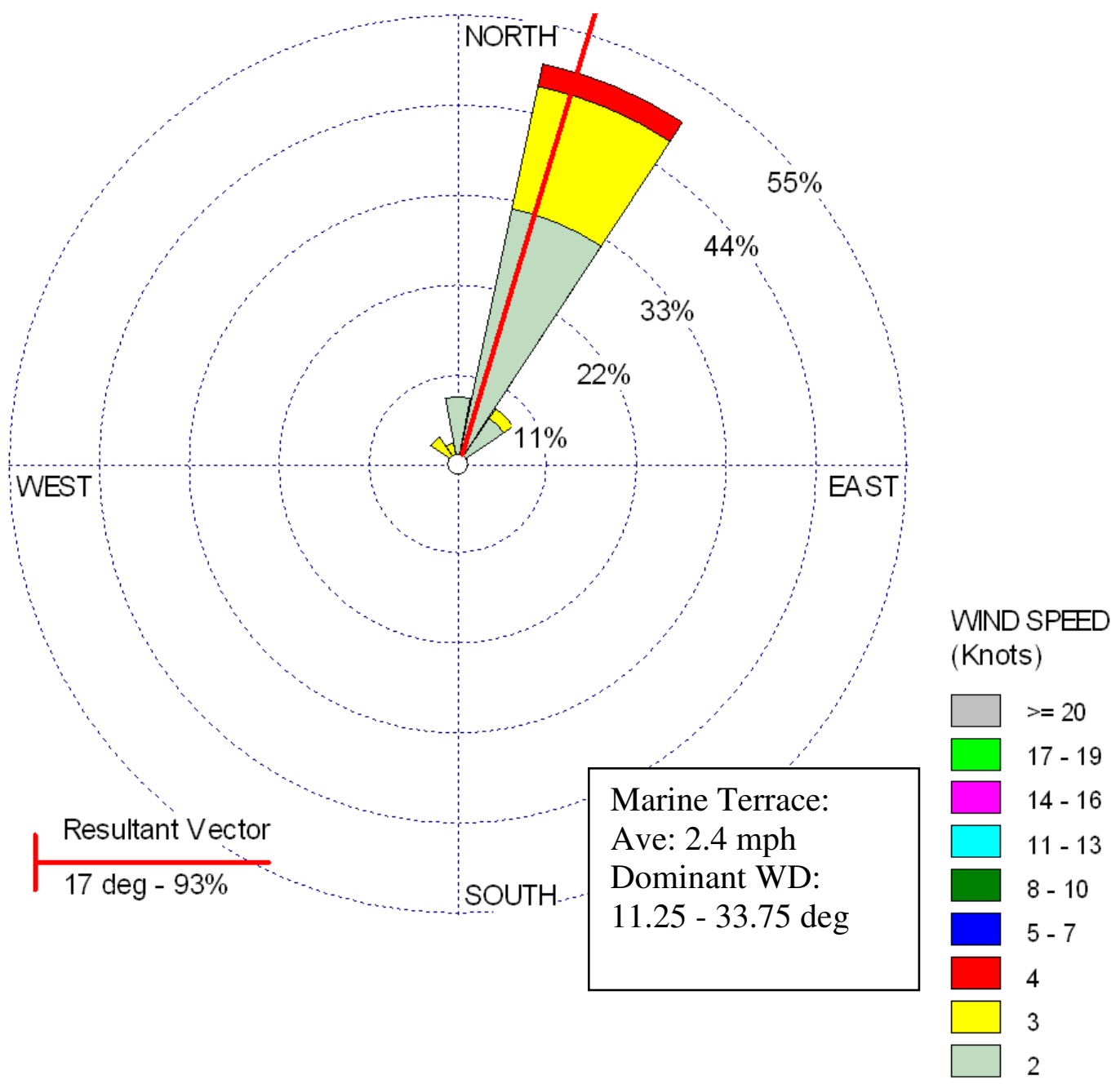

Calms: $25.00 \%$ 


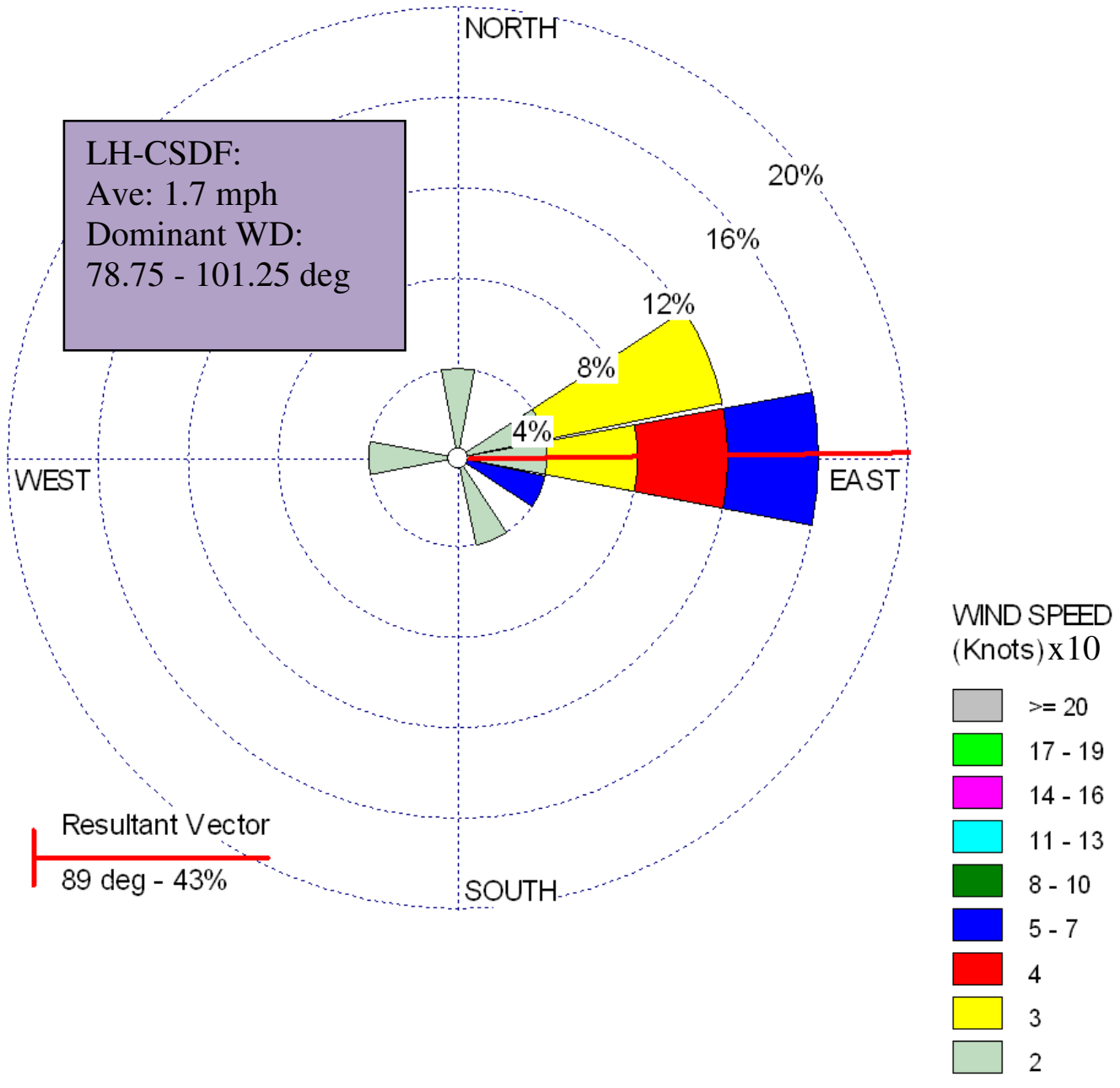

Calms: $56.00 \%$ 


\section{IOP 2a: February 18, 2009}

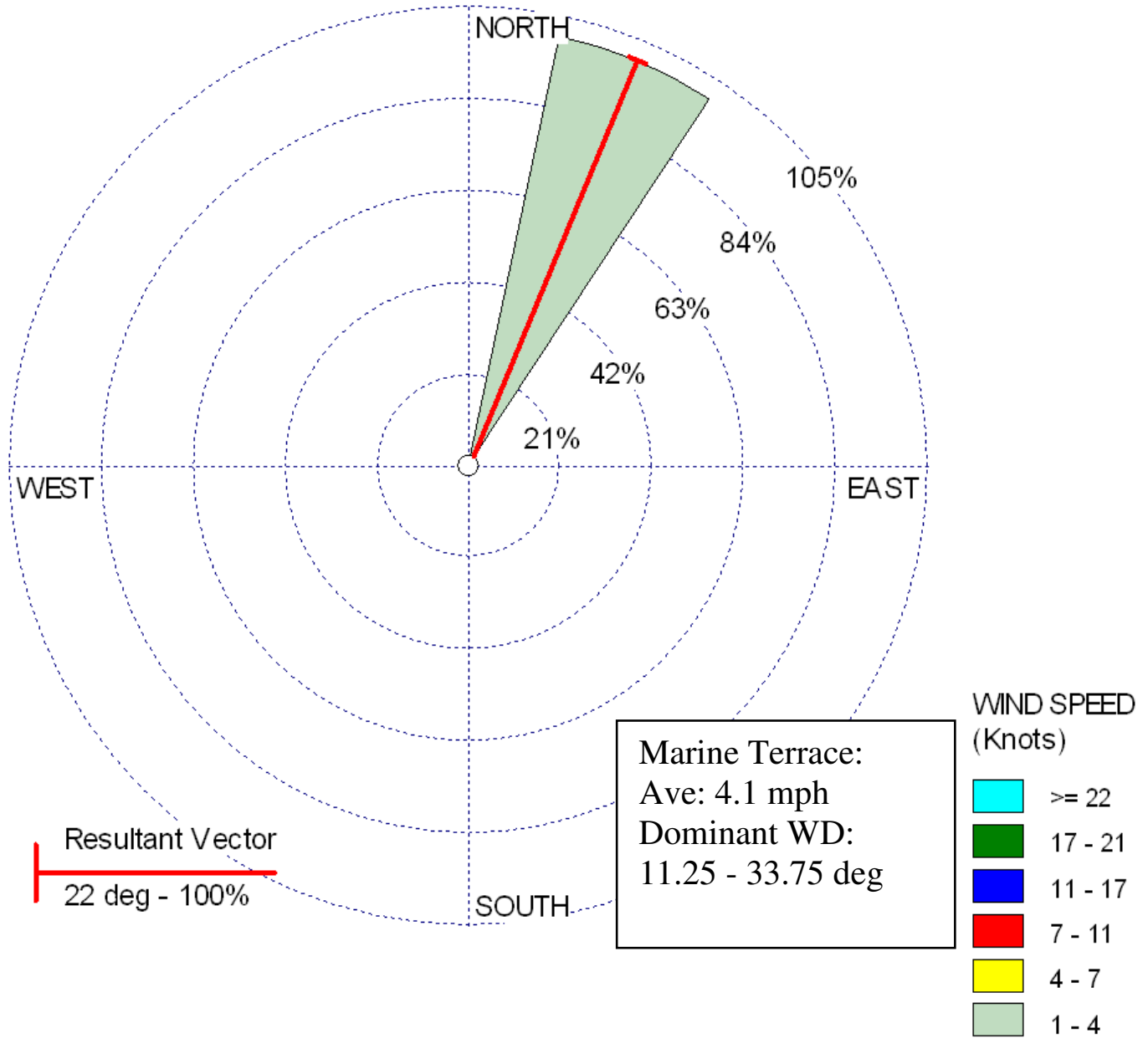

Calms: $0.00 \%$ 


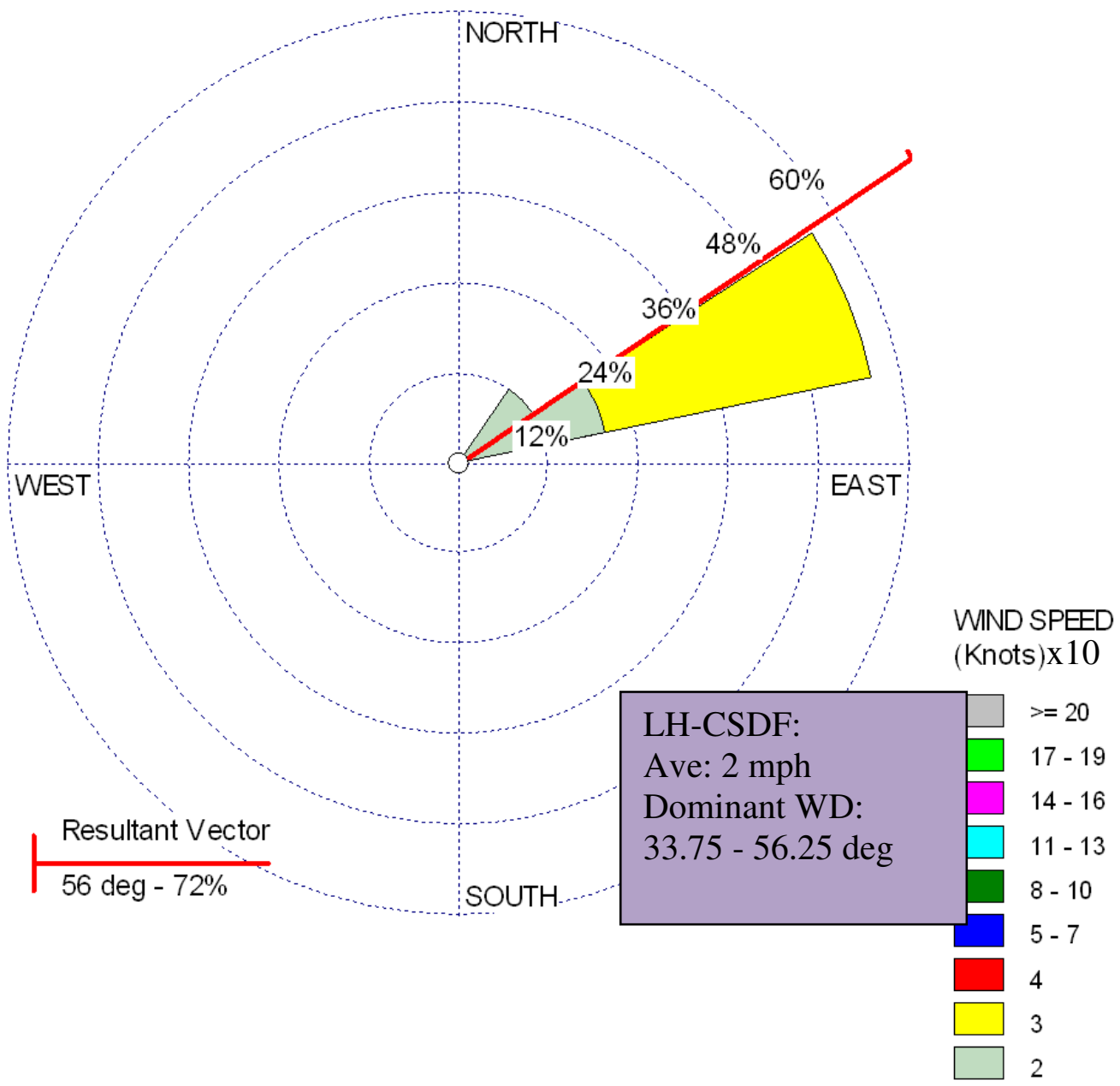

Calms: $32.00 \%$ 


\section{IOP 3a: February 26, 2009}

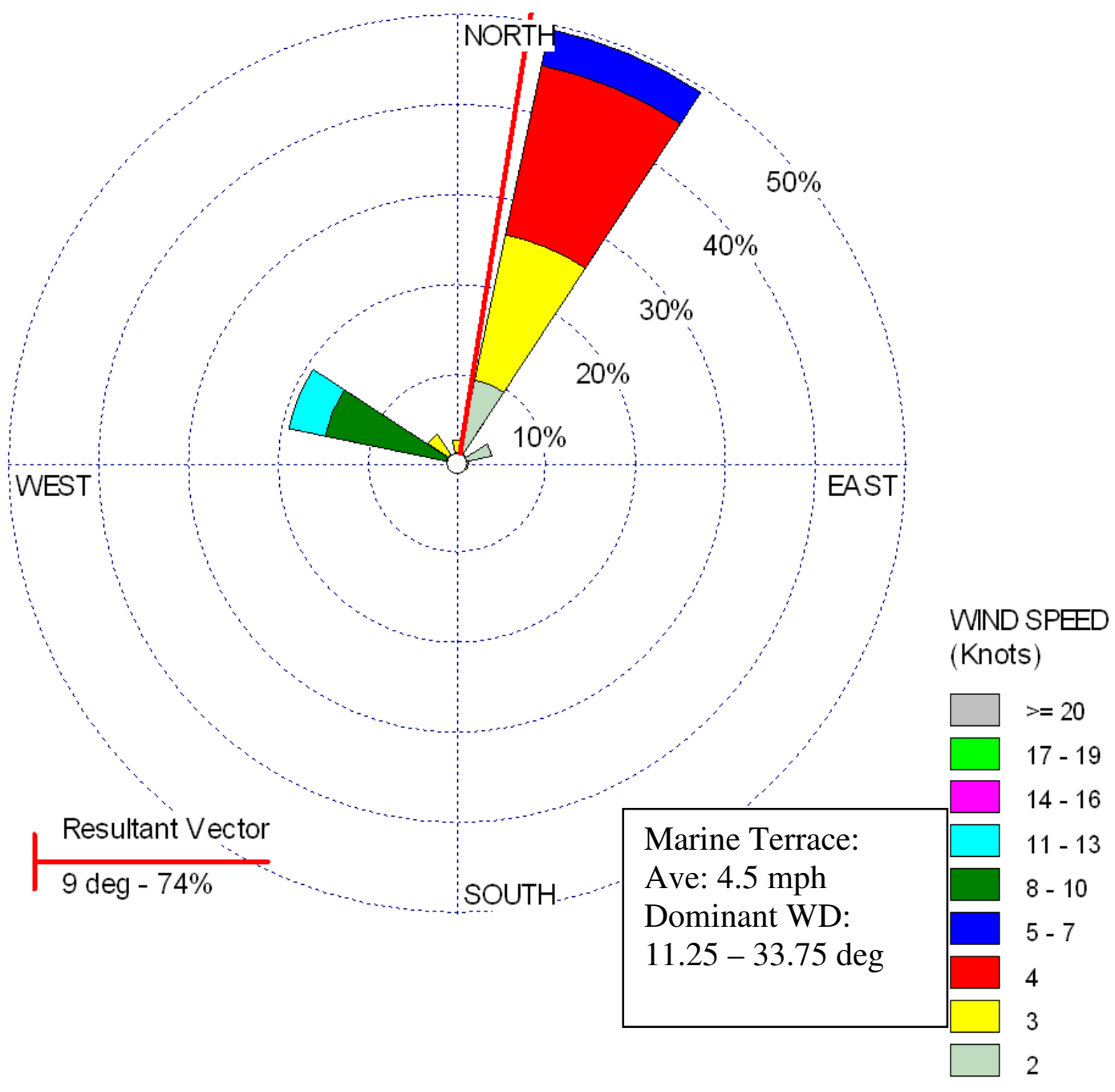

Calms: $16.44 \%$ 


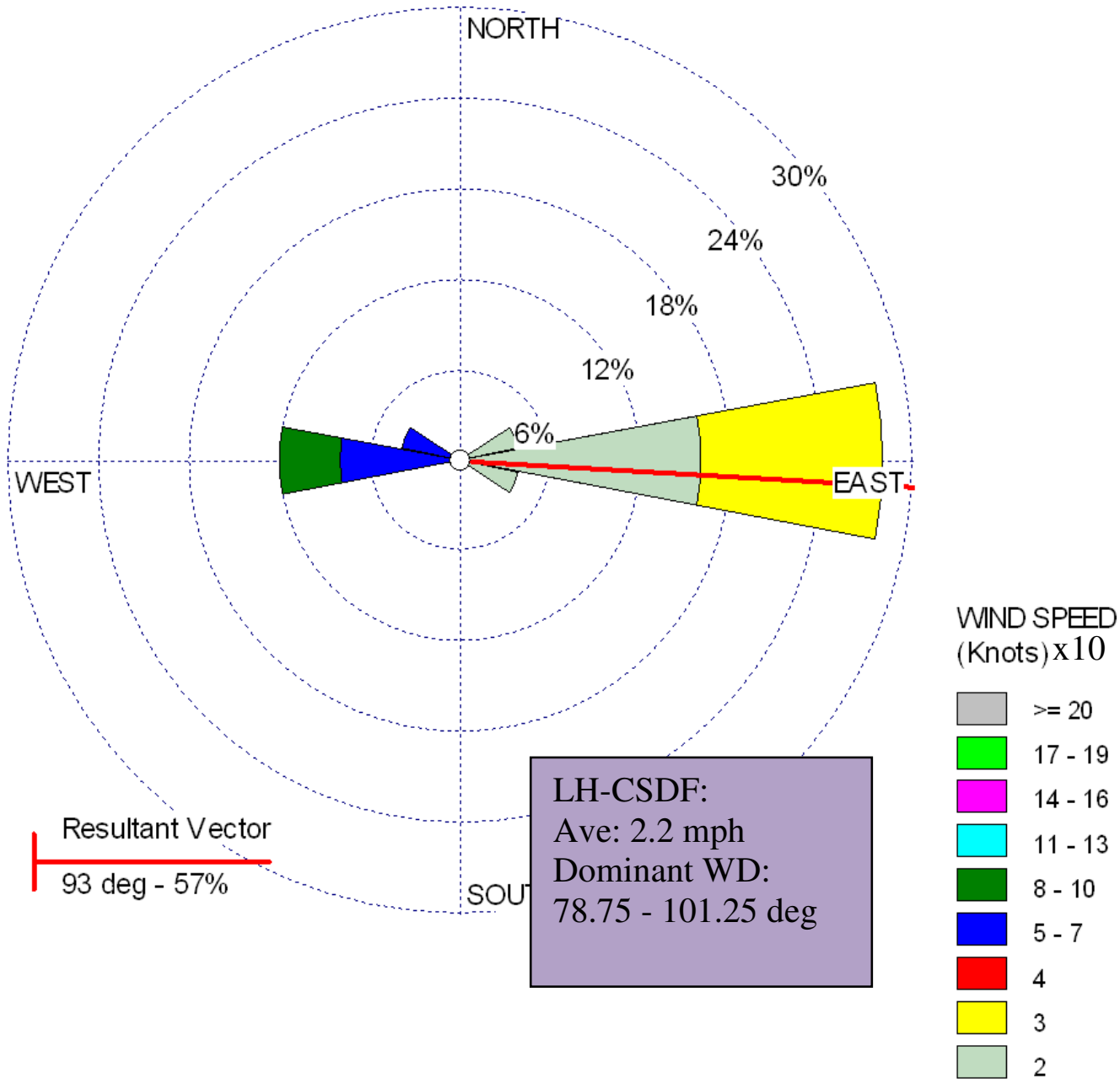

Calms: $48.00 \%$ 


\section{IOP 4a: February 27, 2009}

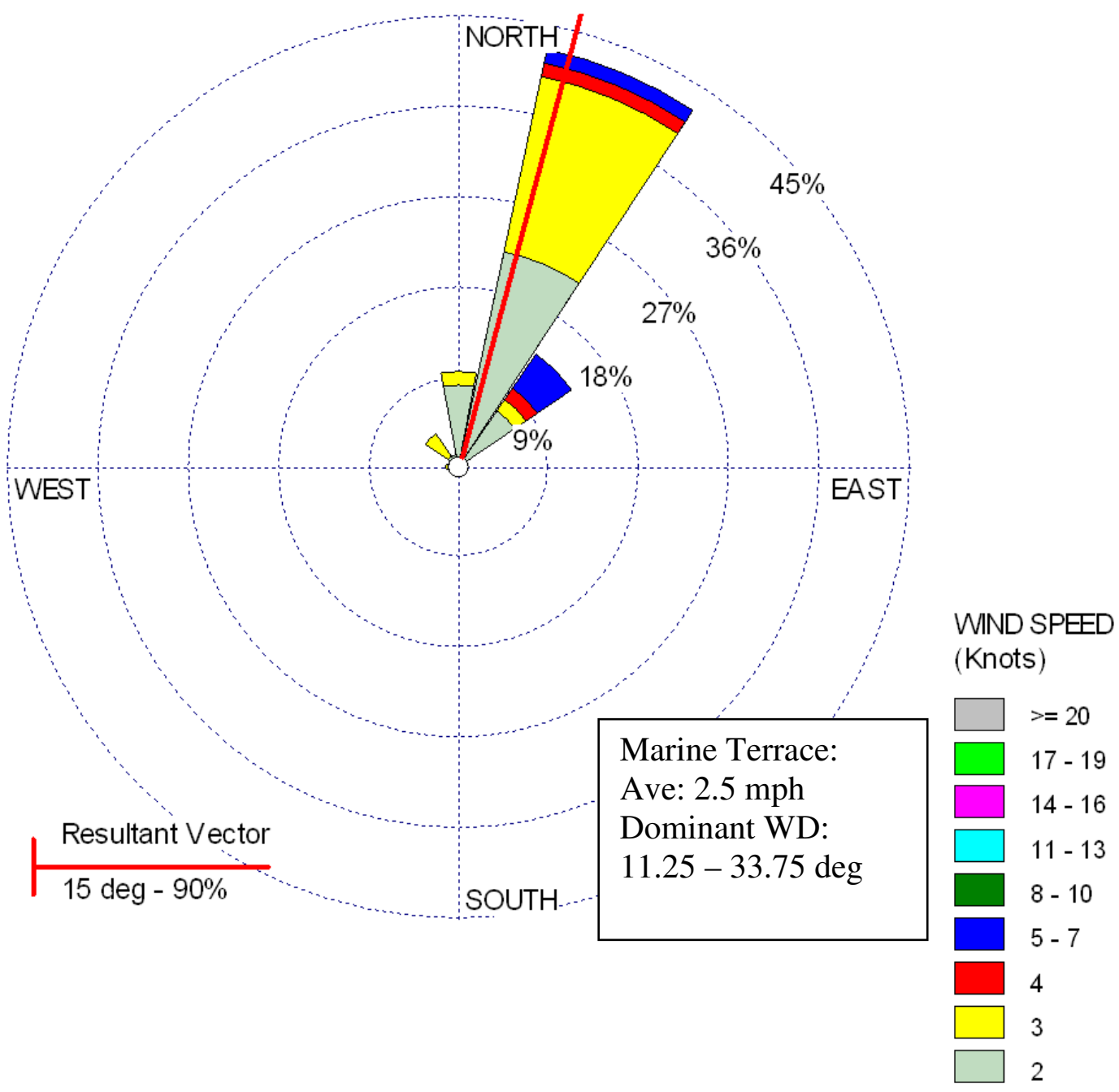

Calms: $27.40 \%$ 


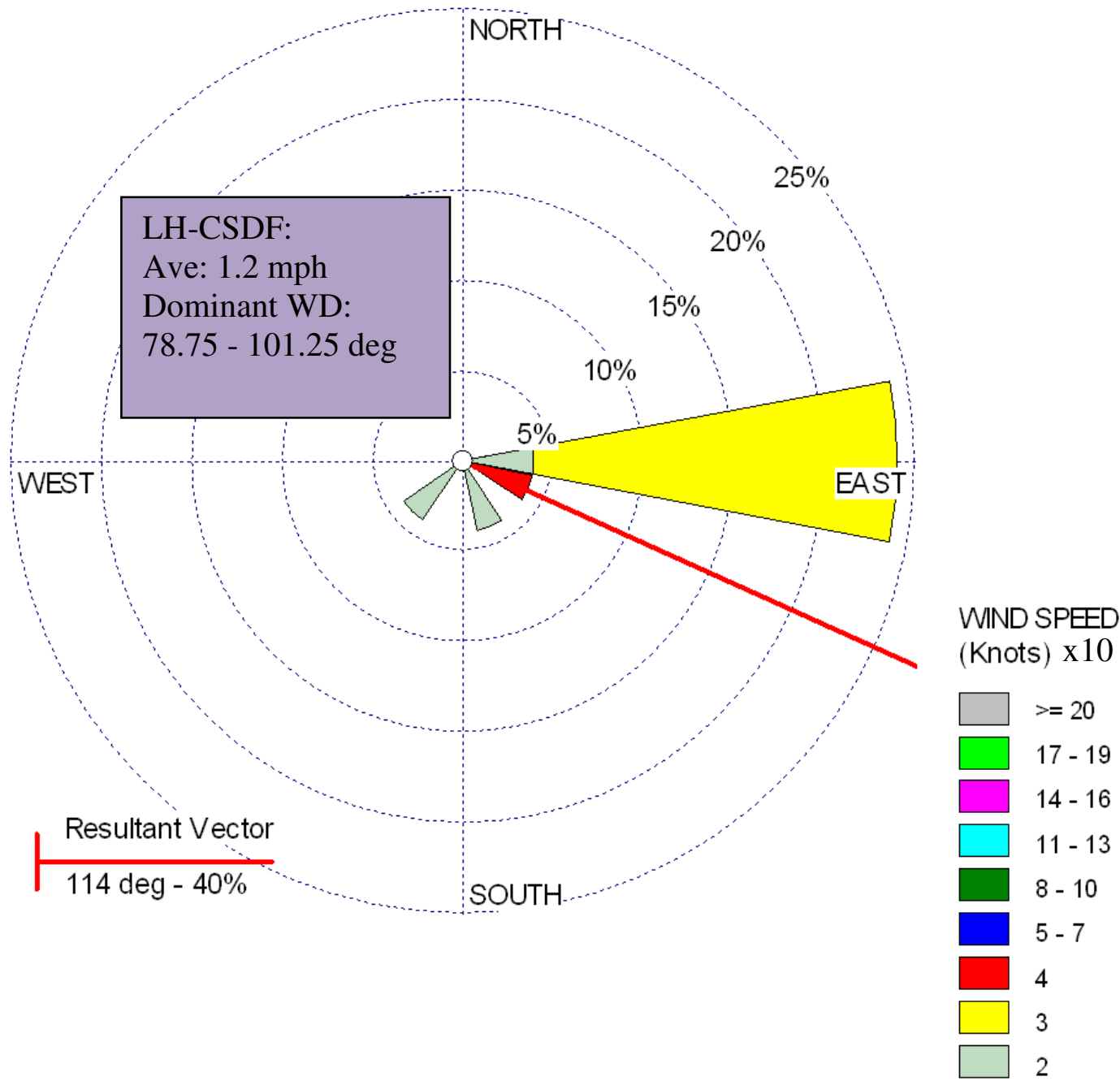

Calms: $64.00 \%$ 


\section{IOP 2b: January 23, 2010}

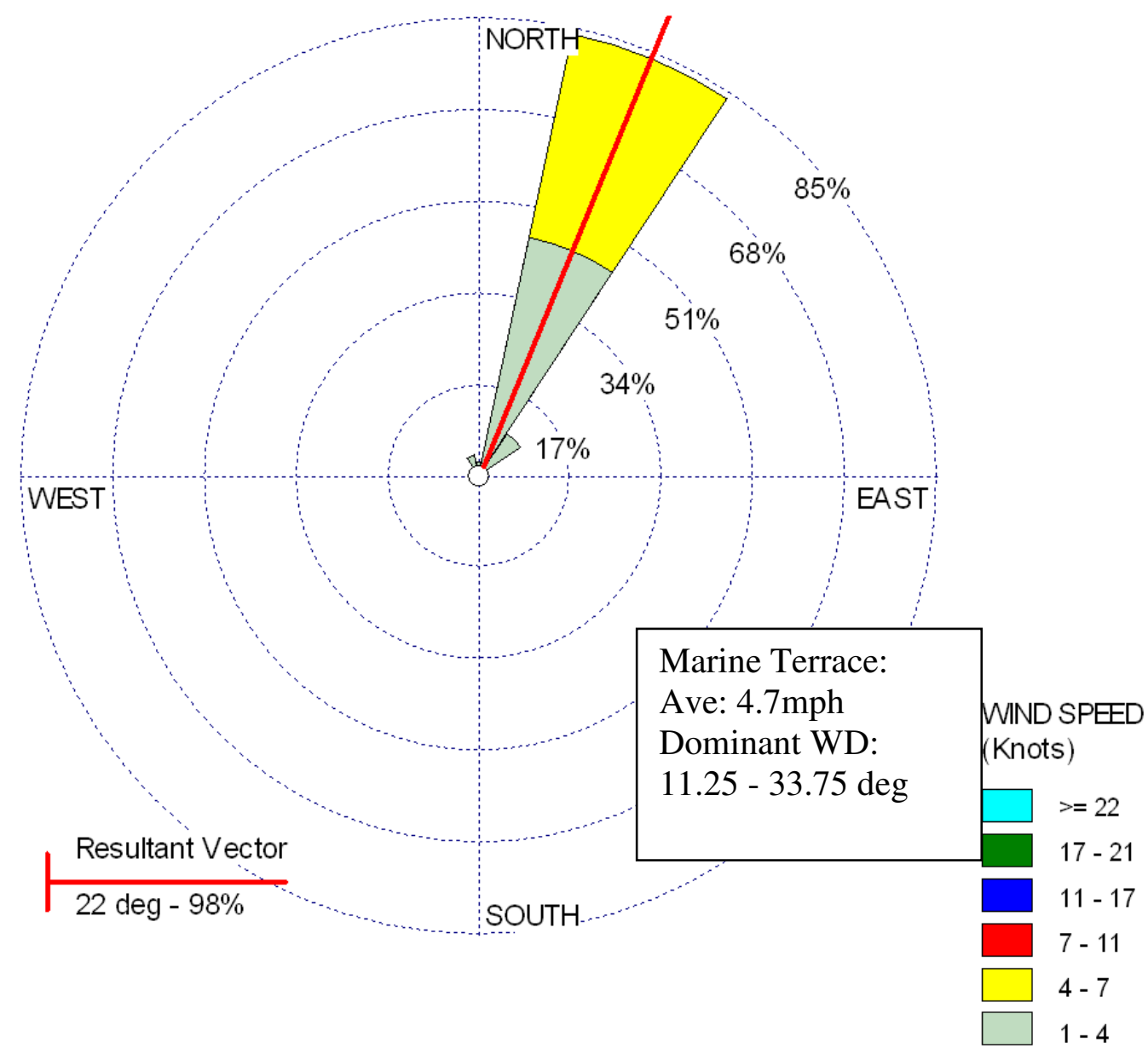

Calms: $0.00 \%$ 


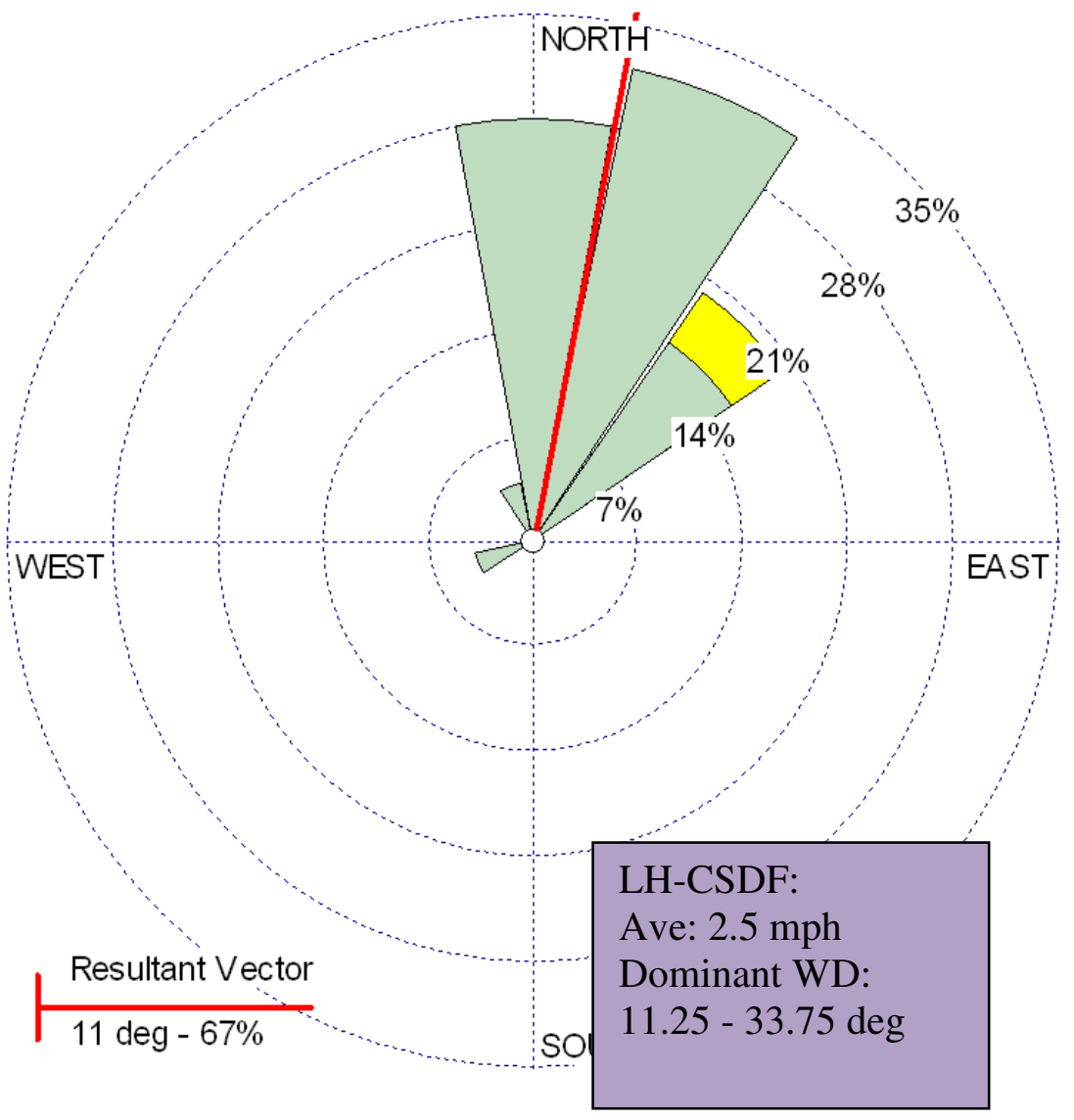

WIND SPEED

(Knots) X10

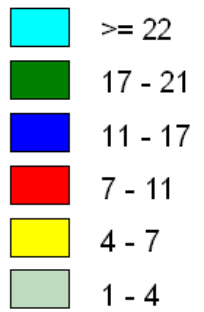

Calms: $12.00 \%$ 


\section{IOP 3b: January 30, 2010}

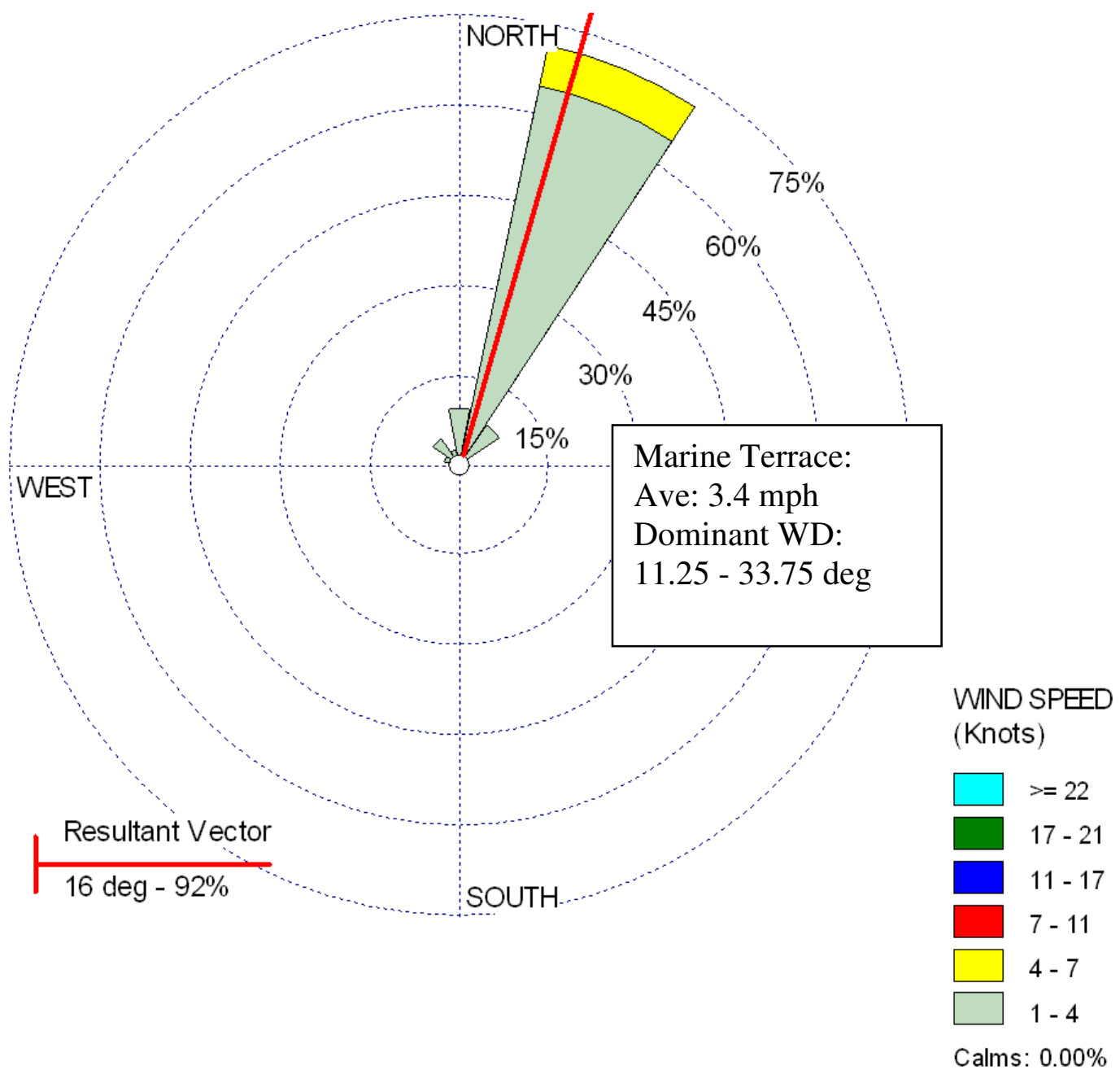




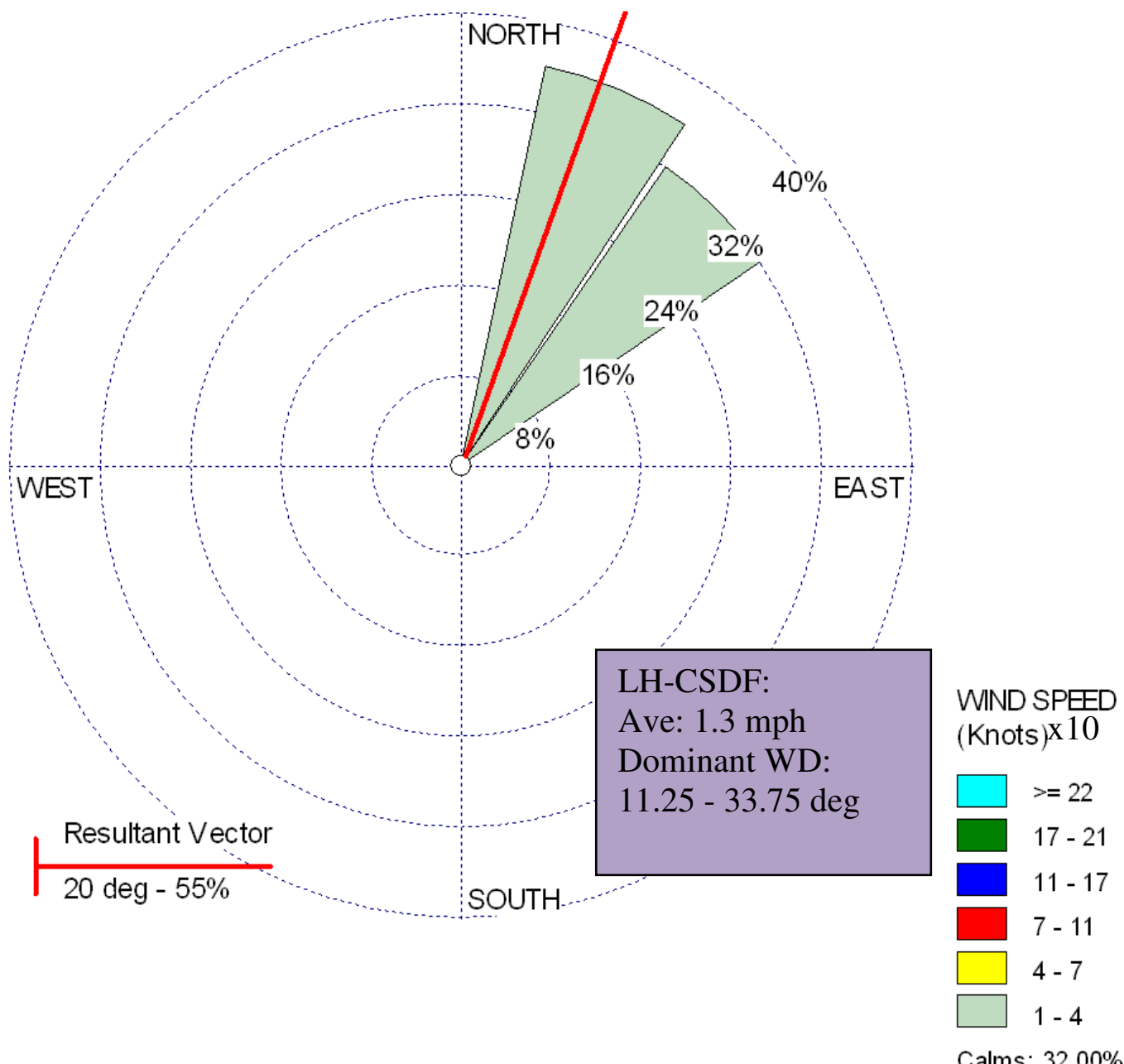

Calms: $32.00 \%$ 


\section{IOP 4b: February 13, 2010 -AM inversions}

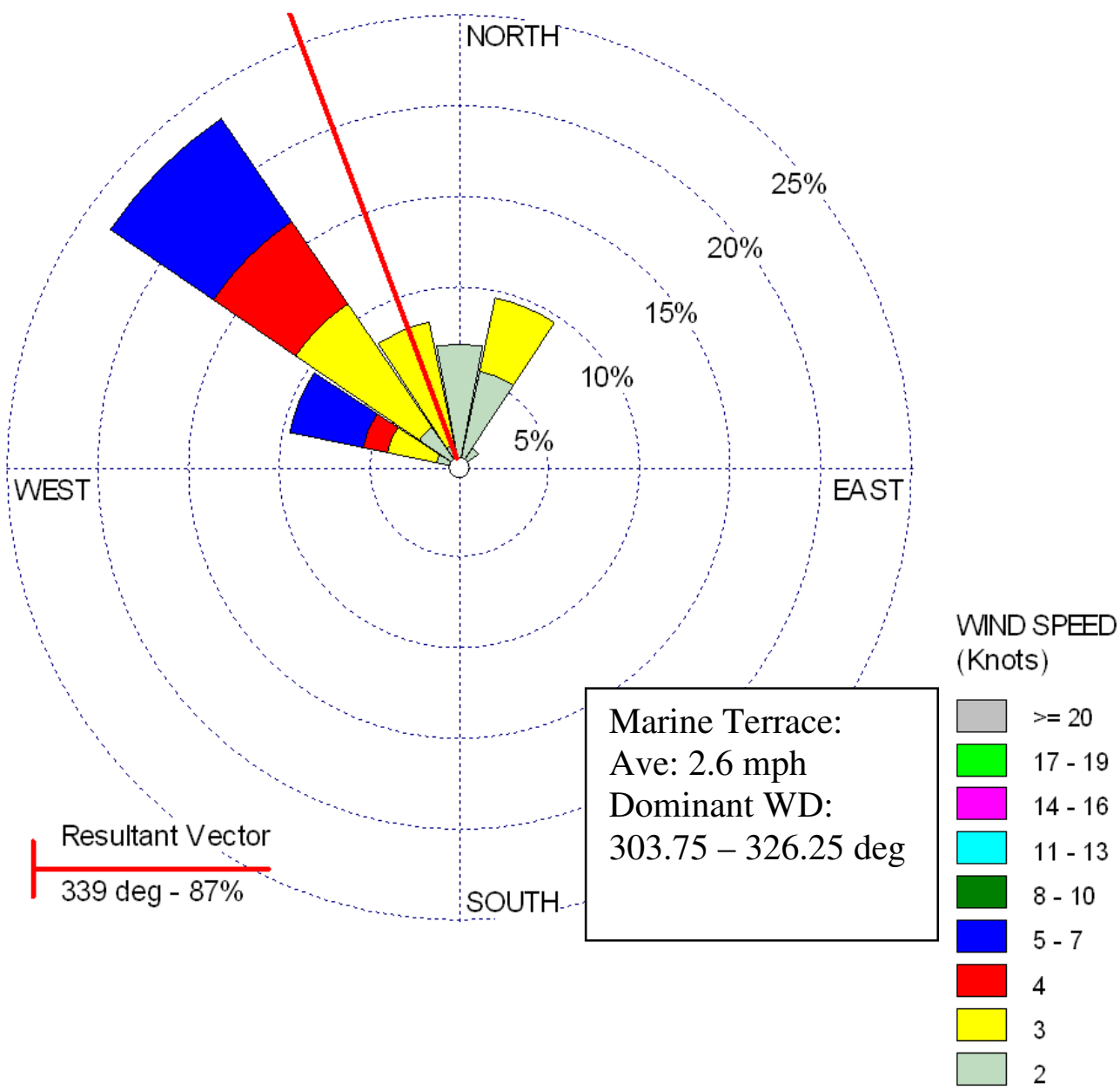

Calms: $41.10 \%$ 


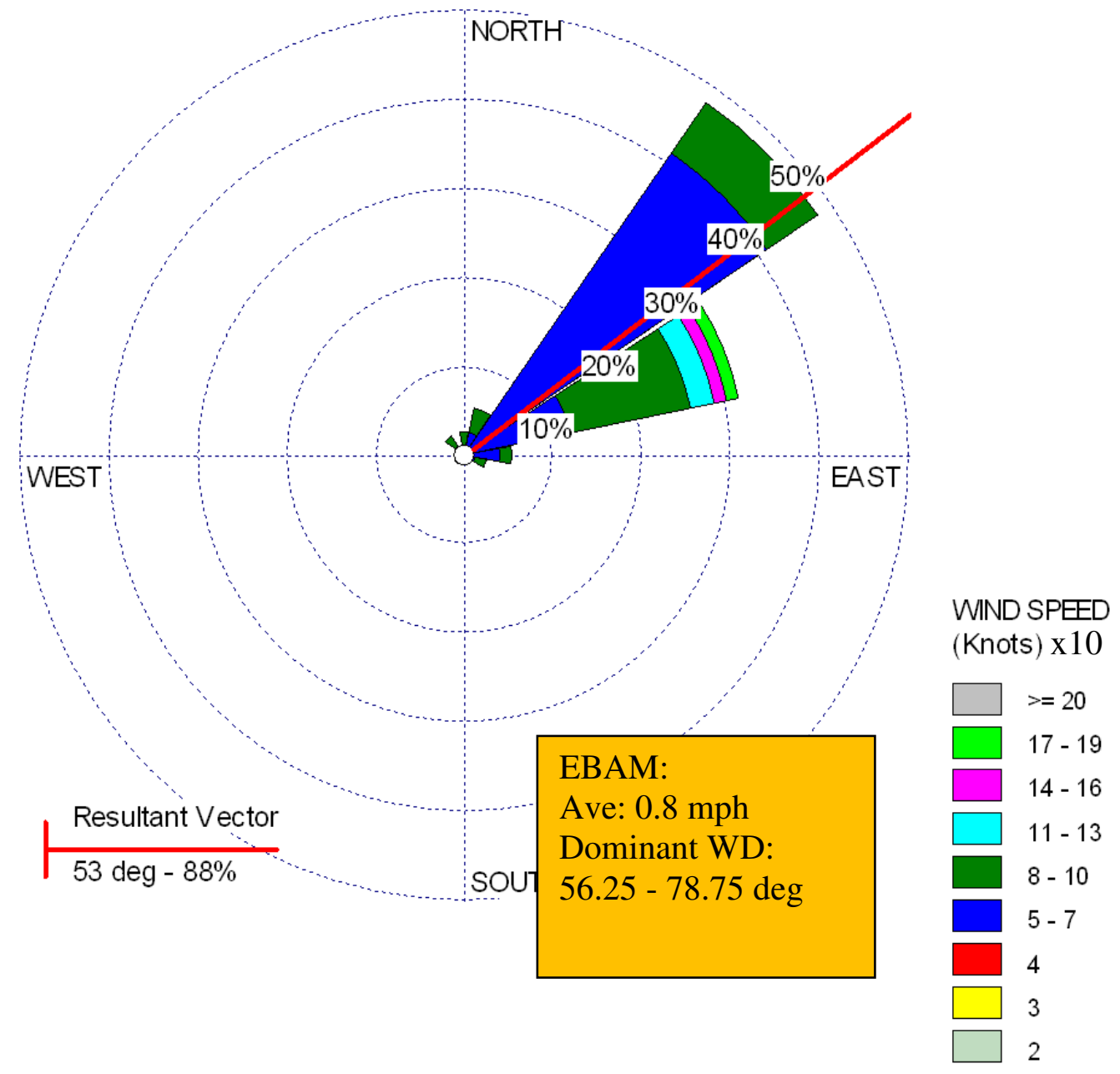

Calms: $0.00 \%$ 


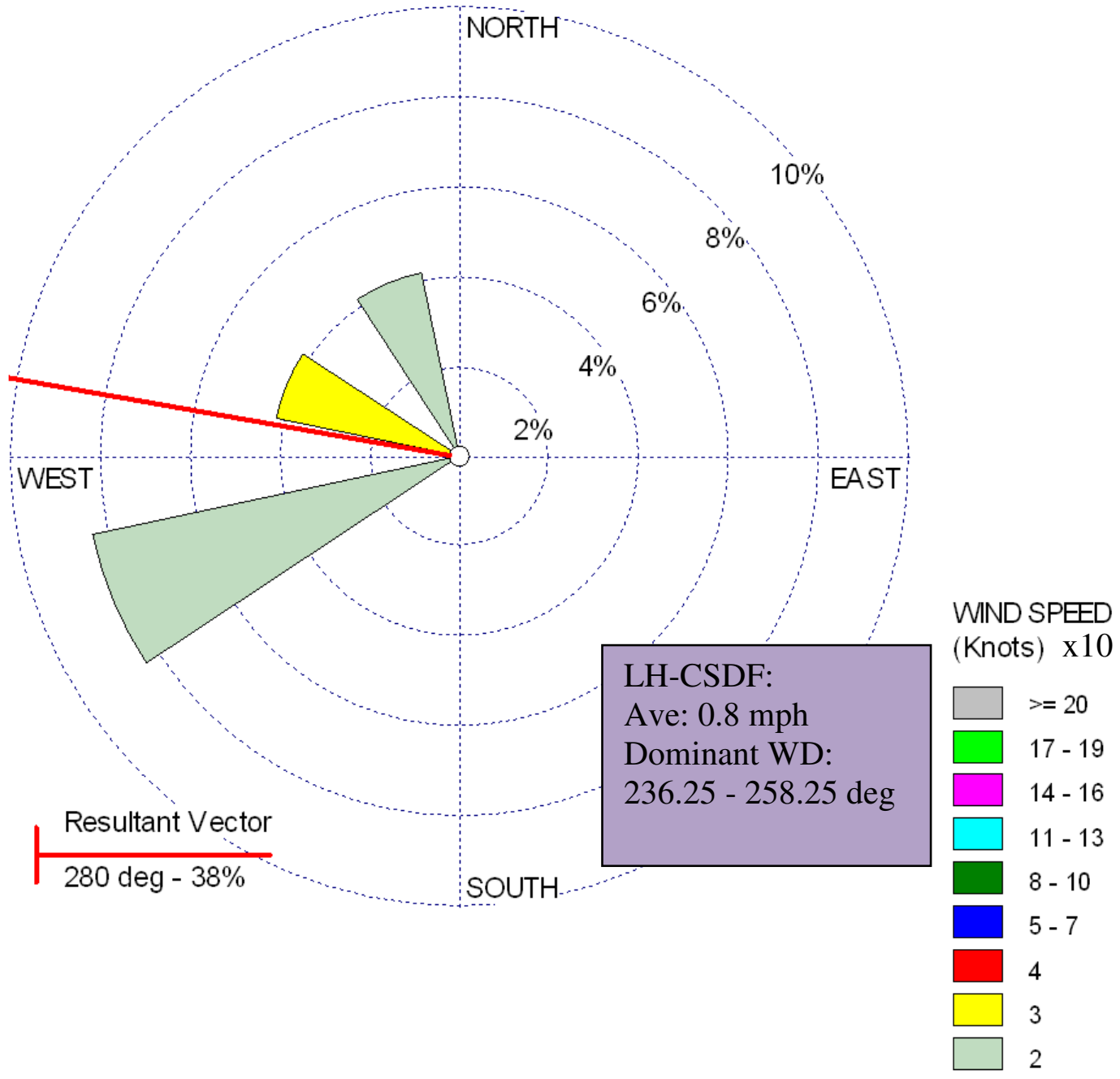

Calms: $83.33 \%$ 
IOP 5b: February 28, 2010

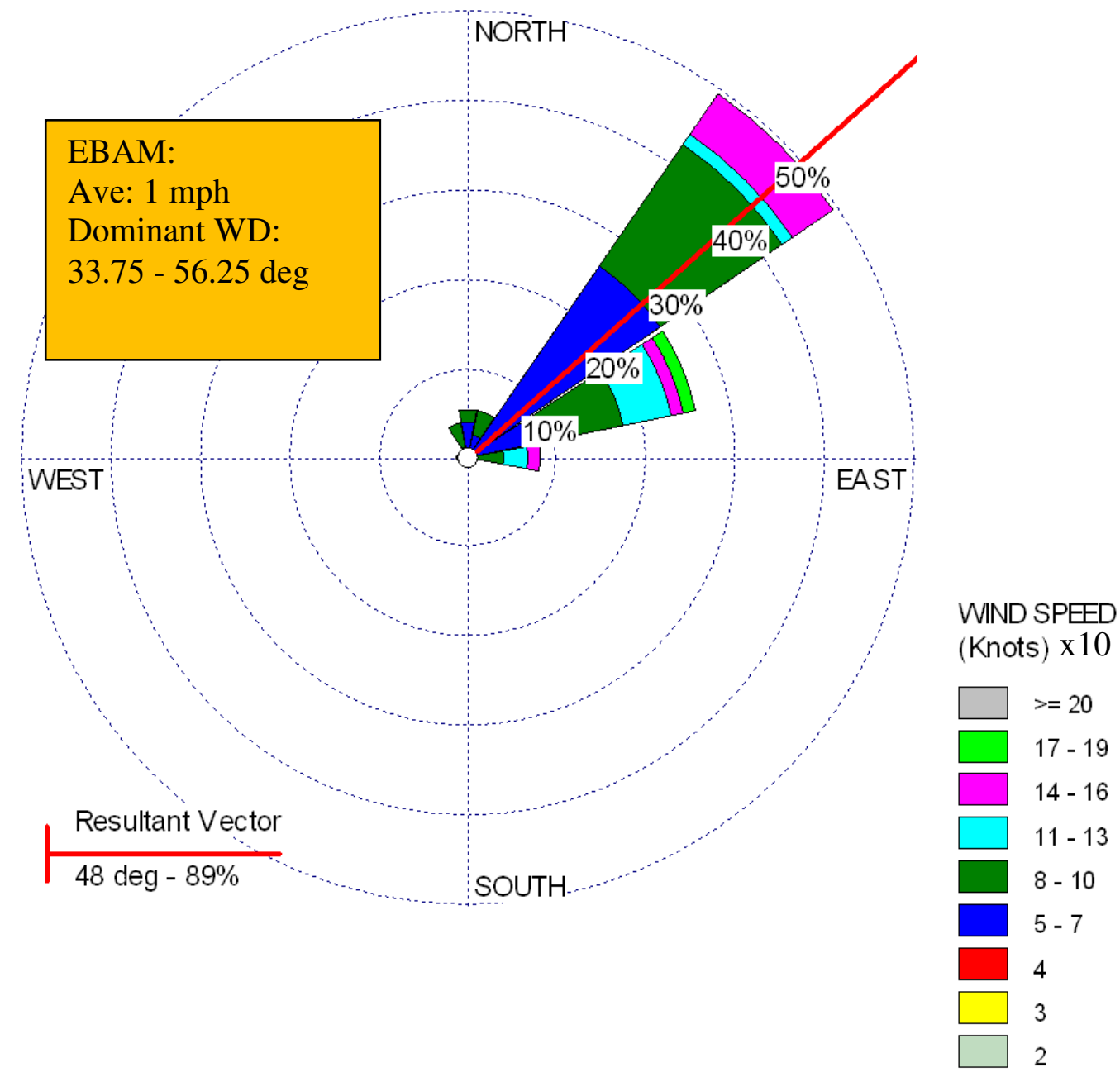

Calms: $0.00 \%$ 


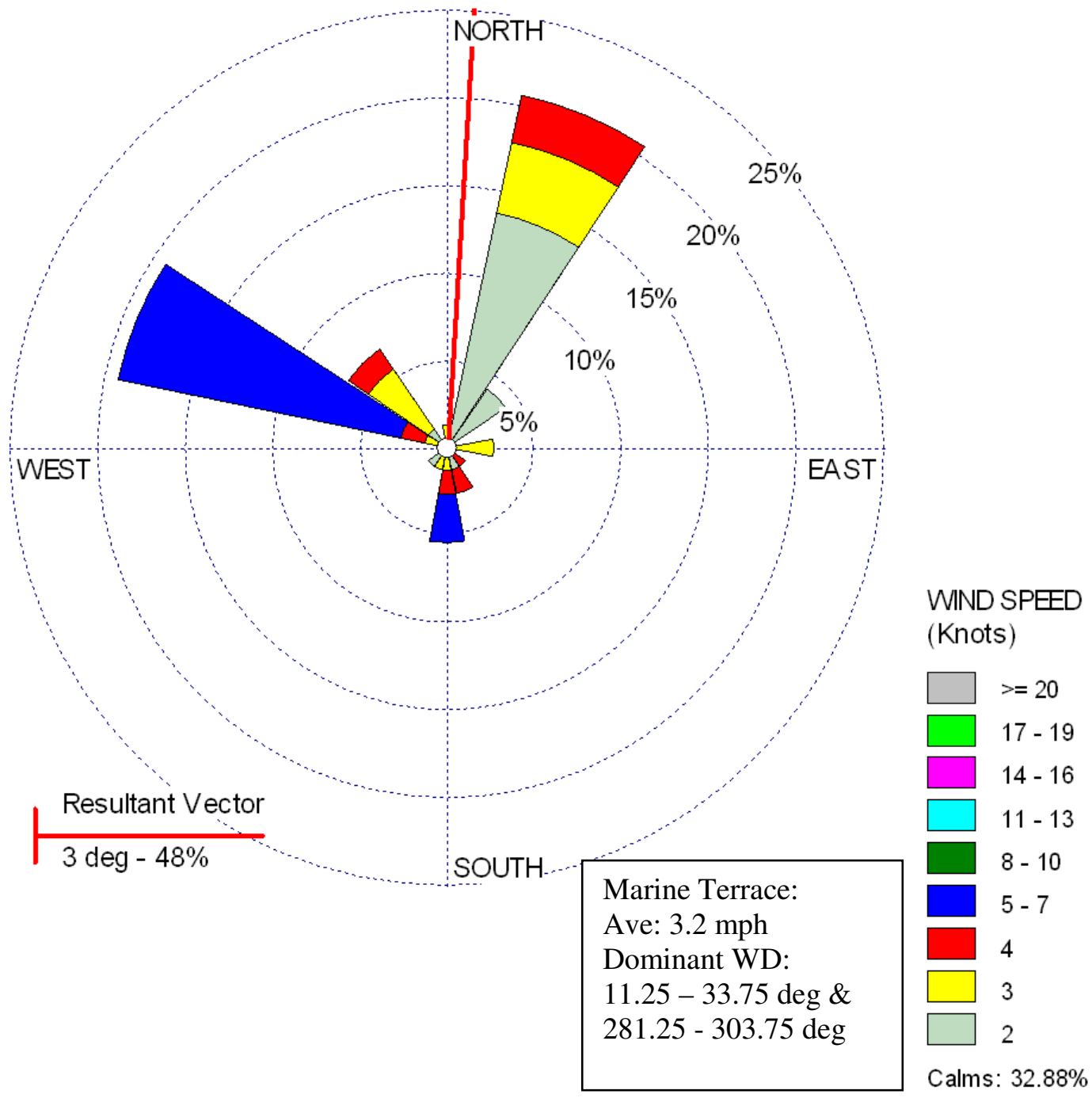




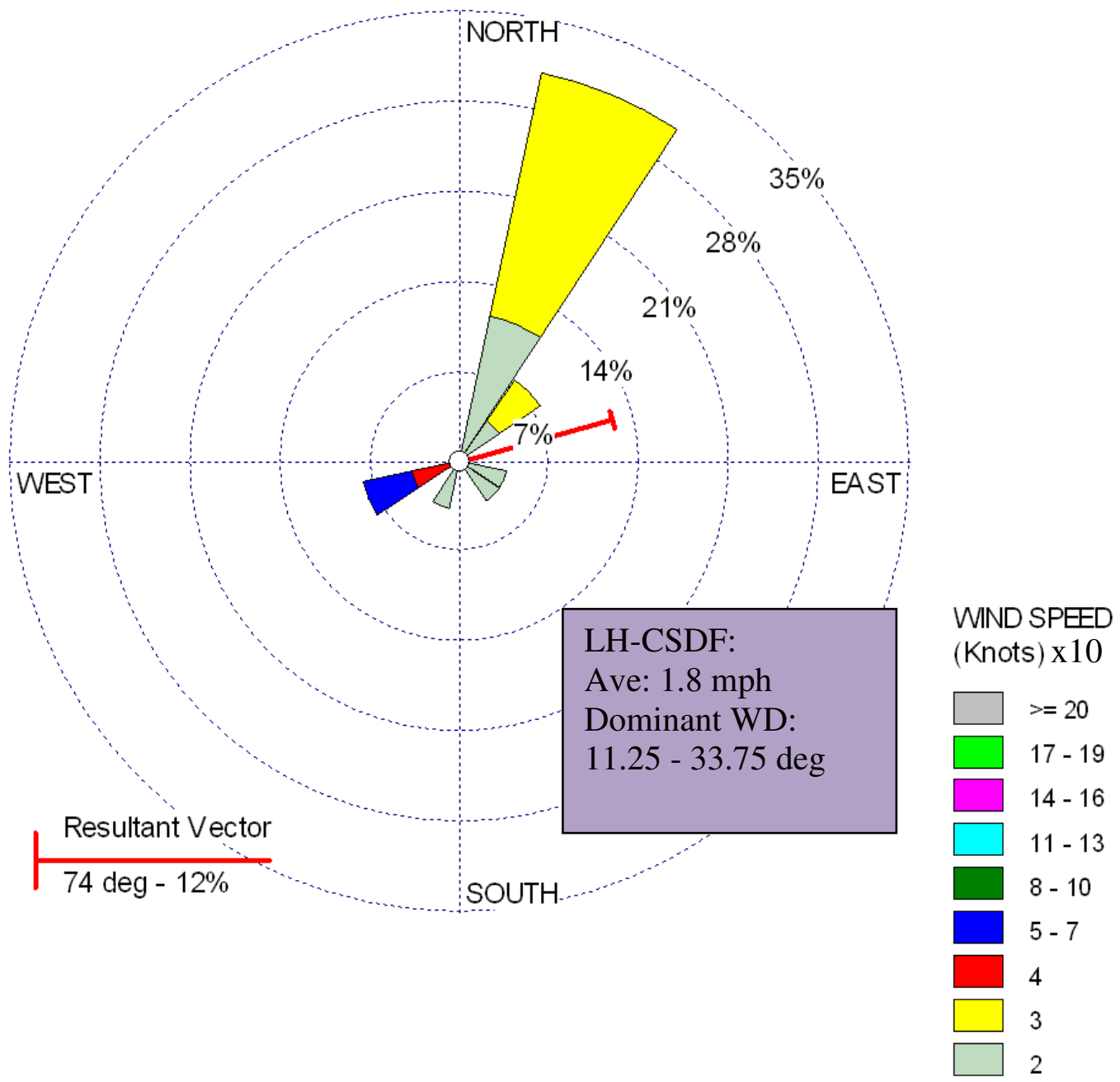

Calms: $42.31 \%$ 
IOP 6b: March 4, 2010

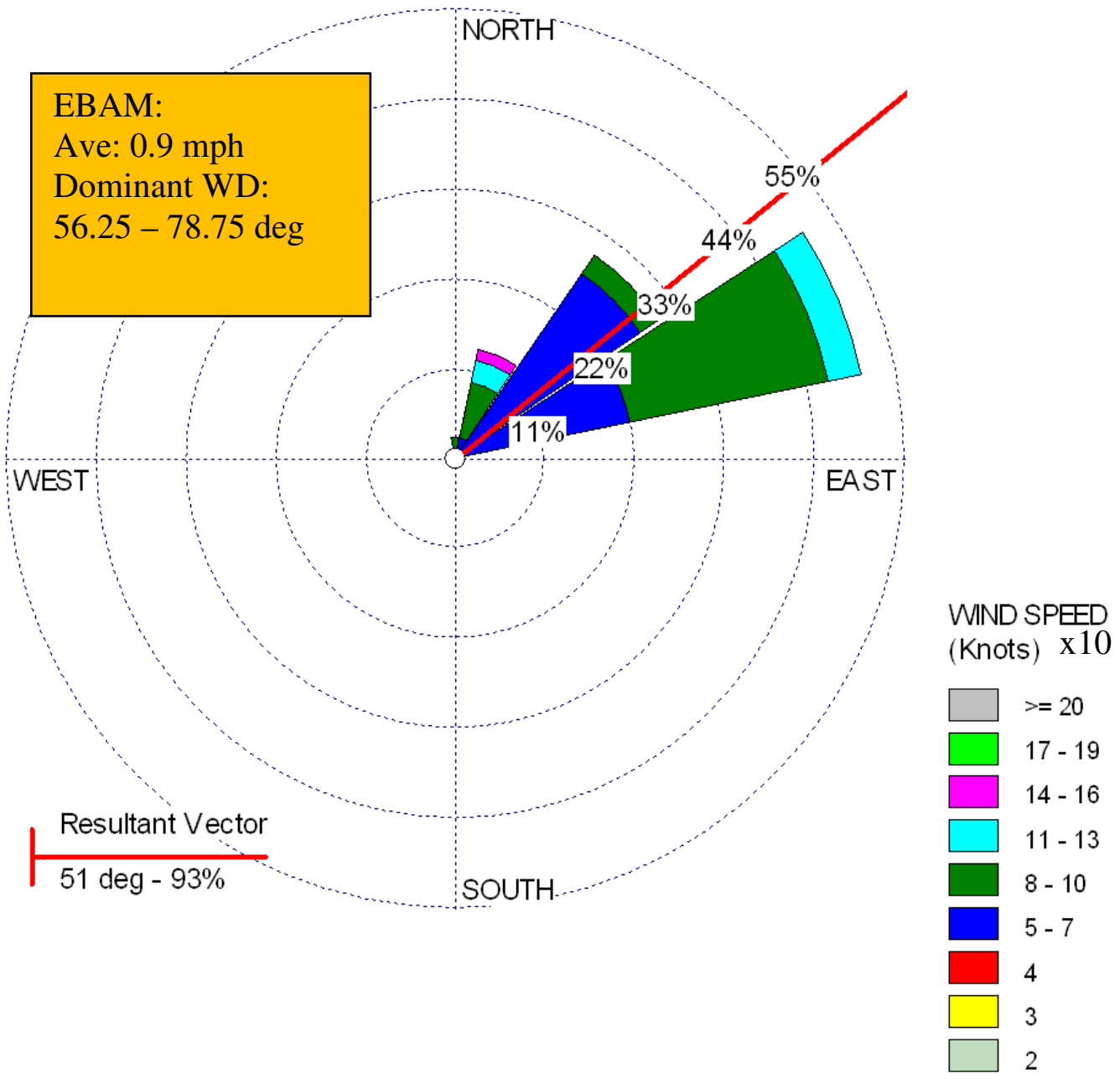

Calms: $0.00 \%$ 


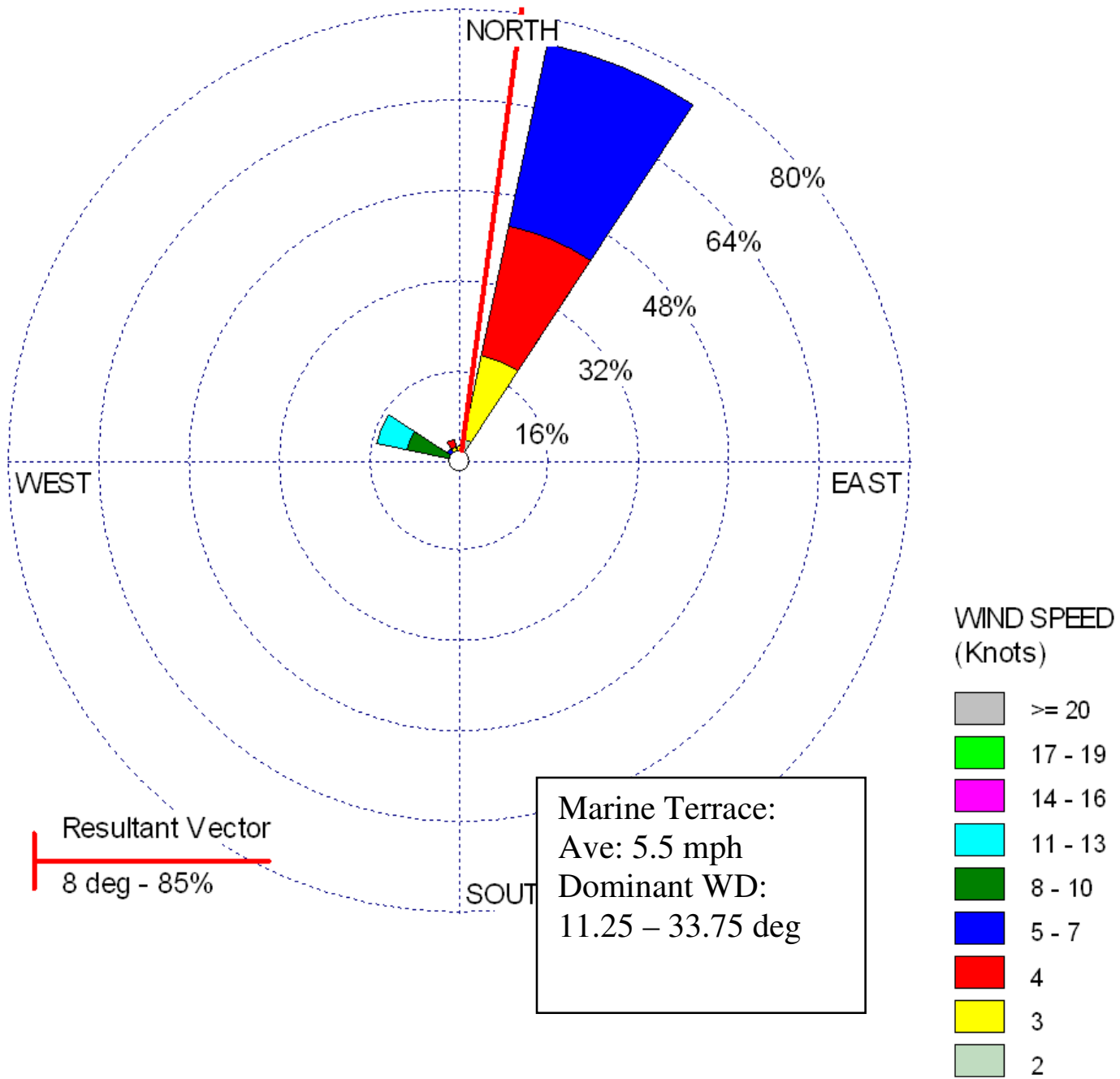

Calms: $0.00 \%$ 


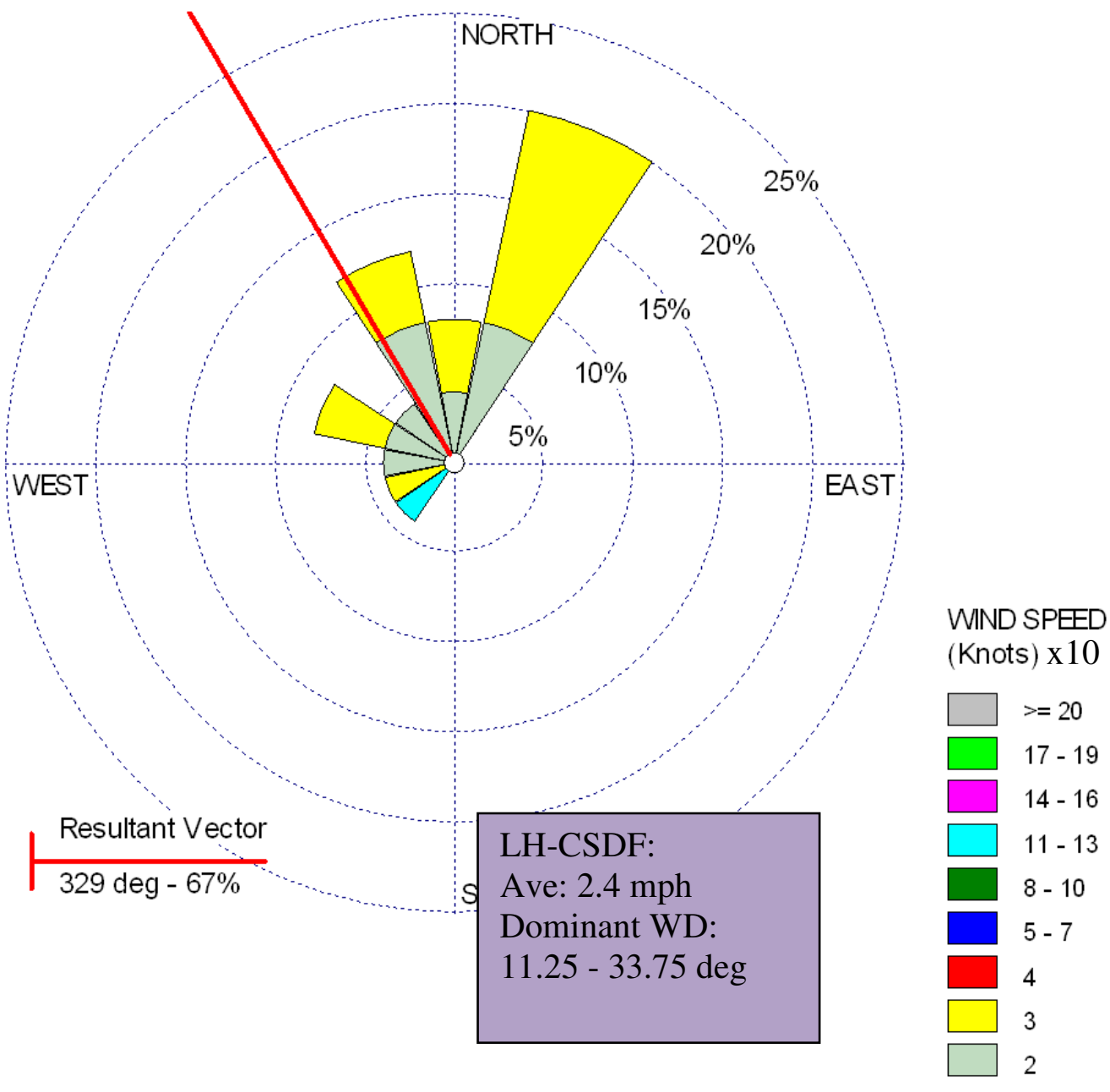

Calms: $36.00 \%$ 
IOP 7b: March 11, 2010 - mostly AM inversions

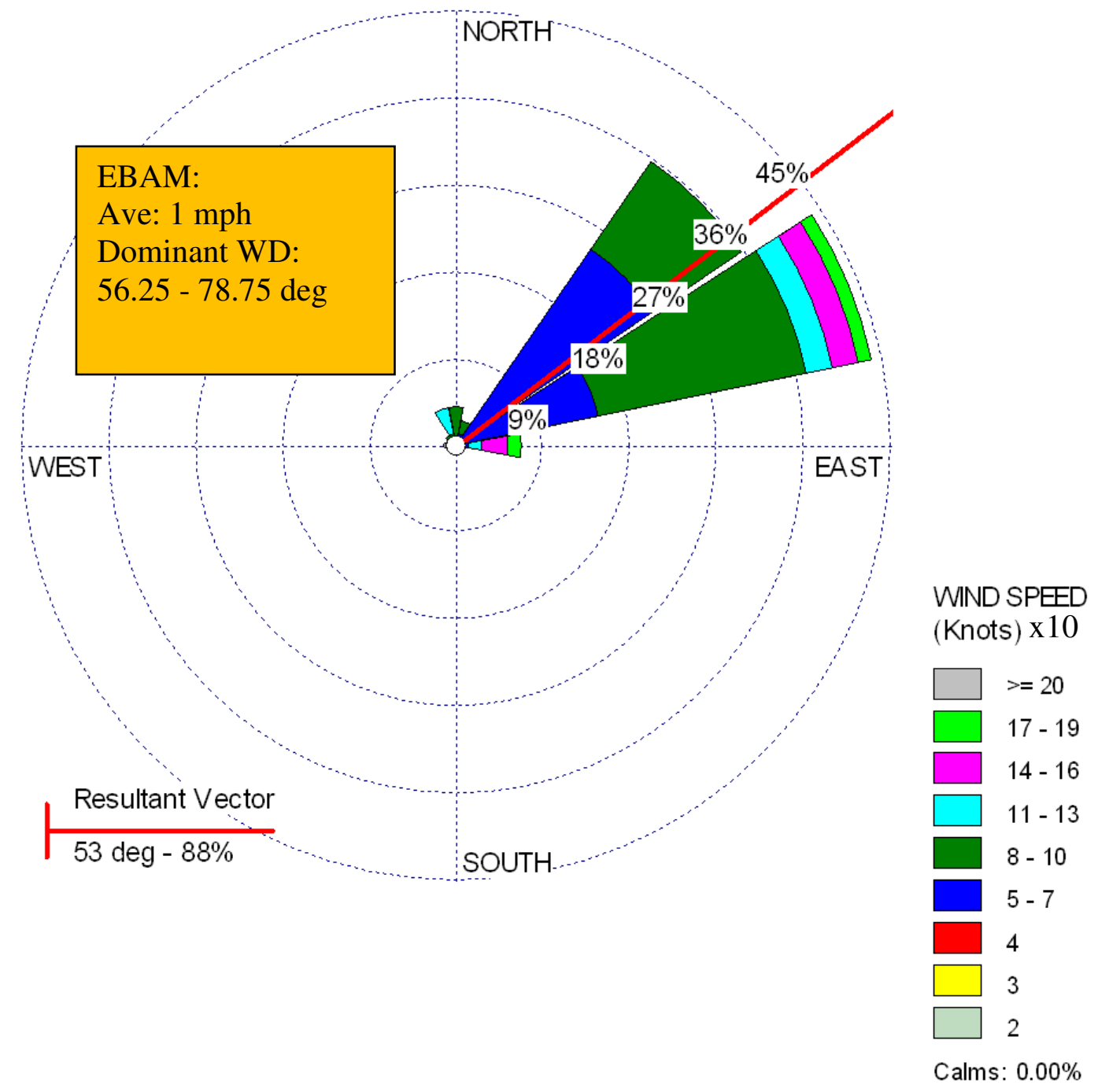




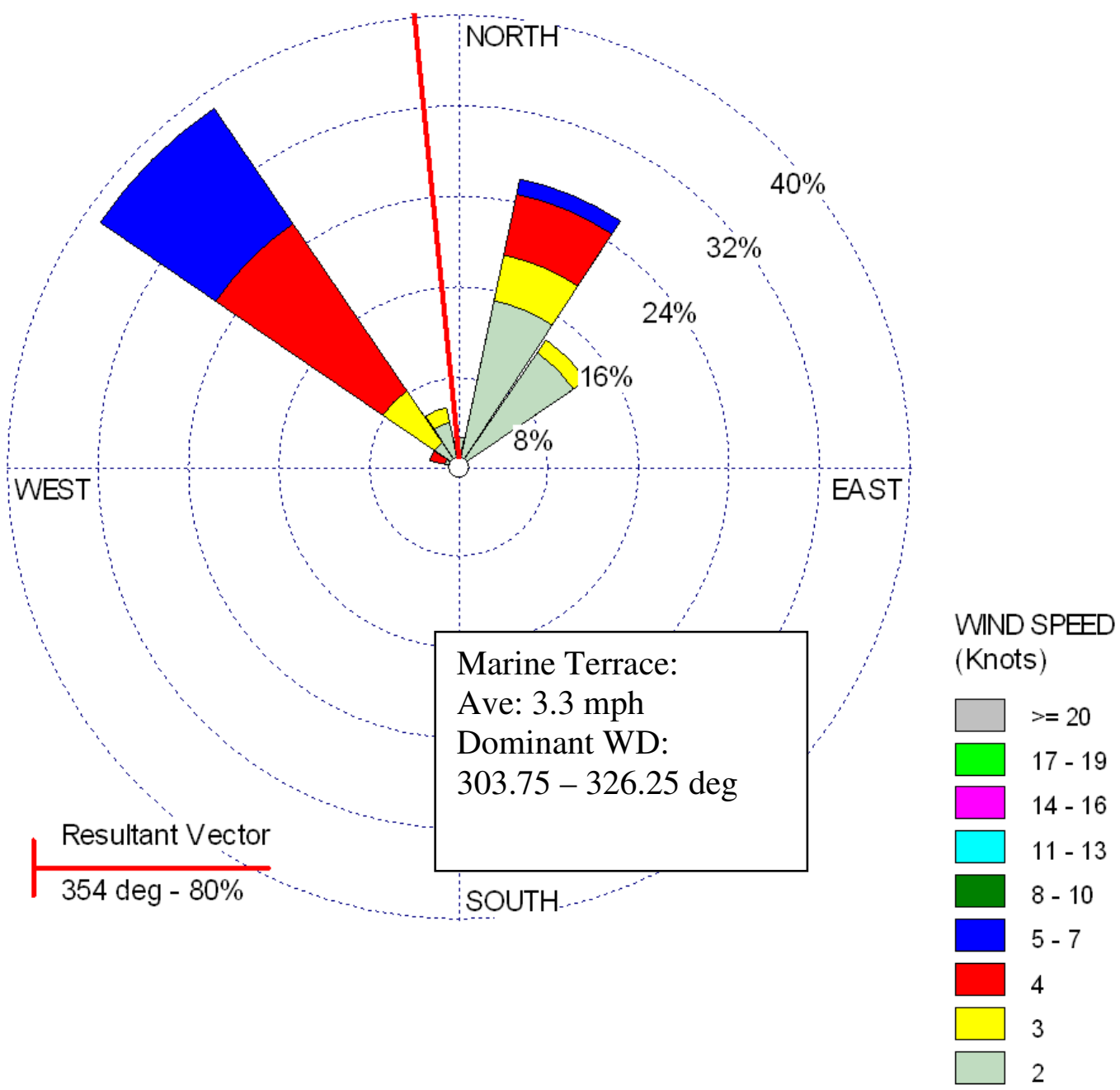

Calms: $10.96 \%$ 


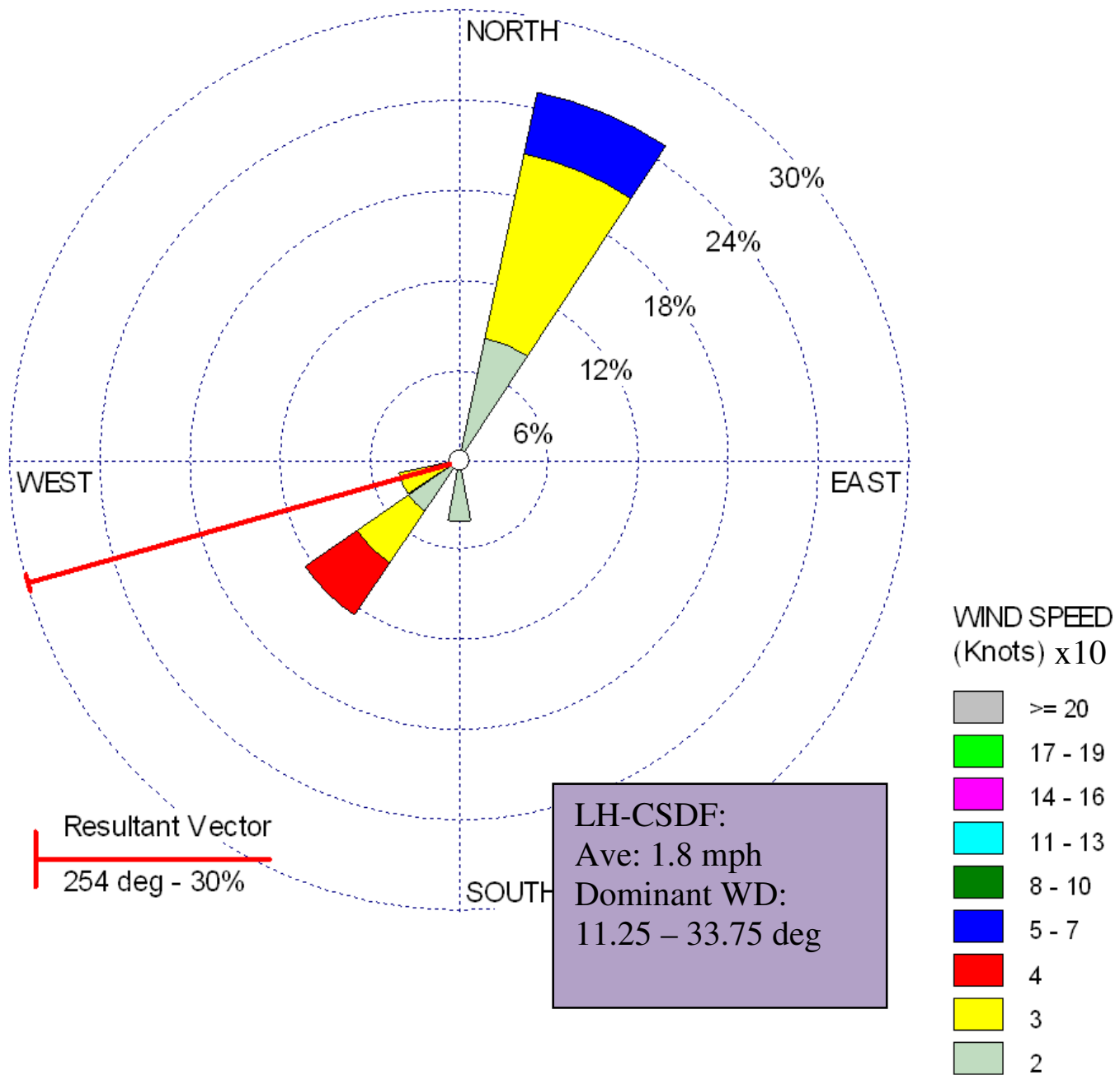

Calms: $54.17 \%$ 
IOP 8b: March 15, 2010

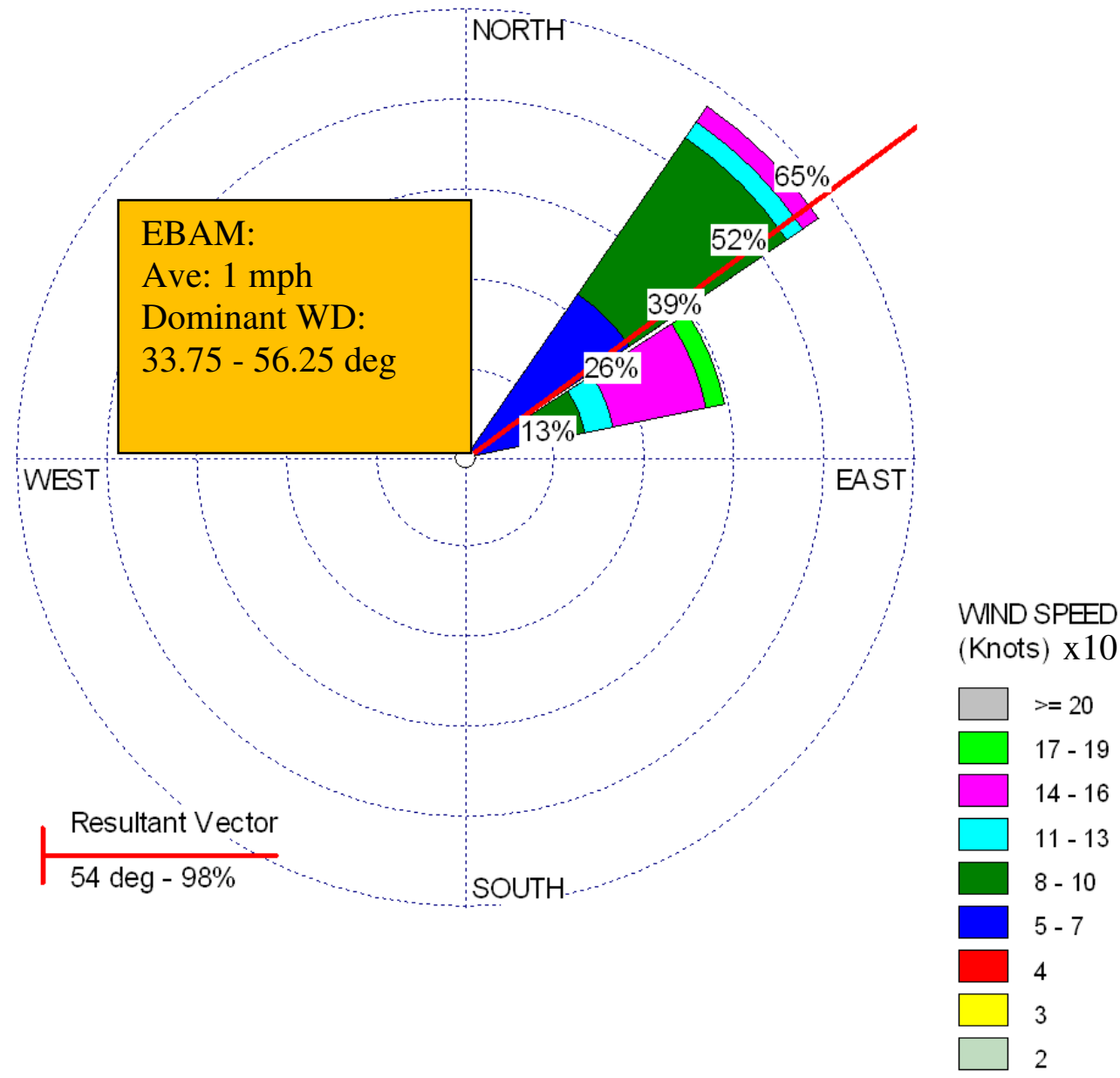

Calms: $0.00 \%$ 


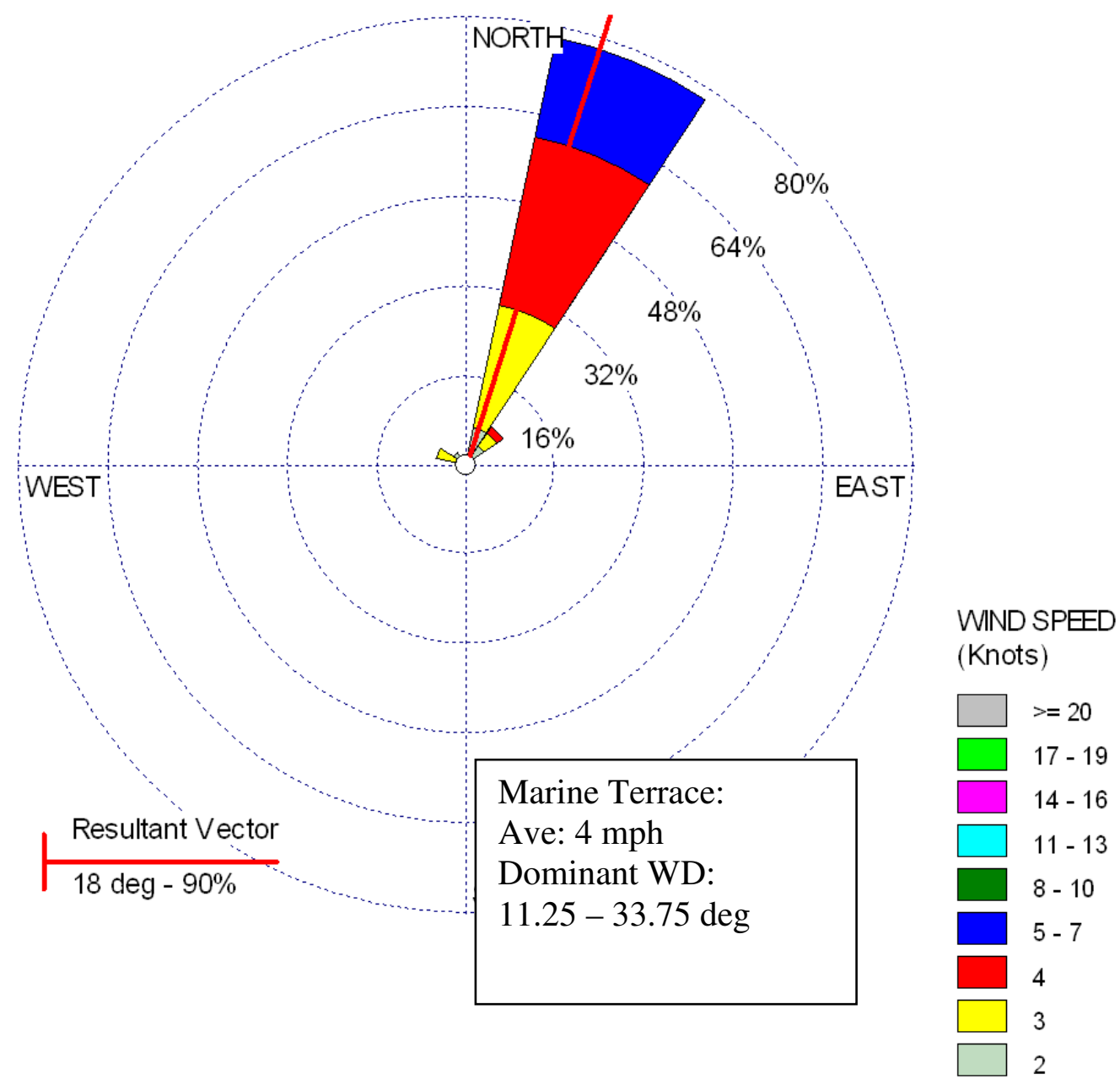

Calms: $4.17 \%$ 


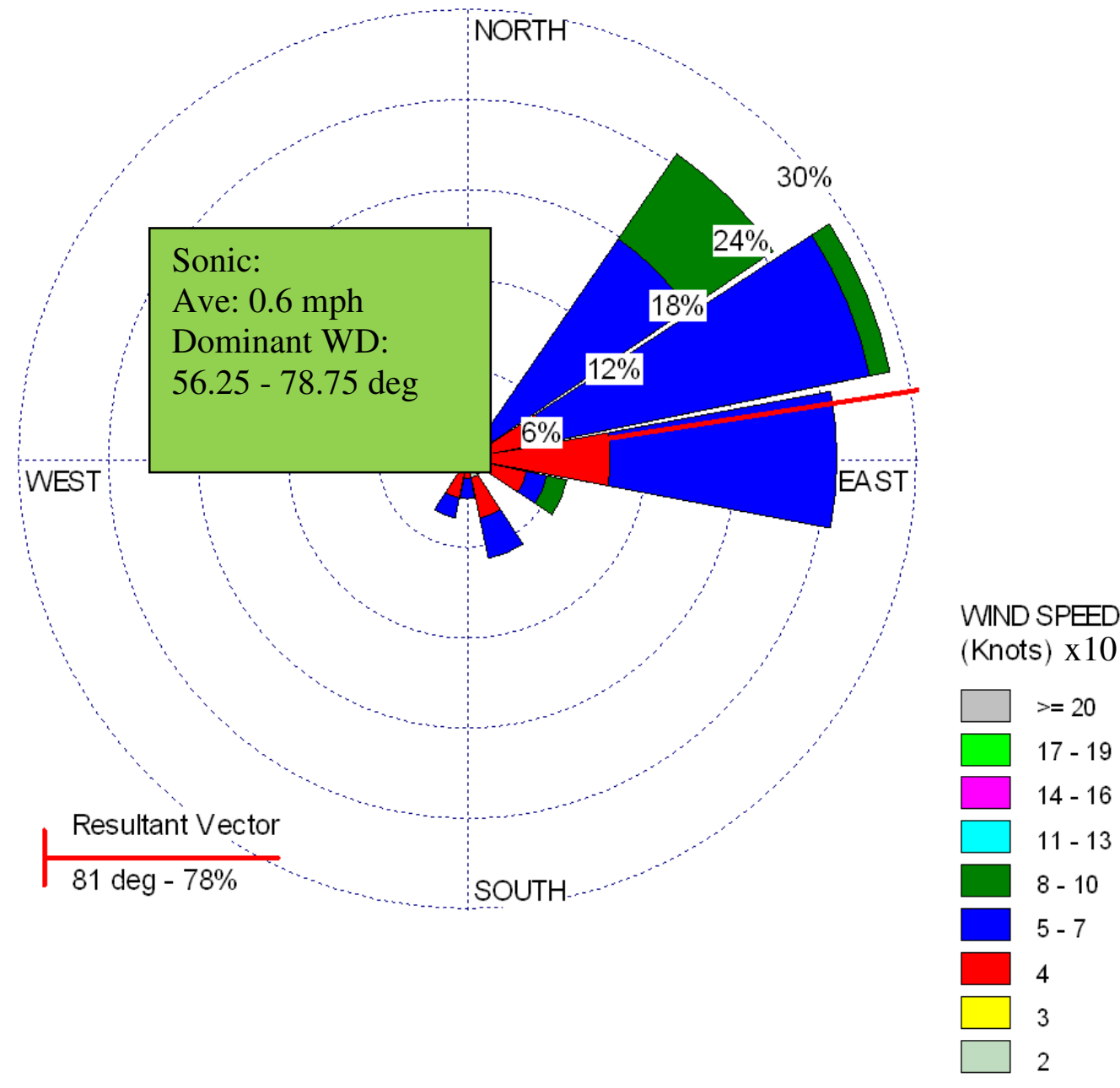

Calms: $0.00 \%$ 


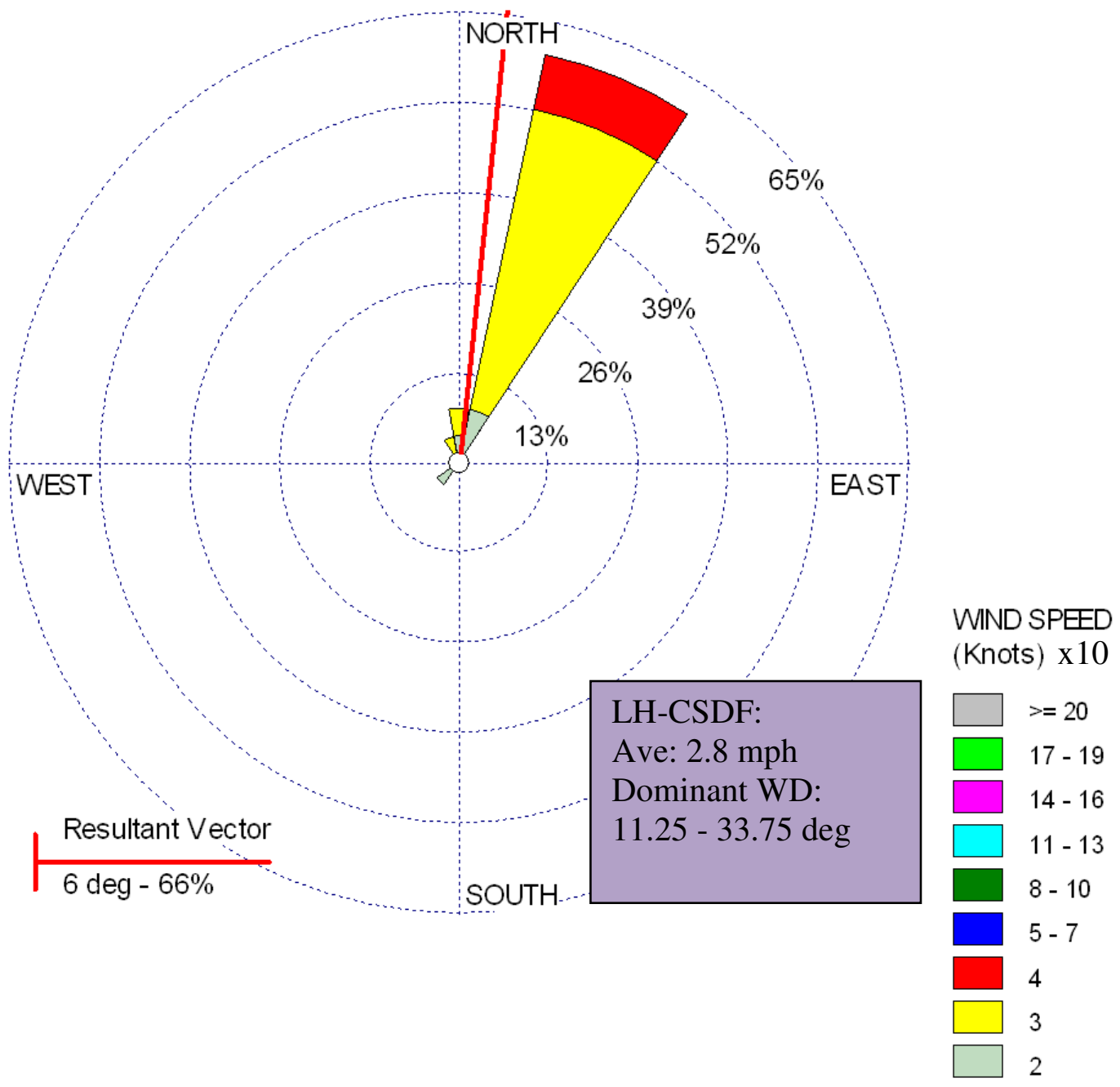

Calms: $24.00 \%$ 


\section{IOP 9b: April 3, 2010}

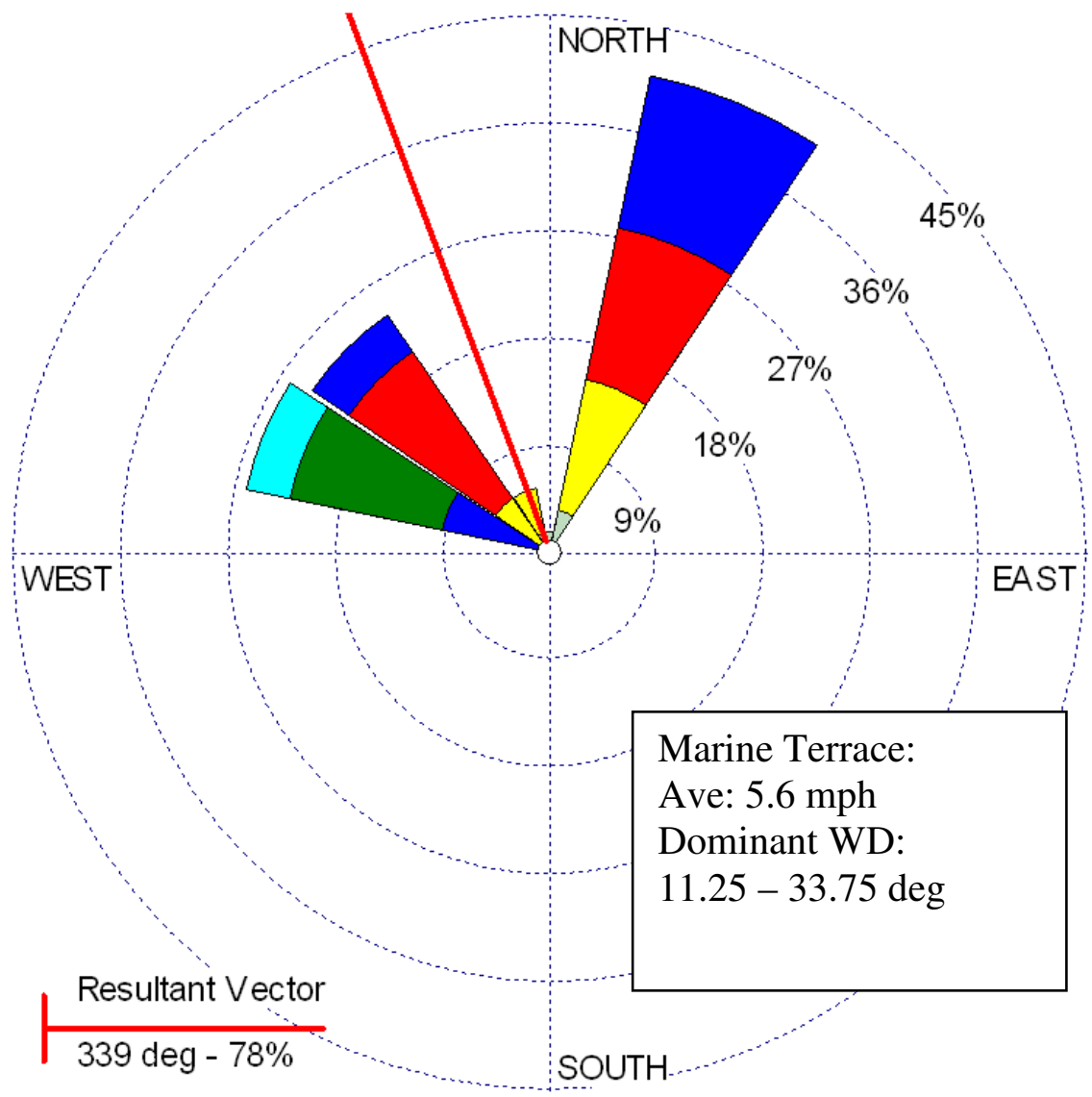

WIND SPEED (Knots)

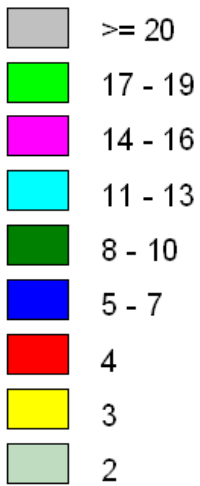

Calms: $1.85 \%$ 


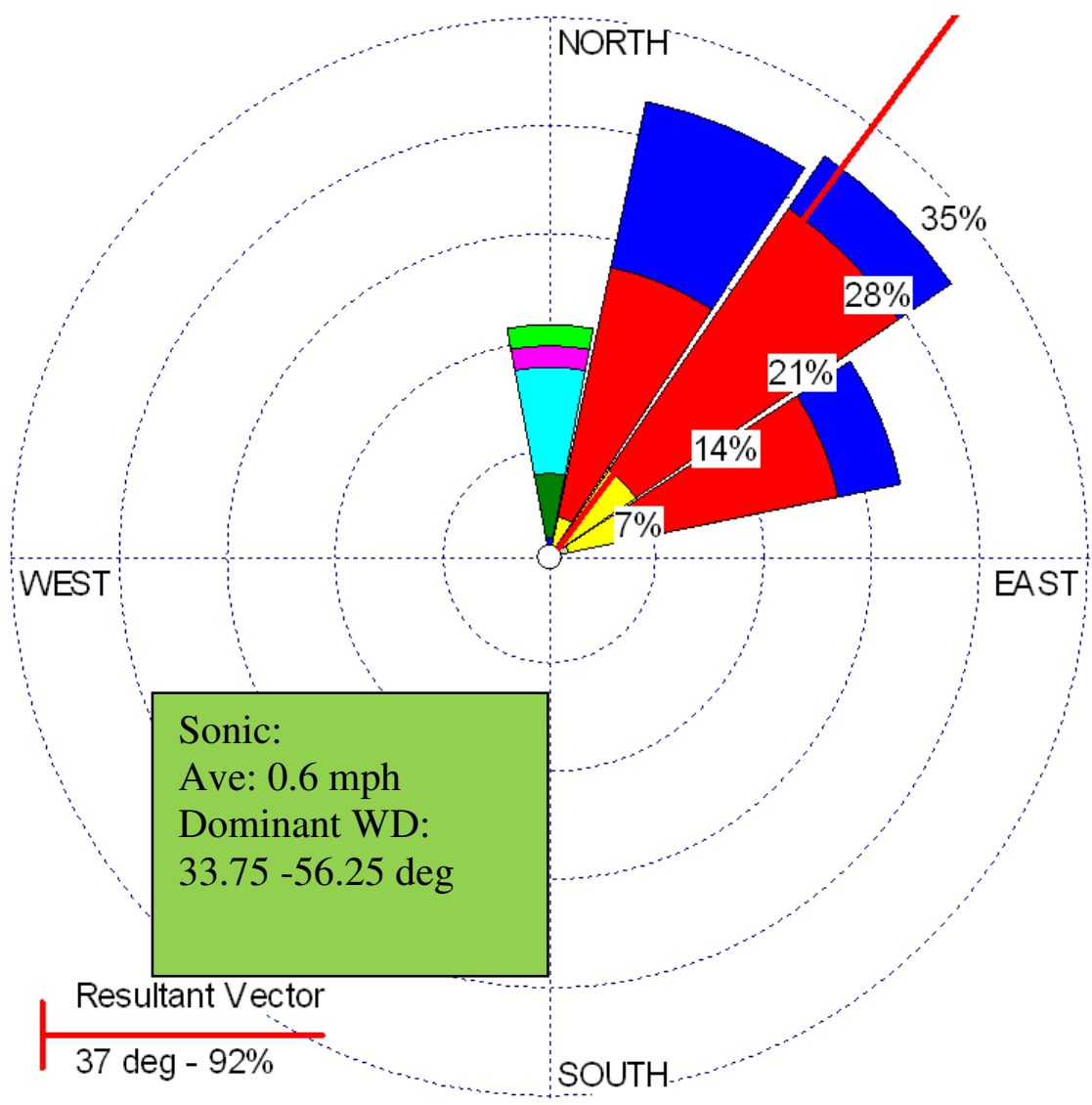

WIND SPEED

(Knots) X 10

$\begin{array}{ll}\square & >=20 \\ \square & 17-19 \\ & 14-16 \\ \square & 11-13 \\ \square & 8-10 \\ & 5-7 \\ \square & 4 \\ \square & 3 \\ \square & 2\end{array}$

Calms: $0.00 \%$ 


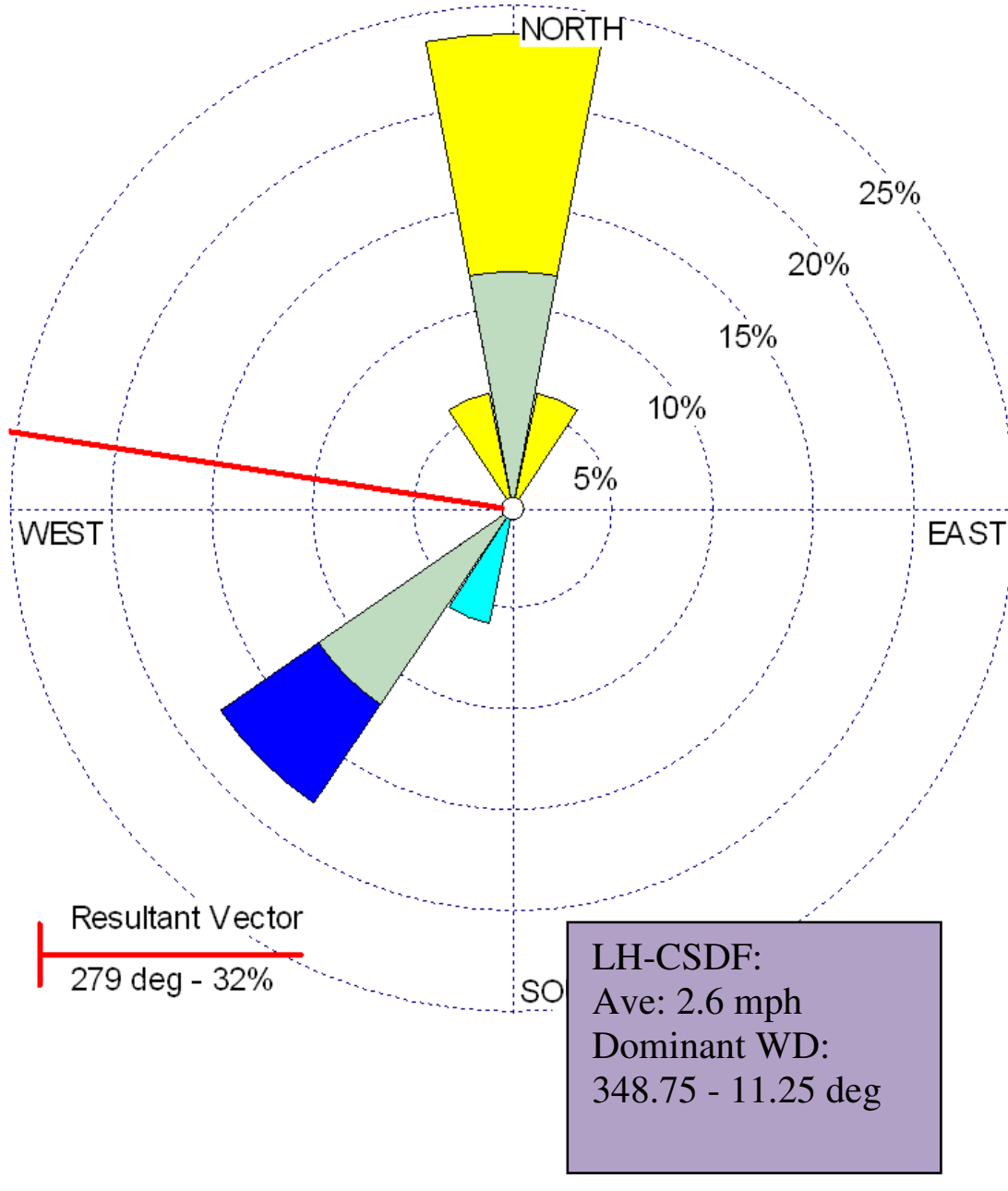

WIND SPEED

(Knots) x 10

\begin{tabular}{ll}
\hline & $>=20$ \\
$\square$ & $17-19$ \\
$\square$ & $14-16$ \\
$\square$ & $11-13$ \\
$\square$ & $8-10$ \\
$\square$ & $5-7$ \\
$\square$ & 4 \\
$\square$ & 3 \\
$\square$ & 2
\end{tabular}

Calms: $41.18 \%$ 


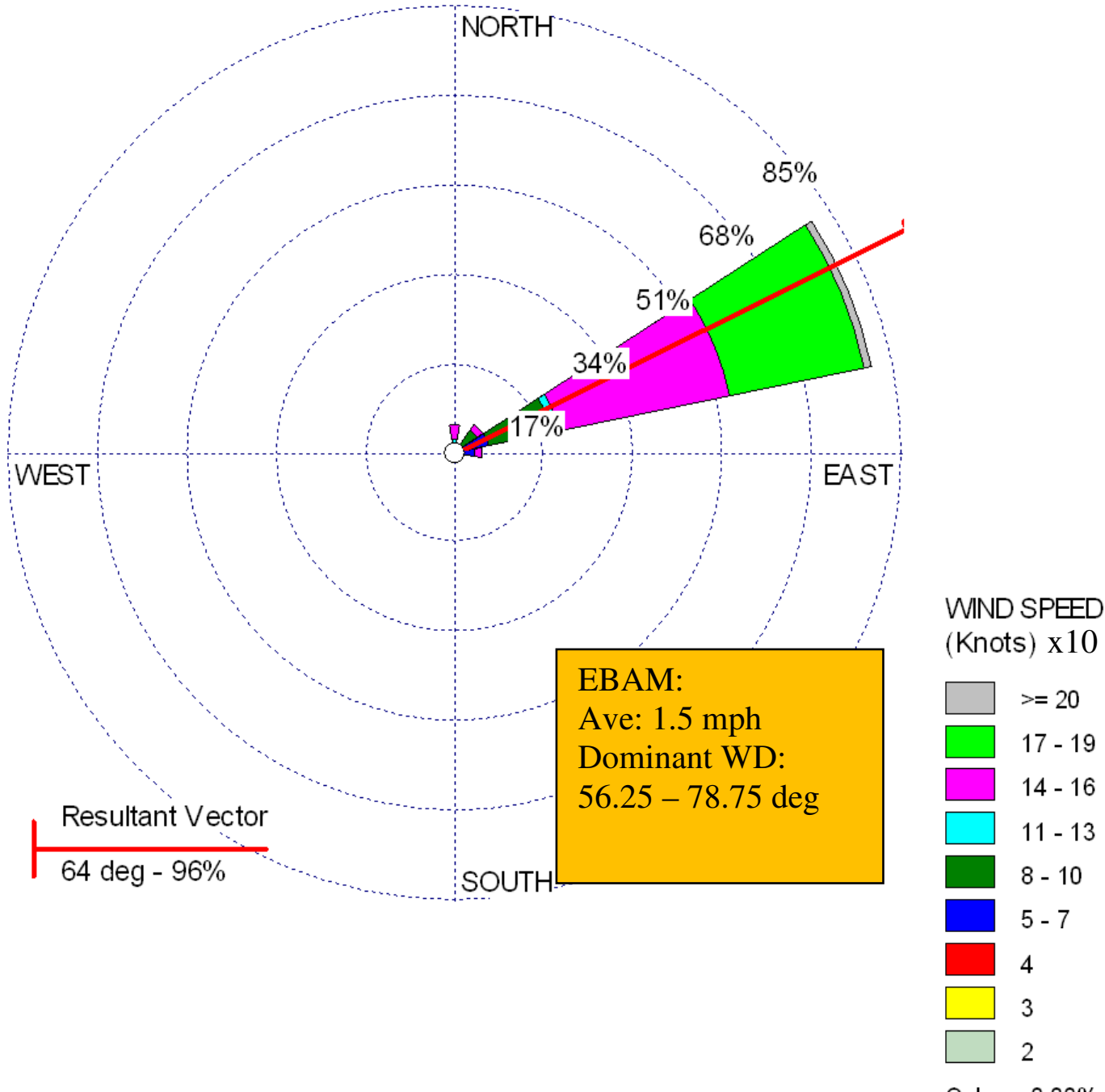

Calms: $0.00 \%$ 


\section{IOP 10b: April 23, 2010}

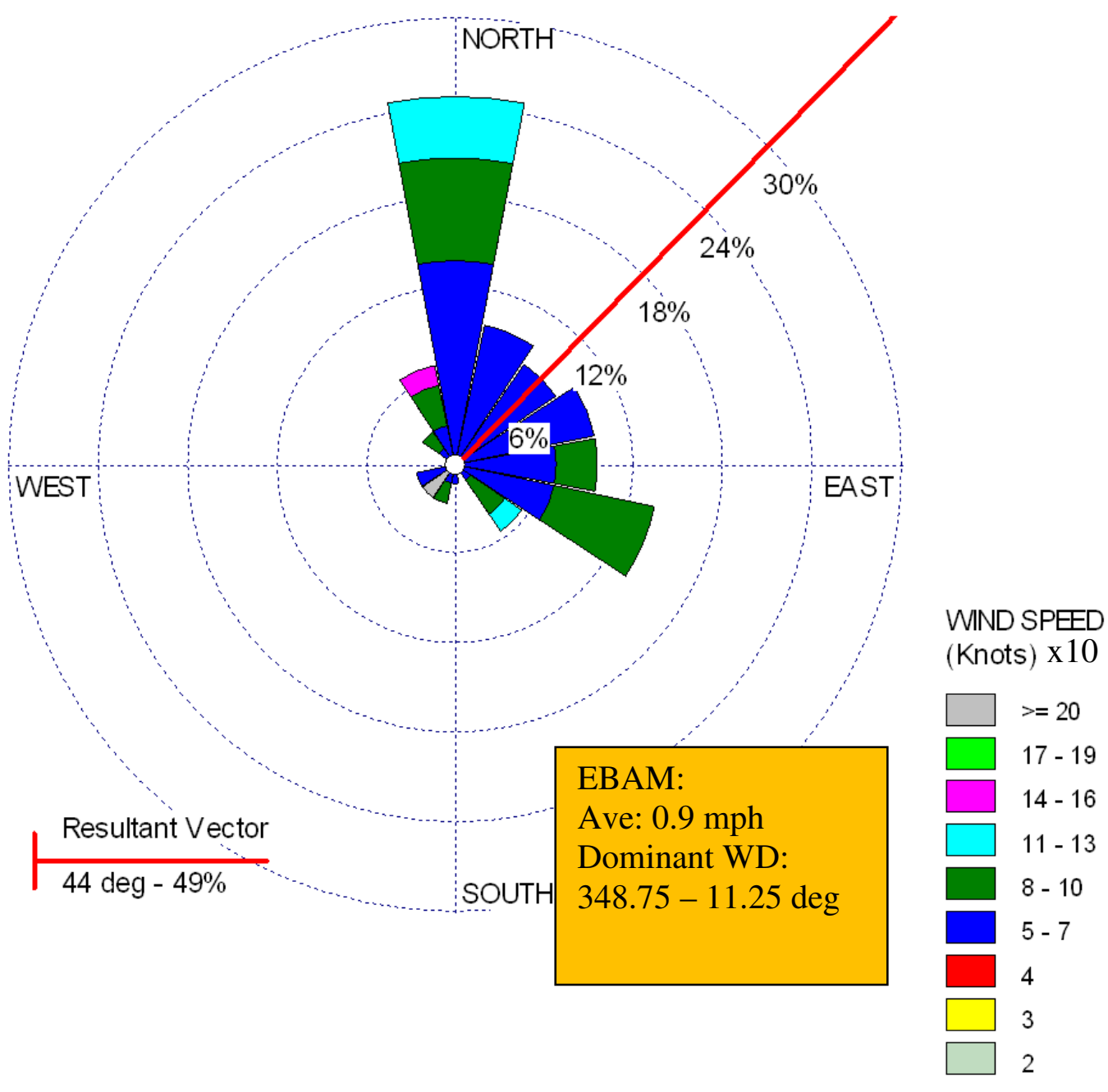

Calms: $0.00 \%$ 


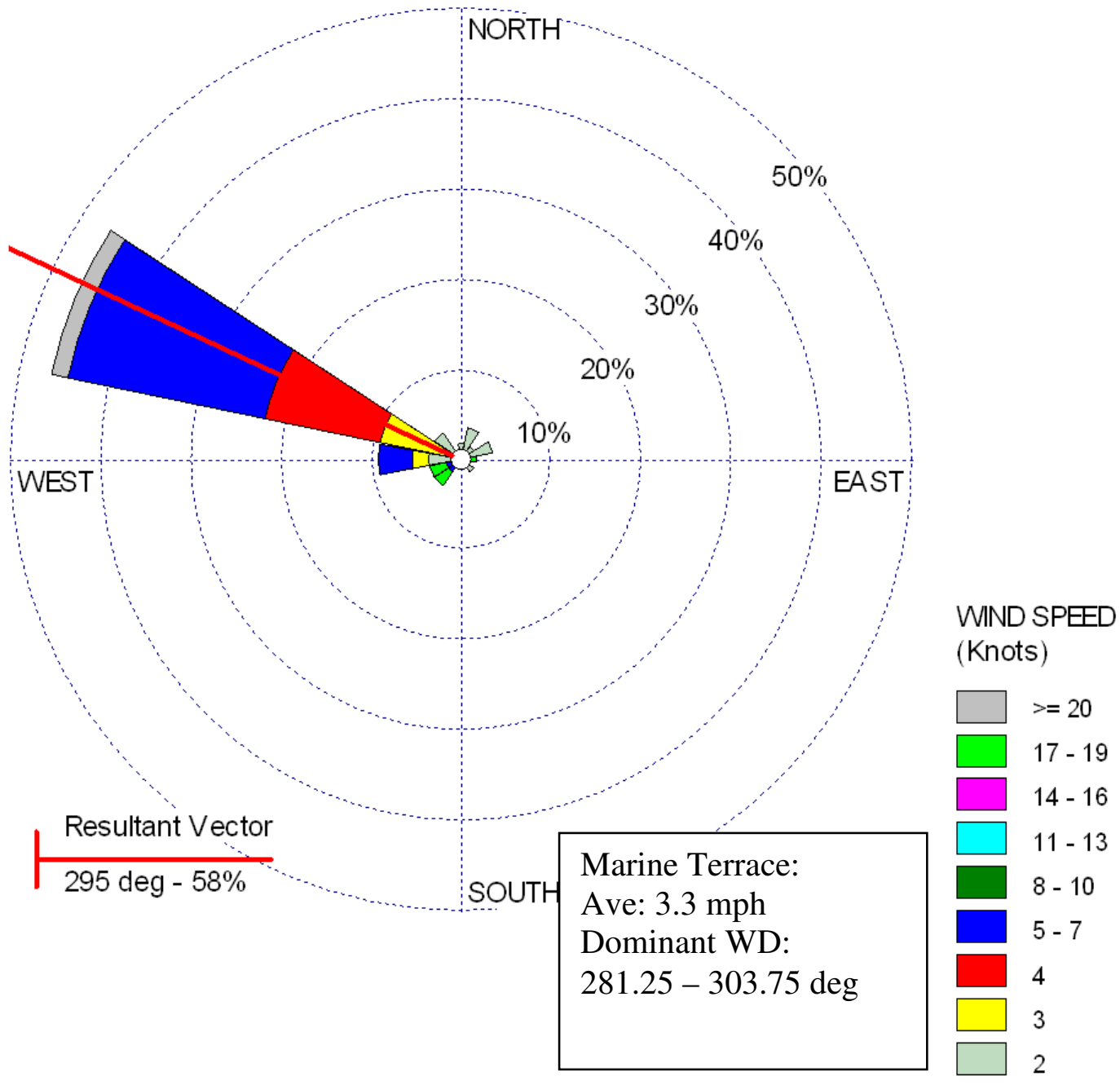

Calms: $18.52 \%$ 


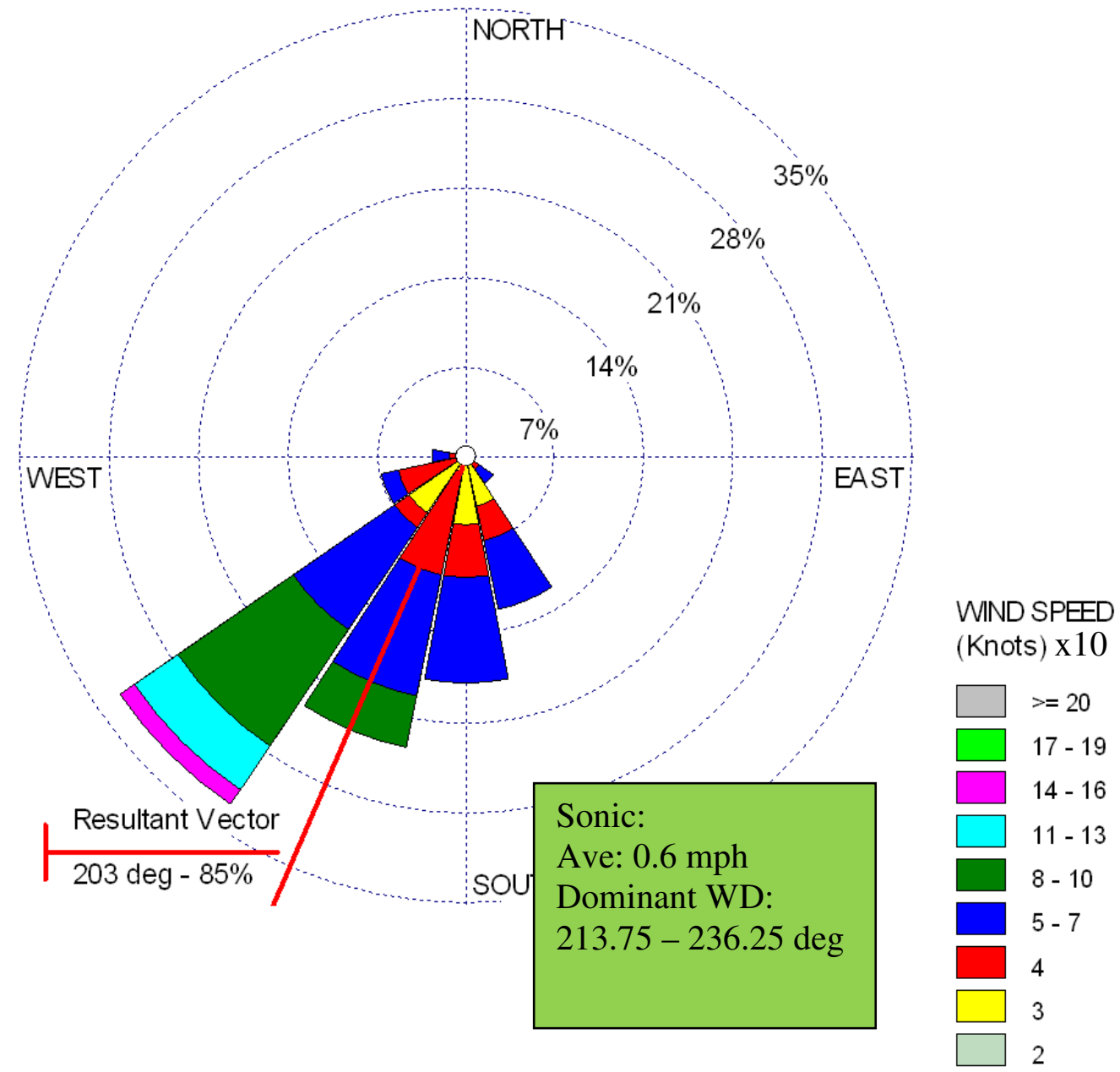

Calms: $1.37 \%$ 


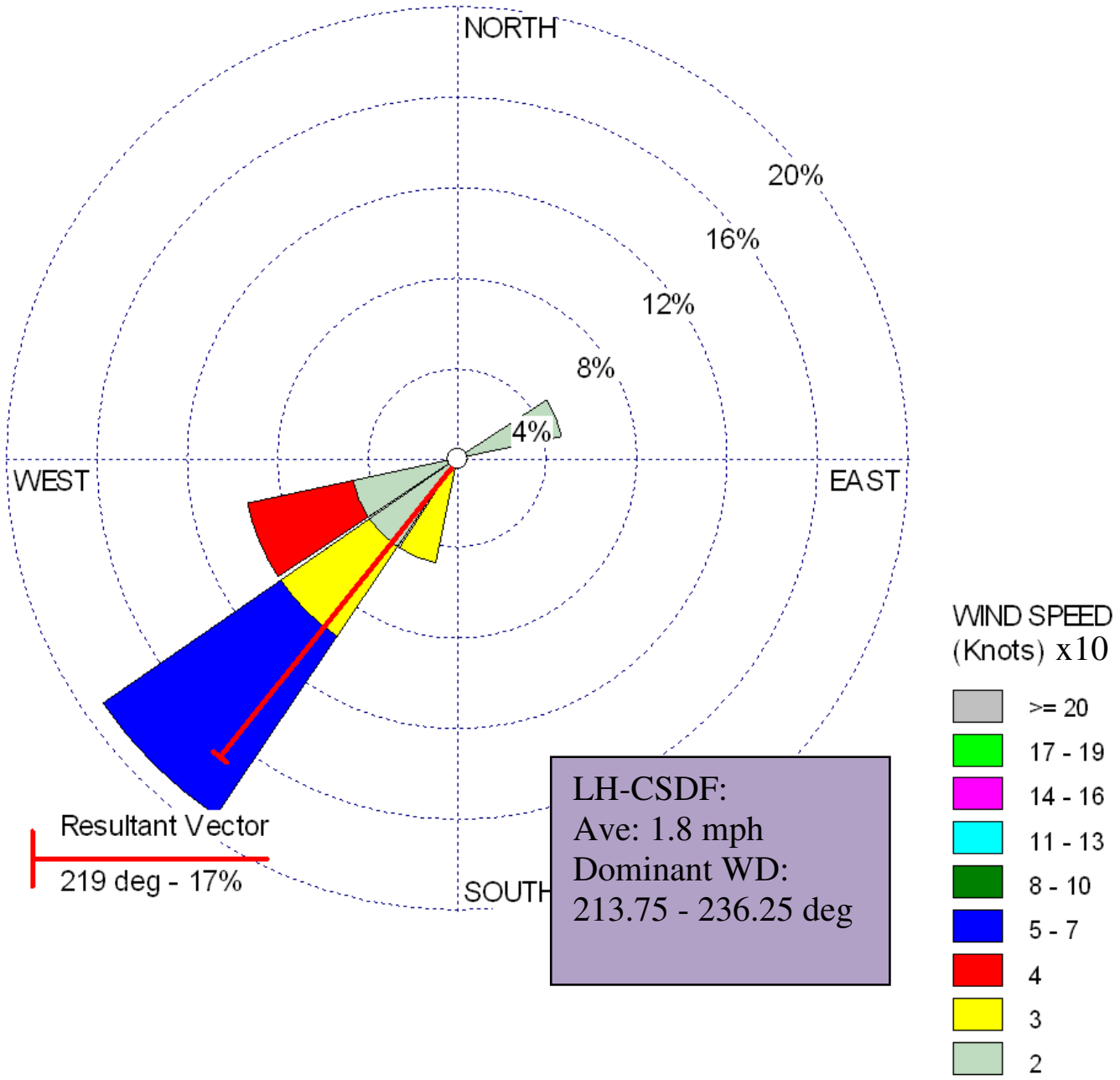

Calms: $61.90 \%$ 


\section{Temperature Profiles}

\section{IOP 1a}

ESRL Physical Sciences Division Wind Profiling Radar

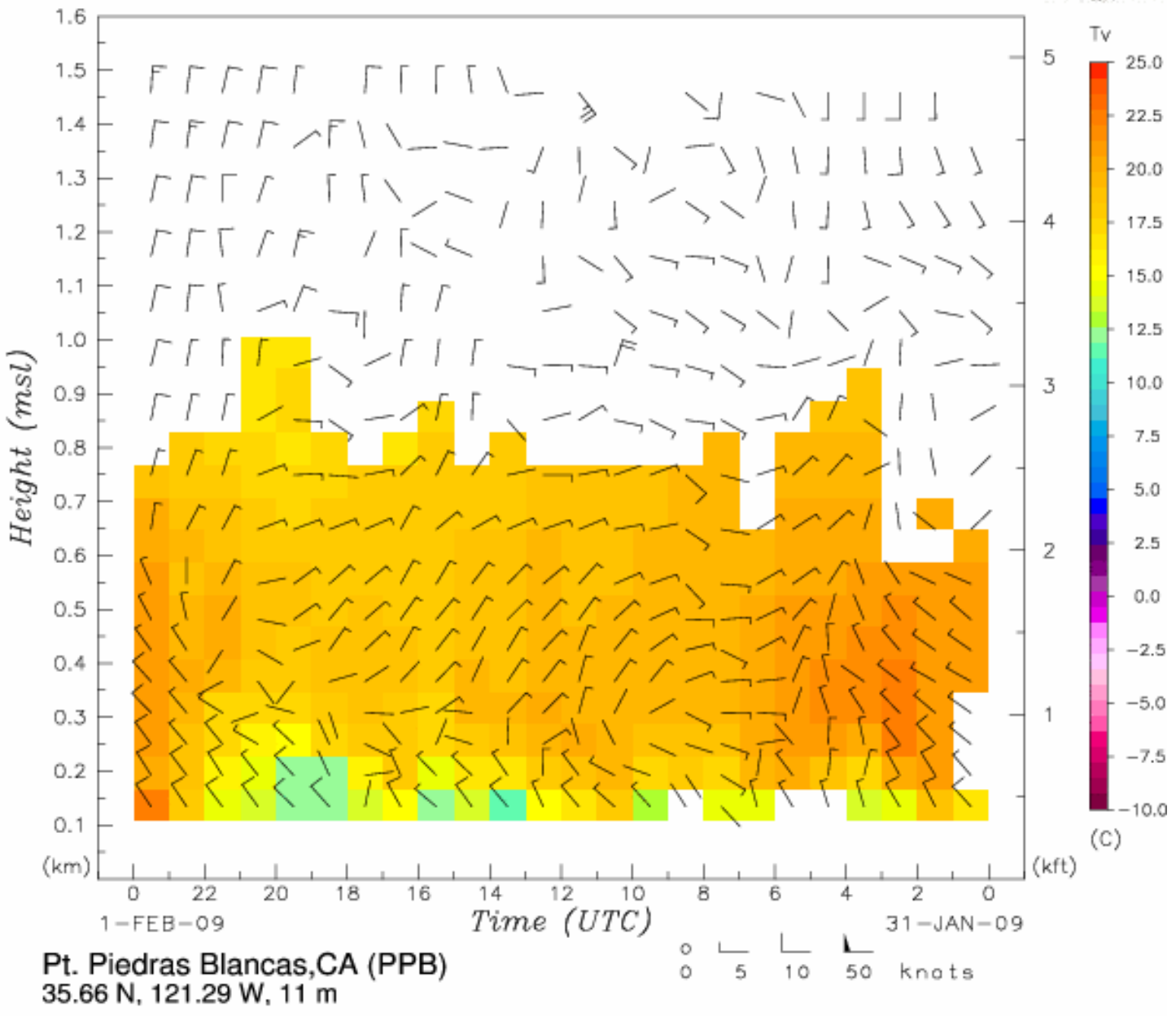




\section{ESRL Physical Sciences Division Wind Profiling Radar}

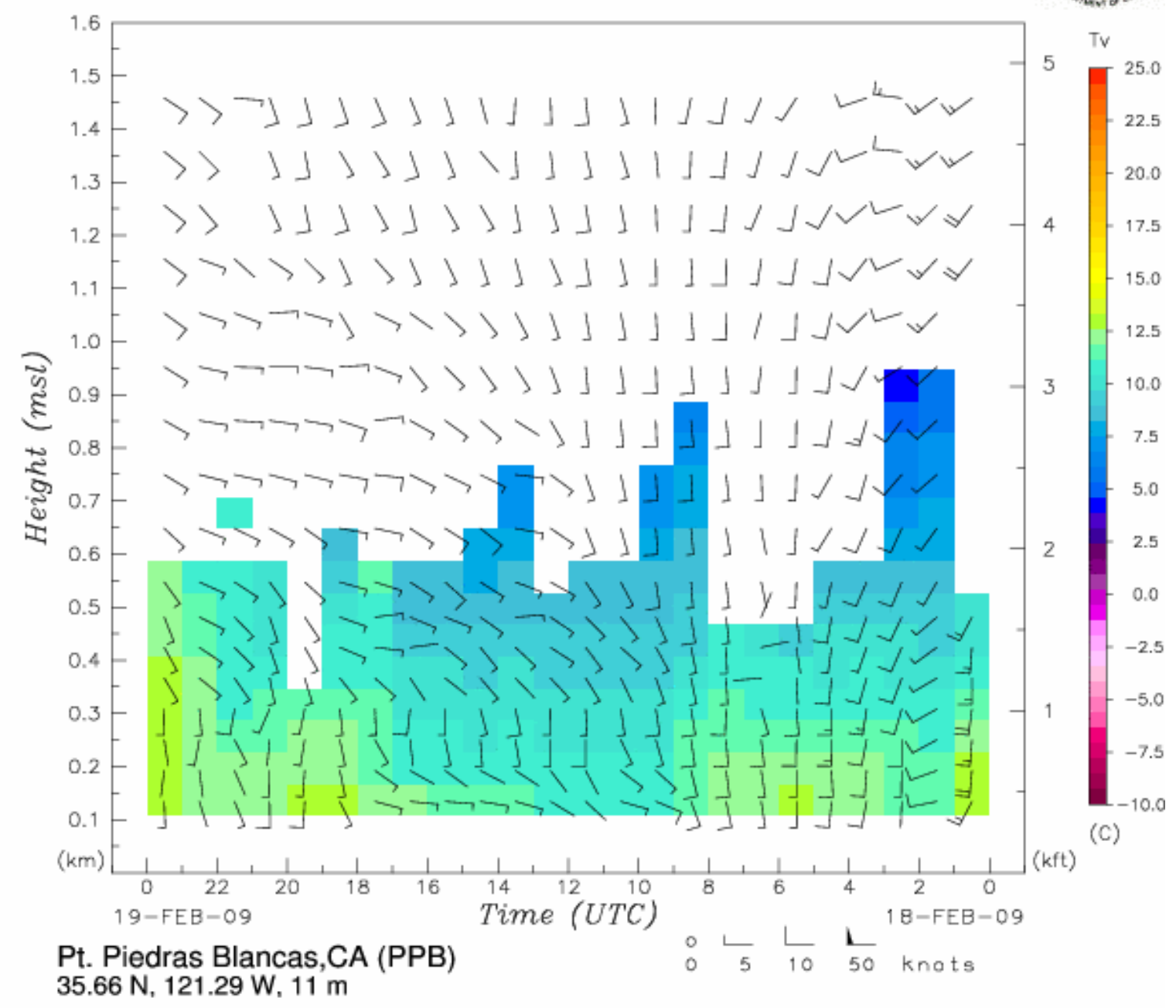




\section{IOP 3a}

\section{ESRL Physical Sciences Division Wind Profiling Radar}

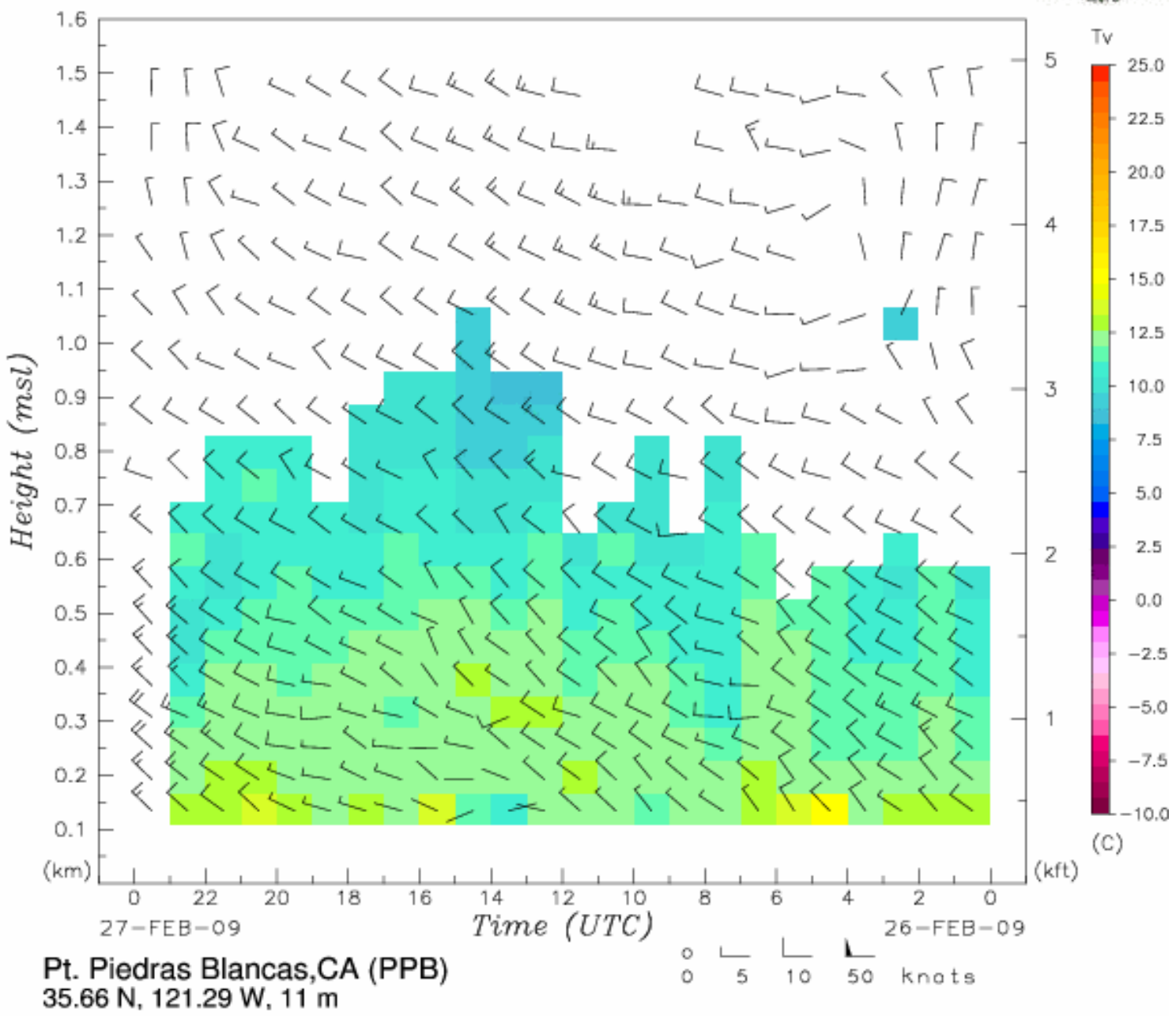




\section{IOP 4a}

ESRL Physical Sciences Division Wind Profiling Radar

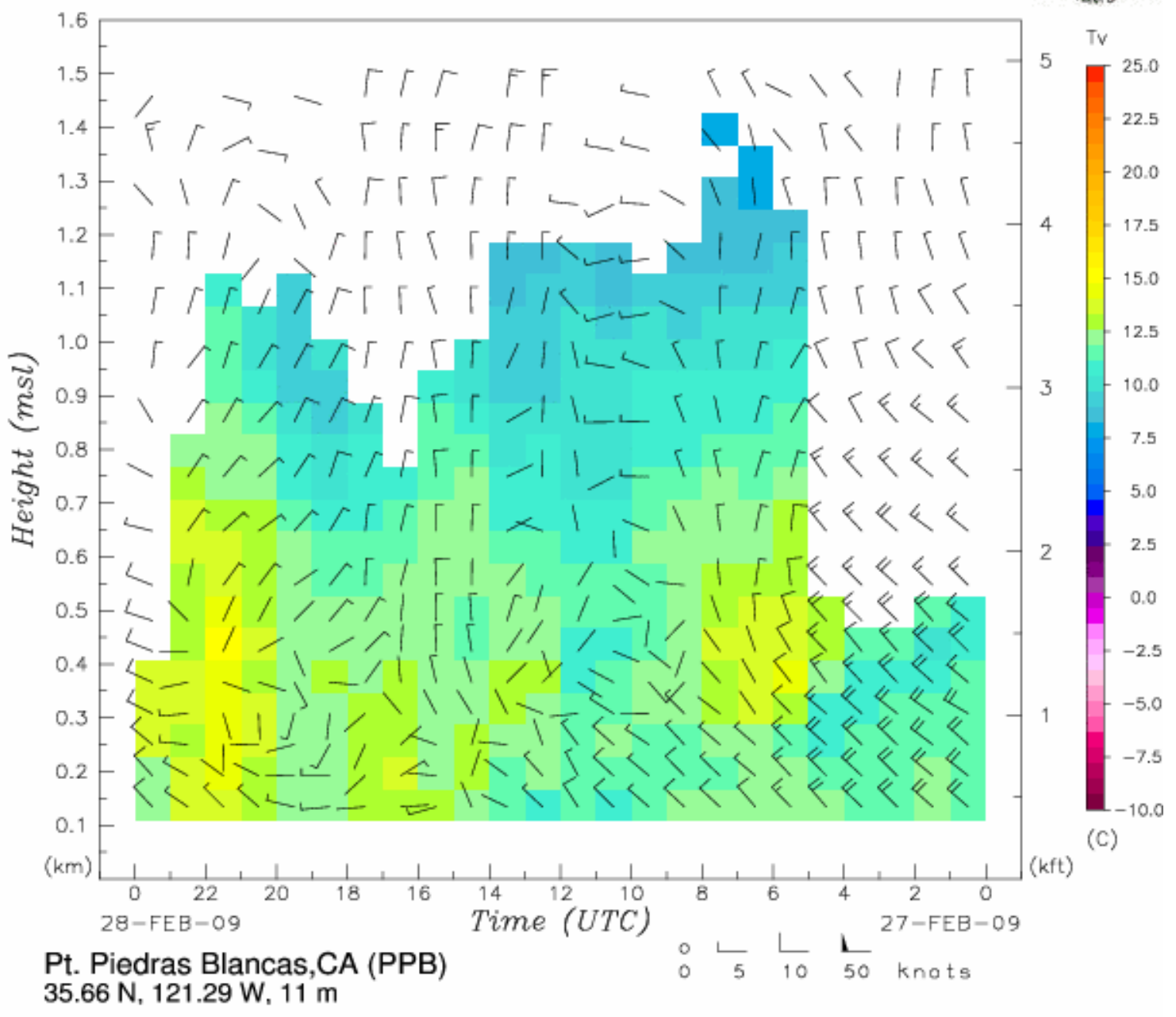




\section{IOP 2b}

ESRL Physical Sciences Division Wind Profiling Radar

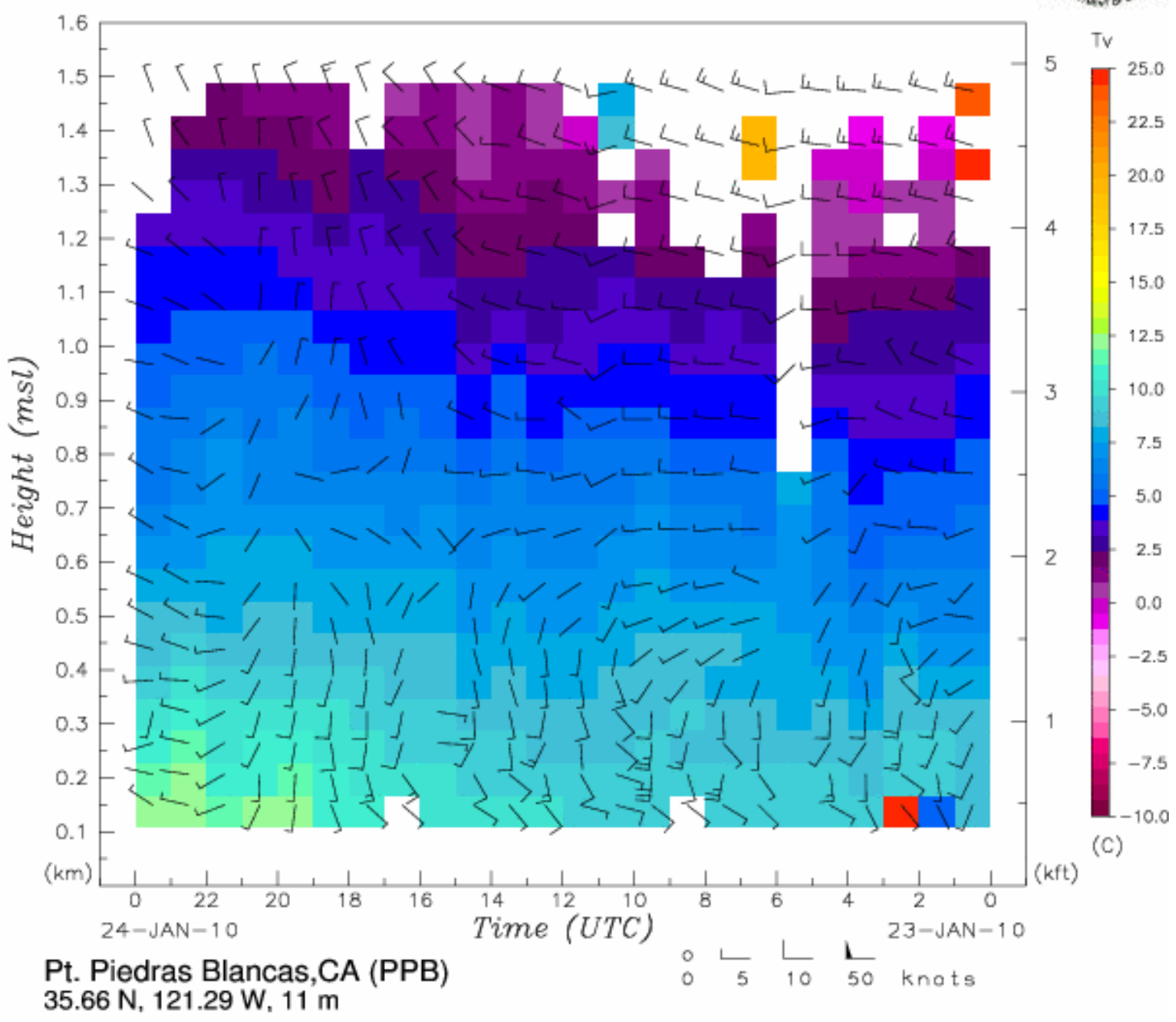




\section{IOP 3b}

\section{ESRL Physical Sciences Division Wind Profiling Radar}

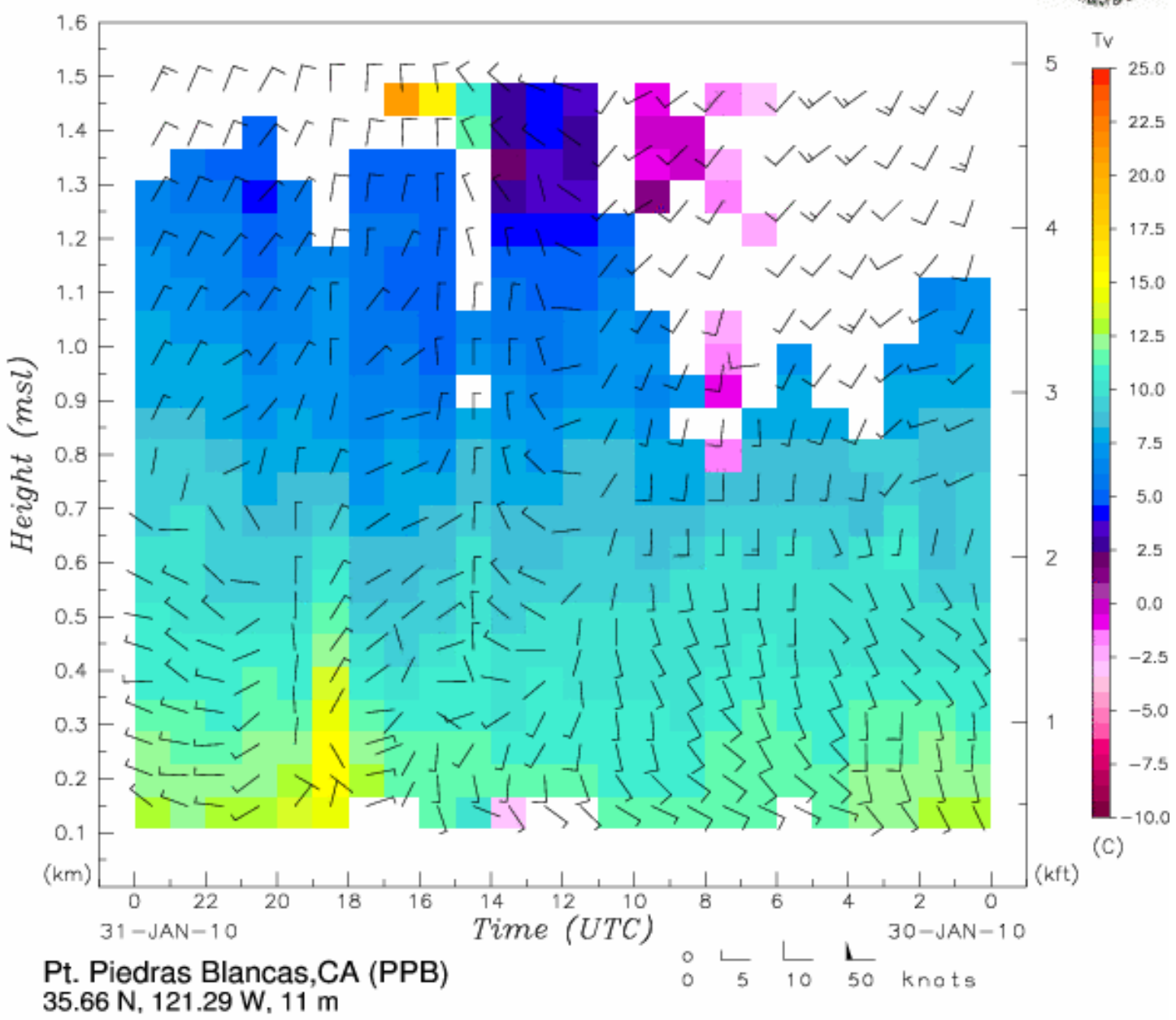




\section{IOP4b}

\section{ESRL Physical Sciences Division Wind Profiling Radar}

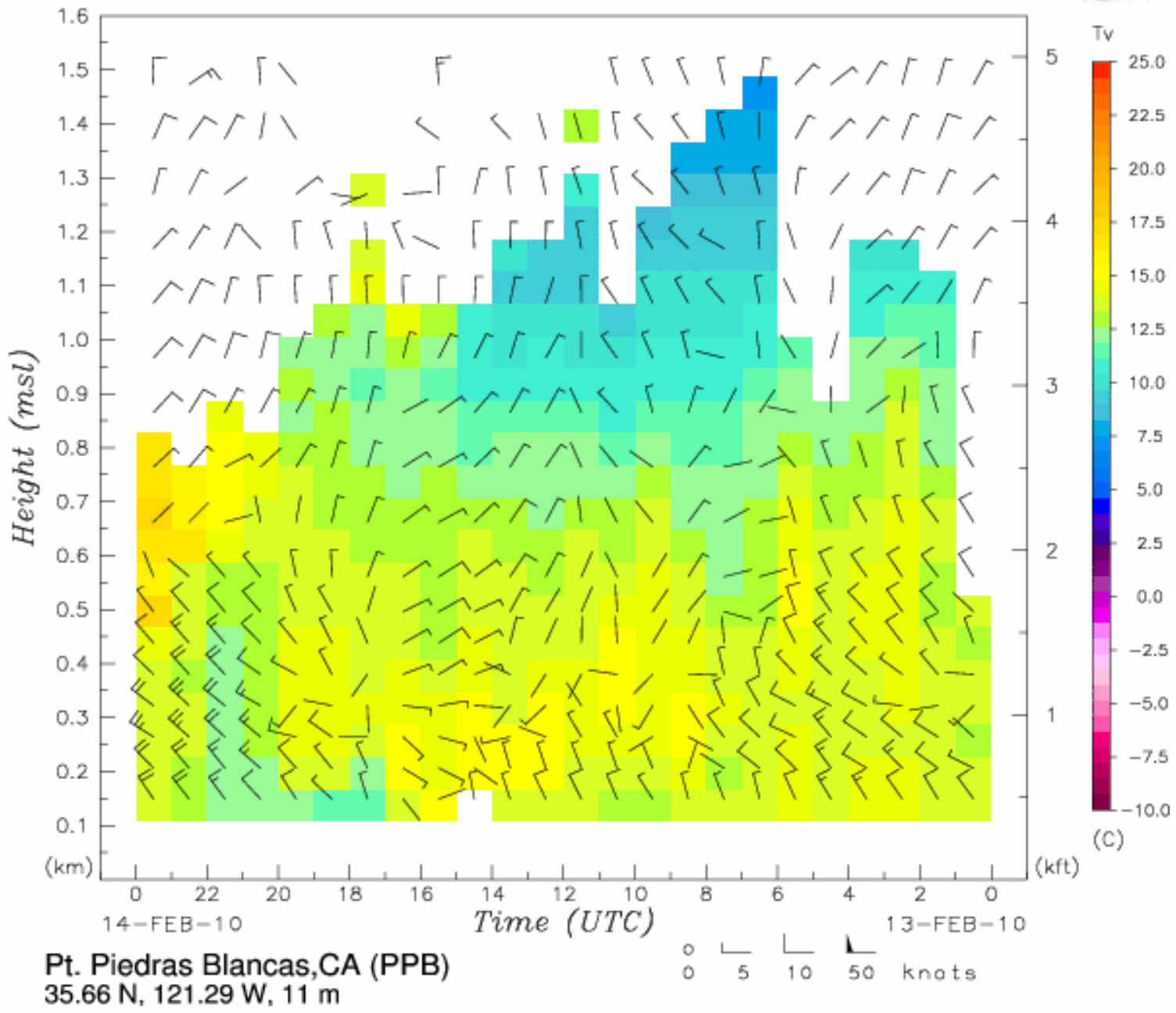




\section{IOP 5b}

\section{ESRL Physical Sciences Division Wind Profiling Radar}

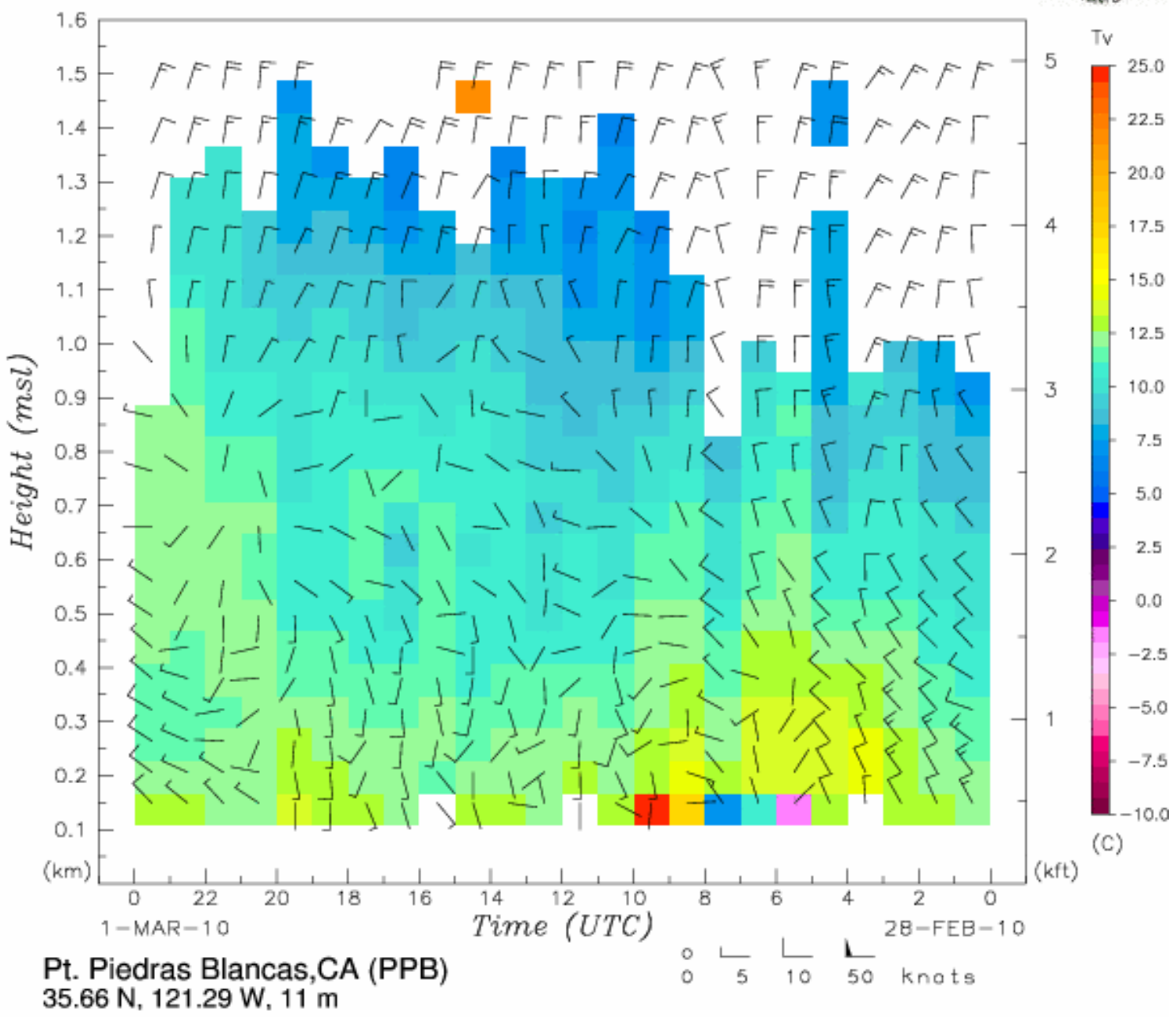




\section{IOP 6b}

\section{ESRL Physical Sciences Division Wind Profiling Radar}

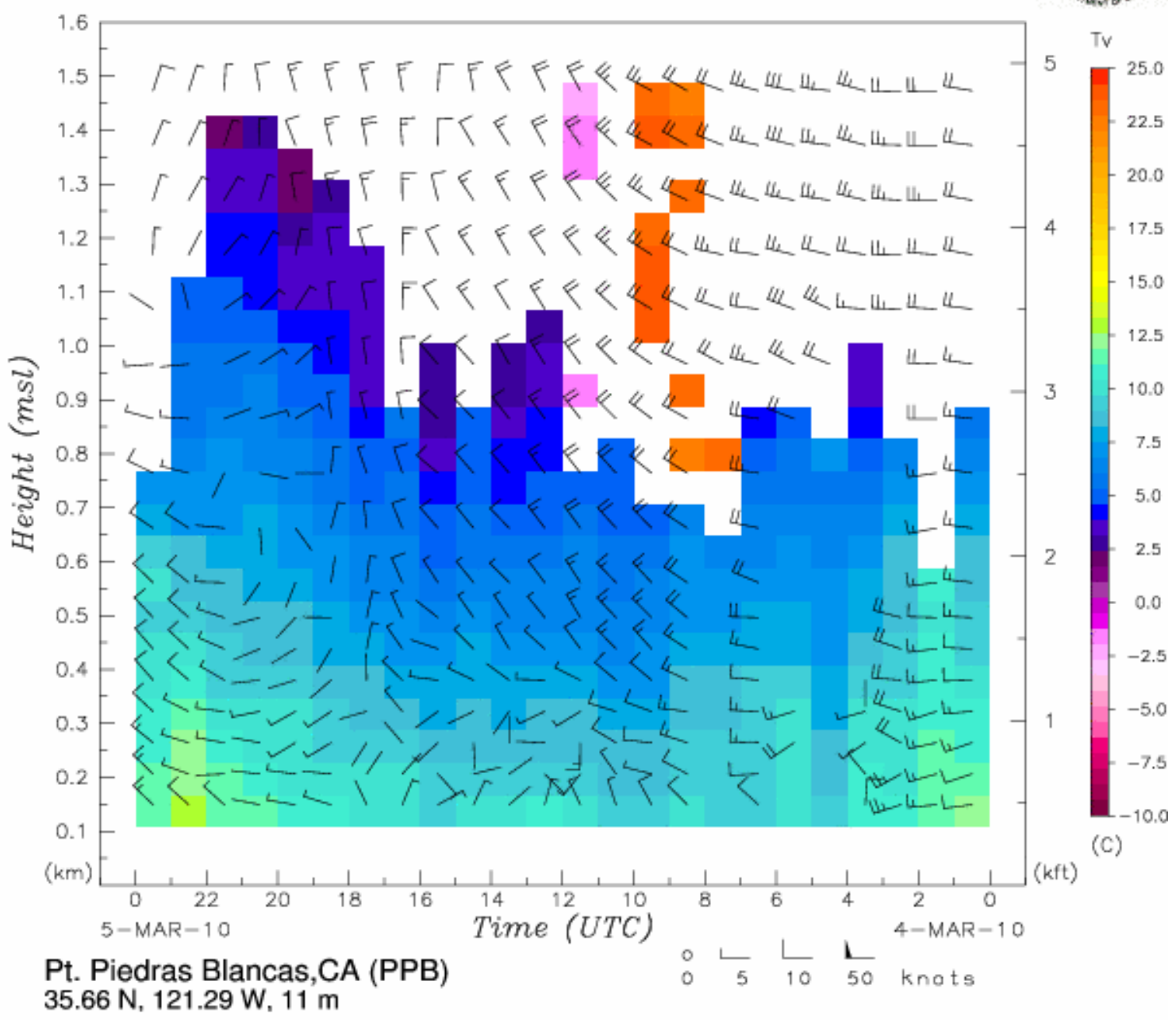




\section{IOP 7b}

\section{ESRL Physical Sciences Division Wind Profiling Radar}

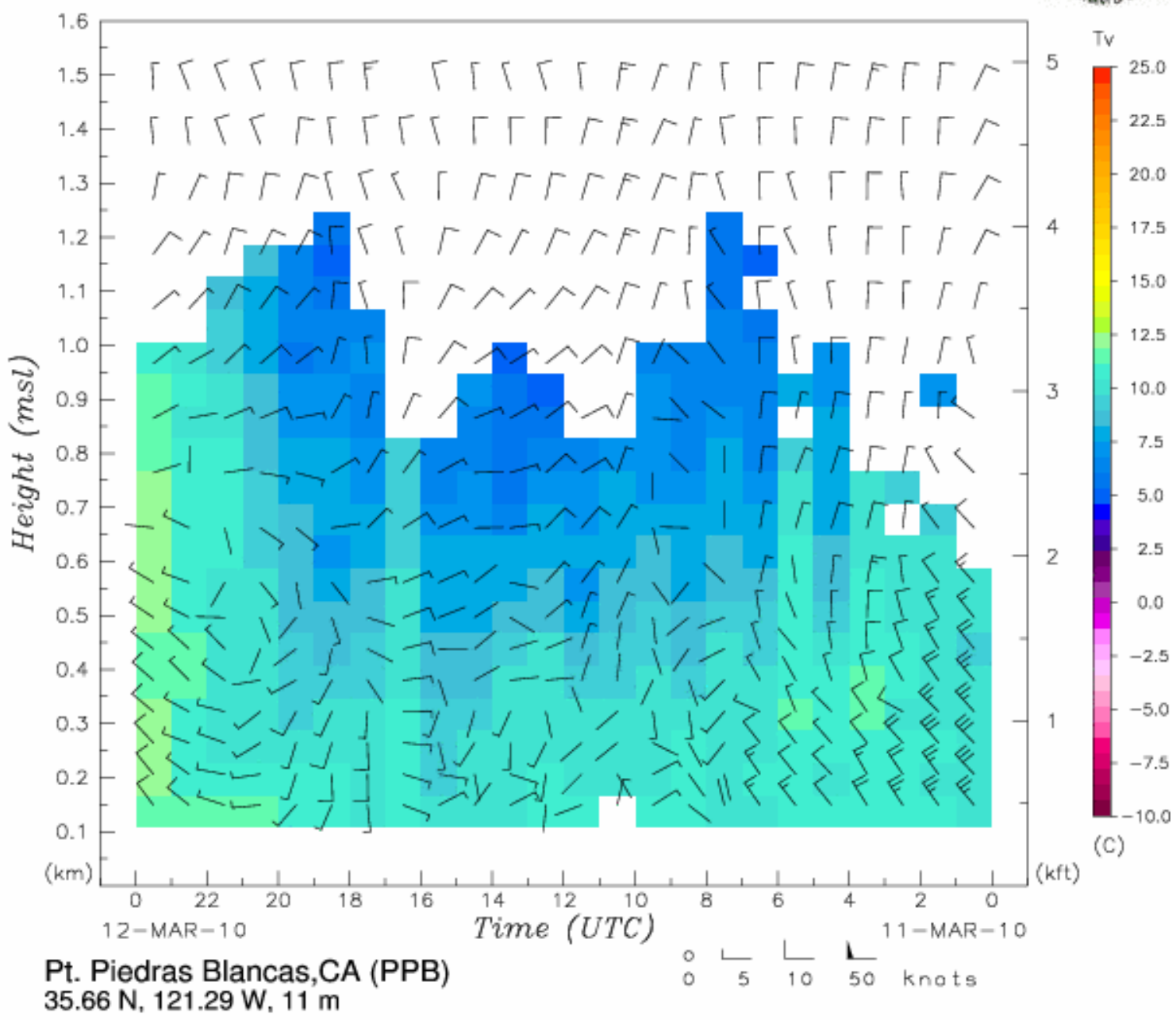




\section{IOP 8b}

\section{ESRL Physical Sciences Division}

Wind Profiling Radar

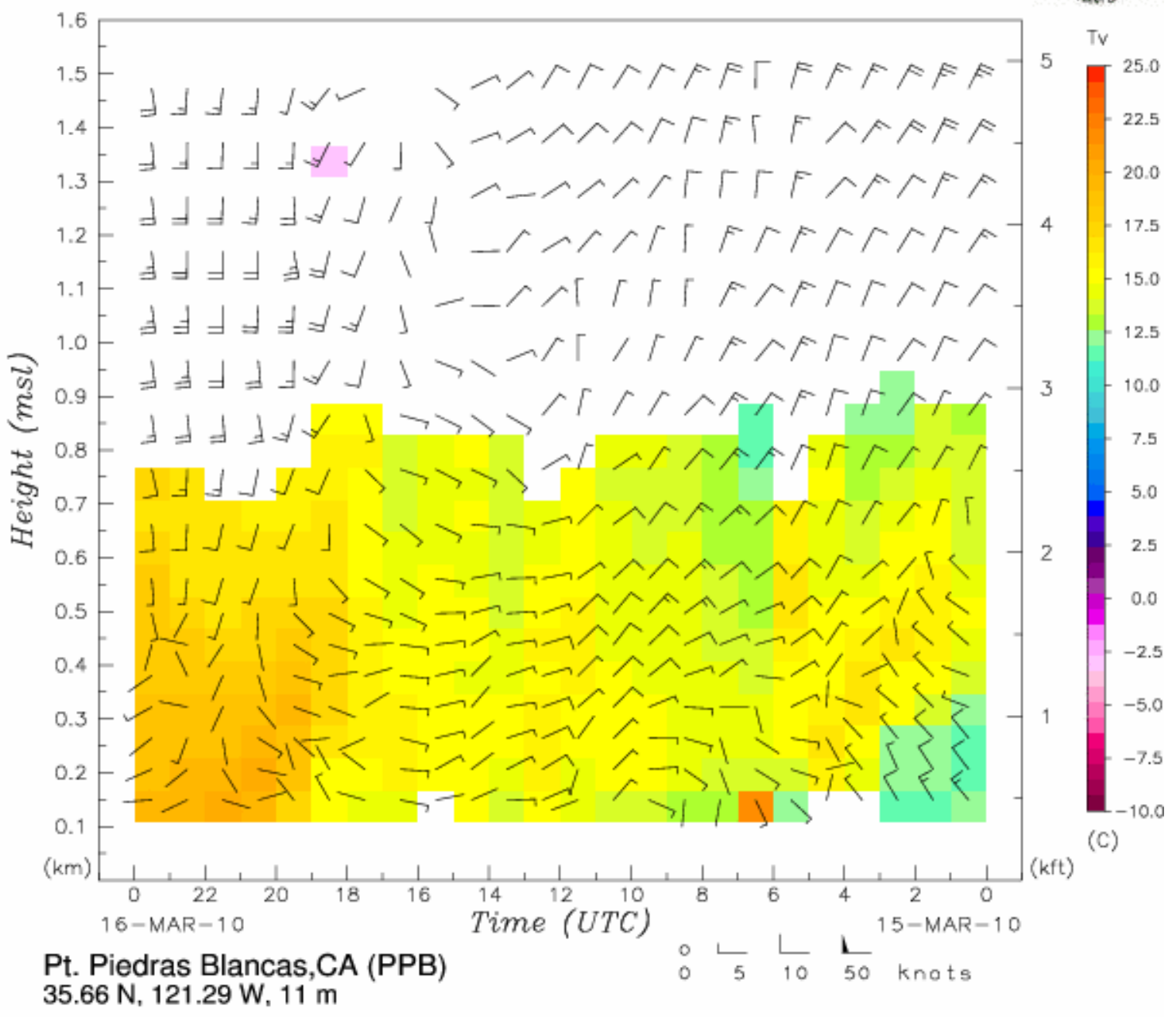




\section{IOP 9b}

\section{ESRL Physical Sciences Division Wind Profiling Radar}

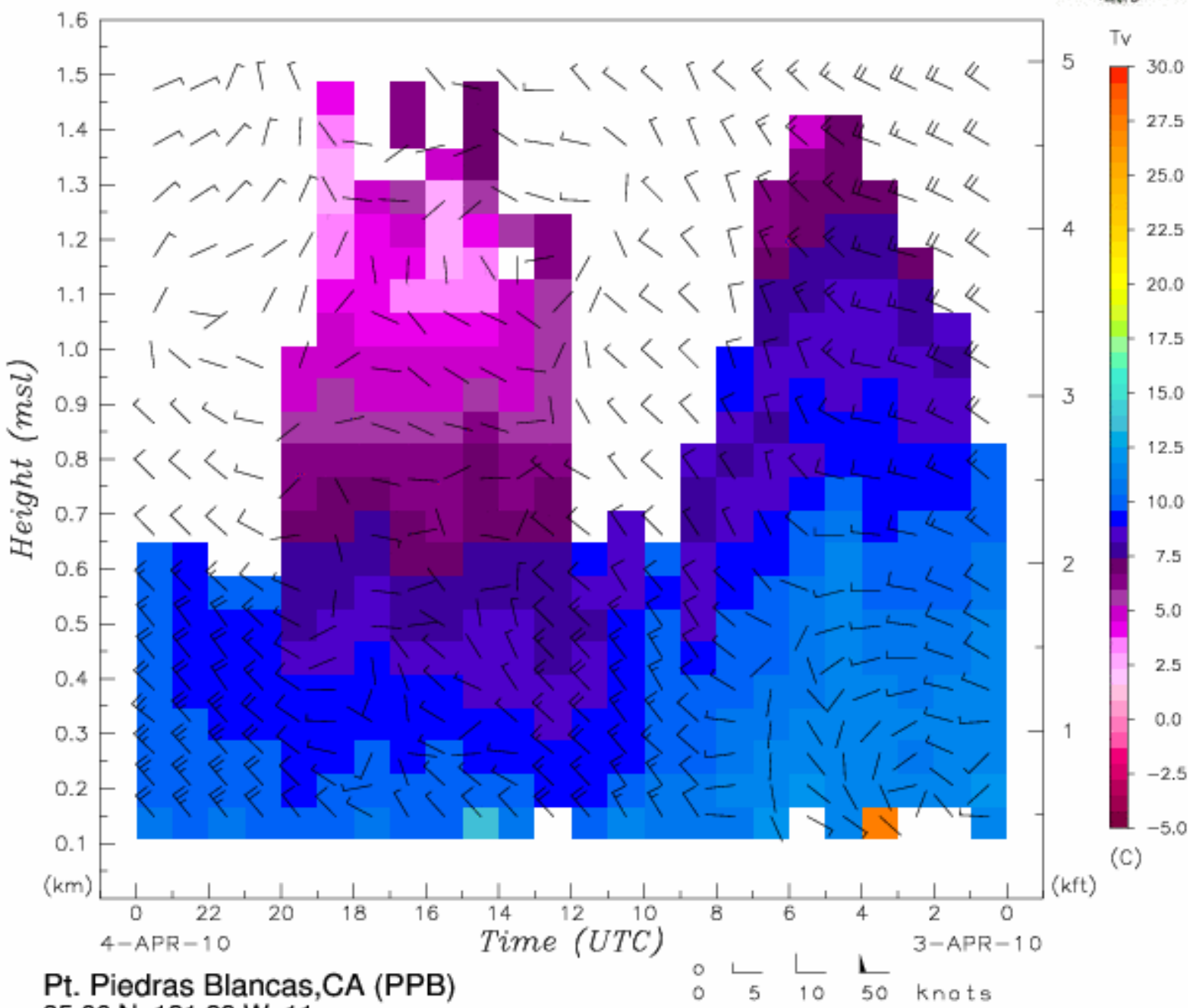

$35.66 \mathrm{~N}, 121.29 \mathrm{~W}, 11 \mathrm{~m}$ 


\section{Appendix F. Graphic Summary for all IOPs}

All IOPs are summarized here to include PEM and aethalometer locations,

concentrations, and spectral dependence, in addition to average wind speed, dominant wind direction, and burning source locations. PEM concentration boxes colored brown indicate an AAE value greater than 1.2 (wood smoke), colored purple indicate an AAE of less than 1.2 (traffic exhaust), and colored pink and labeled LOD indicate limit of detection.

\section{IOP 1a: January 31, 2009}

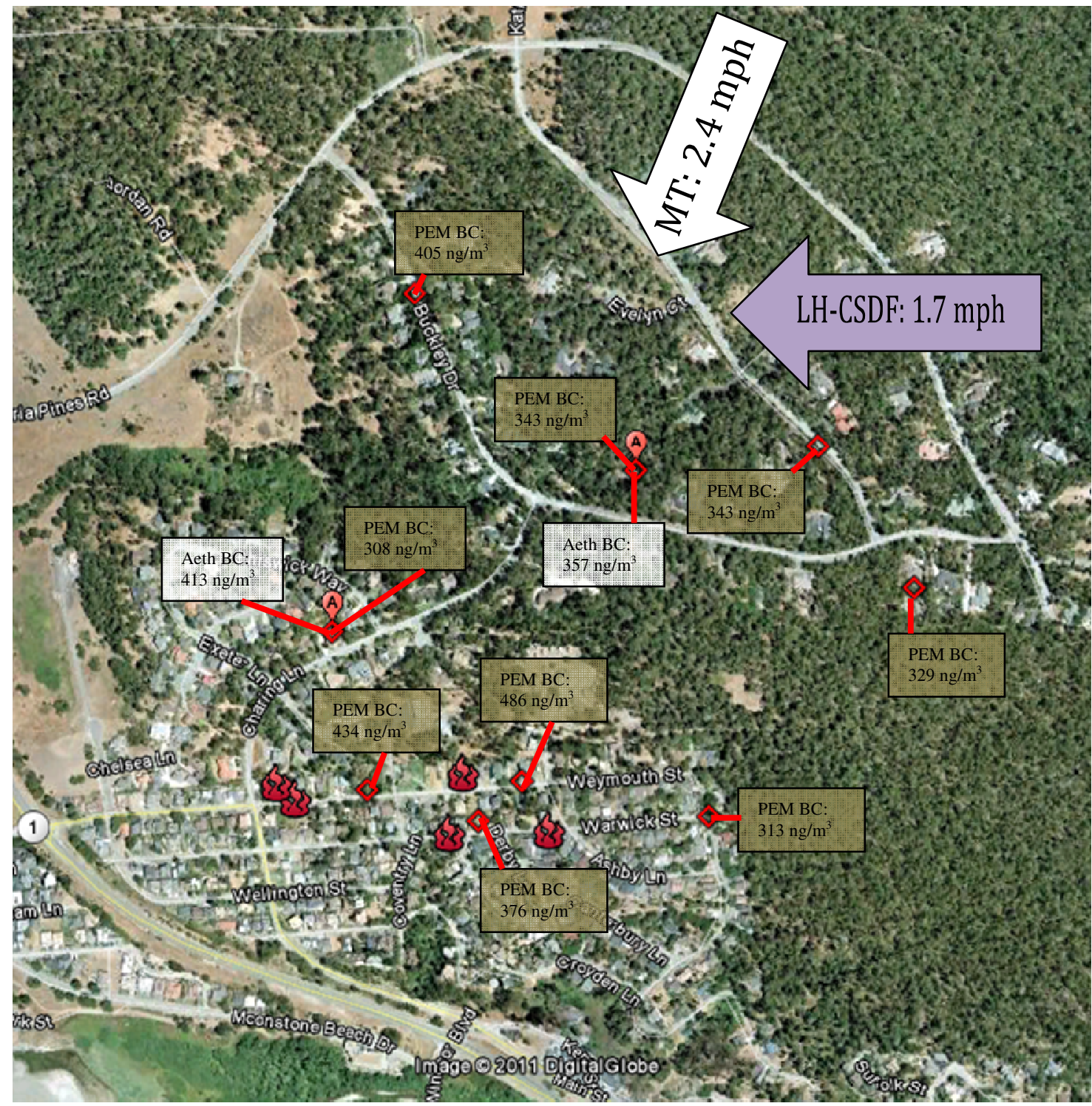




\section{IOP 2a: February 18, 2009}

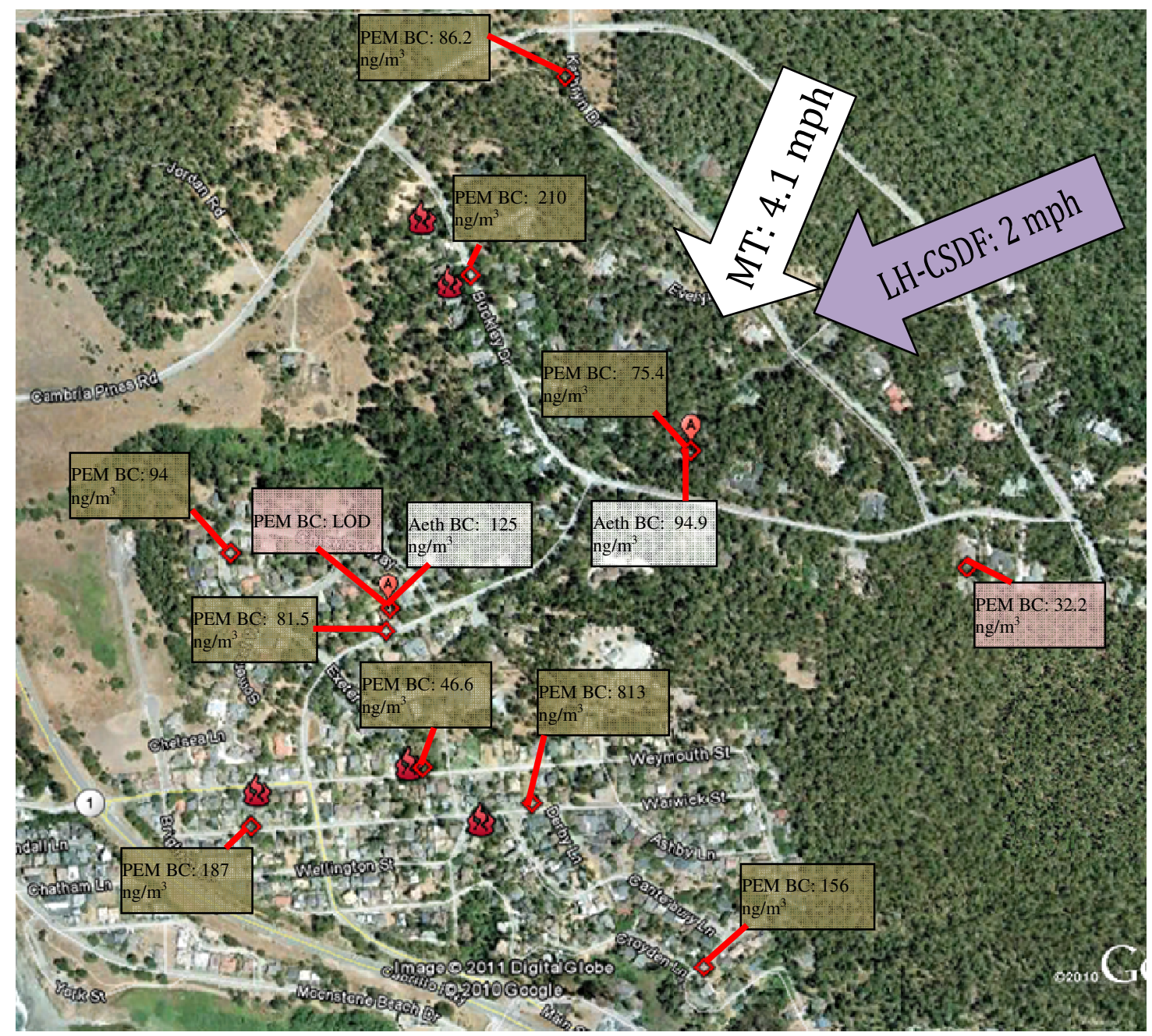




\section{IOP 3a: February 26, 2009}

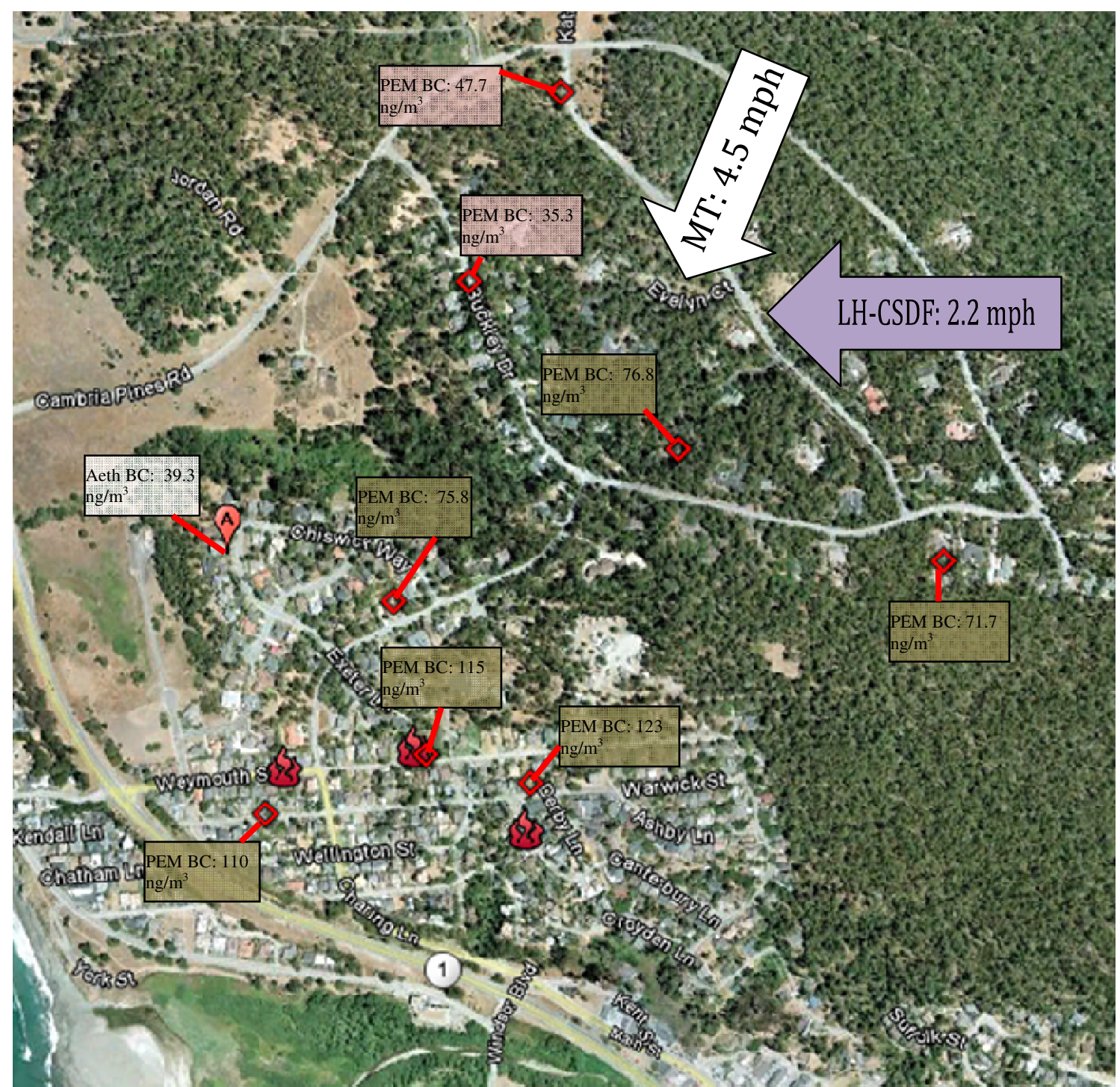




\section{IOP 4a: February 27, 2009}

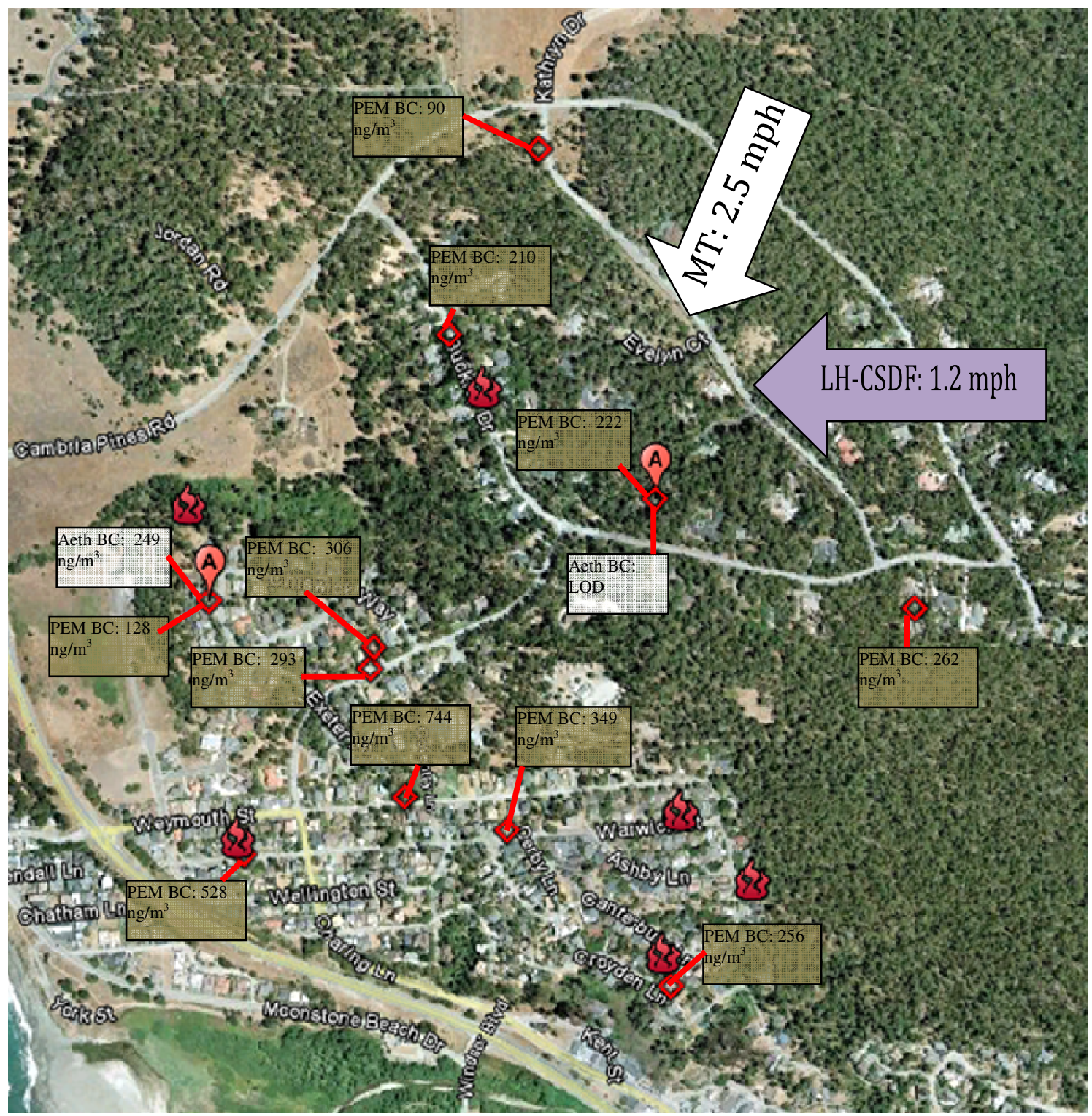




\section{IOP 7a: March 15, 2009}

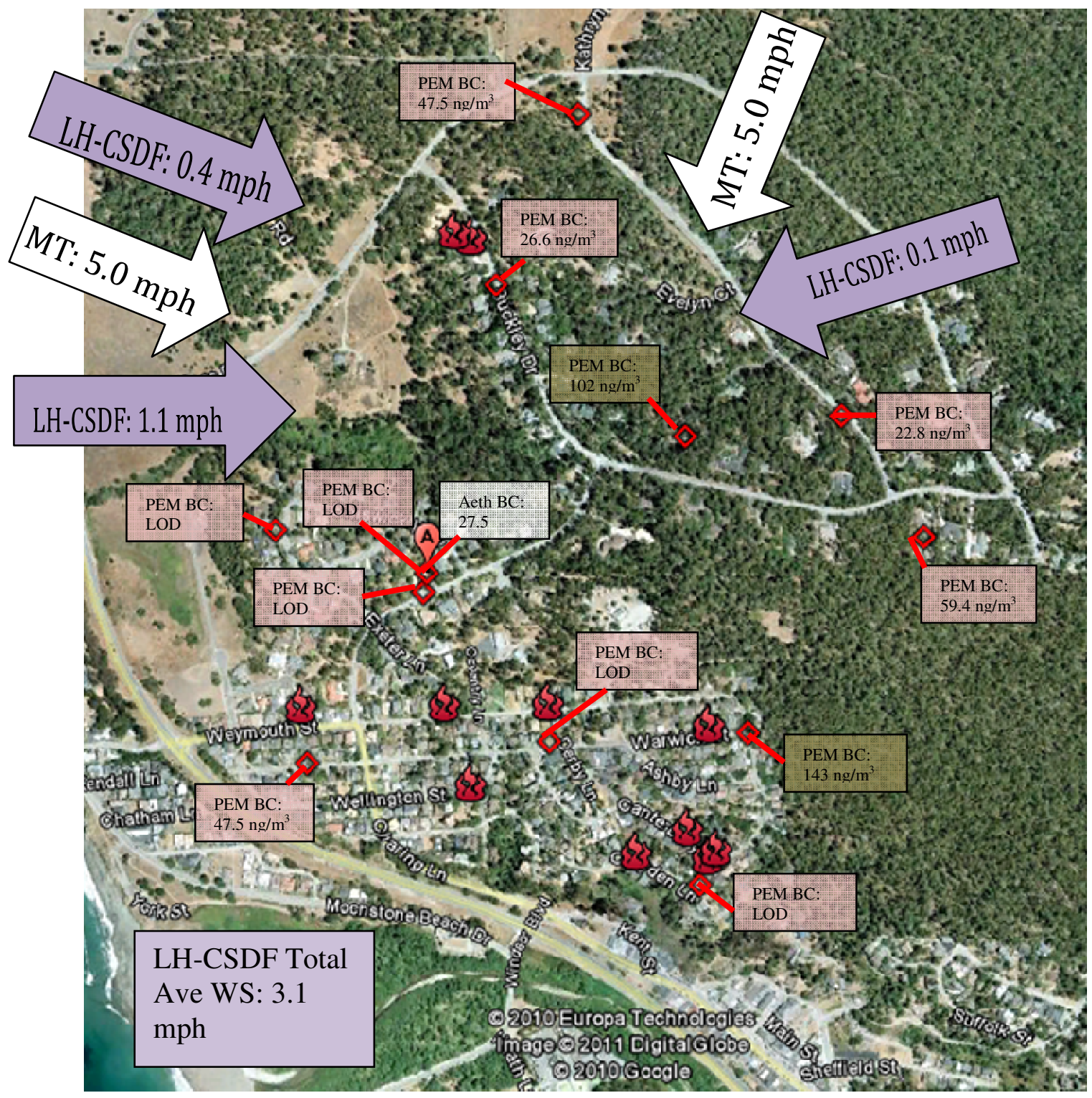




\section{IOP 8a: March 20, 2009}

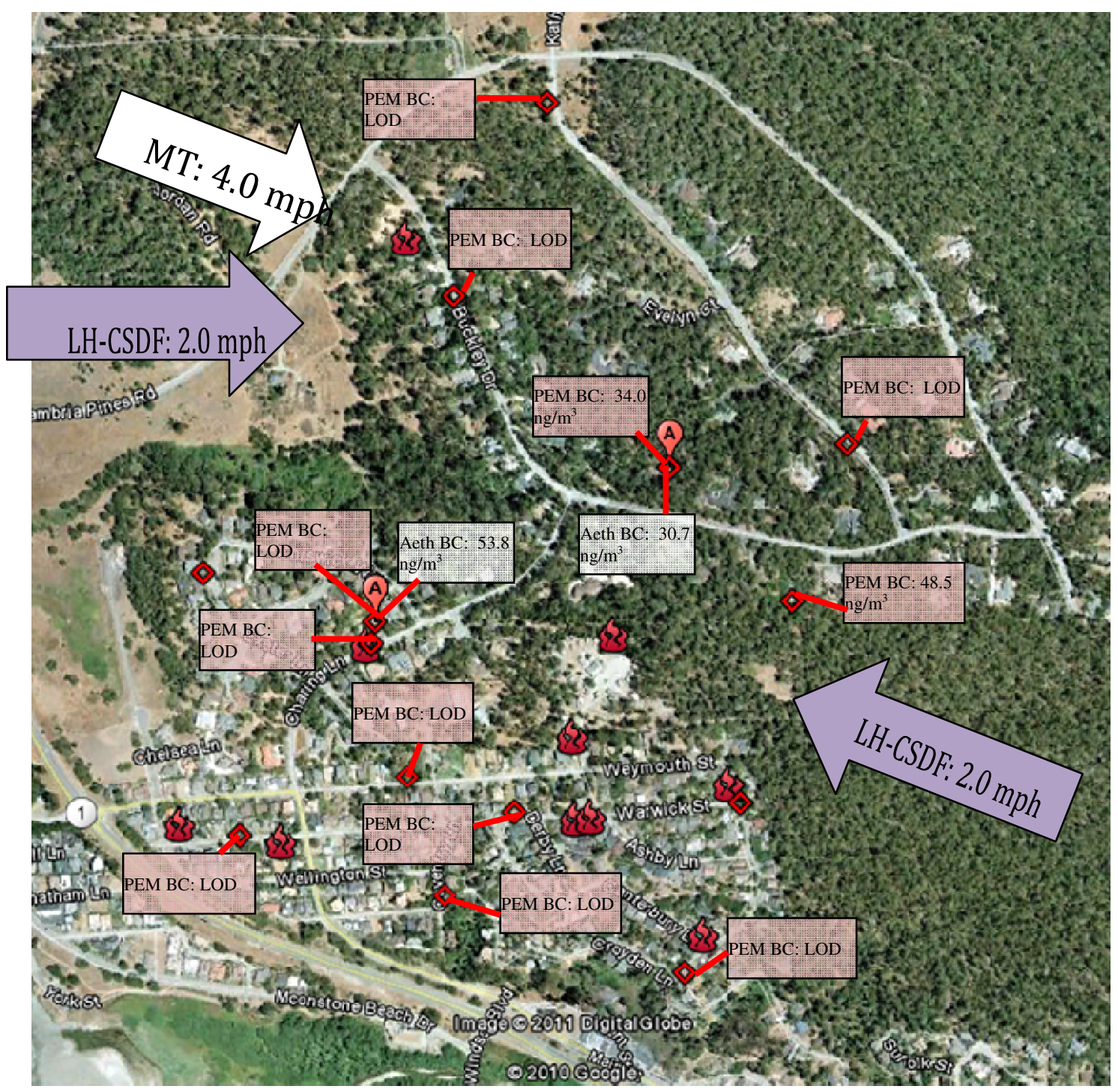




\section{IOP 2b: January 23, 2010}

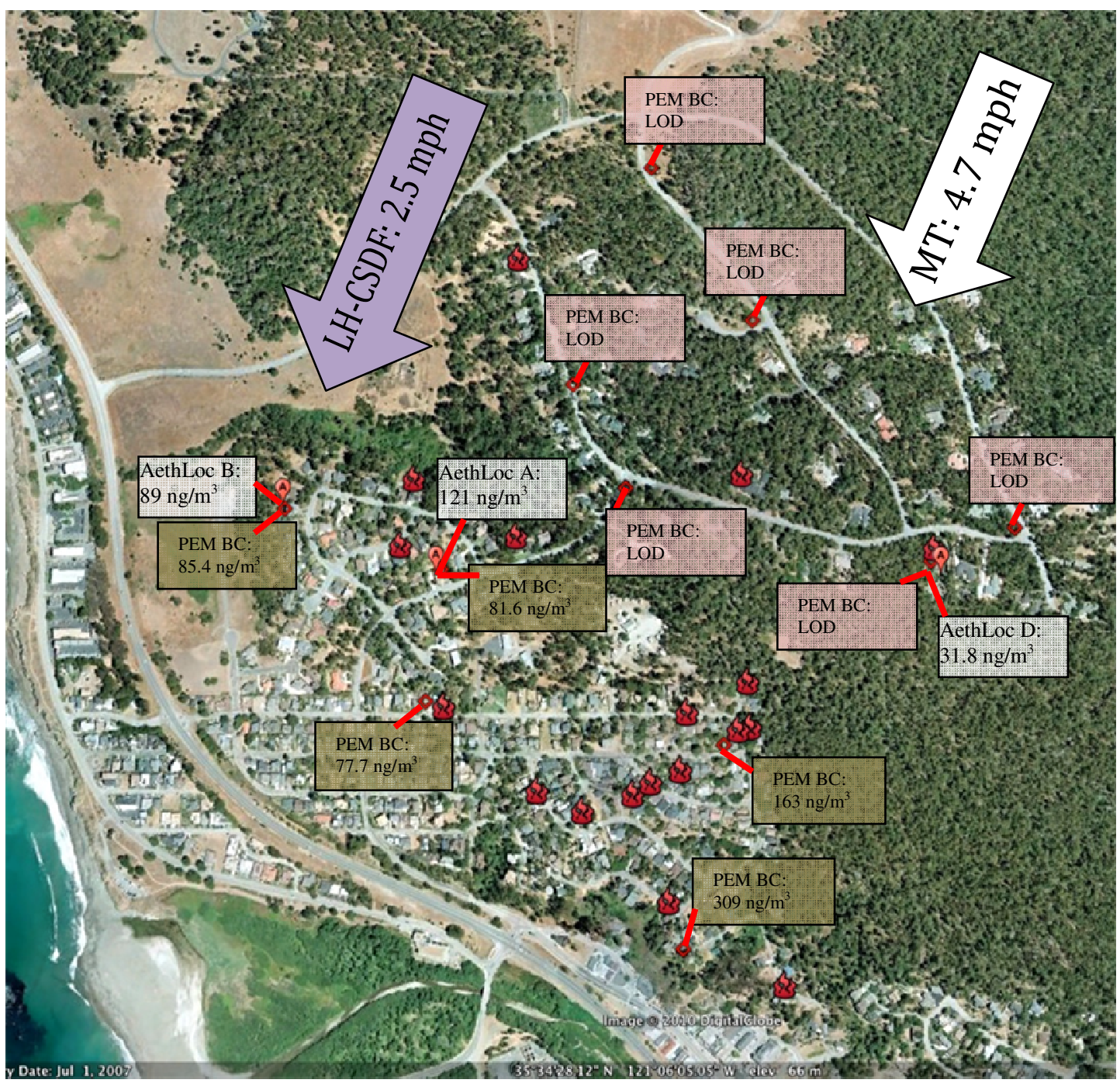


IOP 3b: January 30, 2010

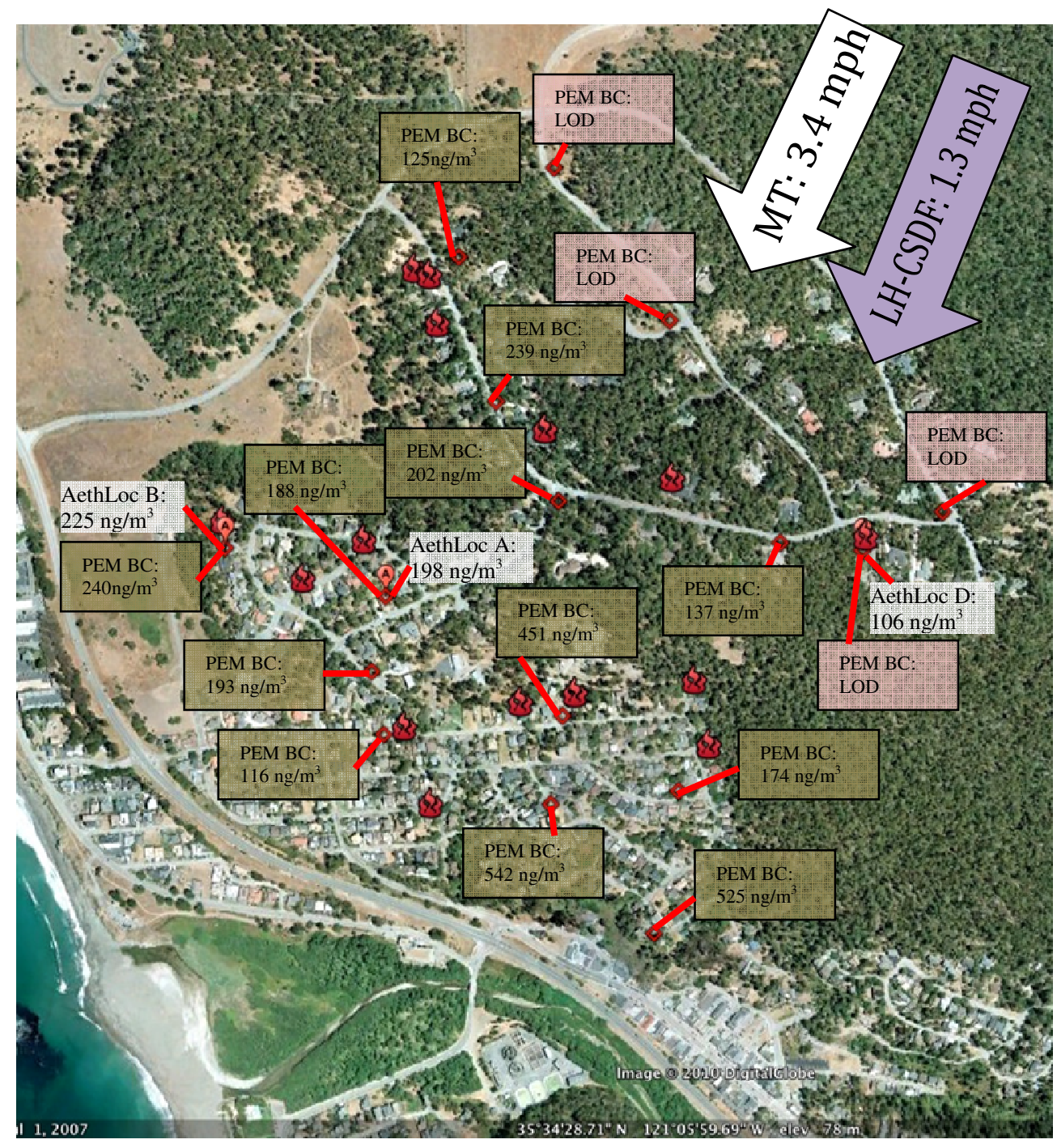




\section{IOP 4b: February 13, 2010 -AM inversions}

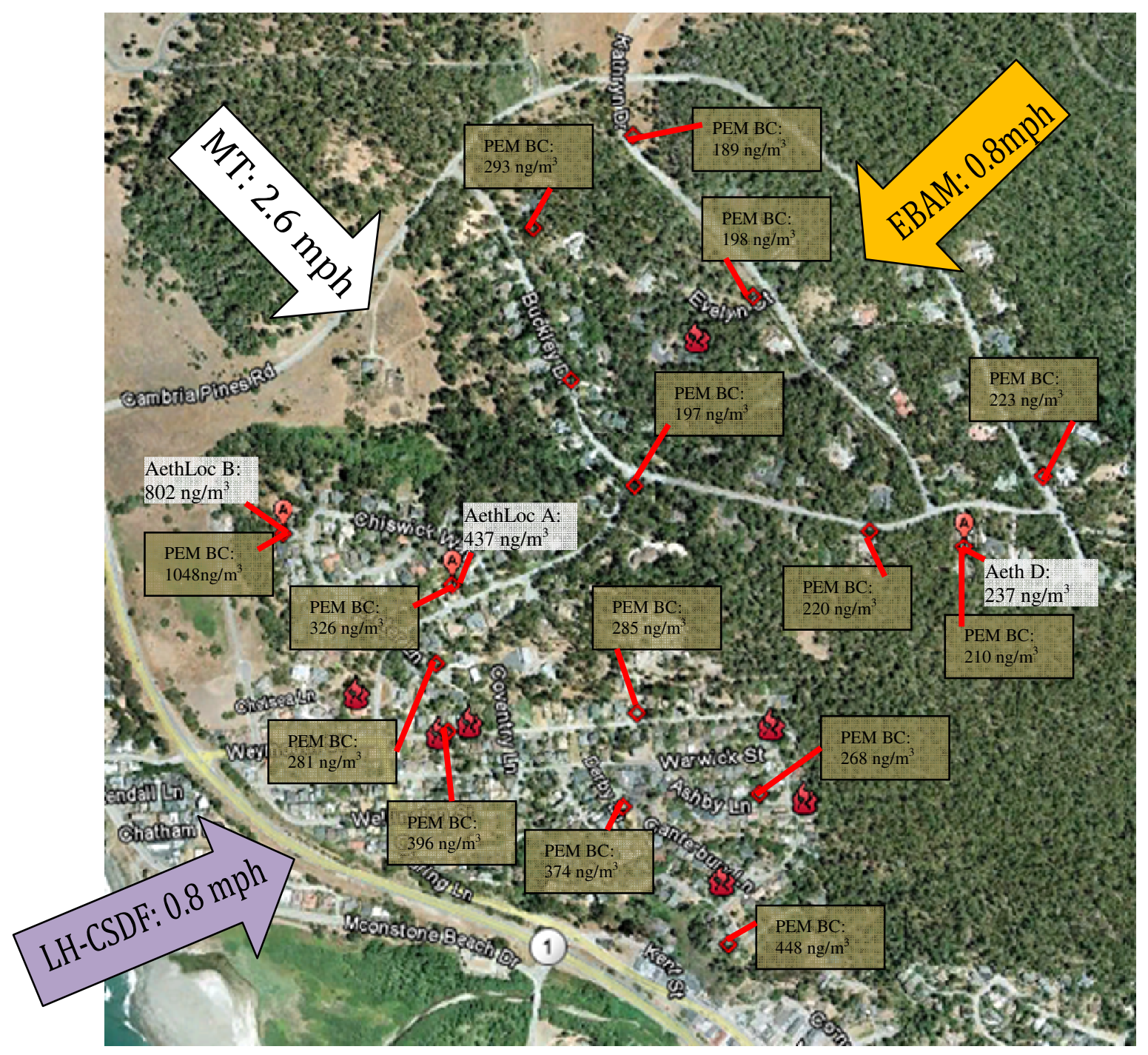


IOP 5b: February 28, 2010

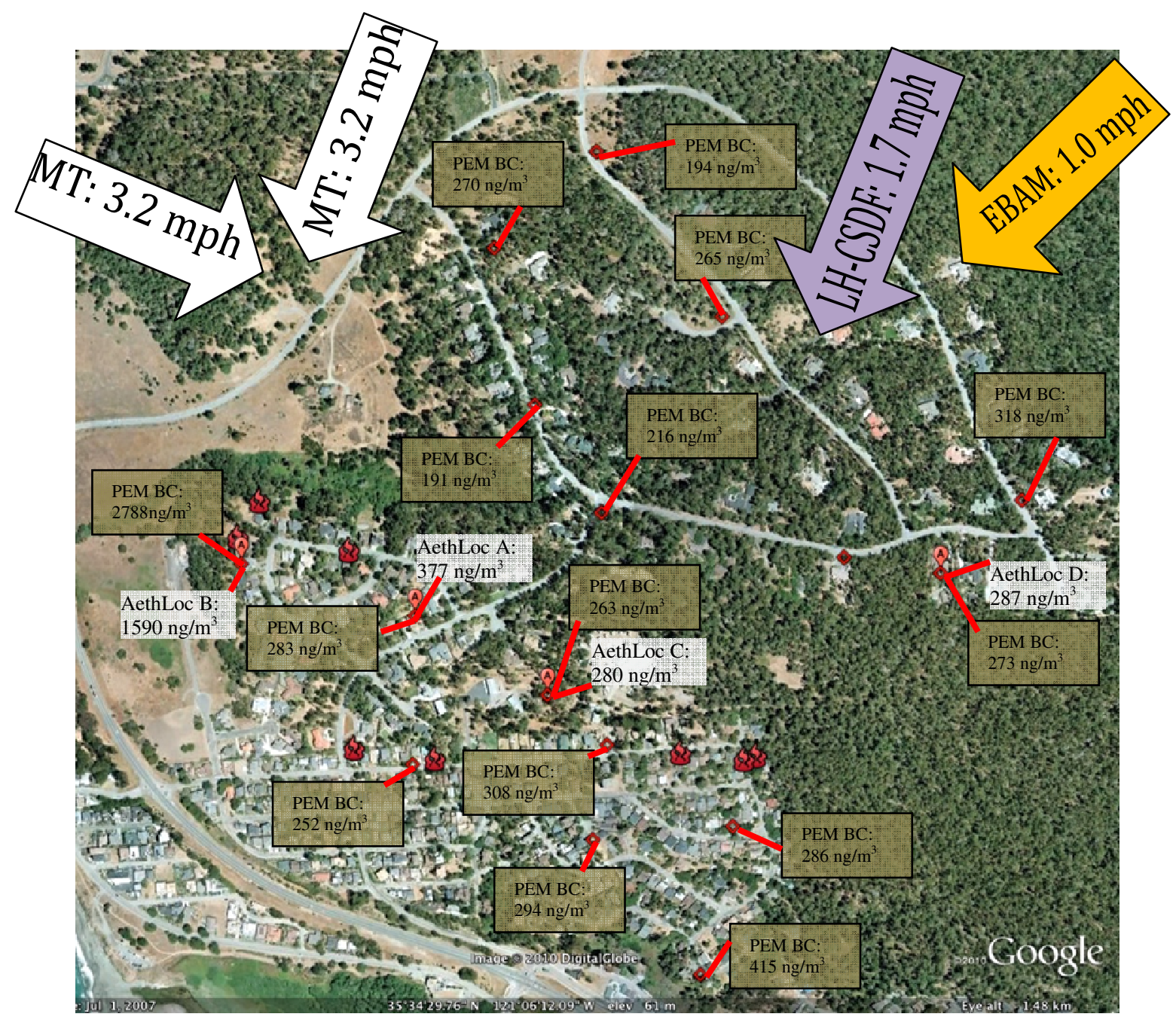


IOP 6b: March 4, 2010

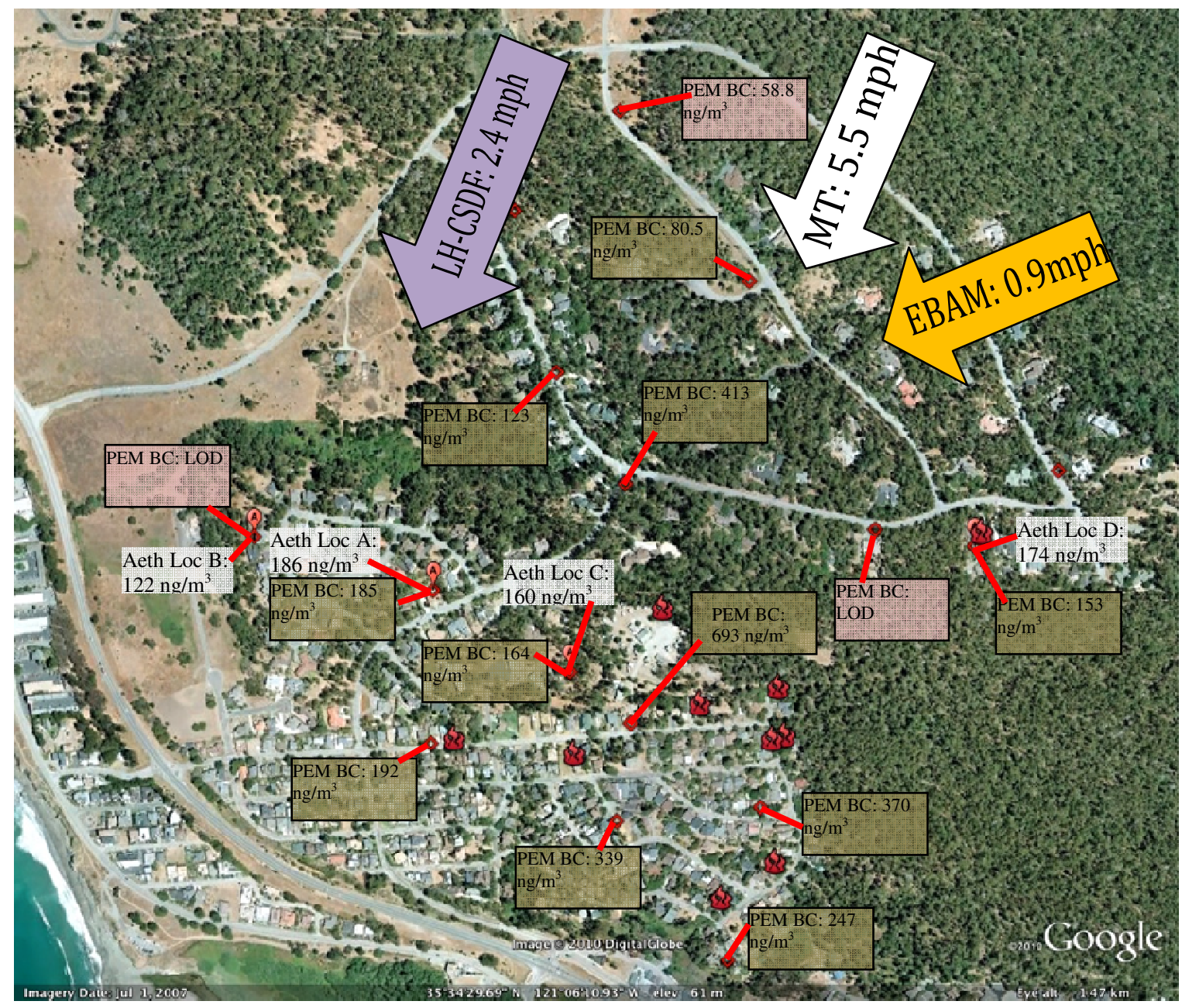




\section{IOP 7b: March 11, 2010 - mostly AM inversions}

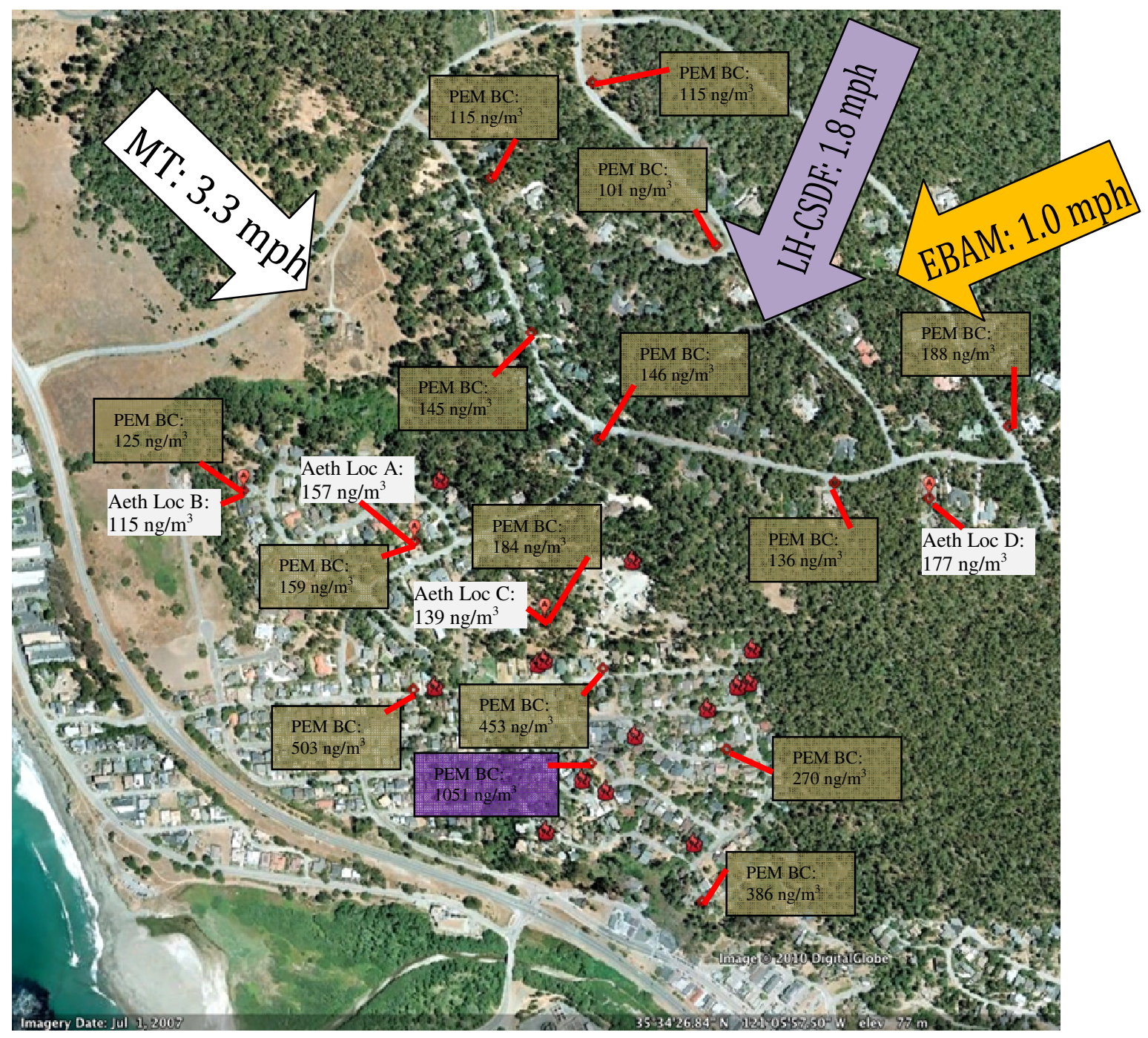


IOP 8b: March 15, 2010

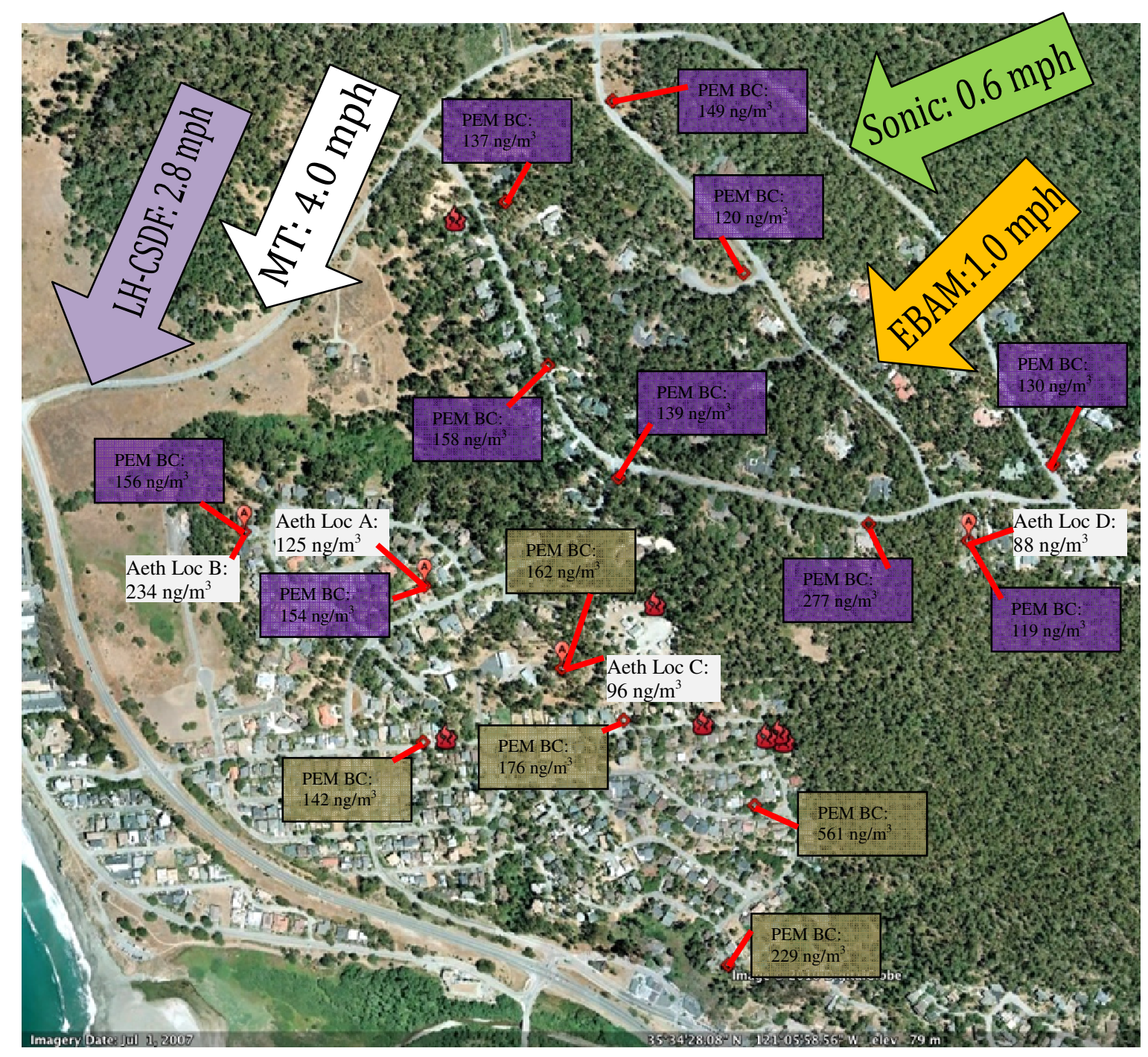




\section{IOP 9b: April 3, 2010}

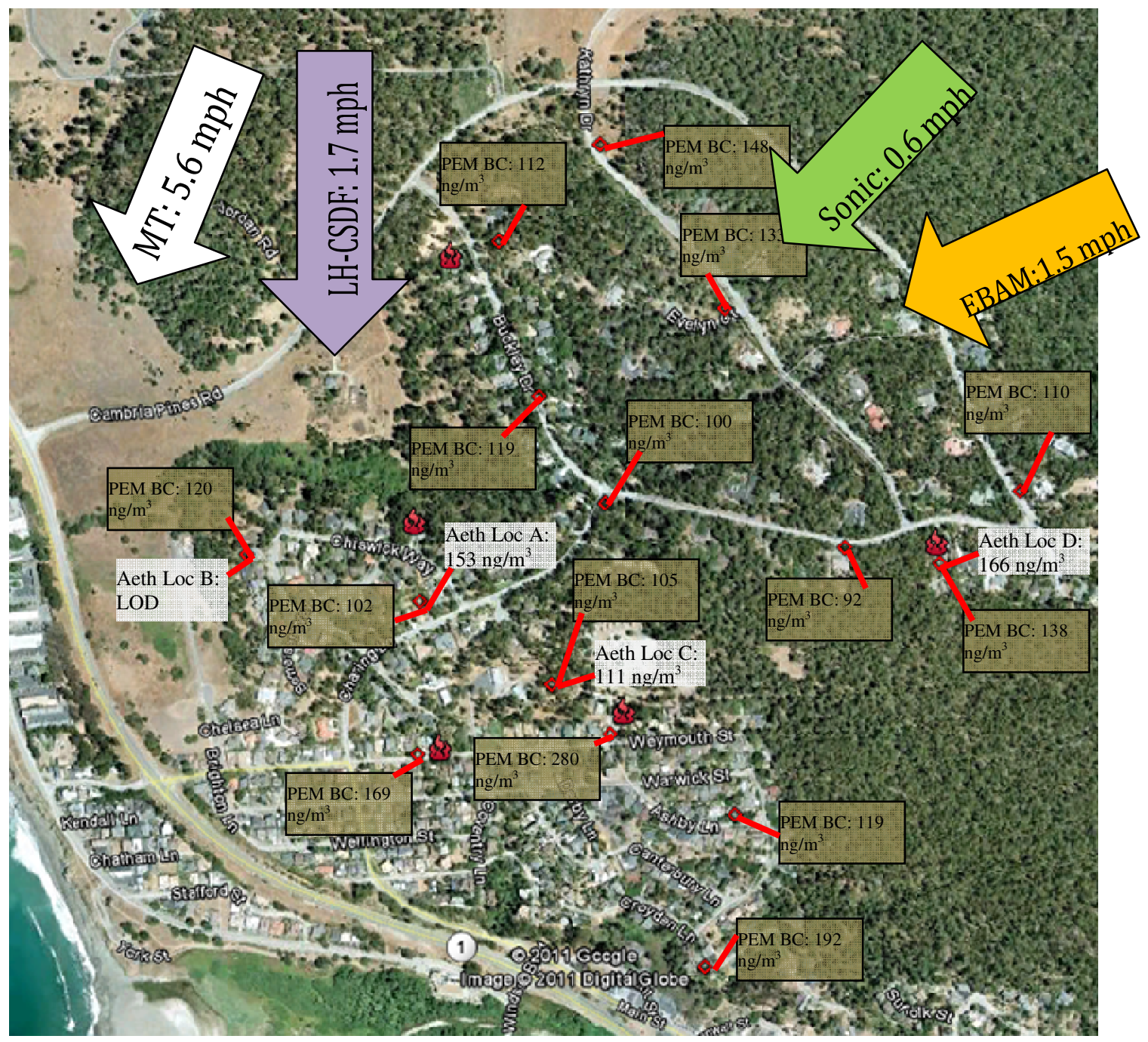




\section{IOP 10b: April 23, 2010}

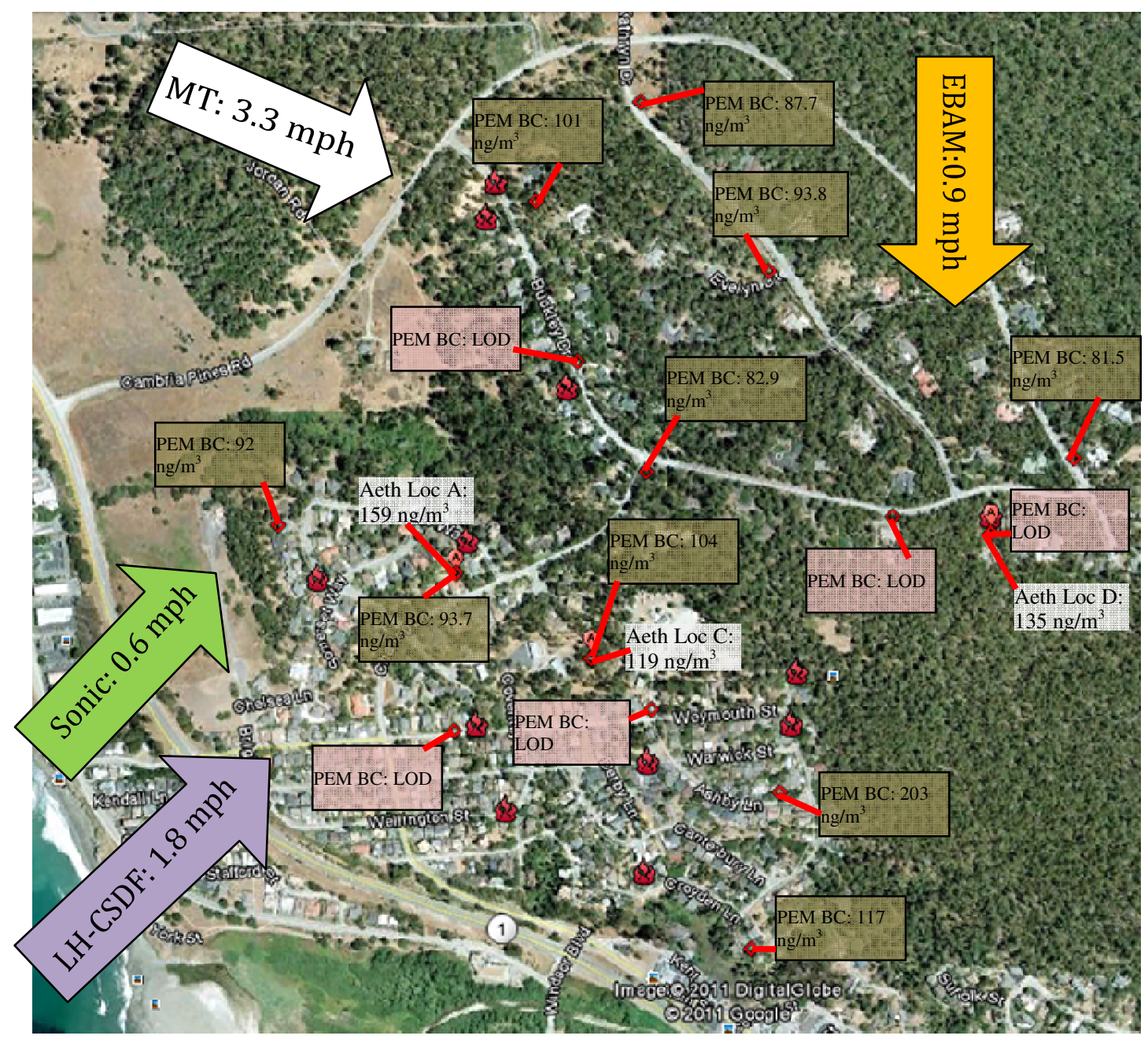




\section{Appendix G. Aethalometer BC Regression Models}

Minitab output for average aethalometer BC concentration regression models.

\section{Average BC Concentration}

\section{Linear EBAM Model}

\section{Regression Analysis: BCave^^-.7 versus lagged1_BCav, Time Interva, ...}

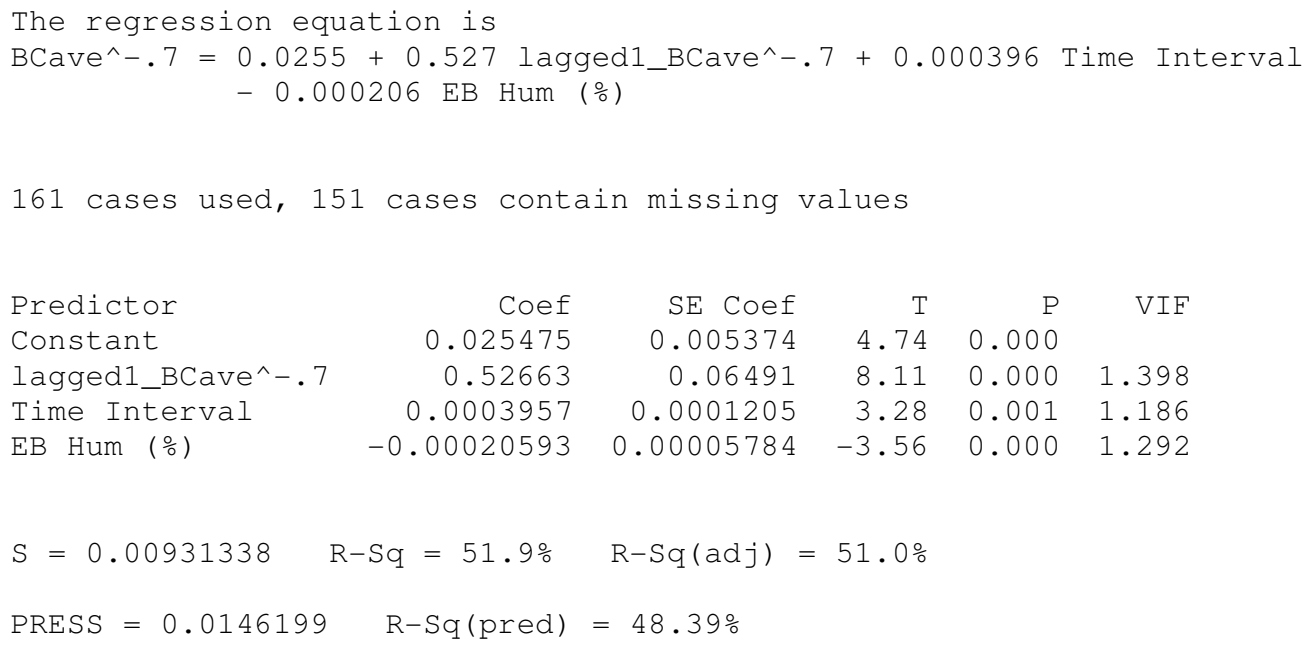


$R$ denotes an observation with a large standardized residual.

$\mathrm{X}$ denotes an observation whose $\mathrm{X}$ value gives it large leverage.

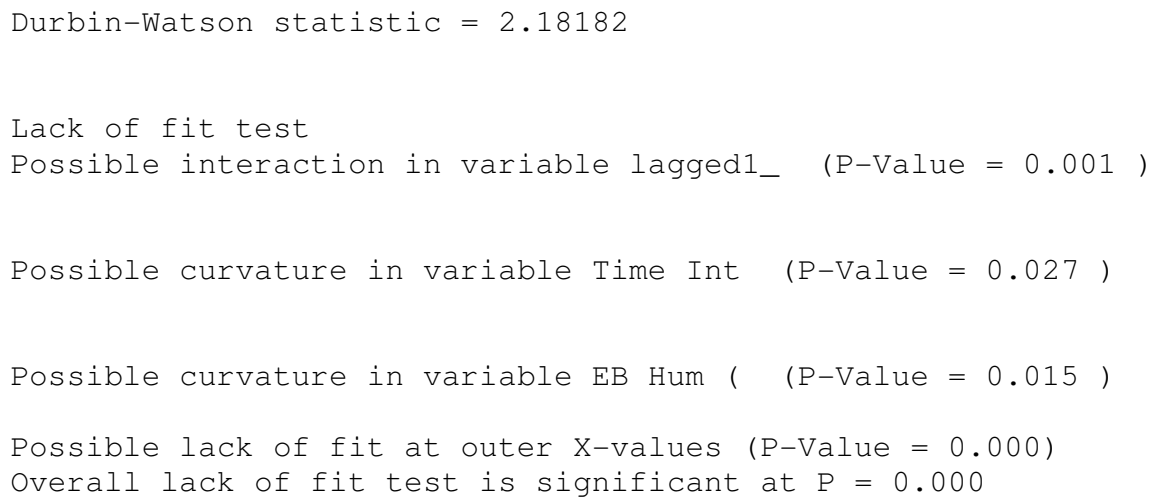

\section{Residual Plots for BCave^-.7}

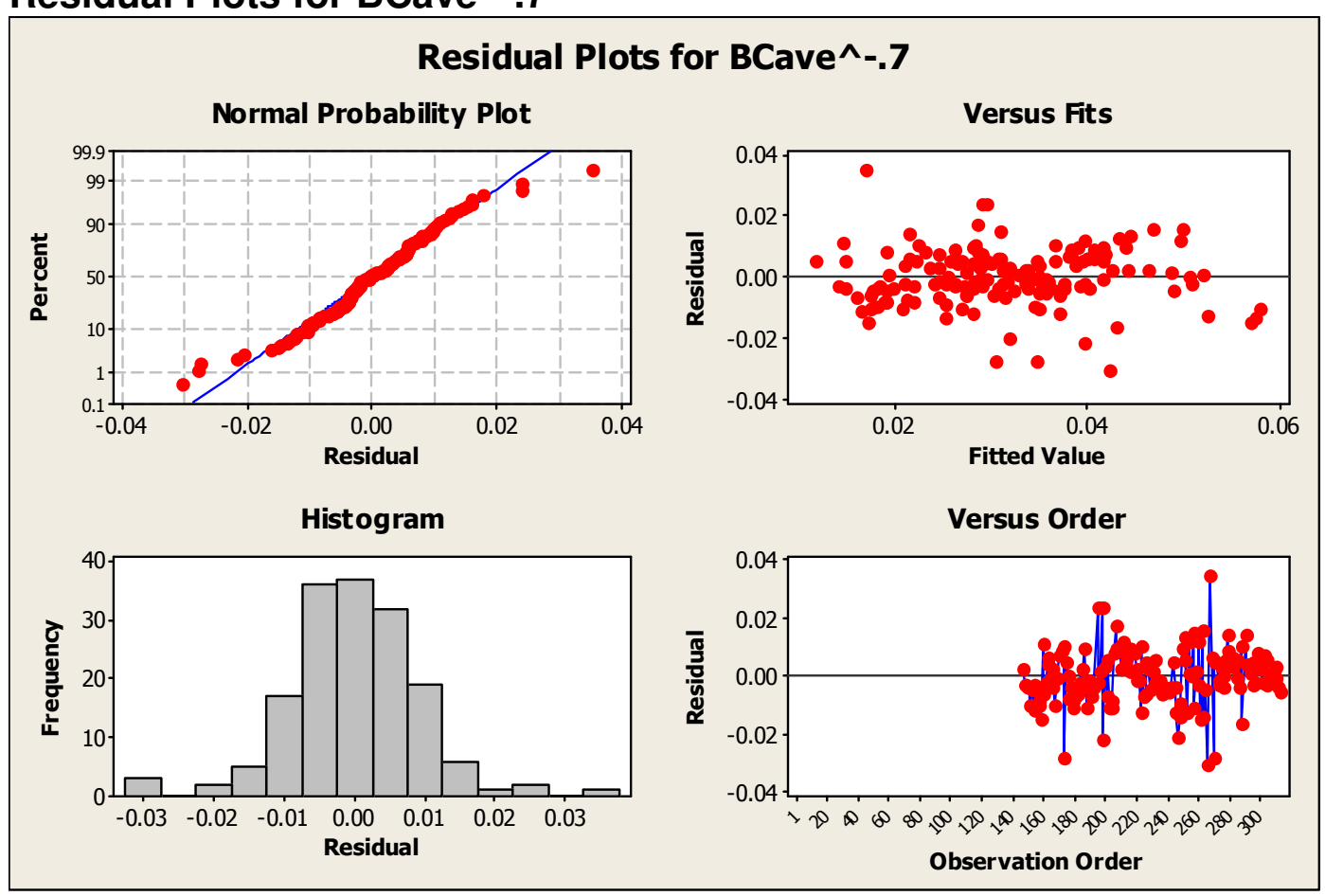



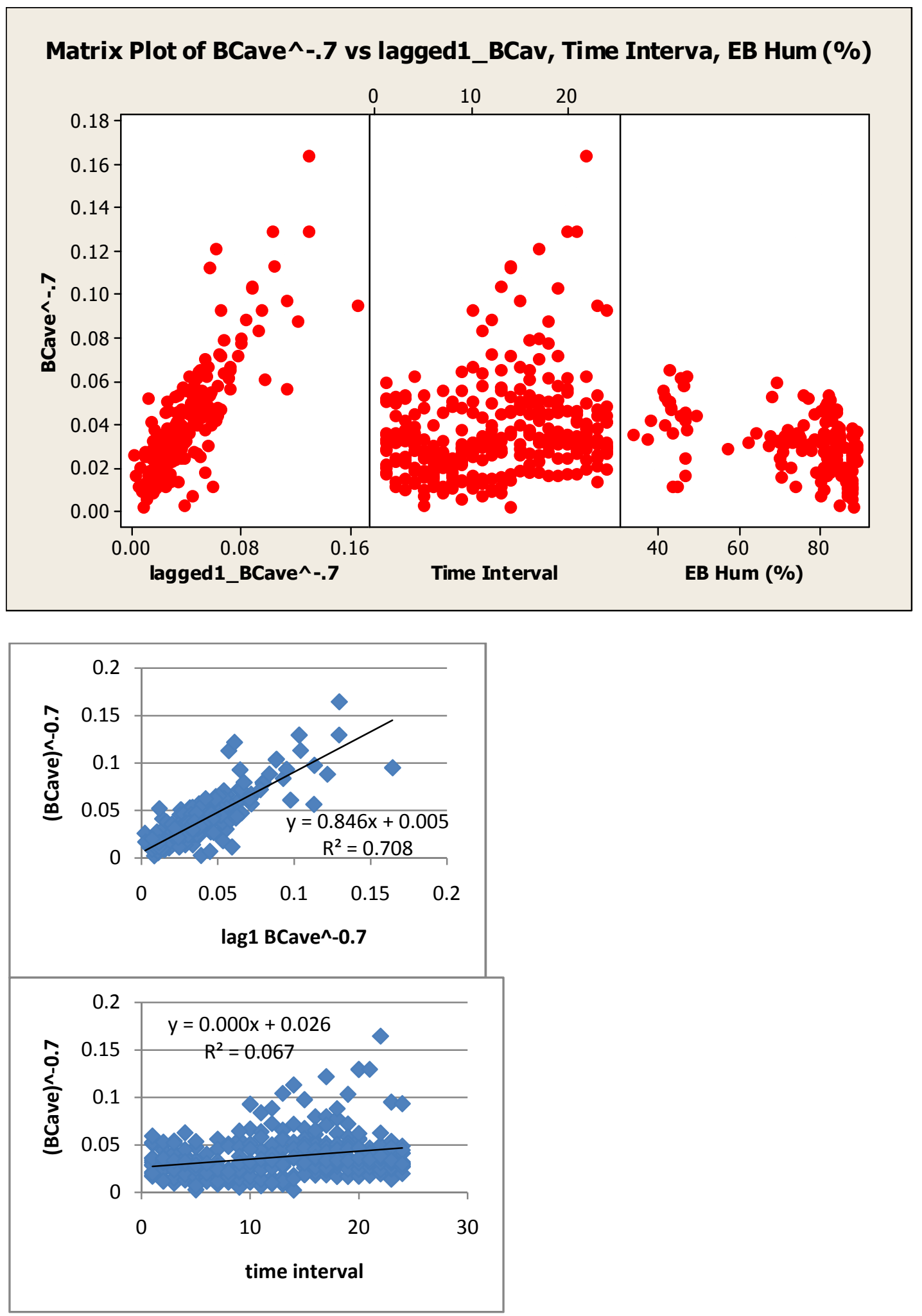


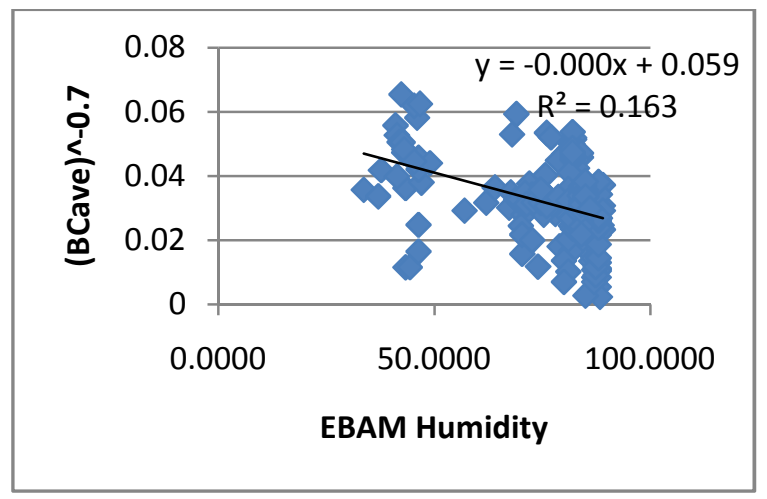

\section{Non-linear EBAM Model

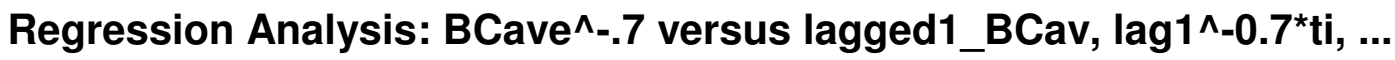

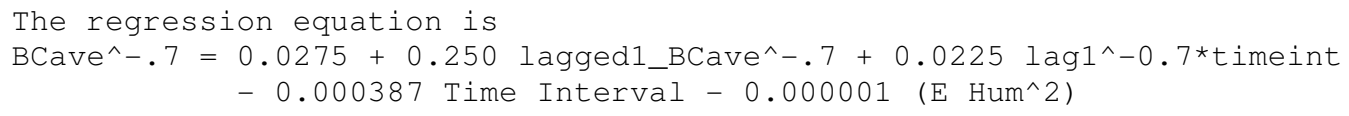

Unusual Observations 


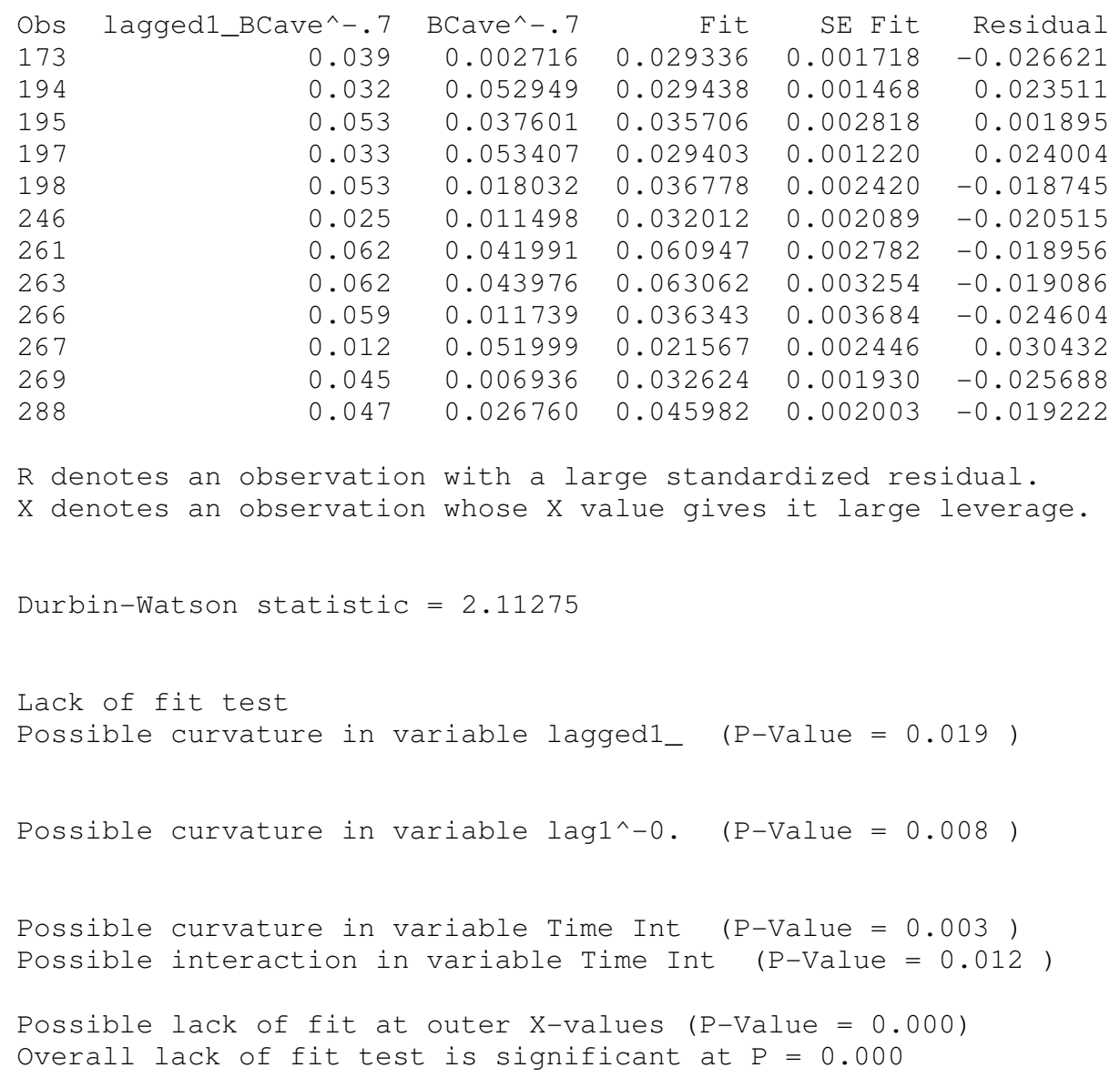

$R$ denotes an observation with a large standardized residual.

$\mathrm{X}$ denotes an observation whose $\mathrm{X}$ value gives it large leverage.

Durbin-Watson statistic $=2.11275$

Lack of fit test

Possible curvature in variable laggedl_ $(\mathrm{P}-$ Value $=0.019$ )

Possible curvature in variable $\operatorname{lag} 1^{\wedge}-0 . \quad(\mathrm{P}-\mathrm{Value}=0.008)$

Possible curvature in variable Time Int (P-Value $=0.003$ )

Possible interaction in variable Time Int (P-Value $=0.012$ )

Possible lack of fit at outer X-values (P-Value $=0.000$ )

Overall lack of fit test is significant at $P=0.000$

\section{Residual Plots for BCave^^-.7}



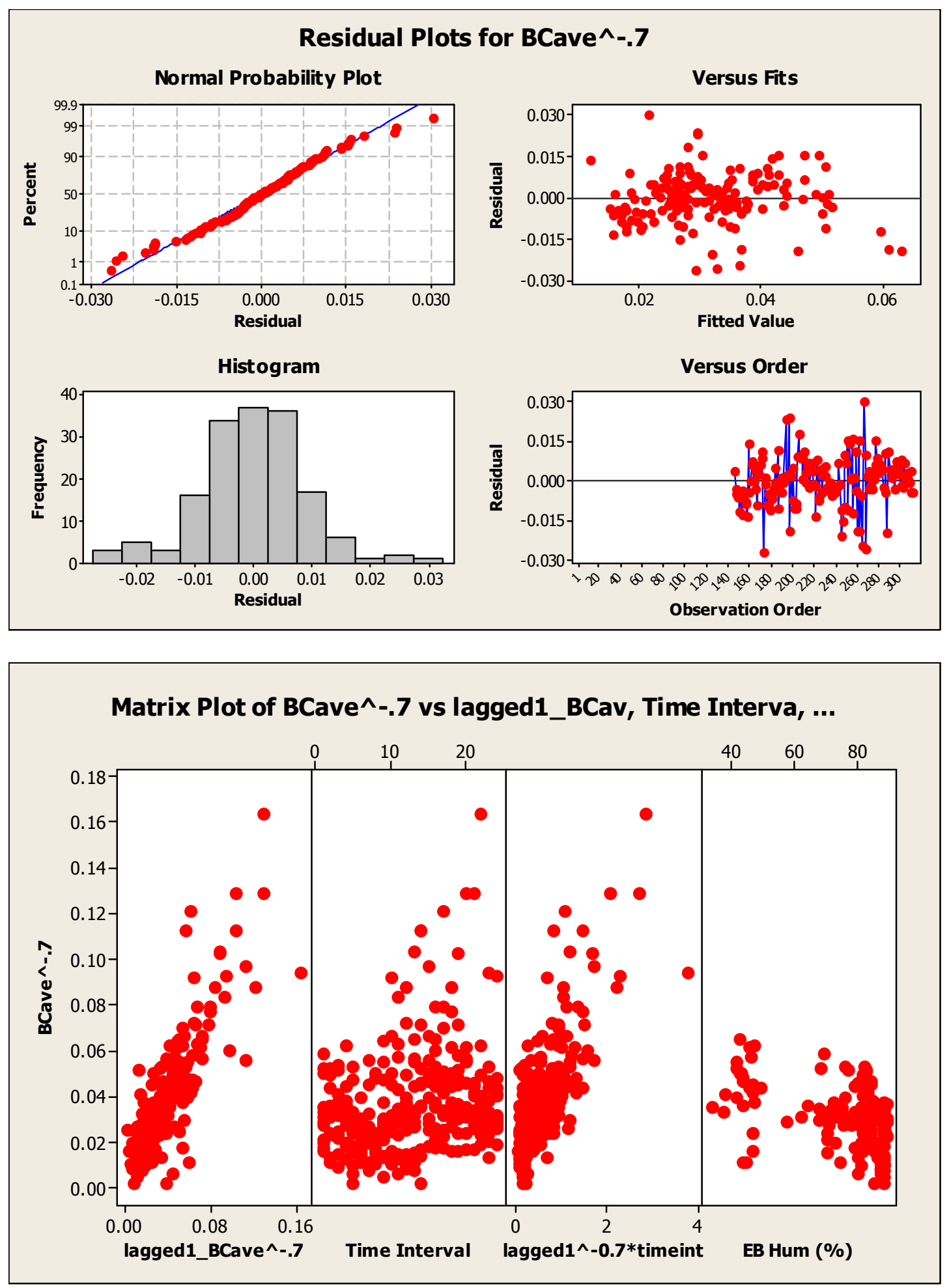

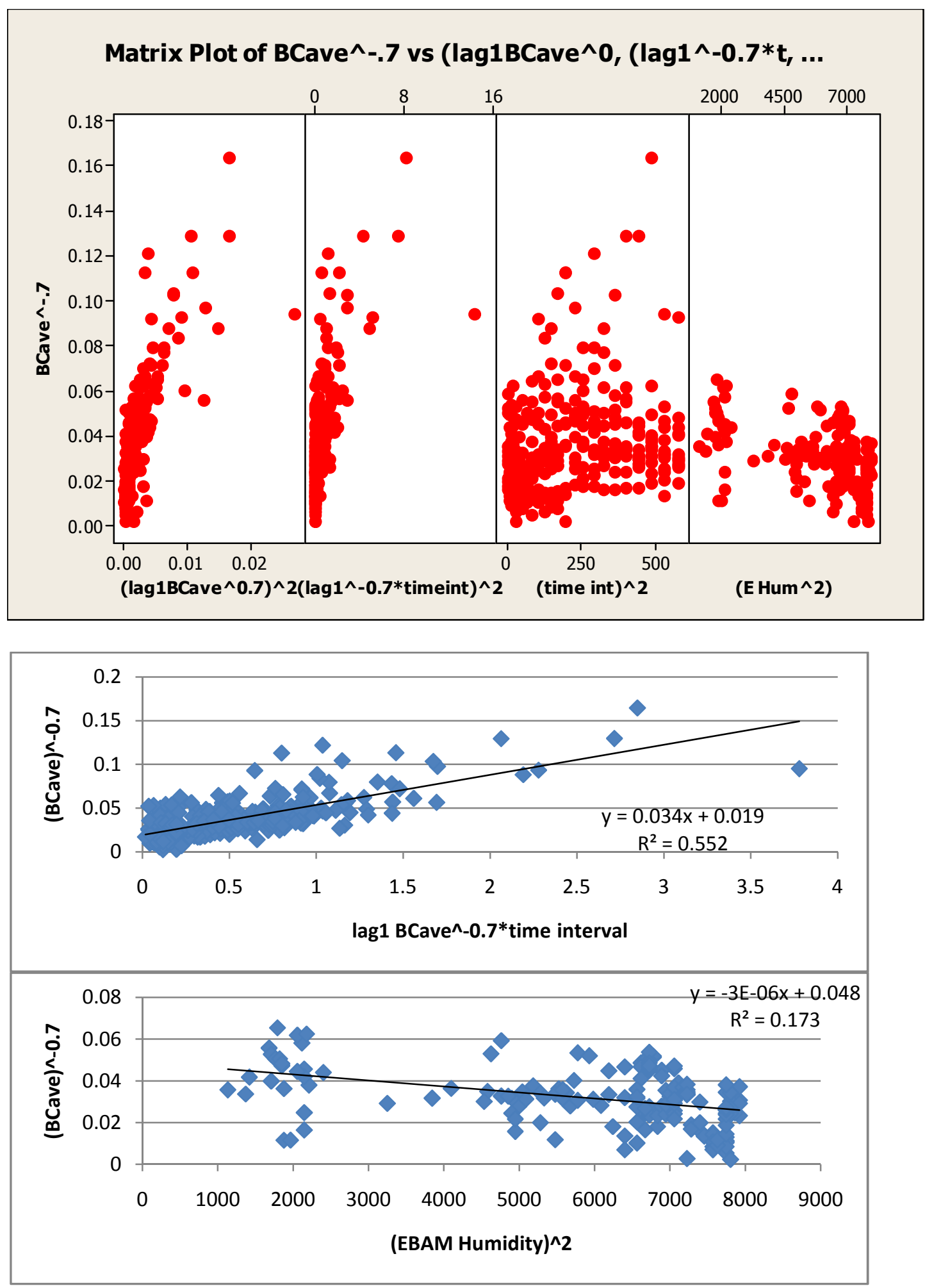


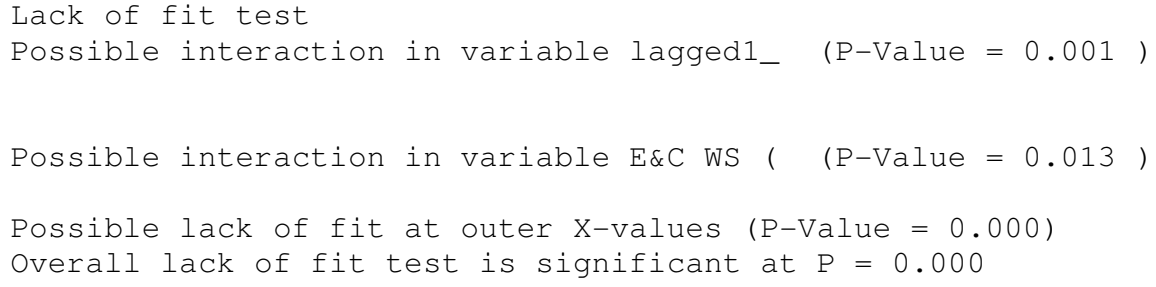

\section{Residual Plots for BCave^^-.7}

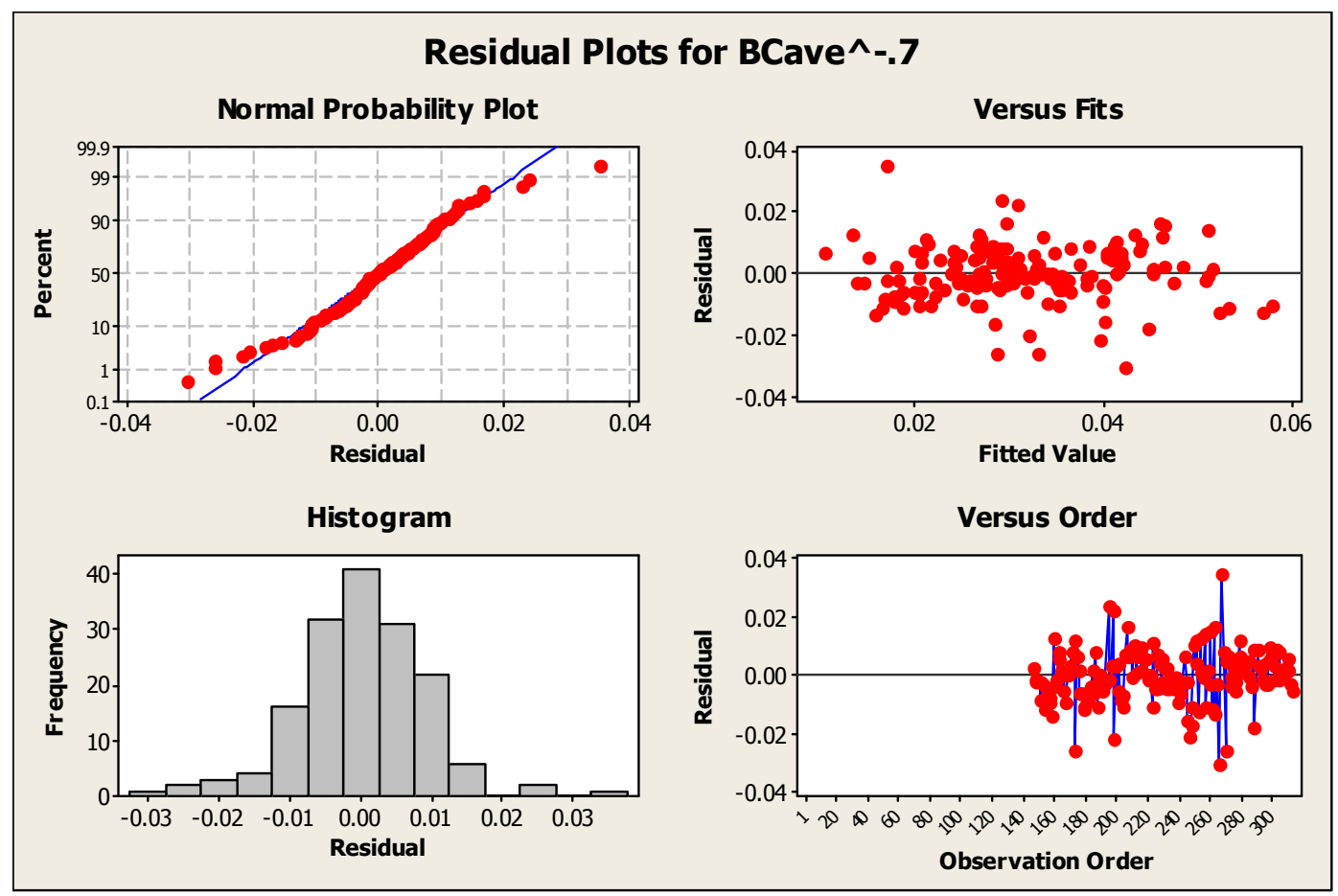



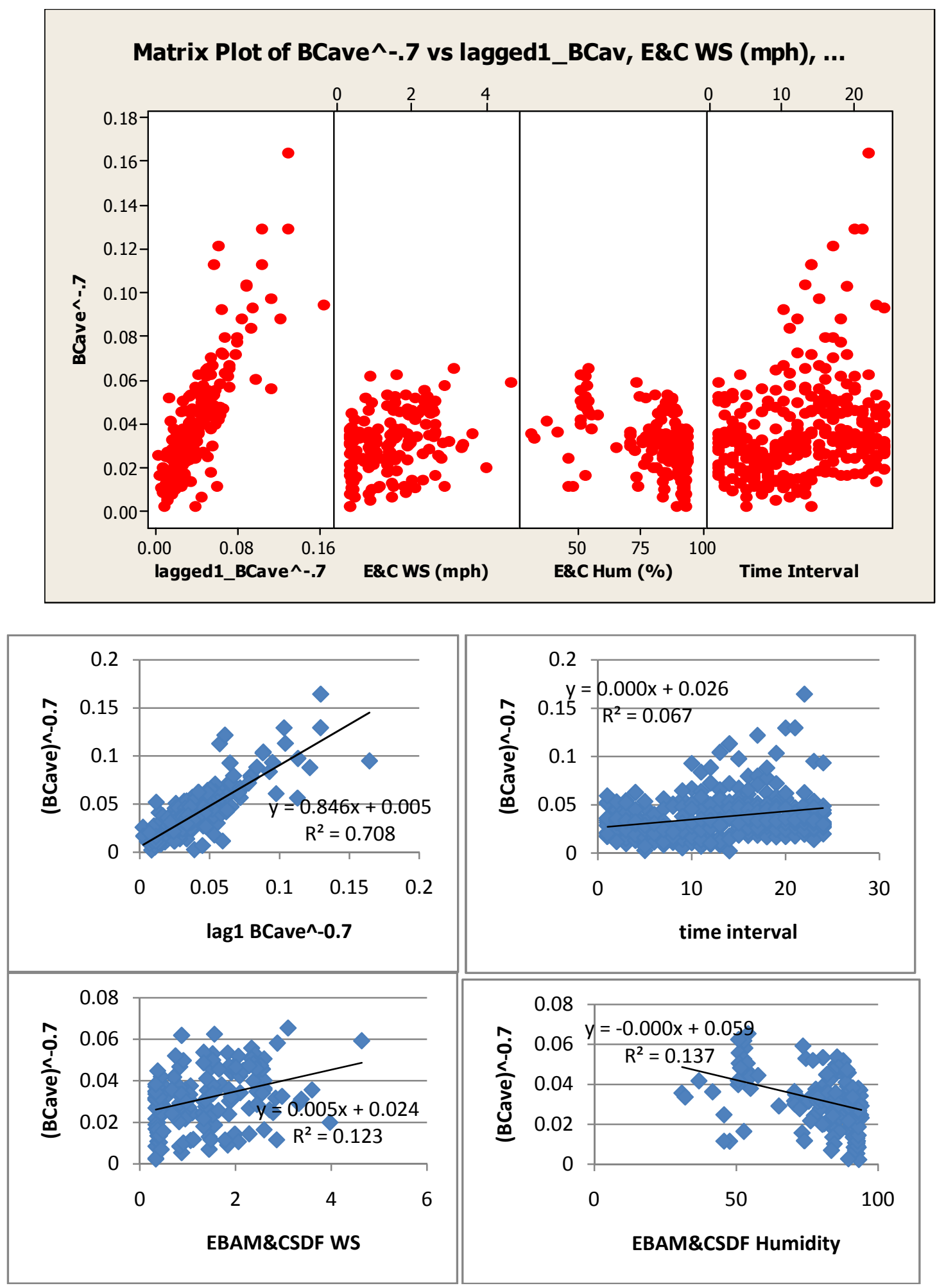

Non-linear EBAM \& CSDF Combination

Regression Analysis: BCave^^${ }^{\wedge} .7$ versus lagged1_BCav, Time Interva, ...

The regression equation is 


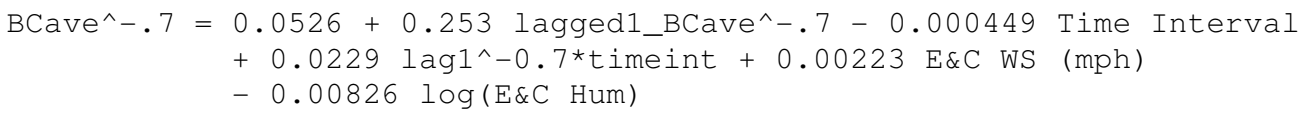

161 cases used, 151 cases contain missing values

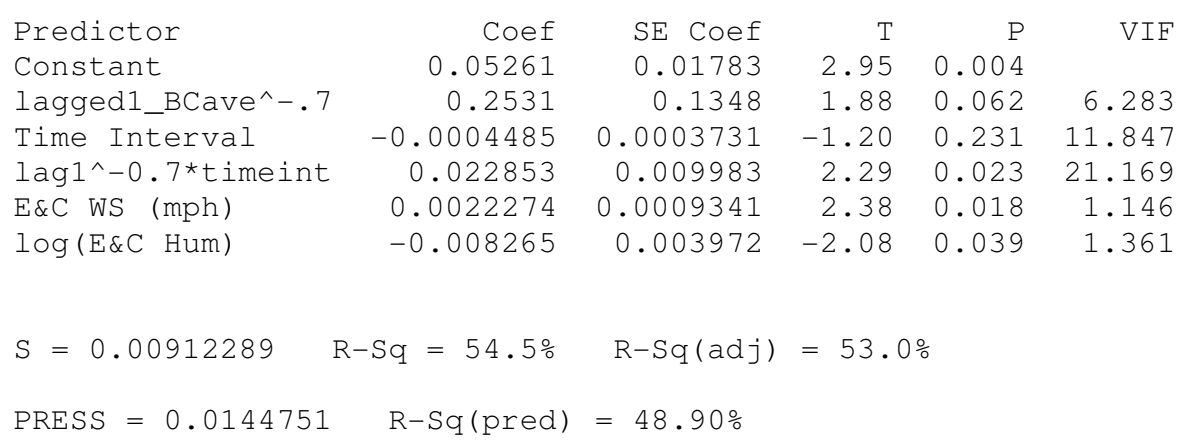

Analysis of Variance

$\begin{array}{lrrrrr}\text { Source } & \text { DF } & \text { SS } & \text { MS } & \text { F } & \text { P } \\ \text { Regression } & 5 & 0.0154263 & 0.0030853 & 37.07 & 0.000 \\ \text { Residual Error } & 155 & 0.0129002 & 0.0000832 & & \\ \text { Total } & 160 & 0.0283265 & & \end{array}$

There are no replicates.

Minitab cannot do the lack of fit test based on pure error.

$\begin{array}{lrr}\text { Source } & \text { DF } & \text { Seq SS } \\ \text { lagged1_BCave^-.7 } & 1 & 0.0131082 \\ \text { Time Interval } & 1 & 0.0005008 \\ \text { lag1^-0.7*timeint } & 1 & 0.0007828 \\ \text { E\&C WS (mph) } & 1 & 0.0006741 \\ \log (\text { E\&C Hum) } & 1 & 0.0003603\end{array}$

Unusual Observations

$\begin{array}{lrrrrrr}\text { Obs lagged1_BCave^-.7 } & \text { BCave^-.7 } & \text { Fit } & \text { SE Fit } & \text { Residual } & \text { St Resid } \\ 173 & 0.039 & 0.002716 & 0.028297 & 0.001829 & -0.025581 & -2.86 \mathrm{R} \\ 194 & 0.032 & 0.052949 & 0.028940 & 0.001478 & 0.024009 & 2.67 \mathrm{R} \\ 197 & 0.033 & 0.053407 & 0.030474 & 0.001378 & 0.022932 & 2.54 \mathrm{R} \\ 198 & 0.053 & 0.018032 & 0.037113 & 0.002433 & -0.019080 & -2.17 \mathrm{R} \\ 242 & 0.036 & 0.033654 & 0.034717 & 0.003740 & -0.001064 & -0.13 \\ 243 & 0.034 & 0.041760 & 0.033859 & 0.003135 & 0.007900 & 0.92 \times \\ 246 & 0.025 & 0.011498 & 0.031610 & 0.002171 & -0.020113 & -2.27 \mathrm{X} \\ 247 & 0.011 & 0.011542 & 0.028999 & 0.003077 & -0.017457 & -2.03 \mathrm{RX} \\ 261 & 0.062 & 0.041991 & 0.057077 & 0.003328 & -0.015086 & -1.78 \\ 263 & 0.062 & 0.043976 & 0.062065 & 0.003274 & -0.018090 & -2.12 \mathrm{RX} \\ 266 & 0.059 & 0.011739 & 0.036338 & 0.003667 & -0.024599 & -2.94 \mathrm{RX} \\ 267 & 0.012 & 0.051999 & 0.020798 & 0.002469 & 0.031201 & 3.55 \mathrm{R} \\ 269 & 0.045 & 0.006936 & 0.031255 & 0.002055 & -0.024319 & -2.74 \mathrm{R} \\ 288 & 0.047 & 0.026760 & 0.047748 & 0.002081 & -0.020988 & -2.36 \mathrm{R}\end{array}$

$R$ denotes an observation with a large standardized residual.

$\mathrm{X}$ denotes an observation whose $\mathrm{X}$ value gives it large leverage. 


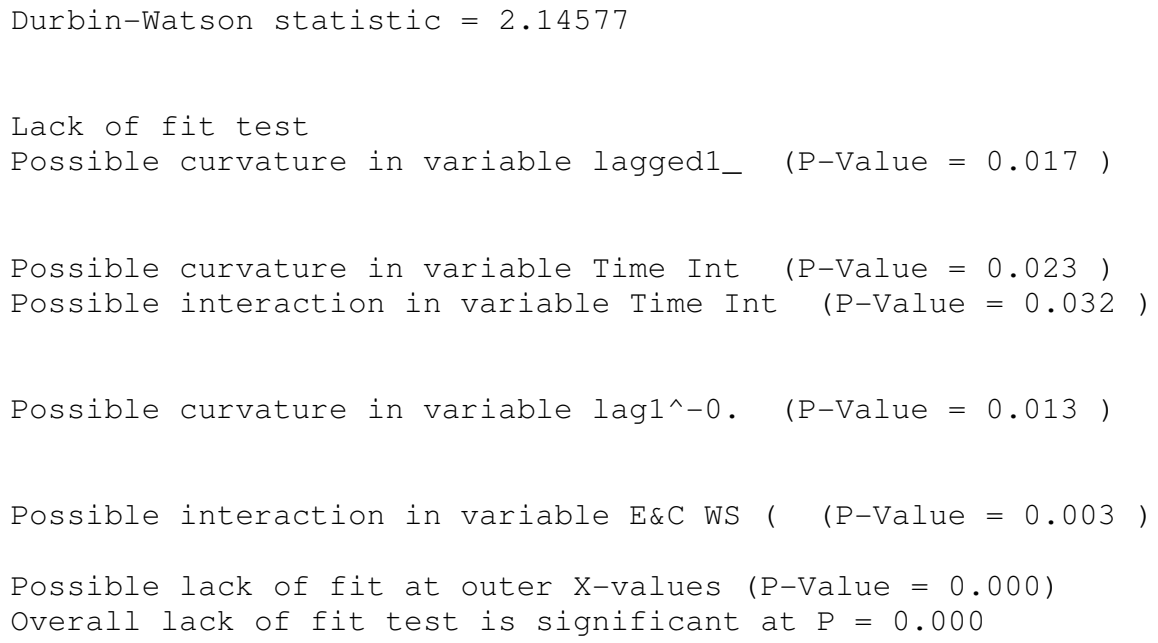

\section{Residual Plots for BCave^^-.7}

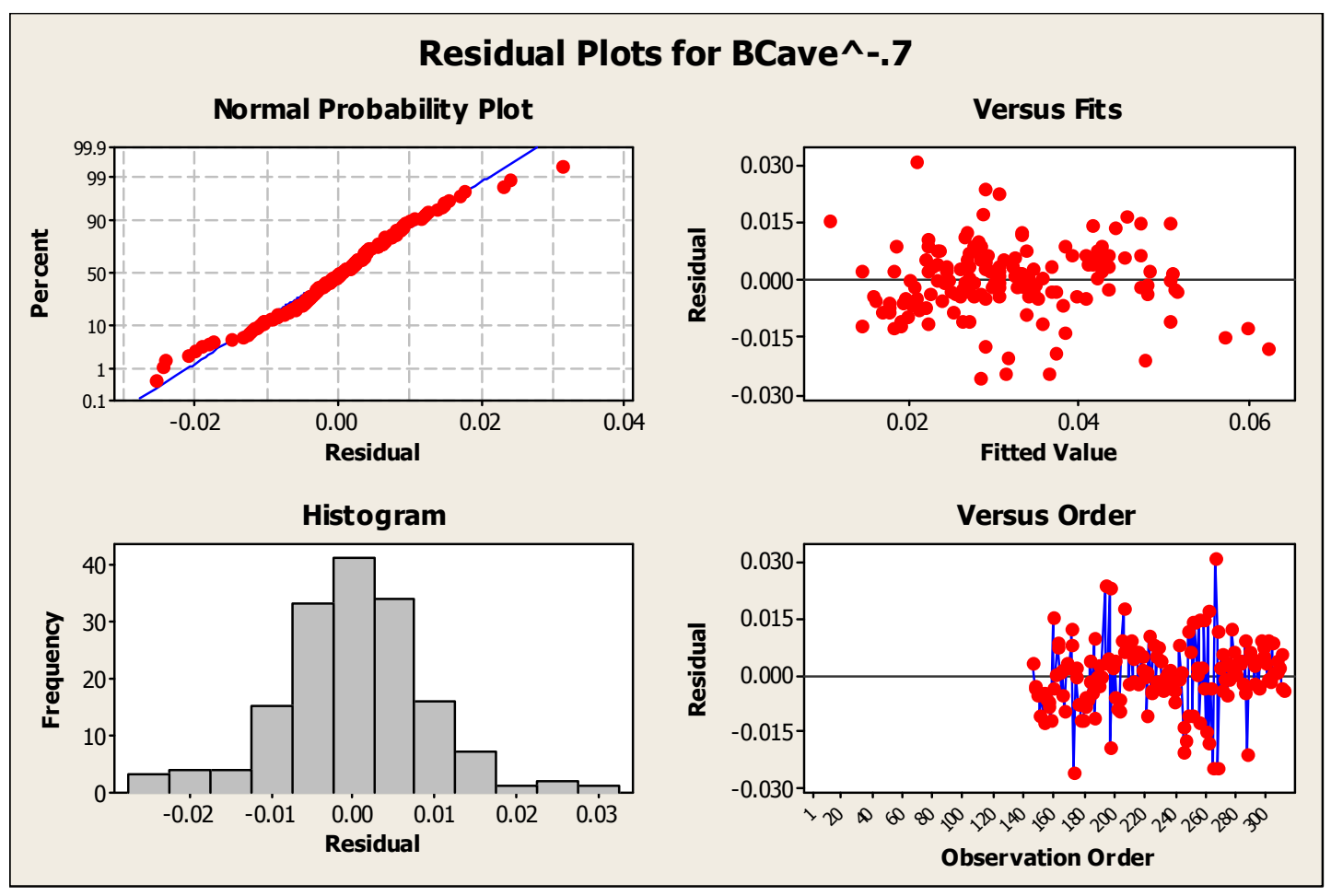


Matrix Plot of BCave^^-.7 vs lag1^^-0.7*ti, E\&C WS (mph), $\log (E \& C$ Hum)
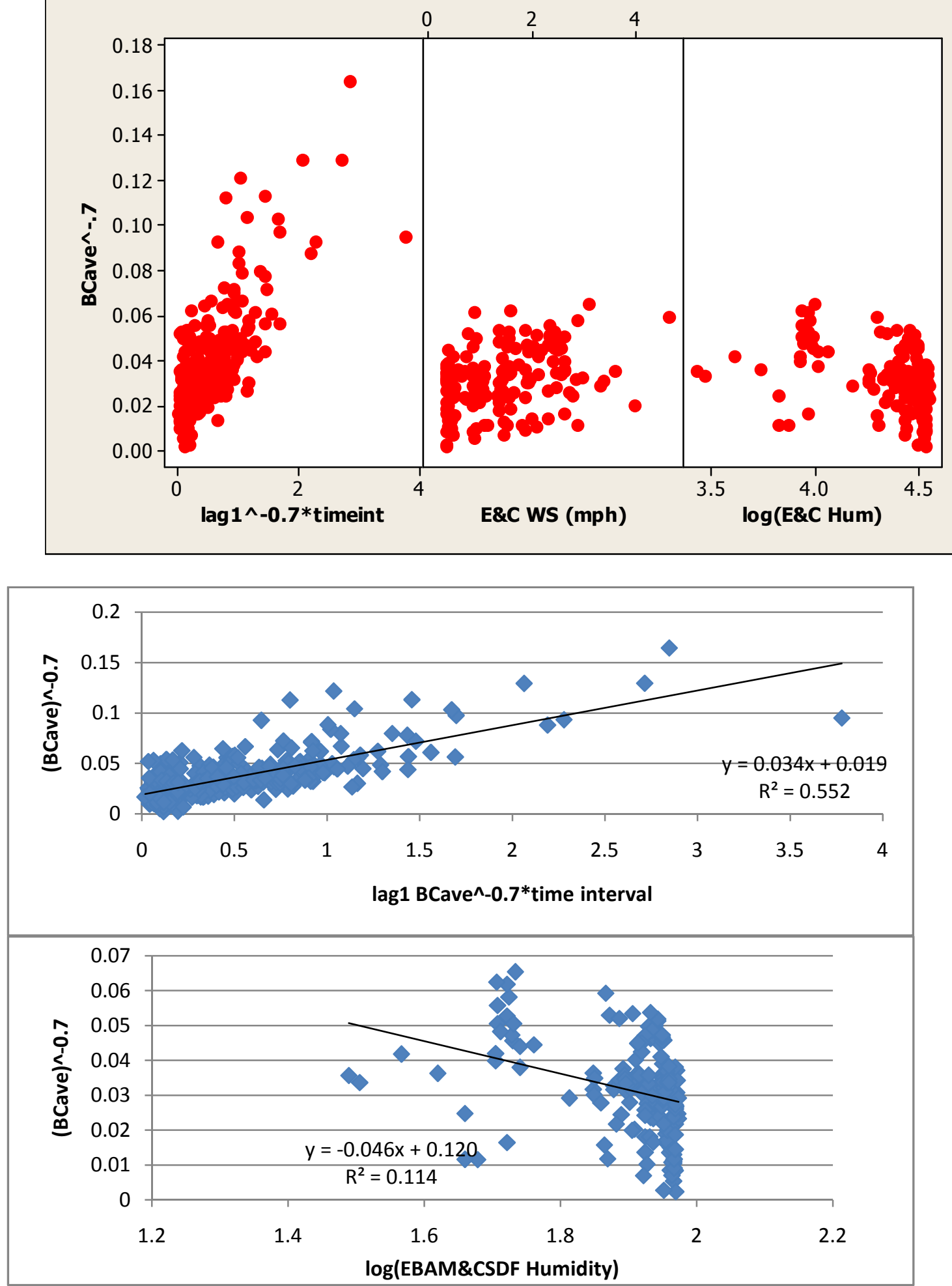


\section{BC Deviation}

\section{Linear CSDF Model \\ Regression Analysis: BCdev^0.5 versus lagged1_BCde, CSDF WS (mph,}

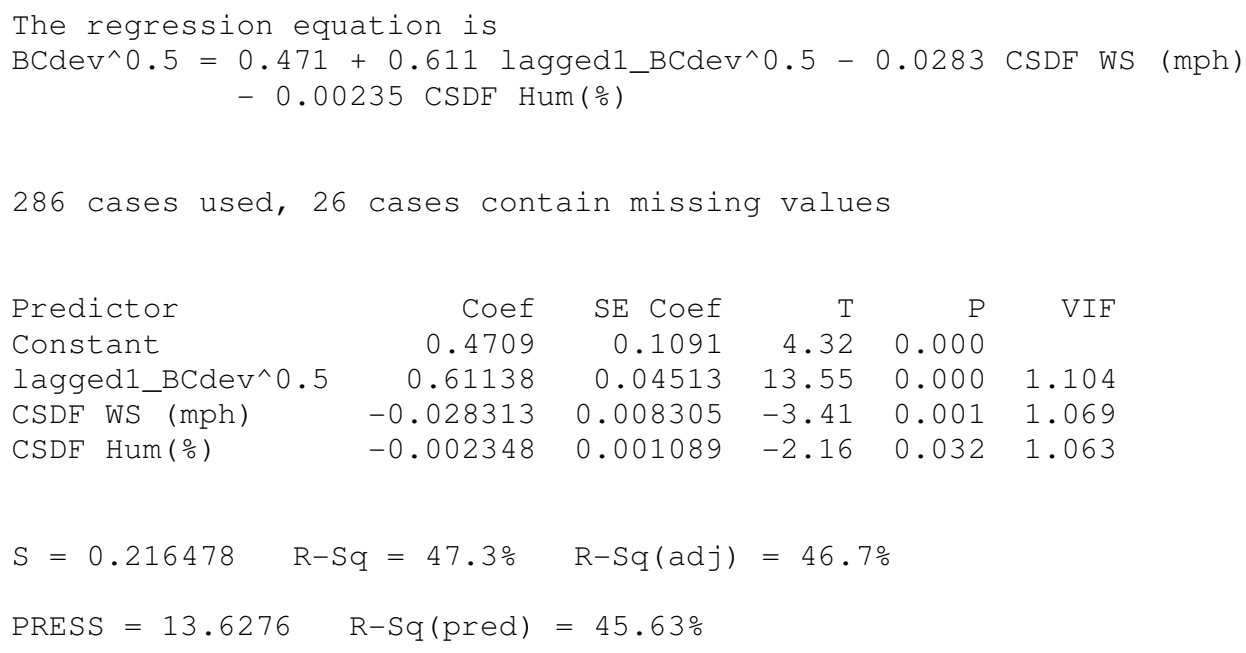

$\begin{array}{rrrrrrr}\text { Obs lagged1_BCdev^0.5 } & \text { BCdev^0.5 } & \text { Fit } & \text { SEFit } & \text { Residual } & \text { St Resid } \\ 5 & 0.54 & 0.3654 & 0.4166 & 0.0446 & -0.0512 & -0.24 \mathrm{X} \\ 32 & 0.79 & 0.0964 & 0.6893 & 0.0163 & -0.5929 & -2.75 \mathrm{R} \\ 72 & 0.12 & 0.7511 & 0.3180 & 0.0329 & 0.4331 & 2.02 \mathrm{R} \\ 74 & 1.13 & 0.8738 & 0.7665 & 0.0514 & 0.1072 & 0.51 \mathrm{X} \\ 90 & 0.98 & 0.1408 & 0.8490 & 0.0261 & -0.7082 & -3.30 \mathrm{R} \\ 159 & 1.27 & 0.4328 & 1.0183 & 0.0371 & -0.5855 & -2.74 \mathrm{R} \\ 173 & 0.83 & 1.3952 & 0.7601 & 0.0220 & 0.6350 & 2.95 \mathrm{R} \\ 242 & 0.59 & 0.9188 & 0.7699 & 0.0708 & 0.1490 & 0.73 \mathrm{X} \\ 243 & 0.92 & 0.8679 & 0.9481 & 0.0594 & -0.0802 & -0.39 \mathrm{X} \\ 244 & 0.87 & 0.4724 & 0.9075 & 0.0554 & -0.4351 & -2.08 \mathrm{RX} \\ 245 & 0.47 & 0.3890 & 0.5408 & 0.0501 & -0.1518 & -0.72 \mathrm{X} \\ 247 & 0.95 & 1.2704 & 0.8252 & 0.0483 & 0.4452 & 2.11 \mathrm{RX} \\ 248 & 1.27 & 0.9713 & 0.9958 & 0.0461 & -0.0245 & -0.12 \mathrm{X} \\ 261 & 0.44 & 0.6372 & 0.6116 & 0.0445 & 0.0256 & 0.12 \mathrm{X}\end{array}$




$\begin{array}{rrrrrrr}284 & 0.33 & 0.8523 & 0.3908 & 0.0180 & 0.4615 & 2.14 \mathrm{R} \\ 288 & 0.32 & 1.0421 & 0.3588 & 0.0201 & 0.6833 & 3.17 \mathrm{R} \\ 290 & 0.96 & 0.2560 & 0.6768 & 0.0450 & -0.4208 & -1.99 \mathrm{X}\end{array}$

$R$ denotes an observation with a large standardized residual.

$X$ denotes an observation whose $X$ value gives it large leverage.

Durbin-Watson statistic $=2.29582$

Lack of fit test

Possible curvature in variable lagged _ $(\mathrm{P}-$ Value $=0.000)$

Possible interaction in variable CSDF Hum (P-Value $=0.015$ )

Overall lack of fit test is significant at $\mathrm{P}=0.000$

\section{Residual Plots for BCdev^0.5}

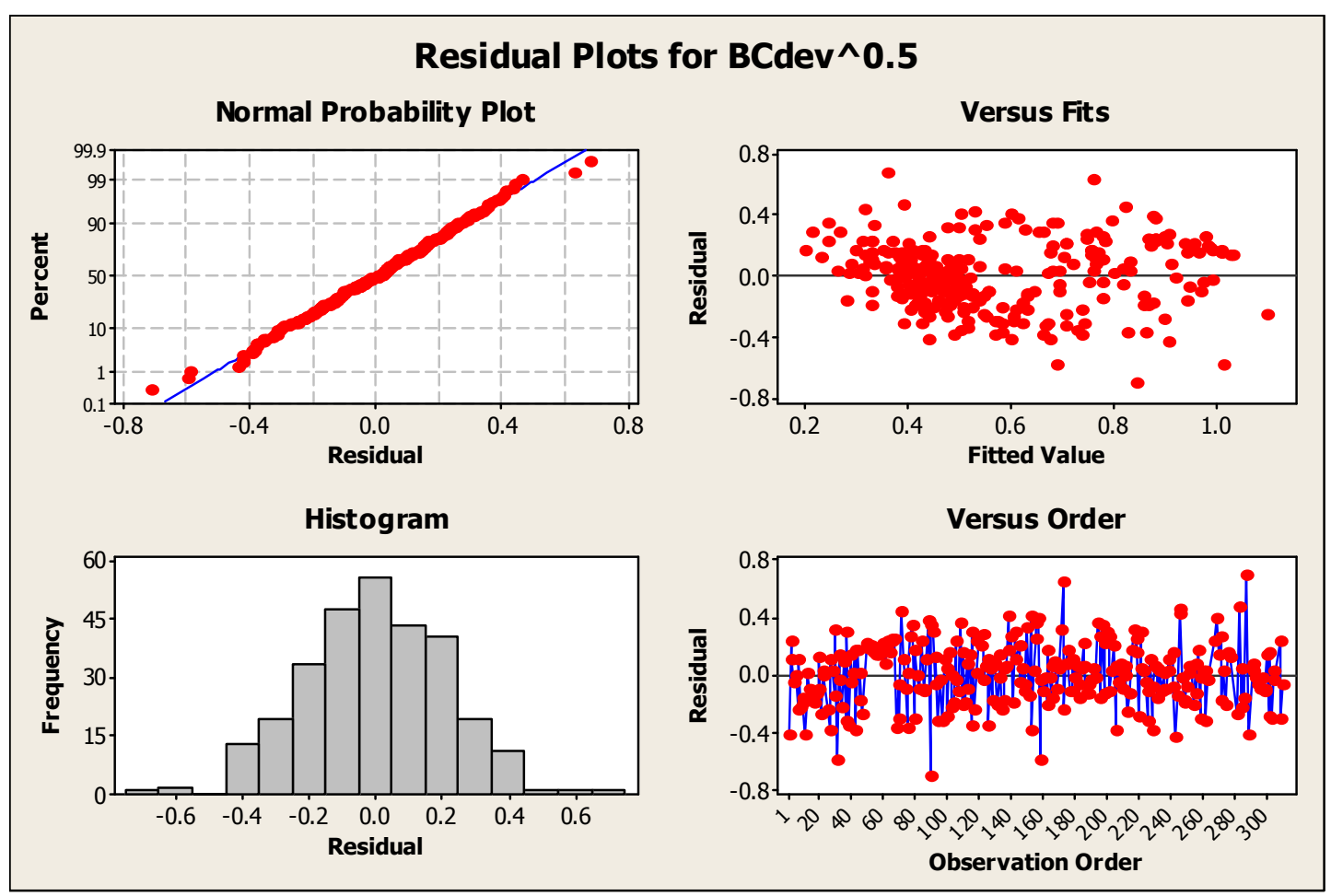




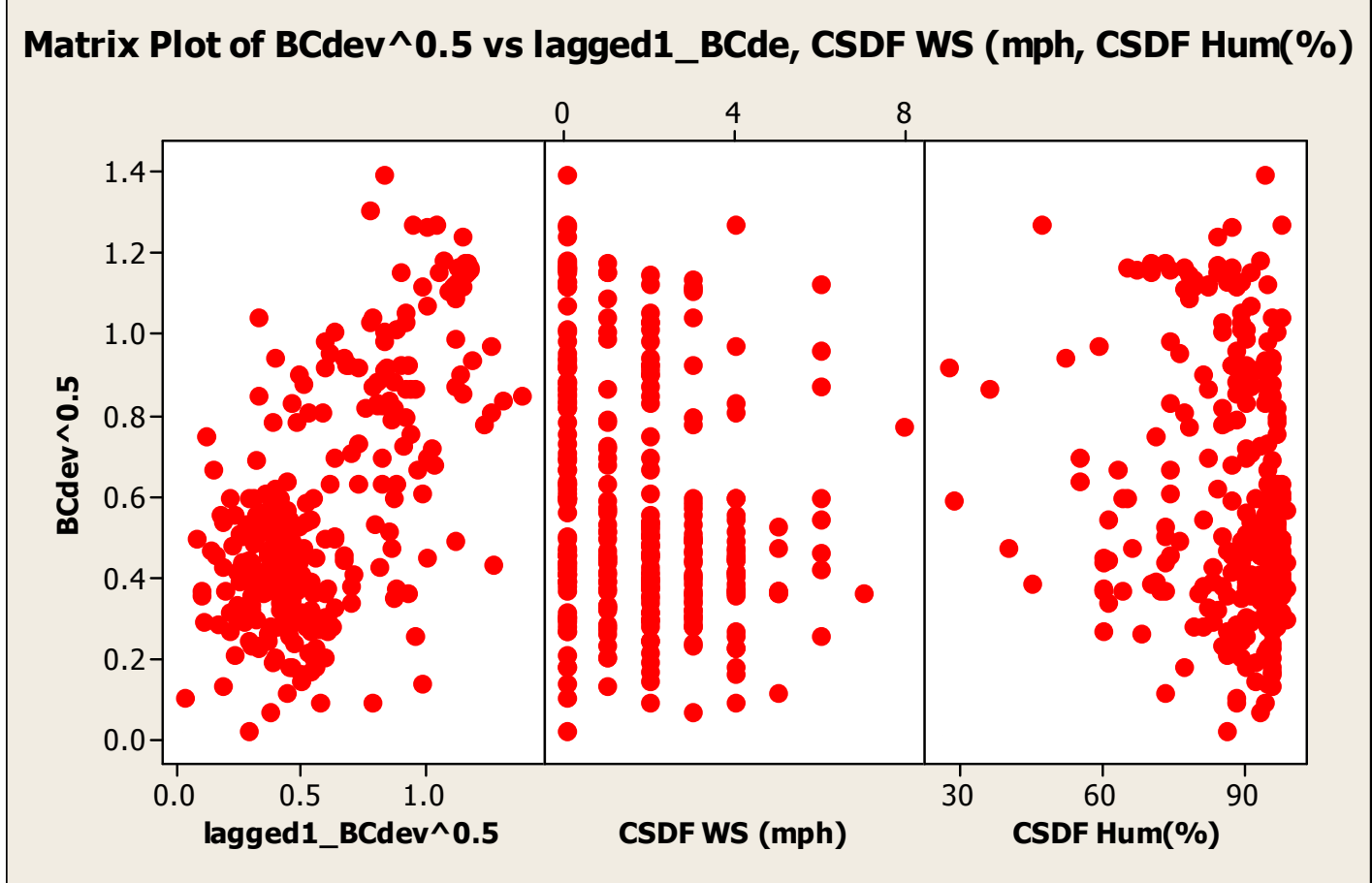




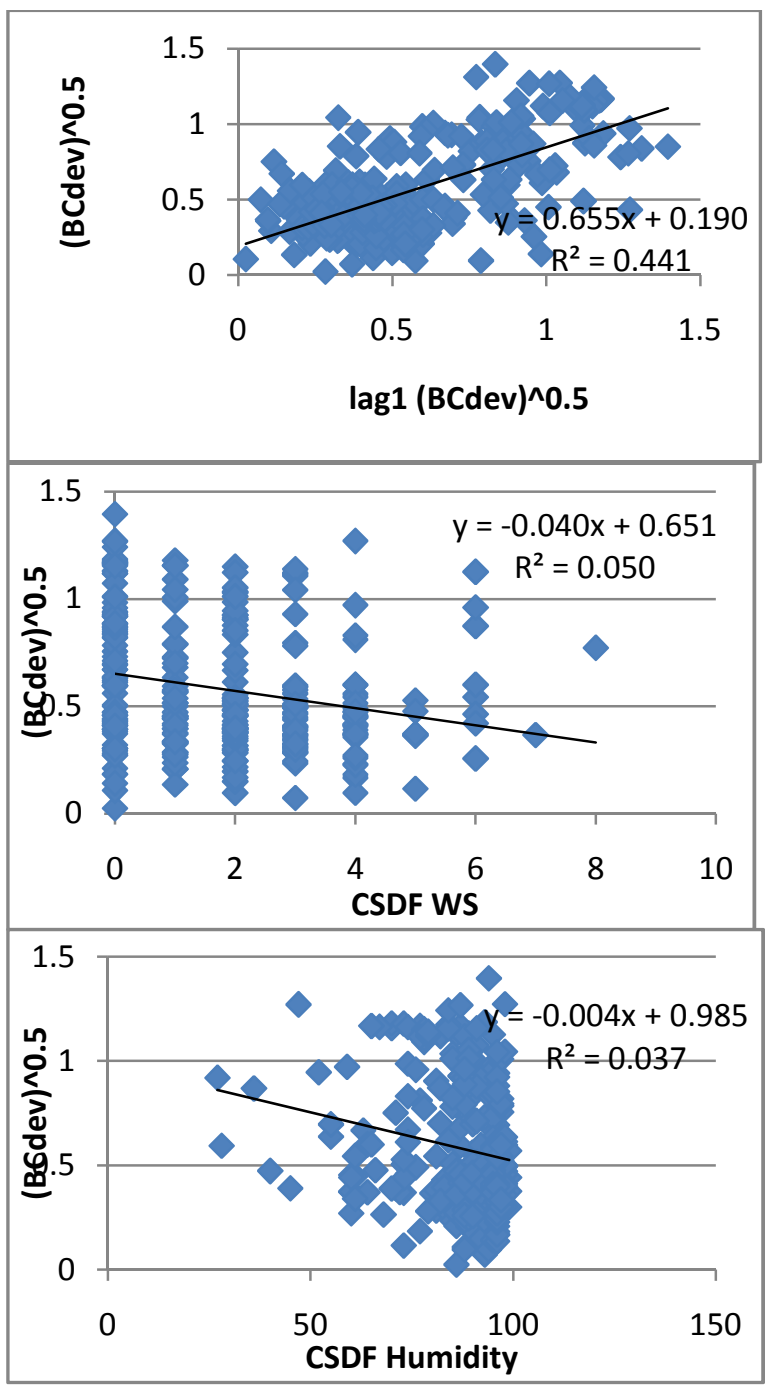




\section{Appendix H. PEM BC Linear Regression Models Average BC Concentration}

\section{Marine Terrace Station Results}

Regression Analysis: BCave^^.4 versus MT Wind Spee, MT sin(Wind , ...

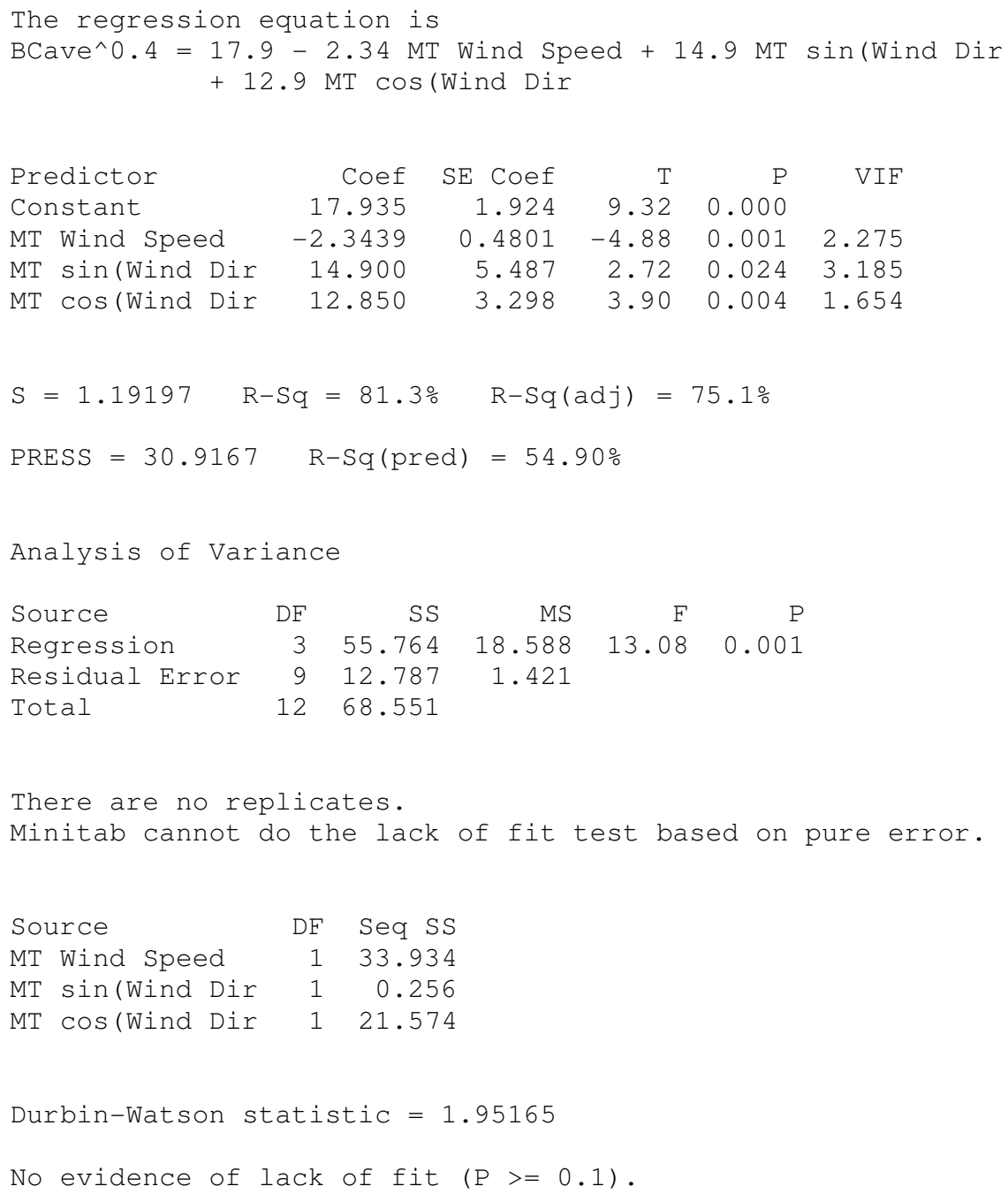

\section{Residual Plots for BCave^^0.4}




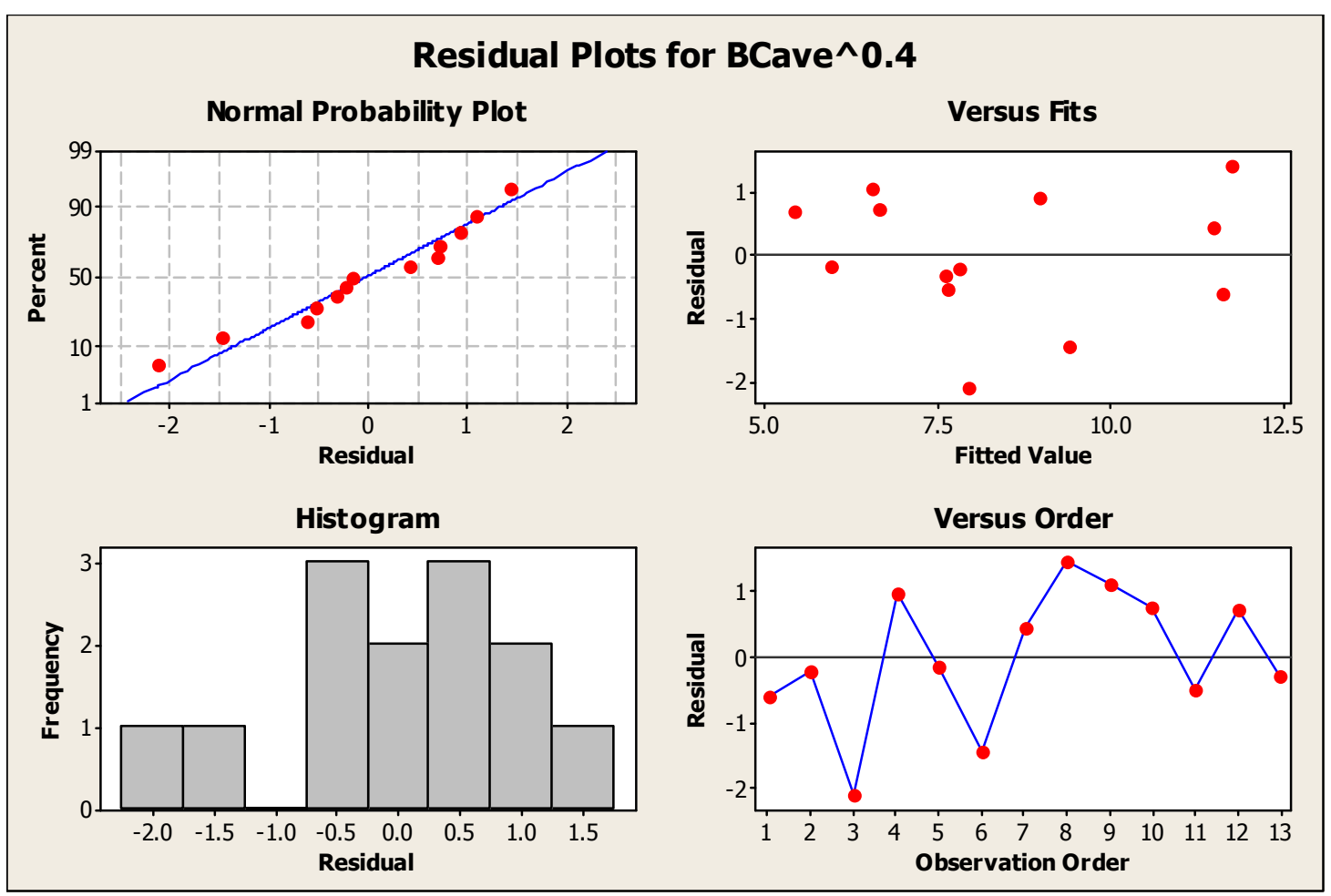

Matrix Plot of BCave^0.4 vs MT Wind Spee, MT $\sin ($ Wind, MT $\cos$ (Wind

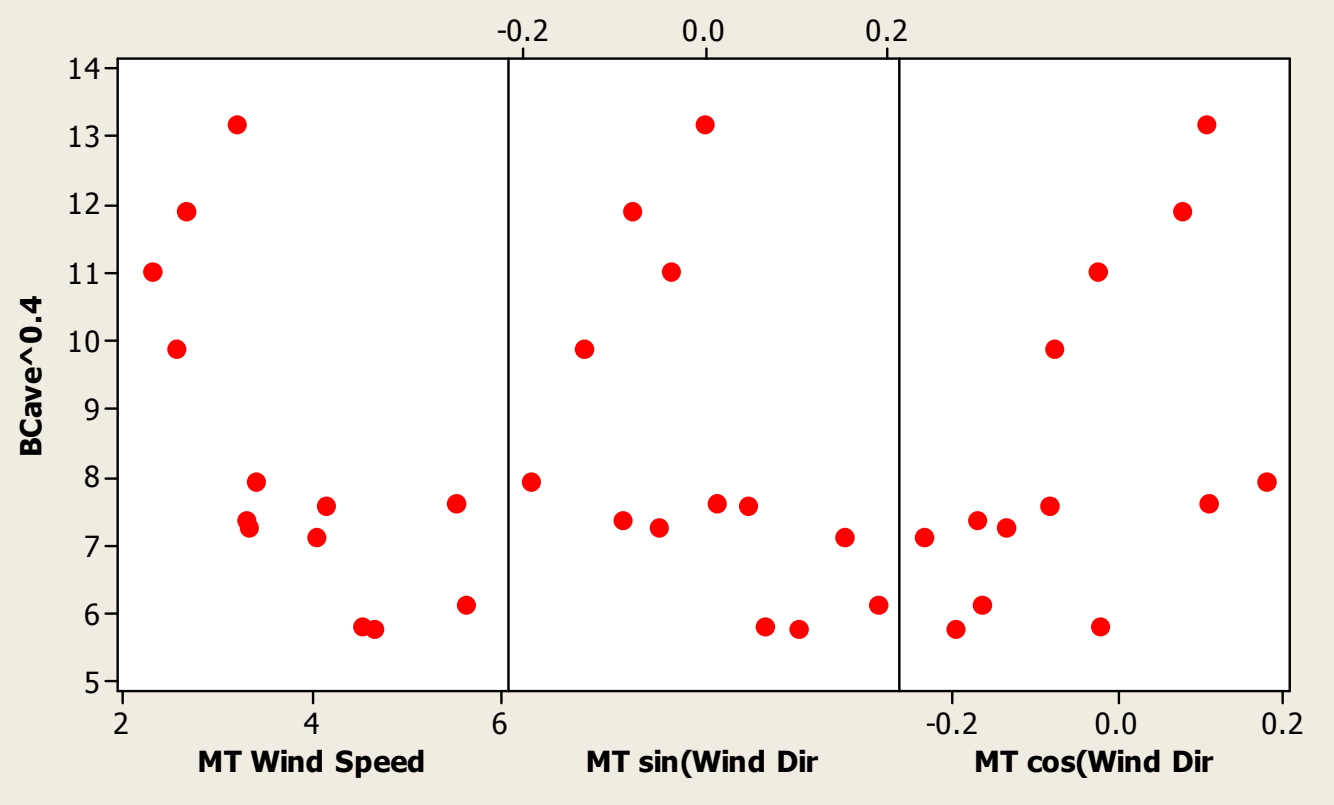



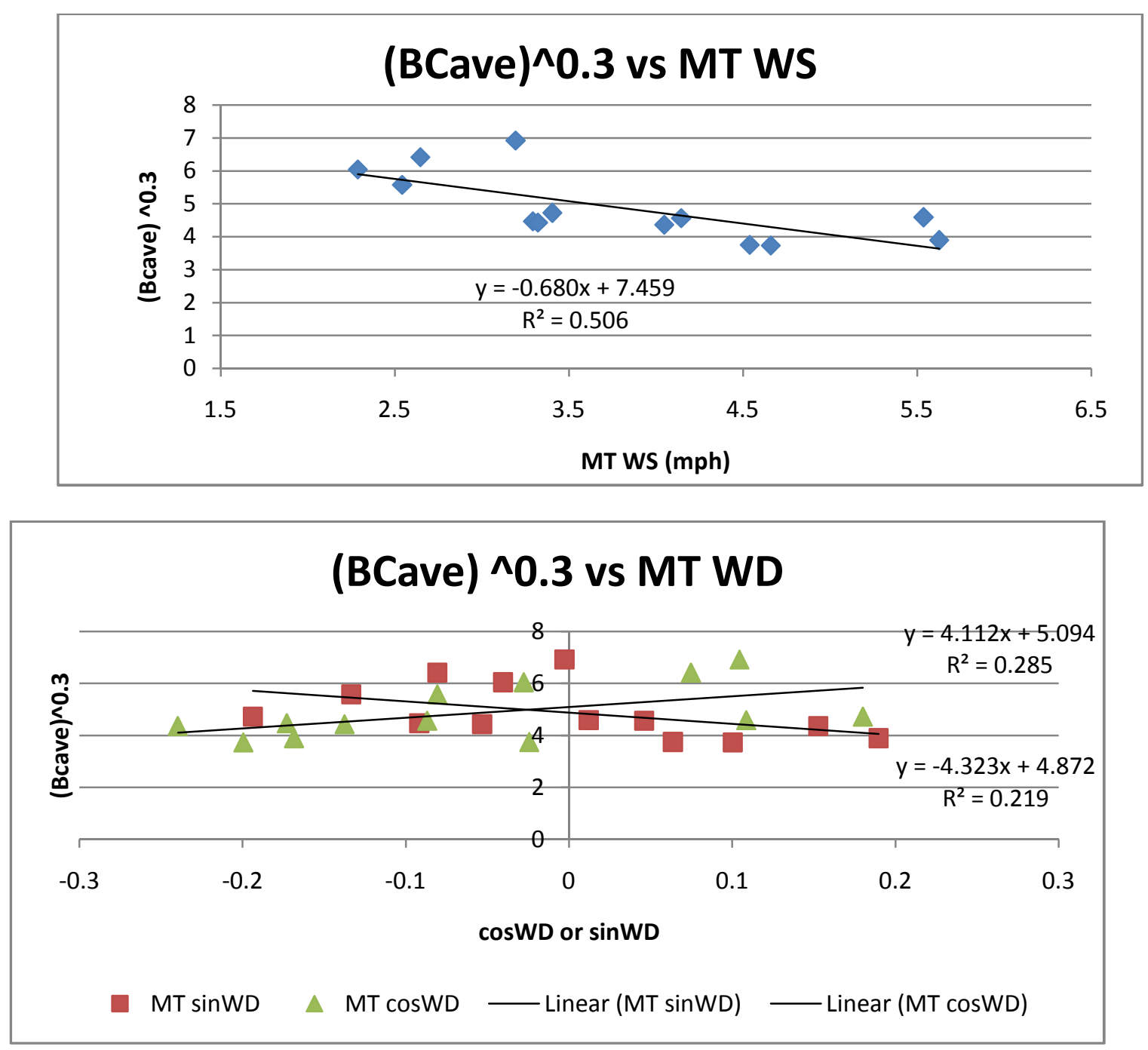

\section{CSDF Station Results}

Regression Analysis: In(BCave) versus CSDF Windspeed (mph)

The regression equation is

$\ln ($ BCave $)=6.71-0.778 \mathrm{CSDF}$ Windspeed (mph)

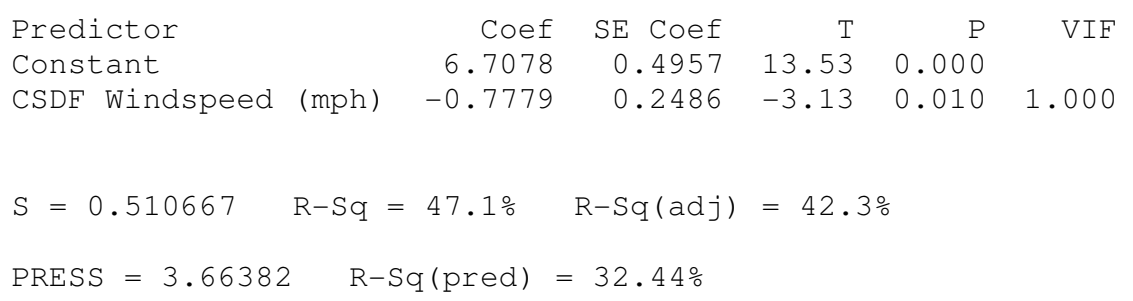




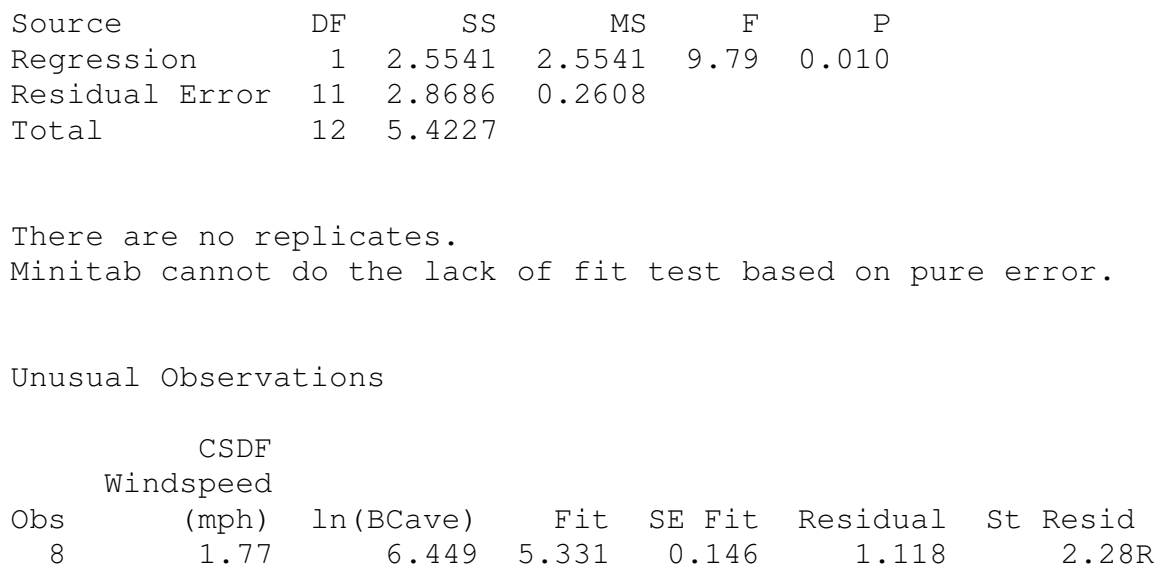

Residual Plots for In(BCave)

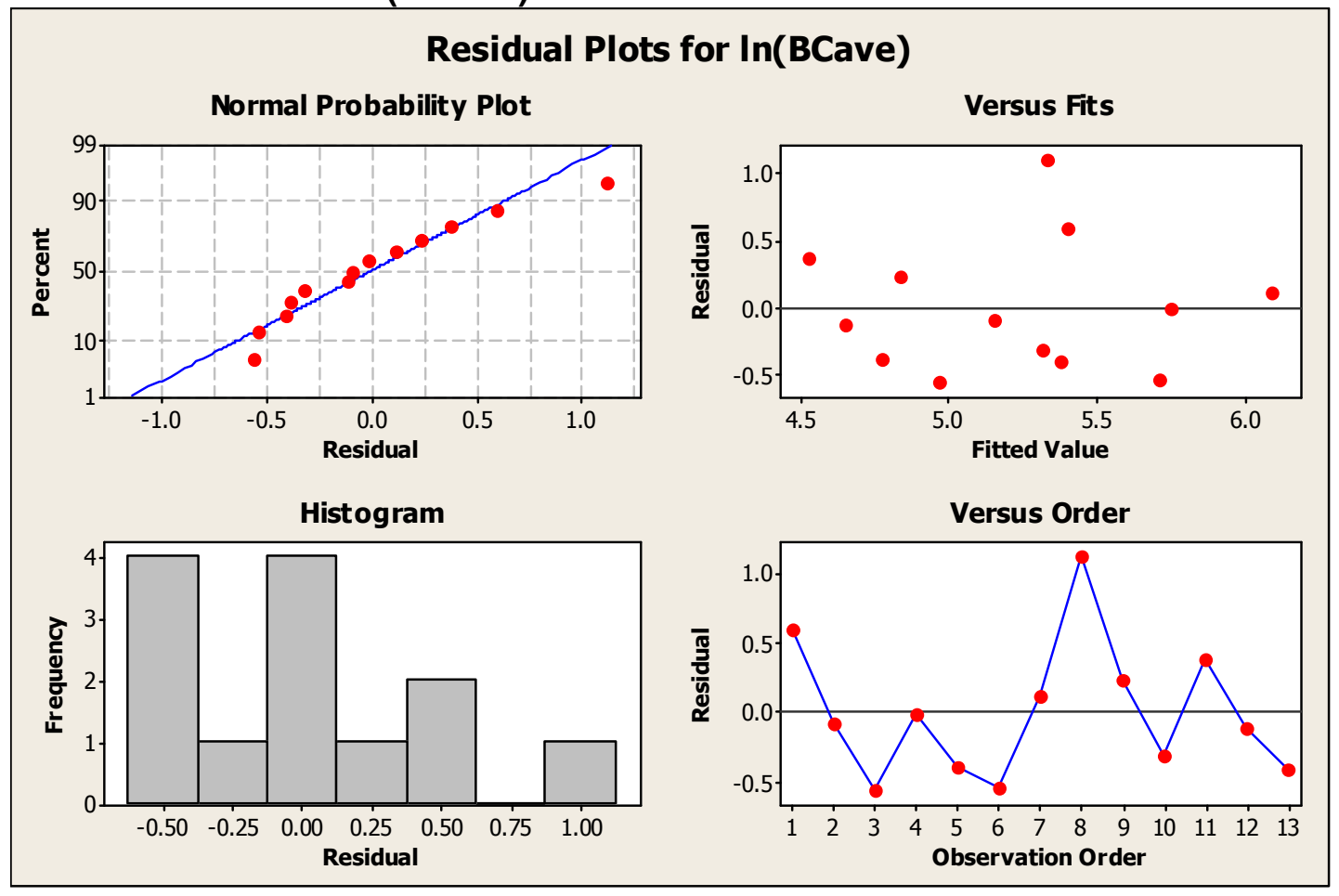




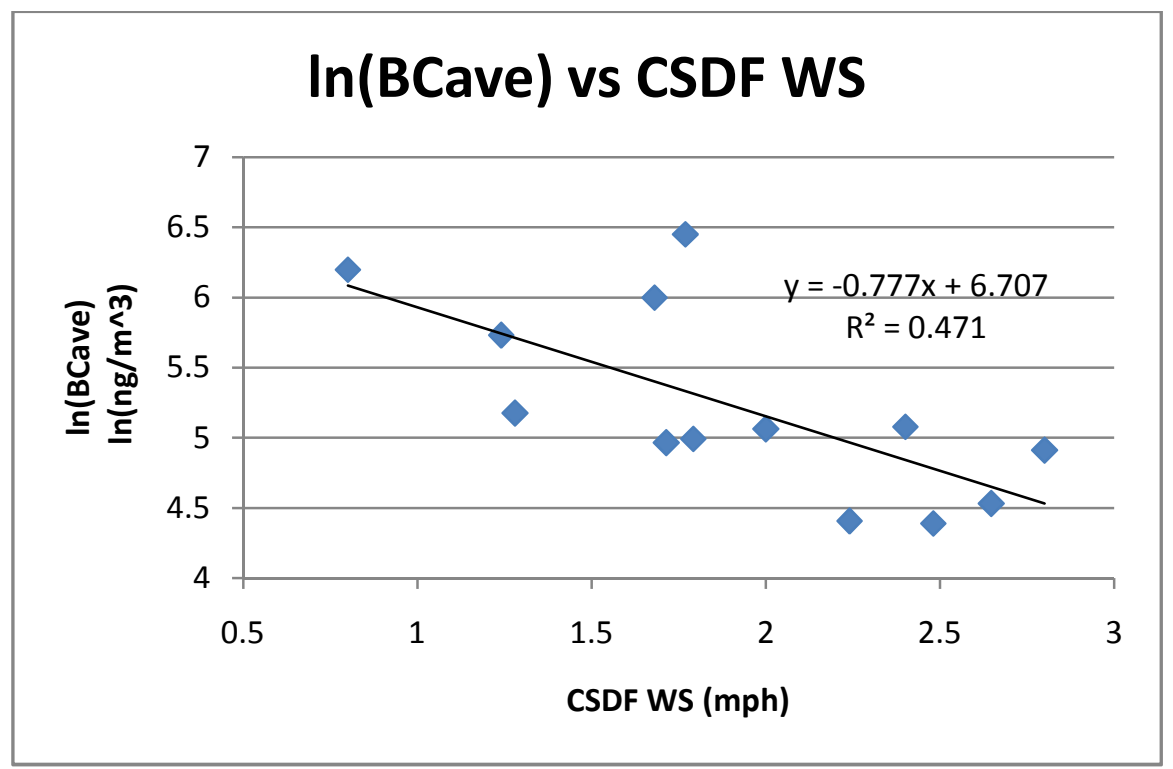

\section{MT \& CSDF Combination Results \\ Regression Analysis: In(BCave) versus Wind Speed (mph), Ave sin(WD) MT+C}

The regression equation is

$\ln ($ BCave $)=7.25-0.725$ Wind Speed (mph) MT+CSDF +2.60 Ave sin(WD) MT+CSDF

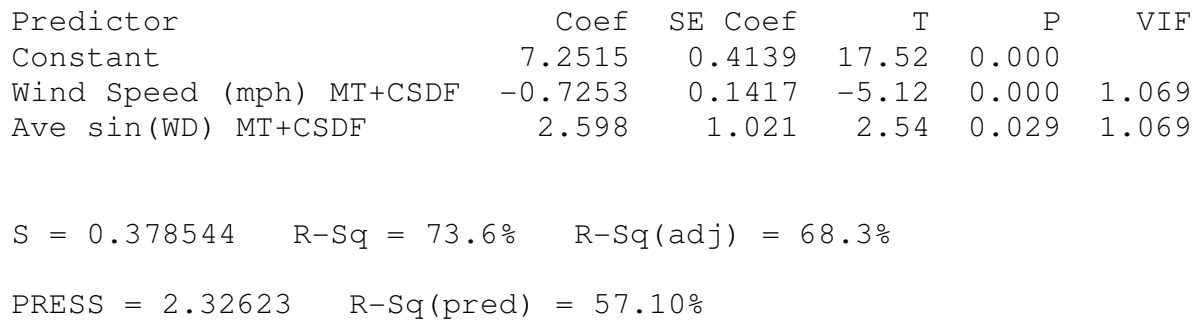


Unusual Observations

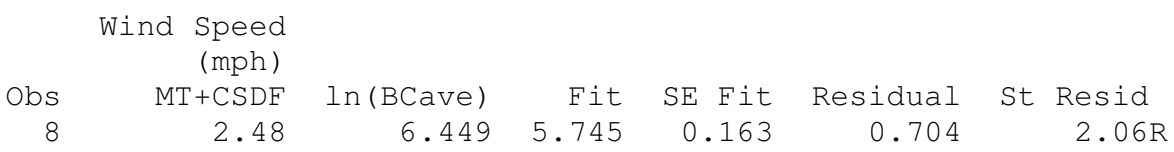

$\mathrm{R}$ denotes an observation with a large standardized residual.

Durbin-Watson statistic $=1.66475$

No evidence of lack of fit $(P>=0.1)$.

\section{Residual Plots for In(BCave)}

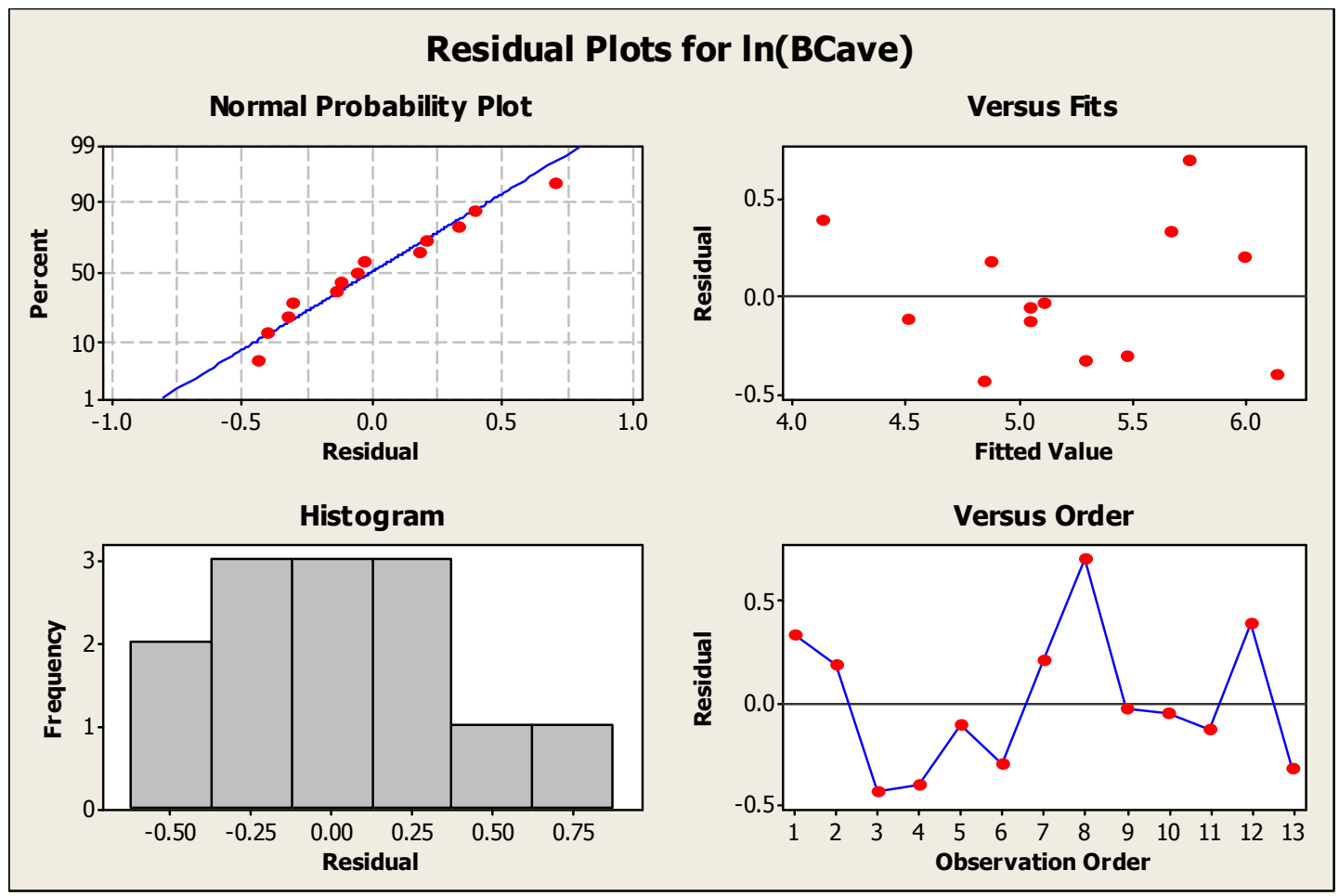



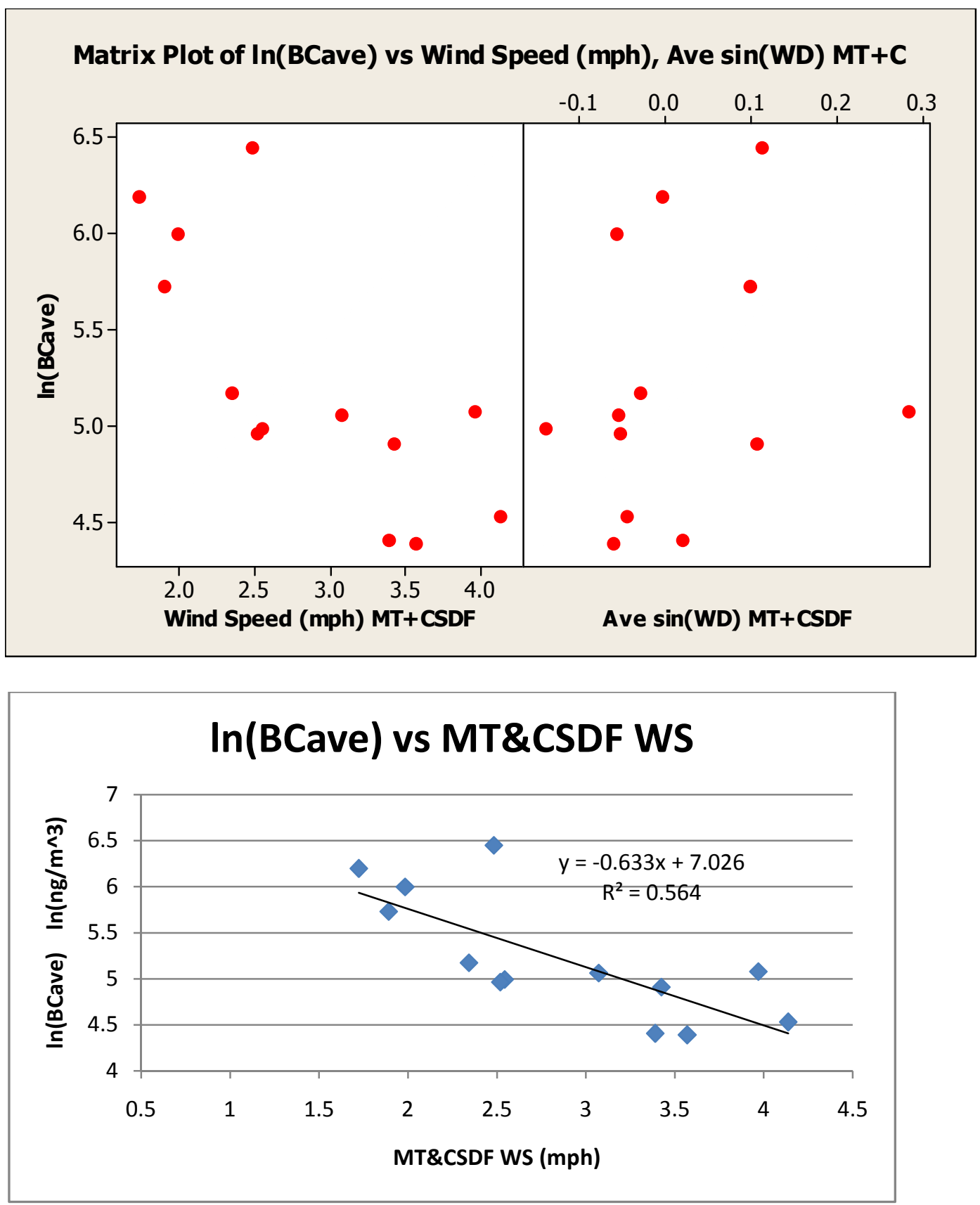


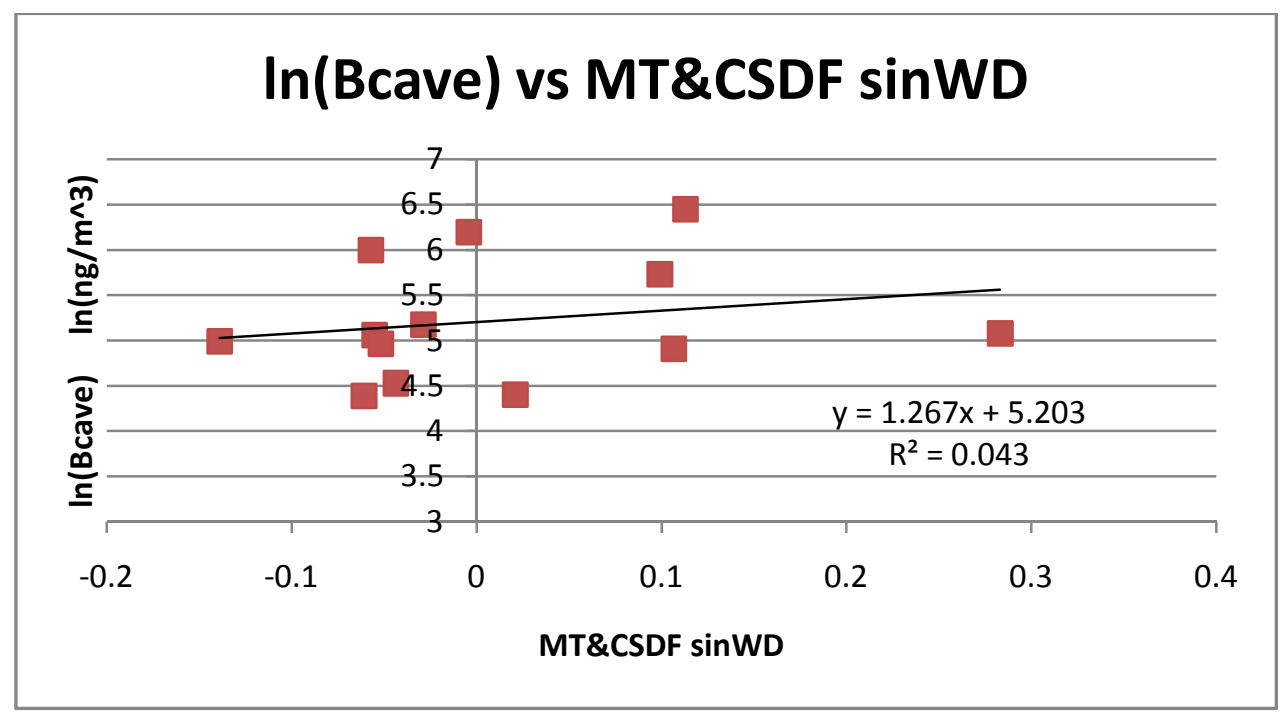

\section{BC Deviation}

\section{Impact of Number of Sources}

\section{Regression Analysis: In(dev) versus Num Sources}

The regression equation is

$\ln (\mathrm{dev})=-1.02+0.0762$ Num Sources

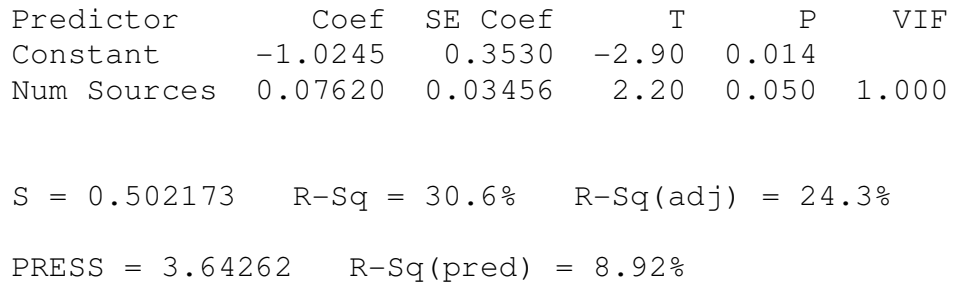

Residual Plots for $\ln (\mathrm{dev})$ 

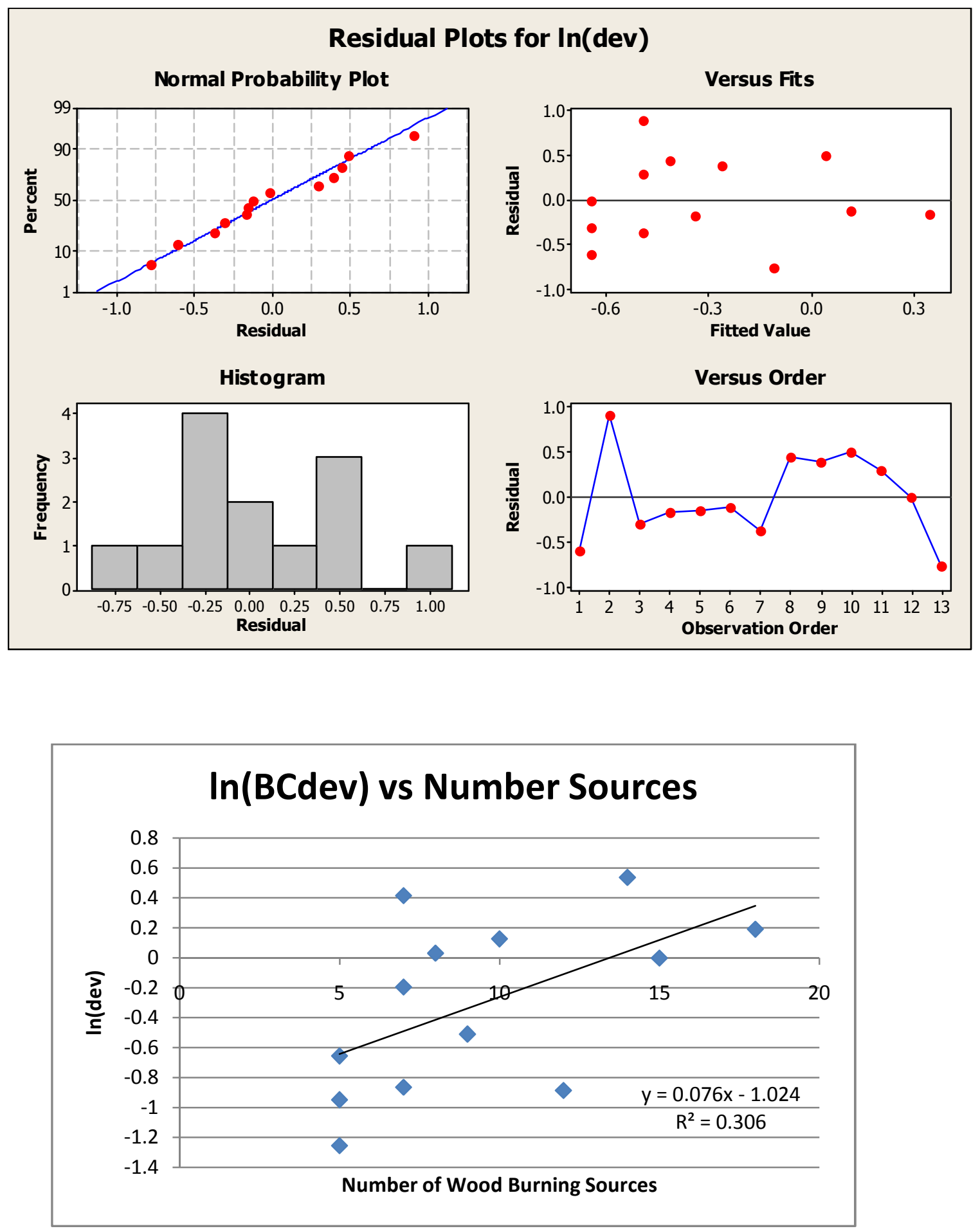

Directional Regression Analysis

General Linear Model: InPEMBC versus Location, IOP

Factor Type Levels Values 


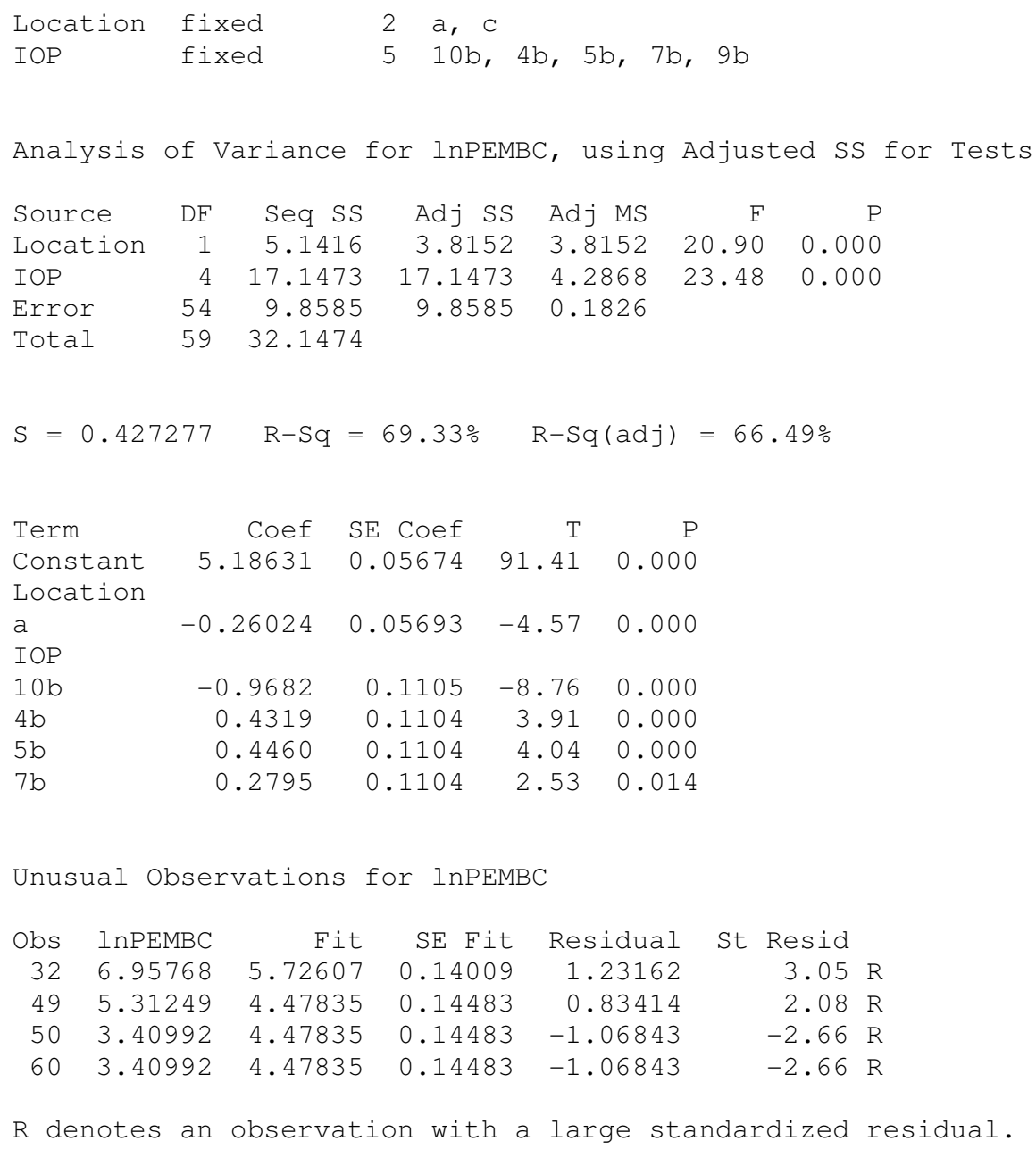

\section{Residual Plots for InPEMBC}



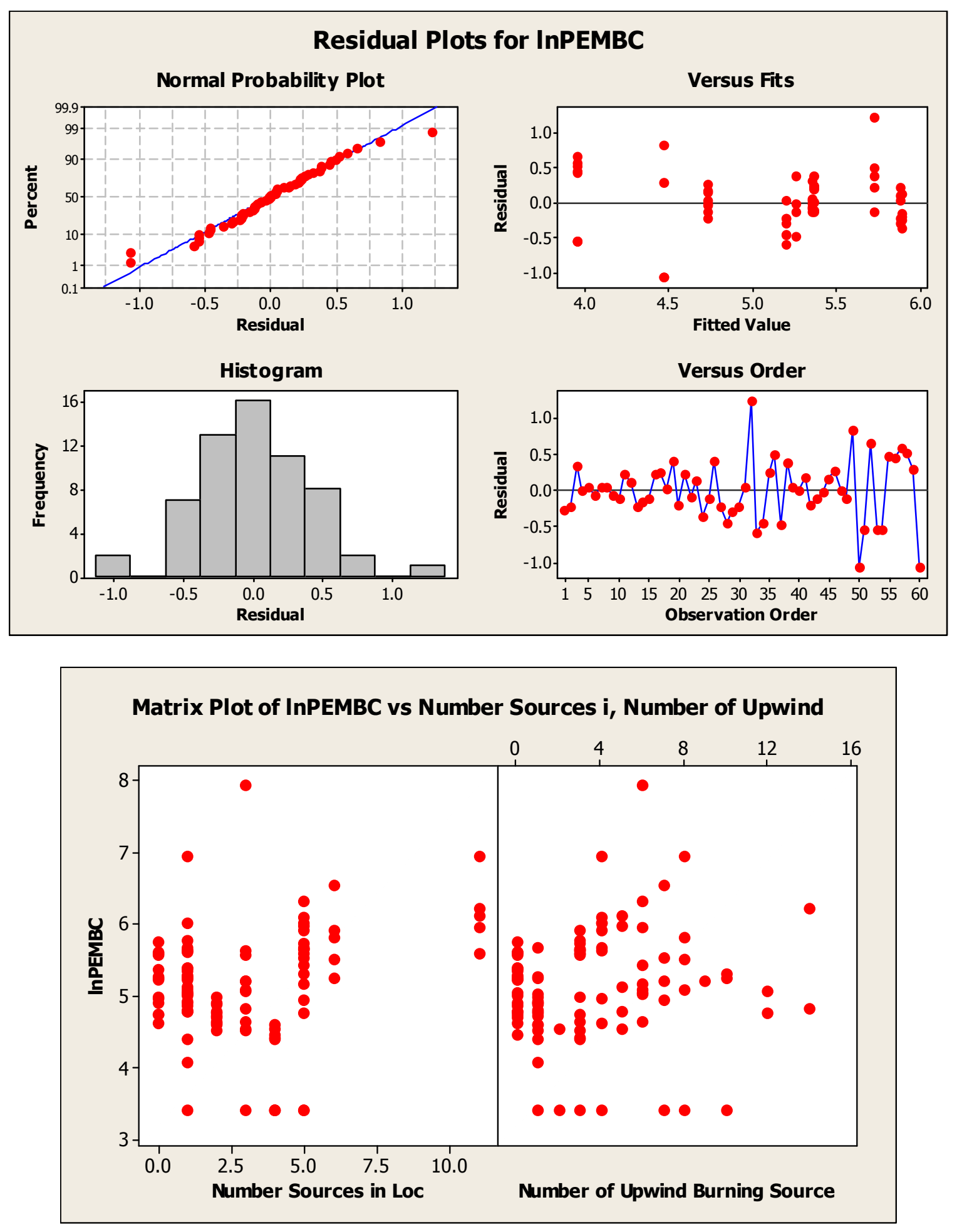


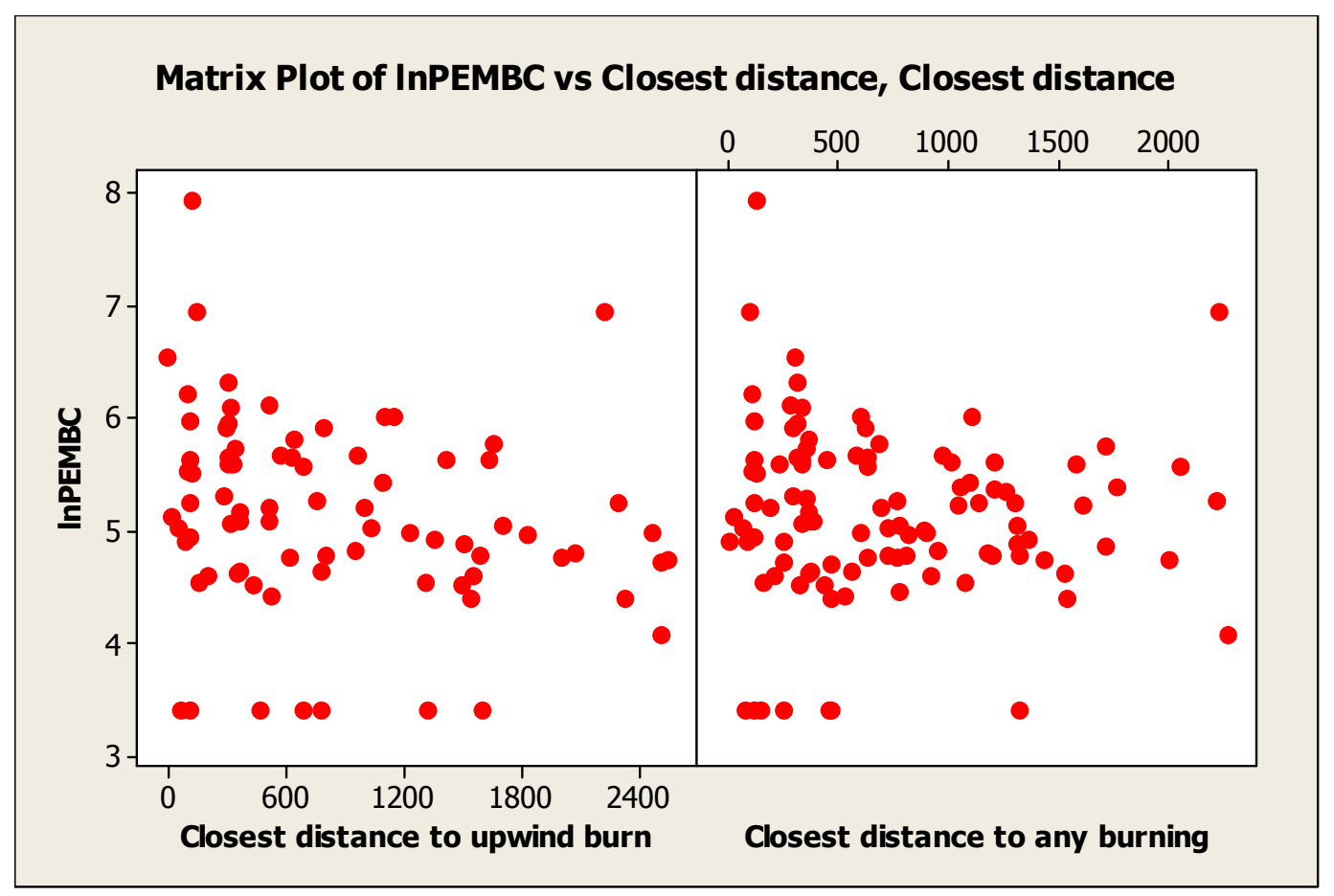

Matrix Plot of InPEMBC vs Smallest angle from domi, Proximity to Roads

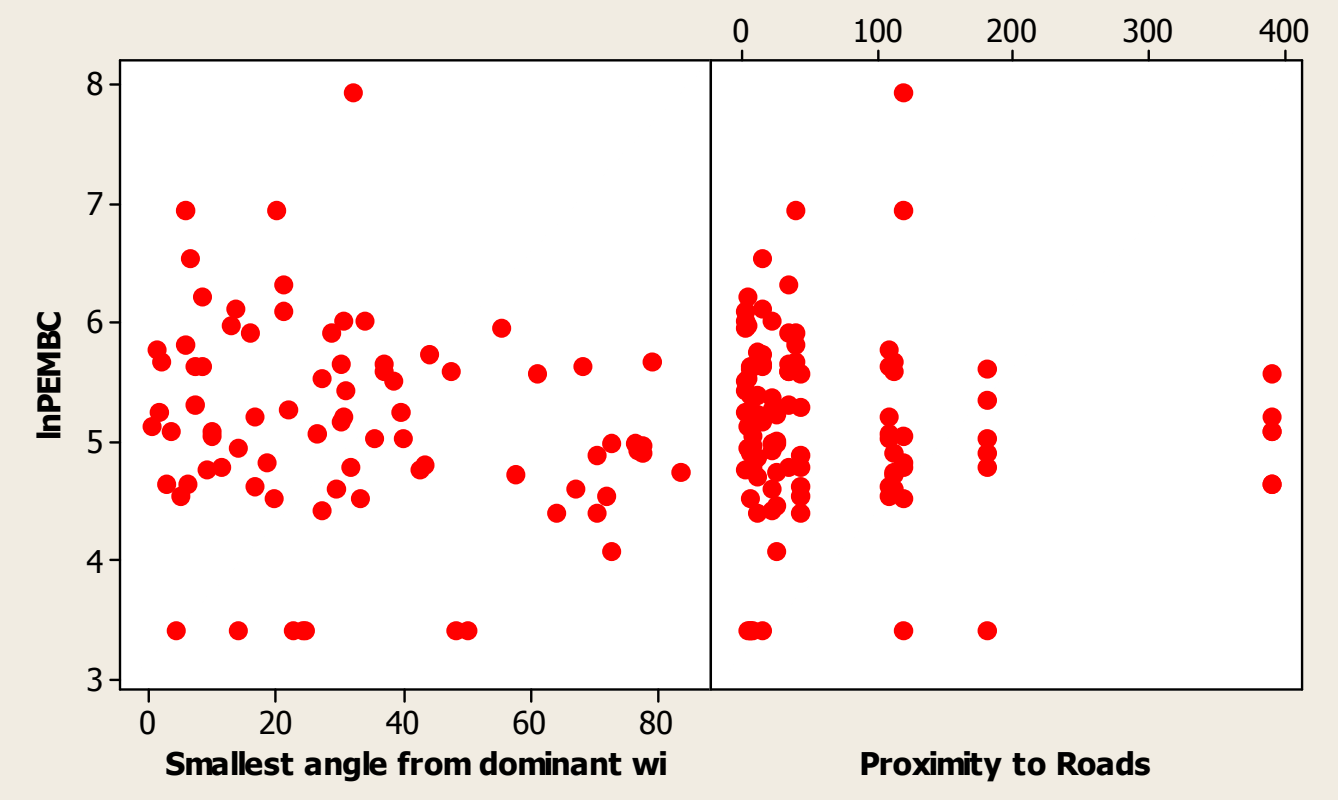

\section{ANOVA Test for Location}

One-way ANOVA: InPEMBC versus Location

$\begin{array}{lrrrrr}\text { Source } & \text { DF } & \text { SS } & \text { MS } & \text { F } & P \\ \text { Location } & 2 & 8.744 & 4.372 & 8.60 & 0.000 \\ \text { Error } & 101 & 51.334 & 0.508 & & \\ \text { Total } & 103 & 60.078 & & & \end{array}$




$\begin{array}{lr}\text { Level } & \mathrm{N} \\ \mathrm{a} & 51 \\ \mathrm{~b} & 21 \\ \mathrm{C} & 32\end{array}$

Mean
4.8791
5.2060
5.5427

StDev

Individual 95\% CIs For Mean Based on Pooled StDev

$----+---------+---------+---------+-----$

0.5921

0.9181

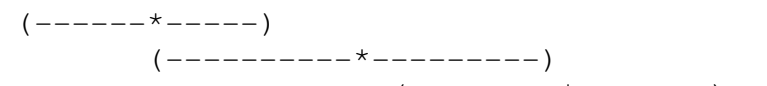

0.7394

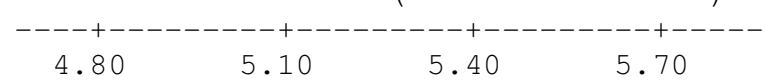

Pooled StDev $=0.7129$

Grouping Information Using Tukey Method

Location N Mean Grouping

c $\quad 32 \quad 5.5427$ A

b $\quad 21 \quad 5.2060$ A B

a $\quad 51 \quad 4.8791 \quad$ B

Means that do not share a letter are significantly different.

Tukey 95\% Simultaneous Confidence Intervals

All Pairwise Comparisons among Levels of Location

Individual confidence level $=98.06 \%$

Location = a subtracted from:

Location Lower Center Upper

$\begin{array}{llll}\text { b } & -0.1122 & 0.3270 & 0.7661\end{array}$

c $\quad 0.2816 \quad 0.6636 \quad 1.0456$

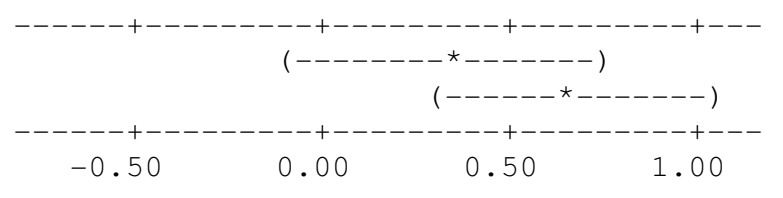

Location $=$ b subtracted from:

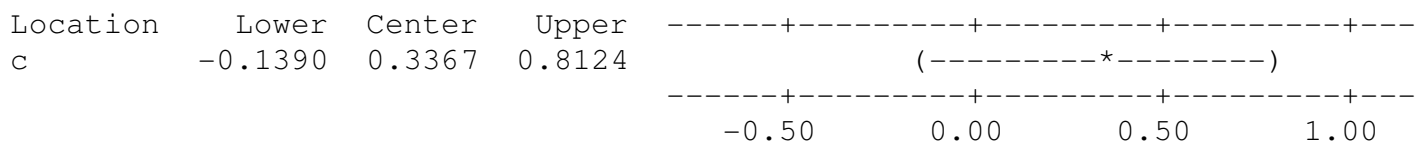

\section{Residual Plots for InPEMBC}




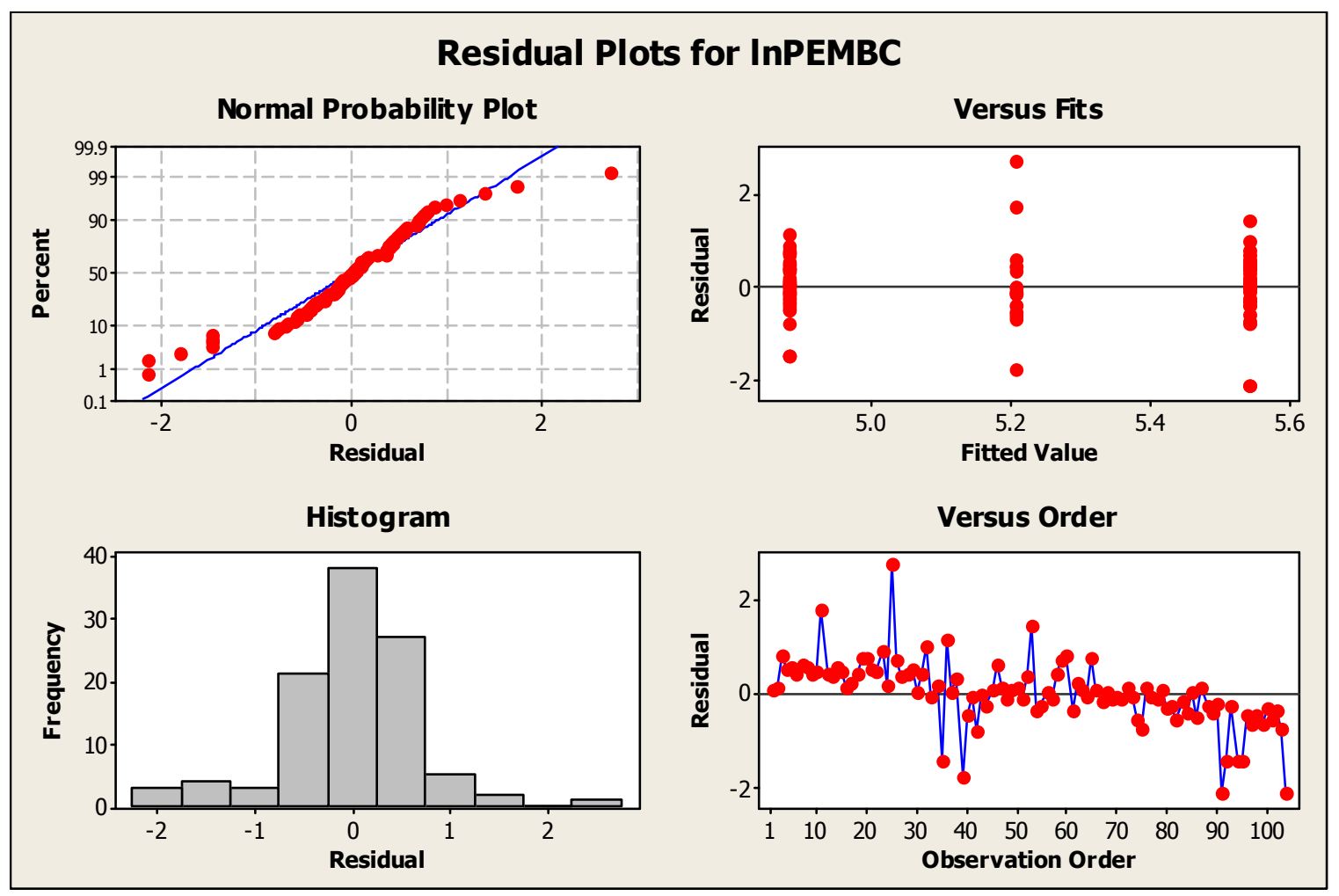

\section{ANOVA Tests for IOP One-way ANOVA: InPEMBC versus IOP}

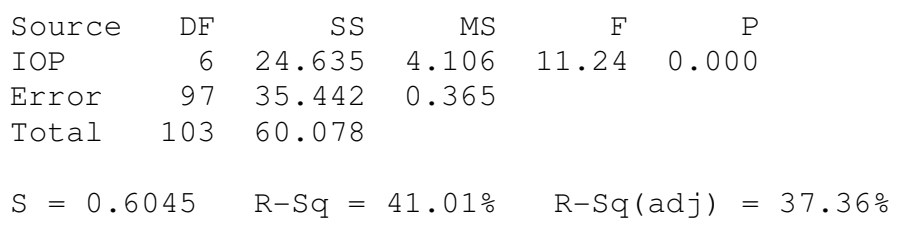
4.20
4.80
5.40
6.00

Pooled StDev $=0.6045$ 


\begin{tabular}{lrrrlll} 
IOP & N & Mean & \multicolumn{2}{l}{ Grouping } \\
5b & 15 & 5.7479 & A & & \\
4b & 15 & 5.6853 & A & B & & \\
7b & 15 & 5.3457 & A & B & C & \\
8b & 15 & 5.1364 & A & B & C & \\
6b & 14 & 5.0373 & & B & C & \\
9b & 15 & 4.8662 & & & C & D \\
10b & 15 & 4.2187 & & & D
\end{tabular}

Means that do not share a letter are significantly different.

Tukey 95\% Simultaneous Confidence Intervals

All Pairwise Comparisons among Levels of IOP

Individual confidence level $=99.67 \%$

$\begin{array}{lrrr}\text { IOP }= & 10 \mathrm{~b} \text { subtracted } & \text { from: } \\ \text { IOP } & \text { Lower } & \text { Center } & \text { Upper } \\ \text { 4b } & 0.8018 & 1.4666 & 2.1315 \\ \text { 5b } & 0.8644 & 1.5293 & 2.1942 \\ \text { 6b } & 0.1420 & 0.8187 & 1.4953 \\ \text { 7b } & 0.4621 & 1.1270 & 1.7919 \\ \text { 8b } & 0.2529 & 0.9177 & 1.5826 \\ \text { 9b } & -0.0174 & 0.6475 & 1.3124\end{array}$

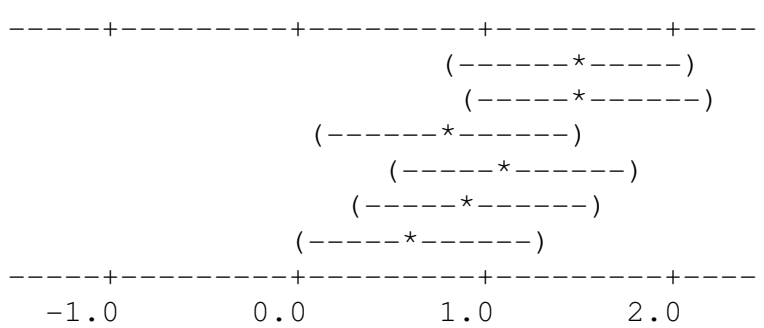

$I O P=4 b$ subtracted from:

$\begin{array}{lrrr}\text { IOP } & \text { Lower } & \text { Center } & \text { Upper } \\ \text { 5b } & -0.6022 & 0.0627 & 0.7275 \\ \text { 6b } & -1.3246 & -0.6480 & 0.0287 \\ \text { 7b } & -1.0045 & -0.3396 & 0.3252 \\ \text { 8b } & -1.2138 & -0.5489 & 0.1160 \\ \text { 9b } & -1.4840 & -0.8191 & -0.1543\end{array}$

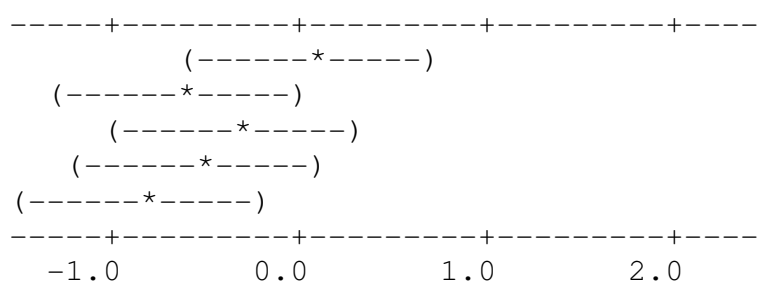

$\mathrm{IOP}=5 \mathrm{~b}$ subtracted from:

$\begin{array}{lrrr}\text { IOP } & \text { Lower } & \text { Center } & \text { Upper } \\ 6 \mathrm{~b} & -1.3873 & -0.7106 & -0.0340 \\ 7 \mathrm{~b} & -1.0672 & -0.4023 & 0.2626 \\ 8 \mathrm{~b} & -1.2764 & -0.6115 & 0.0533 \\ 9 \mathrm{~b} & -1.5467 & -0.8818 & -0.2169\end{array}$

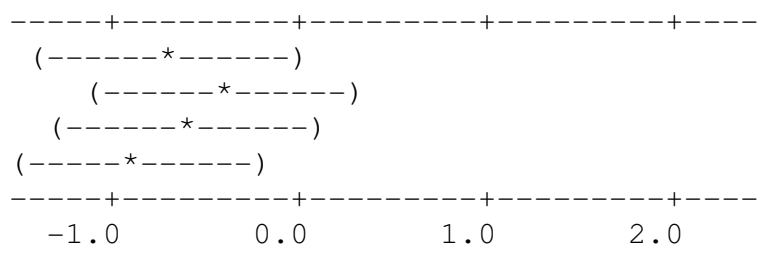

$\mathrm{IOP}=6 \mathrm{~b}$ subtracted from:

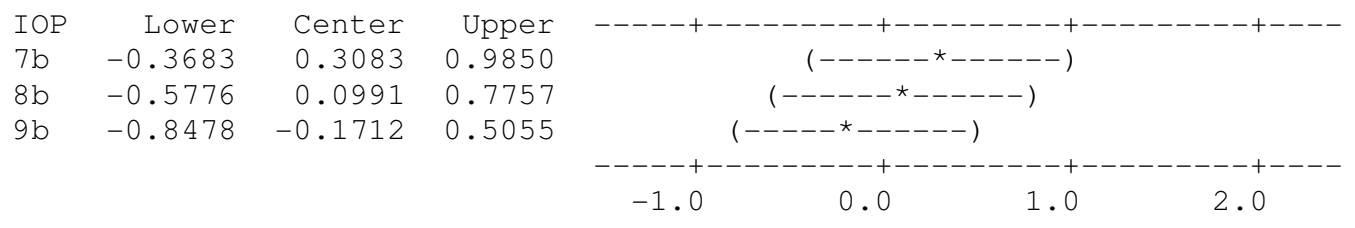



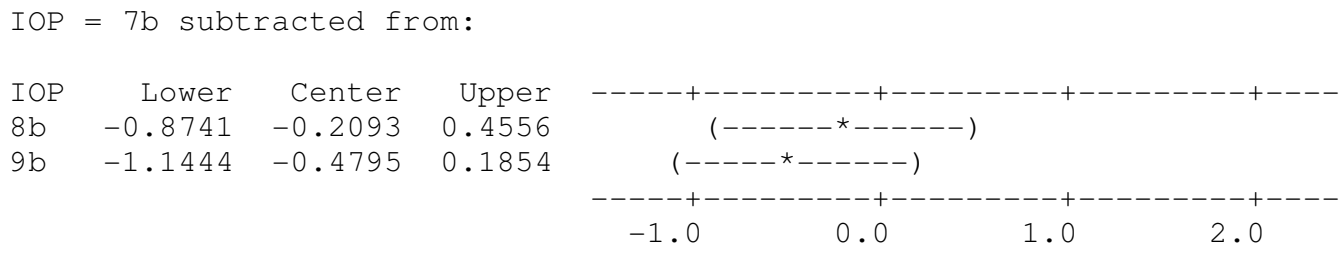

$\mathrm{IOP}=8 \mathrm{~b}$ subtracted from:
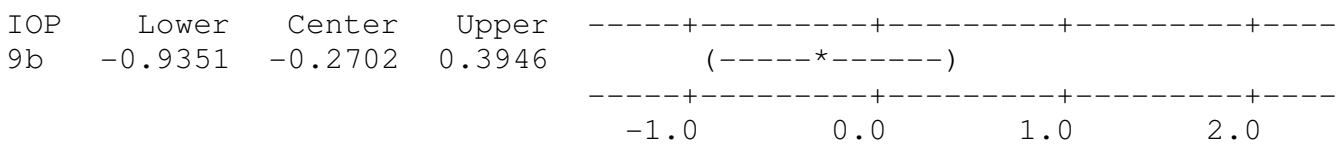

\section{Residual Plots for InPEMBC}

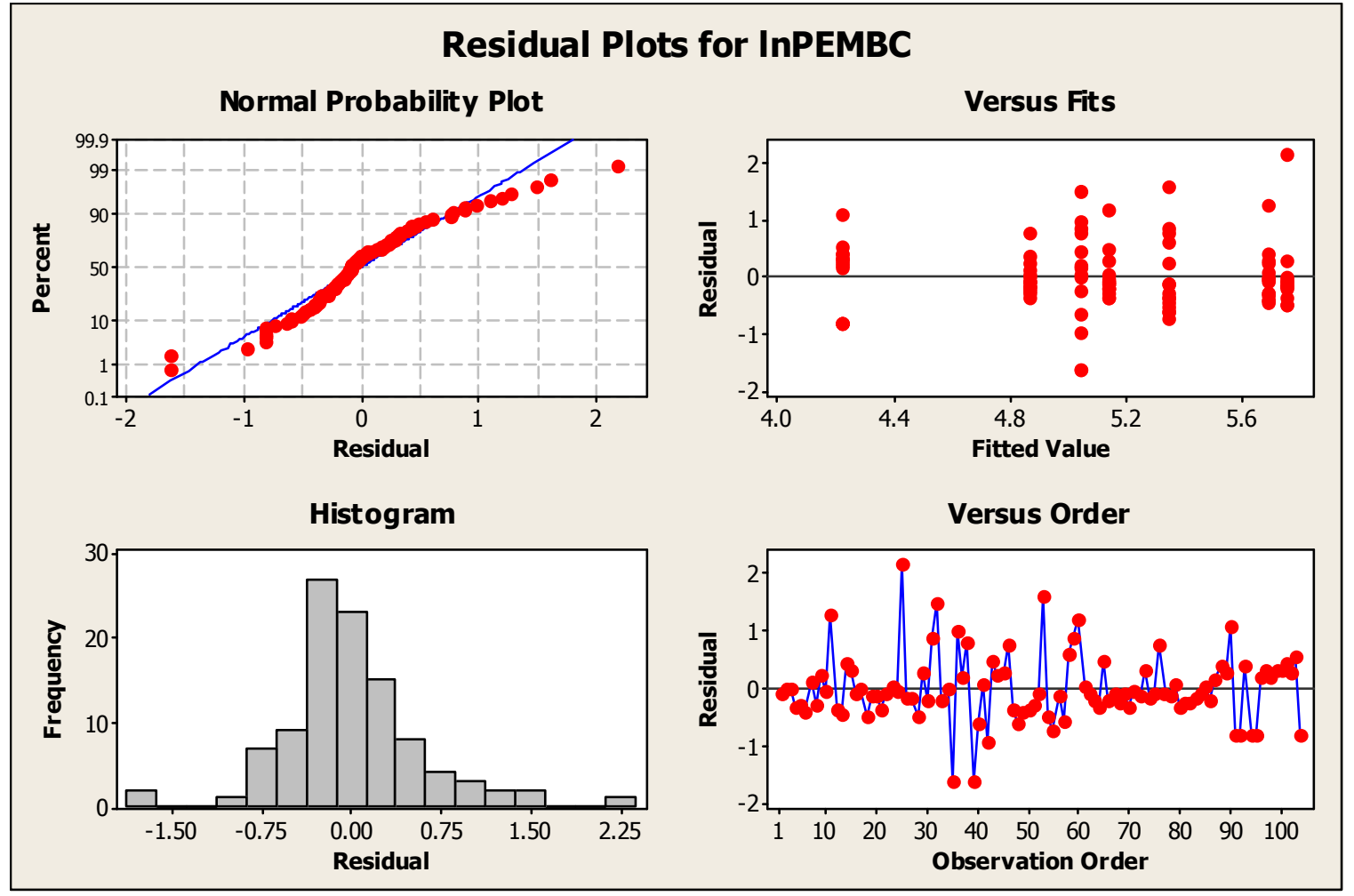




\section{Appendix I. ISC-PRIME and ISCST3 Dispersion Modeling}

\section{Summary of ISC Modeled BC concentrations and Modeled-to-Measured Ratios}

The table below summarizes modeled and measured BC concentrations at each PEM location in addition to the ratio of the modeledto-measured concentration. Ratios listed as 0 were found were modeled concentrations were measured zero and where modeled concentrations were very small. In cases where modeled concentrations are small, ratios of 0.00 mean less than 0.005 .

\begin{tabular}{|c|c|c|c|c|c|c|}
\hline \multirow[b]{2}{*}{ IOP } & \multirow[b]{2}{*}{ Site Label } & \multirow{2}{*}{$\begin{array}{c}\text { Measured } \\
\text { PEM BC } \\
\left(\mathrm{ng} / \mathrm{m}^{3}\right)\end{array}$} & \multicolumn{2}{|c|}{ ISC-PRIME } & \multicolumn{2}{|r|}{ ISCST3 } \\
\hline & & & $\mathrm{BC}\left(\mathrm{ng} / \mathrm{m}^{3}\right)$ & modeled/measured BC & $\mathrm{BC}\left(\mathrm{ng} / \mathrm{m}^{3}\right)$ & $\begin{array}{c}\text { modeled/measured } \\
\text { BC }\end{array}$ \\
\hline $1 \mathrm{a}$ & W48 & 434 & 49.0 & 0.11 & 39.2 & 0.09 \\
\hline $1 a$ & W55 & 376 & 154.4 & 0.41 & 146.6 & 0.39 \\
\hline $1 \mathrm{a}$ & K64 & 343 & 4.7 & 0.01 & 4.7 & 0.01 \\
\hline $1 a$ & $\mathrm{MO}$ & 343 & 9.6 & 0.03 & 9.6 & 0.03 \\
\hline $1 \mathrm{a}$ & B65 & 405 & 5.6 & 0.01 & 5.6 & 0.01 \\
\hline $1 a$ & cow & 486 & 149.9 & 0.31 & 88.4 & 0.18 \\
\hline $1 \mathrm{a}$ & $\mathrm{C}$ & 308 & 24.8 & 0.08 & 24.8 & 0.08 \\
\hline $1 a$ & B3 & 329 & 17.9 & 0.05 & 17.9 & 0.05 \\
\hline $1 \mathrm{a}$ & WS & 313 & 64.8 & 0.21 & 64.8 & 0.21 \\
\hline $2 a$ & E44 & 94 & 2.2 & 0.02 & 2.0 & 0.02 \\
\hline $2 a$ & W48 & 47 & 9.8 & 0.21 & 10.4 & 0.22 \\
\hline $2 a$ & W55 & 813 & 9.3 & 0.01 & 108.7 & 0.13 \\
\hline $2 a$ & $\mathrm{MO}$ & 75 & 4.4 & 0.06 & 4.4 & 0.06 \\
\hline $2 a$ & B65 & 210 & 76.0 & 0.36 & 2.6 & 0.01 \\
\hline $2 a$ & CPK & 86 & 2.2 & 0.03 & 2.9 & 0.03 \\
\hline $2 a$ & $\mathrm{CS}$ & 156 & 0.0 & 0.00 & 0.0 & 0.00 \\
\hline $3 a$ & B65 & 110 & 1.6 & 0.01 & 0.0 & 0.00 \\
\hline $3 a$ & CPK & 115 & 11.1 & 0.10 & 0.1 & 0.00 \\
\hline $3 a$ & $\mathrm{CS}$ & 123 & 1.1 & 0.01 & 0.0 & 0.00 \\
\hline $3 a$ & CP1 & 77 & 1.9 & 0.03 & 1.9 & 0.03 \\
\hline $3 a$ & $\mathrm{E} 2$ & 35 & 0.0 & 0.00 & 0.0 & 0.00 \\
\hline $3 a$ & W & 48 & 0.0 & 0.00 & 0.0 & 0.00 \\
\hline
\end{tabular}




\begin{tabular}{|c|c|c|c|c|c|c|}
\hline \multirow[t]{2}{*}{ IOP } & \multirow[t]{2}{*}{ Site Label } & \multirow[t]{2}{*}{$\begin{array}{c}\text { Measured } \\
\text { PEM BC } \\
\left(\mathrm{ng} / \mathrm{m}^{3}\right)\end{array}$} & \multicolumn{2}{|c|}{ ISC-PRIME } & \multicolumn{2}{|r|}{ ISCST3 } \\
\hline & & & $\mathrm{BC}\left(\mathrm{ng} / \mathrm{m}^{3}\right)$ & modeled/measured BC & $\mathrm{BC}\left(\mathrm{ng} / \mathrm{m}^{3}\right)$ & $\begin{array}{c}\text { modeled/measured } \\
\text { BC }\end{array}$ \\
\hline $3 a$ & C & 72 & 0.0 & 0.00 & 0.0 & 0.00 \\
\hline $4 a$ & B65 & 528 & 21.9 & 0.04 & 13.5 & 0.03 \\
\hline $4 a$ & CPK & 744 & 23.5 & 0.03 & 21.1 & 0.03 \\
\hline $4 a$ & CS & 349 & 22.8 & 0.07 & 15.0 & 0.04 \\
\hline $4 a$ & $\mathrm{CP} 1$ & 222 & 5.8 & 0.03 & 5.7 & 0.03 \\
\hline $4 a$ & $\mathrm{E} 2$ & 210 & 5.8 & 0.03 & 6.0 & 0.03 \\
\hline $4 a$ & W & 90 & 2.7 & 0.03 & 2.7 & 0.03 \\
\hline $4 a$ & S1 & 256 & 1.2 & 0.00 & 0.4 & 0.00 \\
\hline $4 a$ & $\mathrm{C}$ & 306 & 12.9 & 0.04 & 10.3 & 0.03 \\
\hline $4 a$ & B3 & 262 & 12.8 & 0.05 & 12.8 & 0.05 \\
\hline $4 a$ & C2 & 293 & 10.9 & 0.04 & 12.2 & 0.04 \\
\hline $4 a$ & E2 & 128 & 2.9 & 0.02 & 2.8 & 0.02 \\
\hline $2 b$ & $\mathrm{CP}$ & 30 & 6.6 & 0.22 & 7.4 & 0.24 \\
\hline $2 b$ & B3 & 85 & 29.5 & 0.35 & 11.0 & 0.13 \\
\hline $2 b$ & B1 & 78 & 53.0 & 0.68 & 6.5 & 0.08 \\
\hline $2 b$ & EK & 82 & 57.6 & 0.71 & 29.7 & 0.36 \\
\hline $2 b$ & $\mathrm{~K}$ & 30 & 117.4 & 3.88 & 12.9 & 0.43 \\
\hline $2 b$ & W & 30 & 1.9 & 0.06 & 6.8 & 0.22 \\
\hline $2 b$ & WA1 & 163 & 85.9 & 0.53 & 23.9 & 0.15 \\
\hline $2 b$ & B3 & 30 & 23.8 & 0.79 & 5.0 & 0.17 \\
\hline $2 b$ & B4 & 30 & 3.2 & 0.11 & 3.9 & 0.13 \\
\hline $2 b$ & CP1 & 30 & 0.0 & 0.00 & 0.0 & 0.00 \\
\hline $2 b$ & B1 & 309 & 10.1 & 0.03 & 6.8 & 0.02 \\
\hline $3 b$ & E2 & 237 & 34.9 & 0.15 & 8.3 & 0.04 \\
\hline $3 b$ & W & 116 & 47.3 & 0.41 & 18.2 & 0.16 \\
\hline $3 b$ & $\mathrm{D}$ & 542 & 55.3 & 0.10 & 40.3 & 0.07 \\
\hline $3 b$ & E1 & 193 & 89.8 & 0.47 & 49.6 & 0.26 \\
\hline $3 b$ & $C$ & 188 & 92.1 & 0.49 & 59.7 & 0.32 \\
\hline $3 b$ & B3 & 30 & 119.2 & 3.94 & 35.7 & 1.18 \\
\hline
\end{tabular}




\begin{tabular}{|c|c|c|c|c|c|c|}
\hline \multirow[t]{2}{*}{ IOP } & \multirow[t]{2}{*}{ Site Label } & \multirow[t]{2}{*}{$\begin{array}{c}\text { Measured } \\
\text { PEM BC } \\
\left(\mathrm{ng} / \mathrm{m}^{3}\right)\end{array}$} & \multicolumn{2}{|c|}{ ISC-PRIME } & \multicolumn{2}{|r|}{ ISCST3 } \\
\hline & & & $\mathrm{BC}\left(\mathrm{ng} / \mathrm{m}^{3}\right)$ & modeled/measured BC & $\mathrm{BC}\left(\mathrm{ng} / \mathrm{m}^{3}\right)$ & $\begin{array}{c}\text { modeled/measured } \\
\text { BC }\end{array}$ \\
\hline $3 b$ & B4 & 137 & 81.1 & 0.59 & 46.3 & 0.34 \\
\hline $3 b$ & $\mathrm{CP}$ & 30 & 26.7 & 0.88 & 32.4 & 1.07 \\
\hline $3 b$ & $\mathrm{~B} 1$ & 239 & 34.7 & 0.15 & 83.4 & 0.35 \\
\hline $3 b$ & B2 & 125 & 11.2 & 0.09 & 11.2 & 0.09 \\
\hline $3 b$ & $\mathrm{~A}$ & 174 & 53.5 & 0.31 & 58.0 & 0.33 \\
\hline $3 b$ & $\mathrm{AW}$ & 451 & 154.9 & 0.34 & 39.6 & 0.09 \\
\hline $3 b$ & $\mathrm{BC}$ & 202 & 38.7 & 0.19 & 26.8 & 0.13 \\
\hline $3 b$ & EK & 30 & 31.9 & 1.05 & 29.6 & 0.98 \\
\hline $3 b$ & $\mathrm{~K}$ & 30 & 1.7 & 0.06 & 1.8 & 0.06 \\
\hline $3 b$ & S1 & 525 & 13.7 & 0.03 & 3.4 & 0.01 \\
\hline $4 b$ & E2 & 1048 & 6.4 & 0.01 & 2.2 & 0.00 \\
\hline $4 b$ & W & 396 & 88.6 & 0.22 & 18.7 & 0.05 \\
\hline $4 b$ & $D$ & 374 & 19.5 & 0.05 & 31.5 & 0.08 \\
\hline $4 b$ & $\mathrm{E} 1$ & 281 & 37.8 & 0.13 & 13.0 & 0.05 \\
\hline $4 b$ & $\mathrm{C}$ & 326 & 19.6 & 0.06 & 18.4 & 0.06 \\
\hline $4 b$ & B3 & 210 & 22.1 & 0.11 & 22.1 & 0.10 \\
\hline $4 b$ & B4 & 222 & 21.5 & 0.10 & 27.7 & 0.12 \\
\hline $4 b$ & $\mathrm{CP}$ & 221 & 14.8 & 0.07 & 14.8 & 0.07 \\
\hline $4 b$ & B2 & 293 & 8.4 & 0.03 & 8.4 & 0.03 \\
\hline $4 b$ & $A$ & 268 & 20.9 & 0.08 & 21.8 & 0.08 \\
\hline $4 b$ & AW & 285 & 12.5 & 0.04 & 14.8 & 0.05 \\
\hline $4 b$ & $\mathrm{BC}$ & 197 & 21.8 & 0.11 & 18.1 & 0.09 \\
\hline $4 b$ & EK & 198 & 16.1 & 0.08 & 10.1 & 0.05 \\
\hline $4 b$ & $\mathrm{~K}$ & 189 & 6.1 & 0.03 & 6.1 & 0.03 \\
\hline $4 b$ & S1 & 448 & 1.4 & 0.00 & 0.8 & 0.00 \\
\hline $5 b$ & E2 & 2788 & 37.6 & 0.01 & 0.1 & 0.00 \\
\hline $5 b$ & W & 252 & 60.0 & 0.24 & 30.9 & 0.12 \\
\hline $5 b$ & $D$ & 294 & 27.3 & 0.09 & 38.6 & 0.13 \\
\hline $5 b$ & C & 283 & 27.4 & 0.10 & 24.5 & 0.09 \\
\hline
\end{tabular}




\begin{tabular}{|c|c|c|c|c|c|c|}
\hline \multirow[t]{2}{*}{ IOP } & \multirow[t]{2}{*}{ Site Label } & \multirow[t]{2}{*}{$\begin{array}{c}\text { Measured } \\
\text { PEM BC } \\
\left(\mathrm{ng} / \mathrm{m}^{3}\right)\end{array}$} & \multicolumn{2}{|c|}{ ISC-PRIME } & \multicolumn{2}{|r|}{ ISCST3 } \\
\hline & & & $\mathrm{BC}\left(\mathrm{ng} / \mathrm{m}^{3}\right)$ & modeled/measured BC & $\mathrm{BC}\left(\mathrm{ng} / \mathrm{m}^{3}\right)$ & $\begin{array}{c}\text { modeled/measured } \\
\text { BC }\end{array}$ \\
\hline $5 b$ & B3 & 273 & 5.8 & 0.02 & 5.8 & 0.02 \\
\hline $5 b$ & $\mathrm{CP}$ & 318 & 5.1 & 0.02 & 5.2 & 0.02 \\
\hline $5 b$ & $\mathrm{~B} 1$ & 191 & 14.6 & 0.08 & 14.6 & 0.08 \\
\hline $5 b$ & B2 & 270 & 0.0 & 0.00 & 0.0 & 0.00 \\
\hline $5 b$ & $\mathrm{~A}$ & 286 & 35.5 & 0.12 & 12.9 & 0.05 \\
\hline $5 b$ & $\mathrm{AW}$ & 308 & 81.5 & 0.26 & 24.1 & 0.08 \\
\hline $5 b$ & $\mathrm{BC}$ & 216 & 16.3 & 0.08 & 16.2 & 0.08 \\
\hline $5 b$ & FS & 263 & 20.8 & 0.08 & 17.9 & 0.07 \\
\hline $5 b$ & EK & 265 & 6.2 & 0.02 & 6.2 & 0.02 \\
\hline $5 b$ & $\mathrm{~K}$ & 194 & 0.0 & 0.00 & 0.0 & 0.00 \\
\hline $5 b$ & S1 & 415 & 4.7 & 0.01 & 1.6 & 0.00 \\
\hline $6 b$ & E2 & 30 & 3.7 & 0.12 & 3.5 & 0.12 \\
\hline $6 b$ & W & 192 & 55.8 & 0.29 & 8.2 & 0.04 \\
\hline $6 b$ & $\mathrm{D}$ & 339 & 13.7 & 0.04 & 13.1 & 0.04 \\
\hline $6 \mathrm{~b}$ & $\mathrm{C}$ & 185 & 22.7 & 0.12 & 17.9 & 0.10 \\
\hline $6 b$ & B3 & 153 & 13.7 & 0.09 & 12.1 & 0.08 \\
\hline $6 b$ & B4 & 30 & 60.5 & 2.00 & 49.1 & 1.62 \\
\hline $6 b$ & $\mathrm{~B} 1$ & 123 & 1.4 & 0.01 & 1.4 & 0.01 \\
\hline $6 b$ & $\mathrm{~A}$ & 370 & 20.2 & 0.05 & 19.6 & 0.05 \\
\hline $6 b$ & $\mathrm{AW}$ & 693 & 85.1 & 0.12 & 49.2 & 0.07 \\
\hline $6 b$ & $\mathrm{BC}$ & 413 & 11.9 & 0.03 & 7.8 & 0.02 \\
\hline $6 \mathrm{~b}$ & FS & 164 & 42.0 & 0.26 & 48.8 & 0.30 \\
\hline $6 b$ & EK & 81 & 5.4 & 0.07 & 5.7 & 0.07 \\
\hline $6 b$ & $\mathrm{~K}$ & 59 & 2.6 & 0.04 & 2.7 & 0.05 \\
\hline $6 b$ & S1 & 247 & 2.4 & 0.01 & 0.7 & 0.00 \\
\hline $7 b$ & E2 & 125 & 22.8 & 0.18 & 7.3 & 0.06 \\
\hline $7 b$ & W & 503 & 70.3 & 0.14 & 20.9 & 0.04 \\
\hline $7 b$ & $D$ & 1051 & 81.0 & 0.08 & 19.7 & 0.02 \\
\hline $7 \mathrm{~b}$ & C & 159 & 41.5 & 0.26 & 29.8 & 0.19 \\
\hline
\end{tabular}




\begin{tabular}{|c|c|c|c|c|c|c|}
\hline \multirow[t]{2}{*}{ IOP } & \multirow[t]{2}{*}{ Site Label } & \multirow[t]{2}{*}{$\begin{array}{c}\text { Measured } \\
\text { PEM BC } \\
\left(\mathrm{ng} / \mathrm{m}^{3}\right)\end{array}$} & \multicolumn{2}{|c|}{ ISC-PRIME } & \multicolumn{2}{|r|}{ ISCST3 } \\
\hline & & & $\mathrm{BC}\left(\mathrm{ng} / \mathrm{m}^{3}\right)$ & modeled/measured BC & $\mathrm{BC}\left(\mathrm{ng} / \mathrm{m}^{3}\right)$ & $\begin{array}{c}\text { modeled/measured } \\
\text { BC }\end{array}$ \\
\hline $7 \mathrm{~b}$ & B4 & 136 & 4.5 & 0.03 & 4.5 & 0.03 \\
\hline $7 \mathrm{~b}$ & $\mathrm{CP}$ & 188 & 6.6 & 0.04 & 6.6 & 0.04 \\
\hline $7 \mathrm{~b}$ & B1 & 145 & 3.4 & 0.02 & 3.4 & 0.02 \\
\hline $7 \mathrm{~b}$ & B2 & 115 & 0.0 & 0.00 & 0.0 & 0.00 \\
\hline $7 \mathrm{~b}$ & $\mathrm{~A}$ & 270 & 54.9 & 0.20 & 104.0 & 0.39 \\
\hline $7 \mathrm{~b}$ & $\mathrm{AW}$ & 453 & 93.9 & 0.21 & 178.7 & 0.39 \\
\hline $7 \mathrm{~b}$ & $\mathrm{BC}$ & 146 & 3.6 & 0.02 & 7.6 & 0.05 \\
\hline $7 \mathrm{~b}$ & FS & 184 & 27.4 & 0.15 & 25.9 & 0.14 \\
\hline $7 \mathrm{~b}$ & EK & 101 & 1.5 & 0.01 & 1.5 & 0.01 \\
\hline $7 \mathrm{~b}$ & $\mathrm{~K}$ & 115 & 0.0 & 0.00 & 0.0 & 0.00 \\
\hline $7 \mathrm{~b}$ & S1 & 386 & 4.5 & 0.01 & 2.1 & 0.01 \\
\hline $8 b$ & E2 & 156 & 1.7 & 0.01 & 1.6 & 0.01 \\
\hline $8 b$ & W & 142 & 21.6 & 0.15 & 5.0 & 0.04 \\
\hline $8 b$ & $\mathrm{C}$ & 154 & 4.8 & 0.03 & 4.8 & 0.03 \\
\hline $8 b$ & B3 & 119 & 15.0 & 0.13 & 16.5 & 0.14 \\
\hline $8 b$ & B4 & 277 & 29.7 & 0.11 & 29.7 & 0.11 \\
\hline $8 b$ & $\mathrm{CP}$ & 130 & 13.2 & 0.10 & 13.8 & 0.11 \\
\hline $8 b$ & $\mathrm{~B} 1$ & 158 & 0.0 & 0.00 & 0.0 & 0.00 \\
\hline $8 \mathrm{~b}$ & B2 & 137 & 42.8 & 0.31 & 133.5 & 0.97 \\
\hline $8 \mathrm{~b}$ & $A$ & 561 & 2.4 & 0.00 & 0.0 & 0.00 \\
\hline $8 b$ & AW & 176 & 86.7 & 0.49 & 44.8 & 0.25 \\
\hline $8 b$ & $\mathrm{BC}$ & 139 & 0.0 & 0.00 & 0.0 & 0.00 \\
\hline $8 \mathrm{~b}$ & FS & 162 & 32.9 & 0.20 & 33.3 & 0.21 \\
\hline $8 b$ & EK & 120 & 0.3 & 0.00 & 0.3 & 0.00 \\
\hline $8 b$ & $\mathrm{~K}$ & 149 & 5.0 & 0.03 & 5.8 & 0.04 \\
\hline $8 \mathrm{~b}$ & S1 & 229 & 0.5 & 0.00 & 0.1 & 0.00 \\
\hline $9 b$ & E2 & 120 & 27.9 & 0.23 & 0.4 & 0.00 \\
\hline $9 b$ & W & 169 & 28.4 & 0.17 & 0.0 & 0.00 \\
\hline $9 b$ & C & 102 & 39.7 & 0.39 & 10.7 & 0.10 \\
\hline
\end{tabular}




\begin{tabular}{|c|c|c|c|c|c|c|}
\hline \multirow[t]{2}{*}{ IOP } & \multirow[t]{2}{*}{ Site Label } & \multirow[t]{2}{*}{$\begin{array}{c}\text { Measured } \\
\text { PEM BC } \\
\left(\mathrm{ng} / \mathrm{m}^{3}\right)\end{array}$} & \multicolumn{2}{|r|}{ ISC-PRIME } & \multicolumn{2}{|r|}{ ISCST3 } \\
\hline & & & $\mathrm{BC}\left(\mathrm{ng} / \mathrm{m}^{3}\right)$ & modeled/measured BC & $\mathrm{BC}\left(\mathrm{ng} / \mathrm{m}^{3}\right)$ & $\begin{array}{c}\text { modeled/measured } \\
\text { BC }\end{array}$ \\
\hline $9 \mathrm{~b}$ & B3 & 137 & 69.0 & 0.50 & 2.4 & 0.02 \\
\hline $9 b$ & B4 & 92 & 54.3 & 0.59 & 25.6 & 0.28 \\
\hline $9 b$ & $\mathrm{CP}$ & 110 & 15.8 & 0.14 & 3.6 & 0.03 \\
\hline $9 b$ & B1 & 119 & 5.5 & 0.05 & 3.7 & 0.03 \\
\hline $9 b$ & $\mathrm{~B} 2$ & 112 & 11.3 & 0.10 & 9.9 & 0.09 \\
\hline $9 \mathrm{~b}$ & $\mathrm{~A}$ & 119 & 11.5 & 0.10 & 19.5 & 0.16 \\
\hline $9 \mathrm{~b}$ & AW & 280 & 91.3 & 0.33 & 4.6 & 0.02 \\
\hline $9 \mathrm{~b}$ & $\mathrm{BC}$ & 100 & 6.0 & 0.06 & 1.6 & 0.02 \\
\hline $9 b$ & FS & 105 & 17.1 & 0.16 & 4.4 & 0.04 \\
\hline $9 b$ & EK & 133 & 3.3 & 0.03 & 3.6 & 0.03 \\
\hline $9 \mathrm{~b}$ & $\mathrm{~K}$ & 148 & 4.9 & 0.03 & 0.5 & 0.00 \\
\hline $9 b$ & S1 & 192 & 1.5 & 0.01 & 0.5 & 0.00 \\
\hline $10 \mathrm{~b}$ & E2 & 92 & 31.1 & 0.34 & 10.2 & 0.11 \\
\hline $10 \mathrm{~b}$ & W & 30 & 31.7 & 1.05 & 10.8 & 0.36 \\
\hline $10 \mathrm{~b}$ & C & 94 & 30.2 & 0.32 & 23.5 & 0.25 \\
\hline $10 \mathrm{~b}$ & B3 & 30 & 63.6 & 2.10 & 27.4 & 0.91 \\
\hline $10 \mathrm{~b}$ & B4 & 30 & 59.6 & 1.97 & 27.1 & 0.90 \\
\hline $10 \mathrm{~b}$ & $\mathrm{CP}$ & 81 & 36.5 & 0.45 & 39.2 & 0.48 \\
\hline $10 \mathrm{~b}$ & B1 & 30 & 20.9 & 0.69 & 7.5 & 0.25 \\
\hline $10 \mathrm{~b}$ & B2 & 101 & 51.9 & 0.51 & 137.0 & 1.36 \\
\hline $10 \mathrm{~b}$ & A & 203 & 25.3 & 0.12 & 24.8 & 0.12 \\
\hline $10 \mathrm{~b}$ & AW & 30 & 27.8 & 0.92 & 23.3 & 0.77 \\
\hline $10 \mathrm{~b}$ & $B C$ & 83 & 41.5 & 0.50 & 56.9 & 0.69 \\
\hline
\end{tabular}




\begin{tabular}{|c|c|c|c|c|c|c|}
\hline \multirow[b]{2}{*}{ IOP } & \multirow[b]{2}{*}{ Site Label } & \multirow{2}{*}{$\begin{array}{c}\text { Measured } \\
\text { PEM BC } \\
\left(\mathrm{ng} / \mathrm{m}^{3}\right)\end{array}$} & \multicolumn{2}{|c|}{ ISC-PRIME } & \multicolumn{2}{|r|}{ ISCST3 } \\
\hline & & & $\mathrm{BC}\left(\mathrm{ng} / \mathrm{m}^{3}\right)$ & modeled/measured BC & $\mathrm{BC}\left(\mathrm{ng} / \mathrm{m}^{3}\right)$ & $\begin{array}{c}\text { modeled/measured } \\
\text { BC }\end{array}$ \\
\hline $10 \mathrm{~b}$ & FS & 104 & 40.0 & 0.38 & 34.7 & 0.33 \\
\hline $10 b$ & EK & 94 & 18.8 & 0.20 & 31.8 & 0.34 \\
\hline $10 \mathrm{~b}$ & K & 88 & 20.3 & 0.23 & 33.4 & 0.38 \\
\hline $10 b$ & S1 & 117 & 6.4 & 0.05 & 4.2 & 0.04 \\
\hline
\end{tabular}




\section{Graphical Summary of ISCST3 and ISC-PRIME Modeled Results}

The modeled concentrations are displayed in the images below along with contours of the concentrations. The concentrations in red text are the highest modeled concentration in that specific case. Concentrations displayed have not been adjusted to account for the $37.5 \%$ burning time over the sampling period (as opposed to $100 \%$ ).

\section{ISC-PRIME}
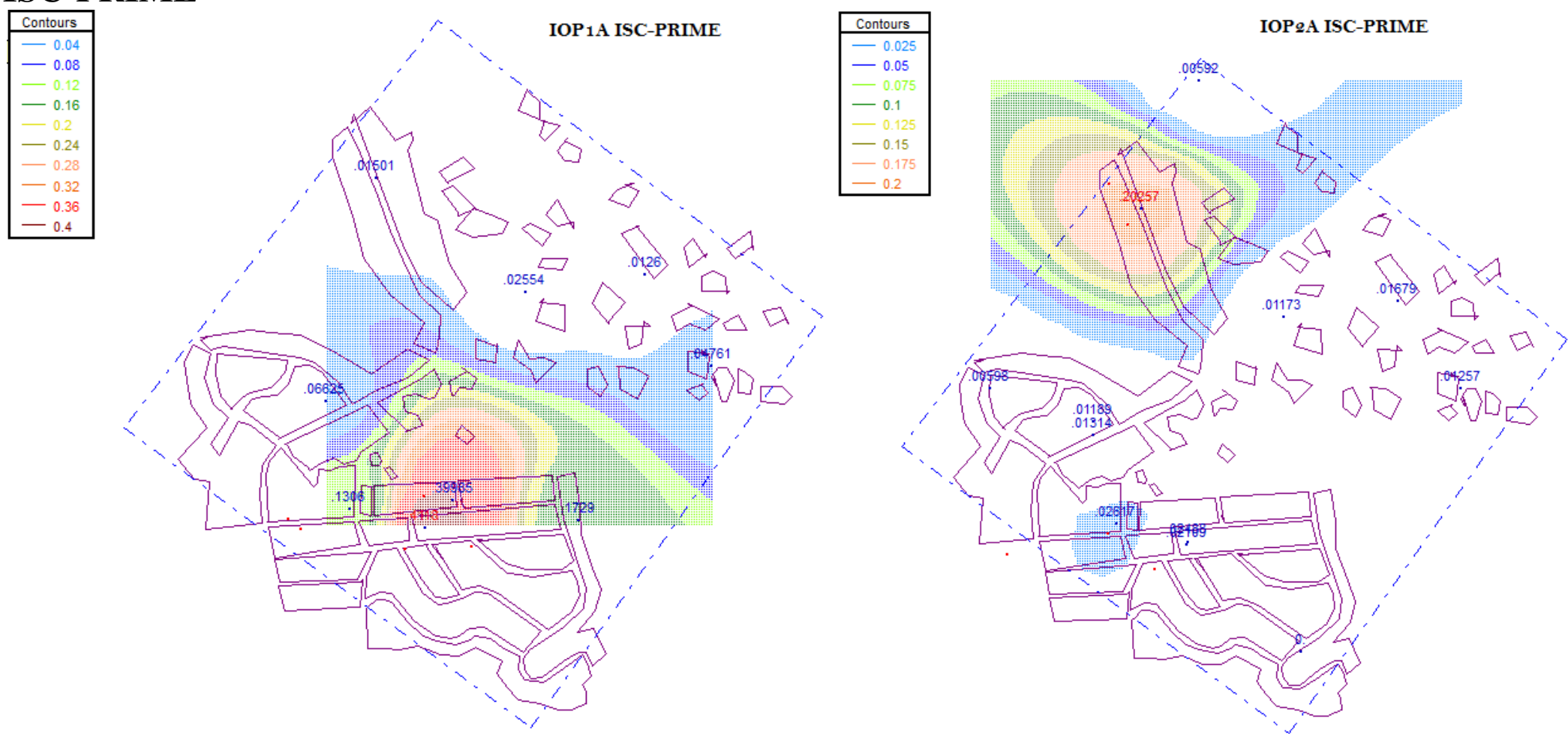

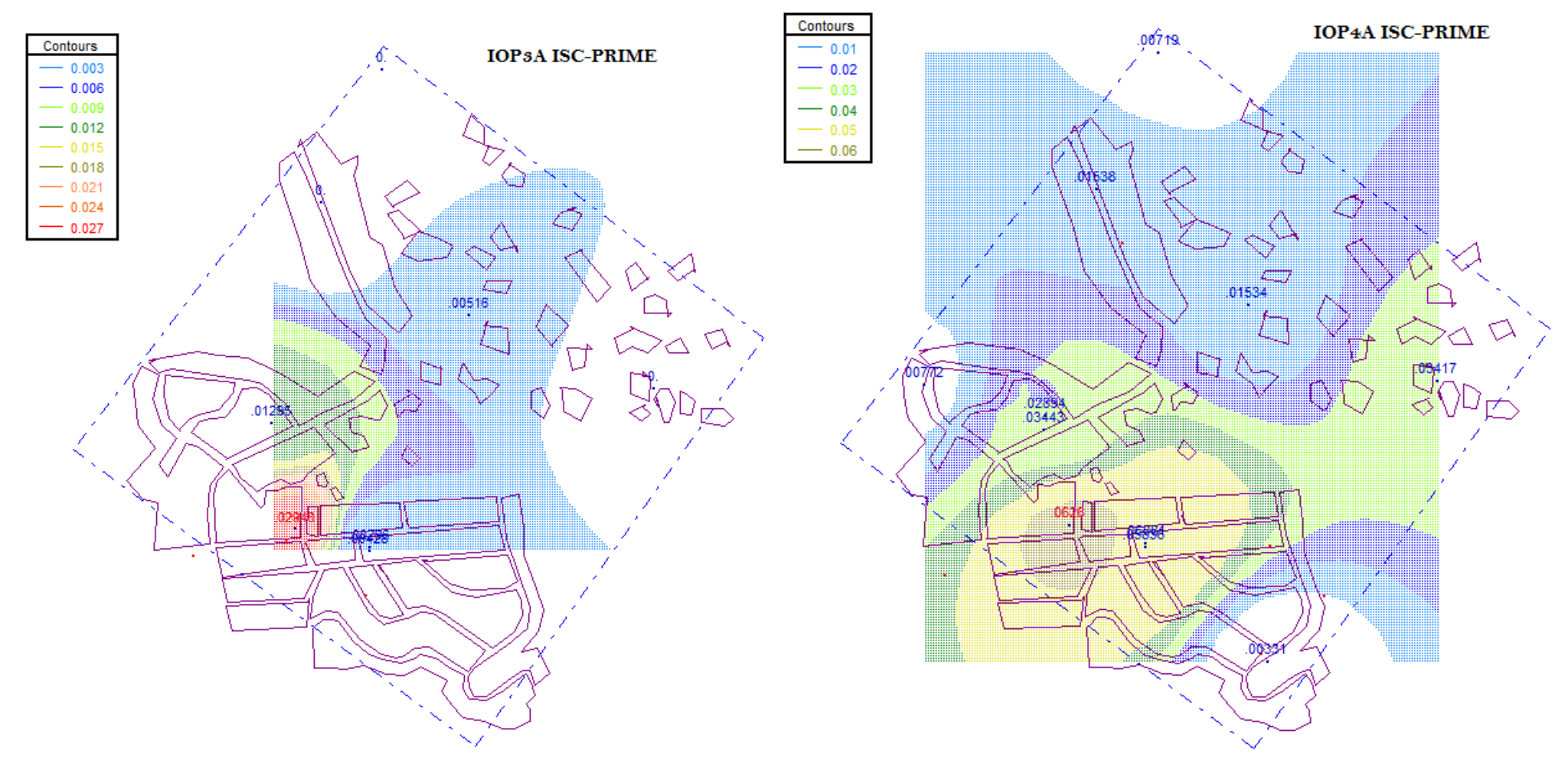


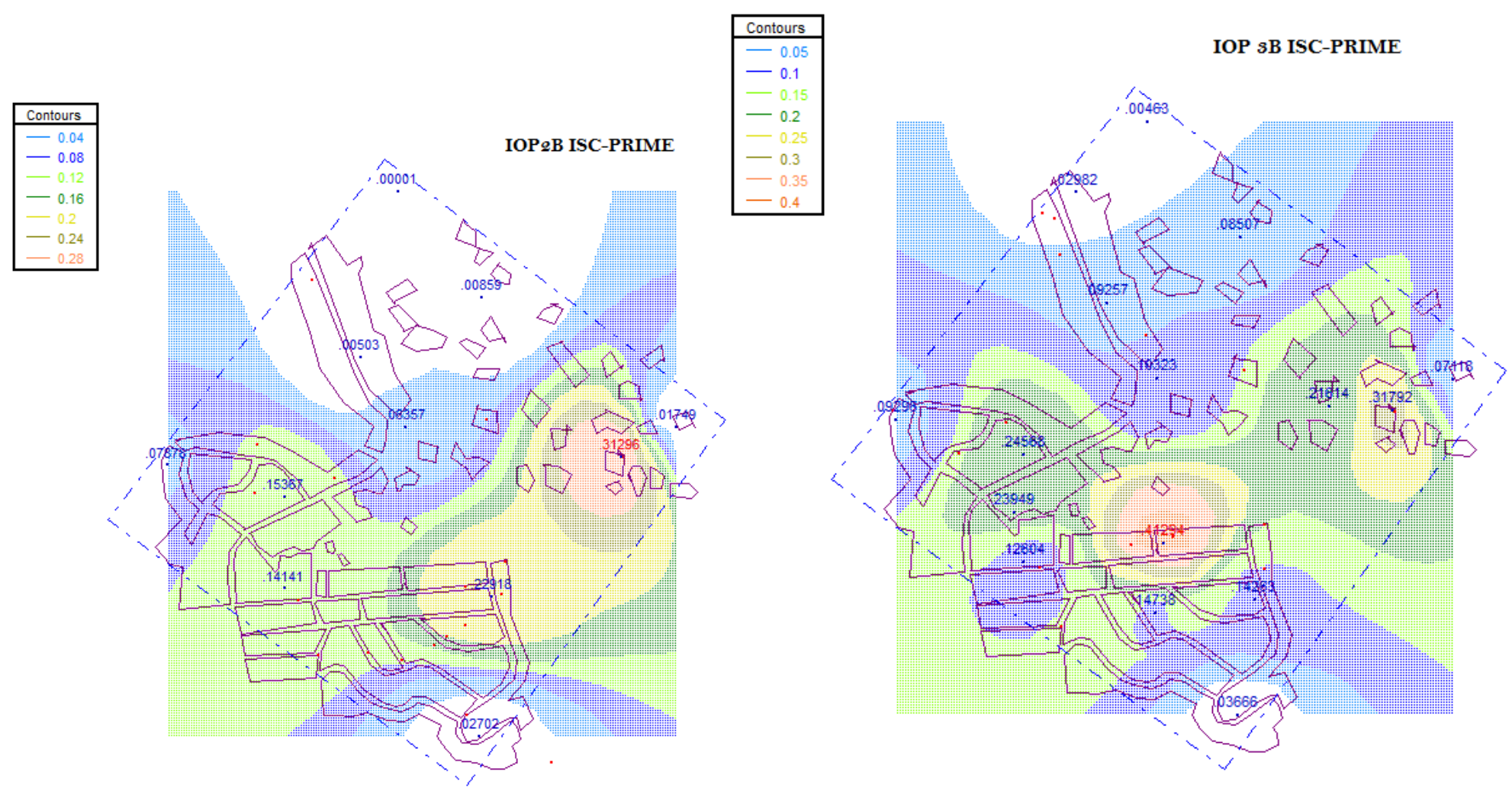




\begin{tabular}{|c|}
\hline Contours \\
\hline-0.025 \\
\hline-0.05 \\
\hline-0.075 \\
\hline-0.1 \\
\hline 二 0.125 \\
\hline - 0.175 \\
\hline 二 0.22 \\
\hline \\
\hline
\end{tabular}
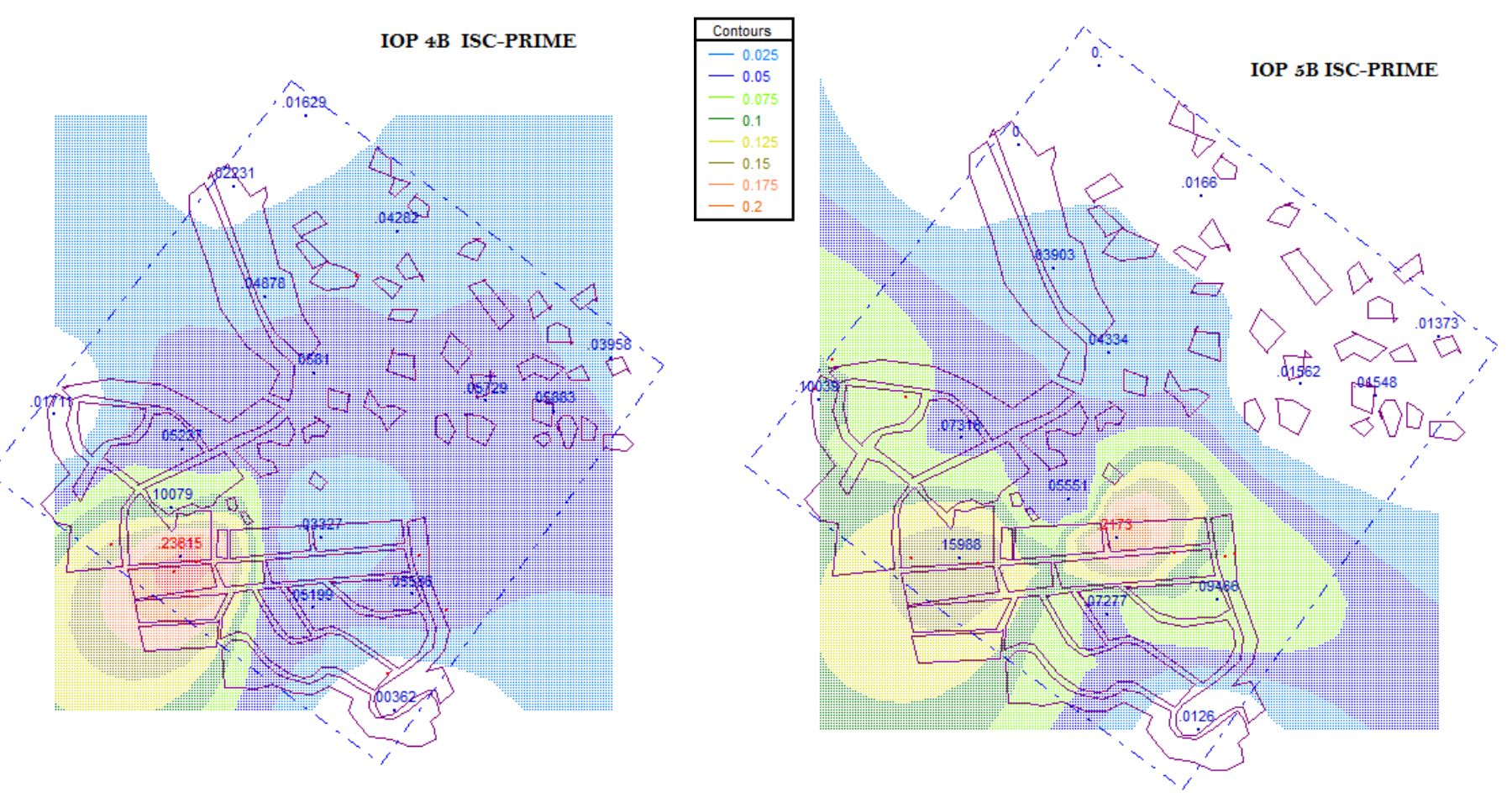

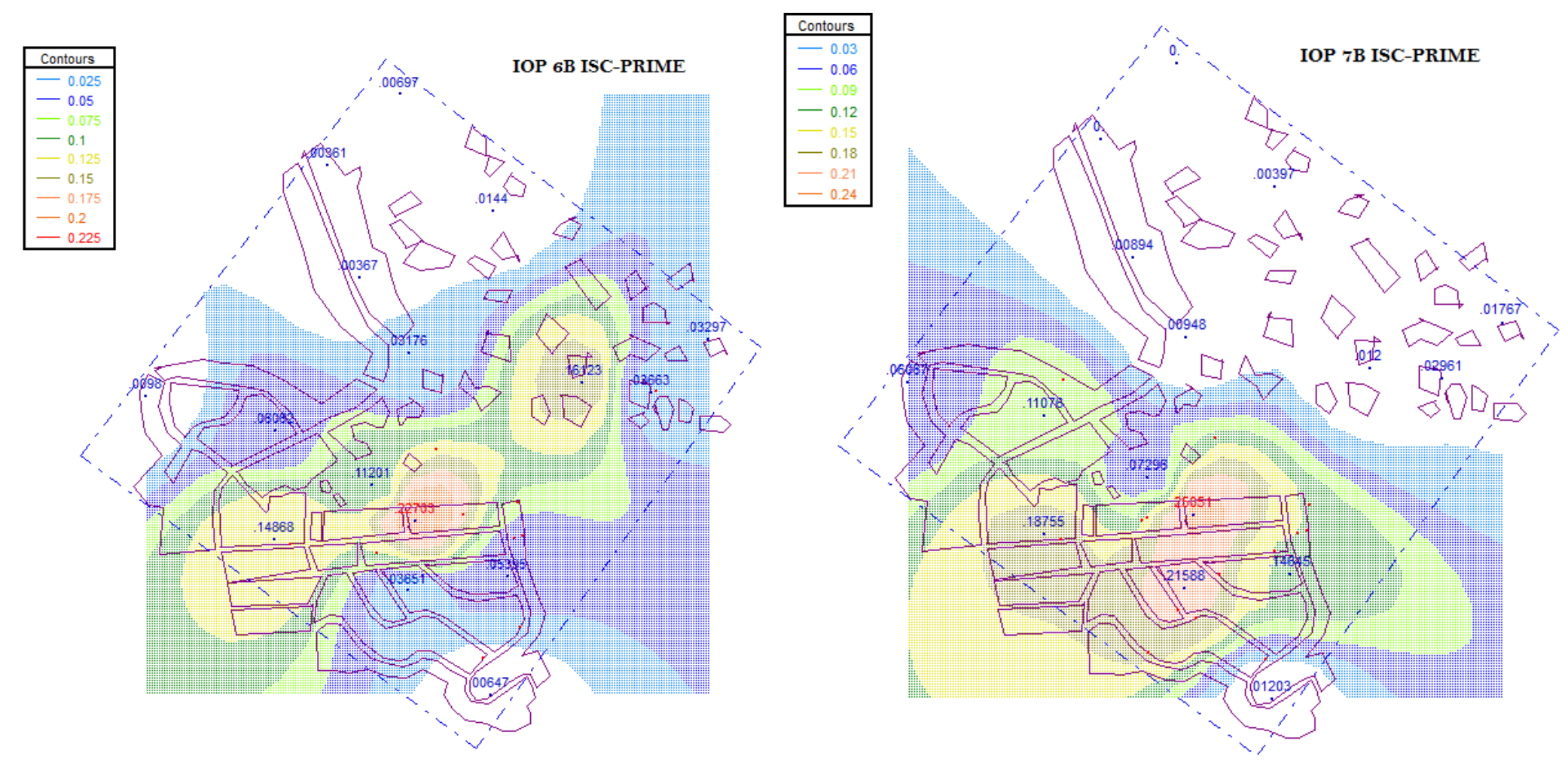


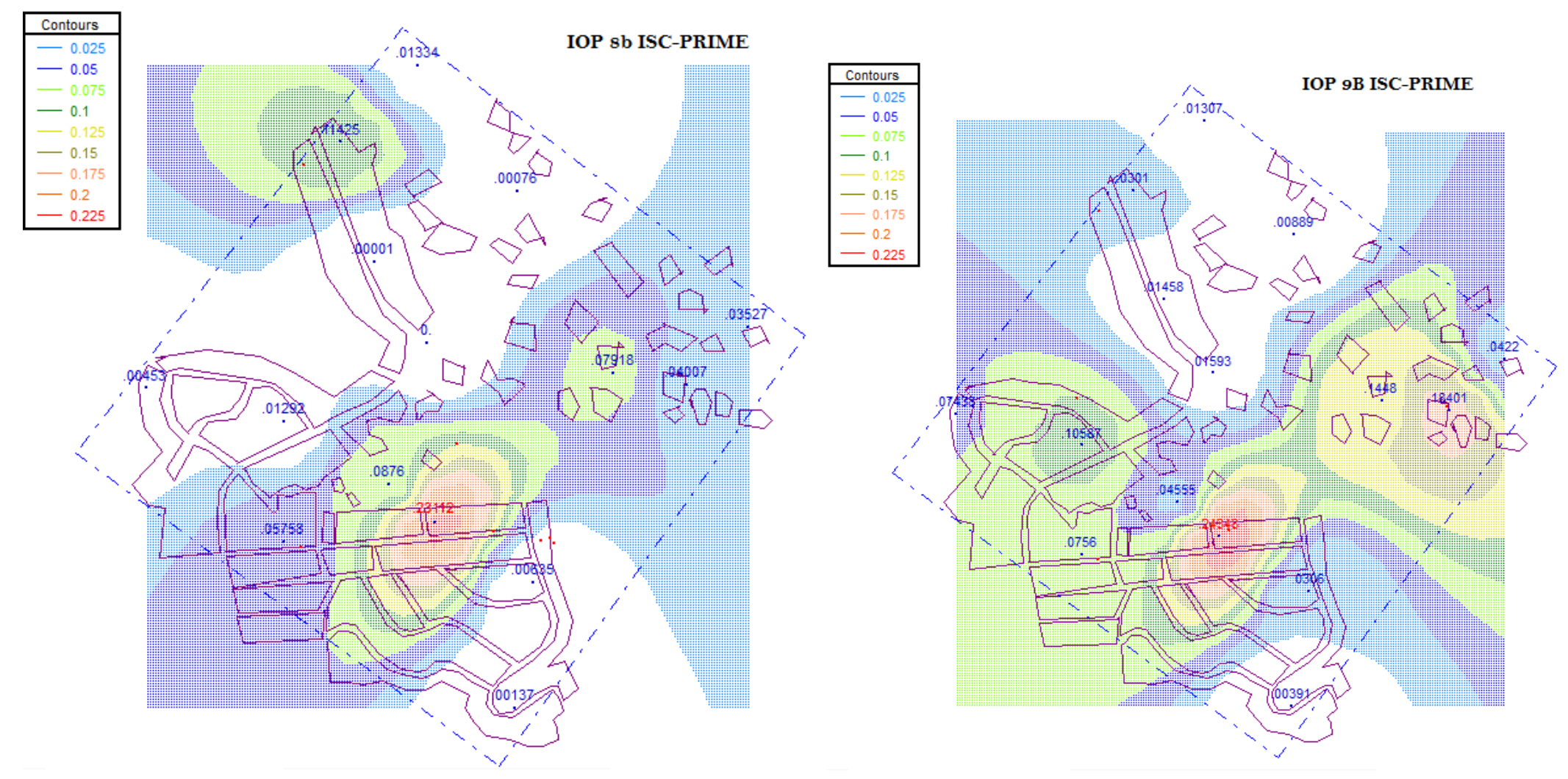




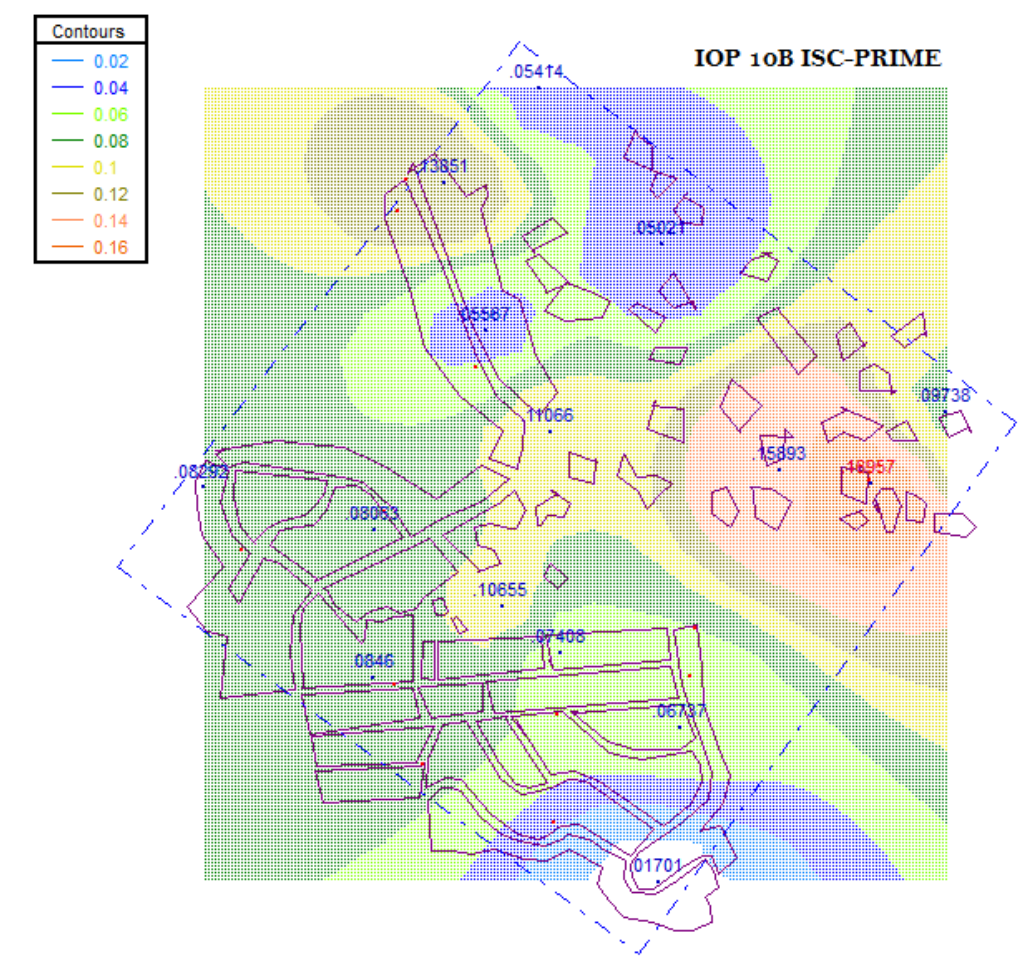




\section{ISCST3}
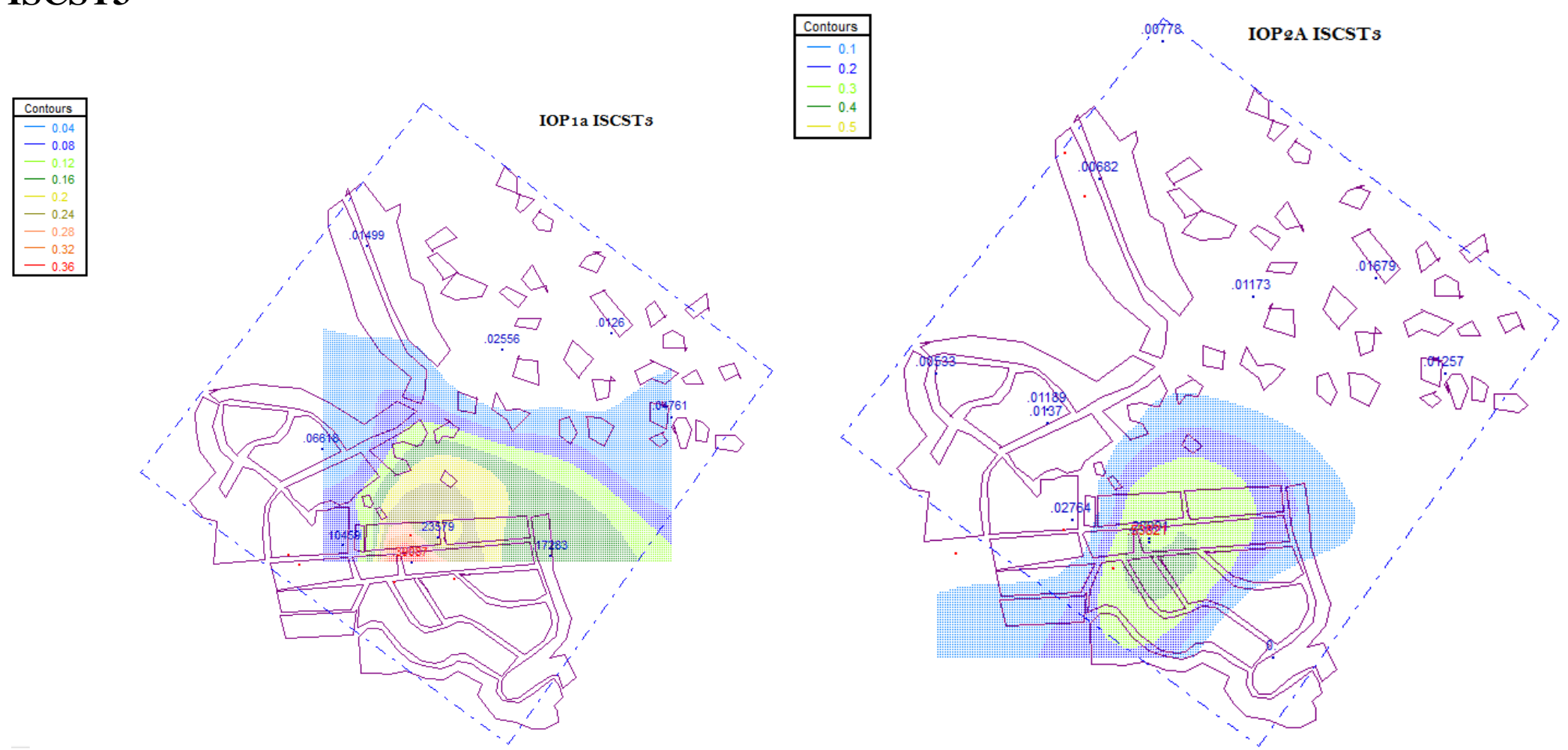


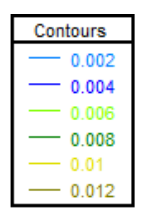

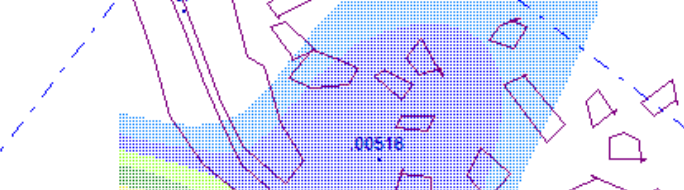
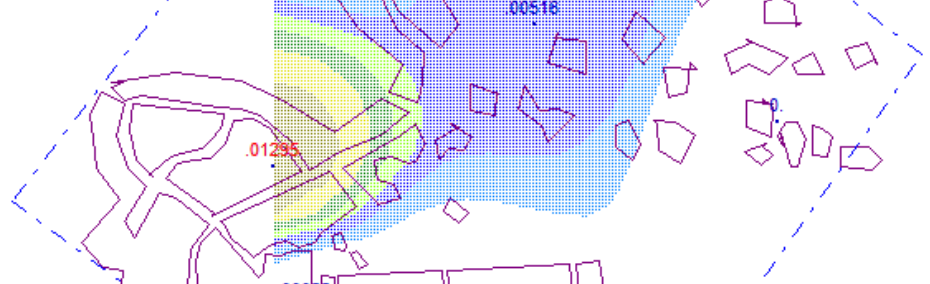

?

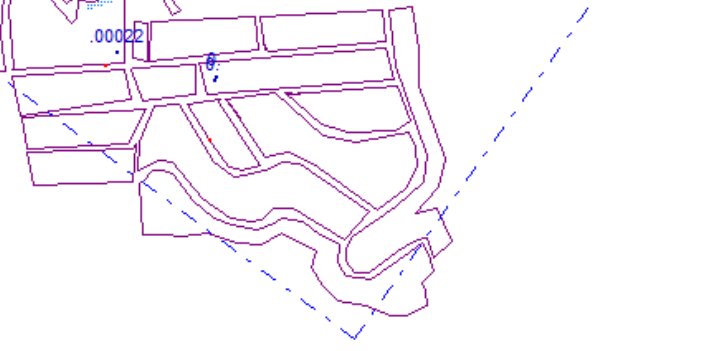

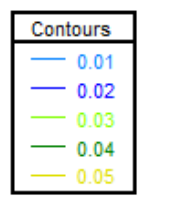

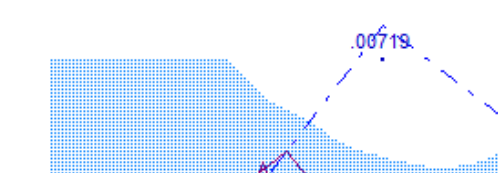

IOP 4A ISCSTs

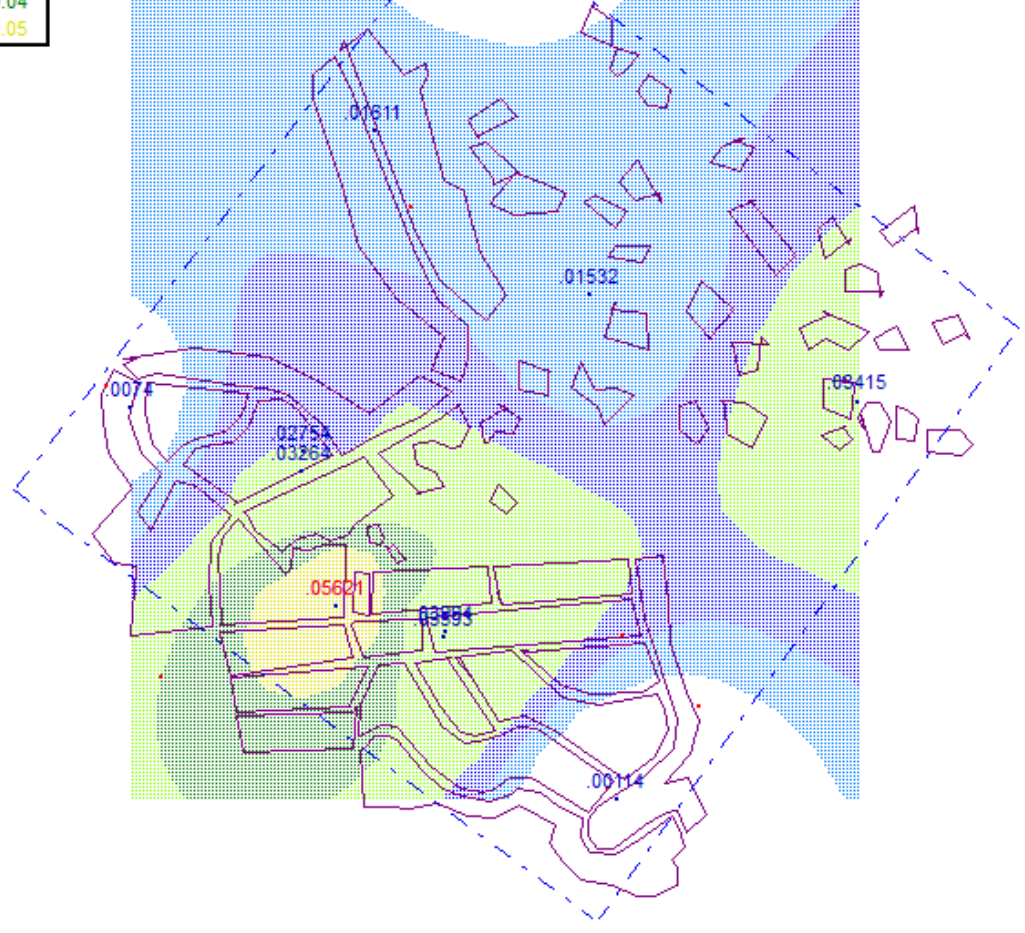




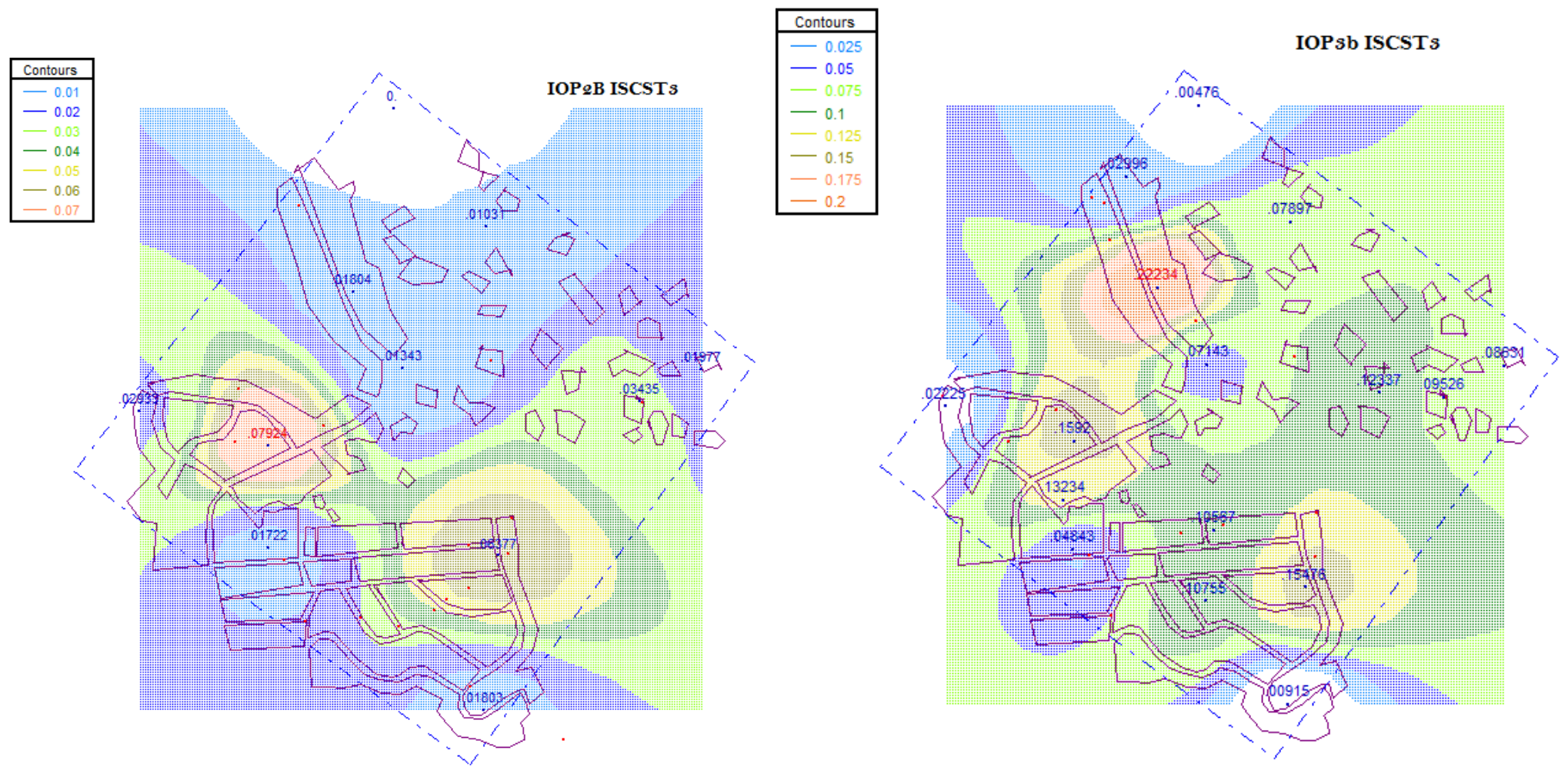




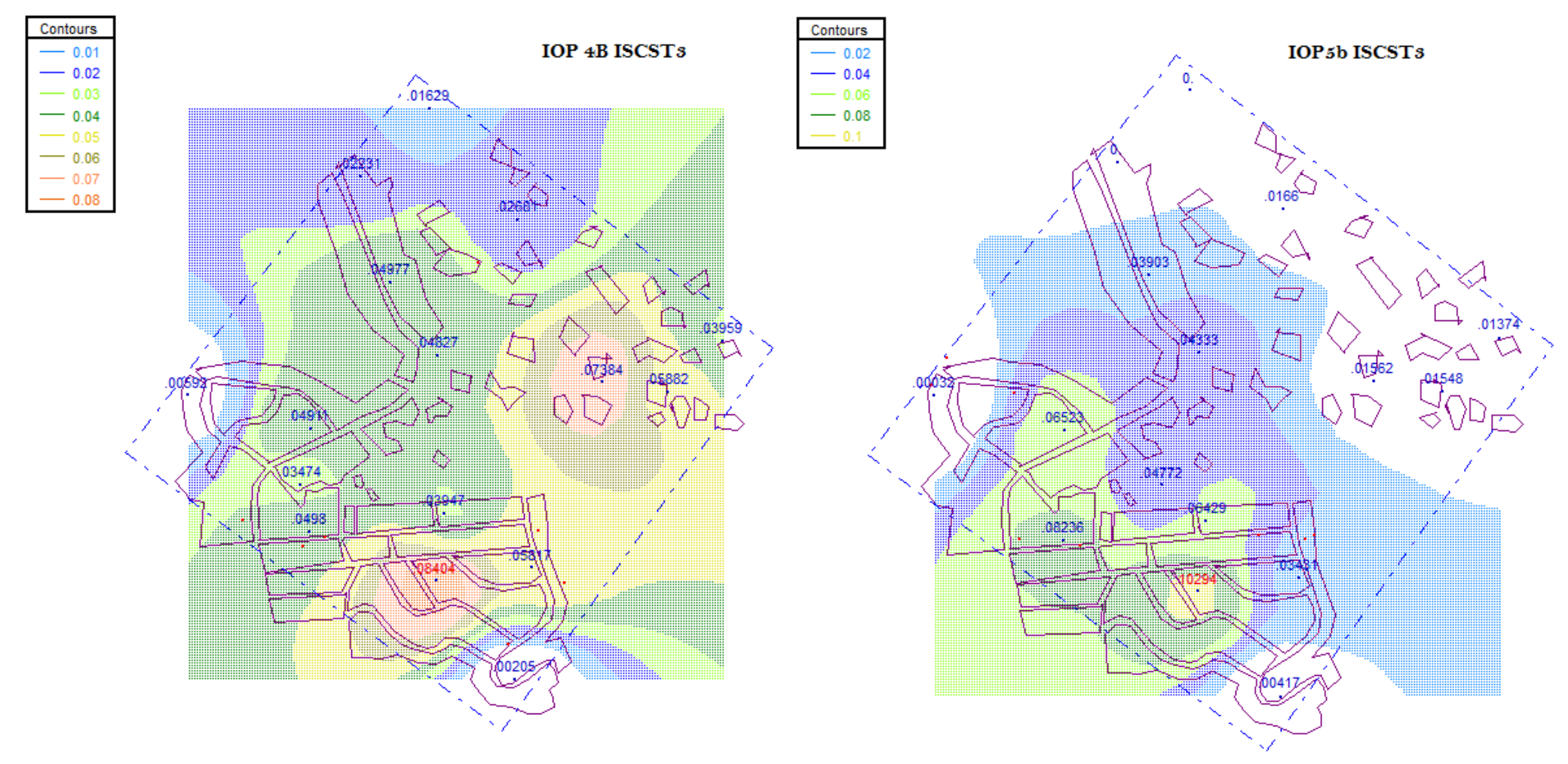



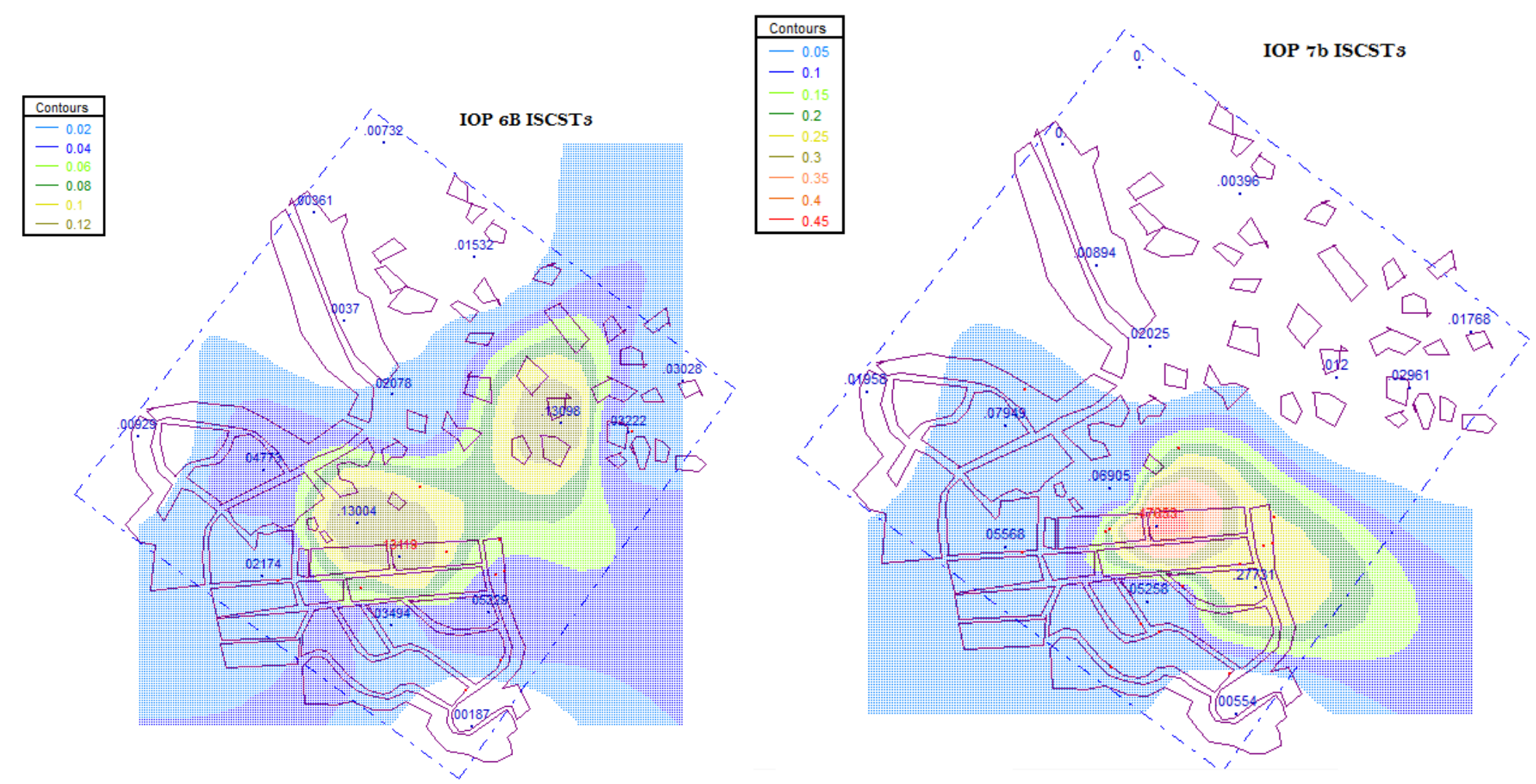


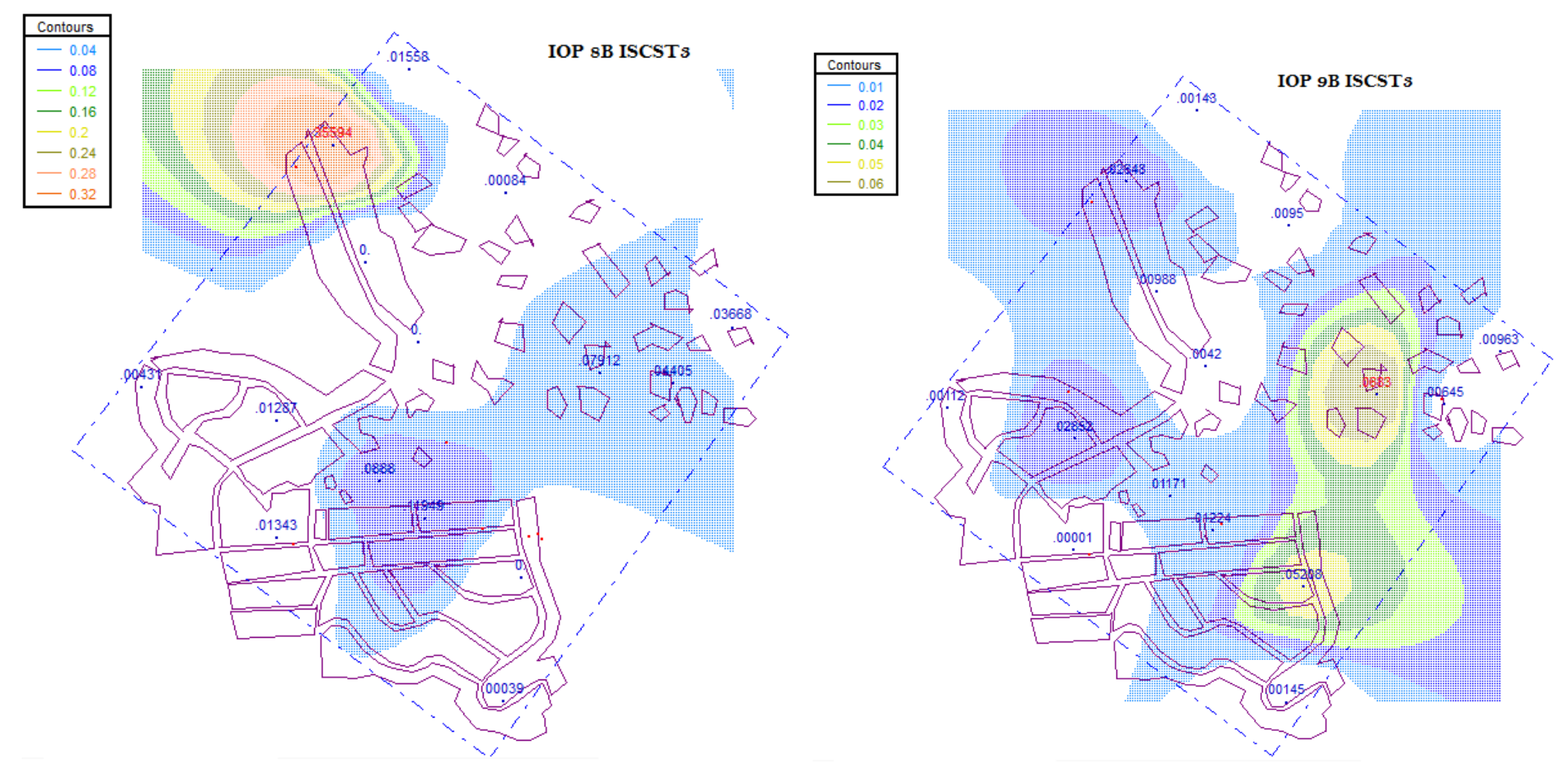




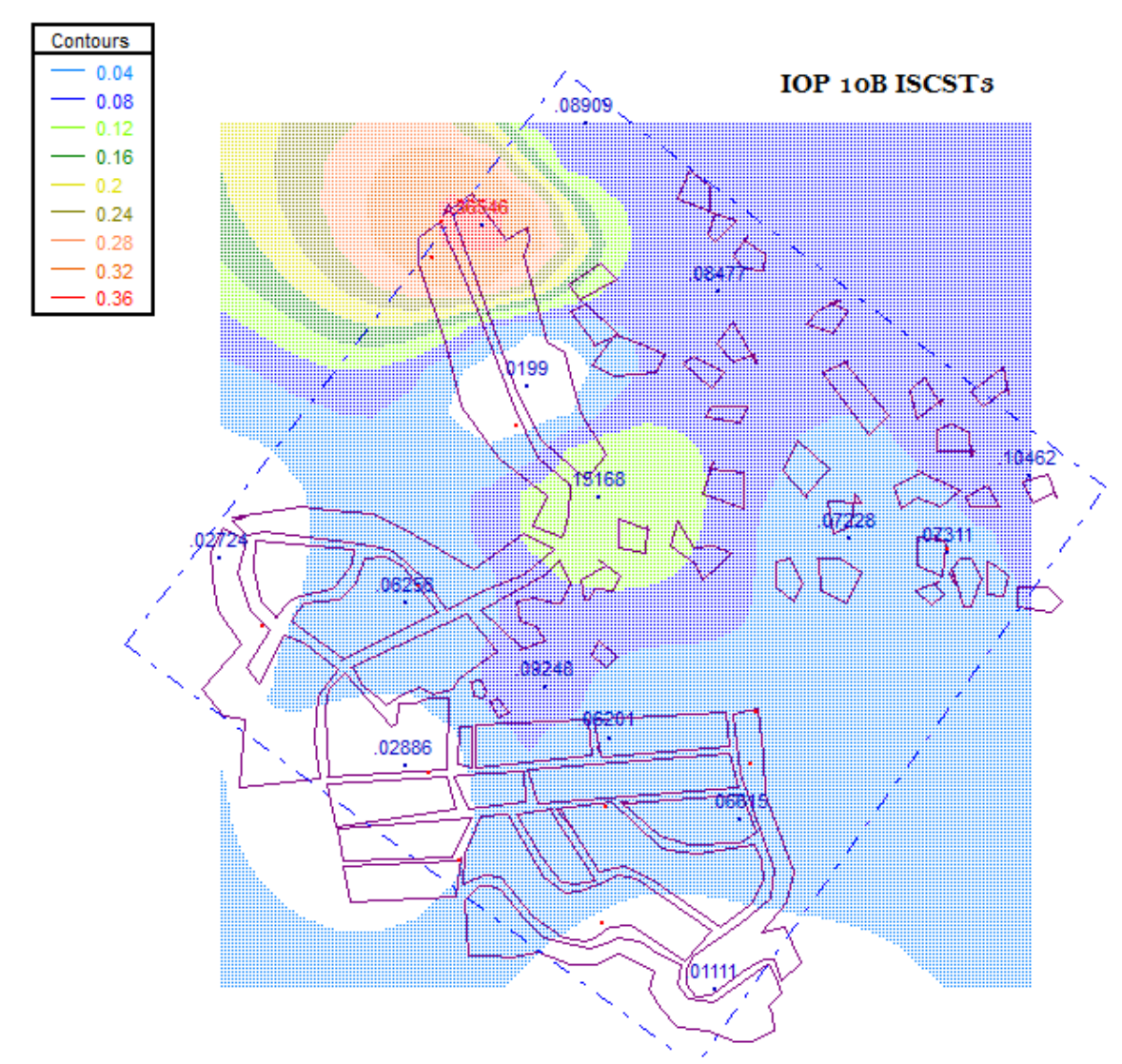




\section{Summary of Corrected ISC Modeled BC concentrations and Modeled- to-Measured Ratios}

The table below summarizes the corrected modeled and measured BC concentrations at each PEM location in addition to the ratio of the modeled-to-measured concentration. Numbers listed as "0" are less than 0.005 .

\begin{tabular}{|c|c|c|c|c|c|c|}
\hline \multirow[b]{2}{*}{ IOP } & \multirow[b]{2}{*}{ Site Label } & \multirow[b]{2}{*}{$\begin{array}{c}\text { Measured } \\
\text { PEM BC } \\
\left(\mathrm{ng} / \mathrm{m}^{3}\right)\end{array}$} & \multicolumn{2}{|c|}{ ISC-PRIME } & \multicolumn{2}{|r|}{ ISCST3 } \\
\hline & & & $\mathrm{BC}\left(\mathrm{ng} / \mathrm{m}^{3}\right)$ & $\begin{array}{c}\text { modeled/measured } \\
\text { BC }\end{array}$ & $\mathrm{BC}\left(\mathrm{ng} / \mathrm{m}^{3}\right)$ & $\begin{array}{c}\text { modeled/measured } \\
\text { BC }\end{array}$ \\
\hline $1 a$ & W48 & 434 & 478 & 1.10 & 714 & 1.6 \\
\hline $1 a$ & W55 & 376 & 1506 & 4.01 & 2668 & 7.1 \\
\hline $1 a$ & K64 & 343 & 46 & 0.13 & 86 & 0.3 \\
\hline $1 a$ & $\mathrm{MO}$ & 343 & 93 & 0.27 & 174 & 0.5 \\
\hline $1 a$ & $\mathrm{~B} 65$ & 405 & 55 & 0.14 & 102 & 0.3 \\
\hline $1 a$ & cow & 486 & 1462 & 3.01 & 1609 & 3.3 \\
\hline $1 a$ & C & 308 & 242 & 0.79 & 452 & 1.5 \\
\hline $1 a$ & B3 & 329 & 174 & 0.53 & 325 & 1.0 \\
\hline $1 a$ & WS & 313 & 632 & 2.02 & 1180 & 3.8 \\
\hline $2 a$ & E44 & 94 & 30 & 0.32 & 36 & 0.4 \\
\hline $2 a$ & W48 & 47 & 96 & 2.06 & 189 & 4.1 \\
\hline $2 a$ & W55 & 813 & 91 & 0.11 & 1979 & 2.4 \\
\hline $2 a$ & $\mathrm{MO}$ & 75 & 43 & 0.57 & 80 & 1.1 \\
\hline $2 a$ & B65 & 210 & 741 & 3.53 & 47 & 0.2 \\
\hline $2 a$ & CPK & 86 & 30 & 0.35 & 53 & 0.6 \\
\hline $2 a$ & CS & 156 & 30 & 0.19 & 30 & 0.2 \\
\hline $3 a$ & W35 & 110 & 30 & 0.28 & 30 & 0.3 \\
\hline $3 a$ & W48 & 115 & 108 & 0.94 & 30 & 0.3 \\
\hline $3 a$ & W55 & 123 & 30 & 0.25 & 30 & 0.2 \\
\hline $3 a$ & $\mathrm{MO}$ & 77 & 30 & 0.39 & 35 & 0.5 \\
\hline $3 a$ & B65 & 35 & 30 & 0.86 & 30 & 0.9 \\
\hline $3 a$ & CPK & 48 & 30 & 0.63 & 30 & 0.6 \\
\hline $3 a$ & B3 & 72 & 30 & 0.42 & 30 & 0.4 \\
\hline $4 a$ & W35 & 528 & 213 & 0.40 & 245 & 0.5 \\
\hline $4 a$ & W48 & 744 & 229 & 0.31 & 384 & 0.5 \\
\hline $4 a$ & W55 & 349 & 222 & 0.64 & 273 & 0.8 \\
\hline $4 a$ & $\mathrm{MO}$ & 222 & 56 & 0.25 & 105 & 0.5 \\
\hline $4 a$ & B65 & 210 & 56 & 0.27 & 110 & 0.5 \\
\hline $4 a$ & CPK & 90 & 30 & 0.34 & 49 & 0.5 \\
\hline $4 a$ & $\mathrm{CS}$ & 256 & 30 & 0.12 & 30 & 0.1 \\
\hline $4 a$ & $\mathrm{C}$ & 222 & 56 & 0.25 & 105 & 0.5 \\
\hline $4 a$ & B3 & 210 & 56 & 0.27 & 110 & 0.5 \\
\hline $4 a$ & $\mathrm{C} 2$ & 90 & 30 & 0.34 & 49 & 0.5 \\
\hline $4 a$ & $\mathrm{E} 2$ & 256 & 30 & 0.12 & 30 & 0.1 \\
\hline $2 b$ & CP1 & 30 & 64 & 2.11 & 135 & 4.5 \\
\hline $2 b$ & E2 & 85 & 288 & 3.37 & 200 & 2.3 \\
\hline $2 b$ & W & 78 & 517 & 6.66 & 118 & 1.5 \\
\hline $2 b$ & $C$ & 82 & 562 & 6.89 & 541 & 6.6 \\
\hline $2 b$ & B3 & 30 & 1144 & 37.81 & 234 & 7.7 \\
\hline $2 b$ & B1 & 30 & 30 & 1.00 & 123 & 4.1 \\
\hline $2 b$ & WA1 & 163 & 838 & 5.15 & 435 & 2.7 \\
\hline $2 b$ & $\mathrm{BC}$ & 30 & 232 & 7.68 & 92 & 3.0 \\
\hline
\end{tabular}




\begin{tabular}{|c|c|c|c|c|c|c|}
\hline \multirow[b]{2}{*}{ IOP } & \multirow[b]{2}{*}{ Site Label } & \multirow{2}{*}{$\begin{array}{c}\text { Measured } \\
\text { PEM BC } \\
\left(\mathrm{ng} / \mathrm{m}^{3}\right)\end{array}$} & \multicolumn{2}{|c|}{ ISC-PRIME } & \multicolumn{2}{|r|}{ ISCST3 } \\
\hline & & & $B C\left(\mathrm{ng} / \mathrm{m}^{3}\right)$ & $\begin{array}{l}\text { modeled/measured } \\
\text { BC }\end{array}$ & $B C\left(\mathrm{ng} / \mathrm{m}^{3}\right)$ & $\begin{array}{c}\text { modeled/measured } \\
\text { BC }\end{array}$ \\
\hline $2 b$ & EK & 30 & 31 & 1.04 & 70 & 2.3 \\
\hline $2 b$ & $\mathrm{~K}$ & 30 & 30 & 1.00 & 30 & 1.0 \\
\hline $2 b$ & S1 & 309 & 99 & 0.32 & 123 & 0.4 \\
\hline $3 b$ & E2 & 237 & 340 & 1.43 & 152 & 0.6 \\
\hline $3 b$ & W & 116 & 461 & 3.96 & 331 & 2.8 \\
\hline $3 b$ & $\mathrm{D}$ & 542 & 539 & 0.99 & 734 & 1.4 \\
\hline $3 b$ & E1 & 193 & 876 & 4.54 & 903 & 4.7 \\
\hline $3 b$ & $\mathrm{C}$ & 188 & 898 & 4.78 & 1087 & 5.8 \\
\hline $3 b$ & B3 & 30 & 1162 & 38.41 & 650 & 21.5 \\
\hline $3 b$ & B4 & 137 & 790 & 5.76 & 842 & 6.1 \\
\hline $3 b$ & $\mathrm{CP} 1$ & 30 & 260 & 8.60 & 589 & 19.5 \\
\hline $3 b$ & B1 & 239 & 338 & 1.42 & 1517 & 6.3 \\
\hline $3 b$ & B2 & 125 & 109 & 0.87 & 204 & 1.6 \\
\hline $3 b$ & $\mathrm{~A}$ & 174 & 521 & 3.00 & 1056 & 6.1 \\
\hline $3 b$ & AW & 451 & 1510 & 3.35 & 721 & 1.6 \\
\hline $3 b$ & $B C$ & 202 & 377 & 1.87 & 488 & 2.4 \\
\hline $3 b$ & EK & 30 & 311 & 10.28 & 539 & 17.8 \\
\hline $3 b$ & $\mathrm{~K}$ & 30 & 30 & 1.00 & 32 & 1.1 \\
\hline $3 b$ & S1 & 525 & 134 & 0.26 & 62 & 0.1 \\
\hline $4 b$ & E2 & 1048 & 63 & 0.06 & 40 & 0.0 \\
\hline $4 b$ & W & 396 & 863 & 2.18 & 340 & 0.9 \\
\hline $4 b$ & $\mathrm{D}$ & 374 & 190 & 0.51 & 574 & 1.5 \\
\hline $4 b$ & E1 & 281 & 369 & 1.31 & 237 & 0.8 \\
\hline $4 b$ & $C$ & 326 & 191 & 0.59 & 335 & 1.0 \\
\hline $4 b$ & B3 & 210 & 215 & 1.02 & 401 & 1.9 \\
\hline $4 b$ & B4 & 222 & 209 & 0.94 & 504 & 2.3 \\
\hline $4 b$ & $\mathrm{CP}$ & 221 & 145 & 0.66 & 270 & 1.2 \\
\hline $4 b$ & B2 & 293 & 82 & 0.28 & 152 & 0.5 \\
\hline $4 b$ & A & 268 & 204 & 0.76 & 397 & 1.5 \\
\hline $4 b$ & AW & 285 & 122 & 0.43 & 269 & 0.9 \\
\hline $4 b$ & $\mathrm{BC}$ & 197 & 212 & 1.08 & 329 & 1.7 \\
\hline $4 b$ & EK & 198 & 157 & 0.79 & 183 & 0.9 \\
\hline $4 b$ & $\mathrm{~K}$ & 189 & 60 & 0.32 & 111 & 0.6 \\
\hline $4 b$ & S1 & 448 & 30 & 0.07 & 30 & 0.1 \\
\hline $5 b$ & E2 & 2788 & 367 & 0.13 & 30 & 0.0 \\
\hline $5 b$ & W & 252 & 585 & 2.32 & 562 & 2.2 \\
\hline $5 b$ & $\mathrm{D}$ & 294 & 266 & 0.91 & 703 & 2.4 \\
\hline $5 b$ & $C$ & 283 & 267 & 0.94 & 445 & 1.6 \\
\hline $5 b$ & B3 & 273 & 57 & 0.21 & 106 & 0.4 \\
\hline $5 b$ & $\mathrm{CP}$ & 318 & 50 & 0.16 & 94 & 0.3 \\
\hline $5 b$ & B1 & 191 & 143 & 0.75 & 266 & 1.4 \\
\hline $5 b$ & B2 & 270 & 30 & 0.11 & 30 & 0.1 \\
\hline $5 b$ & $A$ & 286 & 346 & 1.21 & 234 & 0.8 \\
\hline $5 b$ & AW & 308 & 795 & 2.58 & 439 & 1.4 \\
\hline $5 b$ & $\mathrm{BC}$ & 216 & 158 & 0.73 & 296 & 1.4 \\
\hline $5 b$ & FS & 263 & 203 & 0.77 & 326 & 1.2 \\
\hline $5 b$ & EK & 265 & 61 & 0.23 & 113 & 0.4 \\
\hline $5 b$ & $\mathrm{~K}$ & 194 & 30 & 0.16 & 30 & 0.2 \\
\hline $5 b$ & S1 & 415 & 46 & 0.11 & 30 & 0.1 \\
\hline
\end{tabular}




\begin{tabular}{|c|c|c|c|c|c|c|}
\hline \multirow[b]{2}{*}{ IOP } & \multirow[b]{2}{*}{ Site Label } & \multirow{2}{*}{$\begin{array}{c}\text { Measured } \\
\text { PEM BC } \\
\left(\mathrm{ng} / \mathrm{m}^{3}\right)\end{array}$} & \multicolumn{2}{|c|}{ ISC-PRIME } & \multicolumn{2}{|r|}{ ISCST3 } \\
\hline & & & $\mathrm{BC}\left(\mathrm{ng} / \mathrm{m}^{3}\right)$ & $\begin{array}{c}\text { modeled/measured } \\
\text { BC }\end{array}$ & $\mathrm{BC}\left(\mathrm{ng} / \mathrm{m}^{3}\right)$ & $\begin{array}{c}\text { modeled/measured } \\
\text { BC }\end{array}$ \\
\hline $6 \mathrm{~b}$ & E2 & 30 & 36 & 1.18 & 63 & 2.1 \\
\hline $6 b$ & W & 192 & 544 & 2.83 & 148 & 0.8 \\
\hline $6 b$ & $\mathrm{D}$ & 339 & 133 & 0.39 & 238 & 0.7 \\
\hline $6 b$ & $\mathrm{C}$ & 185 & 222 & 1.20 & 326 & 1.8 \\
\hline $6 b$ & B3 & 153 & 134 & 0.88 & 220 & 1.4 \\
\hline $6 b$ & B4 & 30 & 589 & 19.48 & 894 & 29.5 \\
\hline $6 \mathrm{~b}$ & $\mathrm{~B} 1$ & 123 & 30 & 0.25 & 30 & 0.2 \\
\hline $6 b$ & A & 370 & 197 & 0.53 & 357 & 1.0 \\
\hline $6 b$ & AW & 693 & 830 & 1.20 & 895 & 1.3 \\
\hline $6 b$ & $\mathrm{BC}$ & 413 & 116 & 0.28 & 142 & 0.3 \\
\hline $6 b$ & FS & 164 & 410 & 2.50 & 888 & 5.4 \\
\hline $6 b$ & EK & 81 & 53 & 0.65 & 105 & 1.3 \\
\hline $6 b$ & $\mathrm{~K}$ & 59 & 30 & 0.51 & 50 & 0.9 \\
\hline $6 \mathrm{~b}$ & S1 & 247 & 30 & 0.12 & 30 & 0.1 \\
\hline $7 b$ & E2 & 125 & 222 & 1.77 & 134 & 1.1 \\
\hline $7 b$ & W & 503 & 686 & 1.36 & 380 & 0.8 \\
\hline $7 b$ & $\mathrm{D}$ & 1051 & 789 & 0.75 & 359 & 0.3 \\
\hline $7 b$ & C & 159 & 405 & 2.55 & 543 & 3.4 \\
\hline $7 b$ & B4 & 136 & 44 & 0.32 & 82 & 0.6 \\
\hline $7 b$ & $\mathrm{CP}$ & 188 & 65 & 0.34 & 121 & 0.6 \\
\hline $7 b$ & B1 & 145 & 33 & 0.22 & 61 & 0.4 \\
\hline $7 b$ & B2 & 115 & 30 & 0.26 & 30 & 0.3 \\
\hline $7 b$ & A & 270 & 535 & 1.98 & 1893 & 7.0 \\
\hline $7 b$ & AW & 453 & 916 & 2.02 & 3252 & 7.2 \\
\hline $7 b$ & $B C$ & 146 & 35 & 0.24 & 138 & 0.9 \\
\hline $7 b$ & FS & 184 & 267 & 1.45 & 471 & 2.6 \\
\hline $7 b$ & EK & 101 & 30 & 0.30 & 30 & 0.3 \\
\hline $7 b$ & $\mathrm{~K}$ & 115 & 30 & 0.26 & 30 & 0.3 \\
\hline $7 b$ & S1 & 386 & 44 & 0.11 & 38 & 0.1 \\
\hline $8 b$ & E2 & 156 & 30 & 0.19 & 30 & 0.2 \\
\hline $8 b$ & W & 142 & 211 & 1.48 & 92 & 0.6 \\
\hline $8 b$ & $\mathrm{C}$ & 154 & 47 & 0.31 & 88 & 0.6 \\
\hline $8 b$ & B3 & 119 & 147 & 1.23 & 301 & 2.5 \\
\hline $8 b$ & B4 & 277 & 290 & 1.04 & 540 & 1.9 \\
\hline $8 b$ & $\mathrm{CP}$ & 130 & 129 & 0.99 & 250 & 1.9 \\
\hline $8 b$ & B1 & 158 & 30 & 0.19 & 30 & 0.2 \\
\hline $8 b$ & B2 & 137 & 418 & 3.05 & 2429 & 17.7 \\
\hline $8 b$ & A & 561 & 30 & 0.05 & 30 & 0.1 \\
\hline $8 b$ & AW & 176 & 845 & 4.81 & 816 & 4.6 \\
\hline $8 b$ & $\mathrm{BC}$ & 139 & 30 & 0.22 & 30 & 0.2 \\
\hline $8 b$ & $\mathrm{FS}$ & 162 & 320 & 1.97 & 606 & 3.7 \\
\hline $8 b$ & EK & 120 & 30 & 0.25 & 30 & 0.3 \\
\hline $8 b$ & $\mathrm{~K}$ & 149 & 49 & 0.33 & 106 & 0.7 \\
\hline $8 b$ & $\mathrm{~S} 1$ & 229 & 30 & 0.13 & 30 & 0.1 \\
\hline $9 b$ & E2 & 120 & 272 & 2.26 & 30 & 0.3 \\
\hline $9 b$ & W & 169 & 276 & 1.64 & 30 & 0.2 \\
\hline $9 \mathrm{~b}$ & $\mathrm{C}$ & 102 & 387 & 3.80 & 195 & 1.9 \\
\hline $9 b$ & B3 & 137 & 673 & 4.92 & 44 & 0.3 \\
\hline $9 \mathrm{~b}$ & B4 & 92 & 529 & 5.75 & 466 & 5.1 \\
\hline
\end{tabular}




\begin{tabular}{|c|c|c|c|c|c|c|}
\hline \multirow[b]{2}{*}{ IOP } & \multirow[b]{2}{*}{ Site Label } & \multirow{2}{*}{$\begin{array}{c}\text { Measured } \\
\text { PEM BC } \\
\left(\mathrm{ng} / \mathrm{m}^{3}\right)\end{array}$} & \multicolumn{2}{|c|}{ ISC-PRIME } & \multicolumn{2}{|r|}{ ISCST3 } \\
\hline & & & $B C\left(\mathrm{ng} / \mathrm{m}^{3}\right)$ & $\begin{array}{l}\text { modeled/measured } \\
\text { BC }\end{array}$ & $B C\left(\mathrm{ng} / \mathrm{m}^{3}\right)$ & $\begin{array}{c}\text { modeled/measured } \\
\text { BC }\end{array}$ \\
\hline $9 b$ & $\mathrm{CP}$ & 110 & 154 & 1.40 & 66 & 0.6 \\
\hline $9 b$ & B1 & 119 & 53 & 0.45 & 67 & 0.6 \\
\hline $9 b$ & $\mathrm{~B} 2$ & 112 & 110 & 0.98 & 181 & 1.6 \\
\hline $9 b$ & $A$ & 119 & 112 & 0.94 & 355 & 3.0 \\
\hline $9 b$ & AW & 280 & 890 & 3.18 & 84 & 0.3 \\
\hline $9 b$ & $\mathrm{BC}$ & 100 & 58 & 0.58 & 30 & 0.3 \\
\hline $9 b$ & FS & 105 & 167 & 1.58 & 80 & 0.8 \\
\hline $9 b$ & EK & 133 & 33 & 0.24 & 65 & 0.5 \\
\hline $9 b$ & $\mathrm{~K}$ & 148 & 48 & 0.32 & 30 & 0.2 \\
\hline $9 b$ & S1 & 192 & 30 & 0.16 & 30 & 0.2 \\
\hline $10 \mathrm{~b}$ & E2 & 92 & 303 & 3.30 & 186 & 2.0 \\
\hline $10 \mathrm{~b}$ & W & 30 & 309 & 10.22 & 197 & 6.5 \\
\hline $10 \mathrm{~b}$ & $\mathrm{C}$ & 94 & 295 & 3.15 & 427 & 4.6 \\
\hline $10 \mathrm{~b}$ & B3 & 30 & 620 & 20.49 & 499 & 16.5 \\
\hline $10 \mathrm{~b}$ & B4 & 30 & 581 & 19.20 & 493 & 16.3 \\
\hline $10 \mathrm{~b}$ & $\mathrm{CP}$ & 81 & 356 & 4.37 & 714 & 8.8 \\
\hline $10 \mathrm{~b}$ & B1 & 30 & 204 & 6.73 & 136 & 4.5 \\
\hline $10 \mathrm{~b}$ & B2 & 101 & 506 & 5.02 & 2494 & 24.7 \\
\hline $10 \mathrm{~b}$ & $A$ & 203 & 246 & 1.21 & 451 & 2.2 \\
\hline $10 \mathrm{~b}$ & AW & 30 & 271 & 8.95 & 423 & 14.0 \\
\hline $10 \mathrm{~b}$ & $\mathrm{BC}$ & 83 & 405 & 4.88 & 1035 & 12.5 \\
\hline $10 \mathrm{~b}$ & $\mathrm{FS}$ & 104 & 390 & 3.75 & 631 & 6.1 \\
\hline $10 \mathrm{~b}$ & EK & 94 & 184 & 1.96 & 579 & 6.2 \\
\hline $10 \mathrm{~b}$ & $\mathrm{~K}$ & 88 & 198 & 2.26 & 608 & 6.9 \\
\hline $10 \mathrm{~b}$ & S1 & 117 & 62 & 0.53 & 76 & 0.6 \\
\hline
\end{tabular}

